\title{
Centres of centralizers of unipotent elements in simple algebraic groups
}

\author{
R. Lawther and D.M. Testerman
}

September 15, 2008

\begin{abstract}
The first author is grateful to EPFL for its hospitality and support while this work was carried out.

The second author was supported by the Swiss National Science Foundation grant number PP002-68710/1.
\end{abstract}

\section{Contents}

1 Introduction 2

$\begin{array}{llr}2 & \text { Notation and preliminary results } & 6\end{array}$

3 Reduction of the problem $\quad 11$

4 Classical groups $\quad 14$

5 Exceptional groups: nilpotent orbit representatives 21

6 Associated cocharacters 32

$\begin{array}{llr}7 & \text { The connected centralizer } & 34\end{array}$

8 A composition series for the Lie algebra centralizer 41

$\begin{array}{lll}9 & \text { The Lie algebra of the centre of the centralizer } & 47\end{array}$

$\begin{array}{ll}10 \text { Proofs of the main theorems for exceptional groups } & 65\end{array}$

$\begin{array}{ll}11 \text { Detailed results } & 71\end{array}$

$\begin{array}{lc}\text { References } & 183\end{array}$ 


\section{Introduction}

Let $G$ be a simple algebraic group defined over an algebraically closed field $k$ whose characteristic is either 0 or a good prime for $G$, and let $u \in G$ be unipotent. We study the centralizer $C_{G}(u)$, especially its centre $Z\left(C_{G}(u)\right)$. We calculate the Lie algebra of $Z\left(C_{G}(u)\right)$, in particular determining its dimension; in the case where $G$ is of exceptional type we find the upper central series of the Lie algebra of $R_{u}\left(C_{G}(u)\right)$, writing each term explicitly as a direct sum of indecomposable tilting modules for a reductive complement to $R_{u}\left(C_{G}(u)\right)$ in $C_{G}(u)^{\circ}$.

Work on $C_{G}(u)$ dates back to 1966, when Springer showed in [44] that if $u$ is regular then $C_{G}(u)^{\circ}$ is abelian. Subsequently, Kurtzke established the converse of this result in [17], in the case where $\operatorname{char}(k)$ is either 0 or a good prime for $G$. Further study of $C_{G}(u)$ in the case where $G$ is of exceptional type was undertaken by Chang in [7] and Stuhler in [48] for $G$ of type $G_{2}$, Shinoda in [40] and Shoji in [41] for $G$ of type $F_{4}$, and Mizuno in [27, 28] for $G$ of type $E_{6}, E_{7}$ and $E_{8}$. In more recent work, Sommers in [43], McNinch and Sommers in [25] and Premet in [31] have obtained results on the component group $C_{G}(u) / C_{G}(u)^{\circ}$, while Liebeck and Seitz in [21] have a new approach to the classification of unipotent and nilpotent orbits.

Our interest in $Z\left(C_{G}(u)\right)$ is motivated by the desire to embed $u$ in a connected abelian unipotent subgroup of $G$ satisfying certain uniqueness properties. In [38], Seitz considered this question in the case where either $\operatorname{char}(k)=0$, or $\operatorname{char}(k)$ is a good prime $p$ for $G$ and $u$ has order $p$; he constructed a 1-dimensional connected subgroup $U$ of $G$, intrinsically associated to $u$, such that $u \in U$ and the centralizers in $G$ of $u$, the subgroup $U$ and the Lie algebra of $U$ all coincide. In [34], Proud showed that if $\operatorname{char}(k)$ is a good prime $p$ for $G$, and $u$ has order $p^{t}$, then there exists a closed connected $t$-dimensional abelian unipotent subgroup containing $u$; however the subgroup satisfies no uniqueness properties. A natural candidate for a canonically defined abelian overgroup of a unipotent element $u$ is $Z\left(C_{G}(u)\right)$. In [35], Proud turned to the study of $Z\left(C_{G}(u)\right)$, and in particular proved that if $\operatorname{char}(k)$ is either 0 or a good prime for $G$ then $Z\left(C_{G}(u)\right)^{\circ}$ is unipotent. Seitz carried this further in [39], showing that $Z\left(C_{G}(u)\right)^{\circ}$ has a decomposition into Witt vector groups such that $u$ is contained in one (not uniquely determined) factor; he pointed out that while $Z\left(C_{G}(u)\right)^{\circ}$ is 'of considerable interest ... even the dimension of this subgroup remains a mystery.' We attempt to shed some light upon this mystery here.

Using the existence of a $G$-equivariant homeomorphism between the varieties of unipotent elements of $G$ and nilpotent elements of its Lie algebra (see Theorem 2.2), we replace $u$ by a nilpotent element $e$ and subsequently study $C_{G}(e)$ and $Z\left(C_{G}(e)\right)$; our findings will then equally apply to $C_{G}(u)$ and $Z\left(C_{G}(u)\right)$. We begin by proving some preliminary results valid for all $G$. After dealing fairly quickly with the classical groups, we move on to consider the exceptional groups. For each of these we obtain a set of representatives of the non-zero nilpotent orbits, and proceed with a case-by-case analysis of their centralizers: for each orbit representative $e$ we find a basis for the Lie algebra of $R_{u}\left(C_{G}(e)\right)$ and generators for a reductive complement to $R_{u}\left(C_{G}(e)\right)$ in $C_{G}(e)$.

In order to state the theorems which are direct consequences of our findings, we need to introduce some notation and terminology. Throughout this work we will write $\mathfrak{L}(H)$ for the Lie algebra of an algebraic group $H$. To avoid repetition in the statements of the theorems which follow, we shall say that the pair $(G, e)$ satisfies Hypothesis $(H)$ if

$G$ is a simple algebraic group defined over an algebraically closed field $k$ whose characteristic is either 0 or a good prime for $G$, and $e \in \mathfrak{L}(G)$ is nilpotent.

Given such a pair $(G, e)$, we take a cocharacter $\tau: k^{*} \rightarrow G$ associated to $e$ (see Defini- 
tion 2.6); any two such cocharacters are conjugate by an element of $C_{G}(e)$. From $\tau$ one obtains a unique labelling of the Dynkin diagram of $G$, with labels taken from the set $\{0,1,2\}$, which determines the set of $\tau$-weights on $\mathfrak{L}(G)$ with multiplicities (see $\S 3$ ); the corresponding labelled diagram $\Delta$ is that attached to the $G$-orbit of $e$ in the Bala-CarterPommerening classification of nilpotent orbits in $\mathfrak{L}(G)$. We write $n_{2}(\Delta)$ for the number of labels in $\Delta$ which are equal to 2 .

Note that in two of the results below we shall use dots in labelled diagrams to denote an arbitrary number of unspecified labels, where the underlying group is of type $D_{\ell}$ in both cases; thus $\ldots{ }_{2}^{2}$ will mean any labelled diagram in which the labels of the last two nodes are 2 , and $1 \ldots 1$ will mean one in which the labels of the three endnodes are 1.

Our first result concerns the case where $e$ is distinguished in $\mathfrak{L}(G)$ (see Definition 2.5); we determine $\operatorname{dim} Z\left(C_{G}(e)\right)$ and the action of $\operatorname{im}(\tau)$ on $\mathfrak{L}\left(Z\left(C_{G}(e)\right)\right)$. Note that we may regard $\tau$-weights as integers.

Theorem 1 Let $(G, e)$ satisfy Hypothesis $(H)$, with associated cocharacter $\tau$ and labelled diagram $\Delta$. Let $\ell$ be the rank of $G$, and write $d_{1}, \ldots, d_{\ell}$ for the degrees of the invariant polynomials of the Weyl group of $G$, ordered such that $d_{\ell}$ is $\ell$ if $G$ is of type $D_{\ell}$ and is $\max \left\{d_{i}\right\}$ otherwise, and $d_{i}<d_{j}$ if $i<j<\ell$. Assume $e$ is distinguished in $\mathfrak{L}(G)$. Then

(i) $\operatorname{dim} Z\left(C_{G}(e)\right)=n_{2}(\Delta)=\operatorname{dim} Z\left(C_{G}(\operatorname{im}(\tau))\right)$; and

(ii) the $\tau$-weights on $\mathfrak{L}\left(Z\left(C_{G}(e)\right)\right)$ are the $n_{2}(\Delta)$ integers $2 d_{i}-2$ for $i \in S_{\Delta}$, where

$$
S_{\Delta}= \begin{cases}\left\{1, \ldots, n_{2}(\Delta)-1, \ell\right\} & \text { if } G \text { is of type } D_{\ell} \text { and } \Delta=\ldots{ }_{2}^{2} ; \\ \left\{1, \ldots, n_{2}(\Delta)-1, n_{2}(\Delta)\right\} & \text { otherwise. }\end{cases}
$$

Leaving aside the observation about the action of $\operatorname{im}(\tau)$, we may generalize to the case of nilpotent elements whose cocharacters have labelled diagrams with only even labels.

Theorem 2 Let $(G, e)$ satisfy Hypothesis $(H)$, with associated cocharacter $\tau$ and labelled diagram $\Delta$. Assume $\Delta$ has no label equal to 1 . Then

$$
\operatorname{dim} Z\left(C_{G}(e)\right)=n_{2}(\Delta)=\operatorname{dim} Z\left(C_{G}(\operatorname{im}(\tau))\right) .
$$

Theorem 2 is subsumed by a more general result, the statement of which requires the following. Given a labelled diagram $\Delta$ for the group $G$, we define the 2-free core of $\Delta$ to be the sub-labelled diagram $\Delta_{0}$ obtained by removing from $\Delta$ all labels equal to 2 , together with the corresponding nodes. We let $G_{0}$ be a semisimple algebraic group (of any isogeny type) defined over $k$ whose Dynkin diagram is the underlying diagram of $\Delta_{0}$; thus rank $G_{0}=\operatorname{rank} G-n_{2}(\Delta)$. Note that the assumption that the pair $(G, e)$ satisfies Hypothesis $(\mathrm{H})$ implies that if $\operatorname{char}(k)$ is positive, it is a good prime for $G_{0}$; thus the Bala-Carter-Pommerening classification of nilpotent orbits applies to $\mathfrak{L}\left(G_{0}\right)$, and there is a bijection between the set of nilpotent orbits in $\mathfrak{L}\left(G_{0}\right)$ and the set of labelled diagrams for $G_{0}$ which are unions of labelled diagrams for the simple factors.

Theorem 3 Let $(G, e)$ satisfy Hypothesis $(H)$, with associated cocharacter $\tau$ and labelled diagram $\Delta$. Let $\Delta_{0}$ be the 2 -free core of $\Delta$, with corresponding algebraic group $G_{0}$. Then there exists a nilpotent $G_{0}$-orbit in $\mathfrak{L}\left(G_{0}\right)$ having labelled diagram $\Delta_{0}$. Let $e_{0} \in \mathfrak{L}\left(G_{0}\right)$ be a representative of this orbit. Then

(i) $\operatorname{dim} C_{G}(e)-\operatorname{dim} C_{G_{0}}\left(e_{0}\right)=n_{2}(\Delta)$; and

(ii) $\operatorname{dim} Z\left(C_{G}(e)\right)-\operatorname{dim} Z\left(C_{G_{0}}\left(e_{0}\right)\right)=n_{2}(\Delta)$. 
In fact Theorem 3(ii) follows immediately from the following more general result.

Theorem 4 Let $(G, e)$ satisfy Hypothesis $(H)$, with associated cocharacter $\tau$ and labelled diagram $\Delta$ having labels $a_{1}, \ldots, a_{\ell}$. Then

$$
\operatorname{dim} Z\left(C_{G}(e)\right)=\left\lceil\frac{1}{2} \sum a_{j}\right\rceil+\epsilon, \quad \text { where } \epsilon \in\{0, \pm 1\} .
$$

Moreover the value of $\epsilon$ may be explicitly described as follows. Let $\Delta_{0}$ be the 2 -free core of $\Delta$, with corresponding algebraic group $G_{0}$. Then, provided $\Delta_{0}$ is not the empty diagram, there exists a connected component $\Gamma_{0}$ of $\Delta_{0}$ such that all labels in $\Delta_{0} \backslash \Gamma_{0}$ are 0 . Let $H_{0}$ be the corresponding simple factor of $G_{0}$. Then $\epsilon=0$ with the following exceptions.

$$
\epsilon=1: \begin{array}{c|c}
H_{0} & \Gamma_{0} \\
\hline F_{4} & 1010 \\
E_{7} & 101000,001010 \\
E_{8} & 0000101, \underset{0}{0100001}, \underset{0}{01000100,0010100}
\end{array}
$$

$$
\epsilon=-1: \begin{array}{c|c}
H_{0} & \Gamma_{0} \\
\hline D_{\ell} & 1 \cdots 1 \\
E_{6} & 10101,11011 \\
0 & 101010 \\
E_{7} & 0 \\
E_{8} & 0 \\
1000101, \underset{0}{1010100}
\end{array}
$$

In fact the cases in Theorem 4 where $\epsilon=1$ may be described combinatorially as follows. For a simple algebraic group $H_{0}$ with simple roots $\alpha_{1}, \ldots, \alpha_{\ell}$, write the highest root as $\sum n_{i} \alpha_{i}$; given a labelled diagram $\Gamma_{0}$ for $H_{0}$ with labels $a_{1}, \ldots, a_{\ell} \in\{0,1\}$, we have $\epsilon=1$ precisely if $\left\{i: a_{i}=1\right\}=\left\{j_{1}, j_{2}\right\}$, where $n_{j_{1}}$ and $n_{j_{2}}$ are even and differ by 2 . It seems however to be harder to describe similarly the cases where $\epsilon=-1$.

The information presented here in the case where $G$ is of exceptional type includes an explicit decomposition of $\mathfrak{L}\left(R_{u}\left(C_{G}(e)\right)\right)$ as a direct sum of indecomposable tilting modules for a reductive complement to $R_{u}\left(C_{G}(e)\right)$ in $C_{G}(e)^{\circ}$. We mention here an additional potential application of these results. Consider the case where $k=\mathbb{C}$, and fix an $\mathfrak{s l}_{2}$ triple $(e, h, f)$ in $\mathfrak{L}(G)$. Let $($,$) be a G$-invariant bilinear form on $\mathfrak{L}(G)$ with $(e, f)=1$, and define $\chi \in \mathfrak{L}(G)^{*}$ by setting $\chi(x)=(e, x)$ for all $x \in \mathfrak{L}(G)$. Let $Q_{\chi}$ be the generalized Gelfand-Graev module for the universal enveloping algebra $U(\mathfrak{L}(G))$ associated with the triple $(e, h, f)$ (see [32]); set $H_{\chi}=\operatorname{End}_{\mathfrak{L}(G)}\left(Q_{\chi}\right)^{\text {op }}$. The interest in the algebra $H_{\chi}$ arises in part from its connection with quantizations of certain transverse slices of the nilpotent cone $\mathcal{N}$ of $\mathfrak{L}(G)$. Set $S=e+\operatorname{ker}(\operatorname{ad} f)$, a so-called Slodowy slice to the adjoint orbit $\operatorname{Ad}(G) e$, as in [42]. In [32] Premet shows that, for each algebra homomorphism $\eta: Z\left(H_{\chi}\right) \rightarrow \mathbb{C}$, the algebra $H_{\chi} \otimes_{Z\left(H_{\chi}\right)} \mathbb{C}_{\eta}$ (where $\mathbb{C}_{\eta}$ is the 1-dimensional $Z\left(H_{\chi}\right)$-module induced by $\eta)$ is a quantization of $\mathbb{C}[\mathcal{N} \cap S]$. Moreover, in [33] he considers the case where $e$ is a long root element and gives an explicit presentation of $H_{\chi}$ by generators and relations; he further applies this to study the representation theory of $H_{\chi}$. His calculations use knowledge of the action on $\mathfrak{L}\left(R_{u}\left(C_{G}(e)\right)\right)$ of a reductive complement to $R_{u}\left(C_{G}(e)\right)$ in $C_{G}(e)^{\circ}$. One can hope to treat other nilpotent orbits in a similar fashion.

The remainder of the present work is organized as follows. In $\S 2$ we fix notation and recall results from the literature which will be necessary in what follows. In $\S 3$ we first prove those parts of the main theorems stated here which require no case analysis, and then establish a structural result which, for $e \in \mathfrak{L}(G)$ nilpotent, reduces the determination of $\mathfrak{L}\left(Z\left(C_{G}(e)\right)\right)$ to that of the fixed points of a certain reductive group acting on a certain subalgebra of $\mathfrak{L}(G)$; the identification of this subalgebra is a lengthy but tractable calculation. In $\S 4$ we prove our main theorems for groups $G$ of classical type. In the remainder of the work we therefore restrict our attention to groups $G$ of exceptional type. In $\S 5$ we establish a list of non-zero nilpotent orbit representatives in $\mathfrak{L}(G)$, and thereafter work only with such elements $e$. In $\S 6$ we fix a cocharacter associated to $e$. 
In $\S 7$ we treat the connected centralizer of $e$; we begin with its unipotent radical, whose Lie algebra we determine explicitly, and then exhibit a reductive complement. In $\S 8$ we describe the action of this reductive complement on $\mathfrak{L}\left(C_{G}(e)\right)$. In $\S 9$ we apply the results of the preceding sections, and consider the action of the full centralizer in those cases where it is not connected, to obtain a basis of the Lie subalgebra $\mathfrak{L}\left(Z\left(C_{G}(e)\right)\right)$. In $\S 10$ we provide tables summarizing the results obtained, and prove our main theorems for groups of exceptional type. Finally, in $\S 11$ we describe and present our detailed information for each non-zero nilpotent orbit representative $e$.

The authors are particularly grateful to Professor J.-P. Serre for his close reading of parts of this work and his pertinent comments thereon, which have been most helpful. 


\section{Notation and preliminary results}

In this section, we fix notation which will be used throughout, and recall definitions and results from the literature which play an essential role in what follows.

Let $G$ be a simple algebraic group defined over an algebraically closed field $k$. Fix a maximal torus $T$ of $G$ and write $\Phi=\Phi(G)$ for the root system of $G$ with respect to $T$. Write $\operatorname{dim} T=\ell$, and fix a set of simple roots $\Pi=\Pi(G)=\left\{\alpha_{1}, \ldots, \alpha_{\ell}\right\}$, where we number the roots in the associated Dynkin diagram as in [4]; let $\Phi^{+}$and $\Phi^{-}$be the sets of positive and negative roots determined by $\Pi$. We shall write roots as linear combinations of simple roots, and represent them as $\ell$-tuples of coefficients arranged as in a Dynkin diagram; thus for example if $G$ is of type $E_{8}$ the high root is denoted ${ }_{3}^{2465432}$.

Let $W=N_{G}(T) / T$ be the Weyl group of $G$ with respect to $T$; for $\alpha \in \Phi$, write $w_{\alpha} \in W$ for the reflection associated to $\alpha$, and let $U_{\alpha}$ be the $T$-root subgroup corresponding to $\alpha$. A closed connected semisimple subgroup of $G$ generated by $T$-root subgroups will be called a subsystem subgroup. Note that if $S$ is a torus in $G$, then $C_{G}(S)$ is the Levi factor of a parabolic subgroup of $G$, and the derived group $\left[C_{G}(S), C_{G}(S)\right]$ is a subsystem subgroup. Throughout this work we shall call a Levi factor of a parabolic subgroup of $G$ a Levi subgroup of $G$; as is well known, if $L$ is a Levi subgroup of $G$ then $C_{G}(Z(L))=L$.

By the Chevalley construction $([8])$, there exists a basis $\mathcal{B}$ of $\mathfrak{L}(G)$ of the form $\mathcal{B}=\left\{e_{\alpha}\right.$ : $\alpha \in \Phi\} \cup\left\{h_{\alpha_{i}}: 1 \leq i \leq \ell\right\}$, where $\mathfrak{L}\left(U_{\alpha}\right)=\left\langle e_{\alpha}\right\rangle$ for each $\alpha \in \Phi, \mathfrak{L}(T)=\left\langle h_{\alpha_{i}}: 1 \leq i \leq \ell\right\rangle$, and the structure constants of $\mathfrak{L}(G)$ with respect to the basis $\mathcal{B}$ lie in $\mathbb{Z} \cdot 1_{k}$. There is no canonical choice of structure constants. In the cases where we will need to perform calculations, $\mathfrak{L}(G)$ will be of exceptional type: for $\mathfrak{L}(G)$ of type $G_{2}$, we will use the structure constants of [5, p.211]; for $\mathfrak{L}(G)$ of type $F_{4}$, we will use those given in [41]; for $\mathfrak{L}(G)$ of type $E_{\ell}$, we will use those given in the appendix of [20]. For $\alpha \in \Phi^{+}$we may also write $f_{\alpha}$ for $e_{-\alpha}$.

For each $\alpha \in \Phi$, we fix an isomorphism $x_{\alpha}: \mathbf{G}_{a} \rightarrow U_{\alpha}$ such that $d x_{\alpha}(1)=e_{\alpha}$. The action of $x_{\alpha}(t)$ on $\mathfrak{L}(G)$ with respect to the basis $\mathcal{B}$ is then given as follows. Let $\mathfrak{L}_{\mathbb{C}}(G)=\left(\bigoplus_{v \in \mathcal{B}} \mathbb{Z} v\right) \otimes_{\mathbb{Z}} \mathbb{C}$. For $\gamma \in \mathbb{C}$, let $A_{\alpha}(\gamma)$ be the matrix representing the action of $\exp \left(\gamma\right.$ ad $\left.e_{\alpha}\right)$ on $\mathfrak{L}_{\mathbb{C}}(G)$ with respect to the basis $\{b \otimes 1: b \in \mathcal{B}\}$. For $X$ an indeterminate, there exist polynomials $f_{i j}$ in $\mathbb{Z}[X]$ such that $A_{\alpha}(\gamma)_{i j}=f_{i j}(\gamma)$. Let $A_{\alpha}(X)$ be the $|\mathcal{B}| \times|\mathcal{B}|$ matrix whose $i, j$-entry is $f_{i j}(X)$, and $\bar{A}_{\alpha}(X)$ be the image of $A_{\alpha}(X)$ under the natural homomorphism $\mathbb{Z}[X] \rightarrow\left(\mathbb{Z} \cdot 1_{k}\right)[X]$. For $t \in k$, the matrix $\bar{A}_{\alpha}(t)$ represents the adjoint action of $x_{\alpha}(t)$ on $\mathfrak{L}(G)$ with respect to the basis $\mathcal{B}$.

We define certain specific elements of $N_{G}(T)$. For $\alpha \in \Phi$ and $c \in k^{*}$, set $n_{\alpha}(c)=$ $x_{\alpha}(c) x_{-\alpha}\left(-c^{-1}\right) x_{\alpha}(c), h_{\alpha}(c)=n_{\alpha}(t) n_{\alpha}(1)^{-1}$ and $n_{\alpha}=n_{\alpha}(1)$. Then we have $T=$ $\left\langle h_{\alpha_{i}}(c): c \in k^{*}, 1 \leq i \leq \ell\right\rangle$, and $n_{\alpha} \in N_{G}(T)$ induces on $T$ the reflection associated to $\alpha$; that is, $n_{\alpha} h_{\beta}(c) n_{\alpha}{ }^{-1}=h_{w_{\alpha}(\beta)}(c)$ for all $\beta \in \Phi(G)$ and $c \in k^{*}$.

Recall that a prime $p$ is said to be bad for $G$ if it divides the coefficient of some $\alpha_{i}$ in the high root of $\Phi$, and to be good for $G$ otherwise. Thus if $G$ is of type $A_{\ell}$ then all primes are good; if $G$ is of type $B_{\ell}, C_{\ell}$ or $D_{\ell}$ then 2 is bad; if $G$ is of type $G_{2}, F_{4}, E_{6}$ or $E_{7}$ then 2 and 3 are bad; and if $G$ is of type $E_{8}$ then 2,3 and 5 are bad. A prime $p$ is said to be very good for $G$ if it is good for $G$, and additionally does not divide $\ell+1$ if $G$ is of type $A_{\ell}$.

We now turn to some structural results concerning unipotent elements of $G$ and nilpotent elements of $\mathfrak{L}(G)$. For $H$ a closed nilpotent subgroup of $G$, let $H_{u}$ denote the closed subgroup of unipotent elements in $H$. In [35] Proud studied uniform properties of $Z\left(C_{G}(u)\right)$, for $u$ a unipotent element in a simple algebraic group. He established the following result (subsequently proven differently by Seitz in [39]). 
Theorem 2.1 ([35, 39]) Let $u$ be a unipotent element of $G$.

(a) We have $Z\left(C_{G}(u)\right)=\left(Z\left(C_{G}(u)\right)\right)_{u} \times Z(G)$.

(b) Assume char $(k)$ is either 0 or a good prime for $G$. Then $Z\left(C_{G}(u)\right)^{\circ}=\left(Z\left(C_{G}(u)\right)\right)_{u}=$ $\left(\left(Z\left(C_{G}(u)\right)\right)_{u}\right)^{\circ}$.

In $\S 3$ we shall show that a certain Lie algebra calculation leads to the determination of $\operatorname{dim} Z\left(C_{G}(u)\right)$. In order to establish this, we require a theorem of Springer which describes the connection between the varieties $\mathcal{U}$ of unipotent elements of $G$ and $\mathcal{N}$ of nilpotent elements of $\mathfrak{L}(G)$. A number of variants of this result are known, with slightly different hypotheses and conclusions (see the discussion in [13, 6.20], for example); the version we shall use is the following.

Theorem 2.2 ([23, Proposition 29]) Assume char $(k)$ is either 0 or a good prime for $G$. Then there exists a $G$-equivariant homeomorphism $f: \mathcal{N} \rightarrow \mathcal{U}$.

A map as in Theorem 2.2 will be called a Springer map; its $G$-equivariance means that results proved about centralizers of nilpotent elements apply equally to centralizers of unipotent elements. In his appendix to [24], Serre shows that any two Springer maps give the same bijection between $G$-orbits on $\mathcal{N}$ and on $\mathcal{U}$.

We shall also need the following result of Slodowy on the smoothness of centralizers of unipotent or nilpotent elements.

Theorem 2.3 ([42, p.38]) Assume char $(k)$ is either 0 or a very good prime for G. Given $u \in \mathcal{U}$ and $e \in \mathcal{N}$, we have

$$
\mathfrak{L}\left(C_{G}(u)\right)=C_{\mathfrak{L}(G)}(u) \quad \text { and } \quad \mathfrak{L}\left(C_{G}(e)\right)=C_{\mathfrak{L}(G)}(e) .
$$

We now recall some results on cocharacters and nilpotent elements. Given a cocharacter $\lambda: k^{*} \rightarrow G$, we define an associated grading on $\mathfrak{L}(G)$. Indeed, if $\mathfrak{A} \subseteq \mathfrak{L}(G)$ is any $\operatorname{im}(\lambda)$-invariant subalgebra, for $m \in \mathbb{Z}$ set $\mathfrak{A}(m ; \lambda)=\left\{x \in \mathfrak{A}: \operatorname{Ad} \lambda(c) x=c^{m} x\right\}$; then $\mathfrak{A}=\bigoplus_{m \in \mathbb{Z}} \mathfrak{A}(m ; \lambda)$, and for all $m, n \in \mathbb{Z}$ we have $[\mathfrak{A}(m ; \lambda), \mathfrak{A}(n ; \lambda)] \subseteq \mathfrak{A}(m+n ; \lambda)$. We will denote by $\mathfrak{A}_{+, \lambda}$ the sum of the graded parts $\mathfrak{A}(m ; \lambda)$ with $m>0$.

In addition, we have the following result.

Proposition 2.4 ([46, Proposition 8.4.5]) Given a cocharacter $\lambda: k^{*} \rightarrow G$, set

$$
P_{\lambda}=\left\{x \in G: \lim _{a \rightarrow 0} \lambda(a) x \lambda(a)^{-1} \text { exists }\right\}
$$

then $P_{\lambda}$ is a parabolic subgroup of $G$.

In fact the proof of [46, Proposition 8.4.5] shows that $C_{G}(\operatorname{im}(\lambda))$ is a Levi factor $L_{\lambda}$ of $P_{\lambda}$, and $\mathfrak{L}\left(P_{\lambda}\right)=\mathfrak{L}\left(L_{\lambda}\right) \oplus \mathfrak{L}\left(R_{u}\left(P_{\lambda}\right)\right)$, with $\mathfrak{L}\left(L_{\lambda}\right)=\mathfrak{L}(G)(0 ; \lambda)$ and $\mathfrak{L}\left(R_{u}\left(P_{\lambda}\right)\right)=$ $\bigoplus_{m>0} \mathfrak{L}(G)(m ; \lambda)$. In particular, the subspace $\mathfrak{L}(G)_{+, \lambda}$ consists of nilpotent elements.

Given a nilpotent element, we will choose a certain cocharacter related to it; we require the following definition.

Definition 2.5 Let $L$ be a Levi subgroup of $G$. A unipotent element $u \in L$ is said to be distinguished in $L$ if each torus of $C_{L}(u)$ is contained in $Z(L)$; similarly a nilpotent element $e \in \mathfrak{L}(L)$ is said to be distinguished in $\mathfrak{L}(L)$ if each torus of $C_{L}(e)$ is contained in $Z(L)$. 
In particular, a unipotent or nilpotent element is distinguished in $G$ or $\mathfrak{L}(G)$ precisely if its connected centralizer in $G$ is a unipotent group. It is easy to see that every unipotent element is distinguished in some Levi subgroup of $G$. Indeed, given $u \in \mathcal{U}$ let $S$ be a maximal torus of $C_{G}(u)$; then $u$ is distinguished in $C_{G}(S)$. Similarly we find that every nilpotent element of $\mathfrak{L}(G)$ is distinguished in the Lie algebra of some Levi subgroup of $G$.

The cocharacters with which we will be concerned are as follows.

Definition 2.6 Let e be a nilpotent element in $\mathfrak{L}(G)$. A cocharacter $\tau: k^{*} \rightarrow G$ is called associated to $e$ if

(a) $e \in \mathfrak{L}(G)(2 ; \tau)$; and

(b) there exists a Levi subgroup $L$ of $G$ with $\operatorname{im}(\tau) \subseteq[L, L]$ and e distinguished in $\mathfrak{L}(L)$.

If $\tau$ is a cocharacter associated to a nilpotent element $e \in \mathfrak{L}(G)$, then $\operatorname{im}(\tau)$ normalizes $C_{G}(e)$ and therefore stabilizes $\mathfrak{L}\left(C_{G}(e)\right)$. In $[15, \S 5]$ there is a lengthy discussion of the properties of associated cocharacters and their connection to the structure of the group centralizer of a nilpotent element. We recall here the results which will be used in what follows. The first concerns the existence and conjugacy of cocharacters associated to $e$.

Proposition 2.7 ([15, Lemma 5.3]) Let e be a nilpotent element in $\mathfrak{L}(G)$.

(a) Assume char $(k)$ is either 0 or a good prime for $G$. Then cocharacters associated to $e$ exist.

(b) Two cocharacters associated to e are conjugate under $C_{G}(e)^{\circ}$.

Before stating the other results, we simplify the notation we have introduced for the grading on $\mathfrak{L}(G)$. Usually we have a fixed cocharacter $\tau$ in mind; for an $\operatorname{im}(\tau)$-invariant subalgebra $\mathfrak{A} \subseteq \mathfrak{L}(G)$, we may then write $\mathfrak{A}_{m}$ and $\mathfrak{A}_{+}$for $\mathfrak{A}(m ; \tau)$ and $\mathfrak{A}_{+, \tau}$ respectively.

Proposition 2.8 ([15, Proposition 5.9]) Assume char $(k)$ is either 0 or a good prime for $G$. Let $e \in \mathfrak{L}(G)$ be nilpotent and $\tau$ be a cocharacter associated to $e$. Let $P=P_{\tau}$ be the parabolic subgroup associated to $\tau$ as in Proposition 2.4. Then

(a) the group $P$ depends only on e and not on $\tau$;

(b) $C_{G}(e)=C_{P}(e)$; and

(c) $\overline{\operatorname{Ad}(P) e}=\bigoplus_{m \geq 2} \mathfrak{L}(G)_{m}$.

Assume $\operatorname{char}(k)$ is either 0 or a good prime for $G$, and let $e, \tau$ and $P$ be as in Proposition 2.8. Let $L_{\tau}$ denote the Levi factor $C_{G}(\operatorname{im}(\tau))$ of $P$, and $R_{u}(P)$ the unipotent radical of $P$; then $P$ is the semidirect product (as an algebraic group) of $L_{\tau}$ and $R_{u}(P)$, and we have $\mathfrak{L}\left(L_{\tau}\right)=\mathfrak{L}(G)_{0}$ and $\mathfrak{L}\left(R_{u}(P)\right)=\mathfrak{L}(G)_{+}$. Set $C=C_{G}(e) \cap L_{\tau}$ and $R=$ $C_{G}(e) \cap R_{u}(P)$.

Proposition 2.9 ([15, Proposition 5.10])

(a) Assume char $(k)$ is either 0 or a good prime for $G$. Then $C_{G}(e)$ is the semidirect product (as an algebraic group) of the subgroup $C$ and the normal subgroup $R$.

(b) Assume char $(k)$ is either 0 or a very good prime for $G$. Then $\mathfrak{L}(C)=C_{\mathfrak{L}(G)}(e)_{0}$ and $\mathfrak{L}(R)=C_{\mathfrak{L}(G)}(e)_{+}$. 
(c) The group $R$ is connected and unipotent.

(In fact, the statement of (b) in [15] is slightly different from that given here: there it is assumed that $G$ is simply connected and the characteristic is either 0 or a good prime for $G$. However, the condition on $G$ is only required to ensure $\mathfrak{L}\left(C_{G}(e)\right)=C_{\mathfrak{L}(G)}(e)$, which here follows from Theorem 2.3, given the extra requirement on the field characteristic.)

We note that the proofs of the above results, as given in [15], do not depend upon case-by-case consideration of the list of nilpotent orbits. However, they do rely upon the Bala-Carter-Pommerening classification of $G$-orbits of nilpotent elements in $\mathfrak{L}(G)$, valid in good characteristic, which we now go on to describe.

First we must recall a result of Richardson; the original of this was proved in [36], but the version most useful for the present purposes is due to Steinberg.

Theorem 2.10 ([6, Theorem 5.2.3]) Let $H$ be a connected reductive group defined over an algebraically closed field. Let $P$ be a parabolic subgroup of $H$ with unipotent radical $U$. Let $\mathcal{C}$ be the unique nilpotent orbit in $\mathfrak{L}(H)$ under the adjoint action of $H$ such that $\mathcal{C} \cap \mathfrak{L}(U)$ is an open dense subset of $\mathfrak{L}(U)$. Then $\mathcal{C} \cap \mathfrak{L}(U)$ is a single P-orbit under the adjoint action of $P$ on $\mathfrak{L}(U)$.

In order to describe the Bala-Carter-Pommerening classification, we require the following definition.

Definition 2.11 A parabolic subgroup $P$ of $G$ with unipotent radical $U$ is said to be distinguished if $\operatorname{dim} U /[U, U]=\operatorname{dim} P / U$.

We can now state the theorem.

Theorem 2.12 ([1, 2, 29,30]) Assume char $(k)$ is either 0 or a good prime for $G$.

(a) There is a bijective map between $G$-orbits of distinguished nilpotent elements of $\mathfrak{L}(G)$ and conjugacy classes of distinguished parabolic subgroups of $G$. The $G$-orbit corresponding to a given parabolic subgroup $P$ contains the dense orbit of $P$ acting on the Lie algebra of its unipotent radical.

(b) There is a bijective map between $G$-orbits of nilpotent elements of $\mathfrak{L}(G)$ and $G$-classes of pairs $\left(L, P_{L}\right)$, where $L$ is a Levi subgroup of $G$ and $P_{L}$ is a distinguished parabolic subgroup of $[L, L]$. The $G$-orbit corresponding to a pair $\left(L, P_{L}\right)$ contains the dense orbit of $P_{L}$ acting on the Lie algebra of its unipotent radical.

We note in passing that a recent alternative proof of this theorem, avoiding case analysis, is given by Premet in [31].

This concludes our survey of known results. We end this section with a result which will enable us to work with groups of arbitrary isogeny type. We shall employ the set-up of $[15,2.7]$ : we write $G_{s c}$ for a simply connected group over $k$ of the same type as $G$, and let $\pi: G_{s c} \rightarrow G$ be a surjective morphism of algebraic groups with $\operatorname{ker} \pi \subseteq Z\left(G_{s c}\right)$; then certainly $\pi\left(Z\left(G_{s c}\right)\right)=Z(G)$. Also ker $d \pi$ is an ideal of $\mathfrak{L}\left(G_{s c}\right)$; thus, provided that $\operatorname{char}(k)$ is either 0 or a good prime for $G$, by $[37,1.9]$ we see that $\operatorname{ker} d \pi \subseteq Z\left(\mathfrak{L}\left(G_{s c}\right)\right)$. We write $\mathcal{U}_{s c}$ and $\mathcal{N}_{s c}$ for the varieties of unipotent elements of $G_{s c}$ and nilpotent elements of $\mathfrak{L}\left(G_{s c}\right)$. By [15, Proposition 2.7(a)] the restriction of $d \pi$ to $\mathcal{N}_{s c}$ is an isomorphism $d \pi: \mathcal{N}_{s c} \rightarrow \mathcal{N}$.

The result we shall prove relates centres of group centralizers of elements of $\mathcal{N}_{s c}$ and $\mathcal{N}$; note that Theorem 2.2 implies that Theorem 2.1 applies to these centres. 
Proposition 2.13 Assume char $(k)$ is either 0 or a good prime for $G$. Let e be an element of $\mathcal{N}$, and $e_{s c}$ be the unique element of $\mathcal{N}_{s c}$ with $d \pi\left(e_{s c}\right)=e$.

(a) We have $\pi\left(Z\left(C_{G_{s c}}\left(e_{s c}\right)\right)\right)=Z\left(C_{G}(e)\right)$.

(b) The map $d \pi: \mathfrak{L}\left(Z\left(C_{G_{s c}}\left(e_{s c}\right)\right)^{\circ}\right) \rightarrow \mathfrak{L}\left(Z\left(C_{G}(e)\right)^{\circ}\right)$ is a Lie algebra isomorphism.

(c) Let $\tau: k^{*} \rightarrow G_{s c}$ be a cocharacter associated to $e_{s c}$. Then $\pi \circ \tau: k^{*} \rightarrow G$ is a cocharacter associated to $e$; and the set of $\tau$-weights, with multiplicities, on $\mathfrak{L}\left(Z\left(C_{G_{s c}}\left(e_{s c}\right)\right)\right)$ is the same as the set of $\pi \circ \tau$-weights, with multiplicities, on $\mathfrak{L}\left(Z\left(C_{G}(e)\right)\right)$.

Proof. By [15, Proposition 2.7(a)] we have $\pi\left(C_{G_{s c}}\left(e_{s c}\right)\right)=C_{G}(e)$; it is then immediate that $\pi\left(Z\left(C_{G_{s c}}\left(e_{s c}\right)\right)\right) \subseteq Z\left(C_{G}(e)\right)$. Now take $\pi(x) \in Z\left(C_{G}(e)\right)$. By Theorem 2.1 we have $\pi(x)=\pi(y) \pi(s)$, where $\pi(y) \in \mathcal{U}$ and $\pi(s) \in Z(G)=\pi\left(Z\left(G_{s c}\right)\right)$. Hence $x=v z$, where $v \in \mathcal{U}_{s c}$ and $z \in Z\left(G_{s c}\right)$. Now for all $\pi(g) \in C_{G}(e)$ we have $\pi(x) \pi(g)=\pi(g) \pi(x)$. Hence given $g \in C_{G_{s c}}\left(e_{s c}\right)$ there exists $n_{g} \in \operatorname{ker} \pi$ such that $x g=g x n_{g}$. Thus $v z g=g v z n_{g}$, and so $v g=g v n_{g}$, whence $g^{-1} v g=v n_{g}$; since $v$ is unipotent and $n_{g} \in Z\left(G_{s c}\right)$, the uniqueness of Jordan decomposition forces $n_{g}=1$, so that $x g=g x$. As this is true for all $g \in C_{G_{s c}}\left(e_{s c}\right)$, we have $x \in Z\left(C_{G_{s c}}\left(e_{s c}\right)\right)$, proving (a).

By Theorem 2.1 both $Z\left(C_{G_{s c}}\left(e_{s c}\right)\right)^{\circ}$ and $Z\left(C_{G}(e)\right)^{\circ}$ are unipotent groups, so their Lie algebras consist of nilpotent elements. By (a) the dimensions of these Lie algebras are equal; as $d \pi: \mathcal{N}_{s c} \rightarrow \mathcal{N}$ is bijective, it follows that $d \pi: \mathfrak{L}\left(Z\left(C_{G_{s c}}\left(e_{s c}\right)\right)^{\circ}\right) \rightarrow \mathfrak{L}\left(Z\left(C_{G}(e)\right)^{\circ}\right)$ is a Lie algebra isomorphism, proving (b).

Finally, we observe that $\pi$ maps Levi subgroups of $G_{s c}$ to those of $G$; and if $L_{s c}$ is a Levi subgroup of $G_{s c}$ such that $e_{s c}$ is distinguished in $\mathfrak{L}\left(L_{s c}\right)$ and $\operatorname{im}(\tau) \subseteq\left[L_{s c}, L_{s c}\right]$, then $e$ is distinguished in $\mathfrak{L}\left(\pi\left(L_{s c}\right)\right)$ and $\operatorname{im}(\pi \circ \tau) \subseteq\left[\pi\left(L_{s c}\right), \pi\left(L_{s c}\right)\right]$. Moreover, given $g_{s c} \in G_{s c}$ we have $\pi \circ \operatorname{Int}\left(g_{s c}\right)=\operatorname{Int}\left(\pi\left(g_{s c}\right)\right) \circ \pi$, where Int $: G_{s c} \rightarrow \operatorname{Aut}\left(G_{s c}\right)$ is the morphism sending $g_{s c}$ to the inner automorphism given by $g_{s c}$; taking differentials gives $d \pi \circ \operatorname{Ad}\left(g_{s c}\right)=\operatorname{Ad}\left(\pi\left(g_{s c}\right)\right) \circ d \pi$. Both assertions of (c) follow.

For the most part, in the sections which follow we shall make no assumption on the isogeny type of $G$. However, when treating the classical groups in $\S 4$ it will be convenient to assume that $G$ is $\mathrm{SL}(V), \mathrm{SO}(V)$ or $\mathrm{Sp}(V)$ as appropriate; on the other hand, when concluding the treatment of the exceptional groups in $\S 9$ we shall take $G$ to be of adjoint type. In each case Proposition 2.13 will then imply that our results on $\mathfrak{L}\left(Z\left(C_{G}(e)\right)\right)$ apply to all other isogenous groups. 


\section{Reduction of the problem}

Throughout this section, we take $G$ to be a simple algebraic group defined over an algebraically closed field $k$, and $T$ to be a fixed maximal torus of $G$; to begin with we $\operatorname{assume} \operatorname{char}(k)$ is either 0 or a good prime for $G$. Fix a nilpotent element $e \in \mathfrak{L}(G)$ and an associated cocharacter $\tau: k^{*} \rightarrow T$. As in $\S 2, \tau$ induces a grading on $\mathfrak{L}(G)$; we write $\mathfrak{L}(G)_{+}$for the sum of the strictly positive $\tau$-weight spaces.

Let $P_{\tau}$ be the parabolic subgroup associated to $\tau$ as in Proposition 2.4. In particular $C_{G}(\operatorname{im}(\tau))$ is a Levi factor of $P_{\tau}$. As in the paragraph before Proposition 2.9, we have $C_{G}(e)=R . C$ where $R=C_{G}(e) \cap R_{u}\left(P_{\tau}\right)$ and $C=C_{G}(e) \cap C_{G}(\operatorname{im}(\tau))$.

We begin by proving certain parts of the main theorems of $\S 1$; to do this we must first explain how the cocharacter $\tau$ gives rise to the labelled diagram $\Delta$. For each simple root $\alpha_{i}$, let $a_{i}$ be the $\tau$-weight of the root vector $e_{\alpha_{i}}$, regarded as an integer; thus for all $c \in k^{*}$ we have $\tau(c) e_{\alpha_{i}}=c^{a_{i}} e_{\alpha_{i}}$. The labelled diagram of $\tau$ is the Dynkin diagram of $G$, in which the node corresponding to $\alpha_{i}$ is labelled with $a_{i}$. This is not in general uniquely determined by the nilpotent orbit containing $e$, since conjugation by elements of $W$ need not preserve $\tau$. There is however a unique $W$-conjugate $\tau^{+}$of $\tau$ with the property that all labels in its labelled diagram are non-negative; it is this labelled diagram which is called $\Delta$. By [30,31], $\Delta$ is the diagram which corresponds to the orbit of $e$, and appears for example in the tables of $[6,13.1]$; each of its labels is 0,1 or 2 .

Now the centralizer $C_{G}\left(\operatorname{im}\left(\tau^{+}\right)\right)$is generated by $T$ and the $T$-root subgroups $U_{\alpha}$ such that $\left.\alpha\right|_{\operatorname{im}\left(\tau^{+}\right)}=0$. Hence $C_{G}\left(\operatorname{im}\left(\tau^{+}\right)\right)$is a Levi subgroup whose semisimple rank is equal to the number of zeros in $\Delta$; as $\tau$ is a conjugate of $\tau^{+}$the same is true of $C_{G}(\operatorname{im}(\tau))$. Thus $\operatorname{dim} Z\left(C_{G}(\operatorname{im}(\tau))\right)$ is the number of nonzero entries in $\Delta$; so if $\Delta$ has no label equal to 1 , then $\operatorname{dim} Z\left(C_{G}(\operatorname{im}(\tau))\right)=n_{2}(\Delta)$. As each orbit of distinguished elements of $\mathfrak{L}(G)$ has an even diagram, this proves the second equality in each of Theorems 1(i) and 2.

Next recall from $\S 1$ the definitions of the 2 -free core $\Delta_{0}$ of $\Delta$ and the semisimple group $G_{0}$; note that we may take $G_{0}$ to be the subgroup $\left\langle U_{ \pm \alpha}: \alpha\right.$ a node of $\left.\Delta_{0}\right\rangle$ of $G$. Modulo the existence statement of Theorem 3, we may now prove Theorem 3(i).

Proposition 3.1 Let e have labelled diagram $\Delta$; let $\Delta_{0}$ be the 2 -free core of $\Delta$ and $G_{0}$ be a corresponding semisimple group. Assume that there exists $e_{0} \in \mathfrak{L}\left(G_{0}\right)$ with labelled diagram $\Delta_{0}$. Then $\operatorname{dim} C_{G}(e)-\operatorname{dim} C_{G_{0}}\left(e_{0}\right)=n_{2}(\Delta)$.

Proof. Let $\tau^{+}$be the conjugate of $\tau$ giving rise to $\Delta$. Proposition 2.8 gives

$$
\begin{aligned}
\operatorname{dim} C_{G}(e)=\operatorname{dim} C_{P_{\tau}}(e) & =\operatorname{dim} P_{\tau}-\operatorname{dim} \bigoplus_{m \geq 2} \mathfrak{L}(G)(m ; \tau) \\
& =\operatorname{dim} \mathfrak{L}\left(P_{\tau}\right)-\operatorname{dim} \bigoplus_{m \geq 2} \mathfrak{L}(G)(m ; \tau) \\
& =\operatorname{dim} \mathfrak{L}(G)(0 ; \tau)+\operatorname{dim} \mathfrak{L}(G)(1 ; \tau) \\
& =\operatorname{dim} \mathfrak{L}(G)\left(0 ; \tau^{+}\right)+\operatorname{dim} \mathfrak{L}(G)\left(1 ; \tau^{+}\right) .
\end{aligned}
$$

Similarly we have $\operatorname{dim} C_{G_{0}}\left(e_{0}\right)=\operatorname{dim} \mathfrak{L}\left(G_{0}\right)\left(0 ; \tau_{0}{ }^{+}\right)+\operatorname{dim} \mathfrak{L}\left(G_{0}\right)\left(1 ; \tau_{0}{ }^{+}\right)$, where $\tau_{0}$ is a cocharacter associated to $e_{0}$ and $\tau_{0}{ }^{+}$is the conjugate of $\tau_{0}$ giving rise to $\Delta_{0}$. Taking $G_{0}=\left\langle U_{ \pm \alpha}: \alpha\right.$ a node of $\left.\Delta_{0}\right\rangle \subseteq G$, we see that $\tau^{+}$and $\tau_{0}{ }^{+}$have the same weights on root vectors $e_{\alpha}$ for $\alpha \in \Phi\left(G_{0}\right)$, and the weight of $\tau^{+}$on $e_{\alpha}$ is at least 2 if $\alpha \in \Phi^{+} \backslash \Phi\left(G_{0}\right)$ and at most -2 if $\alpha \in \Phi^{-} \backslash \Phi\left(G_{0}\right)$. Thus $\mathfrak{L}(G)\left(1 ; \tau^{+}\right)=\mathfrak{L}\left(G_{0}\right)\left(1 ; \tau_{0}{ }^{+}\right)$and $\mathfrak{L}(G)\left(0 ; \tau^{+}\right)=$ $\mathfrak{L}\left(G_{0}\right)\left(0 ; \tau_{0}{ }^{+}\right)+\mathfrak{L}(T) ;$ thus $\mathfrak{L}(G)\left(0 ; \tau^{+}\right) / \mathfrak{L}(T) \cong \mathfrak{L}\left(G_{0}\right)\left(0 ; \tau_{0}{ }^{+}\right) / \mathfrak{L}\left(T \cap G_{0}\right)$, and hence $\operatorname{dim} C_{G}(e)-\operatorname{dim} C_{G_{0}}\left(e_{0}\right)=\operatorname{dim} \mathfrak{L}(T)-\operatorname{dim} \mathfrak{L}\left(T \cap G_{0}\right)=\operatorname{rank} G-\operatorname{rank} G_{0}=n_{2}(\Delta)$. 
We now turn to the consideration of $\mathfrak{L}\left(Z\left(C_{G}(e)\right)\right)$. Set

$$
\mathcal{Z}=\left(Z\left(C_{\mathfrak{L}(G)}(e)_{+}\right)\right)^{C},
$$

where the superscript denotes the collection of fixed points under $C$. In the remainder of this section we shall prove that, under a slight strengthening of the assumption on $\operatorname{char}(k)$, we have equality between $\mathfrak{L}\left(Z\left(C_{G}(e)\right)\right)$ and $\mathcal{Z}$. We shall obtain this equality by showing both inclusions; for the first we shall require the following result, which holds under the characteristic assumption stated at the beginning of this section.

Proposition 3.2 We have $\mathfrak{L}\left(Z\left(C_{G}(e)\right)\right) \subseteq \mathfrak{L}(G)_{+}$.

Proof. First let $H$ be a semisimple group defined over $k$, such that $\operatorname{char}(k)$ is either 0 or a good prime for each simple factor of $H$. Let $e_{H}$ be a distinguished nilpotent element in $\mathfrak{L}(H)$, with associated cocharacter $\tau_{H}$. We shall prove that $\mathfrak{L}\left(Z\left(C_{H}\left(e_{H}\right)\right)\right) \subseteq \mathfrak{L}(H)_{+}$; the proof will use various results which are stated for simple groups but hold for semisimple groups.

By Theorem 2.12 there exists a distinguished parabolic subgroup $P \subseteq H$ such that $e$ lies in the dense orbit of $P$ on $\mathfrak{L}\left(R_{u}(P)\right)$. Let $T^{\prime}$ be a maximal torus of $P$. By $[15$, Lemma 5.2] there exist a cocharacter $\tau^{\prime}: k^{*} \rightarrow T^{\prime}$ and a base of the root system with respect to $T^{\prime}$, such that $C_{H}\left(\operatorname{im}\left(\tau^{\prime}\right)\right)$ is a Levi factor of $P$ containing $T^{\prime}$ and simple roots outside $\Phi\left(C_{H}\left(\operatorname{im}\left(\tau^{\prime}\right)\right)\right)$ afford $\tau^{\prime}$-weight 2 ; thus $P=P_{\tau^{\prime}}$ by the proof given in [46] of Proposition 2.4. Now by [6, Proposition 5.8.5] we may assume $e_{H}$ lies in the 2-weight space for $\tau^{\prime}$; as $e_{H}$ is distinguished in $\mathfrak{L}(H)$, the cocharacter $\tau^{\prime}$ is associated to $e_{H}$. By Proposition 2.8 we have $P_{\tau_{H}}=P_{\tau^{\prime}}=P$, and then using [6, Corollary 5.8.6] as well gives $C_{H}\left(e_{H}\right)^{\circ}=C_{P}\left(e_{H}\right)^{\circ} \subseteq R_{u}(P)$ (note that although Proposition 5.8.5 and Corollary 5.8.6 of [6] are stated under the assumption that the characteristic is either 0 or quite large, their proofs are valid provided the characteristic is either 0 or any good prime). Thus certainly $Z\left(C_{H}\left(e_{H}\right)\right)^{\circ} \subseteq R_{u}(P)$, and so $\mathfrak{L}\left(Z\left(C_{H}\left(e_{H}\right)\right)\right)=\mathfrak{L}\left(Z\left(C_{H}\left(e_{H}\right)\right)^{\circ}\right) \subseteq \mathfrak{L}\left(R_{u}(P)\right)=\mathfrak{L}(H)_{+}$ as claimed.

Now let $L$ be a Levi subgroup of $G$ such that $e$ is distinguished in $\mathfrak{L}(L)$ and $\operatorname{im}(\tau) \subseteq$ $[L, L]$. Clearly $Z(L) \subseteq C_{G}(e)$, so $Z\left(C_{G}(e)\right) \subseteq C_{G}(Z(L))=L$. Moreover, by Theorem 2.1 $Z\left(C_{G}(e)\right)^{\circ}$ is a connected unipotent group and hence lies in $[L, L]$; so $Z\left(C_{G}(e)\right)^{\circ} \subseteq$ $Z\left(C_{[L, L]}(e)\right)$. Setting $H=[L, L], e_{H}=e$ and $\tau_{H}=\tau$ in the previous paragraph gives $\mathfrak{L}\left(Z\left(C_{[L, L]}(e)\right)\right) \subseteq \mathfrak{L}([L, L])_{+}$, whence we have $\mathfrak{L}\left(Z\left(C_{G}(e)\right)\right)=\mathfrak{L}\left(Z\left(C_{G}(e)\right)^{\circ}\right) \subseteq$ $\mathfrak{L}\left(Z\left(C_{[L, L]}(e)\right)\right) \subseteq \mathfrak{L}(G)_{+}$

For the remainder of this section we make the slightly stronger assumption that

$$
\operatorname{char}(k) \text { is either } 0 \text { or a very good prime for } G \text {. }
$$

Proposition 2.9 then gives $\mathfrak{L}(R)=C_{\mathfrak{L}(G)}(e)_{+}$and $\mathfrak{L}(C)=C_{\mathfrak{L}(G)}(e)_{0}$.

Proposition 3.3 We have $\mathfrak{L}\left(Z\left(C_{G}(e)\right)\right) \subseteq\left(Z\left(C_{\mathfrak{L}(G)}(e)\right)\right)^{C}$.

Proof. Recall that $\mathfrak{L}(Z(H)) \subseteq Z(\mathfrak{L}(H))$ for an arbitrary closed subgroup $H$ of $G$. Combining this with the fact that $\mathfrak{L}\left(C_{G}(e)\right)=C_{\mathfrak{L}(G)}(e)$ by Theorem 2.3 , we have

$$
\mathfrak{L}\left(Z\left(C_{G}(e)\right)\right) \subseteq Z\left(C_{\mathfrak{L}(G)}(e)\right) .
$$

As $C$ acts trivially on $Z\left(C_{G}(e)\right)$ by conjugation, it also acts trivially on $\mathfrak{L}\left(Z\left(C_{G}(e)\right)\right)$; so

$$
\mathfrak{L}\left(Z\left(C_{G}(e)\right)\right) \subseteq\left(C_{\mathfrak{L}(G)}(e)\right)^{C} .
$$

The result follows. 
The first of our two inclusions is now immediate.

Corollary 3.4 We have $\mathfrak{L}\left(Z\left(C_{G}(e)\right)\right) \subseteq \mathcal{Z}$.

Proof. Apply Propositions 3.2 and 3.3.

In Proposition 3.7 below, we shall show that $C_{G}(e)$ is generated by a certain family of closed commutative subgroups, together with the group $C$. We begin with a lemma.

Lemma 3.5 Let $X, Y \in \mathfrak{L}(G)$ with $[X, Y]=0$. Then $Z\left(C_{G}(Y)\right) \subset C_{G}(X)$, and we have $\left[Z\left(C_{G}(X)\right), Z\left(C_{G}(Y)\right)\right]=1$.

Proof. The group $Z\left(C_{G}(Y)\right)$ acts trivially on $C_{G}(Y)$ and therefore on $\mathfrak{L}\left(C_{G}(Y)\right)$, which by Theorem 2.3 equals $C_{\mathfrak{L}(G)}(Y)$. The latter subalgebra contains $X$ and hence $Z\left(C_{G}(Y)\right) \subseteq C_{G}(X)$, proving the results.

We will also need the following result of [26].

Proposition 3.6 ([26, Theorem A]) If $X \in \mathcal{N}$, then $X \in \mathfrak{L}\left(Z\left(C_{G}(X)\right)\right)$.

Our result on $C_{G}(e)$ is now as follows.

Proposition 3.7 We have $C_{G}(e)=\left\langle C, Z\left(C_{G}(X)\right): X \in C_{\mathfrak{L}(G)}(e)_{+}\right\rangle$.

Proof. Write $H=\left\langle C, Z\left(C_{G}(X)\right): X \in C_{\mathfrak{L}(G)}(e)_{+}\right\rangle$. First note that $\mathfrak{L}(G)_{+}$consists of nilpotent elements and so $C_{\mathfrak{L}(G)}(e)_{+} \subseteq \mathcal{N}$. By Lemma 3.5, for all $X \in C_{\mathfrak{L}(G)}(e)_{+}$ we have $Z\left(C_{G}(X)\right) \subseteq C_{G}(e)$; so $H \subseteq C_{G}(e)$. On the other hand Proposition 3.6 shows that $\mathfrak{L}(H) \supseteq C_{\mathfrak{L}(G)}(e)_{+}=\mathfrak{L}(R)$, and by the definition of $H$ we have $\mathfrak{L}(H) \supseteq \mathfrak{L}(C)$. As $\mathfrak{L}(R) \cap \mathfrak{L}(C)=C_{\mathfrak{L}(G)}(e)_{+} \cap C_{\mathfrak{L}(G)}(e)_{0}=\{0\}$, it follows that

$$
\operatorname{dim} H=\operatorname{dim} \mathfrak{L}(H) \geq \operatorname{dim} \mathfrak{L}(R)+\operatorname{dim} \mathfrak{L}(C)=\operatorname{dim} R+\operatorname{dim} C=\operatorname{dim} C_{G}(e) .
$$

Thus $H^{\circ}=C_{G}(e)^{\circ}$. Since $R$ is connected and lies in $C_{G}(e)$, it lies in $H$; thus we have $H \supseteq\langle C, R\rangle=R . C=C_{G}(e)$, whence $H=C_{G}(e)$ as required.

We may now show the second of our two inclusions.

Proposition 3.8 We have $\mathcal{Z} \subseteq \mathfrak{L}\left(Z\left(C_{G}(e)\right)\right)$.

Proof. Take $v \in \mathcal{Z}$. Lemma 3.5 shows that $Z\left(C_{G}(v)\right)$ commutes with $Z\left(C_{G}(X)\right)$ for all $X \in C_{\mathfrak{L}(G)}(e)_{+}$; as $v \in C_{\mathfrak{L}(G)}(C)$, we have $C \subseteq C_{G}(v)$, and so $Z\left(C_{G}(v)\right)$ also commutes with $C$. By Proposition 3.7 we therefore have $Z\left(C_{G}(v)\right) \subseteq Z\left(C_{G}(e)\right)$. Thus $\mathfrak{L}\left(Z\left(C_{G}(v)\right)\right) \subseteq \mathfrak{L}\left(Z\left(C_{G}(e)\right)\right)$, so by Proposition 3.6 we have $v \in \mathfrak{L}\left(Z\left(C_{G}(e)\right)\right)$.

As a consequence we have our main result of this section.

Theorem 3.9 We have $\mathfrak{L}\left(Z\left(C_{G}(e)\right)\right)=\mathcal{Z}=\left(Z\left(C_{\mathfrak{L}(G)}(e)_{+}\right)\right)^{C}$, so that $\operatorname{dim} Z\left(C_{G}(e)\right)=$ $\operatorname{dim} \mathcal{Z}$. Moreover, if $Z\left(C_{\mathfrak{L}(G)}(e)\right) \subseteq \mathfrak{L}(G)_{+}$then in fact $\mathfrak{L}\left(Z\left(C_{G}(e)\right)\right)=\left(Z\left(C_{\mathfrak{L}(G)}(e)\right)\right)^{C}$.

Proof. The first statement is immediate from Corollary 3.4 and Proposition 3.8. If $Z\left(C_{\mathfrak{L}(G)}(e)\right)$ is contained in $\mathfrak{L}(G)_{+}$, then it lies in $Z\left(C_{\mathfrak{L}(G)}(e)_{+}\right)$; taking fixed points under $C$ and applying Propositions 3.3 and 3.8 gives the second statement.

We conclude this section by noting that, for groups of all types other than $A_{\ell}$, the concepts of 'good prime' and 'very good prime' coincide. 


\section{Classical groups}

Let $G$ be a simple algebraic group of classical type defined over an algebraically closed field $k$ whose characteristic is either 0 or a good prime for $G$. In this section we will prove that the conclusions of the main theorems of $\S 1$ hold for $G$. The arguments employed will be rather different from those employed in the sections which follow, where groups of exceptional type are considered. We begin with the case where $G$ is of type $A_{\ell}$. We then treat the case where $G$ is of type $B_{\ell}, C_{\ell}$ or $D_{\ell}$; here we make use of a result of Yakimova which shortens the argument considerably.

\section{1 $G$ of type $A_{\ell}$}

Let $G$ be of type $A_{\ell}$. By Proposition 2.13 we may assume $G=\operatorname{SL}(V)$, where $V$ is a vector space over $k$ of dimension $\ell+1$; then $\mathfrak{L}(G)=\mathfrak{s l}(V)$, and the action of $G$ on $\mathfrak{L}(G)$ is given by conjugation. Here the distinction between good and very good primes is an obstacle preventing us from using the results of $\S 3$. However, in this case a more direct approach is available.

Take a nilpotent element $e \in \mathfrak{L}(G)$, with associated cocharacter $\tau$ and labelled diagram $\Delta$ having labels $a_{1}, \ldots, a_{\ell}$; let $\Delta_{0}$ be the 2 -free core of $\Delta$, with corresponding algebraic group $G_{0}$. From $[15,3.5$ and 5.4] we see that the collection of $\tau$-weights on $V$ is the union (with multiplicities) of the sets $\{d-1, d-3, \ldots, 3-d, 1-d\}$, where the union runs over the Jordan blocks of $e$ on $V$ and $d$ is the size of the block. Write this collection of $\tau$-weights as $\xi_{1} \geq \xi_{2} \geq \cdots \geq \xi_{\ell} \geq \xi_{\ell+1}$; observe that for each $i$ we have $\xi_{i}+\xi_{\ell+2-i}=0$. From [6, p.393] we see that the $\xi_{i}$ determine the labels of $\Delta$ as follows: for $1 \leq i \leq \ell$ we have $a_{i}=\xi_{i}-\xi_{i+1}$.

We begin by establishing the existence statements of Theorems 3 and 4 . Let $S_{2}(\Delta)=$ $\left\{i: a_{i}=2\right\}$. If $S_{2}(\Delta)$ is empty, then $\Delta_{0}=\Delta$ and we may take $e_{0}=e$ and $\Gamma_{0}=\Delta$; so assume $S_{2}(\Delta)$ is non-empty. The formula above for $a_{i}$ shows that if $i \in S_{2}(\Delta)$ then $\ell+1-i \in S_{2}(\Delta)$; let $j \leq\left\lfloor\frac{\ell+1}{2}\right\rfloor$ be maximal with $j \in S_{2}(\Delta)$.

We have $2=a_{j}=\xi_{j}-\xi_{j+1}$, and hence $V$ has no $\tau$-weight $\xi_{j+1}+1$; so $V$ also has no $\tau$-weight $\xi_{j+1}+2 n+1$ for $n \in \mathbb{N}$. Therefore each $\tau$-weight $\xi_{i}$ for $i<j$ must have the same parity as $\xi_{j}$; so if $i<j$ then $a_{i}=a_{\ell+1-i}=\xi_{i}-\xi_{i+1} \in\{0,2\}$. Thus if $j<\left\lfloor\frac{\ell+1}{2}\right\rfloor$ we may let $\Gamma_{0}$ be the connected component of $\Delta_{0}$ with nodes $\alpha_{j+1}, \ldots, \alpha_{\ell-j}$, and then all labels in $\Delta_{0} \backslash \Gamma_{0}$ are 0 . Moreover if each Jordan block size greater than $\xi_{j+1}+1$ is replaced by $\xi_{j+1}+1$, then the set of numbers obtained (with multiplicities) is the list of Jordan block sizes of a nilpotent element $e_{0}$ of $\mathfrak{L}\left(H_{0}\right)$, where $H_{0}$ is the simple factor of $G_{0}$ corresponding to $\Gamma_{0}$; and the labelled diagram of $e_{0}$, regarded as an element of $\mathfrak{L}\left(G_{0}\right)$, is $\Delta_{0}$. If on the other hand $j=\left\lfloor\frac{\ell+1}{2}\right\rfloor$, then for all $i$ we have $a_{i} \in\{0,2\}$; we may therefore take $e_{0}=0$, and $\Gamma_{0}$ to be any component of $\Delta_{0}$ (provided $\Delta_{0}$ is not the empty diagram). This proves the existence statements of Theorems 3 and 4 .

We now turn to the proof of the dimension formula in Theorem 4, which in this case states that

$$
\operatorname{dim} Z\left(C_{G}(e)\right)=\left\lceil\frac{1}{2} \sum a_{j}\right\rceil
$$

to show this we shall identify $Z\left(C_{G}(e)\right)^{\circ}$ explicitly. Note that we have

$$
\sum a_{j}=\xi_{1}-\xi_{\ell+1}=2 \xi_{1}
$$

Let the Jordan blocks of $e$ on $V$ have sizes $\ell_{1}+1, \ldots, \ell_{n}+1$, where $\ell_{1} \geq \cdots \geq \ell_{n}$ and $\sum_{i=1}^{n}\left(\ell_{i}+1\right)=\ell+1$. There are then vectors $w_{1}, \ldots, w_{n} \in V$, with $e^{\ell_{i}+1} w_{i}=0$ for 
$1 \leq i \leq n$, such that

$$
\left\{e^{s} w_{i}: 1 \leq i \leq n, 0 \leq s \leq \ell_{i}\right\}
$$

is a basis for $V$. Write $V_{i}=\left\langle w_{i}, e w_{i}, \ldots, e^{\ell_{i}} w_{i}\right\rangle$, so that $V=V_{1} \oplus \cdots \oplus V_{n}$. In what follows, we treat elements of both $G$ and $\mathfrak{L}(G)$ as matrices with respect to the given basis, whose elements we order as

$$
e^{\ell_{1}} w_{1}, \ldots, e w_{1}, w_{1}, \quad e^{\ell_{2}} w_{2}, \ldots, e w_{2}, w_{2}, \quad \ldots, \quad e^{\ell_{n}} w_{n}, \ldots, e w_{n}, w_{n} .
$$

We write $E_{i, j}$ for the elementary matrix with 1 in $(i, j)$-position. For $0 \leq i \leq n$ set $s_{i}=\sum_{j=1}^{i}\left(\ell_{j}+1\right)$, so that $s_{0}=0$ and $s_{n}=\ell+1$; then we have $e=\sum_{i=1}^{n} e_{i}$, where for $1 \leq i \leq n$ we set $e_{i}=\sum_{h=1}^{\ell_{i}} E_{s_{i}+h, s_{i}+h+1}$. For each $i$, the nilpotent element $e_{i}$ is regular in $\mathfrak{L}\left(L_{i}\right)$, where $L_{i}=\operatorname{SL}\left(V_{i}\right)$; thus if we let $L$ be the Levi subgroup whose derived group is $L_{1} \ldots L_{n}$ then $e$ is distinguished in $\mathfrak{L}(L)$. Note that we have $\xi_{1}=\ell_{1}$.

We first observe that if $n=1$, so that $e$ is a regular nilpotent element, a straightforward calculation shows that for any $(\ell+1) \times(\ell+1)$ matrix $A=\left(a_{i j}\right)$ we have

$$
A e=e A \Longleftrightarrow a_{i j}=\left\{\begin{array}{ll}
0 & \text { if } i \geq j, \\
a_{1, j-i+1} & \text { if } i<j
\end{array} \Longleftrightarrow A \in\left\langle e^{0}=I, e, e^{2}, \ldots, e^{\ell}\right\rangle .\right.
$$

Now return to the general case; choose $\lambda_{1}, \ldots, \lambda_{n} \in k^{*}$ such that $\lambda_{i} \neq \lambda_{j}$ if $i \neq j$, and $\prod_{i=1}^{n} \lambda_{i}^{\ell_{i}+1}=1$. Let $A=\left(a_{i j}\right)$ be the diagonal matrix with $a_{j j}=\lambda_{i}$ if $s_{i}<j \leq s_{i+1}$; then $A$ lies in $C_{G}(e)$. Hence

$$
Z\left(C_{G}(e)\right) \subseteq C_{G}(A)=\left(\mathrm{GL}\left(V_{1}\right) \oplus \cdots \oplus \mathrm{GL}\left(V_{n}\right)\right) \cap G .
$$

From the regular case, we see that if $z \in Z\left(C_{G}(e)\right)$ then we have $z=\sum_{i=1}^{n} \sum_{j=0}^{\ell_{i}} \mu_{i j} e_{i}^{j}$ for some $\mu_{i j} \in k$. By Theorem 2.1, the elements of $Z\left(C_{G}(e)\right)^{\circ}$ are unipotent, so we have $\mu_{i 0}=1$ for all $i$.

Now given $1 \leq i<n$, set $C_{i}=I+\sum_{h=1}^{\ell_{i+1}+1} E_{s_{i-1}+h, s_{i}+h}$; then we find that $C_{i} \in C_{G}(e)$. Thus if $z \in Z\left(C_{G}(e)\right)^{\circ}$ we must have $z C_{i}=C_{i} z$; equating entries gives $\mu_{i j}=\mu_{i+1, j}$ for all $1 \leq j \leq \ell_{i+1}$. Letting $i$ vary, we see that $z$ is of the form $I+v$ for some $v \in\left\langle e, e^{2}, \ldots, e^{\ell_{1}}\right\rangle$. Conversely, any such matrix clearly lies in $Z\left(C_{G}(e)\right)^{\circ}$; so we have

$$
Z\left(C_{G}(e)\right)^{\circ}=\left\{I+\mu_{1} e+\mu_{2} e^{2}+\cdots+\mu_{\ell_{1}} e^{\ell_{1}}: \mu_{1}, \mu_{2}, \ldots, \mu_{\ell_{1}} \in k\right\} .
$$

Hence we have

$$
\operatorname{dim} Z\left(C_{G}(e)\right)=\operatorname{dim} Z\left(C_{G}(e)\right)^{\circ}=\ell_{1}=\xi_{1}=\left\lceil\frac{1}{2} \sum a_{j}\right\rceil
$$

as required.

It remains to consider Theorem 1(ii). The only distinguished orbit in $\mathfrak{L}(G)$ is that containing regular nilpotent elements; a result of Kostant in [16] says that if $e$ is regular nilpotent then the $\tau$-weights in $C_{\mathfrak{L}(G)}(e)$ are $2 d_{1}-2, \ldots, 2 d_{\ell}-2$, where $d_{1}<\cdots<d_{\ell}$ are the degrees of the invariant polynomials of the Weyl group of $G$. This completes the treatment of groups $G$ of type $A_{\ell}$.

\section{2 $G$ of type $B_{\ell}, C_{\ell}$ or $D_{\ell}$}

Let $G$ be of type $B_{\ell}, C_{\ell}$ or $D_{\ell}$, so that the characteristic of $k$ is not 2. By Proposition 2.13 we may assume $G=\mathrm{SO}(V), \operatorname{Sp}(V)$ or $\mathrm{SO}(V)$, where $V$ is a vector space over $k$ of dimension $2 \ell+1,2 \ell$ or $2 \ell$ respectively; then $\mathfrak{L}(G)=\mathfrak{s o}(V), \mathfrak{s p}(V)$ or $\mathfrak{s o}(V)$, and the 
action of $G$ on $\mathfrak{L}(G)$ is given by conjugation. Take a nilpotent element $e \in \mathfrak{L}(G)$, with associated cocharacter $\tau$ and labelled diagram $\Delta$ having labels $a_{1}, \ldots, a_{\ell}$; let $\Delta_{0}$ be the 2 -free core of $\Delta$, with corresponding algebraic group $G_{0}$. From $[15,3.5$ and 5.4] we see that the collection of $\tau$-weights on $V$ is the union (with multiplicities) of the sets $\{d-1, d-3, \ldots, 3-d, 1-d\}$, where the union runs over the Jordan blocks of $e$ on $V$ and $d$ is the size of the block. Write this collection of $\tau$-weights as $\xi_{1} \geq \xi_{2} \geq \cdots$. From [6, pp.394-396] we see that, with one exception, the $\xi_{i}$ determine the labels of $\Delta$ as follows: for $1 \leq i \leq \ell-1$ we have $a_{i}=\xi_{i}-\xi_{i+1}$, while $a_{\ell}=\xi_{\ell}, 2 \xi_{\ell}$ or $\xi_{\ell-1}+\xi_{\ell}$ according as $G$ is of type $B_{\ell}, C_{\ell}$ or $D_{\ell}$; the single exception is that in type $D_{\ell}$ the labels $a_{\ell-1}$ and $a_{\ell}$, corresponding to the last two nodes of the diagram, may be interchanged if $\xi_{\ell}>0$.

We begin by establishing the existence statements of Theorems 3 and 4 . Let $S_{2}(\Delta)=$ $\left\{i: a_{i}=2\right\}$. If $S_{2}(\Delta)$ is empty, then $\Delta_{0}=\Delta$ and we may take $e_{0}=e$ and $\Gamma_{0}=\Delta$; so assume $S_{2}(\Delta)$ is non-empty and let $j$ be its maximal element.

First suppose $j<\ell$; then we have $2=a_{j}=\xi_{j}-\xi_{j+1}$, and hence $e$ has no $\tau$-weight $\xi_{j+1}+1$. Just as in $\S 4.1$, we see that if $i<j$ then $a_{i}=\xi_{i}-\xi_{i+1} \in\{0,2\}$. Thus we may let $\Gamma_{0}$ be the connected component of $\Delta_{0}$ with nodes $\alpha_{j+1}, \ldots, \alpha_{\ell}$, and then all labels in $\Delta_{0} \backslash \Gamma_{0}$ are 0 . Moreover if each Jordan block size greater than $\xi_{j+1}+1$ is replaced by $\xi_{j+1}+1$, then the parity of the number of blocks of a given size is unchanged, and so the set of numbers obtained (with multiplicities) is the list of Jordan block sizes of a nilpotent element $e_{0}$ of $\mathfrak{L}\left(H_{0}\right)$, where $H_{0}$ is the simple factor of $G_{0}$ corresponding to $\Gamma_{0}$; and the labelled diagram of $e_{0}$, regarded as an element of $\mathfrak{L}\left(G_{0}\right)$, is $\Delta_{0}$.

Now suppose $j=\ell$. If $G$ is of type $B_{\ell}$ then $\xi_{\ell}=2$, while $\xi_{\ell+1}=0$; if $G$ is of type $C_{\ell}$ then $\xi_{\ell}=1$, and so $\xi_{\ell+1}=-\xi_{\ell}=-1$; if $G$ is of type $D_{\ell}$ then $\xi_{\ell-1}+\xi_{\ell}=2$, so $\left(\xi_{\ell-1}, \xi_{\ell}\right)=(1,1)$ or $(2,0)$. In all cases it follows that all $\tau$-weights have the same parity; so again for all $i$ we have $a_{i} \in\{0,2\}$. Hence we may take $e_{0}=0$, and $\Gamma_{0}$ to be any component of $\Delta_{0}$ (provided $\Delta_{0}$ is not the empty diagram). This proves the existence statements of Theorems 3 and 4 .

We now turn to the proof of the dimension formula in Theorem 4. This states that

$$
\operatorname{dim} Z\left(C_{G}(e)\right)=\left\lceil\frac{1}{2} \sum a_{i}\right\rceil+\epsilon, \quad \text { where } \epsilon=\left\{\begin{array}{ll}
-1 & \text { if } \Gamma_{0}=1 \cdots 1 \\
0 & \text { otherwise }
\end{array},\right.
$$

recall that $\Gamma_{0}$ is a connected component of $\Delta_{0}$ such that all labels in $\Delta_{0} \backslash \Gamma_{0}$ are 0 , and dots in labelled diagrams are used to denote an arbitrary number of unspecified labels, so that $1 \ldots{ }_{1}^{1}$ represents any labelled diagram of type $D_{\ell}$ in which the labels of the three endnodes are 1 . Note that we have

$$
\sum a_{i}= \begin{cases}\xi_{1} & \text { if } G \text { is of type } B_{\ell}, \\ \xi_{1}+\xi_{\ell} & \text { if } G \text { is of type } C_{\ell}, \\ \xi_{1}+\xi_{\ell-1} & \text { if } G \text { is of type } D_{\ell} .\end{cases}
$$

Let the Jordan blocks of $e$ on $V$ have sizes $\ell_{1}+1, \ldots, \ell_{n}+1$, where $\ell_{1} \geq \cdots \geq \ell_{n}$ and $\sum_{i=1}^{n}\left(\ell_{i}+1\right)=\ell+1$. There are then vectors $w_{1}, \ldots, w_{n} \in V$, with $e^{\ell_{i}+1} w_{i}=0$ for $1 \leq i \leq n$, such that

$$
\left\{e^{s} w_{i}: 1 \leq i \leq n, 0 \leq s \leq \ell_{i}\right\}
$$

is a basis for $V$. Write $V_{i}=\left\langle w_{i}, e w_{i}, \ldots, e^{\ell_{i}} w_{i}\right\rangle$, so that $V=V_{1} \oplus \cdots \oplus V_{n}$. In what follows, we treat elements of both $G$ and $\mathfrak{L}(G)$ as matrices with respect to the given basis.

We shall make use of work of Yakimova in [51], which identifies the centre of $C_{\mathfrak{L}(G)}(e)$, correcting Proposition 3.5 of [17]. In order to state her result, we must introduce some notation, which in places will differ slightly from hers to avoid clashing with what has already been established. 
Let ( , ) be the non-degenerate symmetric or skew-symmetric bilinear form on $V$ stabilized by $G$; then $\mathfrak{L}(G)$ is the set of $\zeta \in \mathfrak{L}(\operatorname{GL}(V))$ satisfying $(\zeta v, w)=-(v, \zeta w)$ for all $v, w \in V$. As is well known (see for example $[15,1.11]$ ), the vectors $w_{i}$ may be chosen such that there is an involution $i \mapsto i^{\prime}$ on the set $\{1, \ldots, m\}$ satisfying the following conditions:

- $\ell_{i}=\ell_{i^{\prime}}$

- $\left(e^{s} w_{i}, e^{t} w_{j}\right)=0$ unless $j=i^{\prime}$ and $s+t=n_{i}$;

- $i=i^{\prime}$ if and only if $\ell_{i}$ is even if $G$ is of type $B_{\ell}$ or $D_{\ell}$, or odd if $G$ is of type $C_{\ell}$.

The restriction of the form ( , ) to the subspace $V_{i}+V_{i^{\prime}}$ is then non-degenerate. We scale the vectors to ensure that $\left(w_{i}, e^{\ell_{i}} w_{i^{\prime}}\right)=1$ if $i \leq i^{\prime}$.

Given $1 \leq i, j \leq m$ and $s \in \mathbb{Z}$ satisfying $\max \left\{\ell_{j}-\ell_{i}, 0\right\} \leq s \leq \ell_{j}$, we define $\zeta_{i}^{j, s} \in$ $\mathfrak{L}(\mathrm{GL}(V))$ to be the unique map commuting with $e$ which sends $w_{i}$ to $e^{s} w_{j}$ and all other $w_{h}$ to 0 . The maps $\zeta_{i}^{j, s}$ then form a basis of $C_{\mathfrak{L}(\mathrm{GL}(V))}(e)$; with appropriate choices of signs, the maps $\zeta_{i}^{j, \ell_{j}-s} \pm \zeta_{j^{\prime}}^{i^{\prime}, \ell_{i}-s}$ which are non-zero, where $0 \leq s \leq \min \left\{\ell_{i}, \ell_{j}\right\}$, form a basis of $C_{\mathfrak{L}(G)}(e)$.

From $\left[15,3.5\right.$ and 5.4] we see that for all $1 \leq i \leq n$ and $0 \leq s \leq \ell_{i}$, and all $c \in k^{*}$, we have $\tau(c) . e^{s} w_{i}=c^{2 s-n_{i}} e^{s} w_{i}$. Thus for all relevant $i, j, s, t$, and all $c \in k^{*}$, we have

$$
\begin{aligned}
\left((\operatorname{Ad} \tau(c)) \zeta_{i}^{j, s}\right) \cdot e^{t} w_{i} & =\tau(c) \zeta_{i}^{j, s} \tau(c)^{-1} \cdot e^{t} w_{i}=\tau(c) \zeta_{i}^{j, s} \cdot c^{n_{i}-2 t} e^{t} w_{i} \\
& =c^{n_{i}-2 t} \tau(c) \zeta_{i}^{j, s} \cdot e^{t} w_{i}=c^{n_{i}-2 t} \tau(c) \cdot e^{s+t} w_{j} \\
& =c^{n_{i}-2 t} c^{2 s+2 t-n_{j}} e^{s+t} w_{j}=c^{2 s+n_{i}-n_{j}} \zeta_{i}^{j, s} \cdot e^{t} w_{i}
\end{aligned}
$$

as any basis vector $e^{t} w_{h}$ with $h \neq i$ is sent to 0 by both $(\operatorname{Ad} \tau(c)) \zeta_{i}^{j, s}$ and $\zeta_{i}^{j, s}$, we have $(\operatorname{Ad} \tau(c)) \zeta_{i}^{j, s}=c^{2 s+n_{i}-n_{j}} \zeta_{i}^{j, s}$. Therefore the $\tau$-weight of $\zeta_{i}^{j, s}$ is $2 s+\ell_{i}-\ell_{j}$, and hence that of $\zeta_{i}^{j, \ell_{j}-s} \pm \zeta_{j^{\prime}}^{i^{\prime}, \ell_{i}-s}$ is $\left(\ell_{i}-s\right)+\left(\ell_{j}-s\right)$.

Let $Y=\left\langle e, e^{3}, e^{5}, \ldots\right\rangle$; observe that $\operatorname{dim} Y=\left\lceil\frac{1}{2} \xi_{1}\right\rceil$, and that $Y \subset \mathfrak{L}(G)$. The result of Yakimova may then be stated as follows.

Theorem 4.1 ([51, Theorem 2]) With the notation established, if $G$ is of type $B_{\ell}$ or $D_{\ell}$, and $\ell_{1}$ and $\ell_{2}$ are both even with $\ell_{2}>\ell_{3}$, then we have $Z\left(C_{\mathfrak{L}(G)}(e)\right)=Y \oplus\langle x\rangle$, where $x=\zeta_{1}^{2, \ell_{2}}-\zeta_{2}^{1, \ell_{1}}$; in all other cases we have $Z\left(C_{\mathfrak{L}(G)}(e)\right)=Y$.

For convenience we refer to blocks of odd size as 'odd blocks' and blocks of even size as 'even blocks'; thus from Theorem 4.1 we see that $Z\left(C_{\mathfrak{L}(G)}(e)\right)$ properly contains $Y$ precisely when $G$ is of type $B_{\ell}$ or $D_{\ell}$, and the two largest blocks of $e$ are odd and are strictly larger than all others.

As $e^{j}$ has $\tau$-weight $2 j$, and $\zeta_{1}^{2, \ell_{2}}-\zeta_{2}^{1, \ell_{1}}$ has $\tau$-weight $\ell_{1}+\ell_{2}$, Theorem 4.1 also gives $Z\left(C_{\mathfrak{L}(G)}(e)\right) \subseteq \mathfrak{L}(G)_{+}$; thus by Theorem 3.9 we have $\mathfrak{L}\left(Z\left(C_{G}(e)\right)\right)=\left(Z\left(C_{\mathfrak{L}(G)}(e)\right)\right)^{C}$, where $C=C_{G}(\operatorname{im}(\tau)) \cap C_{G}(e)$. So in order to prove that the conclusion of Theorem 4 holds for the groups treated here, we must show that in each case we have

$$
\operatorname{dim}\left(Z\left(C_{\mathfrak{L}(G)}(e)\right)\right)^{C}=\left\lceil\frac{1}{2} \sum a_{i}\right\rceil+\epsilon .
$$

We must thus consider the action of $C$ on $Z\left(C_{\mathfrak{L}(G)}(e)\right)$; as the action of $G$ on its Lie algebra is by conjugation, any element of $G$ which fixes $e$ will clearly fix any power $e^{j}$, whence certainly $Y \subseteq Z\left(C_{\mathfrak{L}(G)}(e)\right)^{C}$. Therefore to determine $Z\left(C_{\mathfrak{L}(G)}(e)\right)^{C}$ it only remains to consider those cases in which $Z\left(C_{\mathfrak{L}(G)}(e)\right)=Y \oplus\langle x\rangle$; so $G$ will be of type $B_{\ell}$ or $D_{\ell}$. 
Proposition 4.2 Assume $Z\left(C_{\mathfrak{L}(G)}(e)\right)=Y \oplus\langle x\rangle$, with $x$ as given in Theorem 4.1; then $x \in\left(Z\left(C_{\mathfrak{L}(G)}(e)\right)\right)^{C}$ if and only if e has exactly two odd blocks.

Proof. First assume there exists some $i>2$ with $\ell_{i}$ even, so that $e$ has a third odd block; let $g$ be the element of $\mathrm{GL}(V)$ which acts as -1 on $V_{1} \oplus V_{i}$ and as 1 on $\bigoplus_{j \neq 1, i} V_{j}$. As each of $V_{1}$ and $V_{i}$ is orthogonal to all other blocks, it is clear that $g$ stabilizes the bilinear form ( , ); as $g$ has determinant 1 we have $g \in G$. Since $g$ acts as a scalar on each block, it lies in $C_{G}(e)$; as $g$ commutes with $\operatorname{im}(\tau)$ it lies in $C$. However, as $g x g^{-1}$ maps $w_{1}$ and $w_{2}$ to $-e^{\ell_{2}} w_{2}$ and $e^{\ell_{1}} w_{1}$ respectively, and sends all other $w_{j}$ to 0 , we have $g x g^{-1}=-x ;$ so $x \notin\left(Z\left(C_{\mathfrak{L}(G)}(e)\right)\right)^{C}$.

Now assume $\ell_{i}$ is odd for all $i>2$, so that $e$ has only the two odd blocks; write $d=\frac{1}{2}\left(\ell_{1}+\ell_{2}\right)$. Set $V^{\prime}=V_{1} \oplus V_{2}$ and $V^{\prime \prime}=V_{3} \oplus \cdots \oplus V_{n}$, so that $V=V^{\prime} \oplus V^{\prime \prime}$. The choice of the vectors $w_{i}$ means that both $V^{\prime}$ and $V^{\prime \prime}$ are non-degenerate subspaces. Let $J$ be the subgroup of $G$ preserving $V^{\prime}$ and fixing $V^{\prime \prime}$ pointwise, and $H$ be the subgroup of $G$ fixing $V^{\prime}$ pointwise and preserving $V^{\prime \prime}$; then $J$ is of type $D_{d+1}$ and $H$ is of type $D_{\ell-d-1}$. We have $e \in \mathfrak{L}(J H)=\mathfrak{L}(J) \oplus \mathfrak{L}(H)$; let $e_{J}$ and $e_{H}$ be the projections of $e$ in $\mathfrak{L}(J)$ and $\mathfrak{L}(H)$, and note that $x \in \mathfrak{L}(J)$. We have $\operatorname{im}(\tau) \subseteq J H$; define $\tau_{J}: k^{*} \rightarrow J$ and $\tau_{H}: k^{*} \rightarrow H$ by letting $\tau_{J}(c)$ and $\tau_{H}(c)$ be the projections of $\tau(c)$ in $J$ and $H$ for all $c \in k^{*}$.

Recall that $\mathfrak{L}(C)=C_{\mathfrak{L}(G)}(e)_{0}$. As mentioned above, with appropriate choices of signs a basis of $C_{\mathfrak{L}(G)}(e)$ is given by the vectors $\zeta_{i}^{j, \ell_{j}-s} \pm \zeta_{j^{\prime}}^{i^{\prime}, \ell_{i}-s}$, of $\tau$-weight $\left(\ell_{i}-s\right)+\left(\ell_{j}-s\right)$; since this value is only 0 if $\ell_{i}=s=\ell_{j}$, which as $\ell_{2}>\ell_{3}$ means that either $i, j \leq 2$ or $i, j \geq 3$, it follows that $C_{\mathfrak{L}(G)}(e)_{0}=C_{\mathfrak{L}(J)}\left(e_{J}\right)_{0} \oplus C_{\mathfrak{L}(H)}\left(e_{H}\right)_{0}$. We shall identify both summands as the Lie algebras of certain subgroups of $J$ and $H$ respectively.

We begin with $C_{\mathfrak{L}(H)}\left(e_{H}\right)_{0}$. Set $K=C_{H}\left(e_{H}\right)=C_{H}(e)$; then we have $C_{K}\left(\operatorname{im}\left(\tau_{H}\right)\right)=$ $C_{K}(\operatorname{im}(\tau))=K \cap L_{\tau}$. As $\operatorname{im}\left(\tau_{H}\right)$ is a torus of $H$ which normalizes $K$, by [3, p.229] we have $C_{\mathfrak{L}(K)}\left(\operatorname{im}\left(\tau_{H}\right)\right)=\mathfrak{L}\left(C_{K}\left(\operatorname{im}\left(\tau_{H}\right)\right)\right)$; moreover Theorem 2.3 gives $C_{\mathfrak{L}(H)}\left(e_{H}\right)=\mathfrak{L}(K)$. Thus

$$
C_{\mathfrak{L}(H)}\left(e_{H}\right)_{0}=C_{C_{\mathfrak{L}(H)}\left(e_{H}\right)}\left(\operatorname{im}\left(\tau_{H}\right)\right)=C_{\mathfrak{L}(K)}\left(\operatorname{im}\left(\tau_{H}\right)\right)=\mathfrak{L}\left(C_{K}\left(\operatorname{im}\left(\tau_{H}\right)\right)\right)=\mathfrak{L}\left(K \cap L_{\tau}\right) .
$$

We now turn to $C_{\mathfrak{L}(J)}\left(e_{J}\right)_{0}$. For $i \in\{1,2\}$ we have $i^{\prime}=i$ since $\ell_{i}$ is even, and it follows that the sign in the vector $\zeta_{i}^{j, \ell_{j}-s} \pm \zeta_{j}^{i, \ell_{i}-s}$ is $(-1)^{s+1}$. Hence if $\ell_{1}>\ell_{2}$ then $C_{\mathfrak{L}(J)}\left(e_{J}\right)_{0}=0$, while if $\ell_{1}=\ell_{2}$ then $C_{\mathfrak{L}(J)}\left(e_{J}\right)_{0}=\langle y\rangle$ where $y=\zeta_{1}^{2,0}-\zeta_{2}^{1,0}$.

Thus suppose for the moment that $\ell_{1}=\ell_{2}=d$. Again, from [15, 3.5 and 5.4] we see that for $j \in\{1,2\}$ and $0 \leq s \leq d$, and for all $c \in k^{*}$, we have $\tau_{J}(c) \cdot e^{s} w_{j}=c^{2 s-d} e^{s} w_{j}$. Take $i \in k$ with $i^{2}=-1$, and define $\lambda: k^{*} \rightarrow J$ as follows: for $c \in k^{*}$ let $\lambda(c)$ be the linear map satisfying

$$
\begin{aligned}
e^{s} w_{1} & \mapsto \frac{1}{2}\left(c+c^{-1}\right) e^{s} w_{1}-\frac{i}{2}\left(c-c^{-1}\right) e^{s} w_{2}, \\
e^{s} w_{2} & \mapsto \frac{i}{2}\left(c-c^{-1}\right) e^{s} w_{1}+\frac{1}{2}\left(c+c^{-1}\right) e^{s} w_{2},
\end{aligned}
$$

for $0 \leq s \leq d$. Write $T_{1}=\operatorname{im}(\lambda)$. A straightforward check reveals that each $\lambda(c)$ preserves the bilinear form $($,$) and has determinant 1$, so $T_{1} \subseteq J$; it is immediate that each $\lambda(c)$ commutes with $e_{J}$, so $T_{1} \subseteq C_{J}\left(e_{J}\right)$ and thus $\mathfrak{L}\left(T_{1}\right) \subseteq \mathfrak{L}\left(C_{J}\left(e_{J}\right)\right)=C_{\mathfrak{L}(J)}\left(e_{J}\right)$. For each $c \in k^{*}$, the map $\lambda(c)$ preserves the subspaces $\left\langle e^{s} w_{1}, e^{s} w_{2}\right\rangle$ for $0 \leq s \leq d$, upon each of which $\operatorname{im}\left(\tau_{J}\right)$ acts as a scalar, so for all $c, c^{\prime} \in k^{*}$ we have $\left[\lambda(c), \tau_{J}\left(c^{\prime}\right)\right]=1$; thus we have $T_{1} \subseteq C_{J}\left(\operatorname{im}\left(\tau_{J}\right)\right)$, whence $\mathfrak{L}\left(T_{1}\right) \subseteq \mathfrak{L}\left(C_{J}\left(\operatorname{im}\left(\tau_{J}\right)\right)=C_{\mathfrak{L}(J)}\left(\operatorname{im}\left(\tau_{J}\right)\right)\right.$, and so $\mathfrak{L}\left(T_{1}\right) \subseteq C_{\mathfrak{L}(J)}\left(e_{J}\right)_{0}$. Since $\operatorname{dim} C_{\mathfrak{L}(J)}\left(e_{J}\right)_{0}=1$ we must have $\mathfrak{L}\left(T_{1}\right)=C_{\mathfrak{L}(J)}\left(e_{J}\right)_{0}$. 
Now return to the general case of $\ell_{1} \geq \ell_{2}$. Set $T_{J}=1$ or $T_{1}$ according as $\ell_{1}>\ell_{2}$ or $\ell_{1}=\ell_{2}$; then by the above we have

$$
\begin{aligned}
\mathfrak{L}(C)=C_{\mathfrak{L}(G)}(e)_{0} & =C_{\mathfrak{L}(J)}\left(e_{J}\right)_{0} \oplus C_{\mathfrak{L}(H)}\left(e_{H}\right)_{0} \\
& =\mathfrak{L}\left(T_{J}\right) \oplus \mathfrak{L}\left(K \cap L_{\tau}\right)=\mathfrak{L}\left(T_{J} .\left(K \cap L_{\tau}\right)\right) .
\end{aligned}
$$

Now $K \cap L_{\tau}=C_{H}(e) \cap L_{\tau} \subseteq C_{G}(e) \cap L_{\tau}=C$, and if $\ell_{1}=\ell_{2}$ then $T_{1} \subseteq C_{J}\left(e_{J}\right) \cap L_{\tau} \subseteq$ $C_{G}(e) \cap L_{\tau}=C$; thus $T_{J} .\left(K \cap L_{\tau}\right) \subseteq C$. Hence $C^{\circ}=\left(T_{J} .\left(K \cap L_{\tau}\right)\right)^{\circ}$; by [6, p.399] we see that in the adjoint group the centralizer of $e$ is connected, so that here we have $C=C^{\circ} \cdot\{ \pm I\}=\left(T_{J} \cdot\left(K \cap L_{\tau}\right)\right)^{\circ} \cdot\{ \pm I\}$. Since $x$ lies in $\mathfrak{L}(J)$ it is fixed by $K \cap L_{\tau} ;$ if $\ell_{1}=\ell_{2}$, an easy check shows that each $\lambda(c)$ commutes with $x$, so that $x$ is also fixed by $T_{J}$; and certainly $x$ is fixed by $-I$. Thus $x$ is fixed by $C$, and we have $x \in\left(Z\left(C_{\mathfrak{L}(G)}(e)\right)\right)^{C}$ as required.

In order to establish the equality $\operatorname{dim}\left(Z\left(C_{\mathfrak{L}(G)}(e)\right)\right)^{C}=\left\lceil\frac{1}{2} \sum a_{i}\right\rceil+\epsilon$, we may now work through the possibilities in turn.

First assume $G$ is of type $B_{\ell}$; then evidently $\epsilon=0$. Since the number of odd blocks is odd, by Proposition 4.2 we have

$$
\operatorname{dim}\left(Z\left(C_{\mathfrak{L}(G)}(e)\right)\right)^{C}=\operatorname{dim} Y=\left\lceil\frac{1}{2} \xi_{1}\right\rceil=\left\lceil\frac{1}{2} \sum a_{i}\right\rceil+\epsilon .
$$

Next assume $G$ is of type $C_{\ell}$; again $\epsilon=0$. As $0 \leq \xi_{\ell}-\xi_{\ell+1} \leq 2$ and $\xi_{\ell+1}=-\xi_{\ell}$, we have $\xi_{\ell} \in\{0,1\}$. If $\xi_{\ell}=0$ then certainly we have

$$
\operatorname{dim}\left(Z\left(C_{\mathfrak{L}(G)}(e)\right)\right)^{C}=\operatorname{dim} Y=\left\lceil\frac{1}{2} \xi_{1}\right\rceil=\left\lceil\frac{1}{2} \sum a_{i}\right\rceil+\epsilon .
$$

If instead $\xi_{\ell}=1$ then all weights are odd, and in particular $\xi_{1}$ is odd; so we have

$$
\operatorname{dim}\left(Z\left(C_{\mathfrak{L}(G)}(e)\right)\right)^{C}=\operatorname{dim} Y=\left\lceil\frac{1}{2} \xi_{1}\right\rceil=\left\lceil\frac{1}{2}\left(\xi_{1}+1\right)\right\rceil=\left\lceil\frac{1}{2} \sum a_{i}\right\rceil+\epsilon .
$$

Finally assume $G$ is of type $D_{\ell}$. As above we have $\xi_{\ell} \in\{0,1\}$; since $0 \leq \xi_{\ell-1}-\xi_{\ell} \leq 2$, and odd weights must occur with even multiplicity, we have four possibilities for the ordered pair $\left(\xi_{\ell-1}, \xi_{\ell}\right):(\mathrm{a})(0,0) ;(\mathrm{b})(1,0) ;(\mathrm{c})(2,0) ;(\mathrm{d})(1,1)$.

(a) If $\left(\xi_{\ell-1}, \xi_{\ell}\right)=(0,0)$ then there are at least four 0 weights, so at least four odd blocks; since $\left(a_{\ell-1}, a_{\ell}\right)=(0,0)$, we have $\epsilon=0$. Thus by Proposition 4.2 we have

$$
\operatorname{dim}\left(Z\left(C_{\mathfrak{L}(G)}(e)\right)\right)^{C}=\operatorname{dim} Y=\left\lceil\frac{1}{2} \xi_{1}\right\rceil=\left\lceil\frac{1}{2} \sum a_{i}\right\rceil+\epsilon
$$

(b) If $\left(\xi_{\ell-1}, \xi_{\ell}\right)=(1,0)$ then there are exactly two 0 weights and some odd weights, so there are exactly two odd blocks and some even blocks; let the high weights of the odd blocks be $2 s$ and $2 t$ with $s \geq t$, and the high weight of the largest even block be $2 r+1$. We have $\left(a_{\ell-1}, a_{\ell}\right)=(1,1)$, so we must consider further to decide the value of $\epsilon$ : we see that $\Gamma_{0}=1 \cdots \frac{1}{1}$ if and only if there exists $i \leq \ell-3$ satisfying $\xi_{i}-\xi_{i+1}=1$, where if $i>1$ we also require $\xi_{i-1}-\xi_{i}=2$. For this to be true, the multiplicity of the weight $\xi_{i}$ must be one, forcing $\xi_{i}$ to be even; so $\xi_{i+1}$ must be the highest odd weight $2 r+1$. It follows that

$$
\epsilon=-1 \Longleftrightarrow 2 s>2 r+1>2 t
$$

We therefore have three subcases. 
(i) If $2 r+1>2 s \geq 2 t$ then $\epsilon=0$ and $\xi_{1}=2 r+1$ is odd; as $Z\left(C_{\mathfrak{L}(G)}(e)\right)=Y$, we have

$$
\operatorname{dim}\left(Z\left(C_{\mathfrak{L}(G)}(e)\right)\right)^{C}=\operatorname{dim} Y=\left\lceil\frac{1}{2} \xi_{1}\right\rceil=\left\lceil\frac{1}{2}\left(\xi_{1}+1\right)\right\rceil=\left\lceil\frac{1}{2} \sum a_{i}\right\rceil+\epsilon .
$$

(ii) If $2 s>2 r+1>2 t$ then $\epsilon=-1$ and $\xi_{1}=2 s$ is even; as $Z\left(C_{\mathfrak{L}(G)}(e)\right)=Y$, we have

$$
\operatorname{dim}\left(Z\left(C_{\mathfrak{L}(G)}(e)\right)\right)^{C}=\operatorname{dim} Y=\left\lceil\frac{1}{2} \xi_{1}\right\rceil=\left\lceil\frac{1}{2}\left(\xi_{1}+1\right)\right\rceil-1=\left\lceil\frac{1}{2} \sum a_{i}\right\rceil+\epsilon .
$$

(iii) If $2 s \geq 2 t>2 r+1$ then $\epsilon=0$ and $\xi_{1}=2 s$ is even; as $Z\left(C_{\mathfrak{L}(G)}(e)\right)=Y \oplus\langle x\rangle$, by Proposition 4.2 we have

$$
\operatorname{dim}\left(Z\left(C_{\mathfrak{L}(G)}(e)\right)\right)^{C}=\operatorname{dim} Y+1=\left\lceil\frac{1}{2} \xi_{1}\right\rceil+1=\left\lceil\frac{1}{2}\left(\xi_{1}+1\right)\right\rceil=\left\lceil\frac{1}{2} \sum a_{i}\right\rceil+\epsilon .
$$

(c) If $\left(\xi_{\ell-1}, \xi_{\ell}\right)=(2,0)$ then there are exactly two 0 weights and no odd weights, so there are exactly two blocks, both of which are odd; since $\left(a_{\ell-1}, a_{\ell}\right)=(2,2)$, we have $\epsilon=0$. As $Z\left(C_{\mathfrak{L}(G)}(e)\right)=Y \oplus\langle x\rangle$, by Proposition 4.2 we have

$$
\operatorname{dim}\left(Z\left(C_{\mathfrak{L}(G)}(e)\right)\right)^{C}=\operatorname{dim} Y+1=\left\lceil\frac{1}{2} \xi_{1}\right\rceil+1=\left\lceil\frac{1}{2}\left(\xi_{1}+2\right)\right\rceil=\left\lceil\frac{1}{2} \sum a_{i}\right\rceil+\epsilon .
$$

(d) If $\left(\xi_{\ell-1}, \xi_{\ell}\right)=(1,1)$ then there are no 0 weights, so no odd blocks; since $\left(a_{\ell-1}, a_{\ell}\right)=$ $(2,0)$ or $(0,2)$, we have $\epsilon=0$. As $Z\left(C_{\mathfrak{L}(G)}(e)\right)=Y$, and $\xi_{1}$ is odd, we have

$$
\operatorname{dim}\left(Z\left(C_{\mathfrak{L}(G)}(e)\right)\right)^{C}=\operatorname{dim} Y=\left\lceil\frac{1}{2} \xi_{1}\right\rceil=\left\lceil\frac{1}{2}\left(\xi_{1}+1\right)\right\rceil=\left\lceil\frac{1}{2} \sum a_{i}\right\rceil+\epsilon
$$

We have therefore shown that in all cases the dimension formula in Theorem 4 holds.

It remains to consider Theorem 1(ii); thus assume now that $e$ is distinguished in $\mathfrak{L}(G)$. We have observed that the basis vectors $e, e^{3}, e^{5}, \ldots$ of $Y$ have $\tau$-weights $2,6,10, \ldots$, and $x=\zeta_{1}^{2, \ell_{2}}-\zeta_{2}^{1, \ell_{1}}$ has $\tau$-weight $\ell_{1}+\ell_{2}$. The degrees $d_{i}$ of the invariant polynomials of the Weyl group of $G$, ordered as in the statement of Theorem 1 , are

$$
\begin{cases}2,4,6, \ldots, 2 \ell-2,2 \ell & \text { if } G \text { is of type } B_{\ell} \text { or } C_{\ell} \\ 2,4,6, \ldots, 2 \ell-2, \ell & \text { if } G \text { is of type } D_{\ell}\end{cases}
$$

Thus if $G$ is of type $B_{\ell}$ or $C_{\ell}$, the first $n_{2}(\Delta)$ of the integers $2 d_{i}-2$ are indeed the $\tau$-weights on $\mathfrak{L}\left(Z\left(C_{G}(e)\right)\right)=Y$. We may therefore assume $G$ is of type $D_{\ell}$. Since $e$ is distinguished in $\mathfrak{L}(G)$, all its blocks are odd and of distinct sizes (see for example [50, Proposition 3.2]). If $e$ has more than two blocks, we are in case (a) above, where $\left(a_{\ell-1}, a_{\ell}\right)=(0,0)$ so that $\Delta=\cdots{ }_{0}^{0}$; here we again have $\mathfrak{L}\left(Z\left(C_{G}(e)\right)\right)=Y$, and the $\tau$-weights are simply the first $n_{2}(\Delta)$ of the integers $2 d_{i}-2$. If however $e$ has just two blocks, we are in case (c) above, where $\left(a_{\ell-1}, a_{\ell}\right)=(2,2)$ so that $\Delta=\ldots{ }_{2}^{2}$; this time we have $x \in \mathfrak{L}\left(Z\left(C_{G}(e)\right)\right)$ of $\tau$-weight $\ell_{1}+\ell_{2}=2 \ell-2=2 d_{\ell}-2$, and the remaining $\tau$-weights are the first $n_{2}(\Delta)-1$ of the integers $2 d_{i}-2$. This completes the proof of Theorem 1 (ii) for $G$ of type $B_{\ell}, C_{\ell}$ or $D_{\ell}$. 


\section{Exceptional groups: nilpotent orbit representatives}

From now on, unless otherwise stated $G$ will be a simple algebraic group of exceptional type defined over an algebraically closed field $k$ whose characteristic is either 0 or a good prime for $G$; again, $T$ will be a fixed maximal torus of $G$. In this section, we produce lists of elements, given in terms of $T$-root vectors, which will be shown to represent the non-zero nilpotent $G$-orbits. In view of the Bala-Carter-Pommerening classification (see Theorem 2.12), it is necessary to determine the $G$-classes of Levi subgroups of $G$.

In most instances, isomorphic Levi subgroups are conjugate, as may be seen from [2]. For exceptional groups, there are two types of cases involving pairs of isomorphic but non-conjugate Levi subgroups; we describe them in terms of the underlying root systems. In the groups $G_{2}$ and $F_{4}$ there are pairs where the root systems are of type $A_{1}, A_{2}$ or $A_{2}+A_{1}$ (the last two only occurring in $F_{4}$ ); in these the two may be distinguished by root length, and we use a tilde to denote a root system consisting of short roots. On the other hand, in $E_{7}$ there are pairs of non-conjugate Levi subgroups where the root systems are of type $3 A_{1}, A_{3}+A_{1}$ or $A_{5}$; in each instance exactly one of the two root systems has a conjugate contained in $\left\langle\alpha_{2}, \alpha_{4}, \alpha_{5}, \alpha_{6}, \alpha_{7}\right\rangle$, and following Dynkin in [10] we denote that particular Levi subgroup with a double prime superscript and the other with a single prime superscript.

We can now explain the notation used in the Bala-Carter-Pommerening classification of nilpotent orbits (and unipotent classes). Let $L$ be a Levi subgroup of $G$, with $[L, L]=$ $L_{1} \ldots L_{t}$ where each $L_{i}$ is simple. A distinguished orbit in $\mathfrak{L}(L)$ is represented by $e_{1}+$ $\cdots+e_{t}$, where each $e_{i}$ is a distinguished element in $\mathfrak{L}\left(L_{i}\right)$. In $\mathfrak{L}\left(L_{i}\right)$ the regular nilpotent orbit corresponds to the Borel subgroup, and is denoted by the type of $L_{i}$ (with a tilde if the root system of $L_{i}$ consists of short roots). For non-regular distinguished nilpotent orbits the notation used is given in Table 1; here each distinguished parabolic subgroup other than the Borel subgroup is indicated by giving the Dynkin diagram with black and white nodes, where the simple roots corresponding to the white nodes form a basis of the root system of the Levi factor. As is customary, the nilpotent orbit concerned is usually denoted $L_{i}\left(a_{j}\right)$ where $j$ is the number of white nodes; if however $L_{i}=E_{8}$ and $j \in\{4,5,6\}$ there are two such distinguished parabolic subgroups, and the corresponding nilpotent orbits are denoted $E_{8}\left(a_{j}\right)$ and $E_{8}\left(b_{j}\right)$. Finally the name of the orbit containing $e$ is obtained by combining those of the elements $e_{i}$; for example, if $t=2$ and the names of the orbits containing $e_{1}$ and $e_{2}$ are $A_{4}$ and $A_{2}$ respectively, then that of the orbit containing $e_{1}+e_{2}$ is $A_{4} A_{2}$. Note that we write $A_{1}^{2}$ for $A_{1} A_{1}$ etc.

We now proceed to obtain the desired lists. For each of the exceptional Lie algebras $\mathfrak{L}(G)$, the second column of Table 2 contains a collection of non-zero nilpotent elements, numbered in the first column for ease of reference. By calculating the Jordan block structure in the adjoint representation one can show that, in each of the exceptional Lie algebras, the nilpotent elements $e$ listed both represent distinct $G$-orbits and have $\operatorname{dim} C_{\mathfrak{L}(G)}(e)$ as given in the third column. Moreover the number of elements equals the number of non-zero nilpotent orbits in $\mathfrak{L}(G)$, the latter being given in good characteristic by the Bala-Carter-Pommerening classification. Therefore for each of the exceptional Lie algebras we have a set of non-zero nilpotent orbit representatives.

The fourth column of Table 2 contains the name of each orbit given by the BalaCarter-Pommerening classification; we must now show that these have been correctly assigned. Let $L$ be a Levi subgroup of $G$, and as before write $[L, L]=L_{1} \ldots L_{t}$ where each $L_{i}$ is simple. It suffices to treat the distinguished orbits in each of the $\mathfrak{L}\left(L_{i}\right)$.

If $L_{i}$ is of type $A_{1}$, with root system $\{ \pm \alpha\}$, clearly a nilpotent orbit representative in $\mathfrak{L}\left(L_{i}\right)$ may be taken to be $e_{\alpha}$. Now assume by induction on the rank of $L_{i}$ that all 
Table 1: Diagrams of distinguished parabolic subgroups

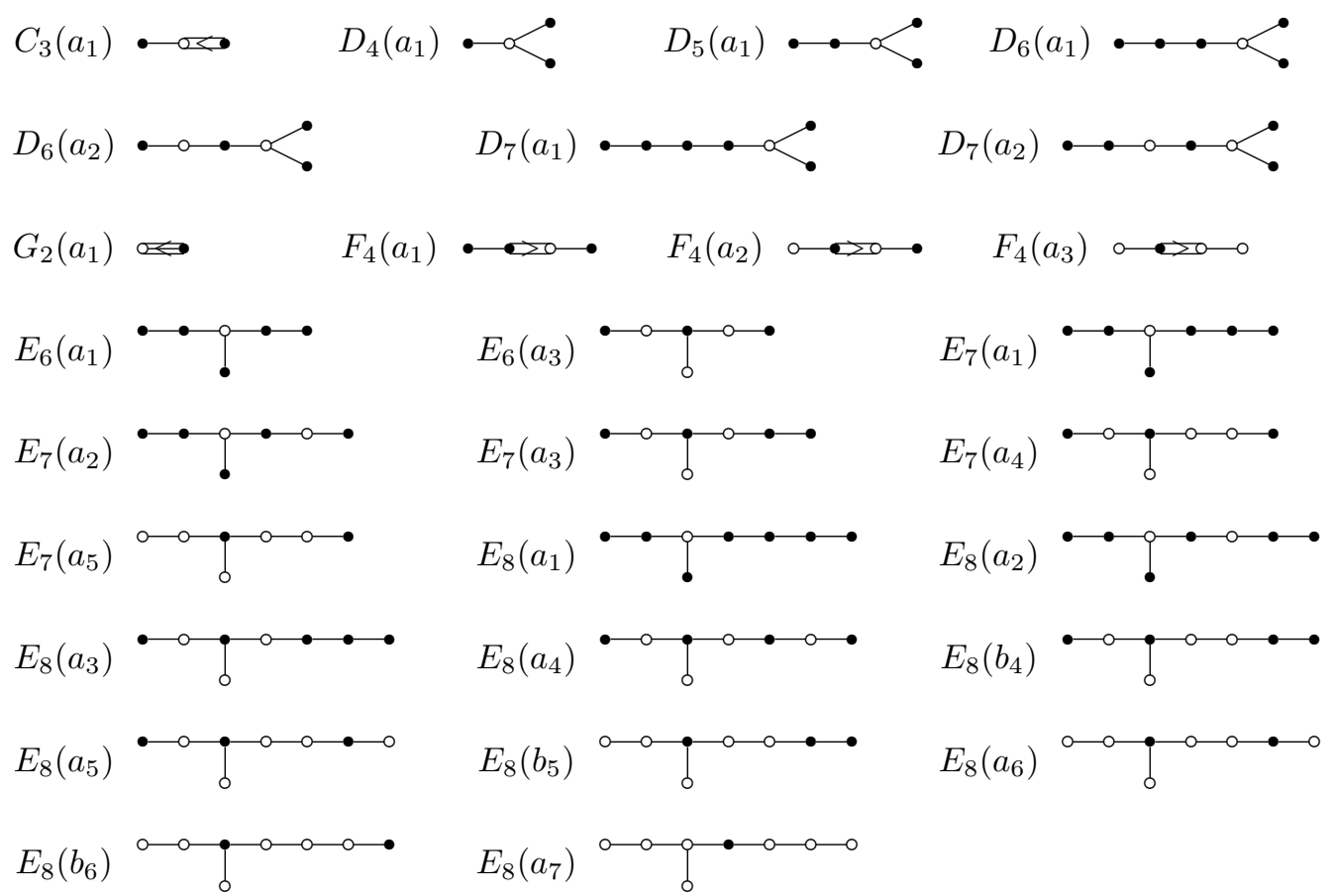

orbits lying in proper Levi subalgebras of $\mathfrak{L}\left(L_{i}\right)$ have been identified; this leaves just the distinguished nilpotent elements in $\mathfrak{L}\left(L_{i}\right)$ to consider. In each case we have a set of non-conjugate distinguished elements in bijection with the set of distinguished parabolic subgroups of $L_{i}$, in such a way that each given element lies in the Lie algebra of the unipotent radical of the corresponding parabolic subgroup. We may now appeal to the following result to deduce that our representatives of non-zero nilpotent orbits of $L_{i}$ are named correctly.

Lemma 5.1 ([50, Lemma 3.3]) Let $H$ be a simple algebraic group and $P_{1}, \ldots, P_{s}$ be a complete list of non-conjugate distinguished parabolic subgroups of $H$. Suppose $x_{1}, \ldots, x_{s}$ are non-conjugate distinguished nilpotent elements of $\mathfrak{L}(H)$ such that $x_{i} \in \mathfrak{L}\left(R_{u}\left(P_{i}\right)\right)$ for $i=1, \ldots, s$. Then $x_{i}$ lies in the dense orbit of $P_{i}$ on $\mathfrak{L}\left(R_{u}\left(P_{i}\right)\right)$ for $i=1, \ldots, s$.

Proof. For $i=1, \ldots, s$ let $\mathcal{O}_{i}$ be the distinguished nilpotent orbit in $\mathfrak{L}(H)$ such that $x_{i} \in \mathcal{O}_{i}$, and $\operatorname{Rich}\left(P_{i}\right)$ denote the nilpotent orbit containing the dense orbit of $P_{i}$ on $\mathfrak{L}\left(R_{u}\left(P_{i}\right)\right)$. As the $P_{i}$ and the $x_{i}$ form complete sets of distinguished parabolic subgroups and representatives of distinguished nilpotent orbits in $\mathfrak{L}(H)$ respectively, either we have $\mathcal{O}_{i}=\operatorname{Rich}\left(P_{i}\right)$ for all $i$, or there exists $j$ such that (after renumbering) we have $\mathcal{O}_{i}=$ $\operatorname{Rich}\left(P_{i+1}\right)$ for $i<j$ and $\mathcal{O}_{j}=\operatorname{Rich}\left(P_{1}\right)$. Now if $n \in \operatorname{Rich}\left(P_{i}\right)$ we have $\operatorname{dim} C_{H}(n)=$ $\operatorname{dim} C_{P_{i}}(n)=\operatorname{dim} P_{i}-\operatorname{dim} R_{u}\left(P_{i}\right)$, while if $n^{\prime} \in \mathfrak{L}\left(R_{u}\left(P_{i}\right)\right)$ with $n^{\prime} \notin \operatorname{Rich}\left(P_{i}\right)$ we have $\operatorname{dim} C_{H}\left(n^{\prime}\right) \geq \operatorname{dim} C_{P_{i}}\left(n^{\prime}\right)>\operatorname{dim} P_{i}-\operatorname{dim} R_{u}\left(P_{i}\right)$. Thus if $\mathcal{O}_{i}=\operatorname{Rich}\left(P_{i+1}\right)$ for $i<j$ and $\mathcal{O}_{j}=\operatorname{Rich}\left(P_{1}\right)$, we have $\operatorname{dim} C_{H}\left(x_{1}\right)<\operatorname{dim} C_{H}\left(x_{2}\right)<\cdots<\operatorname{dim} C_{H}\left(x_{j}\right)<\operatorname{dim} C_{H}\left(x_{1}\right)$, a contradiction; so we must have $\mathcal{O}_{i}=\operatorname{Rich}\left(P_{i}\right)$ for $i=1, \ldots, s$. 
Remark In fact, now that we have shown that the names in the fourth column of Table 2 are correct, it may be seen that in good characteristic the Jordan block structure of each nilpotent element $e$ is the same as that of the unipotent element having the same name; lists of these Jordan block sizes can be found in [18].

We conclude with some additional remarks about our choice of orbit representatives. Firstly, for the regular orbit in $\mathfrak{L}\left(L_{i}\right)$ we have simply taken the sum of the simple root vectors. In the majority of the remaining cases a non-regular distinguished nilpotent element of $\mathfrak{L}\left(L_{i}\right)$ is regular in a naturally defined subalgebra $\mathfrak{L}\left(H^{\sigma}\right)$, where $H$ is a subsystem subgroup of $L_{i}$ and $H^{\sigma}$ is its fixed point subgroup under a (possibly trivial) graph automorphism $\sigma$. If $L_{i}$ is of classical type, the orbits concerned are $C_{3}\left(a_{1}\right)$ and various $D_{r}\left(a_{j}\right)$ for $4 \leq r \leq 7$. An argument analogous to that of [50, Proposition 3.1] shows that the $C_{3}\left(a_{1}\right)$ orbit is represented by a regular element in a subalgebra of type $C_{2}+C_{1}$. Similarly, by [50, Proposition 3.2] the $D_{r}\left(a_{j}\right)$ orbit is represented by a regular element in a subalgebra of type $B_{r-j-1}+B_{j}$; such subalgebras are explicitly given in [50, p.67]. If $L_{i}$ is of exceptional type the orbits concerned are the following.

$\begin{array}{cc}\text { Orbit } & \text { Subalgebra } \\ G_{2}\left(a_{1}\right) & A_{2} \\ F_{4}\left(a_{3}\right) & A_{2} \tilde{A}_{2} \\ F_{4}\left(a_{2}\right) & C_{3} A_{1} \\ F_{4}\left(a_{1}\right) & B_{4} \\ E_{6}\left(a_{3}\right) & A_{5} A_{1} \\ E_{6}\left(a_{1}\right) & C_{4} \\ E_{7}\left(a_{5}\right) & A_{5} A_{2} \\ E_{7}\left(a_{4}\right) & B_{4} B_{1} A_{1} \\ E_{7}\left(a_{3}\right) & D_{6} A_{1} \\ E_{8}\left(a_{7}\right) & A_{4}{ }^{2} \\ E_{8}\left(b_{6}\right) & C_{4} A_{2} \\ E_{8}\left(a_{6}\right) & A_{8} \\ E_{8}\left(b_{5}\right) & E_{6} A_{2} \\ E_{8}\left(a_{5}\right) & B_{6} B_{1} \\ E_{8}\left(a_{4}\right) & D_{8} \\ E_{8}\left(a_{3}\right) & E_{7} A_{1}\end{array}$

This leaves five cases where a distinguished nilpotent element of $\mathfrak{L}\left(L_{i}\right)$ is not regular in any such naturally defined subalgebra, namely $E_{7}\left(a_{2}\right), E_{7}\left(a_{1}\right), E_{8}\left(b_{4}\right), E_{8}\left(a_{2}\right)$ and $E_{8}\left(a_{1}\right)$. Of these, the orbit $E_{8}\left(b_{4}\right)$ is represented by an element of the subalgebra $E_{7} A_{1}$ whose projections in the simple algebras lie in the orbits $E_{7}\left(a_{1}\right)$ and $A_{1}$ respectively. 
Table 2: Nilpotent orbit representatives

\begin{tabular}{|c|l|c|c|}
\hline \multicolumn{4}{|c|}{$G=G_{2}$} \\
\hline Orbit & $e$ & $\operatorname{dim} C_{\mathfrak{L}(G)}(e)$ & Name \\
\hline 1 & $e_{01}$ & 8 & $A_{1}$ \\
2 & $e_{10}$ & 6 & $\tilde{A}_{1}$ \\
3 & $e_{01}+e_{31}$ & 4 & $G_{2}\left(a_{1}\right)$ \\
4 & $e_{10}+e_{01}$ & 2 & $G_{2}$ \\
\hline
\end{tabular}

\begin{tabular}{|c|l|c|c|}
\hline \multicolumn{3}{|c|}{$G=F_{4}$} \\
\hline Orbit & $e$ & $\operatorname{dim} C_{\mathfrak{L}(G)}(e)$ & Name \\
\hline 1 & $e_{1000}$ & 36 & $A_{1}$ \\
2 & $e_{0001}$ & 24 & $\tilde{A}_{1}$ \\
3 & $e_{1000}+e_{0001}$ & $A_{1} \tilde{A}_{1}$ \\
4 & $e_{1000}+e_{0100}$ & $A_{2}$ \\
5 & $e_{0010}+e_{0001}$ & 22 & $\tilde{A}_{2}$ \\
6 & $e_{1000}+e_{0100}+e_{0001}$ & 18 & $A_{2} \tilde{A}_{1}$ \\
7 & $e_{0100}+e_{0010}$ & 16 & $B_{2}$ \\
8 & $e_{0010}+e_{0001}+e_{1000}$ & 16 & $\tilde{A}_{2} A_{1}$ \\
9 & $e_{0001}+e_{0120}+e_{0100}$ & 14 & $C_{3}\left(a_{1}\right)$ \\
10 & $e_{0100}+e_{1120}+e_{1110}+e_{0121}$ & 12 & $F_{4}\left(a_{3}\right)$ \\
11 & $e_{1000}+e_{0100}+e_{0010}$ & 10 & $B_{3}$ \\
12 & $e_{0001}+e_{0010}+e_{0100}$ & 10 & $C_{3}$ \\
13 & $e_{1110}+e_{0001}+e_{0120}+e_{0100}$ & 8 & $F_{4}\left(a_{2}\right)$ \\
14 & $e_{0100}+e_{1000}+e_{0120}+e_{0001}$ & 6 & $F_{4}\left(a_{1}\right)$ \\
15 & $e_{1000}+e_{0100}+e_{0010}+e_{0001}$ & 4 & $F_{4}$ \\
\hline
\end{tabular}


Table 2: Nilpotent orbit representatives (continued)

\begin{tabular}{|c|c|c|c|}
\hline \multicolumn{4}{|c|}{$G=E_{6}$} \\
\hline Orbit & $e$ & $\operatorname{dim} C_{\mathfrak{L}(G)}(e)$ & Name \\
\hline 1 & $e_{10000}$ & 56 & $A_{1}$ \\
\hline 2 & $e_{0}^{10000}+\underset{0}{e 0001}$ & 46 & $A_{1}^{2}$ \\
\hline 3 & $e_{0} \underset{0}{10000}+e_{00100}+e_{00001}$ & 38 & $A_{1}{ }^{3}$ \\
\hline 4 & $e_{0} \underset{0000}{100}+e_{01000}$ & 36 & $A_{2}$ \\
\hline 5 & $e_{0} \underset{0}{10000}+e_{01000}+e_{00000}$ & 32 & $A_{2} A_{1}$ \\
\hline 6 & $e_{0} \underset{0}{1000}+e_{01000}+e_{00010}+e_{00001}$ & 30 & $A_{2}{ }^{2}$ \\
\hline 7 & $e_{00000}+e_{0}^{00100}+e_{0}^{10000}+e_{00001}$ & 28 & $A_{2} A_{1}^{2}$ \\
\hline 8 & $e_{0}^{10000}+\underset{0}{e}+\underset{0}{01000}+e_{00100}$ & 26 & $A_{3}$ \\
\hline 9 & $e_{0} \underset{000}{0}+e_{01000}+e_{00010}+e_{00001}+e_{00000}$ & 24 & $A_{2}{ }^{2} A_{1}$ \\
\hline 10 & $e_{0} \underset{0}{1000}+e_{01000}+e_{00100}+e_{00001}$ & 22 & $A_{3} A_{1}$ \\
\hline 11 & $e_{01000}+e_{00100}+e_{00110}^{0}+e_{00000}+e_{00010}$ & 20 & $D_{4}\left(a_{1}\right)$ \\
\hline 12 & $e_{0}^{10000}+e_{0}^{01000}+e_{00100}+e_{00000}$ & 18 & $A_{4}$ \\
\hline 13 & $e_{01000}+e_{00100}+e_{00000}+e_{00010}$ & 18 & $D_{4}$ \\
\hline 14 & $e_{0} \underset{0}{10000}+e_{01000}+e_{00100}+e_{00000}+e_{00001}$ & 16 & $A_{4} A_{1}$ \\
\hline 15 & $e_{0} e_{10000}+e_{01000}+e_{00100}^{0}+e_{00010}^{0}+e_{00001}$ & 14 & $A_{5}$ \\
\hline 16 & $e_{0} \underset{0}{10000}+e_{01000}+e_{00100}+e_{0}^{00110}+e_{00000}+e_{00010}$ & 14 & $D_{5}\left(a_{1}\right)$ \\
\hline 17 & $e_{01100}+e_{0} \underset{10000}{e}+e_{01110}+e_{00001}^{0}+e_{00110}+e_{00100}$ & 12 & $E_{6}\left(a_{3}\right)$ \\
\hline 18 & $e_{0} \underset{0}{10000}+\underset{0}{e}+\underset{0}{01000}+e_{00100}+e_{00000}+e_{00010}^{0}$ & 10 & $D_{5}$ \\
\hline 19 & $\begin{array}{l}e_{10000}+e_{0} 00001+\underset{0}{0}+\underset{0}{0}+e_{0} 00010+e_{0}^{00110}+e_{01100} \\
\quad+e_{00000} \\
\quad 1\end{array}$ & 8 & $E_{6}\left(a_{1}\right)$ \\
\hline 20 & 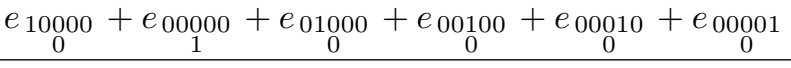 & 6 & $E_{6}$ \\
\hline
\end{tabular}


Table 2: Nilpotent orbit representatives (continued)

\begin{tabular}{|c|c|c|c|}
\hline \multicolumn{4}{|c|}{$G=E_{7}$} \\
\hline Orbit & $e$ & $\operatorname{dim} C_{\mathfrak{L}(G)}(e)$ & Name \\
\hline 1 & $e_{100000}$ & 99 & $A_{1}$ \\
\hline 2 & $e_{100000}+e_{000010}$ & 81 & $A_{1}^{2}$ \\
\hline 3 & $e_{000000}+e_{000100}+e_{000001}$ & 79 & $\left(A_{1}^{3}\right)^{\prime \prime}$ \\
\hline 4 & $e_{0} \underset{00000}{ }+e_{001000}^{00}+e_{0}^{000010}$ & 69 & $\left(A_{1}^{3}\right)^{\prime}$ \\
\hline 5 & $e_{0} 100000+e_{0}^{010000}$ & 67 & $A_{2}$ \\
\hline 6 & $e_{000000}+e_{010000}+e_{000100}+e_{000001}$ & 63 & $A_{1}^{4}$ \\
\hline 7 & $e_{100000}+e_{010000}+e_{000000}$ & 57 & $A_{2} A_{1}$ \\
\hline 8 & $e_{000000}+e_{001000}+e_{100000}+e_{000010}$ & 51 & $A_{2} A_{1}^{2}$ \\
\hline 9 & $\underset{0}{e_{10000}}+e_{010000}+e_{001000}$ & 49 & $A_{3}$ \\
\hline 10 & $e_{0} e_{0}^{100000}+e_{0}^{010000}+e_{0}^{000100}+e_{0}^{000010}$ & 49 & $A_{2}{ }^{2}$ \\
\hline 11 & $e_{0} \underset{100000}{0}+e_{010000}+e_{000000}+e_{000100}+e_{000001}^{0}$ & 49 & $A_{2} A_{1}^{3}$ \\
\hline 12 & $e_{0}^{000100}+e_{0}^{000010}+e_{0}^{000001}+e_{000000}^{0}$ & 47 & $\left(A_{3} A_{1}\right)^{\prime \prime}$ \\
\hline 13 & $e_{0} 100000+e_{010000}+e_{0}^{000100}+e_{0}^{000010}+e_{0}^{000000}$ & 43 & $A_{2}^{2} A_{1}$ \\
\hline 14 & $e_{0} 100000+e_{010000}+e_{001000}+e_{000010}$ & 41 & $\left(A_{3} A_{1}\right)^{\prime}$ \\
\hline 15 & $e_{0}^{010000}+e_{001000}^{0}+e_{0}^{001100}+e_{0}^{000000}+e_{0}^{000100}$ & 39 & $D_{4}\left(a_{1}\right)$ \\
\hline 16 & $\underset{0}{e 000100}+e_{0}^{000010}+e_{000001}^{0}+e_{0}^{000000}+e_{0}^{010000}$ & 39 & $A_{3} A_{1}^{2}$ \\
\hline 17 & $\underset{0}{e} \underset{010000}{e}+e_{0}^{001000}+e_{000000}+e_{000100}^{000}$ & 37 & $D_{4}$ \\
\hline 18 & $\underset{0}{e} e_{010000}+e_{1}^{001000}+e_{0}^{001100}+e_{0}^{000000}+e_{0}^{000100}+e_{0}^{000001}$ & 37 & $D_{4}\left(a_{1}\right) A_{1}$ \\
\hline 19 & $e_{0}^{010000}+e_{0}^{001000}+e_{0}^{000000}+e_{0}^{000010}+e_{0}^{000001}$ & 35 & $A_{3} A_{2}$ \\
\hline 20 & $\underset{0}{e} \underset{100000}{ }+e_{010000}+e_{001000}+e_{000000}$ & 33 & $A_{4}$ \\
\hline 21 & $\underset{0}{e_{000100}}+e_{0}^{000010}+e_{0}^{000001}+e_{100000}+e_{010000}+e_{000000}$ & 33 & $A_{3} A_{2} A_{1}$ \\
\hline 22 & $e_{1}^{000000}+e_{001000}+e_{000100}+e_{000010}+e_{0}^{000001}$ & 31 & $\left(A_{5}\right)^{\prime \prime}$ \\
\hline 23 & $\underset{0}{e_{010000}}+e_{001000}+e_{000000}+e_{000100}+e_{00001}^{000001}$ & 31 & $D_{4} A_{1}$ \\
\hline 24 & $e_{0} e_{0000}+e_{010000}+e_{001000}+e_{000000}+e_{000010}$ & 29 & $A_{4} A_{1}$ \\
\hline 25 & $e_{0} 100000+e_{0}^{010000}+e_{001000}+e_{001100}+e_{000000}+e_{0}^{000100}$ & 27 & $D_{5}\left(a_{1}\right)$ \\
\hline 26 & $e_{0} \underset{0}{100000}+e_{010000}+e_{001000}+e_{000000}+e_{000010}+e_{000001}$ & 27 & $A_{4} A_{2}$ \\
\hline 27 & $\underset{0}{e_{100000}}+e_{010000}^{0}+e_{001000}^{0}+e_{000100}^{0}+e_{00010}^{000}$ & 25 & $\left(A_{5}\right)^{\prime}$ \\
\hline 28 & $e_{1} e_{10000}+e_{001000}^{0}+e_{000100}^{00}+e_{000010}^{00}+e_{000001}+e_{0}^{100000}$ & 25 & $A_{5} A_{1}$ \\
\hline
\end{tabular}


Table 2: Nilpotent orbit representatives (continued)

\begin{tabular}{|c|c|c|c|}
\hline \multicolumn{4}{|c|}{$G=E_{7}$} \\
\hline Orbit & $e$ & $\operatorname{dim} C_{\mathfrak{L}(G)}(e)$ & Name \\
\hline 29 & $\begin{array}{l}e_{100000}+e_{010000}+e_{001000}+e_{001100}+e_{000000}+e_{000100} \\
\quad+e_{000001} \\
\quad e_{0}\end{array}$ & 25 & $D_{5}\left(a_{1}\right) A_{1}$ \\
\hline 30 & $\begin{array}{l}\underset{0}{e_{000001}}+e_{000110}+e_{001000}-e_{011000}+e_{0}^{001100}+e_{000000} \\
\quad+e_{010000}\end{array}$ & 23 & $D_{6}\left(a_{2}\right)$ \\
\hline 31 & $e_{1}^{011000}+e_{0}^{100000}+e_{011100}+e_{000010}+e_{001100}+e_{001000}$ & 23 & $E_{6}\left(a_{3}\right)$ \\
\hline 32 & $e_{0} e_{10000}+e_{010000}+e_{001000}+e_{000000}+e_{0}^{000100}$ & 21 & $D_{5}$ \\
\hline 33 & $\begin{array}{l}e_{0} \underset{0}{111100}+e_{001110}+e_{000001}+e_{011110}+e_{111000}+e_{001000} \\
\quad+e_{011100}\end{array}$ & 21 & $E_{7}\left(a_{5}\right)$ \\
\hline 34 & $e_{0}^{100000}+e_{0} 0000+e_{001000}+e_{000100}+e_{000010}+e_{000001}$ & 19 & $A_{6}$ \\
\hline 35 & $e_{0} e_{100000}+e_{010000}+e_{001000}+e_{000000}+e_{000100}+e_{000001}$ & 19 & $D_{5} A_{1}$ \\
\hline 36 & $\begin{array}{l}\underset{0}{e_{000001}}+e_{000010}+e_{000100}+e_{001000}-e_{0} \\
\quad+e_{010000} \\
\quad \\
0\end{array}$ & 19 & $D_{6}\left(a_{1}\right)$ \\
\hline 37 & $\begin{array}{l}e_{011000}+e_{100000}+e_{011100}+e_{000011}+e_{001110}+e_{000001} \\
\quad+e_{001100}+e_{001000} \\
\quad e_{0}\end{array}$ & 17 & $E_{7}\left(a_{4}\right)$ \\
\hline 38 & $\underset{0}{e} \underset{000001}{e}+e_{000010}+e_{0}^{000100}+e_{0} 001000+e_{000000}+e_{010000}$ & 15 & $D_{6}$ \\
\hline 39 & $\begin{array}{l}\underset{0}{e_{100000}}+e_{000010}+e_{010000}+e_{000100}+e_{001100}+e_{011000} \\
\quad+e_{000000} \\
\quad 1\end{array}$ & 15 & $E_{6}\left(a_{1}\right)$ \\
\hline 40 & $e_{0} e_{10000}+e_{000000}+e_{010000}+e_{001000}+e_{000100}+e_{000010}$ & 13 & $E_{6}$ \\
\hline 41 & $\begin{array}{l}e_{011000}+e_{100000}+e_{011100}+e_{000010}+e_{000001}+e_{001100} \\
\quad+e_{001000} \\
\quad 0\end{array}$ & 13 & $E_{7}\left(a_{3}\right)$ \\
\hline 42 & 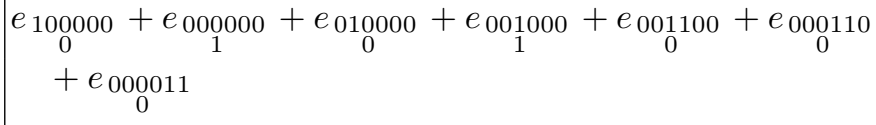 & 11 & $E_{7}\left(a_{2}\right)$ \\
\hline 43 & $\begin{array}{l}e_{100000}+e_{010000}+e_{0}^{011000}+e_{001000}+e_{000100}+e_{000010} \\
\quad+e_{000001}^{e 00} \\
\quad\end{array}$ & 9 & $E_{7}\left(a_{1}\right)$ \\
\hline 44 & 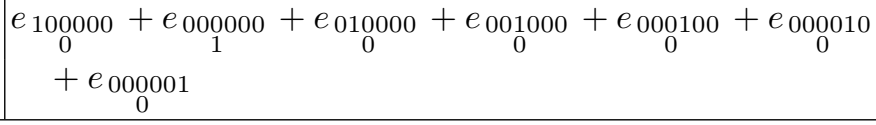 & 7 & $E_{7}$ \\
\hline
\end{tabular}


Table 2: Nilpotent orbit representatives (continued)

\begin{tabular}{|c|c|c|c|c|}
\hline \multicolumn{5}{|c|}{$G=E_{8}$} \\
\hline Orbit & $e$ & & $\operatorname{dim} C_{\mathfrak{L}(G)}(e)$ & Name \\
\hline 1 & $\begin{array}{c}e \\
1000000 \\
0\end{array}$ & & 190 & $A_{1}$ \\
\hline 2 & $e_{0} \underset{1000000}{ }+e_{0000100}$ & & 156 & $A_{1}^{2}$ \\
\hline 3 & $e_{0} e_{000000}+e_{0010000}+e_{0000100}$ & & 136 & $A_{1}{ }^{3}$ \\
\hline 4 & $e_{100000}+e_{0100000}$ & & 134 & $A_{2}$ \\
\hline 5 & $\underset{1}{e 0000000}+e_{0}^{0100000}+e_{0}^{0001000}$ & $+\underset{0}{0000010}$ & 120 & $A_{1}^{4}$ \\
\hline 6 & $\underset{0}{e} \underset{0}{1000000}+e_{0100000}+e_{0000000}$ & & 112 & $A_{2} A_{1}$ \\
\hline 7 & $e_{0000000}+e_{0010000}+e_{0}^{1000000}$ & $+e_{0}^{0000100}$ & 102 & $A_{2} A_{1}^{2}$ \\
\hline 8 & $\underset{0}{e} \underset{0}{e} \underset{0}{1000000}+e_{0100000}+e_{0010000}$ & & 100 & $A_{3}$ \\
\hline 9 & $e_{0} \underset{1000000}{ }+e_{0100000}+e_{0000000}$ & $+e_{0001000}^{000000010}$ & 94 & $A_{2} A_{1}{ }^{3}$ \\
\hline 10 & $e_{0} \underset{00000}{1000}+e_{0100000}+e_{0001000}$ & $+e_{0}^{0000100}$ & 92 & $A_{2}^{2}$ \\
\hline 11 & $e_{0} \underset{1000000}{ }+e_{0100000}+e_{0001000}$ & $+e_{0}^{0000100}+e_{1}^{0000000}$ & 86 & $A_{2}{ }^{2} A_{1}$ \\
\hline 12 & $e_{0} \underset{0}{1000000}+e_{0100000}+e_{0010000}$ & $+e_{0}^{0000100}$ & 84 & $A_{3} A_{1}$ \\
\hline 13 & $\underset{0}{e} 0100000+e_{0010000}+e_{0011000}$ & $+e_{1}^{0000000}+\underset{0}{0001000}$ & 82 & $D_{4}\left(a_{1}\right)$ \\
\hline 14 & $\underset{0}{e x+00000}+e_{0}^{0010000}+e_{0000000}$ & $+e_{0}^{0001000}$ & 80 & $D_{4}$ \\
\hline 15 & $\begin{array}{l}e_{1000000}+e_{0100000}+e_{0001000} \\
\quad+e_{0000001} \\
\quad 0\end{array}$ & $+e_{0}^{0000100}+e_{1}^{0000000}$ & 80 & $A_{2}^{2} A_{1}^{2}$ \\
\hline 16 & $e_{0001000}+e_{0000100}+e_{0000010}$ & $+e_{0000000}+e_{0100000}^{0100}$ & 76 & $A_{3} A_{1}^{2}$ \\
\hline 17 & $\begin{array}{l}e_{0100000}+e_{0010000}+e_{0011000} \\
\quad+e_{0000010} \\
\quad+0\end{array}$ & $+e_{1}^{0000000}+e_{0}^{0001000}$ & 72 & $D_{4}\left(a_{1}\right) A_{1}$ \\
\hline 18 & $\underset{0}{e_{0100000}}+e_{0010000}+e_{0000000}$ & $+e_{0}^{0000100}+e_{0000010}^{000}$ & 70 & $A_{3} A_{2}$ \\
\hline 19 & $e_{0} \underset{0}{1000000}+e_{0100000}+e_{0010000}$ & $+e_{1}^{0000000}$ & 68 & $A_{4}$ \\
\hline 20 & $\begin{array}{l}e_{0001000}+e_{0000100}+e_{0000010} \\
\quad+e_{0000000} \\
1\end{array}$ & $+e_{0}^{1000000}+e_{0}^{0100000}$ & 66 & $A_{3} A_{2} A_{1}$ \\
\hline 21 & $e_{0} \underset{0}{e} 0000+e_{0010000}+e_{0000000}$ & $+e_{0}^{0001000}+e_{0}^{0000010}$ & 64 & $D_{4} A_{1}$ \\
\hline 22 & $\mid \begin{array}{c}e_{0100000}+e_{0010000}+e_{0011000} \\
0 \\
+e_{0000010}+e_{0000001}\end{array}$ & $+e_{1}^{0000000}+e_{0}^{0001000}$ & 64 & $D_{4}\left(a_{1}\right) A_{2}$ \\
\hline 23 & $e_{0} \underset{1000000}{ }+e_{0100000}+e_{0010000}$ & $+e_{1}^{0000000}+e_{0}^{0000100}$ & 60 & $A_{4} A_{1}$ \\
\hline 24 & $\begin{array}{c}e_{1000000}+e_{0100000}+e_{0010000} \\
0 \\
+e_{0000001} \\
0\end{array}$ & $+e_{0}^{0000100}+e_{0}^{0000010}$ & 60 & $A_{3}{ }^{2}$ \\
\hline
\end{tabular}


Table 2: Nilpotent orbit representatives (continued)

\begin{tabular}{|c|c|c|c|c|}
\hline \multicolumn{5}{|c|}{$G=E_{8}$} \\
\hline Orbit & $e$ & & $\operatorname{dim} C_{\mathfrak{L}(G)}(e)$ & Name \\
\hline 25 & $\begin{array}{l}e_{1000000}+e_{0100000}+e_{0010000} \\
\quad+e_{0001000} \\
\quad\end{array}$ & $+e_{0}^{0011000}+e_{000000}$ & 58 & $\overline{D_{5}\left(a_{1}\right)}$ \\
\hline 26 & $\begin{array}{l}e_{0}^{1000000}+e_{0100000}^{0}+e_{0010000} \\
+e_{0000001}^{0}\end{array}$ & $+e_{1}^{0000000}+\underset{0}{0000100}$ & 56 & $A_{4} A_{1}^{2}$ \\
\hline 27 & $\begin{array}{l}e_{1000000}+e_{0100000}+e_{0010000} \\
\quad+e_{0000010} \\
\quad+e_{0}\end{array}$ & $+e_{1}^{0000000}+e_{0}^{0000100}$ & 54 & $A_{4} A_{2}$ \\
\hline 28 & $e_{0} \underset{0}{1000000}+e_{0100000}+e_{0010000}$ & $+e_{0}^{0001000}+e_{0}^{0000100}$ & 52 & $A_{5}$ \\
\hline 29 & $\begin{array}{c}e_{1000000}+e_{0100000}+e_{0010000} \\
0 \\
+e_{0001000}+e_{0000010}\end{array}$ & $+e_{0011000}^{0}+e_{1}^{0000000}$ & 52 & $D_{5}\left(a_{1}\right) A_{1}$ \\
\hline 30 & $\begin{array}{c}e_{0001000}+e_{0000100}+e_{0000010} \\
0 \\
+e_{0100000}+e_{0000000}\end{array}$ & $+e_{0000001}^{0}+e_{0}^{1000000}$ & 52 & $A_{4} A_{2} A_{1}$ \\
\hline 31 & $\begin{array}{l}e_{0100000}+e_{0010000}+e_{0000000} \\
\quad+e_{0000001} \\
\quad\end{array}$ & $+e_{0}^{0001000}+e_{0}^{0000010}$ & 50 & $D_{4} A_{2}$ \\
\hline 32 & $\begin{array}{l}e_{0110000}+e_{1000000}+e_{0111000} \\
\quad+e_{0010000}\end{array}$ & $+e_{0}^{000100}+e_{1}^{0011000}$ & 50 & $E_{6}\left(a_{3}\right)$ \\
\hline 33 & $e_{0} \underset{1000000}{ }+e_{0100000}+e_{0010000}$ & $+e_{1}^{0000000}+e_{0}^{0001000}$ & 48 & $D_{5}$ \\
\hline 34 & $\begin{array}{c}e_{1000000}+e_{0100000}+e_{0010000} \\
0 \\
+e_{0000010}+e_{0000001}^{0}\end{array}$ & $+e_{1}^{0000000}+e_{0}^{0000100}$ & 48 & $A_{4} A_{3}$ \\
\hline 35 & $\begin{array}{l}e_{0000000}+e_{0010000}+e_{0001000} \\
\quad+e_{0}^{1000000} \\
\quad+0\end{array}$ & $+e_{0}^{0000100}+e_{0}^{0000010}$ & 46 & $A_{5} A_{1}$ \\
\hline 36 & $\begin{array}{c}e_{1000000}+e_{0100000}+e_{0010000} \\
0 \\
+e_{0001000}+e_{0000010}+e_{00}\end{array}$ & $+e_{0}^{0011000}+e_{1}^{0000000}$ & 46 & $D_{5}\left(a_{1}\right) A_{2}$ \\
\hline 37 & $\begin{array}{c}e_{0000010}+e_{0001100}+e_{0010000} \\
+e_{0}^{000000}+e_{0100000}^{0}\end{array}$ & $-e_{0}^{0110000}+e_{0011000}^{00}$ & 44 & $D_{6}\left(a_{2}\right)$ \\
\hline 38 & $\begin{array}{c}e_{0110000}+e_{1000000}+e_{0111000} \\
\quad+e_{0010000}+e_{0000001} \\
+e_{0}^{00100}\end{array}$ & $+e_{0}^{000100}+e_{1}^{0011000}$ & 44 & $E_{6}\left(a_{3}\right) A_{1}$ \\
\hline 39 & $\begin{array}{c}e_{1111000}+e_{0011100}+e_{0000010} \\
0 \\
+e_{0010000}+e_{0111000}\end{array}$ & $+e_{0111100}+e_{1110000}$ & 42 & $E_{7}\left(a_{5}\right)$ \\
\hline 40 & $\begin{array}{c}e_{1000000}+e_{0100000}+e_{0010000} \\
0 \\
+e_{0000010} \\
0\end{array}$ & $+e_{1}^{0000000}+e_{0}^{0001000}$ & 40 & $D_{5} A_{1}$ \\
\hline
\end{tabular}


Table 2: Nilpotent orbit representatives (continued)

\begin{tabular}{|c|c|c|c|}
\hline \multicolumn{4}{|c|}{$G=E_{8}$} \\
\hline Orbit & e & $\operatorname{dim} C_{\mathfrak{L}(G)}(e)$ & Name \\
\hline 41 & $\begin{array}{c}e_{1111111}+e_{0121110}+e_{0001000}+e_{1121100}+e_{1221000} \\
\quad+e_{1} \\
+e_{0011111}+e_{0111100}+e_{1111110}\end{array}$ & 40 & $E_{8}\left(a_{7}\right)$ \\
\hline 42 & $\begin{array}{l}e_{0000000}+e_{0010000}+e_{0001000}+e_{0000100}+e_{0000010} \\
\quad+e_{0000001}^{0} \\
\quad+0\end{array}$ & 38 & $A_{6}$ \\
\hline 43 & $\begin{array}{l}e_{0000010}+e_{0000100}+e_{0001000}+e_{0010000}-e_{0110000} \\
\quad+e_{0000000}+e_{0100000} \\
\quad 0\end{array}$ & 38 & $D_{6}\left(a_{1}\right)$ \\
\hline 44 & $\begin{array}{l}e_{0000000}+e_{0010000}+e_{0001000}+e_{0000100}+e_{0000010} \\
\quad+e_{000001}+e_{1000000} \\
\quad+e_{0}\end{array}$ & 36 & $A_{6} A_{1}$ \\
\hline 45 & $\begin{array}{c}e_{0110000}+e_{1000000}+e_{0111000}+e_{0} 0000110 \\
\quad 0 \\
\quad+e_{0000010}^{0}+e_{0011000}+e_{0010000}\end{array}$ & 36 & $E_{7}\left(a_{4}\right)$ \\
\hline 46 & $\begin{array}{l}e_{0} 1000000+e_{0000100}+e_{0100000}+e_{0001000}+e_{0011000} \\
\quad+e_{0110000}+e_{0000000} \\
\quad 1\end{array}$ & 34 & $E_{6}\left(a_{1}\right)$ \\
\hline 47 & $\begin{array}{l}e_{1000000}+e_{0100000}+e_{0}^{0010000}+e_{0}^{0000000}+e_{0001000} \\
\quad+e_{0000010}+e_{0000001} \\
0\end{array}$ & 34 & $D_{5} A_{2}$ \\
\hline 48 & $\begin{array}{l}e_{0000010}+e_{0000100}+e_{0}^{0001000}+e_{0}^{0010000}+e_{0000000} \\
\quad+e_{0100000} \\
\quad 0\end{array}$ & 32 & $D_{6}$ \\
\hline 49 & $\begin{array}{l}e_{1000000}+e_{0000000}+e_{0100000}+e_{0010000}+e_{0001000} \\
\quad+e_{0000100} \\
\quad \\
\quad 0\end{array}$ & 32 & $E_{6}$ \\
\hline 50 & $\begin{array}{c}e_{0000001}+e_{0000010}+e_{0001100}+e_{0010000}-e_{0110000} \\
\quad 0 \\
\quad+e_{0}^{0011000}+e_{0000000}+e_{0100000} \\
0\end{array}$ & 32 & $D_{7}\left(a_{2}\right)$ \\
\hline 51 & $\begin{array}{c}e_{1000000}+e_{0100000}+e_{0010000}+e_{0001000}+e_{0000100} \\
0 \\
+e_{0000010}+e_{0000001} \\
0\end{array}$ & 30 & $A_{7}$ \\
\hline 52 & $\begin{array}{c}e_{0}^{1000000}+e_{0000100}+e_{0100000}+e_{0001000}+e_{0011000} \\
0 \\
\quad+e_{0}^{0110000}+e_{0000000}^{0}+e_{0000001} \\
0\end{array}$ & 30 & $E_{6}\left(a_{1}\right) A_{1}$ \\
\hline 53 & $\begin{array}{l}e_{0110000}^{0}+e_{0}^{1000000}+e_{0}^{0111000}+e_{0000100}+e_{0000010} \\
\quad+e_{1}^{0011000}+e_{0}^{0010000}\end{array}$ & 28 & $E_{7}\left(a_{3}\right)$ \\
\hline 54 & 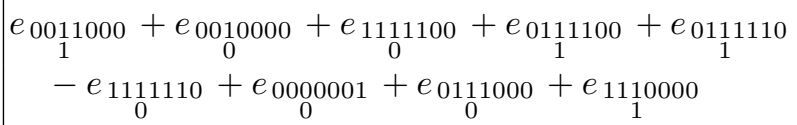 & 28 & $E_{8}\left(b_{6}\right)$ \\
\hline 55 & $\begin{array}{c}e_{0000001}+e_{0000010}+e_{0000100}+e_{0001000}+e_{0010000} \\
0 \\
-e_{0110000}+e_{0000000}+e_{0100000} \\
0\end{array}$ & 26 & $D_{7}\left(a_{1}\right)$ \\
\hline
\end{tabular}


Table 2: Nilpotent orbit representatives (continued)

\begin{tabular}{|c|c|c|c|}
\hline \multicolumn{4}{|c|}{$G=E_{8}$} \\
\hline Orbit & $e$ & $\operatorname{dim} C_{\mathfrak{L}(G)}(e)$ & Name \\
\hline 56 & $\begin{array}{l}e_{1000000}+e_{0000000}+e_{0100000}+e_{0010000}+e_{0001000} \\
0 \\
\quad+e_{0000100}+e_{0000001} \\
0\end{array}$ & 26 & $E_{6} A_{1}$ \\
\hline 57 & $\begin{array}{l}e_{1000000}+e_{0000000}+e_{0100000}+e_{0010000}+e_{0011000}^{000} \\
\quad+e_{0001100}+e_{0000110} \\
\quad 0\end{array}$ & 24 & $E_{7}\left(a_{2}\right)$ \\
\hline 58 & $\begin{array}{l}e_{0011000}+e_{1111100}+e_{0000010}+e_{0111100}+e_{0010000} \\
\quad+e_{0} 0001111+e_{1110000}+e_{0111000} \\
\quad+e_{0}\end{array}$ & 24 & $E_{8}\left(a_{6}\right)$ \\
\hline 59 & $\begin{array}{l}e_{0000001}+e_{0000010}+e_{0000100}+e_{0001000}+e_{0010000} \\
\quad+e_{0000000}+e_{0100000} \\
\quad+0\end{array}$ & 22 & $D_{7}$ \\
\hline 60 & $\begin{array}{l}e_{0011000}+e_{0000001}+e_{1111100}+e_{0000010}+e_{0111100} \\
\quad+e_{0} \\
\quad+e_{0010000}+e_{0111000}+e_{1110000}\end{array}$ & 22 & $E_{8}\left(b_{5}\right)$ \\
\hline 61 & $\begin{array}{l}e_{1000000}+e_{0100000}+e_{0}^{0110000}+e_{0010000}+e_{0001000} \\
\quad+e_{0000100}+e_{0000010} \\
\quad 0\end{array}$ & 20 & $E_{7}\left(a_{1}\right)$ \\
\hline 62 & $\begin{array}{c}e_{0011000}^{0}+e_{0000111}+e_{0}^{0111000}+e_{1000000}+e_{0110000} \\
\quad+e_{0} \\
\quad+e_{0}^{011100}+e_{0001110}+e_{0010000}+e_{0000010}\end{array}$ & 20 & $E_{8}\left(a_{5}\right)$ \\
\hline 63 & $\begin{array}{l}e_{0000001}+e_{0000010}+e_{0000110}+e_{0011100}+e_{0}^{0111000} \\
\quad+e_{1000000}+e_{0110000}+e_{0010000} \\
\quad+e_{0}\end{array}$ & 18 & $E_{8}\left(b_{4}\right)$ \\
\hline 64 & $\begin{array}{l}e_{1000000}+e_{0000000}+e_{0100000}+e_{0010000}+e_{0001000} \\
\quad+e_{0000100}+e_{0000010} \\
\quad 0\end{array}$ & 16 & $E_{7}$ \\
\hline 65 & $\begin{array}{l}e_{0011000}+e_{0000100}+e_{0111000}+e_{1000000}+e_{0110000} \\
\quad+0 \\
\quad+e_{0001110}+e_{0010000}+e_{0000001} \\
0\end{array}$ & 16 & $E_{8}\left(a_{4}\right)$ \\
\hline 66 & $\begin{array}{c}e_{0000001}+e_{0011000}+e_{0000010}+e_{0000100}+e_{0111000} \\
0 \\
+e_{1000000}+e_{0110000}+e_{0010000} \\
0\end{array}$ & 14 & $E_{8}\left(a_{3}\right)$ \\
\hline 67 & $\begin{array}{c}e_{1000000}+e_{0000000}+e_{0100000}+e_{0010000}+e_{0011000} \\
0 \\
+e_{0}^{0001100}+e_{0000110}+e_{0000001} \\
0\end{array}$ & 12 & $E_{8}\left(a_{2}\right)$ \\
\hline 68 & $\begin{array}{c}e_{1000000}+e_{0000000}+e_{1}^{0010000}+e_{0110000}+e_{0001000} \\
\quad+e_{0} \\
\quad+e_{0}^{0000100}+e_{0000010}+e_{0000001} \\
0\end{array}$ & 10 & $E_{8}\left(a_{1}\right)$ \\
\hline 69 & $\begin{array}{c}e_{1000000}+e_{0000000}+e_{0100000}+e_{0010000}+e_{0001000} \\
0 \\
+e_{0000100}+e_{0000010}+e_{0000001} \\
0\end{array}$ & 8 & $E_{8}$ \\
\hline
\end{tabular}




\section{Associated cocharacters}

As before, $G$ will be a simple algebraic group of exceptional type defined over an algebraically closed field $k$ whose characteristic is either 0 or a good prime for $G$, and $T$ will be a fixed maximal torus of $G$. In this section, for each of the non-zero nilpotent orbit representatives in $\mathfrak{L}(G)$ listed in Table 2, we will describe our choice of an associated cocharacter $\tau: k^{*} \rightarrow T$, and obtain its labelled diagram $\Delta$.

Let $e$ be a non-zero nilpotent orbit representative, distinguished in $\mathfrak{L}(L)$, where $L$ is a Levi subgroup of $G$. Write $[L, L]=L_{1} \ldots L_{t}$, a product of simple factors. To indicate our choice of Levi subgroup, we shall give a Dynkin diagram with black and white nodes, such that the simple roots corresponding to the black nodes form a basis of the root system of $[L, L]$; this information is provided at the top of the page for $e$ in $\S 11$.

For each simple group $H$ occurring as a simple factor in some Levi subgroup of an exceptional group, Table 3 gives a list of cocharacters, one for each orbit of distinguished elements in $\mathfrak{L}(H)$; in this table the cocharacter $\tau: k^{*} \rightarrow T$ is defined by giving the expression $\tau(c)=\prod_{i=1}^{\ell} h_{\alpha_{i}}\left(c^{k_{i}}\right)$.

Note that the given cocharacters are invariant under all graph automorphisms of $H$. We thus obtain cocharacters $\tau_{1}, \ldots, \tau_{t}$, one for each simple factor, and the cocharacter which we will associate to $e$ is the product of the $\tau_{i}$; that is, $\tau(c)=\prod_{i=1}^{t} \tau_{i}(c)$ for all $c \in k^{*}$. In order to show that $\tau$ is an associated cocharacter for the element $e$, by Definition 2.6 we must verify that $\operatorname{im}(\tau) \subseteq[L, L] \cap T$ and $\tau(c) e=c^{2} e$ for all $c \in k^{*}$; the first of these is true by construction, while the second is a simple calculation.

At the top of the page for $e$ in $\S 11$ we shall represent $\tau$ by its labelled diagram, as explained at the beginning of $\S 3$; we shall also give the labelled diagram $\Delta$ of the nilpotent orbit containing $e$.

Table 3: Cocharacters in simple factors

\begin{tabular}{|c|l|}
\hline Orbit & Cocharacter \\
\hline$A_{1}$ & $h_{\alpha_{1}}(c)$ \\
$A_{2}$ & $h_{\alpha_{1}}\left(c^{2}\right) h_{\alpha_{2}}\left(c^{2}\right)$ \\
$A_{3}$ & $h_{\alpha_{1}}\left(c^{3}\right) h_{\alpha_{2}}\left(c^{4}\right) h_{\alpha_{3}}\left(c^{3}\right)$ \\
$A_{4}$ & $h_{\alpha_{1}}\left(c^{4}\right) h_{\alpha_{2}}\left(c^{6}\right) h_{\alpha_{3}}\left(c^{6}\right) h_{\alpha_{4}}\left(c^{4}\right)$ \\
$A_{5}$ & $h_{\alpha_{1}}\left(c^{5}\right) h_{\alpha_{2}}\left(c^{8}\right) h_{\alpha_{3}}\left(c^{9}\right) h_{\alpha_{4}}\left(c^{8}\right) h_{\alpha_{5}}\left(c^{5}\right)$ \\
$A_{6}$ & $h_{\alpha_{1}}\left(c^{6}\right) h_{\alpha_{2}}\left(c^{10}\right) h_{\alpha_{3}}\left(c^{12}\right) h_{\alpha_{4}}\left(c^{12}\right) h_{\alpha_{5}}\left(c^{10}\right) h_{\alpha_{6}}\left(c^{6}\right)$ \\
$A_{7}$ & $h_{\alpha_{1}}\left(c^{7}\right) h_{\alpha_{2}}\left(c^{12}\right) h_{\alpha_{3}}\left(c^{15}\right) h_{\alpha_{4}}\left(c^{16}\right) h_{\alpha_{5}}\left(c^{15}\right) h_{\alpha_{6}}\left(c^{12}\right) h_{\alpha_{7}}\left(c^{7}\right)$ \\
$B_{2}$ & $h_{\alpha_{1}}\left(c^{4}\right) h_{\alpha_{2}}\left(c^{3}\right)$ \\
$B_{3}$ & $h_{\alpha_{1}}\left(c^{6}\right) h_{\alpha_{2}}\left(c^{10}\right) h_{\alpha_{3}}\left(c^{6}\right)$ \\
$C_{3}$ & $h_{\alpha_{1}}\left(c^{5}\right) h_{\alpha_{2}}\left(c^{8}\right) h_{\alpha_{3}}\left(c^{9}\right)$ \\
$C_{3}\left(a_{1}\right)$ & $h_{\alpha_{1}}\left(c^{3}\right) h_{\alpha_{2}}\left(c^{4}\right) h_{\alpha_{3}}\left(c^{5}\right)$ \\
\hline
\end{tabular}


Table 3: Cocharacters in simple factors (continued)

\begin{tabular}{|c|c|}
\hline Orbit & Cocharacter \\
\hline$D_{4}$ & $h_{\alpha_{1}}\left(c^{6}\right) h_{\alpha_{2}}\left(c^{10}\right) h_{\alpha_{3}}\left(c^{6}\right) h_{\alpha_{4}}\left(c^{6}\right)$ \\
\hline$D_{4}\left(a_{1}\right)$ & $h_{\alpha_{1}}\left(c^{4}\right) h_{\alpha_{2}}\left(c^{6}\right) h_{\alpha_{3}}\left(c^{4}\right) h_{\alpha_{4}}\left(c^{4}\right)$ \\
\hline$D_{5}$ & $h_{\alpha_{1}}\left(c^{8}\right) h_{\alpha_{2}}\left(c^{14}\right) h_{\alpha_{3}}\left(c^{18}\right) h_{\alpha_{4}}\left(c^{10}\right) h_{\alpha_{5}}\left(c^{10}\right)$ \\
\hline$D_{5}\left(a_{1}\right)$ & $h_{\alpha_{1}}\left(c^{6}\right) h_{\alpha_{2}}\left(c^{10}\right) h_{\alpha_{3}}\left(c^{12}\right) h_{\alpha_{4}}\left(c^{7}\right) h_{\alpha_{5}}\left(c^{7}\right)$ \\
\hline$D_{6}$ & $h_{\alpha_{1}}\left(c^{10}\right) h_{\alpha_{2}}\left(c^{18}\right) h_{\alpha_{3}}\left(c^{24}\right) h_{\alpha_{4}}\left(c^{28}\right) h_{\alpha_{5}}\left(c^{15}\right) h_{\alpha_{6}}\left(c^{15}\right)$ \\
\hline$D_{6}\left(a_{1}\right)$ & $h_{\alpha_{1}}\left(c^{8}\right) h_{\alpha_{2}}\left(c^{14}\right) h_{\alpha_{3}}\left(c^{18}\right) h_{\alpha_{4}}\left(c^{20}\right) h_{\alpha_{5}}\left(c^{11}\right) h_{\alpha_{6}}\left(c^{11}\right)$ \\
\hline$D_{6}\left(a_{2}\right)$ & $h_{\alpha_{1}}\left(c^{6}\right) h_{\alpha_{2}}\left(c^{10}\right) h_{\alpha_{3}}\left(c^{14}\right) h_{\alpha_{4}}\left(c^{16}\right) h_{\alpha_{5}}\left(c^{9}\right) h_{\alpha_{6}}\left(c^{9}\right)$ \\
\hline$D_{7}$ & $h_{\alpha_{1}}\left(c^{12}\right) h_{\alpha_{2}}\left(c^{22}\right) h_{\alpha_{3}}\left(c^{30}\right) h_{\alpha_{4}}\left(c^{36}\right) h_{\alpha_{5}}\left(c^{40}\right) h_{\alpha_{6}}\left(c^{21}\right) h_{\alpha_{7}}\left(c^{21}\right)$ \\
\hline$D_{7}\left(a_{1}\right)$ & $h_{\alpha_{1}}\left(c^{10}\right) h_{\alpha_{2}}\left(c^{18}\right) h_{\alpha_{3}}\left(c^{24}\right) h_{\alpha_{4}}\left(c^{28}\right) h_{\alpha_{5}}\left(c^{30}\right) h_{\alpha_{6}}\left(c^{16}\right) h_{\alpha_{7}}\left(c^{16}\right)$ \\
\hline$D_{7}\left(a_{2}\right)$ & $h_{\alpha_{1}}\left(c^{8}\right) h_{\alpha_{2}}\left(c^{14}\right) h_{\alpha_{3}}\left(c^{18}\right) h_{\alpha_{4}}\left(c^{22}\right) h_{\alpha_{5}}\left(c^{24}\right) h_{\alpha_{6}}\left(c^{13}\right) h_{\alpha_{7}}\left(c^{13}\right)$ \\
\hline$E_{6}$ & $h_{\alpha_{1}}\left(c^{16}\right) h_{\alpha_{2}}\left(c^{22}\right) h_{\alpha_{3}}\left(c^{30}\right) h_{\alpha_{4}}\left(c^{42}\right) h_{\alpha_{5}}\left(c^{30}\right) h_{\alpha_{6}}\left(c^{16}\right)$ \\
\hline$E_{6}\left(a_{1}\right)$ & $h_{\alpha_{1}}\left(c^{12}\right) h_{\alpha_{2}}\left(c^{16}\right) h_{\alpha_{3}}\left(c^{22}\right) h_{\alpha_{4}}\left(c^{30}\right) h_{\alpha_{5}}\left(c^{22}\right) h_{\alpha_{6}}\left(c^{12}\right)$ \\
\hline$E_{6}\left(a_{3}\right)$ & $h_{\alpha_{1}}\left(c^{8}\right) h_{\alpha_{2}}\left(c^{10}\right) h_{\alpha_{3}}\left(c^{14}\right) h_{\alpha_{4}}\left(c^{20}\right) h_{\alpha_{5}}\left(c^{14}\right) h_{\alpha_{6}}\left(c^{8}\right)$ \\
\hline$E_{7}$ & $h_{\alpha_{1}}\left(c^{34}\right) h_{\alpha_{2}}\left(c^{49}\right) h_{\alpha_{3}}\left(c^{66}\right) h_{\alpha_{4}}\left(c^{96}\right) h_{\alpha_{5}}\left(c^{75}\right) h_{\alpha_{6}}\left(c^{52}\right) h_{\alpha_{7}}\left(c^{27}\right)$ \\
\hline$E_{7}\left(a_{1}\right)$ & $h_{\alpha_{1}}\left(c^{26}\right) h_{\alpha_{2}}\left(c^{37}\right) h_{\alpha_{3}}\left(c^{50}\right) h_{\alpha_{4}}\left(c^{72}\right) h_{\alpha_{5}}\left(c^{57}\right) h_{\alpha_{6}}\left(c^{40}\right) h_{\alpha_{7}}\left(c^{21}\right)$ \\
\hline$E_{7}\left(a_{2}\right)$ & $h_{\alpha_{1}}\left(c^{22}\right) h_{\alpha_{2}}\left(c^{31}\right) h_{\alpha_{3}}\left(c^{42}\right) h_{\alpha_{4}}\left(c^{60}\right) h_{\alpha_{5}}\left(c^{47}\right) h_{\alpha_{6}}\left(c^{32}\right) h_{\alpha_{7}}\left(c^{17}\right)$ \\
\hline$E_{7}\left(a_{3}\right)$ & $h_{\alpha_{1}}\left(c^{18}\right) h_{\alpha_{2}}\left(c^{25}\right) h_{\alpha_{3}}\left(c^{34}\right) h_{\alpha_{4}}\left(c^{50}\right) h_{\alpha_{5}}\left(c^{39}\right) h_{\alpha_{6}}\left(c^{28}\right) h_{\alpha_{7}}\left(c^{15}\right)$ \\
\hline$E_{7}\left(a_{4}\right)$ & $h_{\alpha_{1}}\left(c^{14}\right) h_{\alpha_{2}}\left(c^{19}\right) h_{\alpha_{3}}\left(c^{26}\right) h_{\alpha_{4}}\left(c^{38}\right) h_{\alpha_{5}}\left(c^{29}\right) h_{\alpha_{6}}\left(c^{20}\right) h_{\alpha_{7}}\left(c^{11}\right)$ \\
\hline$E_{7}\left(a_{5}\right)$ & $h_{\alpha_{1}}\left(c^{10}\right) h_{\alpha_{2}}\left(c^{15}\right) h_{\alpha_{3}}\left(c^{20}\right) h_{\alpha_{4}}\left(c^{30}\right) h_{\alpha_{5}}\left(c^{23}\right) h_{\alpha_{6}}\left(c^{16}\right) h_{\alpha_{7}}\left(c^{9}\right)$ \\
\hline$E_{8}$ & $h_{\alpha_{1}}\left(c^{92}\right) h_{\alpha_{2}}\left(c^{136}\right) h_{\alpha_{3}}\left(c^{182}\right) h_{\alpha_{4}}\left(c^{270}\right) h_{\alpha_{5}}\left(c^{220}\right) h_{\alpha_{6}}\left(c^{168}\right) h_{\alpha_{7}}\left(c^{114}\right) h_{\alpha_{8}}\left(c^{58}\right)$ \\
\hline$E_{8}\left(a_{1}\right)$ & $h_{\alpha_{1}}\left(c^{72}\right) h_{\alpha_{2}}\left(c^{106}\right) h_{\alpha_{3}}\left(c^{142}\right) h_{\alpha_{4}}\left(c^{210}\right) h_{\alpha_{5}}\left(c^{172}\right) h_{\alpha_{6}}\left(c^{132}\right) h_{\alpha_{7}}\left(c^{90}\right) h_{\alpha_{8}}\left(c^{46}\right)$ \\
\hline$E_{8}\left(a_{2}\right)$ & $h_{\alpha_{1}}\left(c^{60}\right) h_{\alpha_{2}}\left(c^{88}\right) h_{\alpha_{3}}\left(c^{118}\right) h_{\alpha_{4}}\left(c^{174}\right) h_{\alpha_{5}}\left(c^{142}\right) h_{\alpha_{6}}\left(c^{108}\right) h_{\alpha_{7}}\left(c^{74}\right) h_{\alpha_{8}}\left(c^{38}\right)$ \\
\hline$E_{8}\left(a_{3}\right)$ & $h_{\alpha_{1}}\left(c^{52}\right) h_{\alpha_{2}}\left(c^{76}\right) h_{\alpha_{3}}\left(c^{102}\right) h_{\alpha_{4}}\left(c^{152}\right) h_{\alpha_{5}}\left(c^{124}\right) h_{\alpha_{6}}\left(c^{96}\right) h_{\alpha_{7}}\left(c^{66}\right) h_{\alpha_{8}}\left(c^{34}\right)$ \\
\hline$E_{8}\left(a_{4}\right)$ & $h_{\alpha_{1}}\left(c^{44}\right) h_{\alpha_{2}}\left(c^{64}\right) h_{\alpha_{3}}\left(c^{86}\right) h_{\alpha_{4}}\left(c^{128}\right) h_{\alpha_{5}}\left(c^{104}\right) h_{\alpha_{6}}\left(c^{80}\right) h_{\alpha_{7}}\left(c^{54}\right) h_{\alpha_{8}}\left(c^{28}\right)$ \\
\hline$E_{8}\left(b_{4}\right)$ & $h_{\alpha_{1}}\left(c^{40}\right) h_{\alpha_{2}}\left(c^{58}\right) h_{\alpha_{3}}\left(c^{78}\right) h_{\alpha_{4}}\left(c^{116}\right) h_{\alpha_{5}}\left(c^{94}\right) h_{\alpha_{6}}\left(c^{72}\right) h_{\alpha_{7}}\left(c^{50}\right) h_{\alpha_{8}}\left(c^{26}\right)$ \\
\hline$E_{8}\left(a_{5}\right)$ & $h_{\alpha_{1}}\left(c^{36}\right) h_{\alpha_{2}}\left(c^{52}\right) h_{\alpha_{3}}\left(c^{70}\right) h_{\alpha_{4}}\left(c^{104}\right) h_{\alpha_{5}}\left(c^{84}\right) h_{\alpha_{6}}\left(c^{64}\right) h_{\alpha_{7}}\left(c^{44}\right) h_{\alpha_{8}}\left(c^{22}\right)$ \\
\hline$E_{8}\left(b_{5}\right)$ & $h_{\alpha_{1}}\left(c^{32}\right) h_{\alpha_{2}}\left(c^{48}\right) h_{\alpha_{3}}\left(c^{64}\right) h_{\alpha_{4}}\left(c^{96}\right) h_{\alpha_{5}}\left(c^{78}\right) h_{\alpha_{6}}\left(c^{60}\right) h_{\alpha_{7}}\left(c^{42}\right) h_{\alpha_{8}}\left(c^{22}\right)$ \\
\hline$E_{8}\left(a_{6}\right)$ & $h_{\alpha_{1}}\left(c^{28}\right) h_{\alpha_{2}}\left(c^{42}\right) h_{\alpha_{3}}\left(c^{56}\right) h_{\alpha_{4}}\left(c^{84}\right) h_{\alpha_{5}}\left(c^{68}\right) h_{\alpha_{6}}\left(c^{52}\right) h_{\alpha_{7}}\left(c^{36}\right) h_{\alpha_{8}}\left(c^{18}\right)$ \\
\hline$E_{8}\left(b_{6}\right)$ & $h_{\alpha_{1}}\left(c^{24}\right) h_{\alpha_{2}}\left(c^{36}\right) h_{\alpha_{3}}\left(c^{48}\right) h_{\alpha_{4}}\left(c^{72}\right) h_{\alpha_{5}}\left(c^{58}\right) h_{\alpha_{6}}\left(c^{44}\right) h_{\alpha_{7}}\left(c^{30}\right) h_{\alpha_{8}}\left(c^{16}\right)$ \\
\hline$E_{8}\left(a_{7}\right)$ & $h_{\alpha_{1}}\left(c^{16}\right) h_{\alpha_{2}}\left(c^{24}\right) h_{\alpha_{3}}\left(c^{32}\right) h_{\alpha_{4}}\left(c^{48}\right) h_{\alpha_{5}}\left(c^{40}\right) h_{\alpha_{6}}\left(c^{30}\right) h_{\alpha_{7}}\left(c^{20}\right) h_{\alpha_{8}}\left(c^{10}\right)$ \\
\hline$F_{4}$ & $h_{\alpha_{1}}\left(c^{22}\right) h_{\alpha_{2}}\left(c^{42}\right) h_{\alpha_{3}}\left(c^{30}\right) h_{\alpha_{4}}\left(c^{16}\right)$ \\
\hline$F_{4}\left(a_{1}\right)$ & $h_{\alpha_{1}}\left(c^{14}\right) h_{\alpha_{2}}\left(c^{26}\right) h_{\alpha_{3}}\left(c^{18}\right) h_{\alpha_{4}}\left(c^{10}\right)$ \\
\hline$F_{4}\left(a_{2}\right)$ & $h_{\alpha_{1}}\left(c^{10}\right) h_{\alpha_{2}}\left(c^{20}\right) h_{\alpha_{3}}\left(c^{14}\right) h_{\alpha_{4}}\left(c^{8}\right)$ \\
\hline$F_{4}\left(a_{3}\right)$ & $h_{\alpha_{1}}\left(c^{6}\right) h_{\alpha_{2}}\left(c^{12}\right) h_{\alpha_{3}}\left(c^{8}\right) h_{\alpha_{4}}\left(c^{4}\right)$ \\
\hline$G_{2}$ & $h_{\alpha_{1}}\left(c^{6}\right) h_{\alpha_{2}}\left(c^{10}\right)$ \\
\hline$G_{2}\left(a_{1}\right)$ & $h_{\alpha_{1}}\left(c^{2}\right) h_{\alpha_{2}}\left(c^{4}\right)$ \\
\hline
\end{tabular}




\section{The connected centralizer}

Again, $G$ will be a simple algebraic group of exceptional type defined over an algebraically closed field $k$ whose characteristic is either 0 or a good prime for $G$, and $T$ will be a fixed maximal torus of $G$. Let $e \in \mathfrak{L}(G)$ be a non-zero nilpotent orbit representative listed in Table 2, with associated cocharacter $\tau: k^{*} \rightarrow T$ and Levi subgroup $L$ of $G$ as given at the top of the page for $e$ in $\S 11$, so that $T \subset L$ and $e$ is distinguished in $\mathfrak{L}(L)$. Let $P$ be the parabolic subgroup corresponding to $\tau$ as defined in $\S 2$. Recall that we have $C_{G}(e)=R . C$, where $R=C_{G}(e) \cap R_{u}(P)$ and $C=C_{G}(e) \cap C_{G}(\operatorname{im}(\tau))$, with $\mathfrak{L}(R)=C_{\mathfrak{L}(G)}(e)_{+}$and $\mathfrak{L}(C)=C_{\mathfrak{L}(G)}(e)_{0}$. In this section we will obtain detailed information about both $\mathfrak{L}(R)$ and $C^{\circ}$.

\subsection{A basis of $C_{\mathfrak{L}(G)}(e)_{+}$and its upper central series}

We begin with $\mathfrak{L}(R)=C_{\mathfrak{L}(G)}(e)_{+}$; we shall first obtain a basis of this subalgebra. In order to do this we introduce a second grading on $\mathfrak{L}(G)$, which will lead to a refinement of that defined by the cocharacter $\tau$.

We have the torus $Z(L)^{\circ}$; write $X\left(Z(L)^{\circ}\right)$ for its character group. For $\chi \in X\left(Z(L)^{\circ}\right)$ write

$$
\mathfrak{L}(G)^{\chi}=\left\{v \in \mathfrak{L}(G):(\operatorname{Ad} t) v=\chi(t) v \text { for all } t \in Z(L)^{\circ}\right\}
$$

for the corresponding $Z(L)^{\circ}$-weight space of $\mathfrak{L}(G)$; we then have the grading

$$
\mathfrak{L}(G)=\bigoplus_{\chi \in X\left(Z(L)^{\circ}\right)} \mathfrak{L}(G)^{\chi} .
$$

Since $Z(L)^{\circ} \subseteq T$, each $\mathfrak{L}(G)^{\chi}$ for $\chi \neq 0$ has a basis of root vectors, while $\mathfrak{L}(G)^{0}=\mathfrak{L}(L)$; indeed, given $\beta, \gamma \in \Phi$ the root vectors $e_{\beta}$ and $e_{\gamma}$ lie in the same $Z(L)^{\circ}$-weight space if and only if $\beta-\gamma$ is a linear combination of roots in $\Pi([L, L])$.

As both $\operatorname{im}(\tau)$ and $Z(L)^{\circ}$ are subtori of $T$, each preserves the weight spaces of the other; accordingly we may decompose $\mathfrak{L}(G)$ into $\operatorname{im}(\tau) Z(L)^{\circ}$-weight spaces. Given a pair $(m, \chi) \in \mathbb{Z} \times X\left(Z(L)^{\circ}\right)$, we write

$$
\mathfrak{L}(G)_{m}^{\chi}=\mathfrak{L}(G)_{m} \cap \mathfrak{L}(G)^{\chi}
$$

we then have the grading

$$
\mathfrak{L}(G)=\bigoplus_{(m, \chi) \in \mathbb{Z} \times X\left(Z(L)^{\circ}\right)} \mathfrak{L}(G)_{m}^{\chi} .
$$

This is a refinement of the grading defined by $\tau$; for each pair $(m, \chi) \neq(0,0)$ the $\operatorname{im}(\tau) Z(L)^{\circ}$-weight space $\mathfrak{L}(G)_{m}^{\chi}$ has a basis consisting of root vectors.

Proposition 7.1 For $v \in \mathfrak{L}(G)$, write $v=\sum v_{m}^{\chi}$, where the sum runs over all pairs $(m, \chi) \in \mathbb{Z} \times X\left(Z(L)^{\circ}\right)$ and $v_{m}^{\chi} \in \mathfrak{L}(G)_{m}^{\chi}$. Then $v \in C_{\mathfrak{L}(G)}(e)$ if and only if $v_{m}^{\chi} \in$ $C_{\mathfrak{L}(G)}(e)$ for all $(m, \chi) \in \mathbb{Z} \times X\left(Z(L)^{\circ}\right)$.

Proof. Observe that $Z(L)^{\circ}$ lies in $C_{G}(e)$ and hence stabilizes $C_{\mathfrak{L}(G)}(e)$; recall that $\operatorname{im}(\tau)$ also stabilizes $C_{\mathfrak{L}(G)}(e)$. Thus $\operatorname{im}(\tau) Z(L)^{\circ}$ acts on $C_{\mathfrak{L}(G)}(e)$; the result follows.

We note that for $(m, \chi) \in \mathbb{Z} \times X\left(Z(L)^{\circ}\right)$, we have $\operatorname{ad}(e): \mathfrak{L}(G)_{m}^{\chi} \rightarrow \mathfrak{L}(G)_{m+2}^{\chi}$. If we take bases of the kernels of these maps for all pairs $(m, \chi)$ with $m>0$, by Proposition 2.9(b) we see that their union is the desired basis of $C_{\mathfrak{L}(G)}(e)_{+}=\bigoplus_{m>0} C_{\mathfrak{L}(G)}(e)_{m}$. In particular, $\operatorname{dim} C_{\mathfrak{L}(G)}(e)_{+}$is the cardinality of this basis. 
We now consider the upper central series

$$
0 \subset Z_{1}\left(C_{\mathfrak{L}(G)}(e)_{+}\right) \subset Z_{2}\left(C_{\mathfrak{L}(G)}(e)_{+}\right) \subset \cdots,
$$

where we recall that for a Lie algebra $\mathfrak{A}$ we have $Z_{1}(\mathfrak{A})=Z(\mathfrak{A})$, and for $n \geq 2$ the term $Z_{n}(\mathfrak{A})$ contains $Z_{n-1}(\mathfrak{A})$ and satisfies $Z\left(\mathfrak{A} / Z_{n-1}(\mathfrak{A})\right)=Z_{n}(\mathfrak{A}) / Z_{n-1}(\mathfrak{A})$. We note that both $\operatorname{im}(\tau)$ and $Z(L)^{\circ}$ act on $C_{\mathfrak{L}(G)}(e)_{+}$as Lie algebra automorphisms, and hence stabilize each subspace $Z_{n}\left(C_{\mathfrak{L}(G)}(e)_{+}\right)$. So a vector $v$ in $C_{\mathfrak{L}(G)}(e)_{+}$lies in $Z_{n}\left(C_{\mathfrak{L}(G)}(e)_{+}\right)$ if and only if, for each pair $(m, \chi) \in \mathbb{Z} \times X\left(Z(L)^{\circ}\right)$, the projection $v_{m}^{\chi}$ of $v$ into the $\operatorname{im}(\tau) Z(L)^{\circ}$-weight space $\mathfrak{L}(G)_{m}^{\chi}$ lies in $Z_{n}\left(C_{\mathfrak{L}(G)}(e)_{+}\right)$; in particular we have

$$
Z_{n}\left(C_{\mathfrak{L}(G)}(e)_{+}\right)=\bigoplus_{m>0} Z_{n, m}
$$

where for convenience we write $Z_{n, m}=\left(Z_{n}\left(C_{\mathfrak{L}(G)}(e)_{+}\right)\right)_{m}$. We may thus successively identify the terms $Z_{n}\left(C_{\mathfrak{L}(G)}(e)_{+}\right)$for $n=1,2, \ldots$ by calculating commutators of vectors in the basis of $C_{\mathfrak{L}(G)}(e)_{+}$just obtained. Arguing by induction on $n$, one sees that if $n+m$ is greater than the largest $\tau$-weight on $\mathfrak{L}(G)$, or more generally if there are fewer than $n$ distinct $\tau$-weights on $\mathfrak{L}(G)$ which are larger than $m$, then $C_{\mathfrak{L}(G)}(e)_{m}$ lies in $Z_{n}\left(C_{\mathfrak{L}(G)}(e)_{+}\right)$ and so $Z_{n, m}=C_{\mathfrak{L}(G)}(e)_{m}$.

Example To illustrate the above, we take an example in a classical group. Let $G=A_{5}$ and set $e=e_{10000}+e_{01000}+e_{00001}$; then $e$ is distinguished in the Lie algebra of the Levi subgroup $L$ of type $A_{2} A_{1}$ having simple system $\left\{\alpha_{1}, \alpha_{2}, \alpha_{5}\right\}$. We may take the associated cocharacter $\tau$ to be given by $\tau(c)=h_{\alpha_{1}}\left(c^{2}\right) h_{\alpha_{2}}\left(c^{2}\right) h_{\alpha_{5}}(c)$ for $c \in k^{*}$, since we then clearly have $\operatorname{im}(\tau) \subseteq[L, L] \cap T$ and $\tau(c) e=c^{2} e$ for all $c \in k^{*}$. We shall give bases of the various spaces $\mathfrak{L}(G)_{m}^{\chi}$ in a table, in which the rows are labelled by the values of $m$ and the columns correspond to the $Z(L)^{\circ}$-weights $\chi$ : for convenience we may represent each such $\chi$ as a pair $\left(n_{3}, n_{4}\right)$, where the root vectors $e_{\beta}$ lying in $\mathfrak{L}(G)^{\chi}$ are those for which

\begin{tabular}{|c|c|c|c|c|c|c|c|}
\hline & $(-1,-1)$ & $(-1,0)$ & $(0,-1)$ & $(0,0)$ & $(0,1)$ & $(1,0)$ & $(1,1)$ \\
\hline-4 & & & & $f_{11000}$ & & & \\
\hline-3 & $f_{11111}$ & & & & & & $e_{00110}$ \\
\hline-2 & & $f_{11100}$ & & $f_{10000}, f_{01000}, f_{00001}$ & & $e_{00100}$ & \\
\hline-1 & $f_{11110}, f_{01111}$ & & $f_{00011}$ & & $e_{00010}$ & & $e_{01110}, e_{00111}$ \\
\hline 0 & & $f_{01100}$ & & $h_{\alpha_{1}}, h_{\alpha_{2}}, h_{\alpha_{3}}, h_{\alpha_{4}}, h_{\alpha_{3}}$ & & $e_{01100}$ & \\
\hline 1 & $f_{01110}, f_{00111}$ & & $f_{00010}$ & & $e_{00011}$ & & $e_{11110}, e_{01111}$ \\
\hline 2 & & $f_{00100}$ & & $e_{10000}, e_{01000}, e_{00001}$ & & $e_{11100}$ & \\
\hline 3 & $f_{00110}$ & & & & & & $e_{11111}$ \\
\hline 4 & & & & $e_{11000}$ & & & \\
\hline
\end{tabular}
the coefficients in $\beta$ of $\alpha_{3}$ and $\alpha_{4}$ are $n_{3}$ and $n_{4}$ respectively.

By taking the kernels of the maps $\operatorname{ad}(e): \mathfrak{L}(G)_{m}^{\chi} \rightarrow \mathfrak{L}(G)_{m+2}^{\chi}$ for $m>0$, we obtain the

\begin{tabular}{|c|c|c|c|c|c|c|}
\hline & $(-1,-1)$ & $(-1,0)(0,-1)$ & $(0,0)$ & $\overline{(0,1)}$ & $(1,0)$ & $(1,1)$ \\
\hline 1 & $f_{01110}+f_{00111}$ & $f_{00010}$ & & $e_{00011}$ & & $e_{11110}+e_{01111}$ \\
\hline 2 & & $f_{00100}$ & $e_{10000}+e_{01000}, e_{00001}$ & & $e_{11100}$ & \\
\hline 3 & $f_{00110}$ & & & & & $e_{11111}$ \\
\hline 4 & & & $e_{11000}$ & & & \\
\hline
\end{tabular}
following table giving a basis of $C_{\mathfrak{L}(G)}(e)_{+}$.

Thus $\operatorname{dim} C_{\mathfrak{L}(G)}(e)_{+}=11$. Taking commutators then shows that $Z_{n, 2}=\langle e\rangle$ or $C_{\mathfrak{L}(G)}(e)_{2}$ according as $n \leq 2$ or $n>2$, while if $m \neq 2$ then $Z_{n, m}=0$ or $C_{\mathfrak{L}(G)}(e)_{m}$ according as $n+m \leq 4$ or $n+m>4$. 


\subsection{Identifying $C^{\circ}$}

We now turn to the subgroup $C^{\circ}$, which was shown to be reductive by Premet in [31, Theorem 2.3]. In fact, we shall not need to appeal to Premet's result: we shall obtain explicit generators for $C^{\circ}$, as these will be needed for the calculations in the following sections; consequently we shall show independently that $C^{\circ}$ is reductive, and give its rank and the type of its root system.

We have $\operatorname{dim} C=\operatorname{dim} C_{G}(e)-\operatorname{dim} R$, which is now known since the first term on the right is given in Table 2 while the second is equal to the dimension of $C_{\mathfrak{L}(G)}(e)_{+}$. The following result shows that the reductive rank of $C$ is equal to $\ell-\operatorname{rank}[L, L]$.

Lemma 7.2 The torus $Z(L)^{\circ}$ is a maximal torus of $C_{G}(e)$, and hence of $C$.

Proof. Let $S$ be a maximal torus of $C_{G}(e)$ with $Z(L)^{\circ} \subseteq S$; then $S \subset C_{G}\left(Z(L)^{\circ}\right)=L$. But then if $Z(L)^{\circ} \neq S$, we must have $S \cap[L, L]$ of positive dimension, contradicting the fact that $e$ is distinguished in $\mathfrak{L}(L)$.

A 1-dimensional $Z(L)^{\circ}$-invariant connected unipotent subgroup of $C$ will be called a $Z(L)^{\circ}$-root subgroup.

In what follows, we will define a set of closed connected simple subgroups $C_{1}, \ldots, C_{r}$, each generated by $Z(L)^{\circ}$-root subgroups and lying in $C_{G}(e) \cap C_{G}(\operatorname{im}(\tau))$. Note that to see that $C_{i} \subseteq C_{G}(\operatorname{im}(\tau))$ it suffices to show that the generating subgroups lie in products of $T$-root subgroups $U_{\alpha}$ with $\operatorname{im}(\tau) \subseteq \operatorname{ker} \alpha$. Consideration of dimensions will show that $C^{\circ}=C_{1} \ldots C_{r} Z(L)^{\circ}$; it will then follow that $C^{\circ}$ is reductive and $\left[C^{\circ}, C^{\circ}\right]=C_{1} \ldots C_{r}$.

To begin with we exclude the orbits in $E_{7}$ or $E_{8}$ labelled $A_{2} A_{1}{ }^{3}, A_{2}{ }^{2} A_{1}{ }^{2}$ and $D_{4}\left(a_{1}\right) A_{2}$; these will be treated separately later.

We define a certain number $s$ of subgroups $C_{i}$ as follows. For each we give a subsystem subgroup $G_{i}$ of $G$ possessing a (possibly trivial) graph automorphism $\sigma$ with the property that

$$
\text { for all } \alpha \in \Phi\left(G_{i}\right) \text {, we have } \alpha \perp\left\{\sigma^{j} \alpha: \sigma^{j} \alpha \neq \alpha\right\} \text {, }
$$

and let $C_{i}$ be the fixed point subgroup $\left(G_{i}\right)^{\sigma}$. We observe that since $C_{i}$ is the fixed point subgroup of a graph automorphism of $G_{i}$, it is a semisimple algebraic group and the type of $\Phi\left(C_{i}\right)$ is easily deduced; indeed, $G_{i}$ is chosen such that the fixed point subgroup $\left(G_{i}\right)^{\sigma}$ is simple. By construction, root elements of $C_{i}$ are products of commuting root elements of $G$. The action of such an element on $\mathfrak{L}(G)$ is hence given by $\exp \left(\operatorname{ad}\left(b_{1} e_{\gamma_{1}}+\cdots+b_{n} e_{\gamma_{n}}\right)\right)$, for some $\gamma_{1}, \ldots, \gamma_{n} \in \Phi(G)$ with $\left[e_{\gamma_{j}}, e_{\gamma_{j^{\prime}}}\right]=0$ for $j \neq j^{\prime}$ and some $b_{1}, \ldots, b_{n} \in k$; a check reveals that $\left[b_{1} e_{\gamma_{1}}+\cdots b_{n} e_{\gamma_{n}}, e\right]=0$. It follows that $C_{i} \subseteq C_{G}(e)$. On the page for $e$ in $\S 11$ we give explicit expressions for a set of root subgroups of $C_{i}$ corresponding to simple roots and their negatives.

At this point we observe that the subgroups $C_{1}, \ldots, C_{s}$ thus defined all commute, and therefore that $\operatorname{dim} C_{1} \ldots C_{s}=\sum_{i=1}^{s} \operatorname{dim} C_{i}$. Moreover we find that if we set $d=$ $\operatorname{dim} C-\operatorname{dim} Z(L)^{\circ}-\sum_{i=1}^{s}\left|\Phi\left(C_{i}\right)\right|$, then $d=0$ or 2 . If $d=0$, we set $r=s$. If on the other hand $d=2$, we set $r=s+1$; this occurs in the following cases.

(i) $G$ of type $F_{4}$ : orbits labelled $A_{1} \tilde{A}_{1}, A_{2} \tilde{A}_{1}$ and $B_{3}$ (numbers 3,6 and 11 respectively);

(ii) $G$ of type $E_{6}$ : orbit labelled $A_{2} A_{1}{ }^{2}$ (number 7);

(iii) $G$ of type $E_{7}$ : orbits labelled $A_{2} A_{1}^{2}, A_{3} A_{2} A_{1}, A_{4} A_{2}, D_{5}\left(a_{1}\right) A_{1}$ and $A_{6}$ (numbers $8,21,26,29$ and 34 respectively);

(iv) $G$ of type $E_{8}$ : orbits labelled $A_{2} A_{1}^{2}, A_{3} A_{2} A_{1}, A_{4} A_{2}, D_{5}\left(a_{1}\right) A_{1}, A_{4} A_{2} A_{1}, A_{4} A_{3}$, $D_{5}\left(a_{1}\right) A_{2}, A_{6}$ and $A_{6} A_{1}$ (numbers $7,20,27,29,30,34,36,42$ and 44 respectively). 
In these cases we proceed to define the group $C_{r}$, which will be of type $A_{1}$, as follows. We define two 1-dimensional subgroups of $G$ as the images of maps $x_{\beta}, x_{-\beta}: k \rightarrow G$, where $x_{\beta}(t)$ and $x_{-\beta}(t)$ for $t \in k$ are expressed as products of root elements of $G$. For $\gamma \in\{ \pm \beta\}$ one checks the following:

(I) $x_{\gamma}$ defines a morphism of algebraic groups $\mathbf{G}_{a} \rightarrow G$;

(II) for all $t \in k$ the element $x_{\gamma}(t)$ fixes $e$ and lies in $C_{G}(\operatorname{im}(\tau))$, and thus lies in $C^{\circ}$;

(III) the subgroup $X_{\gamma}=\left\{x_{\gamma}(t): t \in k\right\}$ is normalized by $Z(L)^{\circ}$, and is therefore a $Z(L)^{\circ}$-root subgroup of $C^{\circ}$;

(IV) the action of $Z(L)^{\circ}$ on $X_{-\gamma}$ is the inverse of that on $X_{\gamma}$.

We then set $C_{r}=\left\langle X_{ \pm \beta}\right\rangle$. Moreover, we observe that in all but two of these cases the roots occurring in the expressions for $x_{ \pm \beta}(t)$ are orthogonal to $\bigcup_{i=1}^{r-1} \Phi\left(G_{i}\right)$; in the remaining cases (the orbits labelled $A_{2} A_{1}^{2}$ in $E_{7}$ and $E_{8}$ ), one checks that $\left[X_{ \pm \beta}, C_{i}\right]=1$ for $i<r$. Thus $C_{1} \ldots C_{r-1} C_{r}$ is a commuting product, so that $\operatorname{dim}\left(C_{r} \cap C_{1} \ldots C_{r-1}\right)=0$; as $\operatorname{dim} C^{\circ}=\operatorname{dim}\left(C_{1} \ldots C_{r-1} Z(L)^{\circ}\right)+2$, we must have $C^{\circ}=C_{1} \ldots C_{r-1} C_{r} Z(L)^{\circ}$. Thus if we write $J=C_{1} \ldots C_{r-1} Z(L)^{\circ}$, we have $C^{\circ}=J C_{r}$, so $\operatorname{dim} J C_{r}=\operatorname{dim} J+2$, giving

$$
\begin{aligned}
2 & =\operatorname{dim}\left(J C_{r} / J\right) \\
& =\operatorname{dim}\left(C_{r} /\left(C_{r} \cap J\right)\right) \\
& =\operatorname{dim}\left(C_{r} /\left(C_{r} \cap Z(L)^{\circ}\right)\right) \\
& =\operatorname{dim}\left(C_{r} Z(L)^{\circ} / Z(L)^{\circ}\right) \\
& =\operatorname{dim} C_{r} Z(L)^{\circ}-\operatorname{dim} Z(L)^{\circ} .
\end{aligned}
$$

For $\gamma \in\{ \pm \beta\}$ we set $v_{\gamma}=d x_{\gamma}(1)$; then $v_{\gamma}$ spans the Lie algebra $\mathfrak{L}\left(X_{\gamma}\right)$. Let $\mathfrak{M}$ be the subalgebra of $\mathfrak{L}(G)$ generated by $v_{ \pm \beta}$. By calculation we see that $\mathfrak{M} \simeq \mathfrak{s l}_{2}(k)$. Since $\mathfrak{L}\left(C_{r} Z(L)^{\circ}\right)$ contains both $\mathfrak{L}\left(Z(L)^{\circ}\right)$ and $\mathfrak{M}$, and its dimension is $\operatorname{dim} \mathfrak{L}\left(Z(L)^{\circ}\right)+2$ while $\operatorname{dim}\left(\mathfrak{M} \cap \mathfrak{L}\left(Z(L)^{\circ}\right)\right) \leq 1$, we must have $\mathfrak{L}\left(C_{r} Z(L)^{\circ}\right)=\mathfrak{M}+\mathfrak{L}\left(Z(L)^{\circ}\right)$. As this Lie algebra has no non-trivial ideals consisting of nilpotent elements, we see that $C_{r} Z(L)^{\circ}$ is reductive; consideration of dimensions now shows that $C_{r}$ is of type $A_{1}$.

We now turn to the orbits excluded above. Note that the orbit $A_{2} A_{1}{ }^{3}$ occurs in both $E_{7}$ and $E_{8}$ (numbers 11 and 9 respectively); however, it will suffice to treat it in $E_{7}$ since the difference in dimensions between the centralizers in $E_{8}$ and in $E_{7}$ is 3 , which is accounted for by the fact that the centralizer in $E_{8}$ contains $C_{E_{8}}\left(E_{7}\right)=\left\langle X_{ \pm \delta}\right\rangle$ for $\delta={ }_{3}^{2465432}$. The other two orbits $A_{2}{ }^{2} A_{1}{ }^{2}$ and $D_{4}\left(a_{1}\right) A_{2}$ occur only in $E_{8}$ (numbers 15 and 22 respectively).

In each of these three cases we shall give four maps $x_{\beta_{1}}, x_{-\beta_{1}}, x_{\beta_{2}}, x_{-\beta_{2}}: k \rightarrow G$. For $\gamma \in\left\{ \pm \beta_{1}, \pm \beta_{2}\right\}$ one may then check that properties (I)-(IV) above hold, and thus we may write $\gamma: Z(L)^{\circ} \rightarrow k^{*}$ for the root corresponding to the $Z(L)^{\circ}$-root subgroup $X_{\gamma}$. As above, for each $\gamma$ we set $v_{\gamma}=d x_{\gamma}(1)$; then $v_{\gamma}$ spans the Lie algebra $\mathfrak{L}\left(X_{\gamma}\right)$. Let $\mathfrak{M}$ be the subalgebra of $\mathfrak{L}(G)$ generated by $v_{ \pm \beta_{1}}, v_{ \pm \beta_{2}}$; since each of the generating vectors lies in $\mathfrak{L}\left(C^{\circ}\right)$, we have $\mathfrak{M} \subseteq \mathfrak{L}\left(C^{\circ}\right)$. In each case we will find in $\mathfrak{M}$ a set of linearly independent vectors of cardinality equal to $\operatorname{dim} C^{\circ}$; it follows that $\mathfrak{M}=\mathfrak{L}\left(C^{\circ}\right)$ and the vectors found form a basis. We check that this basis satisfies the relations of a simple Lie algebra of type $A_{2}, B_{2}$ or $G_{2}$, having $\beta_{1}$ and $\beta_{2}$ as simple roots. In particular, $\mathfrak{L}\left(C^{\circ}\right)$ has no non-trivial ideal, and so must be simple; hence $C^{\circ}$ is simple and its type is that of $\mathfrak{M}$, with $\left\{\beta_{1}, \beta_{2}\right\}$ a simple system in $\Phi\left(C^{\circ}\right)$. 
We begin with the orbit labelled $A_{2} A_{1}{ }^{3}$ in $E_{7}$. Set

$$
\begin{aligned}
& x_{\beta_{1}}(t)=x_{0}^{111000}(t) x_{001100}(-2 t) x_{112100}\left(-t^{2}\right) x_{111000}(-t) x_{011100}(t), \\
& x_{-\beta_{1}}(t)=x_{-}{ }_{0}^{111000}(2 t) x_{-} 001100(-t) x_{-}{ }_{1}^{112100}\left(t^{2}\right) x_{-} 011000(-t) x_{-} 011100(t) \text {, } \\
& x_{\beta_{2}}(t)=x_{0}^{000110}(t) x_{00011}^{0001)}(t) \\
& x_{-\beta_{2}}(t)=x_{-}{ }_{0}^{000110}(t) x_{-}{ }_{0}^{000011}(t) \text {. }
\end{aligned}
$$

We have

$$
\begin{aligned}
& v_{\beta_{1}}=e_{0}^{111000}-2 e_{001100}-e_{011000}+e_{011100}, \\
& v_{-\beta_{1}}=2 f_{111000}-f_{001100}-f_{011000}+\underset{1}{f 011100}, \\
& v_{\beta_{2}}=e_{0}^{000110}+e_{000011}^{0}, \\
& v_{-\beta_{2}}=\underset{0}{f_{000110}}+\underset{0}{f_{000011}} \text {. }
\end{aligned}
$$

Let

$$
\begin{aligned}
& v_{\beta_{1}+\beta_{2}}=\left[v_{\beta_{2}}, v_{\beta_{1}}\right]=\underset{1}{2 e_{001111}}-\underset{0}{e} \underset{1}{111110}+e_{011110}-e_{011111}, \\
& v_{-\left(\beta_{1}+\beta_{2}\right)}=\left[v_{-\beta_{1}}, v_{-\beta_{2}}\right]=f_{001111}-2 f_{111110}+f_{011110}-f_{011111}, \\
& v_{2 \beta_{1}+\beta_{2}}=\frac{1}{2}\left[v_{\beta_{1}+\beta_{2}}, v_{\beta_{1}}\right]=e_{122110}-2 e_{012211}+e_{112111}-e_{112210}, \\
& v_{-\left(2 \beta_{1}+\beta_{2}\right)}=\frac{1}{2}\left[v_{-\beta_{1}}, v_{-\left(\beta_{1}+\beta_{2}\right)}\right]=2 f_{122110}-f_{1}^{012211}+f_{112111}-f_{112210} \text {, } \\
& v_{3 \beta_{1}+\beta_{2}}=\frac{1}{3}\left[v_{\beta_{1}}, v_{2 \beta_{1}+\beta_{2}}\right]=-e_{123211}-e_{123210}, \\
& v_{-\left(3 \beta_{1}+\beta_{2}\right)}=\frac{1}{3}\left[v_{-\left(2 \beta_{1}+\beta_{2}\right)}, v_{-\beta_{1}}\right]=-f_{123211}-f_{123210}, \\
& v_{3 \beta_{1}+2 \beta_{2}}=\left[v_{\beta_{2}}, v_{3 \beta_{1}+\beta_{2}}\right]=-e_{123321}+e_{123221}, \\
& v_{-\left(3 \beta_{1}+2 \beta_{2}\right)}=\left[v_{-\left(3 \beta_{1}+\beta_{2}\right)}, v_{-\beta_{2}}\right]=-f_{123321}+f_{123221}, \\
& h_{\beta_{1}}=\left[v_{\beta_{1}}, v_{-\beta_{1}}\right]=2 h_{0}^{111000}+2 h_{1}^{001100}+h_{1}^{011000}+h_{0}^{011100}, \\
& h_{\beta_{2}}=\left[v_{\beta_{2}}, v_{-\beta_{2}}\right]=\underset{0}{h_{00110}}+h_{0}^{000011} \text {. }
\end{aligned}
$$

One checks that the 14 vectors $v_{ \pm \beta_{1}}, v_{ \pm \beta_{2}}, v_{ \pm\left(\beta_{1}+\beta_{2}\right)}, v_{ \pm\left(2 \beta_{1}+\beta_{2}\right)}, v_{ \pm\left(3 \beta_{1}+\beta_{2}\right)}, v_{ \pm\left(3 \beta_{1}+2 \beta_{2}\right)}$, $h_{\beta_{1}}, h_{\beta_{2}}$ satisfy the relations of a Lie algebra of type $G_{2}$.

Now consider the orbit labelled $A_{2}^{2} A_{1}^{2}$ in $E_{8}$. Set

$$
\begin{aligned}
& x_{\beta_{1}}(t)=x_{1121100}(t) x_{1221000}(t) x_{1}^{0122100}(-t), \\
& x_{-\beta_{1}}(t)=x_{-} 1121100(t) x_{-} 1221000(t) x_{-} 0122100(-t), \\
& x_{\beta_{2}}(t)=x_{0}^{1111110}(t) x_{0011111}(-2 t) x_{1122221}\left(t^{2}\right) x_{0111110}(-t) x_{0111111}(t), \\
& x_{-\beta_{2}}(t)=x_{-} 1111110(2 t) x_{0} 0011111(-t) x_{-}{ }_{1}^{1122221}\left(-t^{2}\right) x_{-} 0111110(-t) x_{-} 0111111(t) .
\end{aligned}
$$


We have

$$
\begin{aligned}
& v_{\beta_{1}}=e_{1121100}+e_{1221000}-e_{0122100}, \\
& v_{-\beta_{1}}=f_{1121100}+f_{1221000}-f_{0122100}, \\
& v_{\beta_{2}}=e_{0}^{1111110}-2 e_{0011111}-e_{0111110}+e_{0111111} \text {, } \\
& v_{-\beta_{2}}=2 \underset{0}{f_{1111110}}-f_{0011111}-f_{0111110}+f_{0} f_{0} 11111 \text {. }
\end{aligned}
$$

Let

$$
\begin{aligned}
& v_{\beta_{1}+\beta_{2}}=\left[v_{\beta_{1}}, v_{\beta_{2}}\right]=e_{1233210}-2 e_{1232111}-e_{1232210}+e_{1232211}, \\
& v_{-\left(\beta_{1}+\beta_{2}\right)}=\left[v_{-\beta_{2}}, v_{-\beta_{1}}\right]=2 f_{1233210}-f_{1232111}-f_{2} f_{2} 22210+f_{1232211} \text {, } \\
& v_{\beta_{1}+2 \beta_{2}}=\frac{1}{2}\left[v_{\beta_{1}+\beta_{2}}, v_{\beta_{2}}\right]=e_{1343321}-e_{2244321}-e_{2343221}, \\
& v_{-\left(\beta_{1}+2 \beta_{2}\right)}=\frac{1}{2}\left[v_{-\beta_{2}}, v_{-\left(\beta_{1}+\beta_{2}\right)}\right]=f_{1343321}-f_{1244321}-f_{2343221}, \\
& h_{\beta_{1}}=\left[v_{\beta_{1}}, v_{-\beta_{1}}\right]=h_{1121100}+h_{1221000}+h_{0122100} \text {, } \\
& h_{\beta_{2}}=\left[v_{\beta_{2}}, v_{-\beta_{2}}\right]=2 h_{1111110}+2 h_{0011111}+h_{0111110}+h_{0111111} \text {. }
\end{aligned}
$$

One checks that the 10 vectors $v_{ \pm \beta_{1}}, v_{ \pm \beta_{2}}, v_{ \pm\left(\beta_{1}+\beta_{2}\right)}, v_{ \pm\left(\beta_{1}+2 \beta_{2}\right)}, h_{\beta_{1}}, h_{\beta_{2}}$ satisfy the relations of a Lie algebra of type $B_{2}$.

Finally we consider the orbit labelled $D_{4}\left(a_{1}\right) A_{2}$ in $E_{8}$. Set

$$
\begin{aligned}
& x_{\beta_{1}}(t)=x_{0}^{0001111}(3 t) x_{0121100}(t) x_{0}^{0011111}(-t) x_{1}^{011100}(t) x_{0122211}\left(-2 t^{2}\right) \times \\
& x_{1}^{001110}(2 t) x_{0}^{0111110}(-t),
\end{aligned}
$$

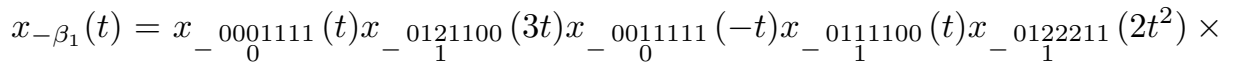

$$
\begin{aligned}
& x_{-} 0011110(t) x_{-}{ }_{0}^{0111110}(-2 t) \text {, } \\
& x_{\beta_{2}}(t)=x_{1111111}(-3 t) x_{1232100}(-t) x_{1121111}(-t) x_{1222100}(t) x_{2343211}\left(-2 t^{2}\right) \times \\
& x_{1122110}(2 t) x_{1221110}(-t),
\end{aligned}
$$

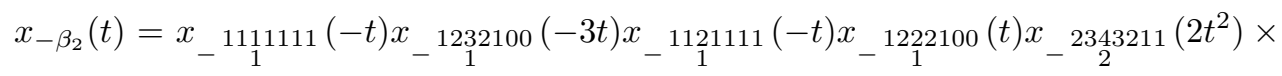

$$
\begin{aligned}
& x_{-}^{1122110}(t) x_{-}{ }_{1}^{1221110}(-2 t) \text {. }
\end{aligned}
$$

We have

$$
\begin{aligned}
& v_{\beta_{1}}=3 e_{0}^{0001111}+e_{0121100}-e_{0011111}+e_{0111100}+2 e_{0011110}-e_{0111110}, \\
& v_{-\beta_{1}}=f_{0001111}+3 f_{0121100}-f_{001111}+f_{0111100}+f_{0011110}-2 f_{0111110}, \\
& v_{\beta_{2}}=-3 e_{1111111}-e_{1232100}-e_{1121111}+e_{1222100}+2 e_{1122110}-e_{1221110} \text {, } \\
& v_{-\beta_{2}}=-f_{1111111}-3 f_{1232100}-f_{1121111}+f_{1222100}+f_{1122110}-2 f_{1221110} \text {. }
\end{aligned}
$$


Let

$$
\begin{aligned}
& v_{\beta_{1}+\beta_{2}}=\frac{1}{2}\left[v_{\beta_{1}}, v_{\beta_{2}}\right]=2 e_{2} 233210-e_{1232221}+e_{1233211}+e_{1232211}, \\
& v_{-\left(\beta_{1}+\beta_{2}\right)}=\frac{1}{2}\left[v_{-\beta_{2}}, v_{-\beta_{1}}\right]=f_{1233210}-2 f_{1232221}+f_{1233211}+f_{1232211}, \\
& h_{\beta_{1}}=\frac{1}{2}\left[v_{\beta_{1}}, v_{-\beta_{1}}\right]=2 h_{0}^{0001111}+2 h_{0}^{0010000}+2 h_{1} h_{111100}+h_{1}^{0011110}+h_{0111110} \text {, } \\
& h_{\beta_{2}}=\frac{1}{2}\left[v_{\beta_{2}}, v_{-\beta_{2}}\right]=2 h_{1111111}+2 h_{0010000}+2 h_{1222100}+h_{1122110}+h_{1221110} .
\end{aligned}
$$

One checks that the 8 vectors $v_{ \pm \beta_{1}}, v_{ \pm \beta_{2}}, v_{ \pm\left(\beta_{1}+\beta_{2}\right)}, h_{\beta_{1}}, h_{\beta_{2}}$ (suitably scaled) satisfy the relations of a Lie algebra of type $A_{2}$.

This completes the determination of $C^{\circ}$ in all cases. 


\section{A composition series for the Lie algebra centralizer}

Once more, $G$ will be a simple algebraic group of exceptional type defined over an algebraically closed field $k$ whose characteristic is either 0 or a good prime for $G$, and $T$ will be a fixed maximal torus of $G$. Let $e \in \mathfrak{L}(G)$ be a non-zero nilpotent orbit representative listed in Table 2, with associated cocharacter $\tau: k^{*} \rightarrow T$ and Levi subgroup $L$ of $G$ as given at the top of the page for $e$ in $\S 11$, so that $T \subset L$ and $e$ is distinguished in $\mathfrak{L}(L)$; write $C=C_{G}(e) \cap C_{G}(\operatorname{im}(\tau))$ as in $\S 2$. In this section, we shall determine the action of $C^{\circ}$ on $C_{\mathfrak{L}(G)}(e)_{+}$; this will enable us in $\S 9$ to find $\left(Z\left(C_{\mathfrak{L}(G)}(e)_{+}\right)\right)^{C}$.

We will in fact give rather more detailed information. As $C$ acts on $C_{\mathfrak{L}(G)}(e)_{+}$as Lie algebra automorphisms, it preserves each term $Z_{n}\left(C_{\mathfrak{L}(G)}(e)_{+}\right)$in the upper central series of $C_{\mathfrak{L}(G)}(e)_{+}$. Moreover as $C$ lies in $C_{G}(\operatorname{im}(\tau))$, it respects the grading $\mathfrak{L}(G)=$ $\bigoplus_{m \in \mathbb{Z}} \mathfrak{L}(G)_{m}$; thus for each $n>0$, it preserves each summand in the decomposition

$$
Z_{n}\left(C_{\mathfrak{L}(G)}(e)_{+}\right)=\bigoplus_{m>0} Z_{n, m}
$$

where as in $\S 7.1$ we write $Z_{n, m}=\left(Z_{n}\left(C_{\mathfrak{L}(G)}(e)_{+}\right)\right)_{m}$. For each $n$ and $m$, we will describe $Z_{n, m}$ as a sum of indecomposable tilting modules for the group $\left[C^{\circ}, C^{\circ}\right]$, the vast majority of which will turn out to be irreducible. For those which are irreducible we shall give a high weight vector; for those few which are reducible we shall give generating vectors.

\subsection{Parametrizing a maximal torus of $\left[C^{\circ}, C^{\circ}\right]$}

We first explain our parametrization of a maximal torus of $\left[C^{\circ}, C^{\circ}\right]$; we shall then go on in $\S 8.2$ to determine the weights of $\left[C^{\circ}, C^{\circ}\right]$ on each $Z_{n, m}$. Recall from $\S 7.2$ that we have $\left[C^{\circ}, C^{\circ}\right]=C_{1} \ldots C_{r}$, a commuting product of simple groups; for each $i$ we have obtained a simple system $\Pi\left(C_{i}\right)$ and the corresponding root subgroups, and we shall now give an appropriate maximal torus of $C_{i}$. For each $\beta \in \Pi\left(C_{i}\right)$, we will define a cocharacter $h_{\beta}: k^{*} \rightarrow T$ with the following properties: its image lies in $C_{i}$, and for all $\beta^{\prime} \in \Pi\left(C_{i}\right)$, $t \in k$ and $c \in k^{*}$ we have $h_{\beta}(c) x_{\beta^{\prime}}(t) h_{\beta}(c)^{-1}=x_{\beta^{\prime}}\left(c^{n_{\beta \beta^{\prime}}} t\right)$, where the $n_{\beta \beta^{\prime}}$ are the integers given by the Cartan matrix of the root system $\Phi\left(C_{i}\right)$. It follows that $h_{\beta}$ is the standard parametrization of the 1-dimensional torus $\left\langle X_{ \pm \beta}\right\rangle \cap T$.

Consider first those $C_{i}$ obtained in $\S 7.2$ as fixed point subgroups of subsystem subgroups $G_{i}$. In these cases, for each $\beta \in \Pi\left(C_{i}\right)$ we obtained subgroups $X_{\beta}$ and $X_{-\beta}$, isomorphic to $\mathbf{G}_{a}$, such that $S L_{2}(k)$ maps surjectively onto $\left\langle X_{ \pm \beta}\right\rangle$; on the page for $e$ in $\S 11$ we give isomorphisms $x_{\gamma}: \mathbf{G}_{a} \rightarrow X_{\gamma}$ for $\gamma \in\{ \pm \beta\}$. For all $\beta \in \Pi\left(C_{i}\right)$ and $c \in k^{*}$, set $n_{\beta}(c)=x_{\beta}(c) x_{-\beta}\left(-c^{-1}\right) x_{\beta}(c)$ and $h_{\beta}(c)=n_{\beta}(c) n_{\beta}(-1)$. In fact, the expression for each such $h_{\beta}$ in terms of the $h_{\alpha}$ for $\alpha \in \Pi(G)$ can be obtained directly as follows. We have $x_{\beta}(t)=\prod_{\gamma \in S_{\beta}} x_{\gamma}\left(f_{\gamma}(t)\right)$ for some non-zero polynomials $f_{\gamma}$, where $S_{\beta}$ is a subset of $\Phi(G)$ in which any two roots are orthogonal; moreover, we observe that each such polynomial $f_{\gamma}$ is linear. This then implies that $h_{\beta}=\prod_{\gamma \in S_{\beta}} h_{\gamma}$; writing each $h_{\gamma}$ in terms of the $h_{\alpha}$ for $\alpha \in \Pi(G)$ gives the desired expression. It is then straightforward to check that the required properties of the $h_{\beta}$ hold.

We now consider the orbits listed under (i)-(iv) in $\$ 7.2$. Here we constructed $C_{r}$ of type $A_{1}$, again giving subgroups $X_{\gamma}$, and explicit isomorphisms $x_{\gamma}: \mathbf{G}_{a} \rightarrow X_{\gamma}$, for $\gamma \in\{ \pm \beta\}$. In each case, we note that $\left\langle X_{ \pm \beta}\right\rangle$ is contained in a subsystem subgroup $H$, which is a commuting product $H=H_{1} \ldots H_{s}$ where each $H_{j}$ is of type $A_{i_{j}}$ for some $i_{j} \in \mathbb{N}$. For each factor $H_{j}$ of type $A_{1}$ or $A_{2}$, we let $\pi_{j}: H \rightarrow H_{j}$ be the projection map and apply the Lemma of [49, pp.301-302] to find a cocharacter $\psi_{j}: k^{*} \rightarrow T \cap \pi_{j}\left(\left\langle X_{ \pm \beta}\right\rangle\right)$, 
such that for all $t \in k$ and $c \in k^{*}$ we have $\psi_{j}(c) \pi_{j}\left(x_{\beta}(t)\right) \psi_{j}(c)^{-1}=\pi_{j}\left(x_{\beta}\left(c^{2} t\right)\right)$. If each $H_{j}$ is of type $A_{1}$ or $A_{2}$, we define $h_{\beta}=\prod_{j=1}^{s} \psi_{j}$. This leaves just the orbits labelled $A_{3} A_{2} A_{1}$ in $E_{7}$ and $E_{8}$ (numbers 21 and 20 respectively), $A_{4} A_{2}$ in $E_{7}$ and $E_{8}$ (numbers 26 and 27 respectively) and $A_{4} A_{2} A_{1}$ in $E_{8}$ (number 30 ). We shall treat together orbits with the same label.

Consider first the $A_{3} A_{2} A_{1}$ orbits. If $G=E_{7}$, we know from $\S 7.2$ that $C^{\circ}$ is simple of type $A_{1}$, and hence is equal to $\left\langle X_{ \pm \beta}\right\rangle$. We define a cocharacter $h_{\beta}: k^{*} \rightarrow T$ by

$$
h_{\beta}(c)=h_{\alpha_{1}}\left(c^{8}\right) h_{\alpha_{2}}\left(c^{12}\right) h_{\alpha_{3}}\left(c^{16}\right) h_{\alpha_{4}}\left(c^{24}\right) h_{\alpha_{5}}\left(c^{18}\right) h_{\alpha_{6}}\left(c^{12}\right) h_{\alpha_{7}}\left(c^{6}\right) ;
$$

one then checks that $\operatorname{im}\left(h_{\beta}\right) \subseteq Z(L)^{\circ} \subseteq C^{\circ}$, and $h_{\beta}(c) x_{\beta}(t) h_{\beta}(c)^{-1}=x_{\beta}\left(c^{2} t\right)$ for all $c \in k^{*}$ and $t \in k$ as required. If instead $G=E_{8}$, then $C^{\circ}$ is a commuting product of the group $\left\langle X_{ \pm \beta}\right\rangle$ above and $C_{G}\left(E_{7}\right)=\left\langle X_{ \pm \delta}\right\rangle$ for $\delta={ }_{3}^{2465432}$, which has already been treated; we take the same cocharacter $h_{\beta}$ for the factor $\left\langle X_{ \pm \beta}\right\rangle$.

Next consider the $A_{4} A_{2}$ orbits. If $G=E_{7}$, again we know from $\S 7.2$ that $C^{\circ}$ is simple of type $A_{1}$, and hence is equal to $\left\langle X_{ \pm \beta}\right\rangle$. Here we define $h_{\beta}$ by

$$
h_{\beta}(c)=h_{\alpha_{1}}\left(c^{6}\right) h_{\alpha_{2}}\left(c^{9}\right) h_{\alpha_{3}}\left(c^{12}\right) h_{\alpha_{4}}\left(c^{18}\right) h_{\alpha_{5}}\left(c^{15}\right) h_{\alpha_{6}}\left(c^{10}\right) h_{\alpha_{7}}\left(c^{5}\right) ;
$$

one then checks that $\operatorname{im}\left(h_{\beta}\right) \subseteq Z(L)^{\circ} \subseteq C^{\circ}$, and $h_{\beta}(c) x_{\beta}(t) h_{\beta}(c)^{-1}=x_{\beta}\left(c^{2} t\right)$ for all $c \in k^{*}$ and $t \in k$ as required. If instead $G=E_{8}$, we proceed exactly as before.

Finally consider the $A_{4} A_{2} A_{1}$ orbit; here $G=E_{8}$. Once more we know from $\S 7.2$ that $C^{\circ}$ is simple of type $A_{1}$, and hence is equal to $\left\langle X_{ \pm \beta}\right\rangle$. This time we define $h_{\beta}$ by

$$
h_{\beta}(c)=h_{\alpha_{1}}\left(c^{10}\right) h_{\alpha_{2}}\left(c^{15}\right) h_{\alpha_{3}}\left(c^{20}\right) h_{\alpha_{4}}\left(c^{30}\right) h_{\alpha_{5}}\left(c^{24}\right) h_{\alpha_{6}}\left(c^{18}\right) h_{\alpha_{7}}\left(c^{12}\right) h_{\alpha_{8}}\left(c^{6}\right) ;
$$

one then checks that $\operatorname{im}\left(h_{\beta}\right) \subseteq Z(L)^{\circ} \subseteq C^{\circ}$, and $h_{\beta}(c) x_{\beta}(t) h_{\beta}(c)^{-1}=x_{\beta}\left(c^{2} t\right)$ for all $c \in k^{*}$ and $t \in k$ as required.

We now consider the orbits treated separately in $\S 7.2$, which are those labelled $A_{2} A_{1}{ }^{3}$ in $E_{7}$ and $E_{8}$ (numbers 11 and 9 respectively), $A_{2}{ }^{2} A_{1}{ }^{2}$ in $E_{8}$ (number 15) and $D_{4}\left(a_{1}\right) A_{2}$ in $E_{8}$ (number 22). With the exception of the $A_{2} A_{1}{ }^{3}$ orbit in $E_{8}$, in each of these cases $C^{\circ}$ is simple of rank 2; in the exceptional case there is an additional $A_{1}$ factor $\left\langle X_{ \pm \delta}\right\rangle$ for $\delta={ }_{3}^{2465432}$, which we may treat as above. We defined $Z(L)^{\circ}$-root subgroups $X_{\beta_{1}}$ and $X_{\beta_{2}}$ of $C^{\circ}$, and showed that $\left\{\beta_{1}, \beta_{2}\right\}$ form a simple system for $\Phi\left(C^{\circ}\right)$. We will now define two cocharacters $h_{\beta_{1}}$ and $h_{\beta_{2}}$; one then checks that $\operatorname{im}\left(h_{\beta_{i}}\right) \subseteq Z(L)^{\circ} \subseteq C^{\circ}$ and $h_{\beta_{i}}(c) x_{\beta_{j}}(t) h_{\beta_{i}}(c)^{-1}=x_{\beta_{j}}\left(c^{n_{\beta_{i} \beta_{j}}} t\right)$ for all $c \in k^{*}, t \in k$ and $i, j \in\{1,2\}$, where the $n_{\beta_{i} \beta_{j}}$ are the integers given by the Cartan matrix, as required.

First consider the $A_{2} A_{1}{ }^{3}$ orbit in both $E_{7}$ and $E_{8}$. For $c \in k^{*}$, set

$$
\begin{aligned}
& h_{\beta_{1}}(c)=h_{\alpha_{1}}\left(c^{2}\right) h_{\alpha_{2}}\left(c^{3}\right) h_{\alpha_{3}}\left(c^{4}\right) h_{\alpha_{4}}\left(c^{6}\right) h_{\alpha_{5}}\left(c^{3}\right), \\
& h_{\beta_{2}}(c)=h_{\alpha_{5}}(c) h_{\alpha_{6}}\left(c^{2}\right) h_{\alpha_{7}}(c) .
\end{aligned}
$$

Now consider the $A_{2}{ }^{2} A_{1}{ }^{2}$ orbit in $E_{8}$. For $c \in k^{*}$, set

$$
\begin{aligned}
& h_{\beta_{1}}(c)=h_{\alpha_{1}}\left(c^{2}\right) h_{\alpha_{2}}\left(c^{3}\right) h_{\alpha_{3}}\left(c^{4}\right) h_{\alpha_{4}}\left(c^{6}\right) h_{\alpha_{5}}\left(c^{4}\right) h_{\alpha_{6}}\left(c^{2}\right), \\
& h_{\beta_{2}}(c)=h_{\alpha_{1}}\left(c^{2}\right) h_{\alpha_{2}}\left(c^{3}\right) h_{\alpha_{3}}\left(c^{4}\right) h_{\alpha_{4}}\left(c^{6}\right) h_{\alpha_{5}}\left(c^{6}\right) h_{\alpha_{6}}\left(c^{6}\right) h_{\alpha_{7}}\left(c^{6}\right) h_{\alpha_{8}}\left(c^{3}\right) .
\end{aligned}
$$

Finally consider the $D_{4}\left(a_{1}\right) A_{2}$ orbit in $E_{8}$. For $c \in k^{*}$, set

$$
\begin{aligned}
h_{\beta_{1}}(c) & =h_{\alpha_{2}}\left(c^{3}\right) h_{\alpha_{3}}\left(c^{3}\right) h_{\alpha_{4}}\left(c^{6}\right) h_{\alpha_{5}}\left(c^{6}\right) h_{\alpha_{6}}\left(c^{6}\right) h_{\alpha_{7}}\left(c^{4}\right) h_{\alpha_{8}}\left(c^{2}\right), \\
h_{\beta_{2}}(c) & =h_{\alpha_{1}}\left(c^{6}\right) h_{\alpha_{2}}\left(c^{6}\right) h_{\alpha_{3}}\left(c^{9}\right) h_{\alpha_{4}}\left(c^{12}\right) h_{\alpha_{5}}\left(c^{9}\right) h_{\alpha_{6}}\left(c^{6}\right) h_{\alpha_{7}}\left(c^{4}\right) h_{\alpha_{8}}\left(c^{2}\right) .
\end{aligned}
$$




\subsection{Composition factors of $C_{\mathfrak{L}(G)}(e)_{+}$}

We now turn to the action of $C^{\circ}$ on each subspace $Z_{n, m}=\left(Z_{n}\left(C_{\mathfrak{L}(G)}(e)_{+}\right)\right)_{m}$. For each $m$ we treat the subspaces in the order of increasing values of $n$; note that we have

$$
0 \subset Z_{1, m} \subset Z_{2, m} \subset \cdots,
$$

while $Z_{n, m}=C_{\mathfrak{L}(G)}(e)_{m}$ for sufficiently large $n$. For convenience we write $D=\left[C^{\circ}, C^{\circ}\right]$ from now on, so that we have the central product $C^{\circ}=D Z\left(C^{\circ}\right)^{\circ}$.

We fix a maximal torus and Borel subgroup for the group $D$ as follows. For each of the simple factors $C_{i}$ of $D$, we have given a simple system $\Pi\left(C_{i}\right)$; for each $\beta \in \Pi\left(C_{i}\right)$ we have given a simple root subgroup $X_{\beta}$ and cocharacter $h_{\beta}$. Write $\Pi(D)=\bigcup_{i} \Pi\left(C_{i}\right)$, and set $T_{0}=\left\langle h_{\beta}(c): \beta \in \Pi(D), c \in k^{*}\right\rangle$ and $B_{0}=T_{0}\left\langle X_{\beta}: \beta \in \Pi(D)\right\rangle$. For $\beta \in \Pi(D)$ let $\lambda_{\beta}$ be the fundamental dominant weight corresponding to the simple root $\beta$. For a dominant weight $\lambda$, we fix notation for certain $k D$-modules with high weight $\lambda$ as follows: we write $V_{D}(\lambda)$ for the irreducible module, $W_{D}(\lambda)$ for the Weyl module, and $T_{D}(\lambda)$ for the tilting module (see [14, pp.183, 458] for the definitions of the second and third of these).

For each $n$ and $m$, we find the set of $T_{0}$-weights on $Z_{n, m}$, with multiplicities, using the basis of this subspace obtained by the procedure described in $\S 7.1$. This task is simplified by the fact that $T_{0} \subset Z(L)^{0}$, and hence $T_{0}$ is constant on each of the $Z(L)^{\circ}$-weight spaces $\mathfrak{L}(G)^{\chi}$. We find that, with a small number of exceptions, all such weights are restricted; these exceptions will be indicated and treated later.

Thus assume all $T_{0}$-weights on $Z_{n, m}$ are restricted. Let $\mu_{1}$ be a maximal weight with respect to the ordering imposed by the choice of base $\Pi(D)$. As $\mu_{1}$ is maximal, we deduce the existence of a $D$-composition factor $V_{D}\left(\mu_{1}\right)$ in $Z_{n, m}$. Moreover, in each of the cases which occur, we observe that $\operatorname{dim} V_{D}\left(\mu_{1}\right)=\operatorname{dim} W_{D}\left(\mu_{1}\right)$. In particular, the set of weights of $V_{D}\left(\mu_{1}\right)$ and their multiplicities are the same as in characteristic 0. (We remark here that the modules which occur are small enough to be treated in [22].)

Now if $\operatorname{dim} Z_{n, m}>\operatorname{dim} V_{D}\left(\mu_{1}\right)$, we take the list of $T_{0}$-weights occurring in $Z_{n, m}$ and remove those found in $V_{D}\left(\mu_{1}\right)$ according to their multiplicities; we then iterate the above process, choosing at each stage a weight $\mu_{j}$ maximal among the remaining weights. Again we find that the Weyl module $W_{D}\left(\mu_{j}\right)$ is irreducible. It follows from [14, Proposition II.2.14] that $Z_{n, m}$ decomposes as a direct sum of irreducible submodules, one for each $\mu_{j}$. We now explain how we find a high weight vector for each of the submodules in this direct sum decomposition.

The basis of $Z_{n, m}$ found by the procedure described in $\S 7.1$ is a basis of weight vectors for the torus $Z(L)^{\circ}$, and hence for $T_{0}$. Let $\mu$ be the high weight of a $D$-composition factor of $Z_{n, m}$. We have a basis of the $T_{0}$-weight space $\left(Z_{n, m}\right)_{\mu}$, which we may use to calculate the fixed point space $V_{n, m, \mu}$ of the subgroup $R_{u}\left(B_{0}\right)$ in its action on $\left(Z_{n, m}\right)_{\mu}$. Since $Z\left(C^{\circ}\right)^{\circ}$ commutes with the action of $D$, it stabilizes $V_{n, m, \mu}$; hence there is a basis of $V_{n, m, \mu}$ consisting of $Z\left(C^{\circ}\right)^{\circ}$-weight vectors $v$, and if $n>1$ we may choose this basis to extend that of $V_{n-1, m, \mu}$ already found. We give these vectors in the table on the page for $e$ in $\S 11$.

In fact, for each such high weight vector $v$ one can obtain a basis for the irreducible $D$ submodule which it generates. Firstly, since the Weyl module $W_{D}(\mu)$ is both irreducible and the universal high weight module with high weight $\mu$, it is the only cyclic $D$-module of high weight $\mu$ (up to isomorphism); thus $\langle D v\rangle$ is an irreducible $D$-submodule of high weight $\mu$, and therefore also a $C^{\circ}$-submodule. Now as the weight $\mu$ is restricted, by [9] we see that $\mathfrak{L}(D)$ acts irreducibly on the $D$-module $V_{D}(\mu)$; we thus have $\langle\mathfrak{L}(D) v\rangle=\langle D v\rangle$. The calculation may therefore be performed within $\mathfrak{L}(G)$, which simplifies matters. 
We illustrate the procedure with an example. Let $G=E_{8}$ and take $e$ to be the representative of the $D_{6}$ orbit (number 48). On the page for $e$ in $\S 11$ we have identified $D$ as being of type $B_{2}$, with simple roots $\beta_{1}$ and $\beta_{2}$, where $\beta_{1}={ }_{2}^{2343210}$ and $x_{ \pm \beta_{2}}(t)=$ $x_{ \pm} 0011111(t) x_{ \pm}^{0111111}(-t)$. For $\gamma \in\left\{ \pm \beta_{1}, \pm \beta_{2}\right\}$ set $v_{\gamma}=d x_{\gamma}(1)$, so that

$$
\begin{aligned}
v_{\beta_{1}} & =e_{2343210}, & v_{\beta_{2}} & =e_{0011111}-e_{0111111}, \\
v_{-\beta_{1}} & =f_{2343210}, & v_{-\beta_{2}} & =f_{0011111}-f_{0111111}
\end{aligned} ;
$$

then $\mathfrak{L}(D)$ is the Lie algebra generated by $v_{ \pm \beta_{1}}, v_{ \pm \beta_{2}}$. The table on the page for $e$ lists nine high weight vectors, of which five have $T_{0}$-weight 0 , one has $T_{0}$-weight $\lambda_{1}$ and three have $T_{0}$-weight $\lambda_{2}$. Those with $T_{0}$-weight 0 span trivial $D$-submodules. The $T_{0}$-weights in $V_{D}\left(\lambda_{1}\right)$ are $\lambda_{1}, \lambda_{1}-\beta_{1}, \lambda_{1}-\beta_{1}-\beta_{2}, \lambda_{1}-\beta_{1}-2 \beta_{2}$ and $\lambda_{1}-2 \beta_{1}-2 \beta_{2}$. Thus if we let $w_{1}$ be the high weight vector of $T_{0}$-weight $\lambda_{1}$ lying in $Z_{2,10}$, by successively taking commutators (and scaling where appropriate for convenience) we obtain

$$
\begin{aligned}
w_{1} & =e_{2465431}, \\
w_{2}=\left[v_{-\beta_{1}}, w_{1}\right] & =e_{0122221}, \\
w_{3}=\left[v_{-\beta_{2}}, w_{2}\right] & =e_{0011110}^{0}-e_{0111110}, \\
w_{4}=\frac{1}{2}\left[v_{-\beta_{2}}, w_{3}\right] & =\underset{0000001}{f_{000}}, \\
w_{5}=-\left[v_{-\beta_{1}}, w_{4}\right] & =\underset{2343211}{f_{23}}
\end{aligned}
$$

hence the $D$-submodule is

$$
\left\langle e_{3}^{2465431}, e_{1}^{0122221}, e_{1}^{0011110}-e_{0}^{0111110}, \underset{0}{0000001}, f_{2343211}\right\rangle .
$$

The $T_{0}$-weights in $V_{D}\left(\lambda_{2}\right)$ are $\lambda_{2}, \lambda_{2}-\beta_{2}, \lambda_{2}-\beta_{1}-\beta_{2}$ and $\lambda_{2}-\beta_{1}-2 \beta_{2}$. Thus if we let $x_{1}$ be the high weight vector of $T_{0}$-weight $\lambda_{1}$ lying in $Z_{3,5}$, by successively taking commutators we obtain

$$
\begin{aligned}
& x_{1}=e_{1343211}-e_{2243221}+e_{1233321}, \\
& x_{2}=\left[v_{-\beta_{2}}, x_{1}\right]=e_{1232110}-e_{122210}+e_{1232100}, \\
& x_{3}=\left[v_{-\beta_{1}}, x_{2}\right]=f_{1111100}+f_{1121000}+f_{1111110}, \\
& x_{4}=\left[v_{-\beta_{2}}, x_{3}\right]=f_{1122221}-f_{1222211}+f_{1232111} ;
\end{aligned}
$$

hence the $D$-submodule is

$$
\begin{gathered}
\left\langle e_{1343211}-e_{1243221}+e_{1233321}, e_{1232110}-e_{1222210}+e_{1232100}\right. \\
f_{1} \\
\left.f_{111100}+f_{1121000}+f_{111110}, f_{1122221}-f_{1222211}+f_{1232111}\right\rangle \\
1
\end{gathered}
$$

Similarly we find that the $D$-submodules forming $Z_{2,9}$ and $Z_{1,15}$ are respectively

$$
\left\langle e_{1343321}-e_{1244321}, e_{1233210}-e_{1232210}, f_{1111000}+f_{1110000}, f_{1122111}-f_{1221111}\right\rangle
$$

and

$$
\left\langle e_{1354321}, e_{1343210}, f_{1000000}, f_{1111111}\right\rangle .
$$


We now consider the instances of non-restricted $T_{0}$-weights in some $C_{\mathfrak{L}(G)}(e)_{m}$; here we must have $\operatorname{char}(k)=p>0$. The cases concerned are as follows:

(i) if $G=E_{7}$ and $e$ lies in the orbit labelled $A_{3} A_{2} A_{1}$ (number 21), the $D$-modules $Z_{n, 2}$ for $n \geq 3$ and $Z_{n, 4}$ for $n \geq 2$ are non-restricted when $p \in\{5,7\}$ and $p=5$ respectively;

(ii) if $G=E_{7}$ and $e$ lies in the orbit labelled $A_{4} A_{2}$ (number 26), the $D$-module $Z_{n, 4}$ for $n \geq 3$ is non-restricted when $p=5$;

(iii) if $G=E_{8}$ and $e$ lies in the orbit labelled $A_{3} A_{2} A_{1}$ (number 20), the $D$-module $Z_{n, 2}$ for $n \geq 5$ is non-restricted when $p=7$.

We first note that case (iii) will be covered by the treatment of case (i). In each of cases (i) and (ii), $C^{\circ}=D$ is of type $A_{1}$ and so the $T_{0}$-weights may be regarded as integers. Moreover, as the $T_{0}$-weights on $\mathfrak{L}(G)$ are all at most $2 p-2$, the $A_{1}$ subgroups are 'good' in the sense of [38], which then shows that $\mathfrak{L}(G)$ is a direct sum of tilting modules for $D$. Such a tilting module $T_{D}(a)$ of high weight $a$ has a composition series of the form $0 \subset W_{1} \subset W_{2} \subset W_{3}$, where $W_{1} \simeq W_{3} / W_{2} \simeq V_{D}(2 p-2-a)$ and $W_{2} / W_{1} \simeq V_{D}(a)$, and is generated by the union of the $T_{0}$-weight spaces of weights $a$ and $2 p-2-a$.

In both cases (i) and (ii), we note that the $T_{0}$-weights occurring in $Z_{n, 4}$ (with multiplicities) are $6,4,2,2,0,0,-2,-2,-4,-6$. If $p=5$, there exists a tilting module summand of high weight 6 ; as $T_{D}(6)$ has dimension 10 , we must have $Z_{n, 4} \simeq T_{D}(6)$. We give bases for the $T_{0}$-weight spaces of weights 2 and 6 in the table on the page for $e$ in $\S 11$.

In case (i), we find that the $T_{0}$-weights occurring in $Z_{n, 2}$ (with multiplicities) are $8,6,4,4,2,2,0,0,0,-2,-2,-4,-4,-6,-8$. We deduce therefore that $Z_{n, 2}$ has a tilting module summand of high weight 8 . We may take as a basis for $Z_{n, 2}$ the following vectors, where in each case the subscript denotes the $T_{0}$-weight:

$$
\begin{aligned}
& w_{8}=e_{234321}, \\
& w_{6}=e_{123321}-e_{123221}, \\
& w_{4}=e_{122210}+e_{122111}, \\
& w_{2}=e_{001111}-e_{011111}-e_{111110}-e_{111100}, \\
& w_{0}=e_{00000}, \quad w_{0}^{\prime}=e_{0} 0000+e_{010000}, \\
& w_{-2}=f_{111000}-f_{011000}-f_{001100}-f_{001110} \\
& w_{-4}=f_{012111}+f_{012210} \text {, } \\
& w_{-6}=f_{123211}+f_{123210}, \\
& w_{-8}=f_{124321} \text {. } \\
& w_{4}{ }^{\prime}=e_{012221}-e_{122210}-e_{112211}, \\
& w_{2}^{\prime}=e_{001111}+e_{011110}+e_{111100}, \\
& w_{0}^{\prime \prime}=e_{000100}+e_{000010}+e_{000001}, \\
& w_{-2}{ }^{\prime}=f_{111000}+f_{011100}+f_{001110}, \\
& w_{-4}{ }^{\prime}=f_{112110}+f_{012111}+f_{122100} \text {, }
\end{aligned}
$$

Now if $p=5$, the $T_{0}$-weights occurring in the tilting module $T_{D}(8)$ (with multiplicities) are $8,6,4,2,0,0,-2,-4,-6,-8$. Since the remaining $T_{0}$-weights in $Z_{n, 2}$ are $4,2,0,-2,-4$, we must have $Z_{n, 2} \simeq T_{D}(8) \oplus V_{D}(4)$ (whereas $Z_{1,2}=Z_{2,2}=\langle e\rangle \simeq V_{D}(0)$, so that $Z_{2,2}$ is not a direct summand of $Z_{3,2}$; this is the only example of such behaviour in the present work). We observe that in $Z_{n, 2}$ the space of fixed points of $T_{0}$-weight 4 is 1-dimensional, spanned by $3 w_{4}+w_{4}{ }^{\prime}$; we therefore list this vector in the table on the page for $e$ in $\S 11$. In order to find generating vectors for $T_{D}(8)$, we will work upward from the bottom of the composition series; it will in fact be convenient to identify a basis of this summand. It is 
clear that $e=w_{0}+w_{0}{ }^{\prime}+w_{0}{ }^{\prime \prime}$ spans $W_{1}$. To complete a basis for $W_{2}$, we apply $x_{-\beta}(t)$ to $w_{8}$ and $x_{\beta}(t)$ to $w_{-8}$ and iterate to obtain

$$
\left\{w_{8}, w_{6}, w_{4}-2 w_{4}{ }^{\prime}, w_{2}-3 w_{2}{ }^{\prime}, w_{-8}, w_{-6}, 3 w_{-4}-2 w_{-4}{ }^{\prime}, w_{-2}-3 w_{-2}{ }^{\prime}\right\} .
$$

To complete a basis of $W_{3}$, it then suffices to find a vector $w$ of $T_{0}$-weight 0 such that the projection of $x_{-\beta}(t) w-w$ into the space of vectors of $T_{0}$-weight -2 is a nonzero multiple of $w_{-2}-3 w_{-2}{ }^{\prime}$; we find that $w=3 w_{0}-w_{0}^{\prime}$ has this property. We therefore list the vectors $w_{8}, e$ and $w$ in the table on the page for $e$ in $\S 11$.

If instead $p=7$, the $T_{0}$-weights occurring in the tilting module $T_{D}(8)$ (with multiplicities) are $8,6,4,4,2,2,0,0,-2,-2,-4,-4,-6,-8$; thus $Z_{n, 2}$ decomposes as a direct sum $T_{D}(8) \oplus V_{D}(0)$. It is clear that the 1-dimensional irreducible summand is spanned by $e$. We find a basis for the summand $T_{D}(8)$ as in the preceding case; we give bases for the $T_{0}$-weight spaces of weights 4 and 8 in the table on the page for $e$ in $\S 11$.

This completes the consideration of the action of $C^{\circ}$ on the $Z_{n, m}$. 


\section{The Lie algebra of the centre of the centralizer}

As in the preceding sections, $G$ will be a simple algebraic group of exceptional type defined over an algebraically closed field $k$ whose characteristic is either 0 or a good prime for $G$, and $T$ will be a fixed maximal torus of $G$; here, however, for convenience we shall take $G$ of adjoint type. Let $e \in \mathfrak{L}(G)$ be a non-zero nilpotent orbit representative listed in Table 2, with associated cocharacter $\tau: k^{*} \rightarrow T$ and Levi subgroup $L$ of $G$ as given at the top of the page for $e$ in $\S 11$, so that $T \subset L$ and $e$ is distinguished in $\mathfrak{L}(L)$; write $C=C_{G}(e) \cap C_{G}(\operatorname{im}(\tau))$ as in $\S 2$, and $D=\left[C^{\circ}, C^{\circ}\right]$ as in $\S 8.2$. In this section we will complete our calculations by finding a basis for $\mathcal{Z}=\left(Z\left(C_{\mathfrak{L}(G)}(e)_{+}\right)\right)^{C}$, which by Theorem 3.9 is equal to $\mathfrak{L}\left(Z\left(C_{G}(e)\right)\right)$; as explained at the end of $\S 2$, by Proposition 2.13 the results obtained will also be valid for $G$ of simply connected type.

In $\S 7.1$ we obtained the upper central series

$$
0 \subset Z_{1}\left(C_{\mathfrak{L}(G)}(e)_{+}\right) \subset Z_{2}\left(C_{\mathfrak{L}(G)}(e)_{+}\right) \subset \cdots ;
$$

for each $n$ we wrote

$$
Z_{n}\left(C_{\mathfrak{L}(G)}(e)_{+}\right)=\bigoplus_{m>0} Z_{n, m},
$$

where $Z_{n, m}=\left(Z_{n}\left(C_{\mathfrak{L}(G)}(e)_{+}\right)\right)_{m}$. In $\S 8.2$ we then wrote each $Z_{n, m}$ as a direct sum of indecomposable tilting modules for $D$; since the generating vectors were chosen to be $Z\left(C^{\circ}\right)^{\circ}$-weight vectors, and $C^{\circ}=D Z\left(C^{\circ}\right)^{\circ}$, each summand is in fact a $C^{\circ}$-submodule. The table on the page for $e$ in $\S 11$ is divided vertically into sections, the last $n$ of which give the $C^{\circ}$-submodules lying in $Z_{n}\left(C_{\mathfrak{L}(G)}(e)_{+}\right)$, along with their $\tau$-weights $m$; in particular the last section of the table lists the $C^{\circ}$-submodules lying in $Z\left(C_{\mathfrak{L}(G)}(e)_{+}\right)$.

Now we observe that $\left(Z\left(C_{\mathfrak{L}(G)}(e)_{+}\right)\right)^{C^{\circ}}$ is simply the sum of the trivial $C^{\circ}$-submodules in $Z\left(C_{\mathfrak{L}(G)}(e)_{+}\right)$. Lemma 7.2 shows that $C^{\circ}=D Z(L)^{\circ}$; we have

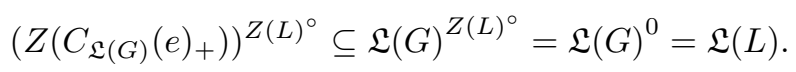

Thus a basis for $\left(Z\left(C_{\mathfrak{L}(G)}(e)_{+}\right)\right)^{C^{\circ}}$ may be formed from the $C^{\circ}$-submodules listed in the last section of the table by taking those vectors which both lie in $\mathfrak{L}(L)$ and generate trivial $D$-submodules. In the fifth column of the table we have written $\mathcal{Z}^{\natural}$ for $\left(Z\left(C_{\mathfrak{L}(G)}(e)_{+}\right)\right)^{C^{\circ}}$, and have indicated its basis vectors; for convenience of reference each such vector is renamed $z_{m}$, with the subscript being the $\tau$-weight, and vectors with the same value of $m$ being distinguished by superscripts.

Finally we complete the determination of $\mathcal{Z}$, whose basis vectors are indicated in the sixth column of the table, by considering the action of the full reductive complement $C$ on $\left(Z\left(C_{\mathfrak{L}(G)}(e)_{+}\right)\right)^{C^{\circ}}$ in the cases where $C / C^{\circ} \neq 1$; it is here that the assumption on the isogeny type of $G$ simplifies matters, since it minimizes the size of the component group $C / C^{\circ}$. Each of the cases must be treated separately, except that in the groups of type $E_{\ell}$ we take together orbits with the same name. We write $A=C / C^{\circ}$; given $c \in C$ we denote its image in $A$ by $\bar{c}$. For the structure of the finite group $A$, we refer to [6, 25].

In each case, we give a set of representatives $c$ of generators of $A$, which we found by explicit calculation; one verifies in each case that $c \in C_{G}(e)$. The elements $c$ which we give are all conjugates of elements of $N_{G}(T)$, and in most cases actually lie in $N_{G}(T)$. We write elements of $N_{G}(T)$ as products $n_{\beta_{1}} \ldots n_{\beta_{s}} t$, where $t \in T$ and $\beta_{1}, \ldots, \beta_{s} \in \Phi$; we write $t$ as a product of elements $h_{i}\left(\lambda_{i}\right)$, where for brevity we use $h_{i}$ to denote $h_{\alpha_{i}}$. If $s>0$, for the convenience of the reader we shall give the image of each relevant root vector under $\operatorname{Ad}(c)$. We take $\omega, \zeta, i \in k \backslash\{1\}$ with $\omega^{3}=\zeta^{5}=-i^{2}=1$, and set $\phi=\zeta^{2}+\zeta^{3}$. 


\section{1 $G$ of type $G_{2}$}

If $G$ is of type $G_{2}$ there is only a single orbit having $A \neq 1$.

\subsubsection{Orbit $G_{2}\left(a_{1}\right)$ (number 3$)$}

Here $e=e_{01}+e_{31}$, which is regular in the Lie algebra of $H$, a long root $A_{2}$ subsystem subgroup of $G$; we have $A \cong \mathcal{S}_{3}$, and $C^{\circ}=1$. We take

$$
\begin{aligned}
& c_{1}=h_{1}(\omega), \\
& c_{2}=n_{10} h_{2}(-1) .
\end{aligned}
$$

Clearly $c_{1} \in Z(H)$. The action of $\operatorname{Ad}\left(c_{2}\right)$ on the root vectors concerned is as follows:

$$
\text { interchanged : } e_{01} \leftrightarrow e_{31} \text {. }
$$

Thus $c_{2}$ acts as a non-trivial graph automorphism of $H$. Hence $A=\left\langle\bar{c}_{1}, \bar{c}_{2}\right\rangle$. We have $\left(Z\left(C_{\mathfrak{L}(G)}(e)_{+}\right)\right)^{C^{\circ}}=\left\langle z_{2}, z_{4}\right\rangle$, where $z_{2}=e$ and $z_{4}=e_{32}$. Since $\operatorname{Ad}\left(c_{2}\right)$ negates $z_{4}$, we have $\mathcal{Z}=\left\langle z_{2}\right\rangle$.

\section{2 $G$ of type $F_{4}$}

If $G$ is of type $F_{4}$ there are 7 orbits having $A \neq 1$.

\subsubsection{Orbit $\tilde{A}_{1}$ (number 2)}

Here $e=e_{0001}$; we have $A \cong \mathcal{S}_{2}$, and $C^{\circ}=A_{3}$ with $\Pi(D)=\{1000,0100,1242\}$. We take

$$
c=n_{0121} h_{3}(-1) .
$$

The action of $\operatorname{Ad}(c)$ on the root vectors concerned is as follows:

$$
\begin{aligned}
\text { fixed : } & e_{0001}, e_{0100} ; \\
\text { interchanged : } & e_{1000} \leftrightarrow e_{1242} .
\end{aligned}
$$

Thus $c$ acts as a non-trivial graph automorphism of $C^{\circ}$. Hence $A=\langle\bar{c}\rangle$. We have $\left(Z\left(C_{\mathfrak{L}(G)}(e)_{+}\right)\right)^{C^{\circ}}=\left\langle z_{2}\right\rangle$, where $z_{2}=e$, so $\mathcal{Z}=\left\langle z_{2}\right\rangle$.

\subsubsection{Orbit $A_{2}$ (number 4$)$}

Here $e=e_{1000}+e_{0100}$; we have $A \cong \mathcal{S}_{2}$, and $C^{\circ}=\tilde{A}_{2}$ with $\Pi(D)=\{0001,1231\}$. We take

$$
c=n_{0110} n_{1120} h_{1}(-1) .
$$

The action of $\operatorname{Ad}(c)$ on the root vectors concerned is as follows:

$$
\text { interchanged : } e_{1000} \leftrightarrow e_{0100}, e_{0001} \leftrightarrow e_{1231} .
$$

Thus $c$ acts as a non-trivial graph automorphism of $C^{\circ}$. Hence $A=\langle\bar{c}\rangle$. We have $\left(Z\left(C_{\mathfrak{L}(G)}(e)_{+}\right)\right)^{C^{\circ}}=\left\langle z_{2}, z_{4}\right\rangle$, where $z_{2}=e$ and $z_{4}=e_{1100}$. Since $\operatorname{Ad}(c)$ negates $z_{4}$, we have $\mathcal{Z}=\left\langle z_{2}\right\rangle$. 


\subsubsection{Orbit $B_{2}$ (number 7$)$}

Here $e=e_{0100}+e_{0010}$; we have $A \cong \mathcal{S}_{2}$, and $C^{\circ}=A_{1}{ }^{2}$ with $\Pi(D)=\{0122,2342\}$. We take

$$
c=n_{1110} h_{4}(-1) \text {. }
$$

The action of $\operatorname{Ad}(c)$ on the root vectors concerned is as follows:

$$
\begin{aligned}
\text { fixed : } & e_{0100}, e_{0010} ; \\
\text { interchanged : } & e_{0122} \leftrightarrow e_{2342} .
\end{aligned}
$$

Thus $c$ acts as a non-trivial graph automorphism of $C^{\circ}$. Hence $A=\langle\bar{c}\rangle$. We have $\left(Z\left(C_{\mathfrak{L}(G)}(e)_{+}\right)\right)^{C^{\circ}}=\left\langle z_{2}, z_{6}\right\rangle$, where $z_{2}=e$ and $z_{6}=e_{0120}$. Since $\operatorname{Ad}(c)$ fixes $z_{6}$, we have $\mathcal{Z}=\left\langle z_{2}, z_{6}\right\rangle$.

\subsubsection{Orbit $C_{3}\left(a_{1}\right)$ (number 9$)$}

Here $e=e_{0001}+e_{0120}+e_{0100}$, which is non-regular distinguished in the Lie algebra of $H$, a $C_{3}$ subsystem subgroup of $G$ with simple system $\{0001,0010,0100\}$; we have $A \cong \mathcal{S}_{2}$, and $C^{\circ}=A_{1}$ with $\Pi(D)=\{2342\}$. We take

$$
c=h_{4}(-1) .
$$

We find that $C_{H}(c) \cong C_{2} C_{1}$, and that $e$ is regular in $C_{H}(c)$. Moreover, as the only element of order 2 in $T \cap C^{\circ}$ is $h_{2}(-1) h_{4}(-1)$ it follows that $c \notin C^{\circ}$. Hence $A=\langle\bar{c}\rangle$. We have $\left(Z\left(C_{\mathfrak{L}(G)}(e)_{+}\right)\right)^{C^{\circ}}=\left\langle z_{2}, z_{6}\right\rangle$, where $z_{2}=e$ and $z_{6}=e_{0122}$. Since $\operatorname{Ad}(c)$ fixes $z_{6}$, we have $\mathcal{Z}=\left\langle z_{2}, z_{6}\right\rangle$.

\subsubsection{Orbit $F_{4}\left(a_{3}\right)$ (number 10$)$}

Here $e=e_{0100}+e_{1120}+e_{1111}+e_{0121}$, which is regular in the Lie algebra of $H$, an $A_{2} \tilde{A}_{2}$ subsystem subgroup of $G$; we have $A \cong \mathcal{S}_{4}$, and $C^{\circ}=1$. We take

$$
\begin{aligned}
& c_{1}=h_{1}(\omega) h_{3}(\omega), \\
& c_{2}=n_{1000} n_{0010} h_{2}(-1) h_{3}(-1), \\
& c_{3}=\left(n_{0011} h_{3}\left(-\frac{2}{3}\right) h_{4}\left(\frac{2}{3}\right)\right)^{u},
\end{aligned}
$$

where

$$
u=x_{0011}\left(-\frac{1}{2}\right) x_{0001}(1) x_{0010}(-1) .
$$

Clearly $c_{1} \in Z(H)$. The action of $\operatorname{Ad}\left(c_{2}\right)$ on the root vectors concerned is as follows:

$$
\text { interchanged : } e_{0100} \leftrightarrow e_{1120}, e_{1111} \leftrightarrow e_{0121} \text {. }
$$

Thus $c_{2}$ acts as a non-trivial graph automorphism of $H$. The action of $c_{3}$ is rather more complicated. Calculation shows that

$$
\operatorname{Ad}(u) e=e_{0100}+e_{0110}+e_{0120}+e_{1120}-\frac{1}{2} e_{0111}+e_{1111}+\frac{3}{2} e_{0121}+\frac{9}{4} e_{0122} .
$$

The action of $\operatorname{Ad}\left(c_{3}{ }^{-1}\right)$ on the root vectors concerned is as follows:

fixed : $e_{0120}, e_{1120}, e_{0111}, e_{1111}$;

$$
\text { interchanged : } e_{0100} \leftrightarrow \frac{9}{4} e_{0122}, e_{0110} \leftrightarrow \frac{3}{2} e_{0121} \text {. }
$$


Thus $c_{3} \in C_{G}(e)$. Hence $A=\left\langle\bar{c}_{1}, \bar{c}_{2}, \bar{c}_{3}\right\rangle$. (Indeed, we see that $c_{1}{ }^{3}=c_{2}{ }^{2}=c_{3}{ }^{2}=1$, with $c_{1}^{c_{2}}=c_{1}^{-1},\left[c_{2}, c_{3}\right]=1$ and $\left(c_{1} c_{3}\right)^{3}=1$; we may thus identify $c_{1}, c_{2}$ and $c_{3}$ with the elements $\left(\begin{array}{lll}1 & 2 & 3\end{array}\right),\left(\begin{array}{ll}1 & 2\end{array}\right)$ and $\left(\begin{array}{ll}1 & 2\end{array}\right)\left(\begin{array}{ll}3 & 4\end{array}\right)$ of $S_{4}$.) We have $\left(Z\left(C_{\mathfrak{L}(G)}(e)_{+}\right)\right)^{C^{\circ}}=\left\langle z_{2}, z_{6}{ }^{1}, z_{6}{ }^{2}\right\rangle$, where $z_{2}=e, z_{6}{ }^{1}=e_{1342}$ and $z_{6}{ }^{2}=e_{2342}$. Since $\operatorname{Ad}\left(c_{1}\right)$ multiplies $z_{6}{ }^{1}$ by $\omega^{2}$ and $z_{6}{ }^{2}$ by $\omega$, we have $\mathcal{Z}=\left\langle z_{2}\right\rangle$.

\subsubsection{Orbit $F_{4}\left(a_{2}\right)$ (number 13 )}

Here $e=e_{1110}+e_{0001}+e_{0120}+e_{0100}$, which is regular in the Lie algebra of $H$, a $C_{3} A_{1}$ subsystem subgroup of $G$; we have $A \cong \mathcal{S}_{2}$, and $C^{\circ}=1$. We take

$$
c=h_{2}(-1) \text {. }
$$

Clearly $c \in Z(H)$. Hence $A=\langle\bar{c}\rangle$. We have $\left(Z\left(C_{\mathfrak{L}(G)}(e)_{+}\right)\right)^{C^{\circ}}=\left\langle z_{2}, z_{10}{ }^{1}, z_{10}{ }^{2}\right\rangle$, where $z_{2}=e, z_{10}{ }^{1}=e_{1342}$ and $z_{10}{ }^{2}=e_{2342}$. Since $\operatorname{Ad}(c)$ negates $z_{10}{ }^{1}$ and fixes $z_{10}{ }^{2}$, we have $\mathcal{Z}=\left\langle z_{2}, z_{10}{ }^{2}\right\rangle$.

\subsubsection{Orbit $F_{4}\left(a_{1}\right)$ (number 14 )}

Here $e=e_{0100}+e_{1000}+e_{0120}+e_{0001}$, which is regular in the Lie algebra of $H$, a $B_{4}$ subsystem subgroup of $G$; we have $A \cong \mathcal{S}_{2}$, and $C^{\circ}=1$. We take

$$
c=h_{4}(-1) \text {. }
$$

Clearly $c \in Z(H)$. Hence $A=\langle\bar{c}\rangle$. We have $\left(Z\left(C_{\mathfrak{L}(G)}(e)_{+}\right)\right)^{C^{\circ}}=\left\langle z_{2}, z_{10}, z_{14}\right\rangle$, where $z_{2}=e, z_{10}=e_{1222}-e_{1242}$ and $z_{14}=e_{2342}$. Since $\operatorname{Ad}(c)$ fixes both $z_{10}$ and $z_{14}$, we have $\mathcal{Z}=\left\langle z_{2}, z_{10}, z_{14}\right\rangle$.

This completes the consideration of the group $F_{4}$.

\section{3 $G$ of type $E_{n}$}

According as $G$ is of type $E_{6}, E_{7}$ or $E_{8}$ there are 3,13 or 32 orbits having $A \neq 1$. As stated above, we treat together orbits in different groups having the same label. Note that we will write all roots as lying in $\Phi\left(E_{8}\right)$.

\subsubsection{Orbit $A_{2}$ (number 4 in $E_{6}, 5$ in $E_{7}, 4$ in $E_{8}$ )}

Here $e=e_{1000000}+e_{0100000}$; we have $A \cong \mathcal{S}_{2}$, and $C^{\circ}=A_{2}{ }^{2}, A_{5}$ or $E_{6}$ respectively with $\Pi(D)=\left\{\begin{array}{c}0001000 \\ 0\end{array}, \underset{0}{000001},{ }_{0}^{0000100},{ }_{0}^{0000010},{ }_{1}^{1232100},{ }_{1}^{0000000}\right\} \cap \Phi(G)$. We take

$$
c=n_{0111000} n_{0110000} n_{1121000} h_{1}(-1) h_{2}(-1) h_{5}(-1) .
$$

The action of $\operatorname{Ad}(c)$ on the root vectors concerned is as follows:

$$
\begin{aligned}
& \text { fixed : } \underset{0}{0000001}, \underset{0}{0000010} \\
& \text { interchanged : } e_{0} 1000000 \leftrightarrow \underset{0}{e_{0100000}}, \underset{0}{0001000} \leftrightarrow \underset{1}{e_{0000000}}, e_{0000100}^{0} \leftrightarrow e_{1232100} \text {. }
\end{aligned}
$$

Thus $c$ acts as a non-trivial graph automorphism of $C^{\circ}$. Hence $A=\langle\bar{c}\rangle$. We have $\left(Z\left(C_{\mathfrak{L}(G)}(e)_{+}\right)\right)^{C^{\circ}}=\left\langle z_{2}, z_{4}\right\rangle$, where $z_{2}=e$ and $z_{4}=\underset{0}{e} e_{0}^{110000}$. Since $\operatorname{Ad}(c)$ negates $z_{4}$, we have $\mathcal{Z}=\left\langle z_{2}\right\rangle$. 


\subsubsection{Orbit $A_{2} A_{1}$ (number 7 in $E_{7}, 6$ in $E_{8}$ )}

Here $e=e_{1000000}+e_{0100000}+e_{0000000}$; we have $A \cong \mathcal{S}_{2}$, and $C^{\circ}=A_{3} T_{1}$ or $A_{5}$ respectively with $\Pi(D)=\left\{\begin{array}{c}0000001 \\ 0\end{array}, \underset{0}{0}{ }_{0}^{0000010},{ }_{0}^{0000100}, \underset{0}{0001000},{ }_{3}^{2464321}\right\} \cap \Phi(G)$. We take

$$
c=n_{1121110} n_{1122100} n_{1343210} h_{3}(-1) h_{5}(-1) h_{7}(-1) .
$$

The action of $\operatorname{Ad}(c)$ on the root vectors concerned is as follows:

$$
\text { fixed : } e_{0}^{0000000}, \underset{0}{e_{0} 000100 ;}
$$

interchanged : $e_{0} e_{0} 00000 \leftrightarrow e_{0} e_{010000}, e_{000001} \leftrightarrow e_{2464321}, e_{0000010} \leftrightarrow e_{0001000}$

Thus $c$ acts as a non-trivial graph automorphism of $C^{\circ}$. Hence $A=\langle\bar{c}\rangle$. We have $\left(Z\left(C_{\mathfrak{L}(G)}(e)_{+}\right)\right)^{C^{\circ}}=\left\langle z_{2}, z_{4}\right\rangle$, where $z_{2}=e$ and $z_{4}=e_{0} 1100000$. Since $\operatorname{Ad}(c)$ negates $z_{4}$, we have $\mathcal{Z}=\left\langle z_{2}\right\rangle$.

\subsubsection{Orbit $A_{2}{ }^{2}$ (number 10 in $E_{8}$ )}

Here $e=\underset{0}{e_{1000000}}+e_{0} e_{0} 0000+e_{0001000}+e_{000100} ;$ we have $A \cong \mathcal{S}_{2}$, and $C^{\circ}=G_{2}{ }^{2}$. In this case $C^{\circ}$ is the fixed point subgroup under a non-trivial graph automorphism of a $D_{4}{ }^{2}$ subsystem subgroup $K$ with $\Pi(K)=\left\{\begin{array}{c}1110000 \\ 0\end{array} \underset{1}{0000000}, \underset{0}{0111000}, \underset{0}{0011100},{ }_{1}^{1221110}, \underset{0}{0000001}\right.$, $\left.{ }_{1}^{1122110},{ }_{1}^{0122210}\right\}$. We take

$$
c=n_{0}^{0111111} n_{011110} n_{1122221} h_{3}(-1),
$$

The action of $\operatorname{Ad}(c)$ on the root vectors concerned is as follows:

$$
\begin{aligned}
& \text { fixed : } \underset{0}{e_{0001000}}, \underset{0}{0000100} \text {; } \\
& \text { interchanged : } \underset{0}{e} e_{1000000} \leftrightarrow e_{010000}, \underset{0}{e_{1110000}} \leftrightarrow e_{1221110}, e_{0000000} \leftrightarrow e_{0000001} \text {, } \\
& \underset{0}{e_{0111000}} \leftrightarrow-e_{1122110}, e_{0011100} \leftrightarrow e_{0122210}
\end{aligned}
$$

Thus $c$ acts as a non-trivial graph automorphism of $K$. Hence $A=\langle\bar{c}\rangle$. We have $\left(Z\left(C_{\mathfrak{L}(G)}(e)_{+}\right)\right)^{C^{\circ}}=\left\langle z_{2}\right\rangle$, where $z_{2}=e$, so $\mathcal{Z}=\left\langle z_{2}\right\rangle$.

\subsubsection{Orbit $D_{4}\left(a_{1}\right)$ (number 11 in $E_{6}, 15$ in $E_{7}, 13$ in $E_{8}$ )}

Here $e=\underset{0}{e_{0100000}}+e_{0010000}+e_{0011000}+e_{0000000}+e_{0001000}$, which is non-regular distinguished in the Lie algebra of $H$, a $D_{4}$ subsystem subgroup of $G$ with $\Pi(H)=$ $\left\{\begin{array}{c}0100000 \\ 0\end{array}, \underset{0}{0010000},{ }_{1}^{000000},{ }_{0}^{0001000}\right\}$; we have $A \cong \mathcal{S}_{3}$, and $C^{\circ}=T_{2}, A_{1}{ }^{3}$ or $D_{4}$ respectively with $\Pi(D)=\left\{\begin{array}{c}0000010 \\ 0\end{array}, \underset{0}{0000001},{ }_{1}^{0122210}, \underset{2}{2343210}\right\} \cap \Phi(G)$. We take

$$
\begin{aligned}
& c_{1}=n_{1110000} n_{1111000} h_{2}(-1), \\
& c_{2}=\left(n_{1221100} n_{1122100} h_{1}(-1) h_{2}(-1) h_{6}(-1)\right)^{g},
\end{aligned}
$$

where

$$
g=x_{0010000}\left(\frac{1}{3}\right) n_{0010000} h_{1}(4) h_{2}(-4) h_{3}(16) h_{4}(-48) h_{5}(16) h_{6}(-8) x_{0010000}\left(-\frac{1}{3}\right) .
$$


The action of $\operatorname{Ad}\left(c_{1}\right)$ on the root vectors concerned is as follows:

$$
\begin{aligned}
& \text { fixed : } \underset{0}{e} \underset{00000}{e}, e_{000010}, e_{0000001} ; \\
& \text { negated : } \underset{0}{0010000} \text {; } \\
& \text { interchanged : } e_{1}^{0010000} \leftrightarrow \underset{0}{e 011000}, e_{1}^{0000000} \leftrightarrow e_{0}^{0001000}, e_{0122210} \leftrightarrow e_{2343210}
\end{aligned}
$$

Thus $c_{1}$ acts as a non-trivial graph automorphism of $C^{\circ}$. The action of $c_{2}$ is a little more complicated. Calculation shows that

$$
\operatorname{Ad}(g) e=e_{1}^{0000000}+\underset{0}{e 110000}+e_{0}^{0011000}+e_{0100000}+e_{0001000},
$$

which is still non-regular distinguished in $\mathfrak{L}(H)$, and $\left(C^{\circ}\right)^{g^{-1}}=C^{\circ}$. The action of $\operatorname{Ad}\left(c_{2} g^{-1}\right)$ on the root vectors concerned is as follows:

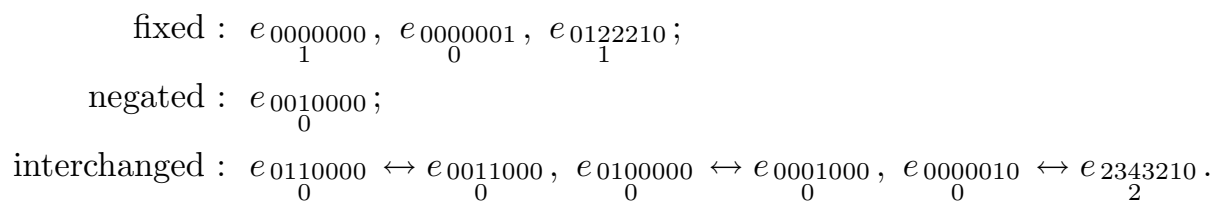

Thus $c_{2}$ also acts as a non-trivial graph automorphism of $C^{\circ}$. Hence $A=\left\langle\bar{c}_{1}, \bar{c}_{2}\right\rangle$. We have $\left(Z\left(C_{\mathfrak{L}(G)}(e)_{+}\right)\right)^{C^{\circ}}=\left\langle z_{2}, z_{6}{ }^{1}, z_{6}{ }^{2}\right\rangle$, where $z_{2}=e, z_{6}{ }^{1}=e_{0111000}$ and $z_{6}{ }^{2}=e_{0121000}$.

Since both $\operatorname{Ad}\left(c_{1}\right)$ and $\operatorname{Ad}\left(c_{2}{ }^{g^{-1}}\right)$ negate $z_{6}{ }^{1}$ and fix $z_{6}{ }^{2}$, and $\operatorname{Ad}(g) z_{6}{ }^{2}=-\frac{3}{2} z_{6}{ }^{1}-\frac{1}{2} z_{6}{ }^{2}$, we have $\mathcal{Z}=\left\langle z_{2}\right\rangle$.

\subsubsection{Orbit $D_{4}\left(a_{1}\right) A_{1}$ (number 18 in $E_{7}, 17$ in $E_{8}$ )}

Here $e=e_{0} 010000+e_{0010000}+e_{0011000}^{0}+e_{0000000}+e_{0}^{0001000}+e_{0}^{0000010}$, which is non-regular distinguished in the Lie algebra of $H$, a $D_{4} A_{1}$ subsystem subgroup of $G$ with $\Pi(H)=$ $\left\{\begin{array}{c}0100000 \\ 0\end{array}{ }_{0}^{0010000},{ }_{1}^{0000000},{ }_{0}^{0001000},{ }_{0}^{0000010}\right\}$; we have $A \cong \mathcal{S}_{2}$ if $G=E_{7}$ or $\mathcal{S}_{3}$ if $G=E_{8}$, and $C^{\circ}=A_{1}{ }^{2}$ or $A_{1}{ }^{3}$ respectively, with $\Pi(D)=\left\{{ }_{1}^{0122210},{ }_{2}^{2343210},{ }_{3}^{2465432}\right\} \cap \Phi(G)$. We take

$$
\begin{aligned}
& c_{1}=\underset{1}{n_{1110000}} n_{0}^{1111000} h_{2}(-1), \\
& c_{2}=\left(n_{1}^{1221111} n_{1122111} h_{3}(i) h_{5}(i)\right)^{g},
\end{aligned}
$$

where

$$
g=x_{0}^{0010000}\left(\frac{1}{3}\right) n_{0}^{0010000} h_{1}(4) h_{2}(-4) h_{3}(16) h_{4}(-48) h_{5}(16) h_{6}(-8) x_{0}^{0010000}\left(-\frac{1}{3}\right) .
$$

The action of $\operatorname{Ad}\left(c_{1}\right)$ on the root vectors concerned is as follows:

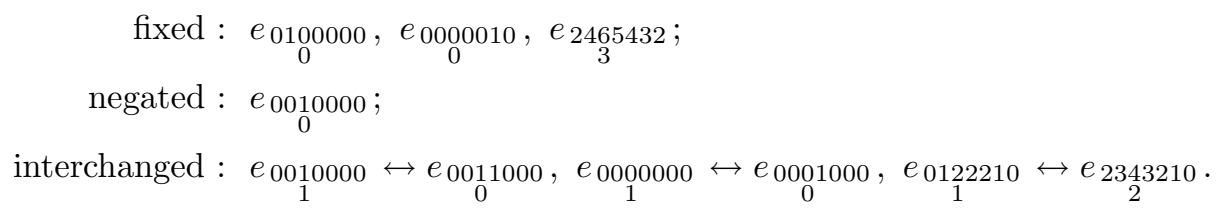


Thus $c_{1}$ acts as a non-trivial graph automorphism of $C^{\circ}$. The action of $c_{2}$ is a little more complicated. Calculation shows that

$$
\operatorname{Ad}(g) e=\underset{1}{e} \underset{0}{000000}+\underset{0}{e_{0110000}}+\underset{0}{e_{0011000}}+\underset{0}{e_{0100000}}+\underset{0}{e_{0001000}}-\frac{1}{8} e_{0000010},
$$

which is still non-regular distinguished in $\mathfrak{L}(H)$, and $\left(C^{\circ}\right)^{g^{-1}}=C^{\circ}$. The action of $\operatorname{Ad}\left(c_{2}{ }^{g^{-1}}\right)$ on the root vectors concerned is as follows:

$$
\begin{aligned}
& \text { fixed : } \underset{1}{0} 000000, \underset{0}{0000010}, e_{2343210} ; \\
& \text { negated : } \underset{0}{00010000} \text {; } \\
& \text { interchanged : } \underset{0}{e_{0110000}} \leftrightarrow \underset{0}{e_{0011000}}, \underset{0}{e_{0100000}} \leftrightarrow \underset{0}{e_{0001000}}, e_{0122210} \leftrightarrow e_{2465432} .
\end{aligned}
$$

Thus $c_{2}$ also acts as a non-trivial graph automorphism of $C^{\circ}$ if $G=E_{8}$. Hence $A=\left\langle\bar{c}_{1}\right\rangle$ if $G=E_{7}$ and $\left\langle\bar{c}_{1}, \bar{c}_{2}\right\rangle$ if $G=E_{8}$. We have $\left(Z\left(C_{\mathfrak{L}(G)}(e)_{+}\right)\right)^{C^{\circ}}=\left\langle z_{2}, z_{6}{ }^{1}, z_{6}{ }^{2}\right\rangle$, where $z_{2}=e$, $z_{6}{ }^{1}=e_{0111000}$ and $z_{6}{ }^{2}=e_{0121000}$. Since both $\operatorname{Ad}\left(c_{1}\right)$ and $\operatorname{Ad}\left(c_{2}{ }^{g^{-1}}\right)$ negate $z_{6}{ }^{1}$ and fix $z_{6}{ }^{2}$, and $\operatorname{Ad}(g) z_{6}{ }^{2}=-\frac{3}{2} z_{6}{ }^{1}-\frac{1}{2} z_{6}{ }^{2}$, we have $\mathcal{Z}=\left\langle z_{2}, z_{6}{ }^{2}\right\rangle$ if $G=E_{7}$ and $\left\langle z_{2}\right\rangle$ if $G=E_{8}$.

\subsubsection{Orbit $A_{3} A_{2}$ (number 19 in $E_{7}, 18$ in $E_{8}$ )}

Here $e=e_{0100000}+e_{0010000}+e_{0000000}+e_{0000100}+e_{0000010} ;$ we have $A \cong \mathcal{S}_{2}$, and $C^{\circ}=A_{1} T_{1}$ or $B_{2} T_{1}$ respectively. In this case $D=J \cap \stackrel{0}{D}$, where $J$ is the fixed point subgroup under a non-trivial graph automorphism of an $A_{3}$ subsystem subgroup $K$ with $\Pi(K)=\left\{\begin{array}{c}0011111 \\ 1\end{array}, \underset{2}{2343210},{ }_{0}^{0111111}\right\}$. We take

$$
c=n_{0011100} n_{0111100} n_{0122110} h_{2}(-1) h_{4}(-1) h_{5}(-1) h_{6}(-1) h_{7}(-1) .
$$

The action of $\operatorname{Ad}(c)$ on the root vectors concerned is as follows:

$$
\text { fixed : } e_{0010000}, e_{2343210} ;
$$

interchanged : $e_{0} e_{0} 00000 \leftrightarrow e_{000000}, e_{0000100} \leftrightarrow e_{0} e_{0} 00010, e_{001111} \leftrightarrow e_{0111111}^{e}$

Thus $c$ acts as a non-trivial graph automorphism of $K$. Hence $A=\langle\bar{c}\rangle$. We have $\left(Z\left(C_{\mathfrak{L}(G)}(e)_{+}\right)\right)^{C^{\circ}}=\left\langle z_{2}, z_{6}\right\rangle$, where $z_{2}=e$ and $z_{6}=e_{0110000}$. Since $\operatorname{Ad}(c)$ fixes $z_{6}$, we have $\mathcal{Z}=\left\langle z_{2}, z_{6}\right\rangle$.

\subsubsection{Orbit $A_{4}$ (number 20 in $E_{7}, 19$ in $E_{8}$ )}

Here $e=\underset{0}{e_{1000000}}+e_{0100000}+e_{0010000}+e_{0000000} ;$ we have $A \cong \mathcal{S}_{2}$, and $C^{\circ}=A_{2} T_{1}$ or $A_{4}$ respectively with $\Pi(D)=\left\{\begin{array}{c}0000001 \\ 0\end{array} \underset{0}{0000010}, \underset{0}{0000100,},{ }_{3}^{2465321}\right\} \cap \Phi(G)$. We take

$$
c=n_{0111100} n_{1111100} n_{1222110} n_{1243210} h_{2}(-1) h_{3}(-1) h_{4}(-1) h_{8}(-1) .
$$

The action of $\operatorname{Ad}(c)$ on the root vectors concerned is as follows:

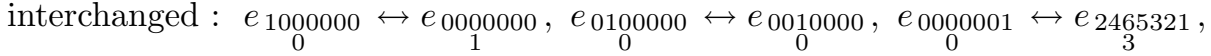

$$
\begin{aligned}
& e_{0000010} \leftrightarrow e_{0000100}
\end{aligned}
$$


(Note that if $G=E_{7}$ we replace $h_{8}(-1)$ by $h_{6}(-1)$ to obtain an element of $G$ whose effect upon those root vectors listed which lie in $\mathfrak{L}(G)$ is the same.) Thus $c$ acts as a non-trivial graph automorphism of $C^{\circ}$. Hence $A=\langle\bar{c}\rangle$. We have $\left(Z\left(C_{\mathfrak{L}(G)}(e)_{+}\right)\right)^{C^{\circ}}=\left\langle z_{2}, z_{6}, z_{8}\right\rangle$, where $z_{2}=e, z_{6}=e_{1110000}-e_{0110000}$ and $z_{8}=e_{1110000}$. Since $\operatorname{Ad}(c)$ fixes $z_{6}$ and negates $z_{8}$, we have $\mathcal{Z}=\left\langle z_{2}, z_{6}\right\rangle$.

\subsubsection{Orbit $D_{4}\left(a_{1}\right) A_{2}$ (number 22 in $E_{8}$ )}

Here $e=\underset{0}{e} e_{0} 0000+e_{1}^{0010000}+e_{0}^{0011000}+e_{1}^{0000000}+e_{0}^{0001000}+e_{0}^{0000010}+e_{0}^{0000001}$, which is non-regular distinguished in the Lie algebra of $H$, a $D_{4} A_{2}$ subsystem subgroup of $G$ with $\Pi(H)=\left\{\begin{array}{c}0100000 \\ 0\end{array}{ }_{0}^{0010000},{ }_{1}^{0000000},{ }_{0}^{0001000},{ }_{0}^{0000010},{ }_{0}^{0000001}\right\}$; we have $A \cong \mathcal{S}_{2}$, and $C^{\circ}=A_{2}$. Here $C^{\circ}$ was found in $\S 7.2$; we have $C^{\circ}=\left\langle X_{ \pm \beta_{1}}, X_{ \pm \beta_{2}}\right\rangle$ where

$$
\begin{aligned}
& x_{\beta_{1}}(t)=x_{0}^{0001111}(3 t) x_{0121100}(t) x_{0}^{0011111}(-t) x_{0111100}(t) x_{1}^{0122211}\left(-2 t^{2}\right) \times \\
& x_{0011110}(2 t) x_{0111110}(-t), \\
& x_{\beta_{2}}(t)=x_{1}^{111111}(-3 t) x_{1}^{1232100}(-t) x_{1}^{1121111}(-t) x_{1}^{1222100}(t) x_{2}^{2343211}\left(-2 t^{2}\right) \times \\
& x_{1122110}(2 t) x_{1221110}(-t) .
\end{aligned}
$$

We take

$$
c=n_{0} 111000 n_{1110000} h_{5}(-1) .
$$

The action of $\operatorname{Ad}(c)$ on the root vectors concerned is as follows:

fixed : $\underset{0}{e} \underset{00000}{e}, \underset{0}{000010}, \underset{0}{0000001}$

negated : $\underset{0}{0010000}$;

interchanged : $e_{0010000} \leftrightarrow e_{0}^{0011000}, e_{0000000} \leftrightarrow e_{0}^{0001000}, e_{0}^{0001111} \leftrightarrow-e_{1111111}$,

$$
\begin{aligned}
& e_{0121100} \leftrightarrow-e_{1232100}, e_{0011111} \leftrightarrow e_{1121111}, e_{0111100} \leftrightarrow e_{1222100} \\
& e_{0011110} \leftrightarrow e_{1122110}, \underset{0}{e_{0111110}} \leftrightarrow e_{1221110}, e_{0122211} \leftrightarrow e_{2343211}
\end{aligned}
$$

Thus $c$ acts as a non-trivial graph automorphism of both $H$ and $C^{\circ}$; we find that $C_{H}(c) \cong$ $B_{2} B_{1} A_{1}$, and that $e$ is regular in $C_{H}(c)$. Hence $A=\langle\bar{c}\rangle$. We have $\left(Z\left(C_{\mathfrak{L}(G)}(e)_{+}\right)\right)^{C^{0}}=$ $\left\langle z_{2}\right\rangle$, where $z_{2}=e$, and so $\mathcal{Z}=\left\langle z_{2}\right\rangle$.

\subsubsection{Orbit $A_{4} A_{1}$ (number 24 in $E_{7}, 23$ in $E_{8}$ )}

Here $e=\underset{0}{e_{100000}}+e_{010000}+e_{0010000}+e_{0000000}+e_{0000100} ;$ we have $A \cong \mathcal{S}_{2}$, and $C^{\circ}=T_{2}$ or $A_{2} T_{1}$ respectively with $\Pi(D)=\left\{\begin{array}{c}0000001 \\ 0\end{array},{ }_{3}^{2465431}\right\} \cap \Phi(G)$. We take

$$
c=n_{0111110} n_{1111110} n_{1222100} n_{1243210} h_{1}(-1) h_{3}(-1) h_{4}(-1) h_{6}(-1) h_{8}(-1) .
$$

The action of $\operatorname{Ad}(c)$ on the root vectors concerned is as follows:

$$
\begin{aligned}
& \text { fixed : } e_{0000100} \text {; } \\
& \text { interchanged : } e_{0} 1000000 \leftrightarrow e_{000000}, \underset{0}{e_{0100000}} \leftrightarrow e_{0}^{0010000}, \underset{0}{0000001} \leftrightarrow e_{2465431} \text {. }
\end{aligned}
$$


(Note that if $G=E_{7}$ we delete the term $h_{8}(-1)$ to obtain an element of $G$ whose effect upon those root vectors listed which lie in $\mathfrak{L}(G)$ is the same.) Thus $c$ acts as a non-trivial graph automorphism of the $A_{2}$ subsystem subgroup containing $D$. Hence $A=\langle\bar{c}\rangle$. We have $\left(Z\left(C_{\mathfrak{L}(G)}(e)_{+}\right)\right)^{C^{\circ}}=\left\langle z_{2}, z_{8}\right\rangle$, where $z_{2}=e$ and $z_{8}=e_{1110000}$. Since $\operatorname{Ad}(c)$ negates $z_{8}$, we have $\mathcal{Z}=\left\langle z_{2}\right\rangle$.

\subsubsection{Orbit $D_{5}\left(a_{1}\right)$ (number 25 in $E_{7}, 25$ in $E_{8}$ )}

Here $e=\underset{0}{e} e_{0} 00000+e_{0100000}+e_{0010000}+e_{0}^{0011000}+e_{0}^{0000000}+e_{0}^{0001000}$, which is nonregular distinguished in the Lie algebra of $H$, a $D_{5}$ subsystem subgroup of $G$ with $\Pi(H)=\left\{\begin{array}{c}1000000 \\ 0\end{array}, \underset{0}{0100000}, \underset{0}{0010000}, \underset{1}{0000000}, \underset{0}{0001000}\right\}$; we have $A \cong \mathcal{S}_{2}$, and $C^{\circ}=A_{1} T_{1}$ or $A_{3}$ respectively with $\Pi(D)=\left\{\begin{array}{c}0000001 \\ 0\end{array} \underset{0}{0000010}, \underset{3}{2465421}\right\} \cap \Phi(G)$. We take

$$
c=n_{1233210} n_{1232210} h_{1}(-1) h_{2}(-1) h_{4}(-1) .
$$

The action of $\operatorname{Ad}(c)$ on the root vectors concerned is as follows:

$$
\begin{aligned}
& \text { fixed : } \underset{0}{1000000}, e_{0100000}, e_{0000010} ; \\
& \text { negated : } e_{0010000} \text {; } \\
& \text { interchanged : } e_{1}^{0010000} \leftrightarrow \underset{0}{e} 0011000, \underset{1}{e} e_{0}^{00000} \leftrightarrow \underset{0}{e_{0001000}}, e_{0}^{000001} \leftrightarrow e_{2465421}
\end{aligned}
$$

Thus $c$ acts as a non-trivial graph automorphism of both $H$ and the $A_{3}$ subsystem subgroup containing $C^{\circ}$; we find that $C_{H}(c) \cong B_{3} B_{1}$, and that $e$ is regular in $C_{H}(c)$. Hence $A=\langle\bar{c}\rangle$. We have $\left(Z\left(C_{\mathfrak{L}(G)}(e)_{+}\right)\right)^{C^{\circ}}=\left\langle z_{2}, z_{8}, z_{10}\right\rangle$, where $z_{2}=e, z_{8}=e_{1111000}$ and $z_{10}=e_{1221000}$. Since $\operatorname{Ad}(c)$ negates $z_{8}$ and fixes $z_{10}$, we have $\mathcal{Z}=\left\langle z_{2}, z_{10}\right\rangle$.

\subsubsection{Orbit $A_{4} A_{1}{ }^{2}$ (number 26 in $E_{8}$ )}

Here $e=e_{1000000}+e_{0100000}+e_{0010000}+e_{0000000}+e_{0000100}+e_{0000001} ;$ we have $A \cong \mathcal{S}_{2}$, and $C^{\circ}=A_{1} T_{1}$. In this case $D$ is the fixed point subgroup under a non-trivial graph automorphism of an $A_{1}{ }^{2}$ subsystem subgroup $K$ with $\Pi(K)=\left\{\begin{array}{c}0000110,0000011 \\ 0\end{array}\right\}$. We take

$$
c=n_{1222210} n_{1222111} n_{1354321} n_{2354321} h_{1}(-1) h_{3}(-1) h_{4}(-1) h_{6}(-1) .
$$

The action of $\operatorname{Ad}(c)$ on the root vectors concerned is as follows:

$$
\begin{aligned}
& \text { interchanged : } e_{0} 1000000 \leftrightarrow e_{0} 0000000, e_{0}^{0100000} \leftrightarrow \underset{0}{e_{0010000}}, e_{0000100} \leftrightarrow e_{0} 000001 \\
& e_{0}^{0000110} \leftrightarrow \underset{0}{e 000011}
\end{aligned}
$$

Thus $c$ acts as a non-trivial graph automorphism of $K$. Hence $A=\langle\bar{c}\rangle$. We have $\left(Z\left(C_{\mathfrak{L}(G)}(e)_{+}\right)\right)^{C^{\circ}}=\left\langle z_{2}, z_{8}\right\rangle$, where $z_{2}=e$ and $z_{8}=e_{1110000}$. Since $\operatorname{Ad}(c)$ negates $z_{8}$, we have $\mathcal{Z}=\left\langle z_{2}\right\rangle$. 


\subsubsection{Orbit $D_{4} A_{2}$ (number 31 in $E_{8}$ )}

Here $e=e_{0100000}+e_{0010000}+e_{0000000}+e_{0001000}+e_{0000010}+e_{0000001} ;$ we have $A \cong \mathcal{S}_{2}$, and $C^{\circ}=A_{2}$. In this case $C^{\circ}$ is the fixed point subgroup under a non-trivial graph automorphism of an $A_{2}{ }^{2}$ subsystem subgroup $K$ with $\Pi(K)=\left\{\begin{array}{c}1110000 \\ 1\end{array}, \underset{2}{1244321}, \underset{0}{1111000},{ }_{2}^{1343321}\right\}$. We take

$$
c=n_{1}^{001110} n_{0}^{0111110} n_{0122211} h_{2}(-1) h_{4}(-1) h_{5}(-1) h_{6}(-1) .
$$

The action of $\operatorname{Ad}(c)$ on the root vectors concerned is as follows:

$$
\begin{aligned}
& \text { fixed : } e_{0010000}, \underset{0}{0001000} \text {; } \\
& \text { interchanged : } e_{0100000} \leftrightarrow e_{0000000}, e_{0000010} \leftrightarrow e_{0000001}, e_{1110000} \leftrightarrow e_{1343321} \text {, } \\
& e_{1244321} \leftrightarrow-e_{0}^{1111000}
\end{aligned}
$$

Thus $c$ acts as a non-trivial graph automorphism of $K$. Hence $A=\langle\bar{c}\rangle$. We have $\left(Z\left(C_{\mathfrak{L}(G)}(e)_{+}\right)\right)^{C^{\circ}}=\left\langle z_{2}, z_{10}\right\rangle$, where $z_{2}=e$ and $z_{10}=\underset{1}{0121000}$. Since $\operatorname{Ad}(c)$ fixes $z_{10}$, we have $\mathcal{Z}=\left\langle z_{2}, z_{10}\right\rangle$.

\subsubsection{Orbit $E_{6}\left(a_{3}\right)$ (number 17 in $E_{6}, 31$ in $E_{7}, 32$ in $E_{8}$ )}

Here $e=e_{0110000}+e_{100000}+e_{0111000}+e_{0000100}+e_{0011000}+e_{0010000}$, which is regular in the Lie algebra of $H$, an $A_{5} A_{1}$ subsystem subgroup of $G$; we have $A \cong \mathcal{S}_{2}$, and $C^{\circ}=1, A_{1}$ or $G_{2}$ respectively. In this case $C^{\circ}=J \cap G$, where $J$ is the fixed point subgroup under a non-trivial graph automorphism of a $D_{4}$ subsystem subgroup $K$ with $\Pi(K)=\left\{\begin{array}{c}1122110 \\ 1\end{array} \underset{0}{0000001}, \underset{1}{0122210}, \underset{1}{1221110}\right\}$. We take

$$
c=h_{4}(-1) \text {. }
$$

Clearly $c \in Z(H)$. Moreover, as $c \in E_{6}$ it follows that $c \notin C^{\circ}$. Hence $A=\langle\bar{c}\rangle$. If $G=E_{7}$ or $E_{8}$ we have $\left(Z\left(C_{\mathfrak{L}(G)}(e)_{+}\right)\right)^{C^{\circ}}=\left\langle z_{2}, z_{10}{ }^{1}, z_{10}{ }^{2}\right\rangle$, where $z_{10}{ }^{1}=e_{1232100}$ and $z_{10}^{2}=e_{1232100}$; if $G=E_{6}$ we have an additional vector $z_{8}=e_{12211}+e_{11221}$. Since $\operatorname{Ad}(c)$ fixes $z_{10}{ }^{2}$ (and $z_{8}$ if $G=E_{6}$ ) and negates $z_{10}{ }^{1}$, we have $\mathcal{Z}=\left\langle z_{2}, z_{8}, z_{10}{ }^{2}\right\rangle$ if $G=E_{6}$ and $\left\langle z_{2}, z_{10}^{2}\right\rangle$ if $G=E_{7}$ or $E_{8}$.

\subsubsection{Orbit $D_{6}\left(a_{2}\right)$ (number 37 in $\left.E_{8}\right)$}

Here $e=e_{0000010}+e_{0001100}+e_{0010000}-e_{0110000}+e_{0011000}+e_{0000000}+e_{0100000}$, which is non-regular distinguished in the Lie algebra of $H$, a $D_{6}$ subsystem subgroup of $G$ with $\Pi(H)=\left\{\begin{array}{c}0000010 \\ 0\end{array}, \underset{0}{0000100}, \underset{0}{0001000}, \underset{0}{0010000}, \underset{1}{0000000}, \underset{0}{0100000}\right\}$; we have $A \cong \mathcal{S}_{2}$, and $C^{\circ}=A_{1}^{2}$ with $\Pi(D)=\left\{\begin{array}{c}2343210 \\ 2\end{array}, \underset{3}{2465432}\right\}$. We take

$$
c=n_{1}^{0011111} n_{0}^{0111111} h_{4}(-1) h_{5}(-1) .
$$

The action of $\operatorname{Ad}(c)$ on the root vectors concerned is as follows:

$$
\begin{aligned}
& \text { fixed : } \underset{0}{e 000010}, \underset{0}{e 0001100}, \underset{0}{e 0011000} \\
& \text { negated : } e_{0010000}, e_{0001000}, e_{0000100} \\
& \text { interchanged : } e_{0010000} \leftrightarrow-e_{0}^{0110000}, \underset{1}{e_{0000000}} \leftrightarrow \underset{0}{e_{0100000}}, e_{2343210} \leftrightarrow e_{2465432}
\end{aligned}
$$


Thus $c$ acts as a non-trivial graph automorphism of both $H$ and $C^{\circ}$; we find that $C_{H}(c) \cong$ $B_{3} B_{2}$, and that $e$ is regular in $C_{H}(c)$. Hence $A=\langle\bar{c}\rangle$. We have $\left(Z\left(C_{\mathfrak{L}(G)}(e)_{+}\right)\right)^{C^{\circ}}=$ $\left\langle z_{2}, z_{10}{ }^{1}, z_{10}{ }^{2}\right\rangle$, where $z_{2}=e, z_{10}{ }^{1}=e_{0122110}$ and $z_{10}{ }^{2}=e_{0122210}$. Since $\operatorname{Ad}(c)$ negates $z_{10}{ }^{1}$ and fixes $z_{10^{2}}{ }^{2}$, we have $\mathcal{Z}=\left\langle z_{2}, z_{10}{ }^{2}\right\rangle$.

\subsubsection{Orbit $E_{6}\left(a_{3}\right) A_{1}$ (number 38 in $E_{8}$ )}

Here $e=e_{0110000}+e_{0}^{1000000}+e_{0}^{0111000}+e_{0}^{0000100}+e_{0011000}+e_{0}^{0010000}+e_{000001}$, which is regular in the Lie algebra of $H$, an $A_{5} A_{1}{ }^{2}$ subsystem subgroup of $G$; we have $A \cong \mathcal{S}_{2}$, and $C^{\circ}=A_{1}$. In this case $C^{\circ}$ is the fixed point subgroup under a non-trivial graph automorphism of an $A_{1}{ }^{3}$ subsystem subgroup $K$ with $\Pi(K)=\left\{\begin{array}{c}1244321 \\ 2\end{array} \underset{2}{1343321},{ }_{2}^{2343221}\right\}$. We take

$$
c=h_{4}(-1) .
$$

Clearly $c \in Z(H)$. Moreover, as $c \in E_{6}$ it follows that $c \notin C^{\circ}$. Hence $A=\langle\bar{c}\rangle$. We have $\left(Z\left(C_{\mathfrak{L}(G)}(e)_{+}\right)\right)^{C^{\circ}}=\left\langle z_{2}, z_{10}{ }^{1}, z_{10}{ }^{2}\right\rangle$, where $z_{2}=e, z_{10}{ }^{1}=e_{1232100}$ and $z_{10}{ }^{2}=e_{1232100}$. Since $\operatorname{Ad}(c)$ negates $z_{10}{ }^{1}$ and fixes $z_{10}{ }^{2}$, we have $\mathcal{Z}=\left\langle z_{2}, z_{10}{ }^{2}\right\rangle$.

\subsubsection{Orbit $E_{7}\left(a_{5}\right)$ (number 33 in $E_{7}, 39$ in $E_{8}$ )}

Here $e=e_{0} 111000+e_{0011100}+e_{0000010}+e_{0111100}+e_{1110000}+e_{0010000}+e_{0111000}$, which is regular in the Lie algebra of $H$, an $A_{5} A_{2}$ subsystem subgroup of $G$; we have $A \cong \mathcal{S}_{3}$, and $C^{\circ}=1$ or $A_{1}$ respectively with $\Pi(D)=\left\{\begin{array}{c}2465432 \\ 3\end{array}\right\} \cap \Phi(G)$. We take

$$
\begin{aligned}
& c_{1}=h_{2}(\omega) h_{3}(\omega) h_{5}(\omega), \\
& c_{2}=n_{1}^{0000000} n_{0100000}^{n_{0001000}} h_{3}(-1) h_{4}(-1) .
\end{aligned}
$$

Clearly $c_{1} \in Z(H)$. The action of $\operatorname{Ad}\left(c_{2}\right)$ on the root vectors concerned is as follows:

$$
\begin{aligned}
& \text { fixed : } \underset{0}{0000010}, e_{2465432} ; \\
& \text { interchanged : } e_{0}^{1111000} \leftrightarrow e_{1110000}, e_{0011100} \leftrightarrow e_{0111100}, e_{0010000}^{0} \leftrightarrow e_{0111000}
\end{aligned}
$$

Thus $c_{2}$ acts as a non-trivial graph automorphism of $H$. Moreover, as $c_{1}, c_{2} \in E_{7}$ it follows that $c_{1}, c_{2} \notin C^{\circ}$. Hence $A=\left\langle\bar{c}_{1}, \bar{c}_{2}\right\rangle$. We have $\left(Z\left(C_{\mathfrak{L}(G)}(e)_{+}\right)\right)^{C^{\circ}}=\left\langle z_{2}, z_{10}{ }^{1}, z_{10}{ }^{2}, z_{10}{ }^{3}\right\rangle$, where $z_{2}=e, z_{10}{ }^{1}=e_{1243210}, z_{10}{ }^{2}=e_{1343210}$ and $z_{10}{ }^{3}=e_{2343210}$. Since $\operatorname{Ad}\left(c_{1}\right)$ fixes $z_{10}{ }^{3}$ and multiplies $z_{10}{ }^{2}$ by $\omega$ and $z_{10}{ }^{1}$ by $\omega^{2}$, and $\operatorname{Ad}\left(c_{2}\right)$ fixes $z_{10}{ }^{3}$, we have $\mathcal{Z}=\left\langle z_{2}, z_{10}{ }^{3}\right\rangle$.

\subsubsection{Orbit $E_{8}\left(a_{7}\right)$ (number 41 in $E_{8}$ )}

Here $e=e_{0} \underset{111111}{ }+e_{0121110}+e_{0001000}+e_{1121100}+e_{1221000}+e_{0011111}+e_{0111100}+e_{1111110}$, which is regular in the Lie algebra of $H$, an $A_{4}{ }^{2}$ subsystem subgroup of $G$; we have $A \cong \mathcal{S}_{5}$, and $C^{\circ}=1$. We take

$$
\begin{aligned}
& c_{1}=h_{2}\left(\zeta^{2}\right) h_{3}\left(\zeta^{4}\right) h_{4}(\zeta) h_{6}\left(\zeta^{4}\right) h_{7}(\zeta) h_{8}\left(\zeta^{2}\right), \\
& c_{2}=\underset{0}{n_{0100000}} n_{0010000}^{00000000} n_{0000100} n_{0000010} n_{0000001} \\
& \times h_{1}(-1) h_{3}(-1) h_{5}(-1) h_{6}(-1) h_{8}(-1), \\
& c_{3}=\left(n_{1110000} n_{0110000} n_{0000111}^{0} n_{0000010} h\right)^{u},
\end{aligned}
$$


where

$$
\begin{aligned}
& h=h_{1}\left(\frac{2}{5}\right) h_{2}\left(-\frac{2}{5}\right) h_{3}\left(\frac{2(1-3 \phi)}{25}\right) h_{4}\left(\frac{2(1-3 \phi)}{25}\right) h_{6}\left(\frac{3+\phi}{5}\right) h_{7}\left(-\frac{3+\phi}{5}\right) h_{8}\left(-\frac{3+\phi}{5}\right) \text {, } \\
& u=x_{1110000}\left(-\frac{1}{2}+\frac{1}{2} \phi\right) x_{1}^{0110000}(-3-2 \phi) x_{1}^{0010000}(-3-2 \phi) x_{1}^{0000000}(1) x_{0}^{1110000}(1+\phi) \\
& \times x_{0110000}(5+3 \phi) x_{0010000}(4+2 \phi) x_{1100000}\left(1+\frac{1}{2} \phi\right) x_{0100000}(1+\phi) x_{1000000}(\phi) \\
& \times \underset{0}{00000111}(-\phi) \underset{0}{x} 0000100(\phi) \underset{0}{00000011}(-1+\phi) \underset{0}{00000010}(-\phi) x_{0}^{0000001}(-\phi) .
\end{aligned}
$$

Clearly $c_{1} \in Z(H)$. The action of $\operatorname{Ad}\left(c_{2}\right)$ on the root vectors concerned is as follows:

$$
\begin{aligned}
& \text { cycled : } e_{1111111} \mapsto e_{1221000} \mapsto e_{1121100} \mapsto e_{111110} \mapsto e_{1111111} \text {, } \\
& \underset{1}{e_{0121110}} \mapsto e_{0011111} \mapsto \underset{0}{e_{0001000}} \mapsto \underset{0}{e_{0111100}} \mapsto e_{0121110} .
\end{aligned}
$$

Thus $c_{2}$ acts as a non-trivial graph automorphism of $H$. The action of $c_{3}$ is considerably more complicated. Calculation shows that

$$
\begin{aligned}
& \operatorname{Ad}(u) e=\underset{0}{e 0001000}+(4+2 \phi) e_{0}^{0011000}+e_{1}^{0011000}+(5+3 \phi) e_{0}^{0111000}+(2+\phi) e_{1}^{0111000} \\
& +(1+\phi) e_{0}^{1111000}+\left(\frac{1}{2}+\frac{3}{2} \phi\right) e_{1}^{1111000}+(5+3 \phi) e_{1}^{0121000}+(4+3 \phi) e_{1121000} \\
& +\left(5+\frac{5}{2} \phi\right) e_{1} e_{1}^{2} 1000-\phi e_{0}^{0001100}-(2+2 \phi) e_{0}^{0011100}-\phi e_{1}^{0011100} \\
& -(2+2 \phi) e_{0}^{0111100}-\phi e_{0111100}-(1-\phi) e_{0}^{1111100}-\left(\frac{3}{2}-2 \phi\right) e_{111100} \\
& +\phi e_{0}^{0111110}+\phi e_{0111110}+(1-\phi) e_{1111110}+(2-\phi) e_{1111110} \\
& +(3+\phi) e_{0121110}+(5+5 \phi) e_{1121110}+\left(\frac{15}{2}+\frac{5}{2} \phi\right) e_{1221110}+\phi e_{0}^{0001111} \\
& +(2+2 \phi) e_{0011111}+(1+\phi) e_{0011111}+(4+\phi) e_{0111111}+(3+\phi) e_{0111111} \\
& +(1+2 \phi) e_{0}^{1111111}+\left(\frac{5}{2}+\frac{5}{2} \phi\right) e_{1111111}+(5+5 \phi) e_{1}^{0121111} \\
& +(15+5 \phi) e_{1121111}+\left(\frac{25}{2}+\frac{25}{2} \phi\right) e_{1221111} .
\end{aligned}
$$

The action of $\operatorname{Ad}\left(c_{3}{ }^{-1}\right)$ on the root vectors concerned is as follows:

$$
\begin{aligned}
& \text { interchanged : } \underset{0}{e_{0001000}} \leftrightarrow\left(\frac{25}{2}+\frac{25}{2} \phi\right) e_{122111}, \underset{0}{e_{0011000}} \leftrightarrow\left(5-\frac{5}{2} \phi\right) e_{112111} \text {, } \\
& e_{0011000} \leftrightarrow(5+5 \phi) e_{0121111}, e_{0111000} \leftrightarrow\left(-\frac{5}{2}+5 \phi\right) e_{111111}, \\
& e_{0111000} \leftrightarrow(2-\phi) e_{0111111}, e_{1111000} \leftrightarrow(2-\phi) e_{0}^{1111111}, \\
& e_{1111000} \leftrightarrow(2+2 \phi) e_{0111111}, e_{0121000} \leftrightarrow(-1+2 \phi) e_{0011111}, \\
& e_{1121000} \leftrightarrow\left(\frac{4}{5}-\frac{2}{5} \phi\right) e_{0011111}, e_{1221000} \leftrightarrow\left(-\frac{2}{5}+\frac{4}{5} \phi\right) e_{0}^{0001111},
\end{aligned}
$$

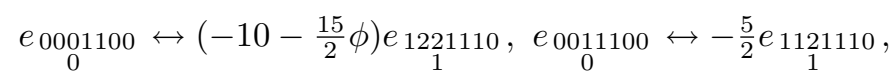

$$
\begin{aligned}
& e_{0011100} \leftrightarrow(-4-3 \phi) e_{0121110}, e_{0111100} \leftrightarrow\left(\frac{1}{2}-\frac{3}{2} \phi\right) e_{1111110}, \\
& e_{0111100} \leftrightarrow-e_{0111110}, e_{1111100} \leftrightarrow-e_{111110}, \\
& e_{1111100} \leftrightarrow\left(-\frac{8}{5}-\frac{6}{5} \phi\right) e_{0111110}
\end{aligned}
$$


Thus $c_{3} \in C_{G}(e)$. Hence $A=\left\langle\bar{c}_{1}, \bar{c}_{2}, \bar{c}_{3}\right\rangle$. (Indeed, we see that $c_{1}{ }^{5}=c_{2}{ }^{4}=c_{3}{ }^{2}=1$, with $c_{1}{ }^{c_{2}}=c_{1}{ }^{3}, c_{2}{ }^{c_{3}}=c_{2}{ }^{-1}$ and $\left(c_{1} c_{3}\right)^{3}=1$; we may thus identify $c_{1}, c_{2}$ and $c_{3}$ with the elements (1 $\left.2 \begin{array}{llll}2 & 4 & 5\end{array}\right),\left(\begin{array}{llll}2 & 3 & 5 & 4\end{array}\right)$ and $(23)(45)$ of $S_{5}$.) We have $\left(Z\left(C_{\mathfrak{L}(G)}(e)_{+}\right)\right)^{C^{\circ}}=$ $\left\langle z_{2}, z_{10}{ }^{1}, z_{10}{ }^{2}, z_{10}{ }^{3}, z_{10}{ }^{4}\right\rangle$, where $z_{2}=e, z_{10}{ }^{1}=e_{2465321}, z_{10}{ }^{2}=e_{2465421}, z_{10}{ }^{3}=e_{2465431}$ and $z_{10}{ }^{4}=e_{2465432}$. Since $\operatorname{Ad}\left(c_{1}\right)$ multiplies $z_{10}{ }^{1}$ by $\zeta, z_{10}{ }^{2}$ by $\zeta^{3}, z_{10}{ }^{3}$ by $\zeta^{4}$ and $z_{10}{ }^{4}$ by $\zeta^{2}$, we have $\mathcal{Z}=\left\langle z_{2}\right\rangle$.

\subsubsection{Orbit $D_{6}\left(a_{1}\right)$ (number 43 in $E_{8}$ )}

Here $e=e_{0}^{0000010}+e_{0}^{0000100}+e_{0}^{0001000}+e_{0010000}-e_{0}^{0110000}+e_{0000000}+e_{0100000}$, which is non-regular distinguished in the Lie algebra of $H$, a $D_{6}$ subsystem subgroup of $G$ with $\Pi(H)=\left\{\begin{array}{c}0000010 \\ 0\end{array},{ }_{0}^{0000100},{ }_{0}^{0001000},{ }_{0}^{0010000},{ }_{1}^{000000},{ }_{0}^{0100000}\right\}$; we have $A \cong \mathcal{S}_{2}$, and $C^{\circ}=A_{1}^{2}$ with $\Pi(D)=\left\{\begin{array}{c}2343210 \\ 2\end{array} \underset{3}{2465432}\right\}$. We take

$$
c=n_{1}^{001111} n_{0111111} h_{2}(i) h_{3}(i) .
$$

The action of $\operatorname{Ad}(c)$ on the root vectors concerned is as follows:

$$
\begin{aligned}
& \text { fixed : } \underset{0}{e 000010}, \underset{0}{e_{0000100}}, \underset{0}{e_{0001000}} \\
& \text { negated : } e_{0010000} \text {; }
\end{aligned}
$$

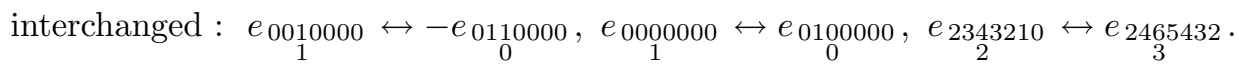

Thus $c$ acts as a non-trivial graph automorphism of both $H$ and $C^{\circ}$; we find that $C_{H}(c) \cong$ $B_{4} B_{1}$, and that $e$ is regular in $C_{H}(c)$. Hence $A=\langle\bar{c}\rangle$. We have $\left(Z\left(C_{\mathfrak{L}(G)}(e)_{+}\right)\right)^{C^{\circ}}=$ $\left\langle z_{2}, z_{10}{ }^{1}, z_{10}{ }^{2}, z_{14}\right\rangle$, where $z_{2}=e, z_{10}{ }^{1}=e_{0121110}+e_{0122100}, z_{10}{ }^{2}=e_{0111110}$ and $z_{14}=$ $e_{0122210}$. Since $\operatorname{Ad}(c)$ negates $z_{10}^{2}$ and fixes $z_{10}{ }^{1}$ and $z_{14}$, we have $\mathcal{Z}=\left\langle z_{2}, z_{10}{ }^{1}, z_{14}\right\rangle$.

\subsubsection{Orbit $E_{7}\left(a_{4}\right)$ (number 37 in $E_{7}, 45$ in $E_{8}$ )}

Here $e=e_{1}^{0110000}+e_{0}^{1000000}+e_{0}^{0111000}+e_{0}^{0000110}+e_{1}^{001100}+e_{0}^{0000010}+e_{0}^{0011000}+e_{0}^{0010000}$, which is non-regular distinguished in the Lie algebra of $H$, a $D_{6} A_{1}$ subsystem subgroup of $G$ with $\Pi(H)=\left\{\begin{array}{c}0110000 \\ 1\end{array}, \underset{0}{1000000}, \underset{0}{0111000}, \underset{0}{0000100}, \underset{0}{0000010},{ }_{1}^{0011000}, \underset{0}{0010000}\right\}$; we have $A \cong \mathcal{S}_{2}$, and $C^{\circ}=1$ or $A_{1}$ respectively with $\Pi(D)=\left\{\begin{array}{c}2465432 \\ 3\end{array}\right\} \cap \Phi(G)$. We take

$$
c=h_{4}(-1) \text {. }
$$

Clearly $c \in Z(H)$. Moreover, as $c \in E_{7}$ it follows that $c \notin C^{\circ}$. Hence $A=\langle\bar{c}\rangle$. We have $\left(Z\left(C_{\mathfrak{L}(G)}(e)_{+}\right)\right)^{C^{\circ}}=\left\langle z_{2}, z_{10}, z_{14}\right\rangle$, where $z_{2}=e, z_{10}=5 e_{1232110}+e_{1232210}+e_{1233210}$ and $z_{14}=e_{2343210}$. Since $\operatorname{Ad}(c)$ fixes $z_{10}$ and $z_{14}$, we have $\mathcal{Z}=\left\langle z_{2}, z_{10}, z_{14}\right\rangle$.

\subsubsection{Orbit $E_{6}\left(a_{1}\right)$ (number 39 in $E_{7}, 46$ in $E_{8}$ )}

Here $e=\underset{0}{e} e_{0} 000000+e_{0}^{0000100}+e_{0}^{010000}+e_{0}^{0001000}+e_{0}^{0011000}+e_{0}^{0110000}+e_{0}^{0000000}$, which is non-regular distinguished in the Lie algebra of $H$, an $E_{6}$ subsystem subgroup of $G$ with 
$\Pi(H)=\left\{\begin{array}{c}1000000 \\ 0\end{array}, \underset{1}{0000000}, \underset{0}{0100000}, \underset{0}{0010000}, \underset{0}{0001000}, \underset{0}{0000100}\right\}$; we have $A \cong \mathcal{S}_{2}$, and $C^{\circ}=T_{1}$ or $A_{2}$ respectively with $\Pi(D)=\left\{\begin{array}{c}0000001 \\ 0\end{array}, \underset{3}{2465431}\right\} \cap \Phi(G)$. We take

$$
c=n_{012210} n_{1122110} n_{1221110} h_{1}(-1) h_{2}(-1) h_{3}(-1) h_{5}(-1) h_{6}(-1) h_{8}(-1) .
$$

The action of $\operatorname{Ad}(c)$ on the root vectors concerned is as follows:

$$
\begin{aligned}
& \text { fixed : } e_{000000} \\
& \text { negated : } \underset{0}{0010000} \text {; } \\
& \text { interchanged : } e_{0} 1000000 \leftrightarrow e_{0000100}, e_{0100000} \leftrightarrow e_{0001000}, e_{0011000} \leftrightarrow e_{0110000} \\
& e_{0}^{0000001} \leftrightarrow e_{2465431}^{e}
\end{aligned}
$$

(Note that if $G=E_{7}$ we delete the term $h_{8}(-1)$ to obtain an element of $G$ whose effect upon those root vectors listed which lie in $\mathfrak{L}(G)$ is the same.) Thus $c$ acts as a nontrivial graph automorphism of both $H$ and the $A_{2}$ subsystem subgroup containing $C^{\circ}$; we find that $C_{H}(c) \cong C_{4}$, and that $e$ is regular in $C_{H}(c)$. Hence $A=\langle\bar{c}\rangle$. We have $\left(Z\left(C_{\mathfrak{L}(G)}(e)_{+}\right)\right)^{C^{\circ}}=\left\langle z_{2}, z_{10}, z_{14}, z_{16}\right\rangle$, where $z_{2}=e, z_{10}=e_{1121100}+e_{1221000}-e_{0122100}$, $z_{14}=e_{1222100}$ and $z_{16}=e_{1232100}$. Since $\operatorname{Ad}(c)$ fixes $z_{10}$ and $z_{14}$ and negates $z_{16}$, we have $\mathcal{Z}=\left\langle z_{2}, z_{10}, z_{14}\right\rangle$.

\subsubsection{Orbit $D_{5} A_{2}$ (number 47 in $E_{8}$ )}

Here $e=e_{1000000}+e_{0100000}+e_{0010000}+e_{0000000}+e_{0}^{0001000}+e_{0000010}+e_{0000001}$; we have $A \cong \mathcal{S}_{2}$, and $C^{\circ}=T_{1}$. We take

$$
c=n_{1233211} n_{1232211} n_{2465431} h_{1}(-1) h_{2}(-1) h_{4}(-1) h_{5}(-1) h_{7}(-1) .
$$

The action of $\operatorname{Ad}(c)$ on the root vectors concerned is as follows:

$$
\begin{aligned}
& \text { fixed : } e_{0} \underset{00000}{ }, e_{0100000}, e_{0010000} ; \\
& \text { interchanged : } e_{1}^{000000} \leftrightarrow \underset{0}{e 0001000}, \underset{0}{0000010} \leftrightarrow \underset{0}{e_{000001}^{0}}
\end{aligned}
$$

Thus $c$ acts as a non-trivial graph automorphism of the $D_{5} A_{2}$ subsystem subgroup in whose Lie algebra $e$ is regular. Hence $A=\langle\bar{c}\rangle$. We have $\left(Z\left(C_{\mathfrak{L}(G)}(e)_{+}\right)\right)^{C^{\circ}}=\left\langle z_{2}, z_{14}\right\rangle$, where $z_{2}=e$ and $z_{14}=e_{1221000}$. Since $\operatorname{Ad}(c)$ fixes $z_{14}$, we have $\mathcal{Z}=\left\langle z_{2}, z_{14}\right\rangle$.

\subsubsection{Orbit $D_{7}\left(a_{2}\right)$ (number 50 in $E_{8}$ )}

Here $e=\underset{0}{e 00001}+e_{000010}+e_{0}^{0001100}+e_{1}^{0010000}-e_{0}^{0110000}+e_{0}^{0011000}+e_{0000000}+e_{0100000}$, which is non-regular distinguished in the Lie algebra of $H$, a $D_{7}$ subsystem subgroup of $G$ with $\Pi(H)=\left\{\begin{array}{c}0000001 \\ 0\end{array}, \underset{0}{0000010}, \underset{0}{0000100}, \underset{0}{0001000}, \underset{0}{0010000}, \underset{1}{0000000}, \underset{0}{0100000}\right\}$; we have $A \cong \mathcal{S}_{2}$, and $C^{\circ}=T_{1}$. We take

$$
c=n_{2354321} n_{2454321} h_{4}(-1) h_{5}(-1) .
$$


The action of $\operatorname{Ad}(c)$ on the root vectors concerned is as follows:

$$
\begin{aligned}
& \text { fixed : } \underset{0}{e_{000001}}, e_{0000010}, e_{0001100}, e_{0011000} ; \\
& \text { negated : } \underset{0}{e 0010000}, \underset{0}{e 001000}, \underset{0}{e 000100 ;} \\
& \text { interchanged : } e_{1}^{0010000} \leftrightarrow-\underset{0}{e} \underset{0}{e} 10000, e_{1}^{0000000} \leftrightarrow e_{0}^{0100000} \text {. }
\end{aligned}
$$

Thus $c$ acts as a non-trivial graph automorphism of $H$; we find that $C_{H}(c) \cong B_{4} B_{2}$, and that $e$ is regular in $C_{H}(c)$. Hence $A=\langle\bar{c}\rangle$. We have $\left(Z\left(C_{\mathfrak{L}(G)}(e)_{+}\right)\right)^{C^{\circ}}=\left\langle z_{2}, z_{14}\right\rangle$, where $z_{2}=e$ and $z_{14}=\underset{1}{e_{012221}}$. Since $\operatorname{Ad}(c)$ fixes $z_{14}$, we have $\mathcal{Z}=\left\langle z_{2}, z_{14}\right\rangle$.

\subsubsection{Orbit $E_{6}\left(a_{1}\right) A_{1}$ (number 52 in $E_{8}$ )}

Here $e=\underset{0}{e}+000000+e_{0}^{0000100}+e_{0}^{0100000}+e_{0}^{0001000}+e_{0}^{0011000}+e_{0110000}+e_{0000000}+e_{0000001}$, which is non-regular distinguished in the Lie algebra of $H$, an $E_{6} A_{1}$ subsystem subgroup of $G$ with $\Pi(H)=\left\{\begin{array}{c}1000000 \\ 0\end{array}, \underset{1}{000000}, \underset{0}{0100000}, \underset{0}{0010000}, \underset{0}{0001000}, \underset{0}{0000100}, \underset{0}{000001}\right\}$; we have $A \cong \mathcal{S}_{2}$, and $C^{\circ}=T_{1}$. We take

$$
c=n_{1244321} n_{1343321} n_{2343221} h_{1}(-1) h_{2}(-1) h_{3}(-1) h_{5}(-1) h_{6}(-1) h_{8}(-1) .
$$

The action of $\operatorname{Ad}(c)$ on the root vectors concerned is as follows:

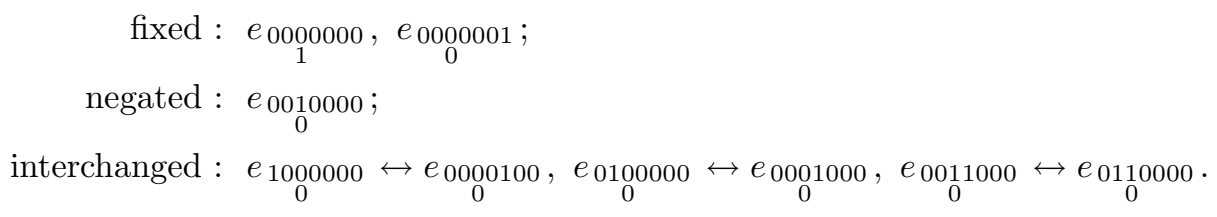

Thus $c$ acts as a non-trivial graph automorphism of $H$; we find that $C_{H}(c) \cong C_{4} A_{1}$, and that $e$ is regular in $C_{H}(c)$. Hence $A=\langle\bar{c}\rangle$. We have $\left(Z\left(C_{\mathfrak{L}(G)}(e)_{+}\right)\right)^{C^{\circ}}=\left\langle z_{2}, z_{14}, z_{16}\right\rangle$, where $z_{2}=e, z_{14}=e_{1222100}$ and $z_{16}=e_{1232100}$. Since $\operatorname{Ad}(c)$ fixes $z_{14}$ and negates $z_{16}$, we have $\mathcal{Z}=\left\langle z_{2}, z_{14}\right\rangle$.

\subsubsection{Orbit $E_{7}\left(a_{3}\right)$ (number 41 in $E_{7}, 53$ in $E_{8}$ )}

Here $e=e_{1}^{0110000}+e_{0} 1000000+e_{0}^{0111000}+e_{0}^{0000100}+e_{0}^{0000010}+e_{1}^{0011000}+e_{0010000}$, which is regular in the Lie algebra of $H$, a $D_{6} A_{1}$ subsystem subgroup of $G$; we have $A \cong \mathcal{S}_{2}$, and $C^{\circ}=1$ or $A_{1}$ respectively with $\Pi(D)=\left\{\begin{array}{c}2465432 \\ 3\end{array}\right\} \cap \Phi(G)$. We take

$$
c=h_{4}(-1) \text {. }
$$

Clearly $c \in Z(H)$. Moreover, as $c \in E_{7}$ it follows that $c \notin C^{\circ}$. Hence $A=\langle\bar{c}\rangle$. If $G=E_{8}$ we have $\left(Z\left(C_{\mathfrak{L}(G)}(e)_{+}\right)\right)^{C^{\circ}}=\left\langle z_{2}, z_{14}, z_{16}, z_{18}\right\rangle$, where $z_{2}=e, z_{14}=e_{223210}+e_{1233210}$, $z_{16}=e_{1243210}$ and $z_{18}=e_{2343210} ;$ if $G=E_{7}$ we have an additional vector $z_{10}=$ $3 e_{122111}+2 e_{123210}+e_{112211}-e_{012221}$. Since $\operatorname{Ad}(c)$ fixes $z_{14}$ and $z_{18}\left(\right.$ and $z_{10}$ if $\left.G=E_{7}\right)$ and negates $z_{16}$, we have $\mathcal{Z}=\left\langle z_{2}, z_{10}, z_{14}, z_{18}\right\rangle$ if $G=E_{7}$ and $\left\langle z_{2}, z_{14}, z_{18}\right\rangle$ if $G=E_{8}$. 


\subsubsection{Orbit $E_{8}\left(b_{6}\right)$ (number 54 in $\left.E_{8}\right)$}

Here $e=e_{1}^{0011000}+e_{0}^{0010000}+e_{0}^{1111100}+e_{1}^{0111100}+e_{1}^{0111110}-e_{0}^{1111110}+e_{0}^{0000001}+$ $e_{0}^{0111000}+e_{1110000}$, which is non-regular distinguished in the Lie algebra of $H$, an $E_{6} A_{2}$ subsystem subgroup of $G$ with $\Pi(H)=\left\{\begin{array}{c}0011000 \\ 1\end{array}, \underset{0}{0000001}, \underset{0}{1111100}, \underset{0}{0000010}, 0111100\right.$, $\left.{ }_{0}^{0010000}, \underset{0}{0111000},{ }_{1}^{1110000}\right\}$ (with the projection of $e$ on the Lie algebra of the $E_{6}$ factor lying in the $E_{6}\left(a_{1}\right)$ orbit — see the comments at the end of $\left.\S 5\right)$; we have $A \cong \mathcal{S}_{3}$, and $C^{\circ}=1$. We take

$$
\begin{aligned}
& c_{1}=h_{1}(\omega) h_{2}(\omega) h_{5}\left(\omega^{2}\right), \\
& c_{2}=n_{0}^{1000000} n_{0000000} n_{0001000}^{0} h_{2}(-1) h_{3}(-1) h_{4}(-1) h_{5}(-1) h_{8}(-1) .
\end{aligned}
$$

Clearly $c_{1} \in Z(H)$. The action of $\operatorname{Ad}\left(c_{2}\right)$ on the root vectors concerned is as follows:

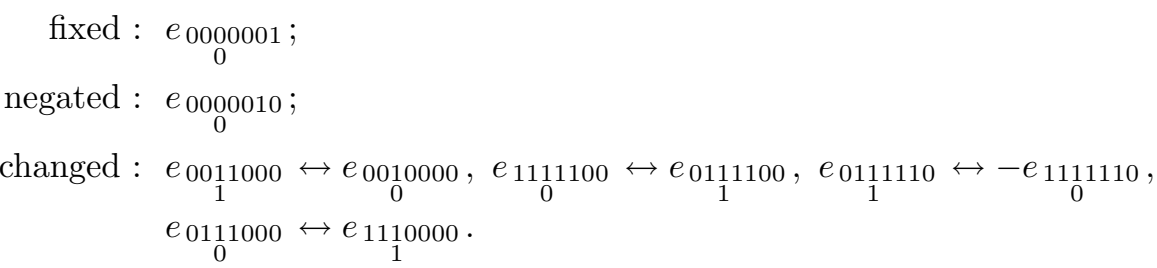

Thus $c_{2}$ acts as a non-trivial graph automorphism of $H$; we find that $C_{H}\left(c_{2}\right) \cong C_{4} A_{1}$, and that $e$ is regular in $C_{H}\left(c_{2}\right)$. Hence $A=\left\langle\bar{c}_{1}, \bar{c}_{2}\right\rangle$. We have $\left(Z\left(C_{\mathfrak{L}(G)}(e)_{+}\right)\right)^{C^{\circ}}=$ $\left\langle z_{2}, z_{14}, z_{16}\right\rangle$, where $z_{2}=e, z_{14}=e_{2465421}$ and $z_{16}=e_{2465432}$. Since $\operatorname{Ad}\left(c_{1}\right)$ fixes both $z_{14}$ and $z_{16}$, and $\operatorname{Ad}\left(c_{2}\right)$ fixes $z_{14}$ but negates $z_{16}$, we have $\mathcal{Z}=\left\langle z_{2}, z_{14}\right\rangle$.

\subsubsection{Orbit $D_{7}\left(a_{1}\right)$ (number 55 in $E_{8}$ )}

Here $e=e_{0}^{0000001}+e_{0}^{000010}+e_{0}^{0000100}+e_{0}^{0001000}+e_{0}^{0010000}-e_{0110000}+e_{0000000}+e_{0100000}$, which is non-regular distinguished in the Lie algebra of $H$, a $D_{7}$ subsystem subgroup of $G$ with $\Pi(H)=\left\{\begin{array}{c}0000001 \\ 0\end{array}, \underset{0}{0000010}, \underset{0}{0000100}, \underset{0}{0001000}, \underset{0}{0010000}, \underset{1}{0000000}, \underset{0}{0100000}\right\}$; we have $A \cong \mathcal{S}_{2}$, and $C^{\circ}=T_{1}$. We take

$$
c=n_{2354321} n_{2454321} h_{2}(-1) h_{4}(-1) h_{6}(-1) h_{8}(-1) .
$$

The action of $\operatorname{Ad}(c)$ on the root vectors concerned is as follows:

$$
\begin{aligned}
& \text { fixed : } \underset{0}{e} e_{000001}, \underset{0}{e_{0000010}}, e_{0000100}, e_{0001000} ; \\
& \text { negated : } e_{0010000} \text {; }
\end{aligned}
$$

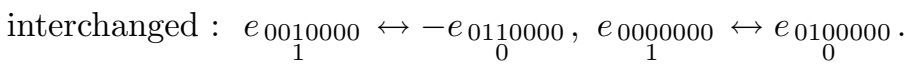

Thus $c$ acts as a non-trivial graph automorphism of $H$; we find that $C_{H}(c) \cong B_{5} B_{1}$, and that $e$ is regular in $C_{H}(c)$. Hence $A=\langle\bar{c}\rangle$. We have $\left(Z\left(C_{\mathfrak{L}(G)}(e)_{+}\right)\right)^{C^{\circ}}=\left\langle z_{2}, z_{14}, z_{18}\right\rangle$, where $z_{2}=e, z_{14}=e_{0122111}+e_{0122210}$ and $z_{18}=e_{1}^{0122221}$. Since $\operatorname{Ad}(c)$ fixes $z_{14}$ and $z_{18}$, we have $\mathcal{Z}=\left\langle z_{2}, z_{14}, z_{18}\right\rangle$. 


\subsubsection{Orbit $E_{8}\left(a_{6}\right)$ (number 58 in $E_{8}$ )}

Here $e=e_{0011000}+e_{1111100}+e_{0000010}+e_{0111100}+e_{0010000}+e_{0001111}+e_{1110000}+e_{0111000}$, which is regular in the Lie algebra of $H$, an $A_{8}$ subsystem subgroup of $G$; we have $A \cong \mathcal{S}_{3}$, and $C^{\circ}=1$. We take

$$
\begin{aligned}
& c_{1}=h_{2}(\omega) h_{3}(\omega) h_{5}(\omega) h_{6}(\omega) h_{8}\left(\omega^{2}\right), \\
& c_{2}=n_{0}^{0000000} n_{0100000}^{n_{0000001}} n_{0001100}^{0} h_{2}(-1) h_{3}(-1) h_{4}(-1) h_{5}(-1) h_{7}(-1) h_{8}(-1) .
\end{aligned}
$$

Clearly $c_{1} \in Z(H)$. The action of $\operatorname{Ad}\left(c_{2}\right)$ on the root vectors concerned is as follows:

$$
\begin{array}{cl}
\text { interchanged : } & e_{0011000} \leftrightarrow e_{0111000}, \underset{0}{e_{1111100}} \leftrightarrow e_{1110000}, e_{0000010} \leftrightarrow e_{0001111}, \\
& e_{0111100} \leftrightarrow e_{0010000}
\end{array}
$$

Thus $c_{2}$ acts as a non-trivial graph automorphism of $H$. Hence $A=\left\langle\bar{c}_{1}, \bar{c}_{2}\right\rangle$. We have $\left(Z\left(C_{\mathfrak{L}(G)}(e)_{+}\right)\right)^{C^{\circ}}=\left\langle z_{2}, z_{14}, z_{18}{ }^{1}, z_{18}{ }^{2}\right\rangle$, where $z_{2}=e, z_{14}=e_{2354321}-e_{2454321}, z_{18}{ }^{1}=$ $e_{2465431}$ and $z_{18}{ }^{2}=e_{2465432}$. Since $\operatorname{Ad}\left(c_{1}\right)$ multiplies $z_{18}{ }^{1}$ by $\omega$ and $z_{18}{ }^{2}$ by $\omega^{2}$ while fixing $z_{14}$, and $\operatorname{Ad}\left(c_{2}\right)$ fixes $z_{14}$, we have $\mathcal{Z}=\left\langle z_{2}, z_{14}\right\rangle$.

\subsubsection{Orbit $E_{8}\left(b_{5}\right)$ (number 60 in $E_{8}$ )}

Here $e=e_{0011000}+e_{000001}+e_{0}^{1111100}+e_{0}^{0000010}+e_{011100}+e_{0010000}+e_{0111000}+e_{1110000}$, which is regular in the Lie algebra of $H$, an $E_{6} A_{2}$ subsystem subgroup of $G$; we have $A \cong \mathcal{S}_{3}$, and $C^{\circ}=1$. We take

$$
\begin{aligned}
& c_{1}=h_{1}(\omega) h_{2}(\omega) h_{5}\left(\omega^{2}\right), \\
& c_{2}=n_{0}^{1000000} n_{0}^{0000000} n_{0}^{0001000} h_{1}(-1) h_{3}(-1) h_{4}(-1) .
\end{aligned}
$$

Clearly $c_{1} \in Z(H)$. The action of $\operatorname{Ad}\left(c_{2}\right)$ on the root vectors concerned is as follows:

$$
\begin{aligned}
& \text { fixed : } e_{0000001}, e_{0000010} \text {; } \\
& \text { interchanged : } e_{1}^{0011000} \leftrightarrow \underset{0}{e 010000}, \underset{0}{e} e_{111100} \leftrightarrow e_{1}^{0111100}, e_{0}^{0111000} \leftrightarrow e_{1110000}
\end{aligned}
$$

Thus $c_{2}$ acts as a non-trivial graph automorphism of $H$. Hence $A=\left\langle\bar{c}_{1}, \bar{c}_{2}\right\rangle$. We have $\left(Z\left(C_{\mathfrak{L}(G)}(e)_{+}\right)\right)^{C^{\circ}}=\left\langle z_{2}, z_{14}, z_{18}{ }^{1}, z_{18}{ }^{2}, z_{22}\right\rangle$, where $z_{2}=e, z_{14}=e_{1243221}+e_{1343321}+$ $e_{2344321}, z_{18}{ }^{1}=e_{2464321}, z_{18}{ }^{2}=e_{2465321}$ and $z_{22}=e_{2465432}$. Since $\operatorname{Ad}\left(c_{1}\right)$ multiplies $z_{18}{ }^{1}$ by $\omega$ and $z_{18}{ }^{2}$ by $\omega^{2}$ while fixing $z_{14}$ and $z_{22}$, and $\operatorname{Ad}\left(c_{2}\right)$ fixes both $z_{14}$ and $z_{22}$, we have $\mathcal{Z}=\left\langle z_{2}, z_{14}, z_{22}\right\rangle$.

\subsubsection{Orbit $E_{8}\left(a_{5}\right)$ (number 62 in $E_{8}$ )}

Here $e=e_{0011000}+e_{0000111}+e_{0111000}+e_{0} 000000+e_{0110000}-e_{0011100}+e_{0001110}+$ $e_{0010000}+\underset{0}{e 000010}$, which is non-regular distinguished in the Lie algebra of $H$, a $D_{8}$ subsystem subgroup of $G$ with $\Pi(H)=\left\{\begin{array}{l}0011000,0000111 \\ 1\end{array}, \underset{0}{0111000}, \underset{0}{1000000}, \underset{1}{0110000}, \underset{0}{0001100}\right.$, $\underset{0}{0010000}, \underset{0}{0000010}\}$; we have $A \cong \mathcal{S}_{2}$, and $C^{\circ}=1$. We take

$$
c=h_{4}(-1) h_{7}(-1)
$$


Clearly $c \in Z(H)$. Hence $A=\langle\bar{c}\rangle$. We have $\left(Z\left(C_{\mathfrak{L}(G)}(e)_{+}\right)\right)^{C^{\circ}}=\left\langle z_{2}, z_{14}, z_{22}{ }^{1}, z_{22}{ }^{2}\right\rangle$, where $z_{2}=e, z_{14}=7 e_{1243221}-e_{1244321}-e_{1343321}-e_{23423210}, z_{22}{ }^{1}=e_{2465431}$ and $z_{22}{ }^{2}=e_{2465432}$. Since $\operatorname{Ad}(c)$ negates $z_{22}{ }^{1}$ and fixes the remaining basis vectors, we have $\mathcal{Z}=\left\langle z_{2}, z_{14}, z_{22}{ }^{2}\right\rangle$.

\subsubsection{Orbit $E_{8}\left(b_{4}\right)$ (number 63 in $\left.E_{8}\right)$}

Here $e=e_{0}^{0000001}+e_{0}^{0000010}+e_{0}^{0000110}+e_{0}^{0011100}+e_{0}^{0111000}+e_{0}^{1000000}+e_{0110000}+e_{0}^{0010000}$, which is non-regular distinguished in the Lie algebra of $H$, an $E_{7} A_{1}$ subsystem subgroup of $G$ with $\Pi(H)=\left\{\begin{array}{c}0000001 \\ 0\end{array},{ }_{1}^{0011000},{ }_{0}^{0000010},{ }_{0}^{0000100},{ }_{0}^{0111000},{ }_{0}^{1000000},{ }_{0}^{0110000},{ }_{1}^{0010000}\right\}$ (with the projection of $e$ on the Lie algebra of the $E_{7}$ factor lying in the $E_{7}\left(a_{1}\right)$ orbit - see the comments at the end of $\S 5$ ); we have $A \cong \mathcal{S}_{2}$, and $C^{\circ}=1$. We take

$$
c=h_{4}(-1) .
$$

Clearly $c \in Z(H)$. Hence $A=\langle\bar{c}\rangle$. We have $\left(Z\left(C_{\mathfrak{L}(G)}(e)_{+}\right)\right)^{C^{\circ}}=\left\langle z_{2}, z_{14}, z_{22}, z_{26}\right\rangle$, where $z_{2}=e, z_{14}=e_{1232221}-e_{1233321}-e_{1343211}-e_{2343210}, z_{22}=e_{2465321}+e_{2465421}$ and $z_{26}=e_{2465432}$. Since $\operatorname{Ad}(c)$ fixes each of the basis vectors, we have $\mathcal{Z}=\left\langle z_{2}, z_{14}, z_{22}, z_{26}\right\rangle$.

\subsubsection{Orbit $E_{8}\left(a_{4}\right)$ (number 65 in $E_{8}$ )}

Here $e=e_{1}^{0011000}+e_{0}^{0000100}+e_{0}^{0111000}+e_{100000}+e_{0}^{0110000}+e_{0}^{0001110}+e_{0}^{0010000}+e_{0000001}^{0}$, which is regular in the Lie algebra of $H$, a $D_{8}$ subsystem subgroup of $G$; we have $A \cong \mathcal{S}_{2}$, and $C^{\circ}=1$. We take

$$
c=h_{4}(-1) h_{8}(-1)
$$

Clearly $c \in Z(H)$. Hence $A=\langle\bar{c}\rangle$. We have $\left(Z\left(C_{\mathfrak{L}(G)}(e)_{+}\right)\right)^{C^{\circ}}=\left\langle z_{2}, z_{14}, z_{22}, z_{26}, z_{28}\right\rangle$, where $z_{2}=e, z_{14}=4 e_{1243210}+3 e_{1233211}-e_{1232211}-e_{1233221}-e_{1232221}, z_{22}=e_{2354321}-$ $e_{2454321}, z_{26}=e_{2465421}$ and $z_{28}=e_{2465432}$. Since $\operatorname{Ad}(c)$ negates $z_{28}$ and fixes the remaining basis vectors, we have $\mathcal{Z}=\left\langle z_{2}, z_{14}, z_{22}, z_{26}\right\rangle$.

\subsubsection{Orbit $E_{8}\left(a_{3}\right)$ (number 66 in $\left.E_{8}\right)$}

Here $e=e_{0}^{0000001}+e_{0011000}+e_{0000010}+e_{0000100}+e_{0}^{0111000}+e_{0}^{1000000}+e_{0110000}+e_{0}^{0010000}$, which is regular in the Lie algebra of $H$, an $E_{7} A_{1}$ subsystem subgroup of $G$; we have $A \cong \mathcal{S}_{2}$, and $C^{\circ}=1$. We take

$$
c=h_{4}(-1) .
$$

Clearly $c \in Z(H)$. Hence $A=\langle\bar{c}\rangle$. We have $\left(Z\left(C_{\mathfrak{L}(G)}(e)_{+}\right)\right)^{C^{\circ}}=\left\langle z_{2}, z_{14}, z_{22}, z_{26}, z_{28}\right.$, $\left.z_{34}\right\rangle$, where $z_{2}=e, z_{14}=e_{012221}-e_{1122211}-e_{1232210}-2 e_{1232111}-e_{1233210}, z_{22}=$ $e_{2343221}-e_{1343321}-e_{1244321}, z_{26}=e_{2354321}-e_{2454321}, z_{28}=e_{2464321}$ and $z_{34}=$ $e_{2465432}$. Since $\operatorname{Ad}(c)$ negates $z_{28}$ and fixes the remaining basis vectors, we have $\mathcal{Z}=$ $\left\langle z_{2}, z_{14}, z_{22}, z_{26}, z_{34}\right\rangle$.

This completes the consideration of the groups $E_{n}$. 


\section{Proofs of the main theorems for exceptional groups}

In this section we use the information obtained in $\S \S 5-9$ to provide proofs of the main results stated in $\S 1$, for groups $G$ of exceptional type. In each case the conclusion is immediate if the nilpotent element $e$ is 0 ; we therefore fix a non-zero nilpotent orbit representative $e \in \mathfrak{L}(G)$ listed in Table 2, and the associated cocharacter $\tau$ as given at the top of the page for $e$ in $\S 11$.

We begin with Theorems 1 and 2; recall that we proved the second equality in each of Theorems 1(i) and 2 at the beginning of $\S 3$. The sixth column of the table on the page for $e$ in $\S 11$ identifies the subalgebra $\mathcal{Z}=\left(Z\left(C_{\mathfrak{L}(G)}(e)_{+}\right)\right)^{C}$, which by Theorem 3.9 is equal to $\mathfrak{L}\left(Z\left(C_{G}(e)\right)\right)$. We first consider those orbits for which all labels of $\Delta$ are even, and thus are either 0 or 2 . It is a straightforward check to see that in each of these cases we have $\operatorname{dim} \mathcal{Z}=n_{2}(\Delta)$. As each distinguished orbit in $\mathfrak{L}(G)$ has an even diagram, this establishes the remainder of Theorems 1(i) and 2. For the proof of Theorem 1(ii), we first recall as in $\S 4.1$ the result of Kostant in [16], which states that if $e$ is regular nilpotent then the $\tau$-weights in $C_{\mathfrak{L}(G)}(e)$ are $2 d_{1}-2, \ldots, 2 d_{\ell}-2$, where $d_{1}<\cdots<d_{\ell}$ are the degrees of the invariant polynomials of the Weyl group of $G$. It is now straightforward to consider the non-regular distinguished orbits in $\mathfrak{L}(G)$ and observe that the $\tau$-weights in $\mathcal{Z}$ are the first $n_{2}(\Delta)$ of these integers; for the convenience of the reader we have in fact listed in Table 4 both the dimension of $\mathcal{Z}$ and the $\tau$-weights, with multiplicities, occurring in $\mathcal{Z}$ for each nilpotent orbit representative.

We therefore turn to the proofs of Theorems 3 and 4 . In some cases these involve information on certain nilpotent orbits in simple Lie algebras of classical type, which were treated in $\S 4$; for convenience we have listed the relevant data in Table 5 .

For each group $G$ of exceptional type, Table 6 has one row for each orbit whose labelled diagram has at least one label equal to 2 . In each case we have specified the orbit name and labelled diagram $\Delta$, and recorded the dimensions of $C_{G}(e)$ and $\mathcal{Z}$. Recall from $\S 1$ the definitions of the 2 -free core $\Delta_{0}$ of $\Delta$ and the semisimple group $G_{0}$; the next entry in the row is the type of the group $G_{0}$. Reference to [2] reveals that the labelled diagram $\Delta_{0}$ corresponds to an orbit $\operatorname{Ad}\left(G_{0}\right) e_{0}$ of nilpotent elements of $\mathfrak{L}\left(G_{0}\right)$, whose name is listed in the next entry; this proves the existence statement of Theorem 3. The last two entries in the row are the dimensions of $C_{G_{0}}\left(e_{0}\right)$ and $\mathcal{Z}_{0}=\mathfrak{L}\left(Z\left(C_{G_{0}}\left(e_{0}\right)\right)\right)$. In each case it is then immediate that $\operatorname{dim} Z\left(C_{G}(e)\right)-\operatorname{dim} Z\left(C_{G_{0}}\left(e_{0}\right)\right)=n_{2}(\Delta)$ (and indeed it is also apparent that $\operatorname{dim} C_{G}(e)-\operatorname{dim} C_{G_{0}}\left(e_{0}\right)=n_{2}(\Delta)$, as proved in Proposition 3.1). This completes the proof of Theorem 3. Finally the proof of Theorem 4 is a case-by-case verification. 
Table 4: $\tau$-weights of vectors in $\mathcal{Z}$ for exceptional groups

\begin{tabular}{|c|c|l||c|c|l|}
\hline \multicolumn{5}{|c|}{$G=G_{2}$} \\
\hline Orbit & $\operatorname{dim} \mathcal{Z}$ & Weights & Orbit & $\operatorname{dim} \mathcal{Z}$ & Weights \\
\hline$A_{1}$ & 1 & 2 & $G_{2}\left(a_{1}\right)$ & 1 & 2 \\
$\tilde{A}_{1}$ & 1 & 2 & $G_{2}$ & 2 & 2,10 \\
\hline
\end{tabular}

\begin{tabular}{|c|c|l||c|c|l||c|c|l|}
\hline \multicolumn{7}{|c|}{$G=F_{4}$} \\
\hline Orbit & $\operatorname{dim} \mathcal{Z}$ & Weights & Orbit & $\operatorname{dim} \mathcal{Z}$ & Weights & Orbit & $\operatorname{dim} \mathcal{Z}$ & Weights \\
\hline$A_{1}$ & 1 & 2 & $A_{2} \tilde{A}_{1}$ & 1 & 2 & $B_{3}$ & 2 & 2,10 \\
$\tilde{A}_{1}$ & 1 & 2 & $B_{2}$ & 2 & 2,6 & $C_{3}$ & 2 & 2,10 \\
$A_{1} \tilde{A}_{1}$ & 1 & 2 & $\tilde{A}_{2} A_{1}$ & 1 & 2 & $F_{4}\left(a_{2}\right)$ & 2 & 2,10 \\
$A_{2}$ & 1 & 2 & $C_{3}\left(a_{1}\right)$ & 2 & 2,6 & $F_{4}\left(a_{1}\right)$ & 3 & $2,10,14$ \\
$\tilde{A}_{2}$ & 1 & 2 & $F_{4}\left(a_{3}\right)$ & 1 & 2 & $F_{4}$ & 4 & $2,10,14,22$ \\
\hline
\end{tabular}

\begin{tabular}{|c|c|l||c|c|l||c|c|l|}
\hline \multicolumn{10}{|c|}{$G=E_{6}$} \\
\hline Orbit & $\operatorname{dim} \mathcal{Z}$ & Weights & Orbit & $\operatorname{dim} \mathcal{Z}$ & Weights & Orbit & $\operatorname{dim} \mathcal{Z}$ & Weights \\
\hline$A_{1}$ & 1 & 2 & $A_{3}$ & 2 & 2,6 & $A_{5}$ & 3 & $2,8,10$ \\
$A_{1}{ }^{2}$ & 1 & 2 & $A_{2}{ }^{2} A_{1}$ & 1 & 2 & $D_{5}\left(a_{1}\right)$ & 3 & $2,8,10$ \\
$A_{1}{ }^{3}$ & 1 & 2 & $A_{3} A_{1}$ & 2 & 2,6 & $E_{6}\left(a_{3}\right)$ & 3 & $2,8,10$ \\
$A_{2}$ & 1 & 2 & $D_{4}\left(a_{1}\right)$ & 1 & 2 & $D_{5}$ & 4 & $2,8,10,14$ \\
$A_{2} A_{1}$ & 2 & 2,4 & $A_{4}$ & 3 & $2,6,8$ & $E_{6}\left(a_{1}\right)$ & 5 & $2,8,10,14,16$ \\
$A_{2}{ }^{2}$ & 2 & 2,4 & $D_{4}$ & 2 & 2,10 & $E_{6}$ & 6 & $2,8,10,14,16,22$ \\
$A_{2} A_{1}{ }^{2}$ & 1 & 2 & $A_{4} A_{1}$ & 2 & 2,8 & & & \\
\hline
\end{tabular}

\begin{tabular}{|c|l|l||c|c|l||c|c|l|}
\hline \multicolumn{1}{|c|}{$G=E_{7}$} \\
\hline Orbit & $\operatorname{dim} \mathcal{Z}$ & Weights & Orbit & $\operatorname{dim} \mathcal{Z}$ & Weights & Orbit & $\operatorname{dim} \mathcal{Z}$ & Weights \\
\hline$A_{1}$ & 1 & 2 & $A_{3} A_{1}{ }^{2}$ & 2 & 2,6 & $E_{6}\left(a_{3}\right)$ & 2 & 2,10 \\
$A_{1}{ }^{2}$ & 1 & 2 & $D_{4}$ & 2 & 2,10 & $D_{5}$ & 3 & $2,10,14$ \\
$\left(A_{1}{ }^{3}\right)^{\prime \prime}$ & 1 & 2 & $D_{4}\left(a_{1}\right) A_{1}$ & 2 & 2,6 & $E_{7}\left(a_{5}\right)$ & 2 & 2,10 \\
$\left(A_{1}{ }^{3}\right)^{\prime}$ & 1 & 2 & $A_{3} A_{2}$ & 2 & 2,6 & $A_{6}$ & 2 & 2,10 \\
$A_{2}$ & 1 & 2 & $A_{4}$ & 2 & 2,6 & $D_{5} A_{1}$ & 3 & $2,10,14$ \\
$A_{1}{ }^{4}$ & 1 & 2 & $A_{3} A_{2} A_{1}$ & 1 & 2 & $D_{6}\left(a_{1}\right)$ & 4 & $2,10,10,14$ \\
$A_{2} A_{1}$ & 1 & 2 & $\left(A_{5}\right)^{\prime \prime}$ & 3 & $2,6,10$ & $E_{7}\left(a_{4}\right)$ & 3 & $2,10,14$ \\
$A_{2} A_{1}{ }^{2}$ & 1 & 2 & $D_{4} A_{1}$ & 2 & 2,10 & $D_{6}$ & 4 & $2,10,14,18$ \\
$A_{3}$ & 2 & 2,6 & $A_{4} A_{1}$ & 1 & 2 & $E_{6}\left(a_{1}\right)$ & 3 & $2,10,14$ \\
$A_{2}{ }^{2}$ & 1 & 2 & $D_{5}\left(a_{1}\right)$ & 2 & 2,10 & $E_{6}$ & 4 & $2,10,14,22$ \\
$A_{2} A_{1}{ }^{3}$ & 1 & 2 & $A_{4} A_{2}$ & 1 & 2 & $E_{7}\left(a_{3}\right)$ & 4 & $2,10,14,18$ \\
$\left(A_{3} A_{1}\right)^{\prime \prime}$ & 2 & 2,6 & $\left(A_{5}\right)^{\prime}$ & 2 & 2,10 & $E_{7}\left(a_{2}\right)$ & 5 & $2,10,14,18,22$ \\
$A_{2}{ }^{2} A_{1}$ & 1 & 2 & $A_{5} A_{1}$ & 2 & 2,10 & $E_{7}\left(a_{1}\right)$ & 6 & $2,10,14,18,22,26$ \\
$\left(A_{3} A_{1}\right)^{\prime}$ & 2 & 2,6 & $D_{5}\left(a_{1}\right) A_{1}$ & 2 & 2,10 & $E_{7}$ & 7 & $2,10,14,18,22,26,34$ \\
$D_{4}\left(a_{1}\right)$ & 1 & 2 & $D_{6}\left(a_{2}\right)$ & 3 & $2,10,10$ & & & \\
\hline
\end{tabular}


Table 4: $\tau$-weights of vectors in $\mathcal{Z}$ for exceptional groups (continued)

\begin{tabular}{|c|l|l||c|l|l|}
\hline \multicolumn{5}{|c|}{$G=E_{8}$} \\
\hline Orbit & dim $\mathcal{Z}$ & Weights & Orbit & dim $\mathcal{Z}$ & Weights \\
\hline$A_{1}$ & 1 & 2 & $D_{5}\left(a_{1}\right) A_{2}$ & 2 & 2,10 \\
$A_{1}{ }^{2}$ & 1 & 2 & $D_{6}\left(a_{2}\right)$ & 2 & 2,10 \\
$A_{1}{ }^{3}$ & 1 & 2 & $E_{6}\left(a_{3}\right) A_{1}$ & 2 & 2,10 \\
$A_{2}$ & 1 & 2 & $E_{7}\left(a_{5}\right)$ & 2 & 2,10 \\
$A_{1}{ }^{4}$ & 1 & 2 & $D_{5} A_{1}$ & 3 & $2,10,14$ \\
$A_{2} A_{1}$ & 1 & 2 & $E_{8}\left(a_{7}\right)$ & 1 & 2 \\
$A_{2} A_{1}{ }^{2}$ & 1 & 2 & $A_{6}$ & 2 & 2,10 \\
$A_{3}$ & 2 & 2,6 & $D_{6}\left(a_{1}\right)$ & 3 & $2,10,14$ \\
$A_{2} A_{1}{ }^{3}$ & 1 & 2 & $A_{6} A_{1}$ & 1 & 2 \\
$A_{2}{ }^{2}$ & 1 & 2 & $E_{7}\left(a_{4}\right)$ & 3 & $2,10,14$ \\
$A_{2}{ }^{2} A_{1}$ & 1 & 2 & $E_{6}\left(a_{1}\right)$ & 3 & $2,10,14$ \\
$A_{3} A_{1}$ & 2 & 2,6 & $D_{5} A_{2}$ & 2 & 2,14 \\
$D_{4}\left(a_{1}\right)$ & 1 & 2 & $D_{6}$ & 3 & $2,14,18$ \\
$D_{4}$ & 2 & 2,10 & $E_{6}$ & 4 & $2,10,14,22$ \\
$A_{2}{ }^{2} A_{1}{ }^{2}$ & 1 & 2 & $D_{7}\left(a_{2}\right)$ & 2 & 2,14 \\
$A_{3} A_{1}{ }^{2}$ & 2 & 2,6 & $A_{7}$ & 2 & 2,14 \\
$D_{4}\left(a_{1}\right) A_{1}$ & 1 & 2 & $E_{6}\left(a_{1}\right) A_{1}$ & 2 & 2,14 \\
$A_{3} A_{2}$ & 2 & 2,6 & $E_{7}\left(a_{3}\right)$ & 3 & $2,14,18$ \\
$A_{4}$ & 2 & 2,6 & $E_{8}\left(b_{6}\right)$ & 2 & 2,14 \\
$A_{3} A_{2} A_{1}$ & 1 & 2 & $D_{7}\left(a_{1}\right)$ & 3 & $2,14,18$ \\
$D_{4} A_{1}$ & 2 & 2,10 & $E_{6} A_{1}$ & 3 & $2,14,22$ \\
$D_{4}\left(a_{1}\right) A_{2}$ & 1 & 2 & $E_{7}\left(a_{2}\right)$ & 4 & $2,14,18,22$ \\
$A_{4} A_{1}$ & 1 & 2 & $E_{8}\left(a_{6}\right)$ & 2 & 2,14 \\
$A_{3}{ }^{2}$ & 1 & 2 & $D_{7}$ & 3 & $2,14,22$ \\
$D_{5}\left(a_{1}\right)$ & 2 & 2,10 & $E_{8}\left(b_{5}\right)$ & 3 & $2,14,22$ \\
$A_{4} A_{1}{ }^{2}$ & 1 & 2 & $E_{7}\left(a_{1}\right)$ & 5 & $2,14,18,22,26$ \\
$A_{4} A_{2}$ & 1 & 2 & $E_{8}\left(a_{5}\right)$ & 3 & $2,14,22$ \\
$A_{5}$ & 2 & 2,10 & $E_{8}\left(b_{4}\right)$ & 4 & $2,14,22,26$ \\
$D_{5}\left(a_{1}\right) A_{1}$ & 2 & 2,10 & $E_{7}$ & 5 & $2,14,22,26,34$ \\
$A_{4} A_{2} A_{1}$ & 1 & 2 & $E_{8}\left(a_{4}\right)$ & 4 & $2,14,22,26$ \\
$D_{4} A_{2}$ & 2 & 2,10 & $E_{8}\left(a_{3}\right)$ & 5 & $2,14,22,26,34$ \\
$E_{6}\left(a_{3}\right)$ & 2 & 2,10 & $E_{8}\left(a_{2}\right)$ & 6 & $2,14,22,26,34,38$ \\
$D_{5}$ & 3 & $2,10,14$ & $E_{8}\left(a_{1}\right)$ & 7 & $2,14,22,26,34,38,46$ \\
$A_{4} A_{3}$ & 1 & 2 & $E_{8}$ & 8 & $2,14,22,26,34,38,46,58$ \\
$A_{5} A_{1}$ & 2 & 2,10 & & & \\
\hline & & & & \\
\hline
\end{tabular}


Table 5: Some nilpotent orbits in classical Lie algebras

\begin{tabular}{|c|c|c|c|c|}
\hline$G$ & $\operatorname{Ad}(G) e$ & $\Delta$ & $\operatorname{dim} C_{G}(e)$ & $\operatorname{dim} Z\left(C_{G}(e)\right)$ \\
\hline$A_{5}$ & $A_{1}$ & 10001 & 25 & 1 \\
$A_{5}$ & $A_{2} A_{1}$ & 11011 & 13 & 2 \\
$B_{3}$ & $B_{1} A_{1}$ & 101 & 9 & 1 \\
$C_{3}$ & $C_{1}$ & 001 & 15 & 1 \\
$D_{4}$ & $A_{1} D_{2}$ & $10_{1}^{1}$ & 12 & 1 \\
$D_{5}$ & $A_{1} D_{2}$ & $101_{0}^{0}$ & 21 & 1 \\
$D_{5}$ & $A_{2} A_{1}$ & $010_{1}^{1}$ & 17 & 2 \\
$D_{6}$ & $A_{1}$ & $0100_{0}^{0}$ & 48 & 1 \\
$D_{6}$ & $A_{1}^{2} D_{2}$ & $1000_{1}^{1}$ & 30 & 1 \\
$D_{6}$ & $A_{2} A_{1}$ & $0101_{0}^{0}$ & 26 & 1 \\
$D_{6}$ & $A_{3} D_{2}$ & $0110_{1}^{1}$ & 18 & 2 \\
$D_{7}$ & $A_{1} D_{2}$ & $10100_{0}^{0}$ & 51 & 1 \\
$D_{7}$ & $A_{3} D_{3}$ & $10110_{1}^{1}$ & 21 & 2 \\
\hline
\end{tabular}

Table 6: 2-free cores for exceptional groups

\begin{tabular}{|c|c|c|c|c|c|c|c|c|}
\hline$G$ & $\operatorname{Ad}(G) e$ & $\Delta$ & $\operatorname{dim} C_{G}(e)$ & $\operatorname{dim} \mathcal{Z}$ & $G_{0}$ & $\operatorname{Ad}\left(G_{0}\right) e_{0}$ & $\operatorname{dim} C_{G_{0}}\left(e_{0}\right)$ & $\operatorname{dim} \mathcal{Z}_{0}$ \\
\hline \multirow[t]{2}{*}{$G_{2}$} & $G_{2}\left(a_{1}\right)$ & 02 & 4 & 1 & $A_{1}$ & $\emptyset$ & 3 & 0 \\
\hline & $G_{2}$ & 22 & 2 & 2 & 1 & $\emptyset$ & 0 & 0 \\
\hline \multirow[t]{9}{*}{$F_{4}$} & $A_{2}$ & 2000 & 22 & 1 & $C_{3}$ & $\emptyset$ & 21 & 0 \\
\hline & $\tilde{A}_{2}$ & 0002 & 22 & 1 & $B_{3}$ & $\emptyset$ & 21 & 0 \\
\hline & $B_{2}$ & 2001 & 16 & 2 & $C_{3}$ & $C_{1}$ & 15 & 1 \\
\hline & $F_{4}\left(a_{3}\right)$ & 0200 & 12 & 1 & $A_{1} \tilde{A}_{2}$ & $\emptyset$ & 11 & 0 \\
\hline & $B_{3}$ & 2200 & 10 & 2 & $\tilde{A}_{2}$ & $\emptyset$ & 8 & 0 \\
\hline & $C_{3}$ & 1012 & 10 & 2 & $B_{3}$ & $B_{1} A_{1}$ & 9 & 1 \\
\hline & $F_{4}\left(a_{2}\right)$ & 0202 & 8 & 2 & $A_{1} \tilde{A}_{1}$ & $\emptyset$ & 6 & 0 \\
\hline & $F_{4}\left(a_{1}\right)$ & 2202 & 6 & 3 & $\tilde{A}_{1}$ & $\emptyset$ & 3 & 0 \\
\hline & $F_{4}$ & 2222 & 4 & 4 & 1 & $\emptyset$ & 0 & 0 \\
\hline \multirow[t]{12}{*}{$E_{6}$} & $A_{2}$ & $\begin{array}{l}00000 \\
2\end{array}$ & 36 & 1 & $A_{5}$ & $\emptyset$ & 35 & 0 \\
\hline & $A_{2}{ }^{2}$ & $\begin{array}{c}20002 \\
0\end{array}$ & 30 & 2 & $D_{4}$ & $\emptyset$ & 28 & 0 \\
\hline & $A_{3}$ & $\underset{2}{10001}$ & 26 & 2 & $A_{5}$ & $A_{1}$ & 25 & 1 \\
\hline & $D_{4}\left(a_{1}\right)$ & $\begin{array}{l}00200 \\
0\end{array}$ & 20 & 1 & $A_{2}^{2} A_{1}$ & $\emptyset$ & 19 & 0 \\
\hline & $A_{4}$ & 20002 & 18 & 3 & $A_{3}$ & $\emptyset$ & 15 & 0 \\
\hline & $D_{4}$ & 00200 & 18 & 2 & $A_{2}{ }^{2}$ & $\emptyset$ & 16 & 0 \\
\hline & $A_{5}$ & $\begin{array}{c}21012 \\
1\end{array}$ & 14 & 3 & $D_{4}$ & $A_{1} D_{2}$ & 12 & 1 \\
\hline & $D_{5}\left(a_{1}\right)$ & $\underset{2}{11011}$ & 14 & 3 & $A_{5}$ & $A_{2} A_{1}$ & 13 & 2 \\
\hline & $E_{6}\left(a_{3}\right)$ & $\begin{array}{c}20202 \\
0\end{array}$ & 12 & 3 & $A_{1}{ }^{3}$ & $\emptyset$ & 9 & 0 \\
\hline & $D_{5}$ & $\frac{20202}{2}$ & 10 & 4 & $A_{1}{ }^{2}$ & $\emptyset$ & 6 & 0 \\
\hline & $E_{6}\left(a_{1}\right)$ & $\begin{array}{c}22022 \\
2\end{array}$ & 8 & 5 & $A_{1}$ & $\emptyset$ & 3 & 0 \\
\hline & $E_{6}$ & $\underset{2}{22222}$ & 6 & 6 & 1 & $\emptyset$ & 0 & 0 \\
\hline
\end{tabular}


Table 6: 2-free cores for exceptional groups (continued)

\begin{tabular}{|c|c|c|c|c|c|c|c|c|}
\hline$G$ & $\operatorname{Ad}(G) e$ & $\Delta$ & $\operatorname{dim} C_{G}(e)$ & $\operatorname{dim} \mathcal{Z}$ & $G_{0}$ & $\operatorname{Ad}\left(G_{0}\right) e_{0}$ & $\operatorname{dim} C_{G_{0}}\left(e_{0}\right)$ & $\operatorname{dim} \mathcal{Z}_{0}$ \\
\hline$E_{7}$ & $\left(A_{1}^{3}\right)^{\prime \prime}$ & $\begin{array}{l}000002 \\
0\end{array}$ & 79 & 1 & $E_{6}$ & $\emptyset$ & 78 & 0 \\
\hline & $A_{2}$ & $\begin{array}{c}200000 \\
0\end{array}$ & 67 & 1 & $D_{6}$ & $\emptyset$ & 66 & 0 \\
\hline & $A_{3}$ & $\begin{array}{c}200010 \\
0\end{array}$ & 49 & 2 & $D_{6}$ & $A_{1}$ & 48 & 1 \\
\hline & $A_{2}{ }^{2}$ & $\begin{array}{l}000020 \\
0\end{array}$ & 49 & 1 & $D_{5} A_{1}$ & $\emptyset$ & 48 & 0 \\
\hline & $A_{2} A_{1}^{3}$ & $\begin{array}{c}000000 \\
2\end{array}$ & 49 & 1 & $A_{6}$ & $\emptyset$ & 48 & 0 \\
\hline & $\left(A_{3} A_{1}\right)^{\prime \prime}$ & 200002 & 47 & 2 & $D_{5}$ & $\emptyset$ & 45 & 0 \\
\hline & $D_{4}\left(a_{1}\right)$ & $\begin{array}{c}020000 \\
0\end{array}$ & 39 & 1 & $A_{5} A_{1}$ & $\emptyset$ & 38 & 0 \\
\hline & $D_{4}$ & $\begin{aligned} 220000 \\
0\end{aligned}$ & 37 & 2 & $A_{5}$ & $\emptyset$ & 35 & 0 \\
\hline & $A_{4}$ & $\begin{array}{c}200020 \\
0\end{array}$ & 33 & 2 & $D_{4} A_{1}$ & $\emptyset$ & 31 & 0 \\
\hline & $A_{3} A_{2} A_{1}$ & $\begin{array}{l}000200 \\
0\end{array}$ & 33 & 1 & $A_{4} A_{2}$ & $\emptyset$ & 32 & 0 \\
\hline & $\left(A_{5}\right)^{\prime \prime}$ & 200022 & 31 & 3 & $D_{4}$ & $\emptyset$ & 28 & 0 \\
\hline & $D_{4} A_{1}$ & $\begin{array}{c}210001 \\
1\end{array}$ & 31 & 2 & $D_{6}$ & $A_{1}^{2} D_{2}$ & 30 & 1 \\
\hline & $D_{5}\left(a_{1}\right)$ & $\begin{array}{c}201010 \\
0\end{array}$ & 27 & 2 & $D_{6}$ & $A_{2} A_{1}$ & 26 & 1 \\
\hline & $A_{4} A_{2}$ & $\begin{array}{c}002000 \\
0\end{array}$ & 27 & 1 & $A_{3} A_{2} A_{1}$ & $\emptyset$ & 26 & 0 \\
\hline & $\left(A_{5}\right)^{\prime}$ & $\begin{array}{c}101020 \\
0\end{array}$ & 25 & 2 & $D_{5} A_{1}$ & $A_{1} D_{2}$ & 24 & 1 \\
\hline & $A_{5} A_{1}$ & $\begin{array}{c}101012 \\
0\end{array}$ & 25 & 2 & $E_{6}$ & $A_{2}^{2} A_{1}$ & 24 & 1 \\
\hline & $D_{5}\left(a_{1}\right) A_{1}$ & $\begin{array}{c}200200 \\
0\end{array}$ & 25 & 2 & $A_{3} A_{2}$ & $\emptyset$ & 23 & 0 \\
\hline & $D_{6}\left(a_{2}\right)$ & $\begin{array}{c}010102 \\
1\end{array}$ & 23 & 3 & $E_{6}$ & $A_{3} A_{1}$ & 22 & 2 \\
\hline & $E_{6}\left(a_{3}\right)$ & $\begin{array}{l}020020 \\
0\end{array}$ & 23 & 2 & $A_{3} A_{1}^{2}$ & $\emptyset$ & 21 & 0 \\
\hline & $D_{5}$ & $\underset{0}{220020}$ & 21 & 3 & $A_{3} A_{1}$ & $\emptyset$ & 18 & 0 \\
\hline & $E_{7}\left(a_{5}\right)$ & $\begin{array}{l}002002 \\
0\end{array}$ & 21 & 2 & $A_{2}^{2} A_{1}$ & $\emptyset$ & 19 & 0 \\
\hline & $A_{6}$ & $\begin{array}{c}002020 \\
0\end{array}$ & 19 & 2 & $A_{2} A_{1}{ }^{3}$ & $\emptyset$ & 17 & 0 \\
\hline & $D_{5} A_{1}$ & $\begin{array}{c}210110 \\
1\end{array}$ & 19 & 3 & $D_{6}$ & $A_{3} D_{2}$ & 18 & 2 \\
\hline & $D_{6}\left(a_{1}\right)$ & $\begin{array}{c}210102 \\
1\end{array}$ & 19 & 4 & $D_{5}$ & $A_{2} A_{1}$ & 17 & 2 \\
\hline & $E_{7}\left(a_{4}\right)$ & $\begin{array}{c}202002 \\
0\end{array}$ & 17 & 3 & $A_{2} A_{1}^{2}$ & $\emptyset$ & 14 & 0 \\
\hline & $D_{6}$ & $\begin{array}{c}210122 \\
1\end{array}$ & 15 & 4 & $D_{4}$ & $A_{1} D_{2}$ & 12 & 1 \\
\hline & $E_{6}\left(a_{1}\right)$ & 202020 & 15 & 3 & $A_{1}^{4}$ & $\emptyset$ & 12 & 0 \\
\hline & $E_{6}$ & 222020 & 13 & 4 & $A_{1}{ }^{3}$ & $\emptyset$ & 9 & 0 \\
\hline & $E_{7}\left(a_{3}\right)$ & $\begin{array}{c}202022 \\
0\end{array}$ & 13 & 4 & $A_{1}{ }^{3}$ & $\emptyset$ & 9 & 0 \\
\hline & $E_{7}\left(a_{2}\right)$ & $\begin{array}{r}220202 \\
2\end{array}$ & 11 & 5 & $A_{1}{ }^{2}$ & $\emptyset$ & 6 & 0 \\
\hline & $E_{7}\left(a_{1}\right)$ & $\frac{220222}{2}$ & 9 & 6 & $A_{1}$ & $\emptyset$ & 3 & 0 \\
\hline & $E_{7}$ & $\frac{222222}{2}$ & 7 & 7 & 1 & $\emptyset$ & 0 & 0 \\
\hline
\end{tabular}


Table 6: 2-free cores for exceptional groups (continued)

\begin{tabular}{|c|c|c|c|c|c|c|c|c|}
\hline$C$ & $\operatorname{Ad}(G) e$ & $\Delta$ & $\operatorname{dim} C_{G}(e)$ & $\operatorname{dim} \mathcal{Z}$ & $G_{0}$ & $\operatorname{Ad}\left(G_{0}\right) e_{0}$ & $\operatorname{dim} C_{G_{0}}\left(e_{0}\right)$ & $\operatorname{dim} \mathcal{Z}_{0}$ \\
\hline \multirow[t]{42}{*}{$E_{8}$} & $A_{2}$ & 0000002 & 134 & 1 & $E_{7}$ & $\emptyset$ & 133 & 0 \\
\hline & $A_{3}$ & $\begin{array}{c}1000002 \\
0\end{array}$ & 100 & 2 & $E_{7}$ & $A_{1}$ & 99 & 1 \\
\hline & $A_{2}{ }^{2}$ & $\begin{array}{c}2000000 \\
0\end{array}$ & 92 & 1 & $D_{7}$ & $\emptyset$ & 91 & 0 \\
\hline & $D_{4}\left(a_{1}\right)$ & $\begin{array}{c}0000020 \\
0\end{array}$ & 82 & 1 & $E_{6} A_{1}$ & $\emptyset$ & 81 & 0 \\
\hline & $D_{4}$ & $\begin{array}{c}0000022 \\
0\end{array}$ & 80 & 2 & $E_{6}$ & $\emptyset$ & 78 & 0 \\
\hline & $A_{4}$ & $\underset{0}{2000002}$ & 68 & 2 & $D_{6}$ & $\emptyset$ & 66 & 0 \\
\hline & $D_{4} A_{1}$ & 0000012 & 64 & 2 & $E_{7}$ & $A_{1}^{4}$ & 63 & 1 \\
\hline & $D_{4}\left(a_{1}\right) A_{2}$ & $\underset{2}{0000000}$ & 64 & 1 & $A_{7}$ & $\emptyset$ & 63 & 0 \\
\hline & $D_{5}\left(a_{1}\right)$ & $\underset{0}{1000102}$ & 58 & 2 & $E_{7}$ & $A_{2} A_{1}$ & 57 & 1 \\
\hline & $A_{4} A_{2}$ & $\begin{array}{c}0000200 \\
0\end{array}$ & 54 & 1 & $D_{5} A_{2}$ & $\emptyset$ & 53 & 0 \\
\hline & $A_{5}$ & 2000101 & 52 & 2 & $D_{7}$ & $A_{1} D_{2}$ & 51 & 1 \\
\hline & $D_{5}\left(a_{1}\right) A_{1}$ & $\begin{array}{c}0010002 \\
0\end{array}$ & 52 & 2 & $E_{7}$ & $A_{2} A_{1}{ }^{2}$ & 51 & 1 \\
\hline & $D_{4} A_{2}$ & $\begin{array}{c}0000002 \\
2\end{array}$ & 50 & 2 & $A_{6}$ & $\emptyset$ & 48 & 0 \\
\hline & $E_{6}\left(a_{3}\right)$ & $\begin{array}{c}2000020 \\
0\end{array}$ & 50 & 2 & $D_{5} A_{1}$ & $\emptyset$ & 48 & 0 \\
\hline & $D_{5}$ & 2000022 & 48 & 3 & $D_{5}$ & $\emptyset$ & 45 & 0 \\
\hline & $D_{5} A_{1}$ & $\begin{array}{c}1001012 \\
0\end{array}$ & 40 & 3 & $E_{7}$ & $A_{3} A_{1}{ }^{2}$ & 39 & 2 \\
\hline & $E_{8}\left(a_{7}\right)$ & $\begin{array}{c}0002000 \\
0\end{array}$ & 40 & 1 & $A_{4} A_{3}$ & $\emptyset$ & 39 & 0 \\
\hline & $A_{6}$ & $\underset{0}{2000200}$ & 38 & 2 & $D_{4} A_{2}$ & $\emptyset$ & 36 & 0 \\
\hline & $D_{6}\left(a_{1}\right)$ & $\begin{array}{c}0100012 \\
1\end{array}$ & 38 & 3 & $E_{7}$ & $D_{4}\left(a_{1}\right) A_{1}$ & 37 & 2 \\
\hline & $E_{7}\left(a_{4}\right)$ & $\begin{array}{c}0010102 \\
0\end{array}$ & 36 & 3 & $E_{7}$ & $A_{3} A_{2}$ & 35 & 2 \\
\hline & $E_{6}\left(a_{1}\right)$ & $\begin{array}{c}2000202 \\
0\end{array}$ & 34 & 3 & $D_{4} A_{1}$ & $\emptyset$ & 31 & 0 \\
\hline & $D_{5} A_{2}$ & $\begin{array}{c}0002002 \\
0\end{array}$ & 34 & 2 & $A_{4} A_{2}$ & $\emptyset$ & 32 & 0 \\
\hline & $D_{6}$ & $\underset{1}{2100012}$ & 32 & 3 & $D_{6}$ & $A_{1}^{2} D_{2}$ & 30 & 1 \\
\hline & $E_{6}$ & $\begin{array}{c}2000222 \\
0\end{array}$ & 32 & 4 & $D_{4}$ & $\emptyset$ & 28 & 0 \\
\hline & $E_{6}\left(a_{1}\right) A_{1}$ & $\begin{array}{c}1010102 \\
0\end{array}$ & 30 & 2 & $E_{7}$ & $A_{4} A_{1}$ & 29 & 1 \\
\hline & $E_{7}\left(a_{3}\right)$ & $\begin{array}{c}2010102 \\
0\end{array}$ & 28 & 3 & $D_{6}$ & $A_{2} A_{1}$ & 26 & 1 \\
\hline & $E_{8}\left(b_{6}\right)$ & $\begin{array}{c}0020002 \\
0\end{array}$ & 28 & 2 & $A_{3} A_{2} A_{1}$ & $\emptyset$ & 26 & 0 \\
\hline & $D_{7}\left(a_{1}\right)$ & $\begin{array}{c}2002002 \\
0\end{array}$ & 26 & 3 & $A_{3} A_{2}$ & $\emptyset$ & 23 & 0 \\
\hline & $E_{6} A_{1}$ & $\begin{array}{c}1010122 \\
0\end{array}$ & 26 & 3 & $E_{6}$ & $A_{2}^{2} A_{1}$ & 24 & 1 \\
\hline & $E_{7}\left(a_{2}\right)$ & $\underset{1}{0101022}$ & 24 & 4 & $E_{6}$ & $A_{3} A_{1}$ & 22 & 2 \\
\hline & $E_{8}\left(a_{6}\right)$ & 0020020 & 24 & 2 & $A_{2}^{2} A_{1}^{2}$ & $\emptyset$ & 22 & 0 \\
\hline & $D_{7}$ & $\begin{array}{c}2101101 \\
1\end{array}$ & 22 & 3 & $D_{7}$ & $A_{3} D_{3}$ & 21 & 2 \\
\hline & $E_{8}\left(b_{5}\right)$ & 0020022 & 22 & 3 & $A_{2}^{2} A_{1}$ & $\emptyset$ & 19 & 0 \\
\hline & $E_{7}\left(a_{1}\right)$ & $\underset{1}{2101022}$ & 20 & 5 & $D_{5}$ & $A_{2} A_{1}$ & 17 & 2 \\
\hline & $E_{8}\left(a_{5}\right)$ & $\underset{0}{2020020}$ & 20 & 3 & $A_{2} A_{1}^{3}$ & $\emptyset$ & 17 & 0 \\
\hline & $E_{8}\left(b_{4}\right)$ & $\begin{array}{l}2020022 \\
0\end{array}$ & 18 & 4 & $A_{2} A_{1}^{2}$ & $\emptyset$ & 14 & 0 \\
\hline & $E_{7}$ & $\underset{1}{2101222}$ & 16 & 5 & $D_{4}$ & $A_{1} D_{2}$ & 12 & 1 \\
\hline & $E_{8}\left(a_{4}\right)$ & $\begin{array}{c}2020202 \\
0\end{array}$ & 16 & 4 & $A_{1}^{4}$ & $\emptyset$ & 12 & 0 \\
\hline & $E_{8}\left(a_{3}\right)$ & $\underset{0}{2020222}$ & 14 & 5 & $A_{1}{ }^{3}$ & $\emptyset$ & 9 & 0 \\
\hline & $E_{8}\left(a_{2}\right)$ & $\begin{array}{r}2202022 \\
2\end{array}$ & 12 & 6 & $A_{1}{ }^{2}$ & $\emptyset$ & 6 & 0 \\
\hline & $E_{8}\left(a_{1}\right)$ & $\underset{2}{2202222}$ & 10 & 7 & $A_{1}$ & $\emptyset$ & 3 & 0 \\
\hline & $E_{8}$ & $\underset{2}{2222222}$ & 8 & 8 & 1 & $\emptyset$ & 0 & 0 \\
\hline
\end{tabular}




\section{Detailed results}

This final section contains the detailed information for exceptional groups obtained as described in the preceding sections. We begin by explaining the manner in which this is presented.

We take the non-zero nilpotent orbits in the order of Table 2. Each such orbit has its own 'page' (which may or may not coincide with a physical page); horizontal lines are used to separate pages where necessary. The page may be regarded as falling into three sections.

The first of the three sections concerns the orbit itself. It begins with the algebraic group $G$, and the number and name of the orbit; it concludes with the chosen orbit representative $e$. In between we specify the Levi subgroup $L$ of $G$ with $e$ distinguished in $\mathfrak{L}(L)$, the cocharacter $\tau$ associated to $e$ with image in $[L, L]$, and the labelled diagram $\Delta$ corresponding to $e$. We represent both $L$ and $\tau$ diagrammatically: for the former, as explained in $\S 6$, we provide a Dynkin diagram of $G$ in which the nodes corresponding to the simple roots of $L$ are coloured black; for the latter, as explained in $\S 3$, we give the $\tau$-weights on simple roots, arranged to occupy the positions of the corresponding nodes in the Dynkin diagram (so that $\Delta$ is obtained by similarly representing the $W$-conjugate $\tau^{+}$of $\tau$; indeed if $L=G$ then $\tau^{+}=\tau$ and so the representation of $\tau$ is simply $\Delta$ ).

The second section concerns the reductive part $C$ of the centralizer $C_{G}(e)$; recall that $C=C_{G}(e) \cap C_{G}(\operatorname{im}(\tau))$ and that $Z(L)^{\circ}$ is a maximal torus of $C$. We first list the isomorphism types of both the connected component $C^{\circ}$ and the component group $C / C^{\circ}$; the former is given as in $\S 7.2$ as a product of simple factors $C_{1}, \ldots, C_{r}$, possibly with a central torus $T_{j}$ of dimension $j \in\{1,2\}$, while generators for the latter are named as in $\S 9$. We then specify both groups completely. For $C^{\circ}$, we let $\left\{\beta_{1}, \beta_{2}, \ldots\right\}$ be a set of simple roots of $C_{1} \ldots C_{r}$, numbered so that those in each simple factor are taken together and occur in the order of [4]. We give root subgroups corresponding to these simple roots and their negatives, using semi-colons to separate each simple factor from the others: for each $i$, if the $Z(L)^{\circ}$-root subgroup $X_{\beta_{i}}$ corresponding to $\beta_{i}$ is in fact a $T$-root subgroup $U_{\alpha}$, we merely write ' $\beta_{i}=\alpha$ '; if however $X_{\beta_{i}}$ is embedded in a product of two or more $T$-root subgroups, we give explicit expressions for the root elements $x_{\beta_{i}}(t)$ and $x_{-\beta_{i}}(t)$. If there is a 1- or 2-dimensional central torus, we give its elements as products of terms $h_{i}\left(\mu^{m_{i}}\right)$ or $h_{i}\left(\mu^{m_{i}} \nu^{n_{i}}\right)$, where we write $h_{i}$ for $h_{\alpha_{i}}$. For $C / C^{\circ}$ we give coset representatives for each of the generators as described in $\S 9$.

The third and final section concerns the Lie algebra of the unipotent radical $R$ of $C_{G}(e)$. Recall that $\mathfrak{L}(R)=C_{\mathfrak{L}(G)}(e)_{+}$, and we have a decomposition

$$
C_{\mathfrak{L}(G)}(e)_{+}=\bigoplus_{m>0} C_{\mathfrak{L}(G)}(e)_{m}
$$

into $\tau$-weight spaces. In $\S 7.1$ we obtained the upper central series

$$
0 \subset Z_{1}\left(C_{\mathfrak{L}(G)}(e)_{+}\right) \subset Z_{2}\left(C_{\mathfrak{L}(G)}(e)_{+}\right) \subset \cdots ;
$$

for each $n$ we wrote

$$
Z_{n}\left(C_{\mathfrak{L}(G)}(e)_{+}\right)=\bigoplus_{m>0} Z_{n, m},
$$

where we set $Z_{n, m}=\left(Z_{n}\left(C_{\mathfrak{L}(G)}(e)_{+}\right)\right)_{m}$. In $\S 8.2$ we then considered the action of $C^{\circ}$ on each $Z_{n, m}$; in each case we obtained a decomposition into a direct sum of indecomposable tilting modules for $D=\left[C^{\circ}, C^{\circ}\right]$, the vast majority of which were irreducible. We provide a table with six columns, usually having one row for each summand; the exceptions are 
the few cases treated separately in $\S 8.2$ involving non-restricted weights when $\operatorname{char}(k)=$ $p \in\{5,7\}$, where a footnote explains how the entries of two rows must be combined and modified if there is a reducible tilting module.

The first column of the table contains the least value of $n$ in which the summand lies. Summands are listed in decreasing order of $n$, and different values of $n$ are separated by horizontal lines; thus the table is divided into sections, of which the $n$th (reading upwards) lists the summands lying in $Z_{n}\left(C_{\mathfrak{L}(G)}(e)_{+}\right)$but not in $Z_{n-1}\left(C_{\mathfrak{L}(G)}(e)_{+}\right)$. The second column contains the $\tau$-weight $m$ of the summand. The third column contains the high weight $\lambda$ of the summand, regarded as a module for $D$; we write $\lambda_{i}$ for the fundamental dominant weight corresponding to the simple root $\beta_{i}$. If in fact $D=1$, we write a dash in place of $\lambda$. The fourth column contains the high weight vector $v$ of the summand. The final two columns are of relevance only to the bottom section of the table, which lists the summands lying in $Z_{1}\left(C_{\mathfrak{L}(G)}(e)_{+}\right)=Z\left(C_{\mathfrak{L}(G)}(e)_{+}\right)$. The fifth column indicates the summands lying in $\mathcal{Z}^{\natural}=\left(Z\left(C_{\mathfrak{L}(G)}(e)_{+}\right)\right)^{C^{\circ}}$, found by taking those contained in $Z\left(C_{\mathfrak{L}(G)}(e)_{+}\right)$which lie in $\mathfrak{L}(L)$ and are trivial $D$-submodules; as explained in $\S 9$, for convenience of reference each such high weight vector $v$ is renamed $z_{m}$, with the subscript being the $\tau$-weight, and vectors with the same value of $m$ being distinguished by superscripts. The sixth column indicates the summands lying in $\mathcal{Z}=\left(Z\left(C_{\mathfrak{L}(G)}(e)_{+}\right)\right)^{C}$, found by taking the vectors in $\mathcal{Z}^{\text {h }}$ fixed by the representatives of the generators of the component group $C / C^{\circ}$; by Theorem 3.9 we have $\mathfrak{L}\left(Z\left(C_{G}(e)\right)\right)=\mathcal{Z}$.

$G_{2}$, orbit 1: $A_{1}$
$L: \quad<$
$\tau:-12$
$\Delta=0 \quad 1$
$e=e_{01}$
$C^{\circ}=\tilde{A}_{1} \quad C / C^{\circ}=1$
$\beta_{1}=21$

\begin{tabular}{|c|c|c|c|c|c|}
\hline$n$ & $m$ & $\lambda$ & $v$ & $\mathcal{Z}^{\natural}$ & $\mathcal{Z}$ \\
\hline \hline 2 & 1 & $3 \lambda_{1}$ & $e_{32}$ & & \\
\hline 1 & 2 & 0 & $e$ & $z_{2}$ & $z_{2}$ \\
\hline
\end{tabular}


$G_{2}$, orbit $2: \tilde{A}_{1}$

$$
\begin{array}{lllllll}
L: \quad & \tau:{ }^{2} & -3 & \Delta=1 & 0 \\
e=e_{10} & & & & & \\
C^{\circ}=A_{1} & C / C^{\circ}=1 & & & \\
\beta_{1}=32 & & & &
\end{array}
$$

\begin{tabular}{|c|c|c|c|c|c|}
\hline$n$ & $m$ & $\lambda$ & $v$ & $\mathcal{Z}^{\natural}$ & $\mathcal{Z}$ \\
\hline \hline 1 & 2 & 0 & $e$ & $z_{2}$ & $z_{2}$ \\
& 3 & $\lambda_{1}$ & $e_{31}$ & & \\
\hline
\end{tabular}

$G_{2}$, orbit 3: $G_{2}\left(a_{1}\right)$

$$
\begin{aligned}
& L: \ll \\
& \tau: 0 \quad 0 \quad 2 \\
& \Delta=0 \quad 2 \\
& e=e_{01}+e_{31} \\
& C^{\circ}=1 \quad C / C^{\circ}=\left\langle c_{1} C^{\circ}, c_{2} C^{\circ}\right\rangle \cong S_{3} \\
& c_{1}=h_{1}(\omega) \text {, } \\
& c_{2}=n_{10} h_{2}(-1)
\end{aligned}
$$

\begin{tabular}{|c|c|c|c|c|c|}
\hline$n$ & $m$ & $\lambda$ & $v$ & $\mathcal{Z}^{\natural}$ & $\mathcal{Z}$ \\
\hline 2 & 2 & - & $e_{11}$ & & \\
& 2 & - & $e_{21}$ & & \\
\hline 1 & 2 & - & $e$ & $z_{2}$ & $z_{2}$ \\
& 4 & - & $e_{32}$ & $z_{4}$ & \\
\hline
\end{tabular}

$G_{2}$, orbit $4: G_{2}$
$L: \longleftrightarrow$
$\tau: 2 \quad 2$
$\Delta=22$
$e=e_{10}+e_{01}$
$C^{\circ}=1 \quad C / C^{\circ}=1$

\begin{tabular}{|c|c|c|c|c|c|}
\hline$n$ & $m$ & $\lambda$ & $v$ & $\mathcal{Z}^{\natural}$ & $\mathcal{Z}$ \\
\hline \hline 1 & 2 & - & $e$ & $z_{2}$ & $z_{2}$ \\
& 10 & - & $e_{32}$ & $z_{10}$ & $z_{10}$ \\
\hline
\end{tabular}


$F_{4}$, orbit 1: $A_{1}$

$$
\begin{aligned}
& L: \quad \begin{array}{llllll}
L & : & 2 & -1 & 0 & 0
\end{array} \quad \Delta=\begin{array}{rrrr}
1 & 0 & 0 & 0
\end{array} \\
& e=e_{1000} \\
& C^{\circ}=C_{3} \quad C / C^{\circ}=1 \\
& \beta_{1}=0010, \beta_{2}=0001, \beta_{3}=1220
\end{aligned}
$$

\begin{tabular}{|c|c|c|c|c|c|}
\hline$n$ & $m$ & $\lambda$ & $v$ & $\mathcal{Z}^{\natural}$ & $\mathcal{Z}$ \\
\hline \hline 2 & 1 & $\lambda_{3}$ & $e_{2342}$ & & \\
\hline 1 & 2 & 0 & $e$ & $z_{2}$ & $z_{2}$ \\
\hline
\end{tabular}

$F_{4}$, orbit $2: \tilde{A}_{1}$

$$
\begin{aligned}
& L: 0-0
\end{aligned}
$$

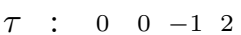

$$
\begin{aligned}
& \Delta=\begin{array}{llll}
0 & 0 & 0 & 1
\end{array}
\end{aligned}
$$

\begin{tabular}{|c|c|c|c|c|c|}
\hline$n$ & $m$ & $\lambda$ & $v$ & $\mathcal{Z}^{\natural}$ & $\mathcal{Z}$ \\
\hline 2 & 1 & $\lambda_{1}$ & $e_{1111}$ & & \\
& 1 & $\lambda_{3}$ & $e_{1232}$ & & \\
\hline 1 & 2 & $\lambda_{2}$ & $e_{1222}$ & & \\
& 2 & 0 & $e$ & $z_{2}$ & $z_{2}$ \\
\hline
\end{tabular}

$F_{4}$, orbit $3: A_{1} \tilde{A}_{1}$

$$
\begin{aligned}
& L: \bullet \gg 0 \text {. } \\
& \tau: \quad 2-1-1 \quad 2 \\
& \Delta=\begin{array}{llll}
0 & 1 & 0 & 0
\end{array} \\
& e=e_{1000}+e_{0001} \\
& C^{\circ}=A_{1}{ }^{2} \quad C / C^{\circ}=1 \\
& \beta_{1}=1242 ; x_{\beta_{2}}(t)=x_{1110}(t) x_{0111}(t) x_{1221}\left(\frac{1}{2} t^{2}\right) \text {, } \\
& x_{-\beta_{2}}(t)=x_{-1110}(2 t) x_{-0111}(2 t) x_{-1221}\left(-2 t^{2}\right)
\end{aligned}
$$

\begin{tabular}{|c|c|c|c|c|c|}
\hline$n$ & $m$ & $\lambda$ & $v$ & $\mathcal{Z}^{\natural}$ & $\mathcal{Z}$ \\
\hline \hline 3 & 1 & $\lambda_{1}+4 \lambda_{2}$ & $e_{2342}$ & & \\
\hline 2 & 2 & $4 \lambda_{2}$ & $e_{1222}$ & & \\
\hline 1 & 2 & 0 & $e$ & $z_{2}$ & $z_{2}$ \\
& 3 & $\lambda_{1}$ & $e_{1122}$ & & \\
\hline
\end{tabular}


$F_{4}$, orbit 4: $A_{2}$

$$
\begin{aligned}
& L: \bullet \quad \begin{array}{lllllllllll}
\tau & : & 2 & 2 & -2 & 0 & \Delta= & 2 & 0 & 0 & 0
\end{array} \\
& e=e_{1000}+e_{0100} \\
& C^{\circ}=\tilde{A}_{2} \quad C / C^{\circ}=\left\langle c C^{\circ}\right\rangle \cong S_{2} \\
& \beta_{1}=0001, \beta_{2}=1231 \\
& c=n_{0110} n_{1120} h_{1}(-1)
\end{aligned}
$$

\begin{tabular}{|c|c|c|c|c|c|}
\hline$n$ & $m$ & $\lambda$ & $v$ & $\mathcal{Z}^{\natural}$ & $\mathcal{Z}$ \\
\hline \hline 2 & 2 & $2 \lambda_{1}$ & $e_{1222}$ & & \\
& 2 & $2 \lambda_{2}$ & $e_{2342}$ & & \\
\hline 1 & 2 & 0 & $e$ & $z_{2}$ & $z_{2}$ \\
& 4 & 0 & $e_{1100}$ & $z_{4}$ & \\
\hline
\end{tabular}

$F_{4}$, orbit 5: $\tilde{A}_{2}$

$$
\begin{aligned}
& L: \quad 0 \quad 20
\end{aligned}
$$

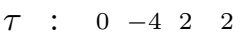

$$
\begin{aligned}
& \Delta=\begin{array}{llll}
0 & 0 & 0 & 2
\end{array} \\
& e=e_{0010}+e_{0001} \\
& C^{\circ}=G_{2} \quad C / C^{\circ}=1 \\
& x_{ \pm \beta_{1}}(t)=x_{ \pm 0111}(t) x_{ \pm 0120}(-t), \beta_{2}=1000
\end{aligned}
$$

\begin{tabular}{|c|c|c|c|c|c|}
\hline$n$ & $m$ & $\lambda$ & $v$ & $\mathcal{Z}^{\natural}$ & $\mathcal{Z}$ \\
\hline \hline 1 & 2 & 0 & $e$ & $z_{2}$ & $z_{2}$ \\
& 4 & $\lambda_{1}$ & $e_{1242}$ & & \\
\hline
\end{tabular}

$F_{4}$, orbit $6: A_{2} \tilde{A}_{1}$

$$
\begin{aligned}
& L: \bullet \bullet 0 \\
& \begin{array}{llllll}
\tau & : & 2 & 2 & -3 & 2
\end{array} \\
& \Delta=\begin{array}{llll}
0 & 0 & 1 & 0
\end{array} \\
& e=e_{1000}+e_{0100}+e_{0001} \\
& C^{\circ}=A_{1} \quad C / C^{\circ}=1 \\
& x_{\beta_{1}}=x_{0122}(2 t) x_{1220}(t) x_{1342}\left(-t^{2}\right) x_{1121}(-t) \text {, } \\
& x_{-\beta_{1}}=x_{-0122}(t) x_{-1220}(2 t) x_{-1342}\left(t^{2}\right) x_{-1121}(-t) \\
& \begin{array}{|c|c|c|c|c|c|}
\hline n & m & \lambda & v & \mathcal{Z}^{\natural} & \mathcal{Z} \\
\hline \hline 4 & 1 & 3 \lambda_{1} & e_{1232} & & \\
\hline 3 & 2 & 4 \lambda_{1} & e_{2342} & & \\
\hline 2 & 3 & \lambda_{1} & e_{1111} & & \\
\hline 1 & 2 & 0 & e & z_{2} & z_{2} \\
& 4 & 2 \lambda_{1} & e_{1222} & & \\
\hline
\end{array}
\end{aligned}
$$


$F_{4}$, orbit $7: B_{2}$

$$
\begin{aligned}
& L: \quad \begin{array}{llllllllll}
L & -4 & 2 & 2 & -3
\end{array} \quad \Delta=\begin{array}{llllll}
2 & 0 & 0 & 1
\end{array} \\
& e=e_{0100}+e_{0010} \\
& C^{\circ}=A_{1}{ }^{2} \quad C / C^{\circ}=\left\langle c C^{\circ}\right\rangle \cong S_{2} \\
& \beta_{1}=0122 ; \beta_{2}=2342 \\
& c=n_{1110} h_{4}(-1)
\end{aligned}
$$

\begin{tabular}{|c|c|c|c|c|c|}
\hline$n$ & $m$ & $\lambda$ & $v$ & $\mathcal{Z}^{\natural}$ & $\mathcal{Z}$ \\
\hline \hline 2 & 3 & $\lambda_{2}$ & $e_{1231}$ & & \\
& 3 & $\lambda_{1}$ & $e_{0121}$ & & \\
\hline 1 & 2 & 0 & $e$ & $z_{2}$ & $z_{2}$ \\
& 4 & $\lambda_{1}+\lambda_{2}$ & $e_{1342}$ & & \\
& 6 & 0 & $e_{0120}$ & $z_{6}$ & $z_{6}$ \\
\hline
\end{tabular}

$F_{4}$, orbit 8: $\tilde{A}_{2} A_{1}$

$$
\begin{aligned}
& L: \bullet-\infty>\bullet \\
& \tau \quad: \quad 2 \quad \begin{array}{llll} 
& 2 & -5 & 2
\end{array} \\
& \Delta=\begin{array}{llll}
0 & 1 & 0 & 1
\end{array} \\
& e=e_{0010}+e_{0001}+e_{1000} \\
& C^{\circ}=A_{1} \quad C / C^{\circ}=1 \\
& x_{ \pm \beta_{1}}(t)=x_{ \pm 1222}(t) x_{ \pm 1231}(-t)
\end{aligned}
$$

\begin{tabular}{|c|c|c|c|c|c|}
\hline$n$ & $m$ & $\lambda$ & $v$ & $\mathcal{Z}^{\natural}$ & $\mathcal{Z}$ \\
\hline \hline 3 & 1 & $3 \lambda_{1}$ & $e_{2342}$ & & \\
& 3 & $\lambda_{1}$ & $e_{1121}-2 e_{0122}$ & & \\
\hline 2 & 2 & 0 & $e_{1000}$ & & \\
& 4 & $2 \lambda_{1}$ & $e_{1242}$ & & \\
\hline 1 & 2 & 0 & $e$ & $z_{2}$ & $z_{2}$ \\
& 5 & $\lambda_{1}$ & $e_{1122}$ & & \\
\hline
\end{tabular}


$F_{4}$, orbit 9: $C_{3}\left(a_{1}\right)$

$$
\begin{aligned}
& L: \quad \begin{array}{lllllll}
L & :-5 & 2 & 0 & 2
\end{array} \quad \Delta=\begin{array}{llll}
1 & 0 & 1 & 0 \\
e=e_{0001}+e_{0120}+e_{0100} &
\end{array} \\
& C^{\circ}=A_{1} \quad C / C^{\circ}=\left\langle c C^{\circ}\right\rangle \cong S_{2} \\
& \beta_{1}=2342 \\
& c=h_{4}(-1)
\end{aligned}
$$

\begin{tabular}{|c|c|c|c|c|c|}
\hline$n$ & $m$ & $\lambda$ & $v$ & $\mathcal{Z}^{\natural}$ & $\mathcal{Z}$ \\
\hline \hline 3 & 2 & 0 & $e_{0110}+e_{0011}$ & & \\
& 2 & 0 & $e_{0100}$ & & \\
\hline 2 & 3 & $\lambda_{1}$ & $e_{1242}-e_{1222}$ & & \\
& 3 & $\lambda_{1}$ & $e_{1232}$ & & \\
& 4 & 0 & $e_{0111}$ & & \\
\hline 1 & 2 & 0 & $e$ & $z_{2}$ & $z_{2}$ \\
& 5 & $\lambda_{1}$ & $e_{1342}$ & & \\
& 6 & 0 & $e_{0122}$ & $z_{6}$ & $z_{6}$ \\
\hline
\end{tabular}

$F_{4}$, orbit 10: $F_{4}\left(a_{3}\right)$

$$
\begin{aligned}
& L: \bullet \bullet \begin{array}{cllllllllllll}
\tau & 0 & 0 & 2 & 0 & 0 & \Delta= & 2 & 0 & 0
\end{array} \\
& e=e_{0100}+e_{1120}+e_{1111}+e_{0121} \\
& C^{\circ}=1 \quad C / C^{\circ}=\left\langle c_{1} C^{\circ}, c_{2} C^{\circ}, c_{3} C^{\circ}\right\rangle \cong S_{4} \\
& c_{1}=h_{1}(\omega) h_{3}(\omega) \text {, } \\
& c_{2}=n_{1000} n_{0010} h_{2}(-1) h_{3}(-1) \text {, } \\
& c_{3}=\left(n_{0011} h_{3}\left(-\frac{2}{3}\right) h_{4}\left(\frac{2}{3}\right)\right)^{u} \text {, } \\
& u=x_{0011}\left(-\frac{1}{2}\right) x_{0001}(1) x_{0010}(-1)
\end{aligned}
$$

\begin{tabular}{|c|c|c|c|c|c|}
\hline$n$ & $m$ & $\lambda$ & $v$ & $\mathcal{Z}^{\natural}$ & $\mathcal{Z}$ \\
\hline \hline 3 & 2 & - & $e_{0110}+e_{1121}-2 e_{0122}$ & & \\
& 2 & - & $e_{1110}+e_{0111}-2 e_{1122}$ & & \\
& 2 & - & $e_{1100}+e_{0110}$ & & \\
& 2 & - & $e_{0120}+e_{1110}$ & & \\
& 2 & - & $e_{0100}+e_{1120}$ & & \\
\hline 2 & 4 & - & $e_{1221}+2 e_{1242}$ & & \\
& 4 & - & $e_{1231}-2 e_{1222}$ & & \\
& 4 & - & $e_{1220}$ & & \\
& 4 & - & $e_{1232}$ & & \\
\hline 1 & 2 & - & $e$ & $z_{2}$ & $z_{2}$ \\
& 6 & - & $e_{1342}$ & $z_{6}{ }^{1}$ & \\
& 6 & - & $e_{2342}$ & $z_{6}{ }^{2}$ & \\
\hline
\end{tabular}


$F_{4}$, orbit 11: $B_{3}$

$$
\begin{aligned}
& L: \bullet \longrightarrow \\
& \begin{array}{llllll}
\tau & : & 2 & 2 & 2 & -6
\end{array} \\
& \Delta=\begin{array}{llll}
2 & 2 & 0 & 0
\end{array} \\
& e=e_{1000}+e_{0100}+e_{0010} \\
& C^{\circ}=A_{1} \quad C / C^{\circ}=1 \\
& x_{\beta_{1}}=x_{1111}(t) x_{0121}(-t) x_{1232}\left(-\frac{1}{2} t^{2}\right), x_{-\beta_{1}}=x_{-1111}(2 t) x_{-0121}(-2 t) x_{-1232}\left(2 t^{2}\right) \\
& \begin{array}{|c|c|c|c|c|c|}
\hline n & m & \lambda & v & \mathcal{Z}^{\natural} & \mathcal{Z} \\
\hline \hline 1 & 2 & 0 & e & z_{2} & z_{2} \\
& 6 & 4 \lambda_{1} & e_{2342} & & \\
& 10 & 0 & e_{1220} & z_{10} & z_{10} \\
\hline
\end{array}
\end{aligned}
$$

$F_{4}$, orbit $12: C_{3}$

$$
\begin{aligned}
& L: \quad 0 \longrightarrow \bullet \\
& e=e_{0001}+e_{0010}+e_{0100} \\
& C^{\circ}=A_{1} \quad C / C^{\circ}=1 \\
& \beta_{1}=2342
\end{aligned}
$$

\begin{tabular}{|c|c|c|c|c|c|}
\hline$n$ & $m$ & $\lambda$ & $v$ & $\mathcal{Z}^{\natural}$ & $\mathcal{Z}$ \\
\hline \hline 3 & 3 & $\lambda_{1}$ & $e_{1231}-e_{1222}$ & & \\
\hline 2 & 6 & 0 & $e_{0120}-e_{0111}$ & & \\
\hline 1 & 2 & 0 & $e$ & $z_{2}$ & $z_{2}$ \\
& 9 & $\lambda_{1}$ & $e_{1342}$ & & \\
& 10 & 0 & $e_{0122}$ & $z_{10}$ & $z_{10}$ \\
\hline
\end{tabular}

$F_{4}$, orbit 13: $F_{4}\left(a_{2}\right)$

$$
\begin{aligned}
& L: \bullet \bullet \bullet \begin{array}{rlllllllllll}
L & : & 0 & 2 & 0 & 2 & \Delta= & 0 & 0 & 2
\end{array} \\
& e=e_{1110}+e_{0001}+e_{0120}+e_{0100} \\
& C^{\circ}=1 \quad C / C^{\circ}=\left\langle c C^{\circ}\right\rangle \cong S_{2} \\
& c=h_{2}(-1)
\end{aligned}
$$

\begin{tabular}{|c|c|c|c|c|c|}
\hline$n$ & $m$ & $\lambda$ & $v$ & $\mathcal{Z}^{\natural}$ & $\mathcal{Z}$ \\
\hline \hline 5 & 2 & - & $e_{0110}+e_{0011}+e_{1120}-3 e_{1100}$ & & \\
& 2 & - & $e_{0100}$ & & \\
\hline 4 & 4 & - & $e_{0111}+e_{1220}$ & & \\
\hline 3 & 6 & - & $e_{0122}-e_{1231}$ & & \\
\hline 2 & 8 & - & $e_{1222}-e_{1242}$ & & \\
\hline 1 & 2 & - & $e$ & $z_{2}$ & $z_{2}$ \\
& 10 & - & $e_{1342}$ & $z_{10}$ & \\
& 10 & - & $e_{2342}$ & $z_{10}{ }^{2}$ & $z_{10}{ }^{2}$ \\
\hline
\end{tabular}


$F_{4}$, orbit 14: $F_{4}\left(a_{1}\right)$

$$
\begin{aligned}
& L: \bullet \longrightarrow \\
& \tau: \begin{array}{lllll}
\tau & 2 & 2 & 0 & 2
\end{array} \\
& \Delta=\begin{array}{llll}
2 & 2 & 0 & 2
\end{array}
\end{aligned}
$$

\begin{tabular}{|c|c|c|c|c|c|}
\hline$n$ & $m$ & $\lambda$ & $v$ & $\mathcal{Z}^{\natural}$ & $\mathcal{Z}$ \\
\hline \hline 3 & 4 & - & $e_{1110}+e_{0111}$ & & \\
& 6 & - & $e_{1220}-e_{1121}+2 e_{0122}$ & & \\
\hline 2 & 10 & - & $e_{1232}$ & & \\
\hline 1 & 2 & - & $e$ & $z_{2}$ & $z_{2}$ \\
& 10 & - & $e_{1222}-e_{1242}$ & $z_{10}$ & $z_{10}$ \\
& 14 & - & $e_{2342}$ & $z_{14}$ & $z_{14}$ \\
\hline
\end{tabular}

$F_{4}$, orbit $15: F_{4}$

$$
\begin{aligned}
& L: \bullet \longrightarrow \bullet \begin{array}{rllllll}
\tau & : & 2 & 2 & 2 & 2
\end{array} \quad \Delta=\begin{array}{llll}
2 & 2 & 2 & 2
\end{array} \\
& e=e_{1000}+e_{0100}+e_{0010}+e_{0001} \\
& C^{\circ}=1 \quad C / C^{\circ}=1
\end{aligned}
$$

\begin{tabular}{|c|c|c|c|c|c|}
\hline$n$ & $m$ & $\lambda$ & $v$ & $\mathcal{Z}^{\natural}$ & $\mathcal{Z}$ \\
\hline \hline 1 & 2 & - & $e$ & $z_{2}$ & $z_{2}$ \\
& 10 & - & $e_{1220}-e_{1121}+2 e_{0122}$ & $z_{10}$ & $z_{10}$ \\
& 14 & - & $e_{1231}-e_{1222}$ & $z_{14}$ & $z_{14}$ \\
& 22 & - & $e_{2342}$ & $z_{22}$ & $z_{22}$ \\
\hline
\end{tabular}


$E_{6}$, orbit 1: $A_{1}$

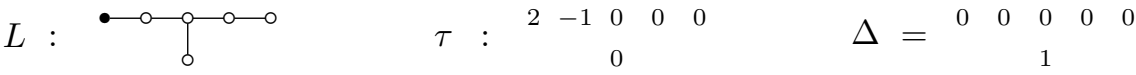

$$
\begin{aligned}
& e=\underset{0}{10000} \\
& C^{\circ}=A_{5} \quad C / C^{\circ}=1 \\
& \beta_{1}=\stackrel{00000}{1}, \beta_{2}=\underset{0}{00100}, \beta_{3}=\underset{0}{00010}, \beta_{4}=\underset{0}{00001}, \beta_{5}=\underset{1}{12210}
\end{aligned}
$$

\begin{tabular}{|c|c|c|c|c|c|}
\hline$n$ & $m$ & $\lambda$ & $v$ & $\mathcal{Z}^{\natural}$ & $\mathcal{Z}$ \\
\hline \hline 2 & 1 & $\lambda_{3}$ & $e_{11221}$ & & \\
\hline 1 & 2 & 0 & $e$ & $z_{2}$ & $z_{2}$ \\
\hline
\end{tabular}

$E_{6}$, orbit 2: $A_{1}^{2}$

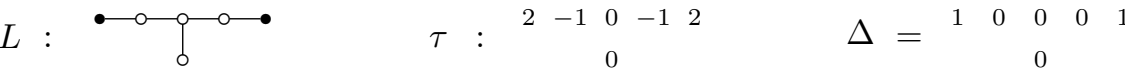

$$
\begin{aligned}
& e=e_{10000}+e_{00001} \\
& C^{\circ}=B_{3} T_{1} \quad C / C^{\circ}=1 \\
& \beta_{1}=\stackrel{00100}{0}, \beta_{2}=\stackrel{00000}{1}, x_{ \pm \beta_{3}}(t)=x_{ \pm} \underset{0}{11110}(t) x_{ \pm}^{01111}(t) \\
& T_{1}=\left\{h_{1}(\mu) h_{3}\left(\mu^{2}\right) h_{5}\left(\mu^{-2}\right) h_{6}\left(\mu^{-1}\right): \mu \in k^{*}\right\}
\end{aligned}
$$

\begin{tabular}{|c|c|c|c|c|c|}
\hline$n$ & $m$ & $\lambda$ & $v$ & $\mathcal{Z}^{\natural}$ & $\mathcal{Z}$ \\
\hline \hline 2 & 1 & $\lambda_{3}$ & $e_{12211}$ & & \\
& & & 1 & & \\
& 1 & $\lambda_{3}$ & $e_{11221}$ & & \\
\hline 1 & 2 & $\lambda_{1}$ & $e_{11211}$ & & \\
& & & 1 & & \\
& 2 & 0 & $e$ & $z_{2}$ & $z_{2}$ \\
\hline
\end{tabular}

$E_{6}$, orbit $3: A_{1}{ }^{3}$

$$
\begin{aligned}
& \begin{array}{lc}
L: & \bullet \\
e=e_{10000}+e_{00100}
\end{array} \\
& \tau: \begin{array}{ccccccccc}
2 & -2 & 2 & -2 & 2
\end{array} \quad \Delta=\begin{array}{lllll}
0 & 0 & 1 & 0 & 0
\end{array} \\
& e=e_{0} \underset{000}{000100}+e_{0001}^{0000} \\
& C^{\circ}=A_{2} A_{1} \quad C / C^{\circ}=1
\end{aligned}
$$

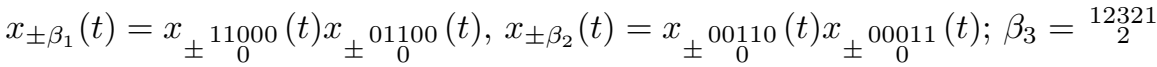

\begin{tabular}{|c|c|c|c|c|c|}
\hline$n$ & $m$ & $\lambda$ & $v$ & $\mathcal{Z}^{\natural}$ & $\mathcal{Z}$ \\
\hline \hline 3 & 1 & $\lambda_{1}+\lambda_{2}+\lambda_{3}$ & $e_{12321}$ & & \\
\hline 2 & 2 & $\lambda_{1}+\lambda_{2}$ & $\underset{1}{e_{11111}}$ & & \\
\hline 1 & 2 & 0 & $e$ & $z_{2}$ & $z_{2}$ \\
& 3 & $\lambda_{3}$ & $e_{11211}$ & & \\
\hline
\end{tabular}


$E_{6}$, orbit 4: $A_{2}$

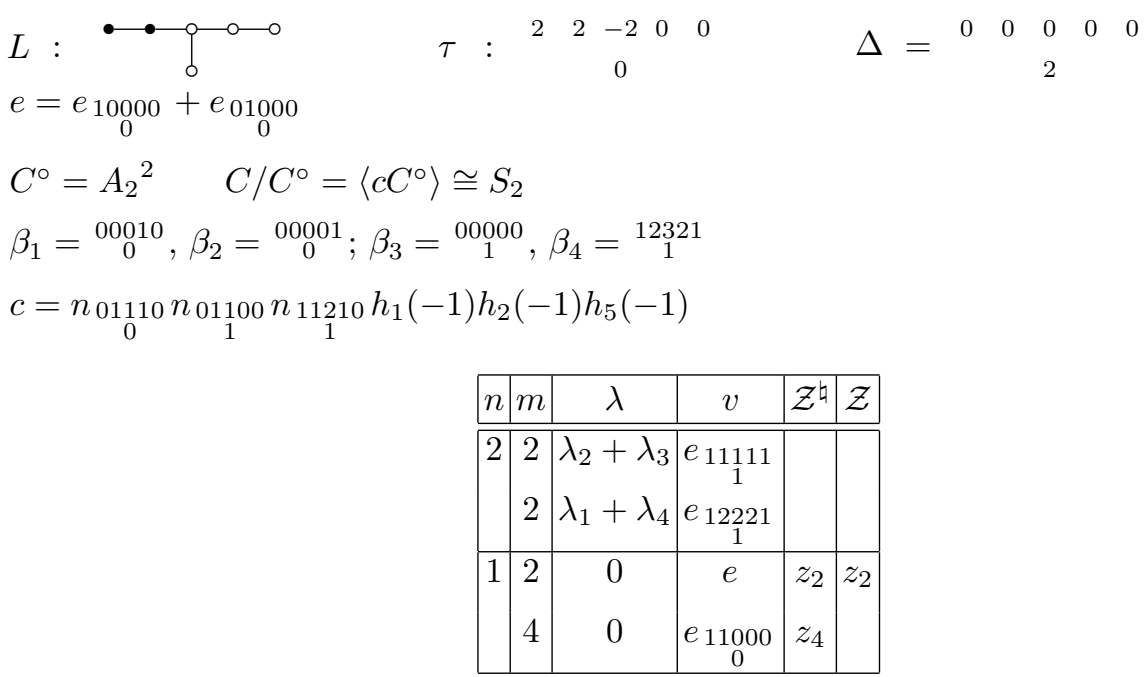

$E_{6}$, orbit 5: $A_{2} A_{1}$

$$
\begin{aligned}
& L: \bullet \begin{array}{c}
\tau \\
L
\end{array} \\
& e=e_{10000}+e_{01000}+e_{00000} \\
& C^{\circ}=A_{2} T_{1} \quad C / C^{\circ}=1 \\
& \beta_{1}=\underset{0}{00010}, \beta_{2}=\underset{0}{00001} \\
& T_{1}=\left\{h_{1}\left(\mu^{2}\right) h_{2}\left(\mu^{3}\right) h_{3}\left(\mu^{4}\right) h_{4}\left(\mu^{6}\right) h_{5}\left(\mu^{4}\right) h_{6}\left(\mu^{2}\right): \mu \in k^{*}\right\}
\end{aligned}
$$

\begin{tabular}{|c|c|c|c|c|c|}
\hline$n$ & $m$ & $\lambda$ & $v$ & $\mathcal{Z}^{\natural}$ & $\mathcal{Z}$ \\
\hline \hline 4 & 1 & $\lambda_{1}$ & $f_{01100}-f_{00100}$ & & \\
& 1 & $\lambda_{2}$ & $e_{11111}-e_{01111}$ & & \\
& & & 0 & 1 \\
12321 & & \\
& 0 & $f_{12}$ & & \\
& 1 & 0 & $e_{12321}$ & & \\
\hline 3 & 2 & $\lambda_{2}$ & $f_{01210}$ & & \\
& 2 & $\lambda_{1}$ & $e_{12221}$ & & \\
& 2 & 0 & $e_{00000}$ & & \\
\hline 2 & 3 & $\lambda_{1}$ & $f_{00100}$ & & \\
& 3 & $\lambda_{2}$ & $e_{11111}$ & & \\
\hline 1 & 2 & 0 & & & \\
& 4 & 0 & $e_{11000}$ & $z_{4}$ & $z_{4}$ \\
\hline
\end{tabular}


$E_{6}$, orbit $6: A_{2}{ }^{2}$

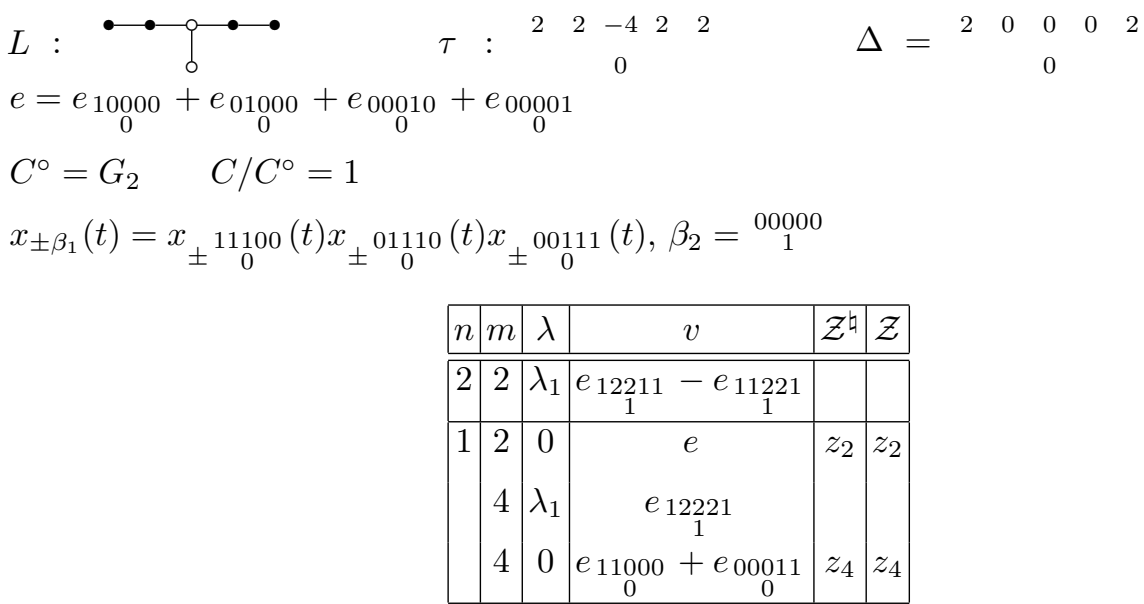

$E_{6}$, orbit 7: $A_{2} A_{1}{ }^{2}$

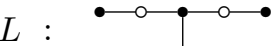

$$
\begin{aligned}
& \tau \quad: \begin{array}{lllll}
2 & -3 & 2 & -3 & 2
\end{array} \\
& \Delta=\begin{array}{lllll}
0 & 1 & 0 & 1 & 0
\end{array} \\
& e=e_{00000}+e_{00100}+e_{10000}+e_{00001} \\
& C^{\circ}=A_{1} T_{1} \quad C / C^{\circ}=1 \\
& x_{\beta_{1}}(t)=x_{11111}(2 t) x_{01210}(-t) x_{12321}\left(-t^{2}\right) x_{11110}(t) x_{01111}(-t) \text {, } \\
& x_{-\beta_{1}}(t)=x_{-}^{11111}(t) x_{-} 01210(-2 t) x_{-}{ }_{1}^{12321}\left(t^{2}\right) x_{-}{ }_{1}^{11110}(t) x_{-} 01111(-t) \\
& T_{1}=\left\{h_{1}(\mu) h_{3}\left(\mu^{2}\right) h_{5}\left(\mu^{-2}\right) h_{6}\left(\mu^{-1}\right): \mu \in k^{*}\right\}
\end{aligned}
$$

\begin{tabular}{|c|c|c|c|c|c|}
\hline$n$ & $m$ & $\lambda$ & $v$ & $\mathcal{Z}^{\natural}$ & $\mathcal{Z}$ \\
\hline 4 & 1 & $\begin{array}{l}3 \lambda_{1} \\
3 \lambda_{1}\end{array}$ & $\begin{array}{l}e_{12211} \\
1 \\
e_{11221}\end{array}$ & & \\
\hline 3 & 2 & $\begin{array}{l}4 \lambda_{1} \\
2 \lambda_{1}\end{array}$ & $\begin{array}{c}e_{12321} \\
2 \\
e_{01211}+e_{11210}\end{array}$ & & \\
\hline 2 & 3 & $\begin{array}{l}\lambda_{1} \\
\lambda_{1}\end{array}$ & $\begin{array}{l}e_{11100} \\
e_{1}^{00111} \\
1\end{array}$ & & \\
\hline 1 & 4 & $\begin{array}{c}0 \\
2 \lambda_{1}\end{array}$ & $\begin{array}{c}e \\
e \underset{1}{11211} \\
\end{array}$ & $z_{2}$ & $z_{2}$ \\
\hline
\end{tabular}


$E_{6}$, orbit 8: $A_{3}$

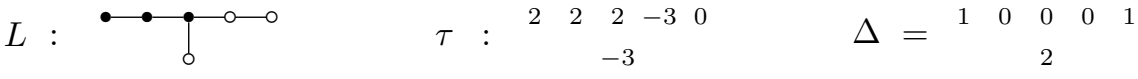

$$
\begin{aligned}
& e=\underset{0}{e} \underset{0}{e 000}+e_{0}^{01000}+e_{00100}^{00} \\
& C^{\circ}=B_{2} T_{1} \quad C / C^{\circ}=1 \\
& \beta_{1}={ }_{0}^{00001}, x_{ \pm \beta_{2}}(t)=x_{ \pm}{ }_{11110}(t) x_{ \pm}^{01210}(-t) \\
& T_{1}=\left\{h_{1}(\mu) h_{3}\left(\mu^{2}\right) h_{4}\left(\mu^{3}\right) h_{5}\left(\mu^{4}\right) h_{6}\left(\mu^{2}\right): \mu \in k^{*}\right\}
\end{aligned}
$$

\begin{tabular}{|c|c|c|c|c|c|}
\hline$n$ & $m$ & $\lambda$ & $v$ & & $\mathcal{Z}$ \\
\hline 5 & 1 & $3 \lambda_{1}$ & $e_{12321}$ & & \\
\hline & 1 & $\lambda_{1}$ & $e_{11100}+e_{11110}+e_{01111}-e_{00111}$ & & \\
\hline 4 & 2 & $2 \lambda_{1}$ & $e_{12211}-e_{11221}$ & & \\
\hline & 2 & 0 & $e_{00000}$ & & \\
\hline 3 & 3 & $\lambda_{1}$ & $e_{11110}+e_{01111}$ & & \\
\hline & 3 & $\lambda_{1}$ & $e_{11110}+e_{11111}$ & & \\
\hline \begin{tabular}{|l|}
2 \\
\end{tabular} & 4 & $2 \lambda_{1}$ & $e_{12221}$ & & \\
\hline & 4 & 0 & $e_{11000}+e_{00011}$ & & \\
\hline 1 & 2 & 0 & $e$ & $z_{2}$ & $z_{2}$ \\
\hline & 5 & $\lambda_{1}$ & $e_{11111}$ & & \\
\hline
\end{tabular}

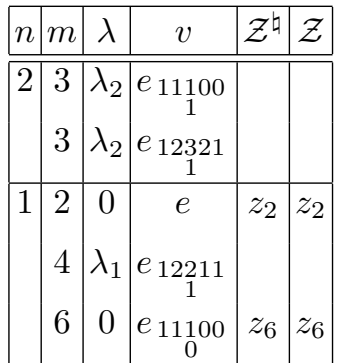

$E_{6}$, orbit 9: $A_{2}^{2} A_{1}$

$$
\begin{aligned}
& L: \bullet \bullet \bullet \bullet \bullet \\
& \tau:{ }^{\prime} \quad \begin{array}{lllll}
2 & 2 & -5 & 2 & 2
\end{array} \\
& \Delta=\begin{array}{lllll}
1 & 0 & 1 & 0 & 1 \\
& & 0 & &
\end{array} \\
& e=\underset{0}{e} \underset{0}{10000}+\underset{0}{e 01000}+\underset{0}{e 00010}+\underset{1}{e 00001}+e_{00000}^{00} \\
& C^{\circ}=A_{1} \quad C / C^{\circ}=1 \\
& x_{ \pm \beta_{1}}(t)=x_{ \pm}{ }_{12210}(t) x_{ \pm}{ }_{1}^{11211}(t) x_{ \pm}{ }_{1}^{01221}(-t)
\end{aligned}
$$


$E_{6}$, orbit 10: $A_{3} A_{1}$

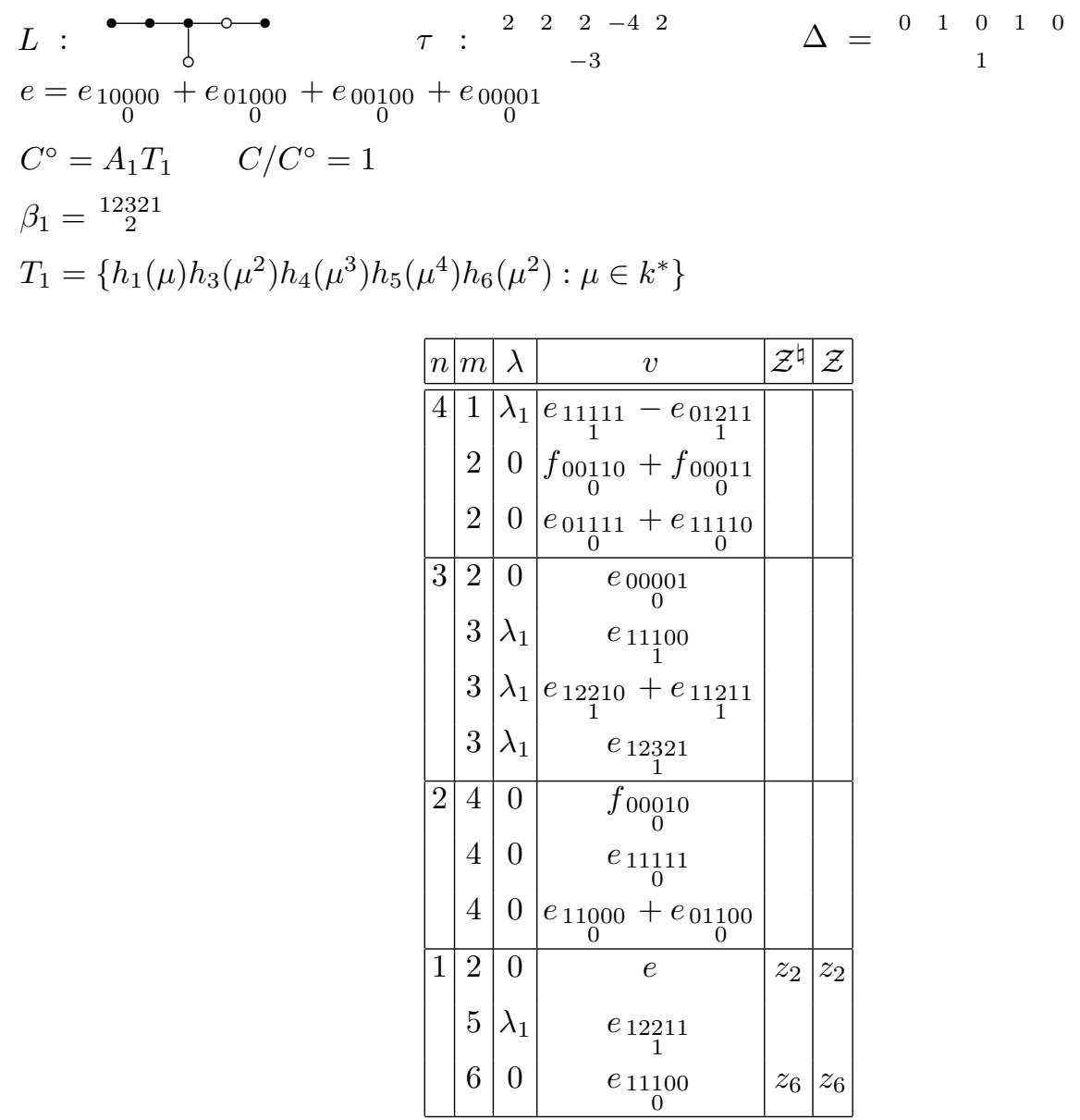


$E_{6}$, orbit 11: $D_{4}\left(a_{1}\right)$

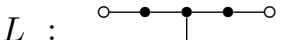

$$
\begin{aligned}
& \tau \quad: \quad \begin{array}{llllll}
-4 & 2 & 0 & 2 & -4
\end{array} \\
& \Delta= \\
& \begin{array}{llll}
0 & 2 & 0 & 0
\end{array} \\
& e=\underset{0}{e 01000}+e_{1}^{00100}+e_{0}^{00110}+e_{1}^{00000}+\underset{0}{e 00010} \\
& C^{\circ}=T_{2} \quad C / C^{\circ}=\left\langle c_{1} C^{\circ}, c_{2} C^{\circ}\right\rangle \cong S_{3} \\
& T_{2}=\left\{h_{1}\left(\mu^{2}\right) h_{2}(\mu \nu) h_{3}\left(\mu^{2} \nu\right) h_{4}\left(\mu^{2} \nu^{2}\right) h_{5}\left(\mu \nu^{2}\right) h_{6}\left(\nu^{2}\right): \mu, \nu \in k^{*}\right\} \\
& c_{1}=n_{11100} n_{11110} h_{2}(-1) \text {, } \\
& c_{2}=\left(n_{12211} n_{11221} h_{1}(-1) h_{2}(-1) h_{6}(-1)\right)^{g}, \\
& g=x_{00100}\left(\frac{1}{3}\right) n_{00100} h_{1}(4) h_{2}(-4) h_{3}(16) h_{4}(-48) h_{5}(16) h_{6}(-8) x_{00100}\left(-\frac{1}{3}\right)
\end{aligned}
$$

\begin{tabular}{|c|c|c|c|c|}
\hline$n \mid n$ & $\lambda$ & $v$ & $\mathcal{Z}^{\natural}$ & $\mathcal{Z}$ \\
\hline \begin{tabular}{l|l}
3 & 2 \\
\end{tabular} & - & $f_{11111}+f_{11211}$ & & \\
\hline 2 & - & $e_{12221}-e_{12321}$ & & \\
\hline 2 & - & $f_{00011}^{1}-f_{00111}^{1}$ & & \\
\hline 2 & - & $e_{01111}^{0}+e_{01211}^{0}$ & & \\
\hline ? & $1-$ & ${ }^{1} f_{11100}^{1}$ & & \\
\hline & . & $\sqrt{11100}$ & & \\
\hline 2 & - & $e_{11110}$ & & \\
\hline 2 & - & $2 e_{0}^{00000}-e_{1}^{00100}+e_{0}^{01100}+e_{0}^{00110}$ & & \\
\hline 2 & - & $e_{00000}+e_{00010}^{000}$ & & \\
\hline \begin{tabular}{|l|l|l|}
2 & 4
\end{tabular} & - & $f_{11111}$ & & \\
\hline 4 & - & $e_{12321}$ & & \\
\hline 4 & - & $f_{00001}$ & & \\
\hline 4 & - & $e_{01221}$ & & \\
\hline 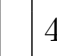 & - & $f_{10000}$ & & \\
\hline 4 & - & $e_{12210}$ & & \\
\hline 4 & - & $e_{01100}+2 e_{00110}-e_{01110}$ & & \\
\hline $1 \mid 2$ & - & $e$ & $z_{2}$ & $z_{2}$ \\
\hline 6 & - & $e_{01110}$ & $z_{6}{ }^{1}$ & \\
\hline 6 & - & $e_{01210}$ & $z_{6}^{2}$ & \\
\hline
\end{tabular}


$E_{6}$, orbit $12: A_{4}$

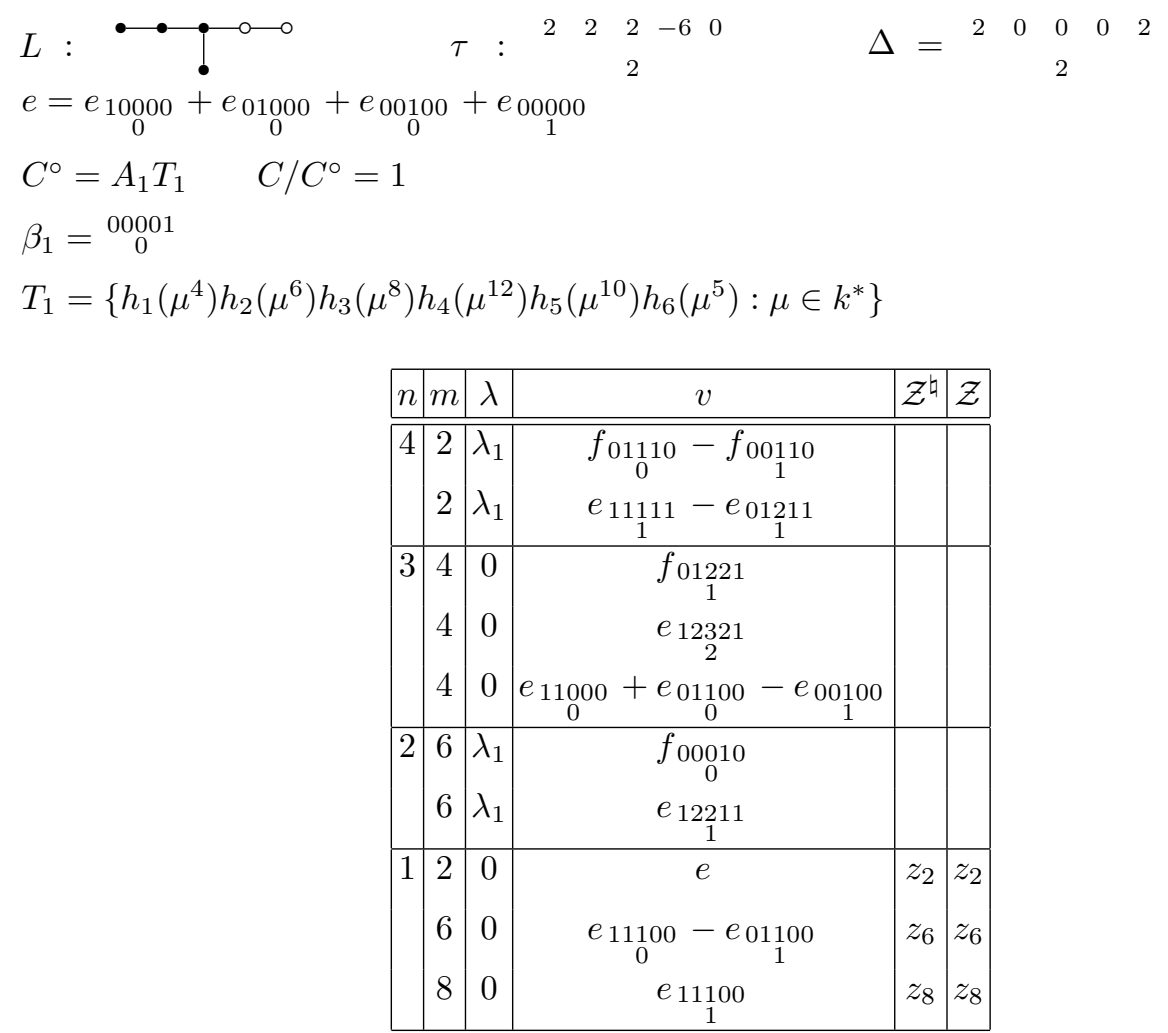

$E_{6}$, orbit $13: D_{4}$

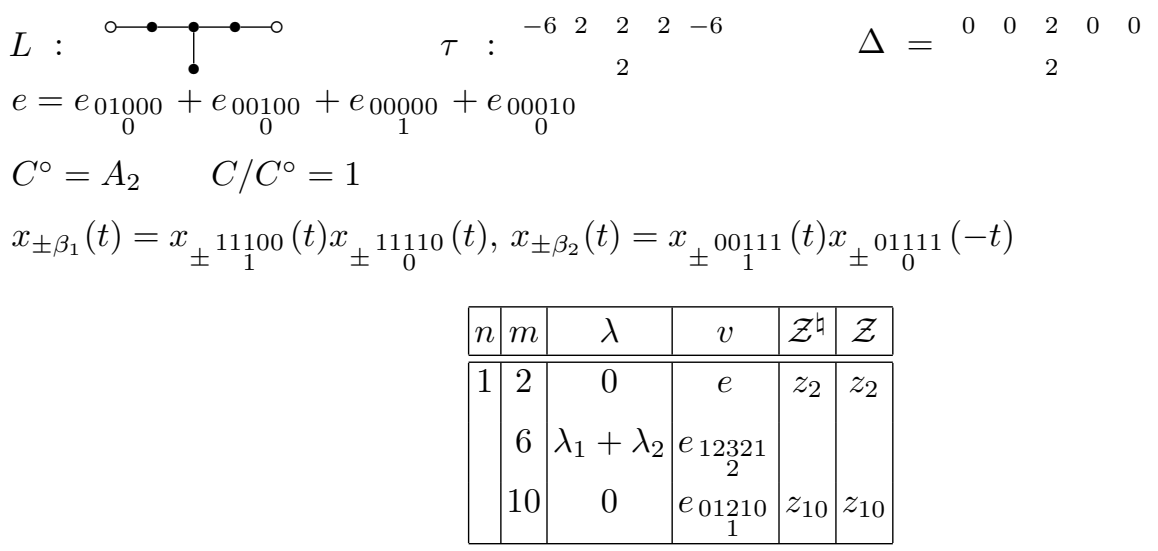


$E_{6}$, orbit 14: $A_{4} A_{1}$

$$
\begin{aligned}
& L: \bullet \bullet \bullet-\bullet \\
& \tau:{ }^{2} \quad \begin{array}{lllll}
2 & 2 & 2 & -7 & 2
\end{array} \\
& \Delta=\begin{array}{lllll}
1 & 1 & 0 & 1 & 1
\end{array} \\
& e=\underset{0}{e} \underset{0}{10000}+e_{0}^{01000}+e_{0}^{00100}+e_{00000}^{0000}+e_{0}^{00001} \\
& C^{\circ}=T_{1} \quad C / C^{\circ}=1 \\
& T_{1}=\left\{h_{1}\left(\mu^{4}\right) h_{2}\left(\mu^{6}\right) h_{3}\left(\mu^{8}\right) h_{4}\left(\mu^{12}\right) h_{5}\left(\mu^{10}\right) h_{6}\left(\mu^{5}\right): \mu \in k^{*}\right\}
\end{aligned}
$$

\begin{tabular}{|c|c|c|c|c|c|}
\hline$n$ & $m$ & $\lambda$ & $v$ & $\mathcal{Z}^{\natural}$ & $\mathcal{Z}$ \\
\hline 8 & 1 & & $\begin{array}{l}f_{01110}+f_{00111}-f_{01111}-2 f_{11110} \\
1 \\
e_{01111}+e_{01210}-e_{11110}-2 e_{1} \\
0 \\
0 \\
0\end{array}$ & & \\
\hline 7 & 2 & - & $e_{00001}$ & & \\
\hline 6 & 3 & - & $\begin{array}{l}f_{01110}-f_{00110} \\
e_{11111}-e_{01211}\end{array}$ & & \\
\hline 5 & $\begin{array}{l}4 \\
4\end{array}$ & - & $\begin{array}{c}f_{01221} \\
e_{12321} \\
e_{2} \\
e_{11000}+e_{0}^{01100}-e_{00100}\end{array}$ & & \\
\hline 4 & $\begin{array}{l}5 \\
5\end{array}$ & - & $\begin{array}{l}f_{00110}+f_{00011} \\
0 \\
e_{12210}+e_{11211}\end{array}$ & & \\
\hline 3 & 6 & - & $e_{0}^{11100}-e_{01100}^{0100}$ & & \\
\hline 2 & $\begin{array}{l}7 \\
7\end{array}$ & - & $\begin{array}{l}f_{00010} \\
0 \\
e_{12211} \\
\end{array}$ & & \\
\hline 1 & 2 & - & $\begin{array}{c}e \\
e_{11100}\end{array}$ & & $\begin{array}{l}z_{2} \\
z_{8}\end{array}$ \\
\hline
\end{tabular}

$E_{6}$, orbit $15: A_{5}$
$L$ :
$\tau \quad: \quad \begin{array}{lllll}2 & 2 & 2 & 2 & 2\end{array}$
$-9$
$\Delta=\begin{array}{lllll}2 & 1 & 0 & 1 & 2\end{array}$
$e=e_{10000}+e_{01000}+e_{0}^{00100}+e_{0}^{00010}+e_{00001}$
$C^{\circ}=A_{1} \quad C / C^{\circ}=1$
$\beta_{1}={ }_{2}^{12321}$ 


\begin{tabular}{|c|c|c|c|c|c|}
\hline$n$ & $m$ & $\lambda$ & $v$ & $\mathcal{Z}^{\natural}$ & $\mathcal{Z}$ \\
\hline 3 & 3 & $\lambda_{1}$ & $e_{12210}+e_{11211}-e_{01221}$ & & \\
\hline 2 & 5 & $\lambda_{1}$ & $\begin{array}{c}\underset{0}{e_{11000}}+e_{01100}+e_{00110}+e_{00011} \\
e_{12211}-e_{11221} \\
1 \\
e_{11100}+e_{01110}+e_{00111}\end{array}$ & & \\
\hline 1 & 10 & $\begin{array}{l}\lambda_{1} \\
0\end{array}$ & $\begin{array}{c}e \\
e_{0}^{e_{1110}+e_{01111}}{ }_{0} \\
e_{12321} \\
e_{11111}\end{array}$ & $\begin{array}{l}z_{2} \\
z_{8}\end{array}$ & $\begin{array}{l}z_{2} \\
z_{8}\end{array}$ \\
\hline
\end{tabular}

$E_{6}$, orbit 16: $D_{5}\left(a_{1}\right)$

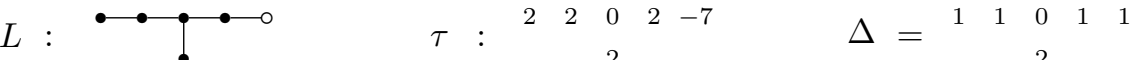

$$
\begin{aligned}
& e=e_{10000}+e_{01000}+e_{00100}+e_{00110}+e_{00000}+e_{00010} \\
& C^{\circ}=T_{1} \quad C / C^{\circ}=1 \\
& T_{1}=\left\{h_{1}\left(\mu^{2}\right) h_{2}\left(\mu^{3}\right) h_{3}\left(\mu^{4}\right) h_{4}\left(\mu^{6}\right) h_{5}\left(\mu^{5}\right) h_{6}\left(\mu^{4}\right): \mu \in k^{*}\right\}
\end{aligned}
$$

\begin{tabular}{|c|c|c|c|c|c|}
\hline$n$ & $m$ & $\lambda$ & $v$ & $\mathcal{Z}^{\natural}$ & $\mathcal{Z}$ \\
\hline \multirow[t]{3}{*}{5} & 1 & - & $f_{11111}+f_{01211}$ & & \\
\hline & 1 & - & $e_{11111}+e_{01221}$ & & \\
\hline & 4 & - & $e_{11100}+e_{01110}-e_{01100}-2 e_{00110}$ & & \\
\hline \multirow[t]{3}{*}{4} & 2 & - & $e_{1}^{00000}+e_{0}^{00010}$ & & \\
\hline & 5 & - & $f_{00011}-\underset{0}{f_{00111}}$ & & \\
\hline & 5 & - & $e_{12221}-e_{12321}$ & & \\
\hline \multirow[t]{2}{*}{3} & 6 & - & $e_{0}^{11110}-e_{01110}+e_{01210}$ & & \\
\hline & 6 & - & $e_{11100}+e_{01110}+e_{01210}$ & & \\
\hline \multirow[t]{2}{*}{2} & 7 & - & $f_{00001}$ & & \\
\hline & 7 & - & $e_{12321}$ & & \\
\hline \multirow[t]{3}{*}{1} & 2 & - & $e$ & $z_{2}$ & $z_{2}$ \\
\hline & 8 & - & $e_{11110}$ & $z_{8}$ & $z_{8}$ \\
\hline & 10 & - & $e_{12210}$ & $z_{10}$ & $z_{10}$ \\
\hline
\end{tabular}


$E_{6}$, orbit 17: $E_{6}\left(a_{3}\right)$

$$
\begin{aligned}
& L: \bullet \bullet \bullet \\
& \tau \quad \cdot \begin{array}{llllll}
2 & 2 & 0 & 2 & 0 & 2
\end{array} \\
& \Delta=\begin{array}{lllll}
2 & 0 & 2 & 0 & 2
\end{array} \\
& e=e_{1}^{01100}+e_{0} 0000+e_{0}^{01110}+e_{0}^{00001}+e_{0}^{00110}+e_{0}^{00100} \\
& C^{\circ}=1 \quad C / C^{\circ}=\left\langle c C^{\circ}\right\rangle \cong S_{2} \\
& c=h_{4}(-1)
\end{aligned}
$$

\begin{tabular}{|c|c|c|c|c|c|}
\hline$n$ & $m$ & $\lambda \mid$ & $v$ & $\mathcal{Z}^{\natural}$ & $\mathcal{Z}$ \\
\hline 5 & $\begin{array}{l}2 \\
2\end{array}$ & - & $\begin{array}{c}e_{0}^{e} 11000+e_{01100}+e_{00110}+e_{00011}-3 e 00100+e_{01110} \\
0 \\
e_{00100} \\
0\end{array}$ & & \\
\hline 4 & 4 & - & $e_{11100}-e_{00111}-e_{01210}$ & & \\
\hline 3 & $\begin{array}{l}4 \\
4 \\
6\end{array}$ & $\begin{array}{l}- \\
- \\
-\end{array}$ & $\begin{array}{c}e_{11110}+e_{01111}-e_{11100}-e_{00111} \\
0 \\
e_{11100}+e_{00111}-e_{11110}-e_{01111} \\
0 \\
0 \\
e_{11111}-e_{12210}-e_{01221} \\
0 \\
1\end{array}$ & & \\
\hline 2 & $\begin{array}{l}6 \\
8\end{array}$ & - & $\begin{array}{l}e_{11210}+e_{01211} \\
e_{11211}+e_{12221}\end{array}$ & & \\
\hline 1 & \begin{tabular}{c|}
2 \\
8 \\
10 \\
10
\end{tabular} & $\begin{array}{l}- \\
- \\
- \\
-\end{array}$ & $\begin{array}{c}e \\
e_{12211}+e_{11221} \\
e_{12321} \\
1 \\
e_{12321}\end{array}$ & $\begin{array}{c}z_{2} \\
z_{8} \\
z_{10}{ }^{1} \\
z_{10}{ }^{2}\end{array}$ & $\begin{array}{c}z_{2} \\
z_{8}\end{array}$ \\
\hline
\end{tabular}


$E_{6}$, orbit 18: $D_{5}$

$$
\begin{aligned}
& L \text { : } \\
& \bullet \bullet \bullet \\
& e=e_{0} \underset{0000}{0}+e_{0}^{01000}+e_{0}^{00100}+e_{1}^{00000}+e_{0}^{00010} \\
& \tau:{ }^{\prime} \quad \begin{array}{llllll}
2 & 2 & 2 & 2 & -10
\end{array} \\
& \Delta=\begin{array}{lllll}
2 & 0 & 2 & 0 & 2 \\
& & 2 & &
\end{array} \\
& C^{\circ}=T_{1} \quad C / C^{\circ}=1 \\
& T_{1}=\left\{h_{1}\left(\mu^{2}\right) h_{2}\left(\mu^{3}\right) h_{3}\left(\mu^{4}\right) h_{4}\left(\mu^{6}\right) h_{5}\left(\mu^{5}\right) h_{6}\left(\mu^{4}\right): \mu \in k^{*}\right\}
\end{aligned}
$$

\begin{tabular}{|c|c|c|c|c|}
\hline \begin{tabular}{|l|l|}
$n$ & $m$ \\
\end{tabular} & \begin{tabular}{|l|l|}
$\lambda$ \\
\end{tabular} & $v$ & $\mathcal{Z}^{\natural}$ & $\mathcal{Z}$ \\
\hline \begin{tabular}{|l|l|l|}
3 & 4
\end{tabular} & $=-$ & $f_{01111}-f_{00111}$ & & \\
\hline 4 & - & $e_{12211}-e_{11221}$ & & \\
\hline 6 & - & $e_{0}^{11100}-e_{01100}-2 e_{0}^{00110}+e_{01110}^{0110}$ & & \\
\hline $\begin{array}{lll}2 & 10\end{array}$ & \begin{tabular}{ll|}
0 & -
\end{tabular} & $f_{00001}$ & & \\
\hline 10 & $0-$ & $e_{12321}$ & & \\
\hline \begin{tabular}{|l|l}
1 & 2 \\
\end{tabular} & - & $e$ & $z_{2}$ & $z_{2}$ \\
\hline 8 & - & $e_{11110}+e_{11100}$ & $z_{8}$ & $z_{8}$ \\
\hline 10 & $0-$ & $e_{1110}-e_{01210}$ & $z_{10}$ & $z_{10}$ \\
\hline 14 & $4-$ & $e_{12210}$ & $z_{14}$ & $z_{14}$ \\
\hline
\end{tabular}

$E_{6}$, orbit 19: $E_{6}\left(a_{1}\right)$

$$
\begin{aligned}
& L: \bullet \bullet \bullet-\bullet: \begin{array}{llllll}
2 & 2 & 0 & 2 & 2
\end{array} \quad \Delta=\begin{array}{lllll}
2 & 2 & 0 & 2 & 2
\end{array} \\
& e=e_{10000}+e_{0}^{00001}+e_{0}^{01000}+e_{0}^{00010}+e_{0}^{00110}+e_{0}^{01100}+e_{00000} \\
& C^{\circ}=1 \quad C / C^{\circ}=1
\end{aligned}
$$

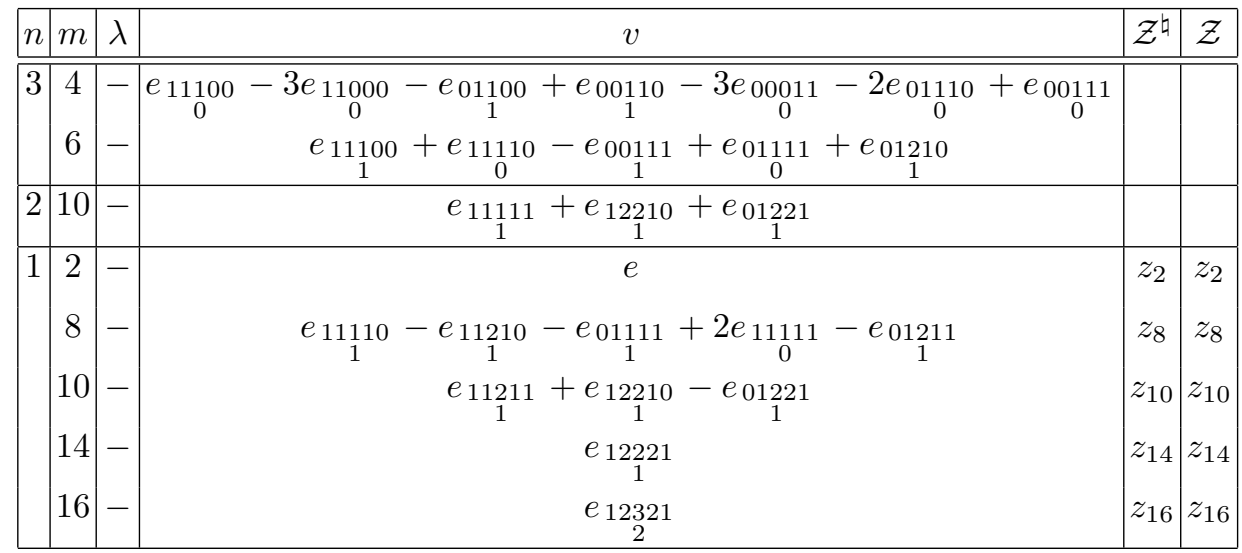


$E_{6}$, orbit 20: $E_{6}$

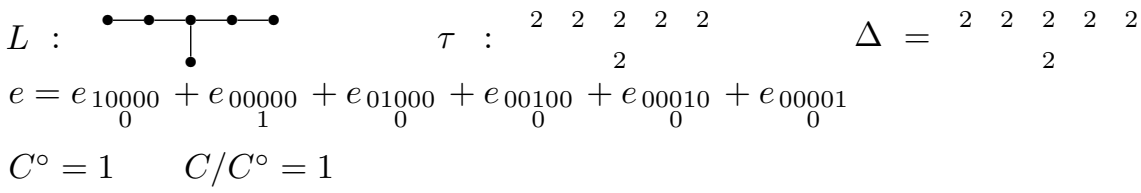

\begin{tabular}{|c|c|c|c|c|c|}
\hline$n$ & $m$ & $\lambda$ & $v$ & $\mathcal{Z}^{\natural}$ & $\mathcal{Z}$ \\
\hline 1 & 2 & -1 & $e$ & $z_{2}$ & $z_{2}$ \\
\hline & 8 & - & $e_{11100}+e_{0}^{11110}+e_{01111}-e_{00111}$ & $z_{8}$ & $z_{8}$ \\
\hline & 10 & - & $e_{11110}+2 e_{0}^{11111}-e_{01111}-e_{01210}$ & $z_{10}$ & $z_{10}$ \\
\hline & 14 & - & $e_{12210}+e_{11211}-e_{1}^{01221}$ & $z_{14}$ & $z_{14}$ \\
\hline & 16 & - & $e_{12211}-e_{11221}^{112}$ & $z_{16}$ & $z_{16}$ \\
\hline & 22 & - & $e_{12321}$ & $z_{22}$ & $z_{2}$ \\
\hline
\end{tabular}


$E_{7}$, orbit 1: $A_{1}$

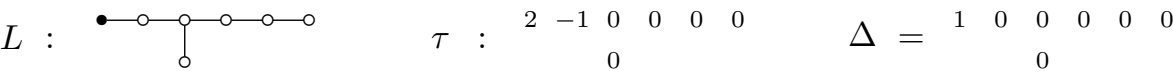

$$
\begin{aligned}
& e=\underset{0}{100000} \\
& C^{\circ}=D_{6} \quad C / C^{\circ}=1 \\
& \beta_{1}=\stackrel{000000}{1}, \beta_{2}=\stackrel{001000}{0}, \beta_{3}=\underset{0}{000100}, \beta_{4}=\underset{0}{000010}, \beta_{5}=\underset{0}{000001}, \beta_{6}=\underset{1}{122100}
\end{aligned}
$$

\begin{tabular}{|c|c|c|c|c|c|}
\hline$n$ & $m$ & $\lambda$ & $v$ & $\mathcal{Z}^{\natural}$ & $\mathcal{Z}$ \\
\hline 2 & 1 & $\lambda_{6}$ & $e_{234321}$ & & \\
\hline 1 & 2 & 0 & $e$ & $z_{2}$ & $z_{2}$ \\
\hline
\end{tabular}

$E_{7}$, orbit 2: $A_{1}{ }^{2}$

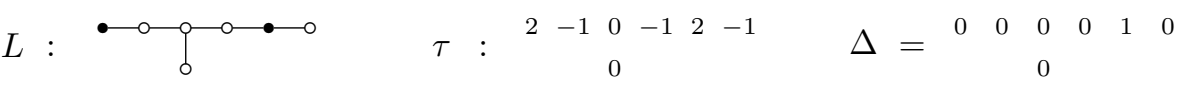

$$
\begin{aligned}
& e=\underset{0}{e 0000}+\underset{0}{000010} \\
& C^{\circ}=B_{4} A_{1} \quad C / C^{\circ}=1 \\
& \beta_{1}=\stackrel{000111}{0}, \beta_{2}={ }_{0}^{001000}, \beta_{3}={ }_{1}^{000000}, x_{ \pm \beta_{4}}(t)=x_{ \pm \underset{0}{111100}}(t) x_{ \pm}^{011110}(t) ; \beta_{5}={ }_{1}^{122111}
\end{aligned}
$$

\begin{tabular}{|c|c|c|c|c|c|}
\hline$n$ & $m$ & $\lambda$ & $v$ & $\mathcal{Z}^{\natural}$ & $\mathcal{Z}$ \\
\hline \hline 2 & 1 & $\lambda_{4}+\lambda_{5}$ & $e_{234321}$ & & \\
\hline 1 & 2 & $\lambda_{1}$ & $e_{112221}$ & & \\
& 2 & 0 & $e$ & $z_{2}$ & $z_{2}$ \\
& 2 & 0 & &
\end{tabular}

$E_{7}$, orbit 3: $\left(A_{1}{ }^{3}\right)^{\prime \prime}$

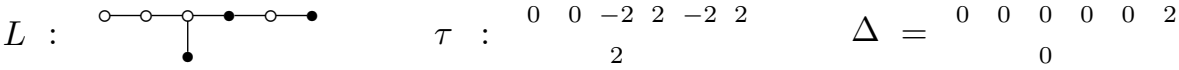

$$
\begin{aligned}
& e=e_{000000}+e_{000100}+e_{000001} \\
& C^{\circ}=F_{4} \quad C / C^{\circ}=1 \\
& \beta_{1}=\stackrel{100000}{0}, \beta_{2}=\stackrel{010000}{0}, x_{ \pm \beta_{3}}(t)=x_{ \pm} 001000(t) x_{ \pm}^{001100}(t) \text {, } \\
& x_{ \pm \beta_{4}}(t)=x_{ \pm \underset{0}{000110}}(t) x_{ \pm \underset{0}{000011}(t)}
\end{aligned}
$$

\begin{tabular}{|c|c|c|c|c|c|}
\hline$n$ & $m$ & $\lambda$ & $v$ & $\mathcal{Z}^{\natural}$ & $\mathcal{Z}$ \\
\hline \hline 1 & 2 & $\lambda_{4}$ & $e_{123321}$ & & \\
& 2 & 0 & $e$ & $z_{2}$ & $z_{2}$ \\
\hline
\end{tabular}


$E_{7}$, orbit $4:\left(A_{1}^{3}\right)^{\prime}$

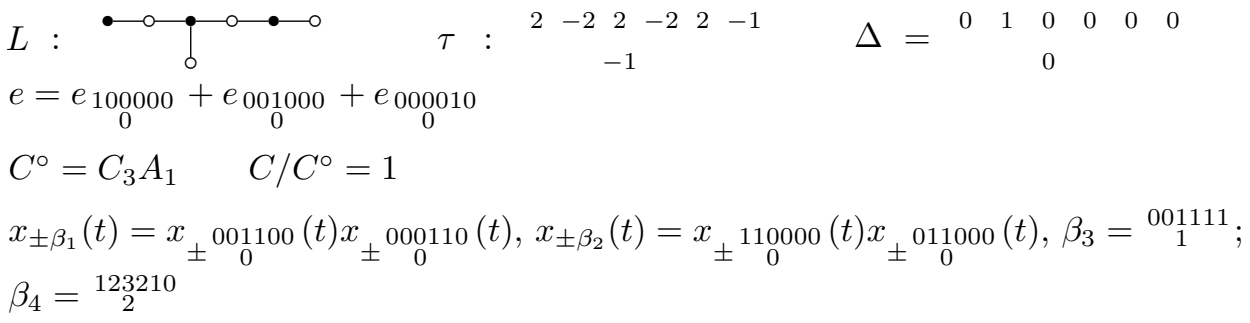

\begin{tabular}{|c|c|c|c|c|c|}
\hline$n$ & $m$ & $\lambda$ & $v$ & $\mathcal{Z}^{\natural}$ & $\mathcal{Z}$ \\
\hline \hline 3 & 1 & $\lambda_{2}+\lambda_{4}$ & $e_{234321}$ & & \\
\hline 2 & 2 & $\lambda_{2}$ & $e_{123221}$ & & \\
\hline 1 & 2 & 0 & $e$ & $z_{2}$ & $z_{2}$ \\
& 3 & $\lambda_{4}$ & $\underset{1}{e_{11210}}$ & & \\
\hline
\end{tabular}

$E_{7}$, orbit 5: $A_{2}$

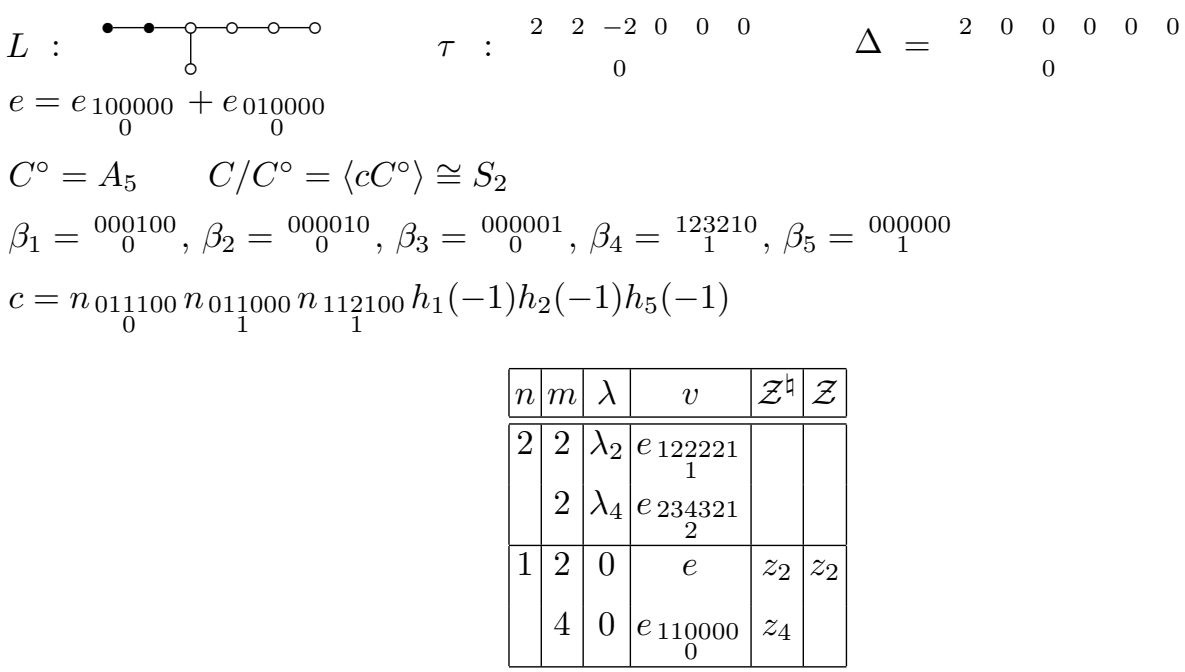

$E_{7}$, orbit 6: $A_{1}{ }^{4}$

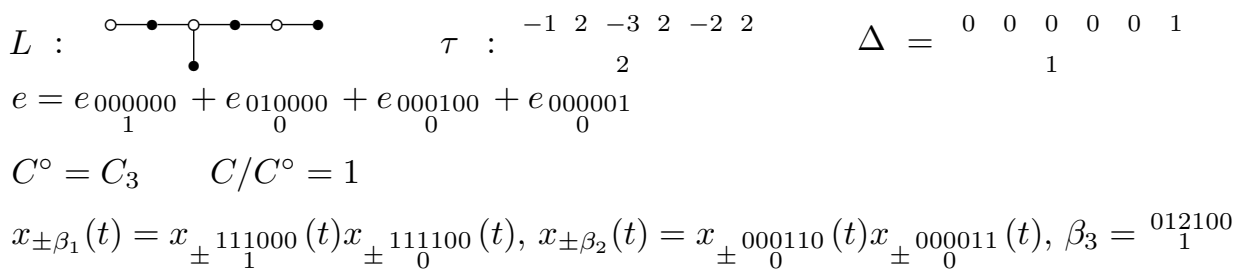




\begin{tabular}{|c|c|c|c|c|c|}
\hline$n$ & $m$ & $\lambda$ & $v$ & $\mathcal{Z}^{\natural}$ & $\mathcal{Z}$ \\
\hline 3 & 1 & $\lambda_{3}$ & $e_{134321}$ & & \\
\hline & 1 & $\lambda_{1}$ & $e_{122210}-e_{122111}+2 e_{112211}$ & & \\
\hline 2 & 2 & $\lambda_{2}$ & $e_{2}^{123321}$ & & \\
\hline & 2 & 0 & $\begin{array}{c}e 010000 \\
0\end{array}$ & & \\
\hline 1 & 2 & 0 & $e$ & $z_{2}$ & $z_{2}$ \\
\hline & 3 & $\lambda_{1}$ & $e_{122211}$ & & \\
\hline
\end{tabular}

$E_{7}$, orbit 7: $A_{2} A_{1}$

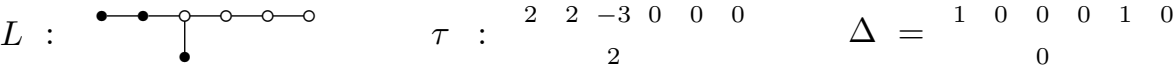

$$
\begin{aligned}
& e=e_{100000}+e_{010000}+e_{000000} \\
& C^{\circ}=A_{3} T_{1} \quad C / C^{\circ}=\left\langle c C^{\circ}\right\rangle \cong S_{2} \\
& \beta_{1}=\underset{0}{000100}, \beta_{2}=\underset{0}{000010}, \beta_{3}=\underset{0}{000001} \\
& T_{1}=\left\{h_{1}\left(\mu^{4}\right) h_{2}\left(\mu^{6}\right) h_{3}\left(\mu^{8}\right) h_{4}\left(\mu^{12}\right) h_{5}\left(\mu^{9}\right) h_{6}\left(\mu^{6}\right) h_{7}\left(\mu^{3}\right): \mu \in k^{*}\right\} \\
& c=n_{112111} n_{112210} n_{134321} h_{3}(-1) h_{5}(-1) h_{7}(-1)
\end{aligned}
$$

\begin{tabular}{|c|c|c|c|c|c|}
\hline$n$ & $m$ & $\lambda$ & $v$ & $\mathcal{Z}^{\natural}$ & $\mathcal{Z}$ \\
\hline \hline 4 & 1 & $\lambda_{1}$ & $f_{011000}-f_{001000}$ & & \\
0 & & & 1 \\
& $\lambda_{3}$ & & \\
$e_{111111}-e_{011111}$ & & \\
& 1 & $\lambda_{123210}$ & & \\
& 1 & $\lambda_{3}$ & $f_{123210}$ & & \\
& 1 & $\lambda_{1}$ & $e_{123321}$ & & \\
\hline 3 & 2 & $\lambda_{2}$ & $f_{012100}$ & & \\
& 2 & $\lambda_{2}$ & $e_{12221}$ & & \\
& 2 & 0 & $f_{124321}$ & & \\
& 2 & 0 & $e_{234321}$ & & \\
& 2 & 0 & 2 & & \\
& 2 & 0 & $e_{000000}$ & & \\
\hline 2 & 3 & $\lambda_{1}$ & $f_{001000}$ & & \\
& 3 & $\lambda_{3}$ & $e_{11111}$ & & \\
\hline 1 & 2 & 0 & & & \\
& 4 & 0 & $e_{110000}$ & $z_{4}$ & \\
& 4 & 0 & $z_{2}$ \\
\end{tabular}




$$
\begin{aligned}
& E_{7} \text {, orbit 8: } A_{2} A_{1}^{2}
\end{aligned}
$$

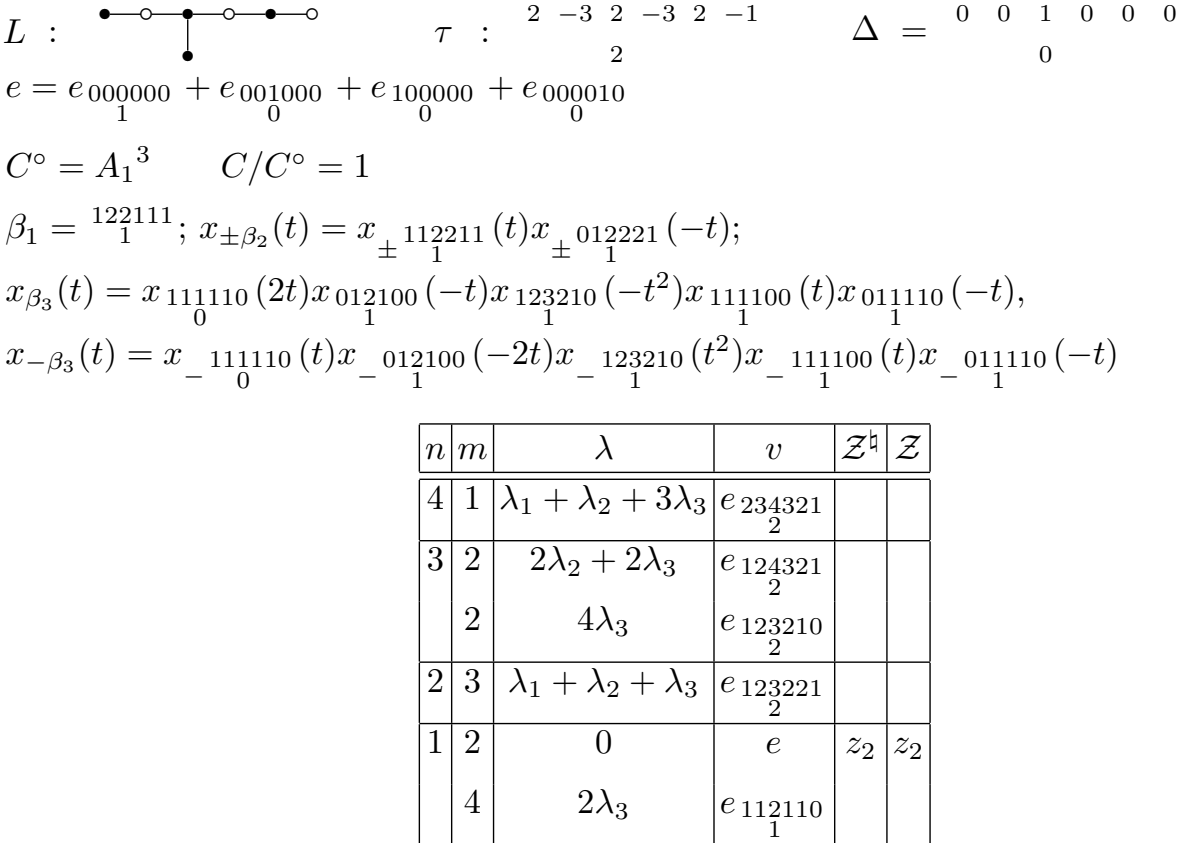

$E_{7}$, orbit 9: $A_{3}$

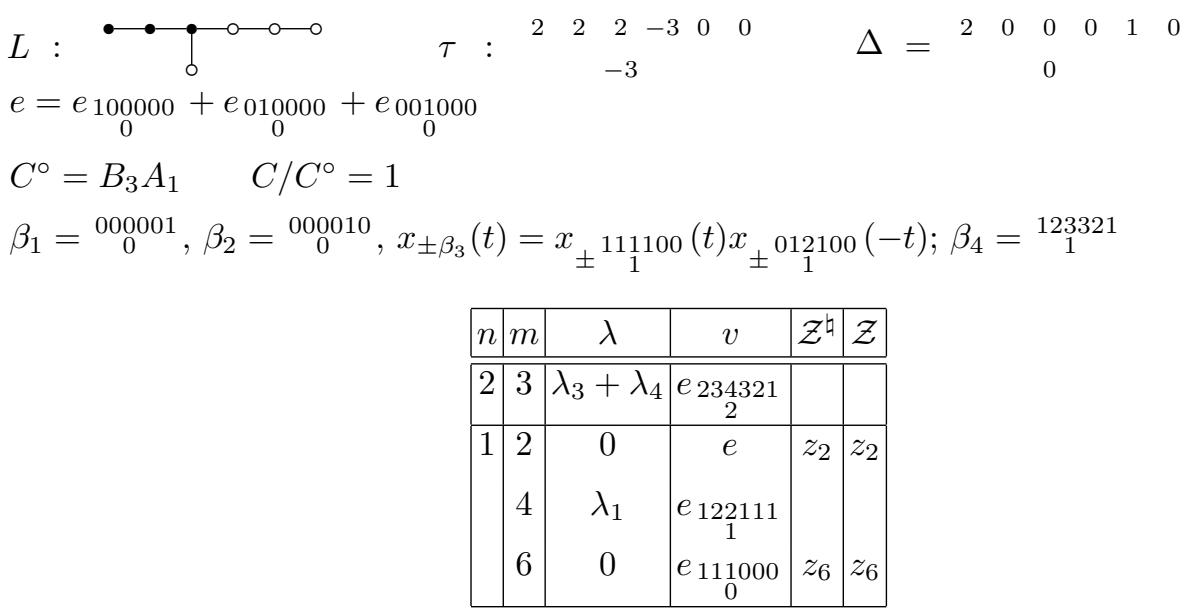


$E_{7}$, orbit 10: $A_{2}{ }^{2}$

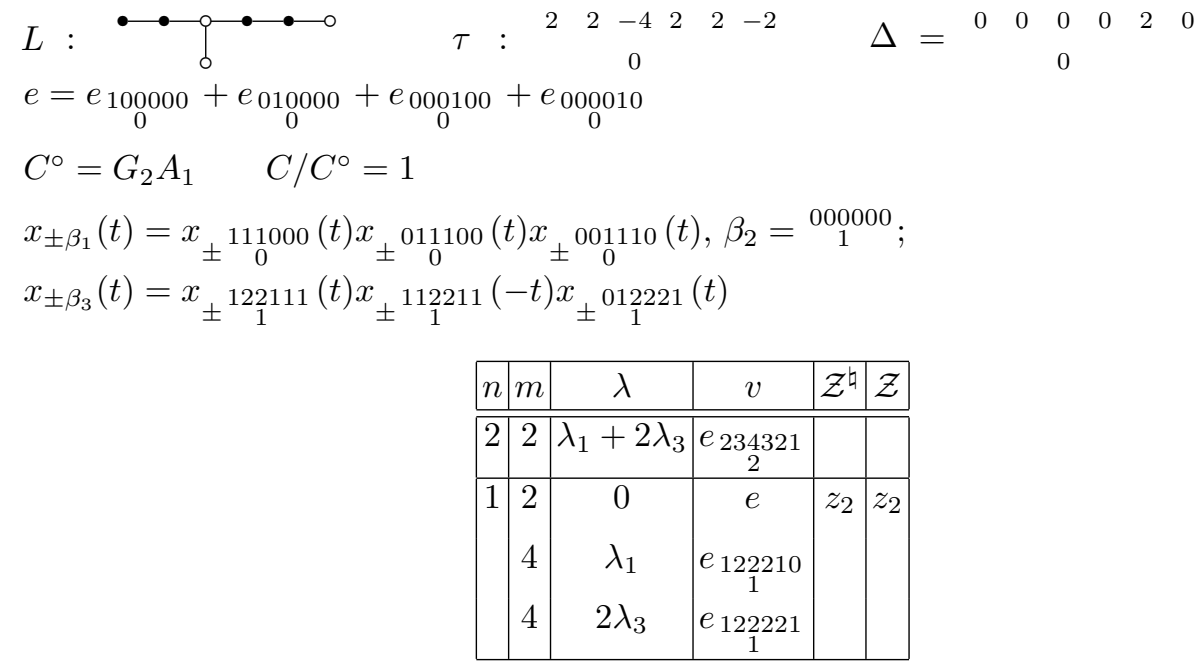

$E_{7}$, orbit 11: $A_{2} A_{1}{ }^{3}$

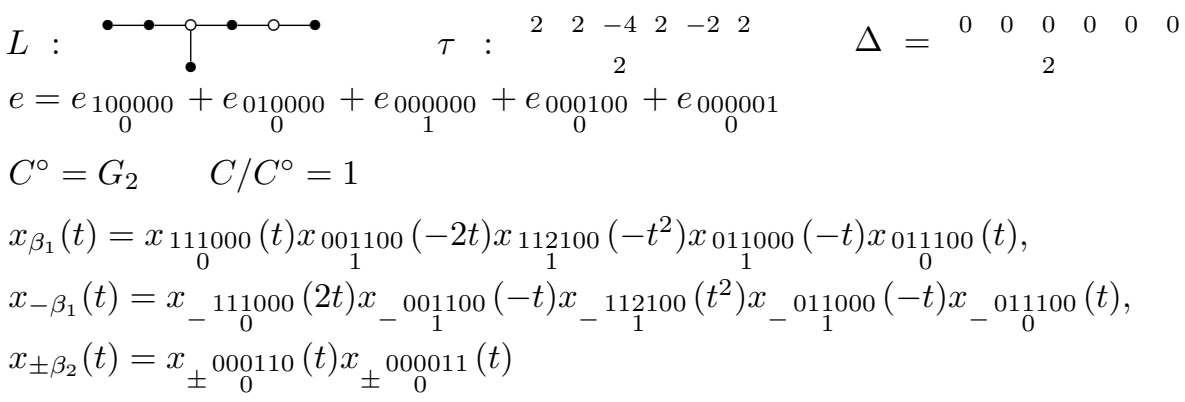

\begin{tabular}{|c|c|c|c|c|c|}
\hline$n$ & $m$ & $\lambda$ & $v$ & $\mathcal{Z}^{\natural}$ & $\mathcal{Z}$ \\
\hline \hline 2 & 2 & $2 \lambda_{1}$ & $e_{234321}$ & & \\
\hline 1 & 2 & 0 & $e$ & $z_{2}$ & $z_{2}$ \\
& 4 & $\lambda_{1}$ & $e_{122211}$ & & \\
& & & & & \\
\hline
\end{tabular}


$E_{7}$, orbit 12: $\left(A_{3} A_{1}\right)^{\prime \prime}$

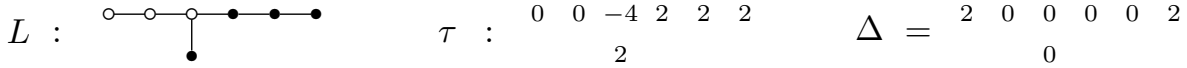

$$
\begin{aligned}
& e=\underset{0}{e 000100}+\underset{0}{e 000010}+\underset{1}{e 000001}+e_{0}^{000000} \\
& C^{\circ}=B_{3} \quad C / C^{\circ}=1 \\
& \beta_{1}={ }_{0}^{01000}, \beta_{2}={ }_{0}^{100000}, x_{ \pm \beta_{3}}(t)=x_{ \pm}{ }_{1}^{012111}(t) x_{ \pm} 012210(t) \\
& \begin{array}{|c|c|c|c|c|c|}
\hline n & m & \lambda & v & \mathcal{Z}^{\natural} & \mathcal{Z} \\
\hline 3 & 2 & \lambda_{3} & e_{123221}-e_{123321} & & \\
& 2 & 0 & e_{000000} & & \\
\hline 2 & 4 & \lambda_{3} & e_{123321} & & \\
\hline 1 & 2 & 0 & e & z_{2} & z_{2} \\
& 4 & \lambda_{1} & e_{122221} & & \\
& 6 & 0 & e_{000111} & z_{6} & z_{6} \\
& 6 & 0 & & \\
\hline
\end{array}
\end{aligned}
$$

$E_{7}$, orbit $13: A_{2}^{2} A_{1}$

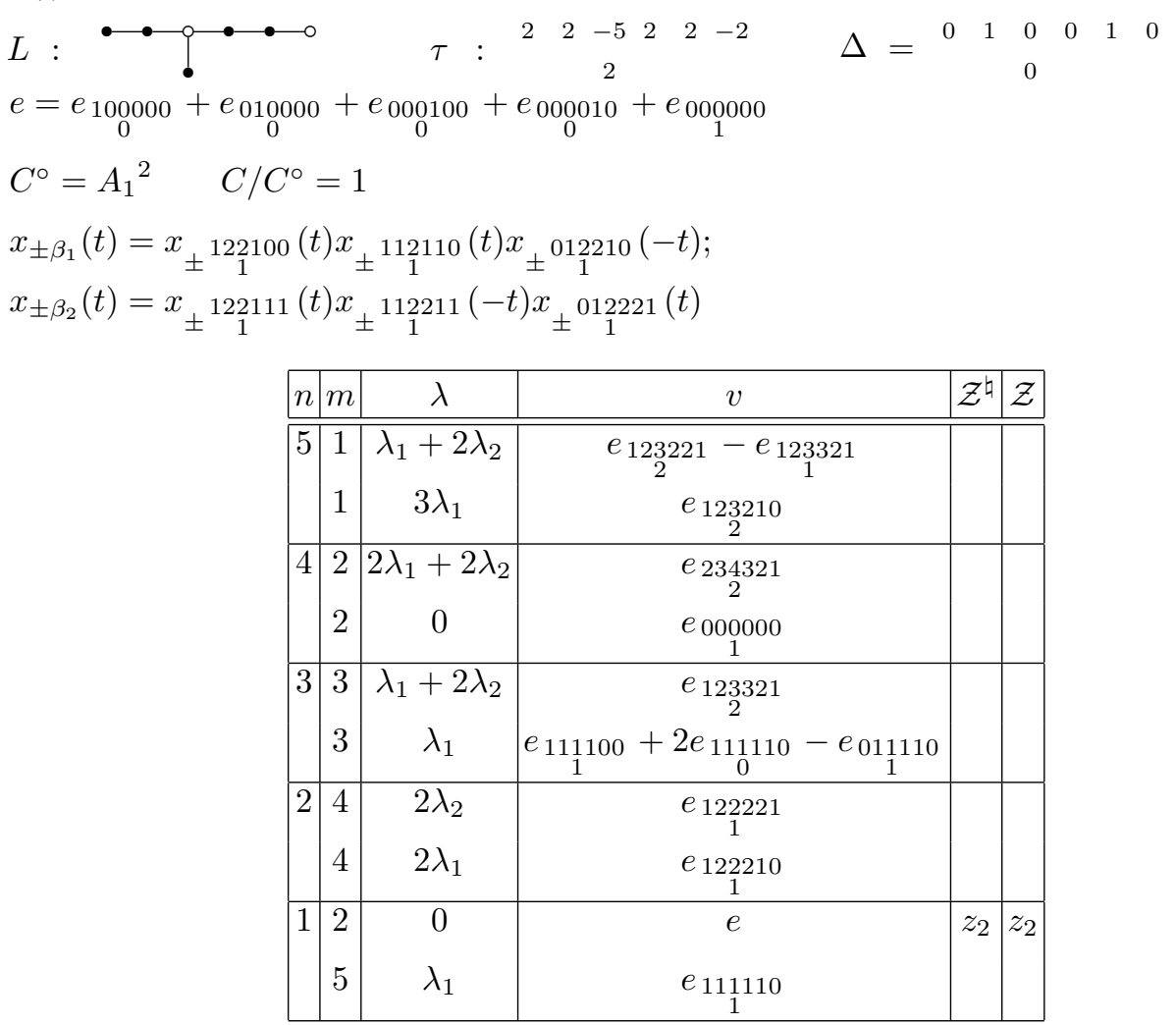


$E_{7}$, orbit 14: $\left(A_{3} A_{1}\right)^{\prime}$

$$
\begin{aligned}
& L: \bullet \longrightarrow \cdots \cdots \\
& \tau \quad \cdot \begin{array}{lllllll}
2 & 2 & 2 & -4 & 2 & -1
\end{array} \\
& \Delta=\begin{array}{llllll}
1 & 0 & 1 & 0 & 0 & 0 \\
& & & 0 & &
\end{array} \\
& e=\underset{0}{e} \underset{0}{100000}+\underset{0}{e 010000}+e_{0}^{001000}+e_{000010}^{000} \\
& C^{\circ}=A_{1}{ }^{3} \quad C / C^{\circ}=1 \\
& \beta_{1}={ }_{2}^{123210} ; \beta_{2}={ }_{1}^{123321} ; x_{ \pm \beta_{3}}(t)=x_{ \pm}{ }_{1}^{111111}(t) x_{ \pm} 012111(-t)
\end{aligned}
$$

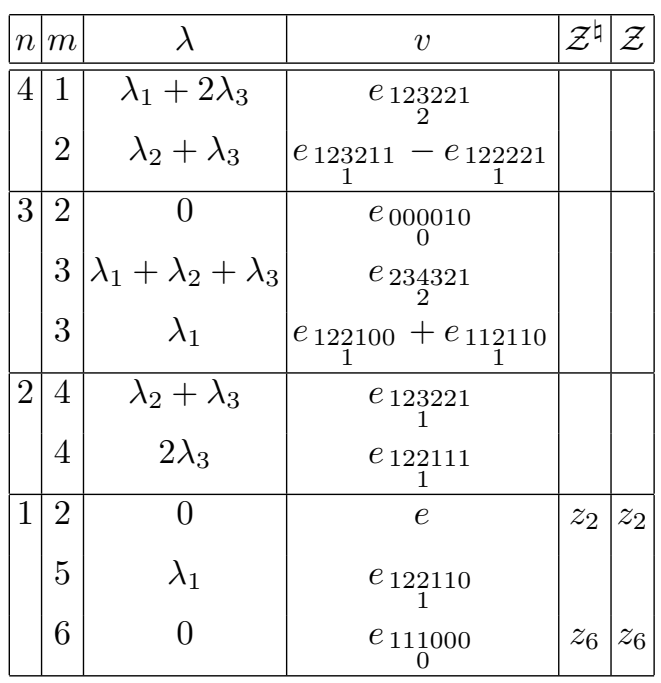


$E_{7}$, orbit 15: $D_{4}\left(a_{1}\right)$

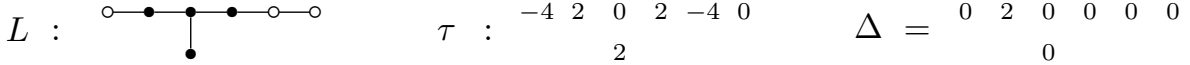

$$
\begin{aligned}
& e=\underset{0}{010000}+e_{1}^{001000}+e_{0}^{001100}+e_{1}^{000000}+e_{0}^{000100} \\
& C^{\circ}=A_{1}{ }^{3} \quad C / C^{\circ}=\left\langle c_{1} C^{\circ}, c_{2} C^{\circ}\right\rangle \cong S_{3} \\
& \beta_{1}={ }_{0}^{000001} ; \beta_{2}={ }_{1}^{012221} ; \beta_{3}={ }_{2}^{234321} \\
& c_{1}=n_{111000} n_{111100} h_{2}(-1) \text {, } \\
& c_{2}=\left(n_{122110} n_{112210} h_{1}(-1) h_{2}(-1) h_{6}(-1)\right)^{g}, \\
& g=x_{0}^{001000}\left(\frac{1}{3}\right) n_{001000} h_{1}(4) h_{2}(-4) h_{3}(16) h_{4}(-48) h_{5}(16) h_{6}(-8) x_{001000}\left(-\frac{1}{3}\right)
\end{aligned}
$$

\begin{tabular}{|c|c|c|c|c|c|}
\hline$n$ & & $\lambda$ & $v$ & $\mathcal{Z}^{\natural}$ & $\mathcal{Z}$ \\
\hline 3 & & $\lambda_{2}+\lambda_{3}$ & $e_{123321}$ & & \\
\hline & 2 & $\lambda_{1}+\lambda_{3}$ & $e_{122211}-e_{123211}$ & & \\
\hline & 2 & $\lambda_{1}+\lambda_{2}$ & $e_{011111}+e_{012111}$ & & \\
\hline & 2 & 0 & $2 e_{000000}-e_{001000}+e_{011000}+e_{001100}$ & & \\
\hline & 2 & 0 & $e_{000000}+e_{000100}$ & & \\
\hline 2 & 4 & $\lambda_{2}+\lambda_{3}$ & $e_{134321}$ & & \\
\hline & 4 & $\lambda_{1}+\lambda_{3}$ & $e_{123211}$ & & \\
\hline & 4 & $\lambda_{1}+\lambda_{2}$ & $e_{012211}$ & & \\
\hline & 4 & 0 & $e_{011000}+2 e_{001100}-e_{011100}$ & & \\
\hline 1 & 2 & 0 & $e$ & $z_{2}$ & $z_{2}$ \\
\hline & 6 & 0 & $e_{011100}$ & $z_{6}{ }^{1}$ & \\
\hline & 6 & 0 & $e_{012100}$ & $z_{6}^{2}$ & \\
\hline
\end{tabular}


$E_{7}$, orbit 16: $A_{3} A_{1}^{2}$

$$
\begin{aligned}
& L: \quad \bullet ? \cdot \bullet \bullet \quad \tau: \begin{array}{cccccc}
-1 & 2 & -5 & 2 & 2 & 2
\end{array} \quad \Delta=\begin{array}{cccccc}
1 & 0 & 0 & 1 & 0 & 1
\end{array} \\
& e=\underset{0}{e 000100}+\underset{0}{e 000010}+\underset{0}{e 00001}+\underset{0}{e 00000}+\underset{0}{00000} \\
& C^{\circ}=A_{1}^{2} \quad C / C^{\circ}=1 \\
& \beta_{1}={ }_{2}^{234321} ; x_{ \pm \beta_{2}}(t)=x_{ \pm} 012111(t) x_{ \pm} 012210(t)
\end{aligned}
$$

\begin{tabular}{|c|c|c|c|c|c|}
\hline \begin{tabular}{l|l}
$n$ & $r$
\end{tabular} & $m$ & $\lambda$ & $v$ & & $\mathcal{Z}$ \\
\hline 6 & $\begin{array}{l}1 \\
1\end{array}$ & $\begin{array}{c}\lambda_{1}+2 \lambda_{2} \\
\lambda_{2}\end{array}$ & $\begin{array}{c}e_{134321} \\
e_{0} \\
e_{011110}+e_{001110}+e_{001111}+2 e_{011100}\end{array}$ & & \\
\hline 5 & $\begin{array}{l}2 \\
2\end{array}$ & $\begin{array}{c}\lambda_{1}+\lambda_{2} \\
0 \\
0\end{array}$ & $\begin{array}{c}e_{123221}-e_{123321} \\
e_{000000} \\
1 \\
e_{010000} \\
0\end{array}$ & & \\
\hline 4 & $\begin{array}{l}3 \\
3\end{array}$ & $\begin{array}{l}\lambda_{1} \\
\lambda_{2} \\
\lambda_{2}\end{array}$ & $\begin{array}{l}e_{122211}-e_{112221} \\
e_{1} \\
e_{1} \\
e_{1}+e_{00111} \\
e_{11110}+e_{011111} \\
01\end{array}$ & & \\
\hline \begin{tabular}{|l|}
3 \\
\end{tabular} & 4 & $\begin{array}{c}\lambda_{1}+\lambda_{2} \\
2 \lambda_{2}\end{array}$ & $\begin{array}{l}e_{123} 2321 \\
2 \\
e_{012221} \\
\end{array}$ & & \\
\hline 2 & 5 & $\lambda_{2}$ & $e_{011111}$ & & \\
\hline 1 & 6 & $\begin{array}{c}0 \\
\lambda_{1} \\
0\end{array}$ & $\begin{array}{c}e \\
e_{12221} \\
e_{000111}\end{array}$ & $z_{2}$ & $z_{2}$ \\
\hline
\end{tabular}

$E_{7}$, orbit 17: $D_{4}$

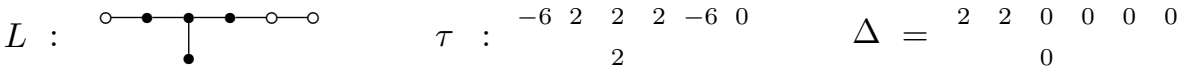

$$
\begin{aligned}
& e=e_{010000}+e_{001000}+e_{000000}+e_{000100} \\
& C^{\circ}=C_{3} \quad C / C^{\circ}=1 \\
& x_{ \pm \beta_{1}}(t)=x_{ \pm}{ }_{1}^{111000}(t) x_{ \pm}{ }_{0}^{111100}(t), x_{ \pm \beta_{2}}(t)=x_{ \pm}{ }_{1}^{00110}(t) x_{ \pm} 011110(-t), \beta_{3}={ }_{0}^{000001}
\end{aligned}
$$

\begin{tabular}{|c|c|c|c|c|c|}
\hline$n$ & $m$ & $\lambda$ & $v$ & $\mathcal{Z}^{\natural}$ & $\mathcal{Z}$ \\
\hline \hline 1 & 2 & 0 & $e$ & $z_{2}$ & $z_{2}$ \\
& 6 & $\lambda_{2}$ & $e_{134321}$ & & \\
& 10 & 0 & $e_{012100}$ & $z_{10}$ & $z_{10}$ \\
\hline
\end{tabular}


$E_{7}$, orbit 18: $D_{4}\left(a_{1}\right) A_{1}$

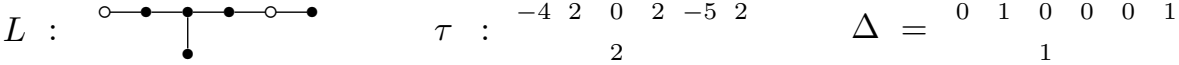

$$
\begin{aligned}
& e=\underset{0}{0010000}+e_{1}^{001000}+e_{0}^{001100}+e_{1}^{000000}+e_{0}^{000100}+e_{0}^{000001} \\
& C^{\circ}=A_{1}{ }^{2} \quad C / C^{\circ}=\left\langle c C^{\circ}\right\rangle \cong S_{2} \\
& \beta_{1}={ }_{1}^{012221} ; \beta_{2}={ }_{2}^{234321} \\
& c=n_{111000} n_{111100} h_{2}(-1)
\end{aligned}
$$

\begin{tabular}{|c|c|c|c|c|c|}
\hline$n \mid r$ & & $\lambda$ & $v$ & $\mathcal{Z}^{\natural}$ & $\mathcal{Z}$ \\
\hline \begin{tabular}{l|l}
6 &
\end{tabular} & 1 & $\begin{array}{l}\lambda_{2} \\
\lambda_{1}\end{array}$ & $\mid \begin{array}{ccc}e_{122111}+e_{123210}-2 e_{112211}-e_{122210} & 1 \\
1 & 1 & 1 \\
e_{011110}+e_{012110}+2 e_{001111}-e_{011111} & 0 \\
1\end{array}$ & & \\
\hline 5 & 21 & $\lambda_{1}+\lambda_{2}$ & $e_{123321}$ & & \\
\hline & 2 & 0 & $2 e_{000000}-e_{001000}+e_{011000}^{010}+e_{001100}$ & & \\
\hline & 2 & 0 & $e_{000000}+e_{0}^{000100}$ & & \\
\hline & 2 & 0 & $\begin{array}{c}e_{000001} \\
0\end{array}$ & & \\
\hline 4 & 3 & $\lambda_{2}$ & $e_{123210}+e_{123211}$ & & \\
\hline & 3 & $\lambda_{2}$ & $e_{122211}-e_{123211}$ & & \\
\hline & 3 & $\lambda_{1}$ & $e_{012111}+e_{012210}$ & & \\
\hline & 3 & $\lambda_{1}$ & $e_{012111}^{e_{1}}+e_{011111}$ & & \\
\hline 3 & 41 & $\lambda_{1}+\lambda_{2}$ & $e_{134321}$ & & \\
\hline & 4 & 0 & $e_{1} e_{11000}+2 e_{001100}-e_{011100}$ & & \\
\hline 2 & 5 & $\lambda_{2}$ & $e_{123211}$ & & \\
\hline & 5 & $\lambda_{1}$ & $e_{012211}^{0}$ & & \\
\hline 1 & 2 & 0 & $e$ & $z_{2}$ & $z_{2}$ \\
\hline & 6 & 0 & $e_{0}^{011100}$ & $z_{6}{ }^{1}$ & \\
\hline & 6 & 0 & $e_{012100}$ & $z_{6}{ }^{2}$ & $z_{6}{ }^{2}$ \\
\hline
\end{tabular}


$E_{7}$, orbit 19: $A_{3} A_{2}$

$$
\begin{aligned}
& L: \quad \bullet \quad \cdots \cdots \cdot \tau: \begin{array}{llllll}
-3 & 2 & 2 & -6 & 2 & 2
\end{array} \quad \Delta=\begin{array}{llllll}
0 & 0 & 1 & 0 & 1 & 0
\end{array} \\
& e=\underset{0}{010000}+\underset{0}{0001000}+e_{0}^{000000}+e_{0}^{000010}+e_{0}^{000001} \\
& C^{\circ}=A_{1} T_{1} \quad C / C^{\circ}=\left\langle c C^{\circ}\right\rangle \cong S_{2} \\
& \beta_{1}={ }_{2}^{234321} \\
& T_{1}=\left\{h_{2}\left(\mu^{3}\right) h_{3}\left(\mu^{3}\right) h_{4}\left(\mu^{6}\right) h_{5}\left(\mu^{6}\right) h_{6}\left(\mu^{4}\right) h_{7}\left(\mu^{2}\right): \mu \in k^{*}\right\} \\
& c=n_{1}^{001110} \underset{0}{011110} n_{012211} h_{2}(-1) h_{4}(-1) h_{5}(-1) h_{6}(-1) h_{7}(-1)
\end{aligned}
$$

\begin{tabular}{|c|c|c|c|c|c|}
\hline$n$ & $m$ & $\lambda$ & $v$ & $\mathcal{Z}^{\natural}$ & $\mathcal{Z}$ \\
\hline 6 & 1 & $\overline{\lambda_{1}}$ & $e_{122100}+e_{112110}+e_{111111}$ & & \\
\hline & 1 , & $\lambda_{1}$ & $e_{123210}+e_{123211}-e_{122221}$ & & \\
\hline 5 & 2 & 0 & $f_{012210}$ & & \\
\hline & 2 & 0 & $e_{012221}$ & & \\
\hline & 2 & 0 & $f_{011100}+f_{001110}+f_{000111}$ & & \\
\hline & 2 & 0 & $e_{1}^{012100}+e_{011110}^{0}+e_{0}^{011111}$ & & \\
\hline & 2 & 0 & $f_{011100}-f_{001100}$ & & \\
\hline & 2 & 0 & $e_{011111}-e_{001111}$ & & \\
\hline & 2 & 0 & $\underset{0}{e} e_{000010}+e_{000001}$ & & \\
\hline 4 & 3 & $\lambda_{1}$ & $e_{111000}$ & & \\
\hline & 3 & $\lambda_{1}$ & $e_{1342321}$ & & \\
\hline & 3 & $\lambda_{1}$ & $e_{122110}+e_{112111}$ & & \\
\hline & 3 & $\lambda_{1}$ & $e_{123211}-e_{123221}$ & & \\
\hline \begin{tabular}{|l|}
3 \\
\end{tabular} & \begin{tabular}{|l|}
4 \\
\end{tabular} & 0 & $f_{001100}+f_{000110}$ & & \\
\hline & 4 & 0 & $e_{012110}+e_{011111}$ & & \\
\hline & 4 & 0 & $e_{0}^{000011}$ & & \\
\hline 2 & 4 & 0 & $e_{011000}-e_{001000}+e_{000011}$ & & \\
\hline & 5 & $\lambda_{1}$ & $e_{122111}$ & & \\
\hline & 5 & $\lambda_{1}$ & $e_{123221}$ & & \\
\hline 1 & 2 & 0 & $e$ & $z_{2}$ & $z_{2}$ \\
\hline & 6 & 0 & $f_{000100}$ & & \\
\hline & 6 & 0 & $e_{012111}$ & & \\
\hline & 6 & 0 & $e_{0}^{011000}$ & $z_{6}$ & 26 \\
\hline
\end{tabular}


$E_{7}$, orbit 20: $A_{4}$

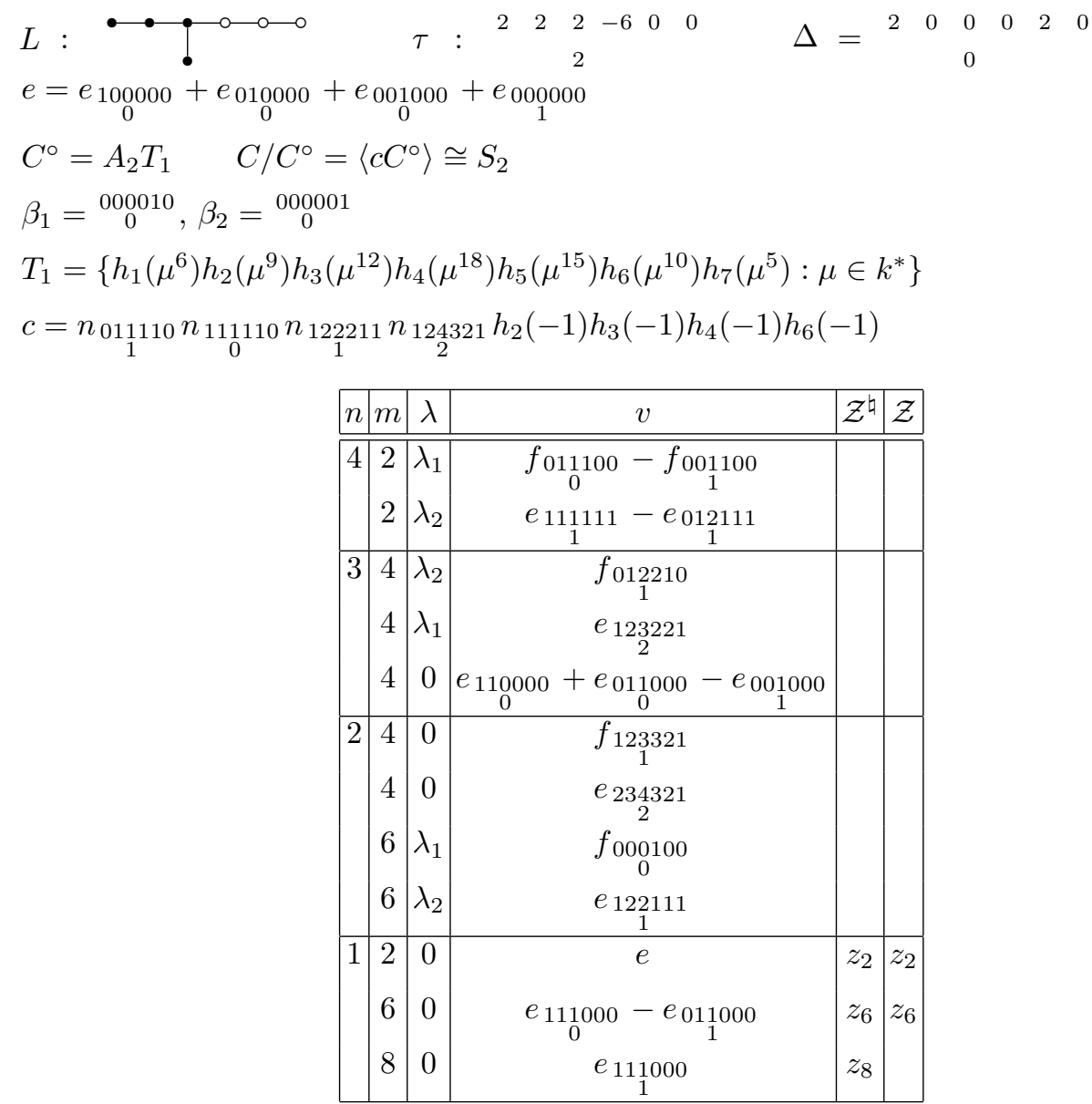

$E_{7}$, orbit 21: $A_{3} A_{2} A_{1}$

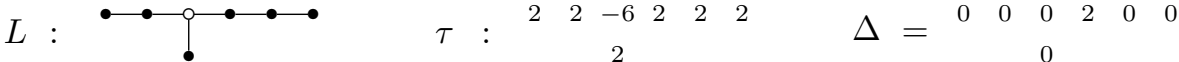

$$
\begin{aligned}
& e=\underset{0}{e_{000100}}+\underset{0}{e_{000010}}+e_{000001}+e_{100000}+e_{010000}+e_{000000} \\
& C^{\circ}=A_{1} \quad C / C^{\circ}=1 \\
& x_{\beta_{1}}(t)=x_{1}^{011100}(2 t) x_{0}^{001111}(t) x_{111000}(3 t) x_{011110}(t) x_{12211}\left(t^{2}\right) x_{112111}\left(\frac{3}{2} t^{2}\right) \times \\
& x_{122110}\left(-\frac{3}{2} t^{2}\right) x_{123211}\left(-2 t^{3}\right) x_{123221}\left(-t^{3}\right) x_{1342321}\left(\frac{9}{4} t^{4}\right) x_{0}^{111100}(t) x_{1}^{001110}(t) \times \\
& x_{112210}\left(-\frac{1}{2} t^{2}\right) \text {, } \\
& x_{-\beta_{1}}(t)=x_{-} 011100(2 t) x_{-}{ }_{0}^{001111}(6 t) x_{-}{ }_{1}^{111000}(2 t) x_{-}{ }_{0}^{011110}(4 t) x_{-}{ }_{0}^{012211}\left(-6 t^{2}\right) \times \\
& x_{-} 112111\left(-6 t^{2}\right) x_{-}{ }_{1}^{122110}\left(4 t^{2}\right) x_{-}{ }_{12}^{223211}\left(-8 t^{3}\right) x_{-}{ }_{1}^{123221}\left(-16 t^{3}\right) x_{-}{ }_{13}^{134321}\left(-36 t^{4}\right) \times \\
& x_{-}{ }_{0}^{111100}(2 t) x_{-} 001110(2 t) x_{-}{ }_{1}^{112210}\left(2 t^{2}\right)
\end{aligned}
$$




\begin{tabular}{|c|c|c|c|c|c|}
\hline$n$ & $m$ & $\lambda$ & $v$ & $\mathcal{Z}^{\natural}$ & $\mathcal{Z}$ \\
\hline \hline 3 & 2 & $8 \lambda_{1}$ & $e_{234321}$ & & \\
& 2 & $4 \lambda_{1}$ & $3 e_{122111}+2 e_{122210}+e_{012221}-e_{112211}$ & & \\
& 2 & 4 & $6 \lambda_{1}$ & $e_{123321}$ & \\
& & $2 \lambda_{1}$ & $e_{111110}-e_{111111}+2 e_{011111}$ & & \\
& 4 & & 0 & & \\
\hline 1 & 2 & 0 & $e$ & $z_{2}$ & $z_{2}$ \\
& 6 & $4 \lambda_{1}$ & $e_{122221}$ & & \\
& & & & \\
\hline
\end{tabular}

If $p=5$, the two summands with $m=2$ and high weights $8 \lambda_{1}$ and 0 are replaced by a single reducible tilting module $T_{A_{1}}\left(8 \lambda_{1}\right)$ generated by the two given vectors together with $3 e_{1}^{000000}-e_{0} 00000-e_{0}^{010000}$; also the two summands with $m=4$ and high weights $6 \lambda_{1}$ and $2 \lambda_{1}$ are replaced by a single reducible tilting module $T_{A_{1}}\left(6 \lambda_{1}\right)$ generated by the two given vectors together with $e_{111110}+e_{11111}$.

If $p=7$, the two summands with $m=2$ and high weights $8 \lambda_{1}$ and $4 \lambda_{1}$ are replaced by a single reducible tilting module $T_{A_{1}}\left(8 \lambda_{1}\right)$ generated by the two given vectors together with $e_{122111}+e_{122210}$.

$E_{7}$, orbit 22: $\left(A_{5}\right)^{\prime \prime}$

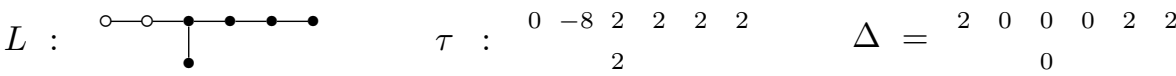

$$
\begin{aligned}
& e=e_{000000}+e_{001000}+e_{000100}^{0}+e_{000010}+e_{000001} \\
& C^{\circ}=G_{2} \quad C / C^{\circ}=1 \\
& x_{ \pm \beta_{1}}(t)=x_{ \pm}^{011110}(t) x_{ \pm}^{011111}(t) x_{ \pm}^{012100}(t), \beta_{2}=\underset{0}{100000}
\end{aligned}
$$

\begin{tabular}{|c|c|c|c|c|c|}
\hline$n$ & $m$ & $\lambda$ & $v$ & $\mathcal{Z}^{\natural}$ & $\mathcal{Z}$ \\
\hline \hline 2 & 4 & $\lambda_{1}$ & $e_{123221}-e_{123321}$ & & \\
\hline 1 & 2 & 0 & $e$ & $z_{2}$ & $z_{2}$ \\
& 6 & 0 & $e_{001100}+e_{001110}+e_{000111}$ & $z_{6}$ & $z_{6}$ \\
& & & 0 & 0 \\
& 8 & $\lambda_{1}$ & $e_{124321}$ & & \\
& & 0 & $e_{001111}$ & $z_{10}$ & $z_{10}$ \\
& 10 & 0 & & & \\
\hline
\end{tabular}


$E_{7}$, orbit 23: $D_{4} A_{1}$

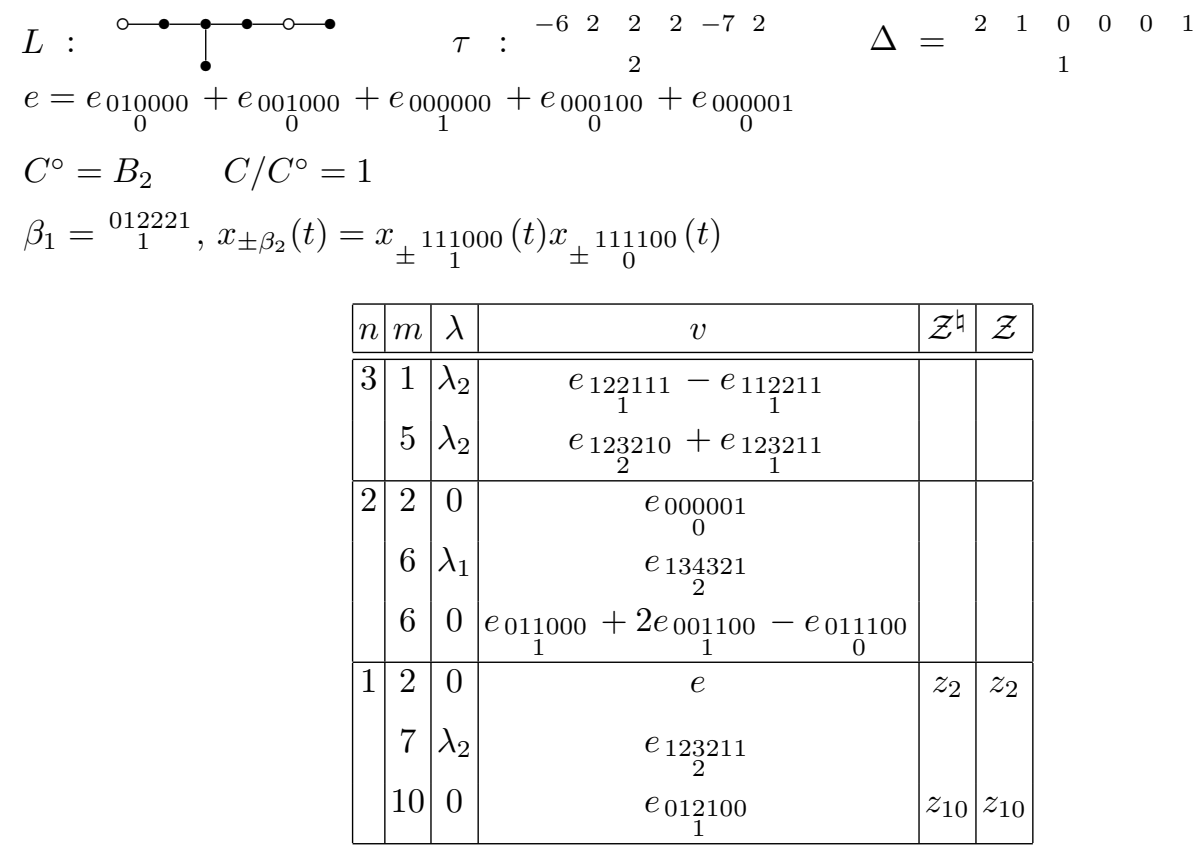

$E_{7}$, orbit $24: A_{4} A_{1}$

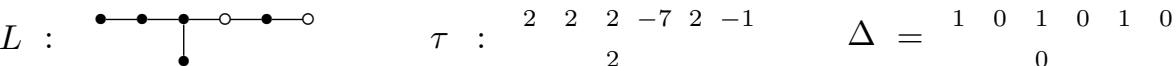

$$
\begin{aligned}
& e=e_{100000}+e_{010000}+e_{001000}+e_{000000}+e_{000010} \\
& C^{\circ}=T_{2} \quad C / C^{\circ}=\left\langle c C^{\circ}\right\rangle \cong S_{2} \\
& T_{2}=\left\{h_{1}\left(\mu^{2}\right) h_{2}\left(\mu^{3}\right) h_{3}\left(\mu^{4}\right) h_{4}\left(\mu^{6}\right) h_{5}\left(\mu^{5}\right) h_{6}\left(\mu^{3} \nu\right) h_{7}\left(\mu \nu^{2}\right): \mu, \nu \in k^{*}\right\} \\
& c=n_{011111} n_{111111} n_{122210} n_{124321} h_{1}(-1) h_{3}(-1) h_{4}(-1) h_{6}(-1)
\end{aligned}
$$




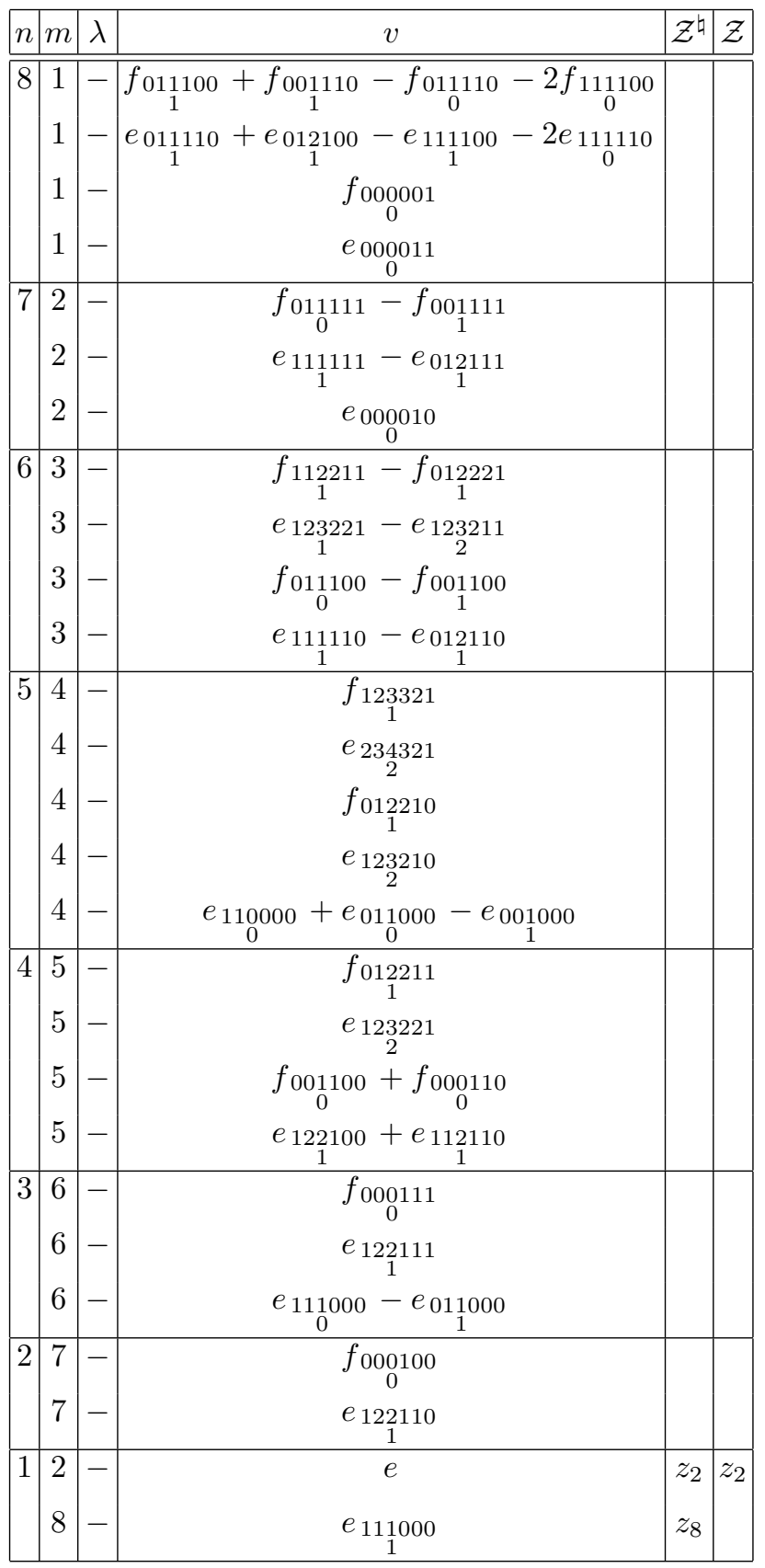


$E_{7}$, orbit 25: $D_{5}\left(a_{1}\right)$

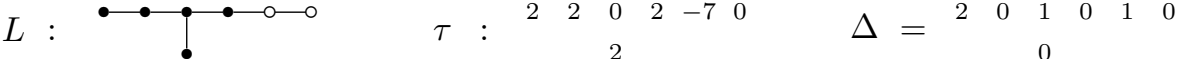

$$
\begin{aligned}
& e=\underset{0}{e} \underset{0}{100000}+e_{1}^{010000}+e_{001000}^{0}+e_{0}^{001100}+e_{1}^{000000}+e_{0}^{000100} \\
& C^{\circ}=A_{1} T_{1} \quad C / C^{\circ}=\left\langle c C^{\circ}\right\rangle \cong S_{2} \\
& \beta_{1}=\underset{0}{00001} \\
& T_{1}=\left\{h_{1}\left(\mu^{2}\right) h_{2}\left(\mu^{3}\right) h_{3}\left(\mu^{4}\right) h_{4}\left(\mu^{6}\right) h_{5}\left(\mu^{5}\right) h_{6}\left(\mu^{4}\right) h_{7}\left(\mu^{2}\right): \mu \in k^{*}\right\} \\
& c=n_{123321} n_{123221} h_{1}(-1) h_{2}(-1) h_{4}(-1)
\end{aligned}
$$

\begin{tabular}{|c|c|c|c|c|c|}
\hline$|n|$ & $m$ & $\lambda$ & $v$ & $\mathcal{Z}^{\natural}$ & $\mathcal{Z}$ \\
\hline 5 & 1 & $\lambda_{1}$ & $f_{111110}+f_{012110}$ & & \\
\hline & 1 & $\lambda_{1}$ & $e_{111111}+e_{012211}$ & & \\
\hline & 4 & 0 & $e_{0}^{111000}+e_{011100}-e_{011000}-2 e_{001100}$ & & \\
\hline 4 & 2 & 0 & $f_{123221}$ & & \\
\hline & 2 & 0 & $e_{123321}$ & & \\
\hline & 2 & 0 & $e_{000000}+e_{000100}$ & & \\
\hline & 5 & $\lambda_{1}$ & $f_{000110}-f_{001110}^{0}$ & & \\
\hline & 5 & $\lambda_{1}$ & $e_{122211}-e_{123211}$ & & \\
\hline 3 & 6 & 0 & $f_{012221}$ & & \\
\hline & 6 & 0 & $e_{234321}$ & & \\
\hline & 6 & 0 & $e_{111100}-e_{011100}+e_{012100}$ & & \\
\hline & 6 & 0 & $e_{111000}+e_{011100}+e_{012100}$ & & \\
\hline 2 & 7 & $\lambda_{1}$ & $f_{000010}$ & & \\
\hline & 7 & $\lambda_{1}$ & $e_{123211}$ & & \\
\hline 1 & 2 & 0 & e & $z_{2}$ & $z_{2}$ \\
\hline & 8 & 0 & $e_{111100}$ & $z_{8}$ & \\
\hline & 10 & 0 & $e_{122100}$ & $z_{10}$ & $z_{10}$ \\
\hline
\end{tabular}


$E_{7}$, orbit 26: $A_{4} A_{2}$

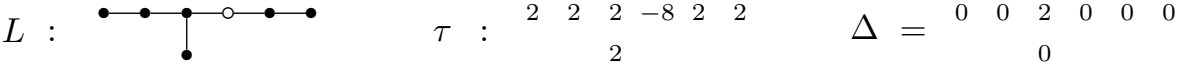

$$
\begin{aligned}
& e=\underset{0}{e}+\underset{0}{00000}+\underset{0}{010000}+e_{0}^{001000}+e_{0}^{000000}+e_{0}^{000010}+e_{00001}^{0000} \\
& C^{\circ}=A_{1} \quad C / C^{\circ}=1 \\
& x_{\beta_{1}}(t)=x_{001111}(3 t) x_{0}^{111110}(-2 t) x_{012100}(t) x_{112221}\left(3 t^{2}\right) x_{123210}\left(-t^{2}\right) x_{124321}\left(-2 t^{3}\right) \times \\
& x_{111100}(-t) x_{0}^{011111}(-2 t) x_{122211}\left(t^{2}\right) x_{011110}(t), \\
& x_{-\beta_{1}}(t)=x_{-}^{001111}(t) x_{-\underset{0}{111110}}(-2 t) x_{-}^{012100}(3 t) x_{-}^{112221}\left(-t^{2}\right) x_{-}^{123210}\left(3 t^{2}\right) \times \\
& x+\underset{2}{124321}\left(-2 t^{3}\right) x_{-} 111100(-2 t) x_{-} \underset{0}{011111}(-t) x_{-}^{122211}\left(-t^{2}\right) x_{-}{ }_{1}^{011110}(t)
\end{aligned}
$$

\begin{tabular}{|c|c|c|c|c|c|}
\hline$n$ & $m$ & $\lambda$ & $v$ & $\mathcal{Z}^{\text {到 }}$ & $\mathcal{Z}$ \\
\hline 4 & 2 & $4 \lambda_{1}$ & $e_{123210}+e_{123211}-e_{122221}$ & & \\
\hline 3 & $\begin{array}{l}4 \\
4\end{array}$ & $\begin{array}{l}6 \lambda_{1} \\
2 \lambda_{1}\end{array}$ & $\begin{array}{c}e_{234321} \\
2 \\
e_{122100}+e_{112110}+2 e_{111111}-e_{012111}\end{array}$ & & \\
\hline 2 & \begin{tabular}{|l|}
6 \\
\end{tabular} & $4 \lambda_{1}$ & $e_{123221}$ & & \\
\hline 1 & $\begin{array}{l}2 \\
8\end{array}$ & $\begin{array}{c}0 \\
2 \lambda_{1}\end{array}$ & $\begin{array}{c}e \\
e_{122111}\end{array}$ & $z_{2}$ & $z_{2}$ \\
\hline
\end{tabular}

If $p=5$, the two summands with $m=4$ and high weights $6 \lambda_{1}$ and $2 \lambda_{1}$ are replaced by a single reducible tilting module $T_{A_{1}}\left(6 \lambda_{1}\right)$ generated by the two given vectors together with $e_{111111}-e_{012111}$.

$E_{7}$, orbit $27:\left(A_{5}\right)^{\prime}$

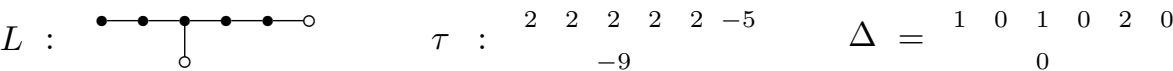

$$
\begin{aligned}
& e=e_{100000}+e_{010000}+e_{001000}+e_{000100}+e_{000010} \\
& C^{\circ}=A_{1}^{2} \quad C / C^{\circ}=1 \\
& \beta_{1}={ }_{2}^{123210} ; x_{ \pm \beta_{2}}(t)=x_{ \pm}{ }_{1}^{122111}(t) x_{ \pm} \underset{1}{112211}(-t) x_{ \pm} \underset{1}{012221}(t)
\end{aligned}
$$




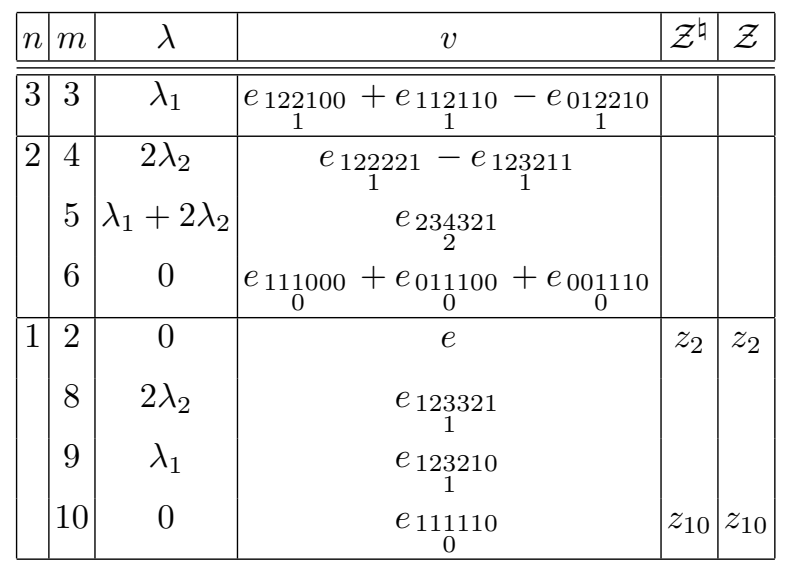

$E_{7}$, orbit 28: $A_{5} A_{1}$

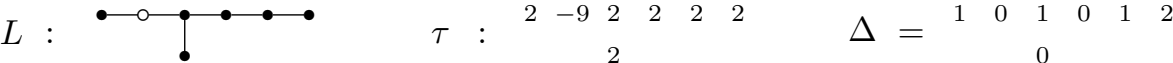

$$
\begin{aligned}
& e=e_{000000}+e_{001000}+e_{000100}+e_{000010}+e_{000001}+e_{100000} \\
& C^{\circ}=A_{1} \quad C / C^{\circ}=1 \\
& x_{ \pm \beta_{1}}(t)=x_{ \pm}{ }_{2}^{123210}(t) x_{ \pm}{ }_{1}^{123211}(t) x_{ \pm}{ }_{1}^{12221}(-t)
\end{aligned}
$$

\begin{tabular}{|c|c|c|c|c|c|}
\hline$n$ & & $\lambda$ & $v$ & $\mathcal{Z}^{\natural}$ & $\mathcal{Z}$ \\
\hline 5 & $\begin{array}{l}1 \\
3\end{array}$ & $\begin{array}{l}3 \lambda_{1} \\
\lambda_{1}\end{array}$ & $\begin{array}{c}e_{234321} \\
e_{112110}-e_{012210}-e_{012111}+2 e_{111111} \\
e_{1}\end{array}$ & & \\
\hline 4 & 2 & $\begin{array}{r}0 \\
2 \lambda_{1}\end{array}$ & $\begin{array}{c}e_{100000} \\
0 \\
e_{123321}-e_{123221} \\
1\end{array}$ & & \\
\hline 3 & $\begin{array}{l}5 \\
7\end{array}$ & $\begin{array}{l}\lambda_{1} \\
\lambda_{1}\end{array}$ & $\begin{array}{l}e_{112210}+e_{112111} \\
e_{1}{ }_{1} 211-e_{012221}\end{array}$ & & \\
\hline 2 & 6 & $\begin{array}{r}0 \\
2 \lambda_{1}\end{array}$ & $\begin{array}{c}e_{001100}^{0}+e_{0}^{001110}+e_{0}^{000111} \\
e_{124321}\end{array}$ & & \\
\hline 1 & $\begin{array}{c}2 \\
9 \\
10\end{array}$ & $\begin{array}{c}0 \\
\lambda_{1} \\
0\end{array}$ & $\begin{array}{c}e \\
e_{112221} \\
e_{1} \\
e_{001111}\end{array}$ & $z_{2}$ & $z_{2}$ \\
\hline
\end{tabular}


$E_{7}$, orbit 29: $D_{5}\left(a_{1}\right) A_{1}$

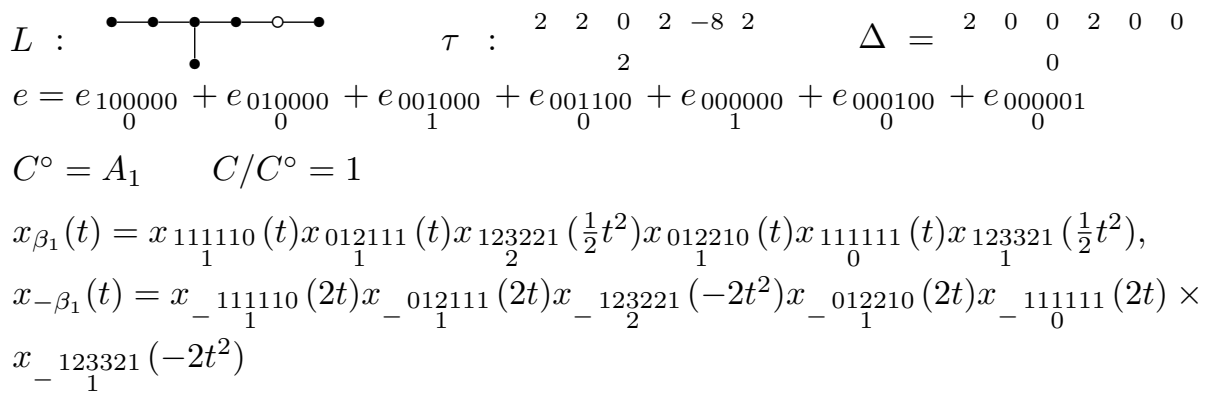

\begin{tabular}{|c|c|c|c|c|c|}
\hline$n$ & $m$ & $\lambda$ & $v$ & $\mathcal{Z}^{\natural}$ & $\mathcal{Z}$ \\
\hline \hline 3 & 2 & $4 \lambda_{1}$ & $e_{123321}$ & & \\
& 2 & 0 & $e_{000000}+e_{000100}+e_{000001}$ & 0 & \\
& & & 1 & & \\
& 4 & $2 \lambda_{1}$ & $e_{12211}+e_{123210}-e_{122210}-2 e_{112211}$ & & \\
\hline 2 & 6 & $4 \lambda_{1}$ & 1 & & \\
& 6 & $2 \lambda_{1}$ & $e_{123210}-e_{123211}+2 e_{122211}$ & & \\
& 2 & 0 & $e$ & $z_{2}$ & $z_{2}$ \\
\hline & 2 & 0 & $e_{123211}$ & & \\
& 8 & $2 \lambda_{1}$ & 2 & & \\
& 10 & 0 & $e_{122100}$ & $z_{10}$ \\
\hline
\end{tabular}


$E_{7}$, orbit 30: $D_{6}\left(a_{2}\right)$

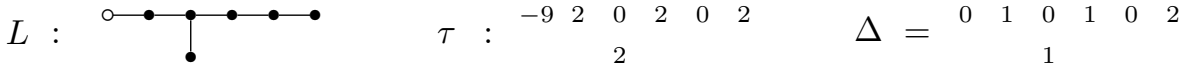

$$
\begin{aligned}
& e=\underset{0}{e 000001}+e_{0}^{000110}+e_{1}^{001000}-e_{0}^{011000}+e_{0}^{001100}+e_{000000}+e_{010000} \\
& C^{\circ}=A_{1} \quad C / C^{\circ}=1 \\
& \beta_{1}=\underset{2}{234321}
\end{aligned}
$$

\begin{tabular}{|c|c|c|c|c|c|}
\hline & $m$ & $\lambda$ & $v$ & $\mathcal{Z}^{\natural}$ & $\mathcal{Z}$ \\
\hline 5 & $\begin{array}{l}2 \\
2\end{array}$ & \begin{tabular}{l|} 
\\
0
\end{tabular} & $\begin{array}{c}e_{000000}-e_{010000}+e_{000100}-e_{001000}-e_{011000}-e_{000011}-e_{001110} \\
0 \\
e_{000000}+e_{010000}+e_{001100}\end{array}$ & & \\
\hline 4 & $\begin{array}{l}3 \\
3 \\
4\end{array}$ & $\begin{array}{c}\lambda_{1} \\
\lambda_{1} \\
0\end{array}$ & $\begin{array}{c}e_{122211}+e_{123221}-e_{122221}-e_{123211} \\
1 \\
e_{122211}+e_{123210} \\
1 \\
e_{001100}+2 e_{011000}+e_{1}^{001110}-e_{0} \\
011100\end{array}$ & & \\
\hline 3 & \begin{tabular}{l|}
5 \\
6 \\
6
\end{tabular} & $\begin{array}{l}\lambda_{1} \\
0 \\
0\end{array}$ & $\begin{array}{c}e_{123221}-e_{123211}+e_{123321} \\
e_{011110}+e_{011111}-e_{012100}-e_{012110} \\
e_{011100}\end{array}$ & & \\
\hline 2 & $\begin{array}{l}6 \\
7 \\
8\end{array}$ & $\begin{array}{c}0 \\
\lambda_{1} \\
0\end{array}$ & 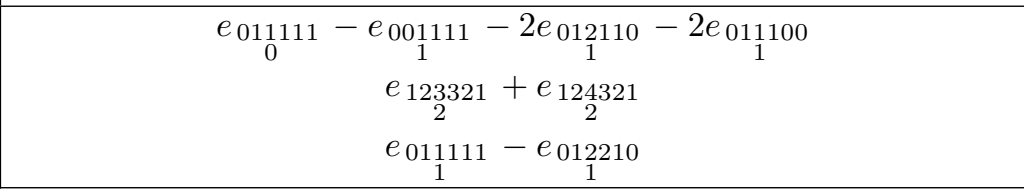 & & \\
\hline 1 & $\begin{array}{c}2 \\
9 \\
10 \\
10\end{array}$ & $\begin{array}{c}0 \\
\lambda_{1} \\
0 \\
0\end{array}$ & $\begin{array}{c}e \\
e \\
e_{134321} \\
e_{2} 012211 \\
1 \\
e_{012221}\end{array}$ & $\begin{array}{l}z_{10}{ }^{1} \\
z_{10}^{2}\end{array}$ & $\begin{array}{l}z_{10}^{1} \\
z_{10}^{2}\end{array} \mid$ \\
\hline
\end{tabular}

$E_{7}$, orbit 31: $E_{6}\left(a_{3}\right)$

$$
\begin{aligned}
& L: \bullet \bullet \bullet \bullet-\tau: \begin{array}{lllllll}
2 & 0 & 2 & 0 & 2 & -8
\end{array} \quad \Delta=\begin{array}{llllll}
0 & 2 & 0 & 0 & 2 & 0
\end{array} \\
& e=e_{011000}+e_{100000}+e_{011100}+e_{000010}+e_{001100}+e_{001000} \\
& C^{\circ}=A_{1} \quad C / C^{\circ}=\left\langle c C^{\circ}\right\rangle \cong S_{2} \\
& x_{ \pm \beta_{1}}(t)=x_{ \pm}{ }_{1}^{122111}(t) x_{ \pm}{ }_{1}^{112211}(t) x_{ \pm}{ }_{1}^{012221}(-t) \\
& c=h_{4}(-1)
\end{aligned}
$$




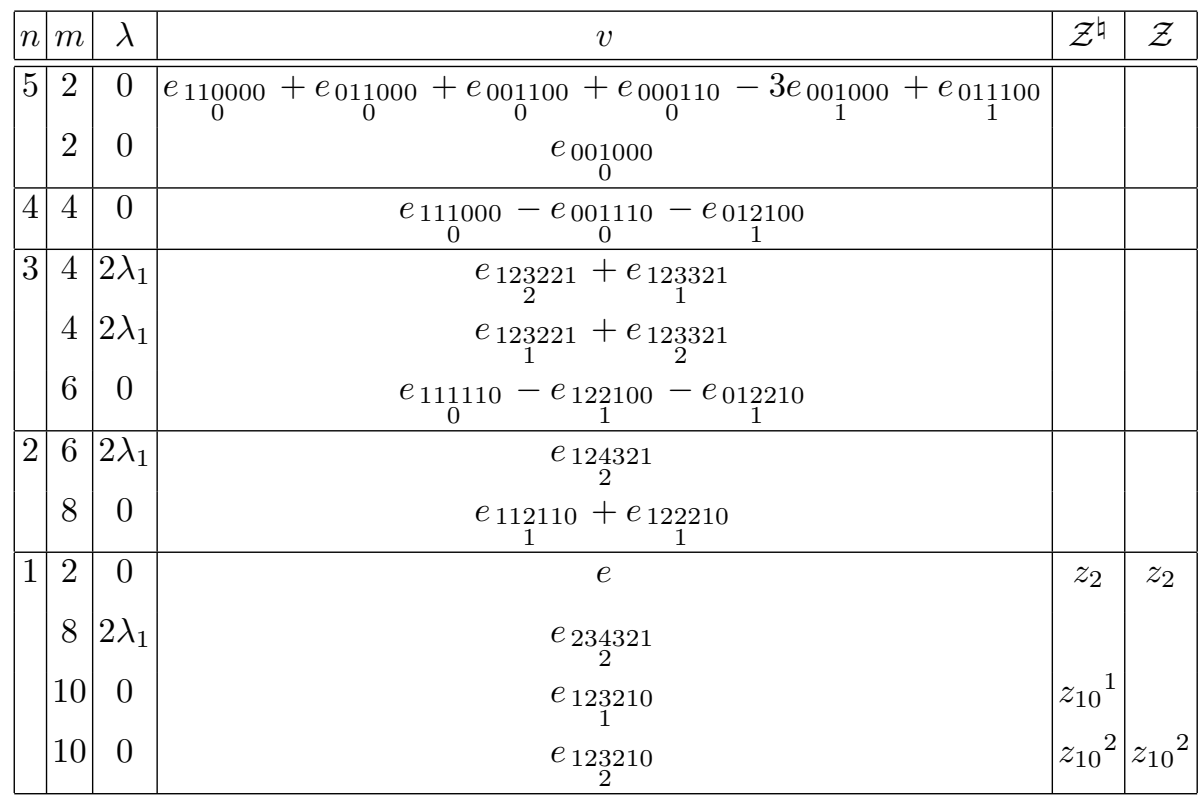

$E_{7}$, orbit $32: D_{5}$

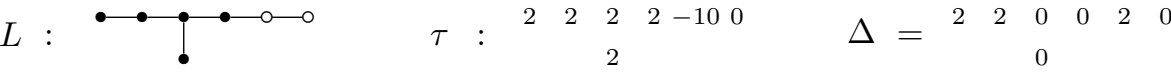

$$
\begin{aligned}
& e=e_{100000}+e_{010000}+e_{001000}+e_{000000}+e_{000100} \\
& C^{\circ}=A_{1}{ }^{2} \quad C / C^{\circ}=1
\end{aligned}
$$

\begin{tabular}{|c|c|c|c|c|}
\hline \begin{tabular}{|l|l|}
$n$ & $m$ \\
\end{tabular} & $\lambda$ & $v$ & $\mathcal{Z}^{\text {দ }}$ & $\mathcal{Z}$ \\
\hline \begin{tabular}{|l|l|}
3 & 4
\end{tabular} & $\lambda_{1}+\lambda_{2}$ & $e_{122111}-e_{112211}$ & & \\
\hline 6 & 0 & $e_{0} e_{111000}-e_{011000}-2 e_{001100}+e_{011100}$ & & \\
\hline \begin{tabular}{|l|l|l|}
2 & 10
\end{tabular} & $0 \lambda_{1}+\lambda_{2} \mid$ & $e_{123211}$ & & \\
\hline \begin{tabular}{|l|l|}
1 & 2 \\
\end{tabular} & 0 & $e$ & $z_{2}$ & $z_{2}$ \\
\hline 8 & $2 \lambda_{2}$ & $e_{234321}$ & & \\
\hline 10 & 0 & $e_{111100}-e_{012100}$ & $z_{10}$ & $z_{10}$ \\
\hline 14 & 0 & $e_{122100}$ & $z_{14}$ & $z_{14}$ \\
\hline
\end{tabular}

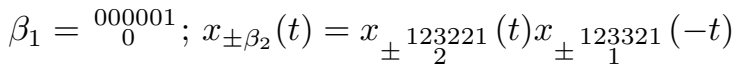


$E_{7}$, orbit $33: E_{7}\left(a_{5}\right)$

$$
\begin{aligned}
& L: \bullet \bullet \longrightarrow \bullet \bullet \\
& \tau \cdot \begin{array}{cccccc}
0 & 0 & 2 & 0 & 0 & 2
\end{array} \\
& \Delta=\begin{array}{llllll}
0 & 0 & 2 & 0 & 0 & 2
\end{array} \\
& e=e_{0}^{111100}+e_{1}^{00110}+e_{0}^{000001}+e_{0}^{011110}+e_{111000}+e_{0}^{001000}+e_{01100}^{0110} \\
& C^{\circ}=1 \quad C / C^{\circ}=\left\langle c_{1} C^{\circ}, c_{2} C^{\circ}\right\rangle \cong S_{3} \\
& c_{1}=h_{2}(\omega) h_{3}(\omega) h_{5}(\omega) \text {, } \\
& c_{2}=n_{000000} n_{010000} n_{000100} h_{3}(-1) h_{4}(-1)
\end{aligned}
$$

\begin{tabular}{|c|c|c|c|c|c|}
\hline$n$ & $m$ & $\lambda$ & $v$ & $\mathcal{Z}^{\natural}$ & $\mathcal{Z}$ \\
\hline 5 & 2 & - & 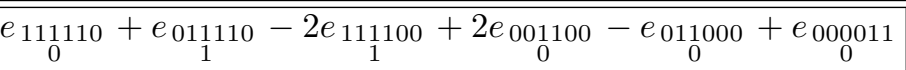 & & \\
\hline & 2 & - & $e_{111110}+e_{0}^{001110}-2 e_{111000}+2 e_{011000}-e_{0}^{001100}+e_{0}^{000111}$ & & \\
\hline & 2 & - & $e_{011000}+e_{001100}-e_{011100}$ & & \\
\hline & 2 & - & $e_{001100}+e_{011000}-e_{001000}^{001}$ & & \\
\hline & 2 & - & $e_{001000}+e_{011100}$ & & \\
\hline 4 & 4 & - & $e_{001111}+e_{112110}-2 e_{122100}-e_{012210}$ & & \\
\hline & 4 & - & $e_{011111}+e_{122210}-2 e_{112100}+e_{012110}$ & & \\
\hline & 4 & - & $e_{011111}-e_{001111}+e_{122110}-e_{112210}$ & & \\
\hline & 4 & - & $e_{012100}$ & & \\
\hline 3 & 6 & - & $e_{122211}+e_{112221}-e_{012111}$ & & \\
\hline & 6 & - & $e_{112111}-e_{122221}+e_{012211}$ & & \\
\hline & 6 & - & $e_{122111}+e_{112211}+e_{012221}$ & & \\
\hline & 6 & - & $e_{012111}+e_{123210}$ & & \\
\hline & 6 & - & $e_{012211}+e_{123210}$ & & \\
\hline 2 & 8 & - & $e_{123221}+e_{123211}$ & & \\
\hline & 8 & - & $e_{123321}+e_{123211}$ & & \\
\hline & 8 & - & $e_{123221}+e_{123321}$ & & \\
\hline 1 & 2 & - & $e$ & $z_{2}$ & $z_{2}$ \\
\hline & 10 . & & $e_{124321}$ & $z_{10}{ }^{1}$ & \\
\hline & 10 & - & $e_{1342}$ & $z_{10}^{2}$ & \\
\hline & 10 . & - & $e_{234321}$ & $z_{10}{ }^{3}$ & $z_{10}{ }^{3}$ \\
\hline
\end{tabular}


$E_{7}$, orbit $34: A_{6}$

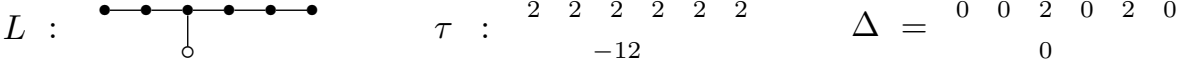

$$
\begin{aligned}
& e=\underset{0}{e} \underset{0}{100000}+\underset{0}{e 010000}+\underset{0}{e 01000}+\underset{0}{e 00100}+e_{0}^{000010}+e_{00001}^{000} \\
& C^{\circ}=A_{1} \quad C / C^{\circ}=1 \\
& x_{\beta_{1}}(t)=x_{012210}(-t) x_{111111}(2 t) x_{123321}\left(-\frac{1}{2} t^{2}\right) x_{11_{1} 110}(t) x_{012111}(-t) x_{122100}(t) \text {, } \\
& x_{-\beta_{1}}(t)=x_{-} 012210(-2 t) x_{-}{ }_{1}^{111111}(t) x_{-} 123321\left(\frac{1}{2} t^{2}\right) x_{-} 112110(t) x_{-} 012111(-t) \times \\
& x_{-} 122100(t)
\end{aligned}
$$

\begin{tabular}{|c|c|c|c|c|c|}
\hline$n$ & $m$ & $\lambda$ & $v$ & $\mathcal{Z}^{\text {b }}$ & $\mathcal{Z}$ \\
\hline 3 & \begin{tabular}{l|l}
4 & 2 \\
\end{tabular} & $2 \lambda_{1}$ & $e_{122111}-e_{112211}+e_{012221}^{01221}$ & & \\
\hline 2 & \begin{tabular}{l|l}
6 & 4 \\
8 & 2
\end{tabular} & $\begin{array}{l}4 \lambda_{1} \\
2 \lambda_{1}\end{array}$ & $\begin{array}{c}e_{234321} \\
2 \\
e_{123211}-e_{122221}\end{array}$ & & \\
\hline 1 & \begin{tabular}{c|c}
2 & \\
10 & \\
12 & 2
\end{tabular} & $\begin{array}{c}0 \\
0 \\
2 \lambda_{1}\end{array}$ & $\begin{array}{c}e \\
e_{0} e_{0}^{111110}+e_{011111} \\
e_{123321}\end{array}$ & $z_{2}$ & $\begin{array}{l}z_{2} \\
z_{10}\end{array}$ \\
\hline
\end{tabular}

$E_{7}$, orbit $35: D_{5} A_{1}$

$$
\begin{aligned}
& L: \bullet \bullet \bullet-\bullet \quad \tau: \begin{array}{rrrrrr}
2 & 2 & 2 & 2 & -11 & 2
\end{array} \quad \Delta=\begin{array}{rrrrrr}
2 & 1 & 0 & 1 & 1 & 0
\end{array} \\
& e=\underset{0}{e_{100000}}+\underset{0}{e_{010000}}+\underset{0}{e_{001000}}+e_{0}^{000000}+e_{0}^{000100}+e_{00001} \\
& C^{\circ}=A_{1} \quad C / C^{\circ}=1 \\
& x_{ \pm \beta_{1}}(t)=x_{ \pm \underset{2}{123221}}(t) x_{ \pm}{ }_{1}^{123321}(-t)
\end{aligned}
$$

\begin{tabular}{|c|c|c|c|c|c|}
\hline$n$ & $m$ & $\lambda$ & $v$ & $\mathcal{Z}^{\natural}$ & $\mathcal{Z}$ \\
\hline \hline 5 & 2 & 0 & $e_{000001}$ & & \\
& 3 & $\lambda_{1}$ & $e_{122110}+e_{112111}-e_{112210}-2 e_{012211}$ & & \\
& 4 & 5 & $\lambda_{1}$ & $e_{122111}-e_{112211}$ & \\
1 & 1 & & \\
& 6 & 0 & $e_{111000}-e_{011000}-2 e_{001100}+e_{011100}$ & & \\
\hline 3 & 8 & $2 \lambda_{1}$ & $e_{234321}$ & \\
& 9 & $\lambda_{1}$ & $e_{123210}+e_{123211}$ & & \\
\hline 2 & 11 & $\lambda_{1}$ & $e_{123211}$ & & \\
\hline 1 & 2 & 0 & $e$ & $z_{2}$ & $z_{2}$ \\
& 10 & 0 & $e_{111100}-e_{012100}$ & $z_{10}$ & $z_{10}$ \\
& 14 & 0 & $e_{122100}$ & $z_{14}$ & $z_{14}$ \\
\hline
\end{tabular}


$E_{7}$, orbit 36: $D_{6}\left(a_{1}\right)$

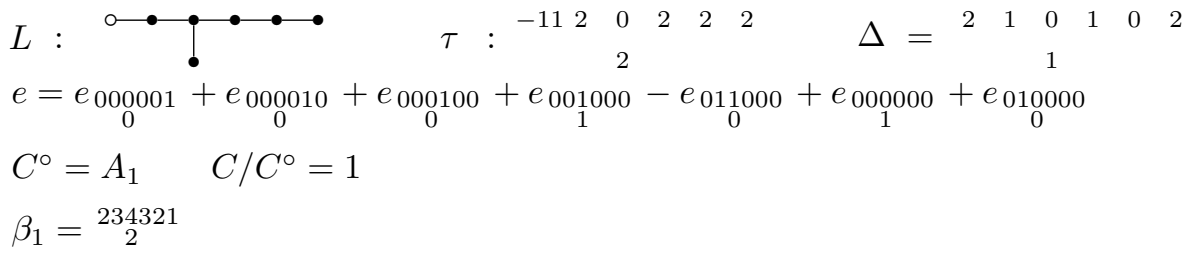

\begin{tabular}{|c|c|c|c|c|c|}
\hline & $m$ & $\lambda$ & $v$ & $\mathcal{Z}^{\natural}$ & $\mathcal{Z}$ \\
\hline 5 & \begin{tabular}{l|}
2 \\
3
\end{tabular} & \begin{tabular}{c|}
0 \\
$\lambda_{1}$
\end{tabular} & \begin{tabular}{|c|c|}
$e_{000000}+e_{010000}$ & 0 \\
1 & \\
$e_{112221}-e_{122211}-e_{123211}-2 e_{123210}$ \\
1
\end{tabular} & & \\
\hline 4 & $\begin{array}{l}5 \\
6 \\
6\end{array}$ & $\begin{array}{c}\lambda_{1} \\
0 \\
0\end{array}$ & $\begin{array}{c}e_{122221}-e_{123211} \\
2 \\
2 e_{011100}+e_{001110}+e_{011110}+e_{001111} \\
1 \\
2 e_{012100}+e_{001110}-e_{011110}+e_{000111} \\
1\end{array} \mid$ & & \\
\hline 3 & $\begin{array}{l}8 \\
9\end{array}$ & $\begin{array}{c}0 \\
\lambda_{1}\end{array}$ & $\begin{array}{c}2 e_{011110}+e_{011111}+e_{001111} \\
e_{123321}+e_{124321}\end{array}$ & & \\
\hline & 11 & $\lambda_{1}$ & $e_{134321}$ & & \\
\hline 1 & $\begin{array}{c}2 \\
10 \\
10 \\
14\end{array}$ & $\begin{array}{l}0 \\
0 \\
0 \\
0\end{array}$ & $\begin{array}{c}e \\
e_{012111}+e_{012210} \\
e_{011111} \\
e_{012221} \\
e_{1}\end{array}$ & $\begin{array}{c}z_{2} \\
z_{10}{ }^{1} \\
z_{10}{ }^{2} \\
z_{14}\end{array}$ & $\begin{array}{c}z_{2} \\
z_{10}{ }^{1} \\
z_{10}{ }^{2} \\
z_{14}\end{array}$ \\
\hline
\end{tabular}


$E_{7}$, orbit $37: E_{7}\left(a_{4}\right)$

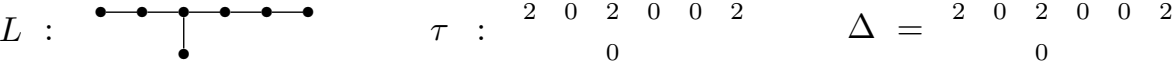

$$
\begin{aligned}
& e=e_{1}^{011000}+e_{0} 00000+e_{0}^{011100}+e_{0}^{000011}+e_{1}^{001110}+e_{0}^{000001}+e_{1}^{001100}+e_{0}^{001000} \\
& C^{\circ}=1 \quad C / C^{\circ}=\left\langle c C^{\circ}\right\rangle \cong S_{2} \\
& c=h_{4}(-1)
\end{aligned}
$$

\begin{tabular}{|c|c|c|c|c|c|}
\hline \begin{tabular}{|l|l|}
$n$ & $n$ \\
\end{tabular} & 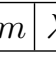 & & $v$ & $\mathcal{Z}^{\natural}$ & $\mathcal{Z}$ \\
\hline \begin{tabular}{|l|l}
7 & 2
\end{tabular} & \begin{tabular}{l|l}
2 & - \\
2 & -
\end{tabular} & & $\begin{array}{c}\underset{0}{110000}+4 e_{001100}+e_{011000}-2 e_{001110}+e_{011100}+2 e_{000111} \\
-6 e_{001000}+e_{011110} \\
1 \\
e_{000001}+e_{001100} \\
0 \\
e_{001000} \\
0\end{array}$ & & \\
\hline 6 & $4-$ & & $\begin{array}{c}e_{111000}-e_{111100}-e_{012110}-e_{011111} \\
0 \\
1 \\
e_{111100}-e_{012100}-2 e_{0} \\
1 \\
1 \\
0\end{array}$ & & \\
\hline 5 & \begin{tabular}{c|c}
6 & - \\
6 & - \\
6 & -
\end{tabular} & - & $\begin{array}{c}e_{122110}+e_{112210}+2 e_{012211}-e_{111111} \\
e_{1} \underset{1}{122100}-e_{112210}-2 e_{012221}-e_{111111} \\
e_{112100}+e_{012111} \\
e_{1}\end{array}$ & & \\
\hline 48 & \begin{tabular}{l|l}
8 & - \\
8 & -
\end{tabular} & & $\begin{array}{c}2 e_{112111}+e_{122211}-e_{122221}-e_{123210} \\
1 \\
2 e_{112211}+e_{122111}-e_{123210} \\
1\end{array}$ & & \\
\hline \begin{tabular}{|l|l|l}
3 & 1
\end{tabular} & 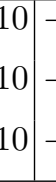 & & $\begin{array}{l}e_{123211}-e_{123221} \\
e_{1} \\
e_{123211}+e_{123321} \\
\underset{2}{e_{123211}} \\
\end{array}$ & & \\
\hline \begin{tabular}{|l|l|}
2 & 1 \\
\end{tabular} & $12-$ & & $e_{124321}$ & & \\
\hline \begin{tabular}{|l|l|l}
1 & 2 \\
1
\end{tabular} & $\begin{array}{l}2 \\
10 \\
14\end{array}$ & - & 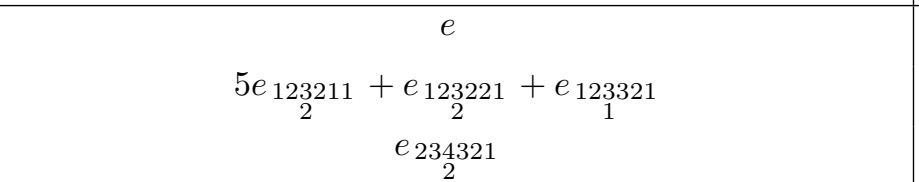 & $\begin{array}{l}z_{2} \\
z_{10} \\
z_{14}\end{array}$ & $\begin{array}{l}z_{2} \\
z_{10} \\
z_{14}\end{array}$ \\
\hline
\end{tabular}


$E_{7}$, orbit $38: D_{6}$

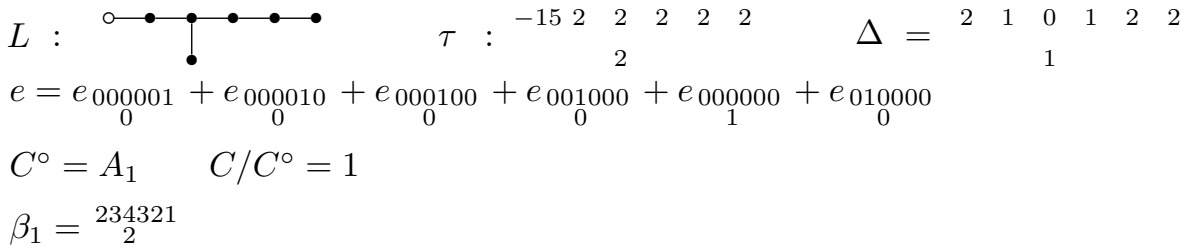

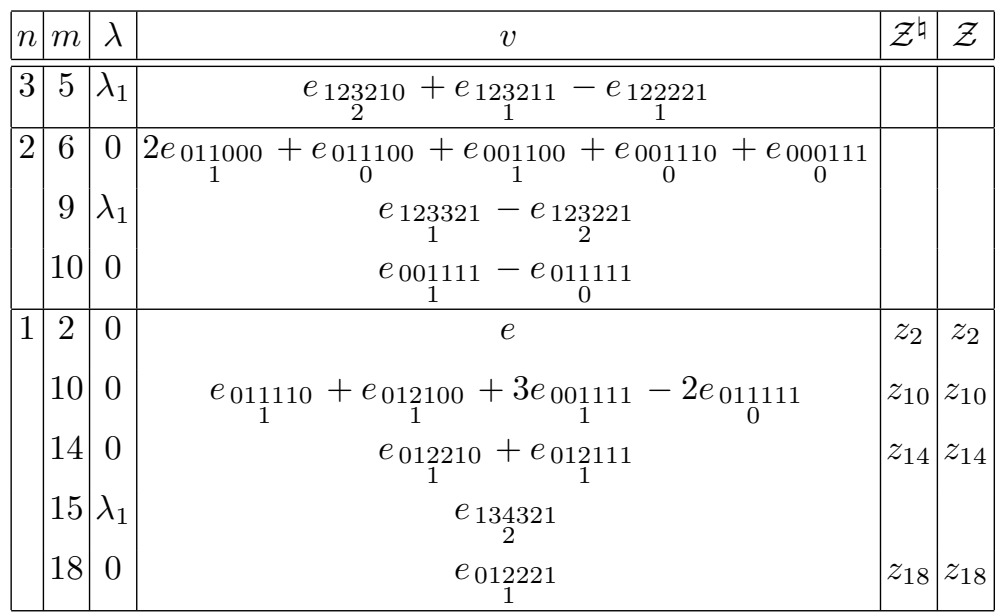

$E_{7}$, orbit 39: $E_{6}\left(a_{1}\right)$
$L: \bullet \bullet \bullet \bullet \bullet$
$\tau \quad \cdot \quad \begin{array}{cccccc}\tau & 2 & 0 & 2 & 2 & -12\end{array}$
$\Delta=$
$\begin{array}{llllll}2 & 0 & 2 & 0 & 2 & 0\end{array}$
$e=e_{100000}+e_{000010}+e_{010000}+e_{000100}+e_{001100}+e_{011000}+e_{000000}$
$C^{\circ}=T_{1} \quad C / C^{\circ}=\left\langle c C^{\circ}\right\rangle \cong S_{2}$
$T_{1}=\left\{h_{1}\left(\mu^{2}\right) h_{2}\left(\mu^{3}\right) h_{3}\left(\mu^{4}\right) h_{4}\left(\mu^{6}\right) h_{5}\left(\mu^{5}\right) h_{6}\left(\mu^{4}\right) h_{7}\left(\mu^{3}\right): \mu \in k^{*}\right\}$
$c=n_{012221} n_{112211} n_{122111} h_{1}(-1) h_{2}(-1) h_{3}(-1) h_{5}(-1) h_{6}(-1)$ 


\begin{tabular}{|c|c|c|c|c|c|}
\hline$n$ & & $\lambda$ & $v$ & $\mathcal{Z}^{\natural}$ & $\mathcal{Z}$ \\
\hline 4 & 4 & -1 & 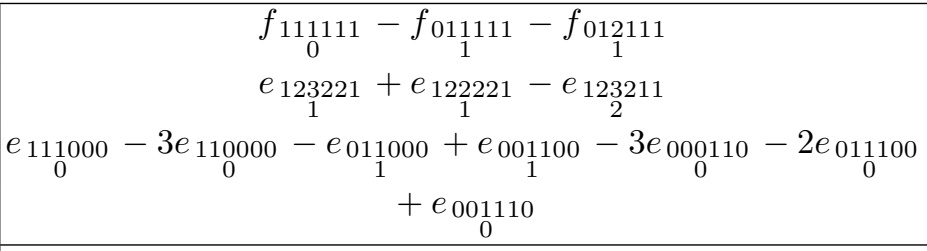 & & \\
\hline 3 & $\begin{array}{l}6 \\
8\end{array}$ & $\begin{array}{l}- \\
-\end{array}$ & $\begin{array}{c}e_{111000}+e_{111100}-e_{001110}+e_{011110}+e_{012100} \\
f_{001111}-f_{000111} \\
0 \\
e_{123321}-e_{124321} \\
e_{111100}-e_{112100}-e_{011110}+2 e_{111110}-e_{012110}\end{array}$ & & \\
\hline 2 & $\begin{array}{l}10 \\
12 \\
12\end{array}$ & $\begin{array}{l}- \\
-\end{array}$ & $\begin{array}{c}e_{111110}+e_{122100}+e_{1}^{012210} \\
1 \\
f_{000001} \\
0 \\
e_{234321} \\
2\end{array}$ & & \\
\hline 1 & $\begin{array}{l}10 \\
14\end{array}$ & $\begin{array}{l}- \\
-\end{array}$ & $\begin{array}{c}e \\
e_{112110}+e_{122100}-e_{012210} \\
e_{122210} \\
e_{123210}\end{array}$ & $\begin{array}{l}z_{2} \\
z_{10} \\
z_{14} \\
z_{16}\end{array}$ & $\begin{array}{l}z_{2} \\
z_{10} \\
z_{14}\end{array}$ \\
\hline
\end{tabular}

$E_{7}$, orbit 40: $E_{6}$

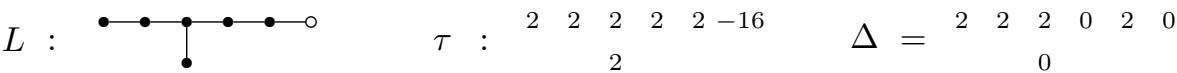

$$
\begin{aligned}
& e=e_{100000}+e_{000000}+e_{010000}+e_{001000}+e_{000100}+e_{000010} \\
& C^{\circ}=A_{1} \quad C / C^{\circ}=1 \\
& x_{ \pm \beta_{1}}(t)=x_{ \pm}{ }_{1}^{122111}(t) x_{ \pm}{ }_{11211}(-t) x_{ \pm}{ }^{012221}(t)
\end{aligned}
$$

\begin{tabular}{|c|c|c|c|c|c|}
\hline$n$ & $m$ & $\lambda$ & $v$ & $\mathcal{Z}^{\natural}$ & $\mathcal{Z}$ \\
\hline \hline 2 & 8 & $2 \lambda_{1}$ & $e_{123221}-e_{123321}$ & & \\
\hline 1 & 2 & 0 & $e$ & $z_{2}$ & $z_{2}$ \\
& 10 & 0 & $e_{111100}+e_{111110}-e_{011110}-e_{012100}$ & $z_{10}$ & $z_{10}$ \\
& 14 & 0 & $e_{122100}+e_{112110}-e_{012210}$ & $z_{14}$ & $z_{14}$ \\
& 16 & $2 \lambda_{1}$ & 1 & & \\
& 22 & 0 & $e_{234321}$ & \\
& & $e_{123210}$ & $z_{22}$ & $z_{22}$ \\
\hline
\end{tabular}


$E_{7}$, orbit $41: E_{7}\left(a_{3}\right)$

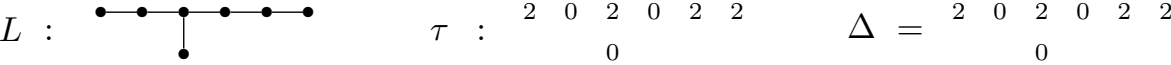

$$
\begin{aligned}
& e=e_{1}^{011000}+e_{100000}+e_{011100}+e_{0}^{000010}+e_{0}^{000001}+e_{001100}+e_{001000}^{000} \\
& C^{\circ}=1 \quad C / C^{\circ}=\left\langle c C^{\circ}\right\rangle \cong S_{2} \\
& c=h_{4}(-1)
\end{aligned}
$$

\begin{tabular}{|c|c|c|c|c|c|}
\hline$n$ & & $\lambda$ & $v$ & $\mathcal{Z}^{\natural}$ & $\mathcal{Z}$ \\
\hline 5 & 2 & - & $\begin{array}{c}e_{001000} \\
e_{001110}+e_{111100}+e_{000111}-3 e_{111000}+e_{011110}+2 e_{012100} \\
0\end{array}$ & & \\
\hline 4 & 6 & - & $e_{0}^{001111}+e_{112100}+e_{012110}^{0}$ & & \\
\hline 3 & 10 & - & $\begin{array}{c}e_{111110}+e_{011111}-2 e_{001111}-e_{122100}-e_{012210} \\
0 \\
e_{111111}-e_{112110}-2 e_{012111}-e_{122210} \\
e_{122111}+e_{123210} \\
1\end{array}$ & & \\
\hline 2 & 14 & - & $\begin{array}{l}e_{112111}-e_{123210} \\
1 \\
e_{123221}+e_{123321} \\
1\end{array}$ & & \\
\hline 1 & 2 & - & $\begin{array}{c}e \\
3 e_{122111}+2 e_{123210}+e_{112211}-e_{012221} \\
e_{123221}+e_{123321} \\
e_{124321} \\
e_{234321}\end{array}$ & $\begin{array}{l}z_{2} \\
z_{10} \\
z_{14} \\
z_{16} \\
z_{18}\end{array}$ & $z_{2}$ \\
\hline
\end{tabular}


$E_{7}$, orbit $42: E_{7}\left(a_{2}\right)$

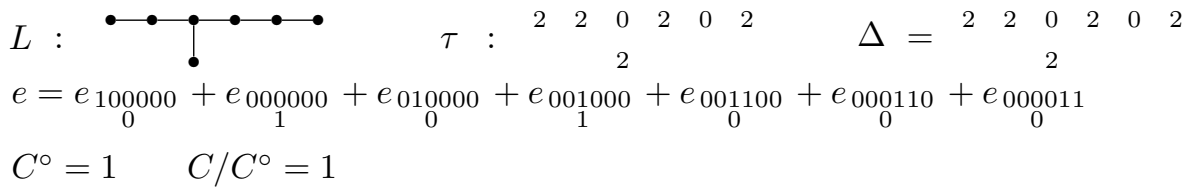

\begin{tabular}{|c|c|c|c|c|c|}
\hline$|n|$ & & $\lambda$ & $v$ & $\mathcal{Z}^{\natural}$ & $\mathcal{Z}$ \\
\hline \begin{tabular}{|l|}
5 \\
\end{tabular} & 2 & & $\begin{array}{c}e_{000000}+e_{000100}+e_{000001} \\
0 \\
2 e_{111000}+3 e_{111110}-e_{111100}+3 e_{012110}+e_{012100}-e_{011110} \\
0 \quad 00 \\
+e_{011100}-e_{011111}+2 e_{001111} \\
1\end{array}$ & & \\
\hline 4 & 8 & - & $e_{111110}-e_{111100}-e_{111111}+e_{012210}-e_{012111}$ & & \\
\hline 3 & $\begin{array}{l}10 \\
14\end{array}$ & - & $\begin{array}{c}e_{111111}+e_{012211} \\
1 \\
e_{123210}+e_{123221}\end{array}$ & & \\
\hline 2 & 16 & - & $e_{123221}-e_{2}{ }_{22321}+e_{123321}$ & & \\
\hline 1 & $\begin{array}{l}2 \\
10 \\
14 \\
18 \\
22\end{array}$ & $\begin{array}{l}- \\
-\end{array}$ & $\begin{array}{c} \\
e \\
e_{111111}+e_{012211}+e_{122100}+e_{112210}+e_{112111}+2 e_{1} \\
e_{12322210}+e_{123211}+e_{122221}-e_{122211} \\
1 \\
e_{123321} \\
e_{2343}\end{array}$ & $\begin{array}{l}z_{2} \\
z_{10} \\
z_{14} \\
z_{18} \\
z_{22}\end{array}$ & $\begin{array}{l}z_{2} \\
z_{10} \\
z_{14} \\
z_{18} \\
z_{22}\end{array}$ \\
\hline
\end{tabular}


$E_{7}$, orbit $43: E_{7}\left(a_{1}\right)$

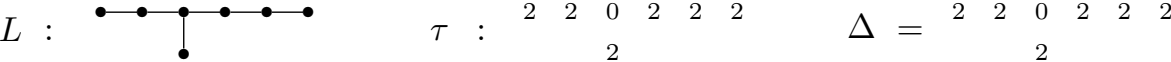

$$
\begin{aligned}
& e=e_{0} 100000+e_{0}^{010000}+e_{0}^{011000}+e_{001000}^{0}+e_{0}^{000100}+e_{0}^{000010}+e_{0}^{000001} \\
& C^{\circ}=1 \quad C / C^{\circ}=1
\end{aligned}
$$

\begin{tabular}{|c|c|c|c|c|}
\hline \begin{tabular}{l|l}
$n$ & $m$ \\
\end{tabular} & $\lambda$ & $v$ & $\mathcal{Z}^{\natural}$ & $\mathcal{Z}$ \\
\hline \begin{tabular}{l|l}
3 & 6
\end{tabular} & - & $\begin{array}{c}2 e_{111000}-e_{111100}-e_{012100}+2 e_{011100}-e_{011110}+2 e_{001110} \\
-3 e_{00111}+2 e_{000111} \\
0 \\
e_{111110}+e_{011111}+e_{122100} \\
1\end{array}$ & & \\
\hline \begin{tabular}{l|l}
2 & 16
\end{tabular} & - & $e_{123210}-e_{123211}+e_{122211}-e_{112221}$ & & \\
\hline \begin{tabular}{l|l}
1 & 2
\end{tabular} & - & $e$ & $z_{2}$ & $z_{2}$ \\
\hline 10 & - & $\begin{array}{c}2 e_{111110}+2 e_{011111}+e_{122100}+e_{012111}+e_{012210}-e_{112110} \\
+2 e_{111111}\end{array}$ & $z_{10}$ & $z_{10}$ \\
\hline 14 & $1-$ & $e_{123210}+e_{122111}-e_{112211}+e_{012221}$ & $z_{14}$ & $z_{14}$ \\
\hline 18 & - & $e_{123211}-e_{122221}$ & $z_{18}$ & $z_{18}$ \\
\hline 22 & - & $e_{124321}-e_{123321}$ & $z_{22}$ & $z_{22}$ \\
\hline 26 & - & $e_{234321}$ & $z_{26}$ & $z_{26}$ \\
\hline
\end{tabular}

$E_{7}$, orbit $44: E_{7}$
$L$ :

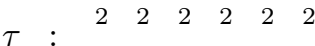
$\Delta=\begin{array}{llllll}2 & 2 & 2 & 2 & 2 & 2\end{array}$
$e=e_{0} 0000+e_{00000}+e_{010000}+e_{001000}+e_{000100}+e_{000010}+e_{000001}$

\begin{tabular}{|c|c|c|c|c|}
\hline$n \mid m$ & $\imath \lambda$ & $v$ & $\mathcal{Z}^{\natural}$ & $\mathcal{Z}$ \\
\hline \begin{tabular}{|l|l|}
1 & 2
\end{tabular} & $2-$ & $e$ & $z_{2}$ & $z_{2}$ \\
\hline 10 & $0-$ & $e_{111100}+2 e_{0}^{111110}-e_{011110}-3 e_{001111}+2 e_{011111}-e_{012100}$ & $z_{10}$ & $z_{10}$ \\
\hline 14 & $4-$ & $e_{122100}+e_{112110}+2 e_{111111}-e_{012111}-e_{1}^{012210}$ & $z_{14}$ & $z_{14}$ \\
\hline 18 & $8-$ & $e_{122111}-e_{112211}+e_{012221}$ & $z_{18}$ & $z_{18}$ \\
\hline 22 & $2-$ & $e_{123210}+e_{123211}-e_{122221}$ & $z_{22}$ & $z_{22}$ \\
\hline 26 & $6-$ & $e_{123221}-e_{123321}$ & $z_{26}$ & $z_{26}$ \\
\hline 34 & $4-$ & $e_{2}^{234321}$ & $z_{34}$ & $z_{34}$ \\
\hline
\end{tabular}
$C^{\circ}=1 \quad C / C^{\circ}=1$ 
$E_{8}$, orbit 1: $A_{1}$

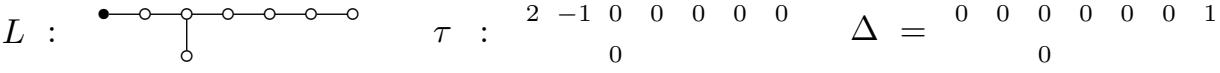

$$
\begin{aligned}
& e=\underset{0}{e} 100000 \\
& C^{\circ}=E_{7} \quad C / C^{\circ}=1 \\
& \beta_{1}=\underset{0}{0000001}, \beta_{2}=\stackrel{1221000}{1}, \beta_{3}=\underset{0}{0000010}, \beta_{4}=\underset{0}{0000100}, \beta_{5}=\underset{0}{0001000}, \beta_{6}=\begin{array}{c}
0010000 \\
0
\end{array} \text {, } \\
& \beta_{7}=000000
\end{aligned}
$$

\begin{tabular}{|c|c|c|c|c|c|}
\hline$n$ & $m$ & $\lambda$ & $v$ & $\mathcal{Z}^{\natural}$ & $\mathcal{Z}$ \\
\hline \hline 2 & 1 & $\lambda_{7}$ & $e_{2354321}$ & & \\
\hline 1 & 2 & 0 & $e$ & $z_{2}$ & $z_{2}$ \\
\hline
\end{tabular}

$E_{8}$, orbit 2: $A_{1}^{2}$

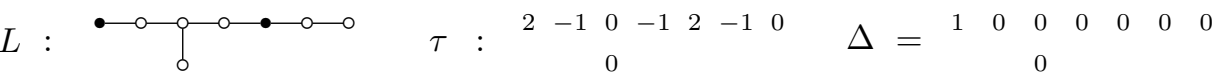

$$
\begin{aligned}
& e=\underset{0}{e} \underset{00000}{e}+\underset{000100}{e} \\
& C^{\circ}=B_{6} \quad C / C^{\circ}=1 \\
& \beta_{1}=\stackrel{1221110}{1}, \beta_{2}=\underset{0}{000001}, \beta_{3}=\underset{0}{0001110}, \beta_{4}=\underset{0}{0010000}, \beta_{5}=\underset{1}{0000000} \text {, }
\end{aligned}
$$

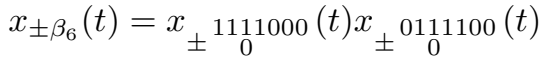

\begin{tabular}{|c|c|c|c|c|c|}
\hline$n$ & $m$ & $\lambda$ & $v$ & $\mathcal{Z}^{\natural}$ & $\mathcal{Z}$ \\
\hline \hline 2 & 1 & $\lambda_{6}$ & $e_{2465421}$ & & \\
\hline 1 & 2 & $\lambda_{1}$ & $e_{2343321}$ & & \\
& 2 & 0 & 2 & & \\
& 2 & 0 & $e$ & $z_{2}$ & $z_{2}$ \\
\hline
\end{tabular}


$E_{8}$, orbit $3: A_{1}{ }^{3}$

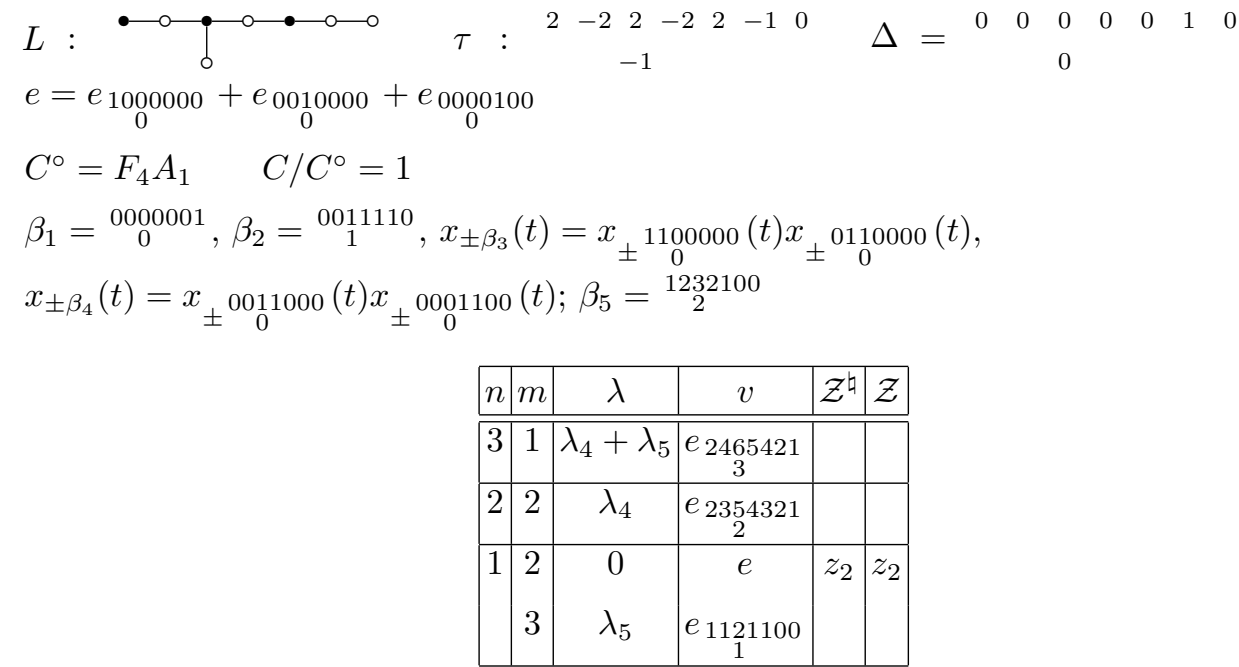

$E_{8}$, orbit $4: A_{2}$

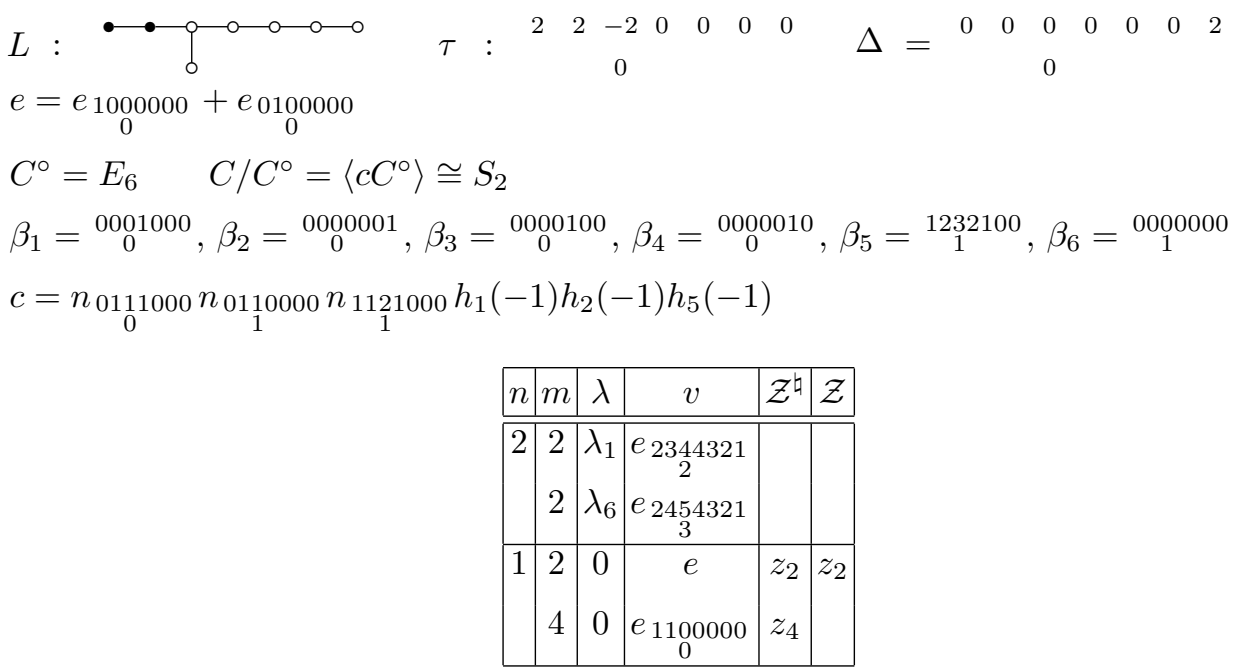


$E_{8}$, orbit 5: $A_{1}{ }^{4}$

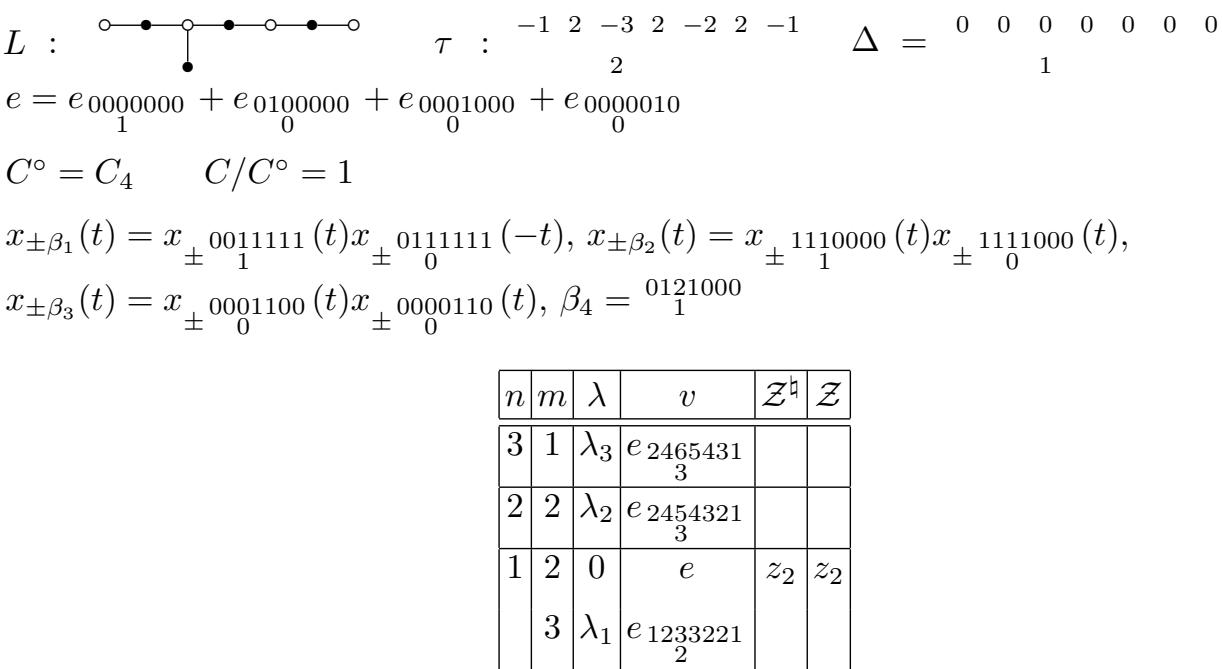

$E_{8}$, orbit 6: $A_{2} A_{1}$

$$
\begin{aligned}
& L: \bullet \bullet-\longrightarrow \\
& e=e_{1000000}+e_{0100000}+e_{0000000} \\
& C^{\circ}=A_{5} \quad C / C^{\circ}=\left\langle c C^{\circ}\right\rangle \cong S_{2} \\
& \beta_{1}=\underset{0}{0000001}, \beta_{2}=\underset{0}{0000010}, \beta_{3}=\underset{0}{0000100}, \beta_{4}=\underset{0}{0001000}, \beta_{5}=\underset{3}{2464321} \\
& c=n_{1121110} n_{1}^{1122100} n_{2}^{1343210} h_{3}(-1) h_{5}(-1) h_{7}(-1)
\end{aligned}
$$

\begin{tabular}{|c|c|c|c|c|c|}
\hline$n$ & $m$ & $\lambda$ & $v$ & $\mathcal{Z}^{\natural}$ & $\mathcal{Z}$ \\
\hline 4 & 1 & $\lambda_{5}$ & $e_{2454321}-e_{2354321}$ & & \\
\hline & 1 & $\lambda_{1}$ & $e_{1111111}-e_{0111111}$ & & \\
\hline & 1 & $\lambda_{3}$ & $e_{1233321}$ & & \\
\hline 3 & 2 & $\lambda_{2}$ & $e_{1222221}$ & & \\
\hline & 2 & $\lambda_{4}$ & $e_{2344321}$ & & \\
\hline & 2 & 0 & $\begin{array}{c}e_{0000000} \\
1\end{array}$ & & \\
\hline 2 & 3 & $\lambda_{5}$ & $e_{2454321}$ & & \\
\hline & 3 & $\lambda_{1}$ & $e_{1111111}$ & & \\
\hline 1 & 2 & 0 & $e$ & $z_{2}$ & $z_{2}$ \\
\hline & 4 & 0 & $\begin{array}{c}e 1100000 \\
0\end{array}$ & $z_{4}$ & \\
\hline
\end{tabular}


$E_{8}$, orbit 7: $A_{2} A_{1}^{2}$

$L$

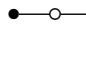

$$
\text { ○一 - — }
$$

$$
\tau: \begin{array}{lllllll}
2 & -3 & 2 & -3 & 2 & -1 & 0
\end{array}
$$

$$
\Delta=\begin{array}{lllllll}
0 & 0 & 0 & 0 & 1 & 0 & 0 \\
& 0 & & & &
\end{array}
$$

$e=e_{1}^{000000}+e_{0}^{0010000}+e_{0}^{1000000}+\underset{0}{e 0000100}$

$C^{\circ}=B_{3} A_{1} \quad C / C^{\circ}=1$

$\beta_{1}={ }_{1}^{1221110}, \beta_{2}={ }_{0}^{0000001}, x_{ \pm \beta_{3}}(t)=x_{ \pm}{ }_{1122110}(t) x_{ \pm} 0122210(-t) ;$

$x_{\beta_{4}}(t)=x_{0}^{1111100}(2 t) x_{0121000}(-t) x_{1232100}\left(-t^{2}\right) x_{111000}(t) x_{1}^{0111100}(-t)$,

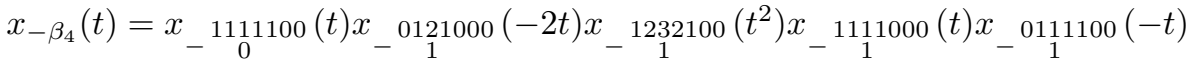

\begin{tabular}{|c|c|c|c|c|c|}
\hline$n$ & $m$ & $\lambda$ & $v$ & $\mathcal{Z}^{\natural}$ & $\mathcal{Z}$ \\
\hline \hline 4 & 1 & $\lambda_{3}+3 \lambda_{4}$ & $e_{2465421}$ & & \\
\hline 3 & 2 & $\lambda_{1}+2 \lambda_{4}$ & $e_{2464321}$ & & \\
& 2 & $4 \lambda_{4}$ & $e_{1232100}$ & & \\
\hline 2 & 3 & $\lambda_{3}+\lambda_{4}$ & $\underset{2}{e_{2354321}}$ & & \\
\hline 1 & 2 & 0 & $e$ & $z_{2}$ & $z_{2}$ \\
& 4 & $2 \lambda_{4}$ & $e_{1121100}$ & & \\
\hline
\end{tabular}

$E_{8}$, orbit 8: $A_{3}$

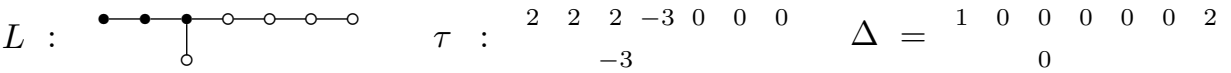

$$
\begin{aligned}
& e=\underset{0}{e_{1000000}}+e_{0100000}+e_{0010000} \\
& C^{\circ}=B_{5} \quad C / C^{\circ}=1 \\
& \beta_{1}={ }_{1}^{1233210}, \beta_{2}=\underset{0}{0000001}, \beta_{3}={ }_{0}^{0000010}, \beta_{4}=\underset{0}{0000100}, \\
& x_{ \pm \beta_{5}}(t)=x_{ \pm}{ }_{11}^{111000}(t) x_{ \pm}{ }_{1}^{0121000}(-t) \\
& \begin{array}{|c|c|c|c|c|c|}
\hline n & m & \lambda & v & \mathcal{Z}^{\natural} & \mathcal{Z} \\
\hline \hline 2 & 3 & \lambda_{5} & e_{2464321} & & \\
\hline 1 & 2 & 0 & e & z_{2} & z_{2} \\
& 4 & \lambda_{1} & e_{2454321} & & \\
& 6 & 0 & e_{1110000} & z_{6} & z_{6} \\
& & & 0 & & \\
\end{array}
\end{aligned}
$$


$E_{8}$, orbit 9: $A_{2} A_{1}{ }^{3}$

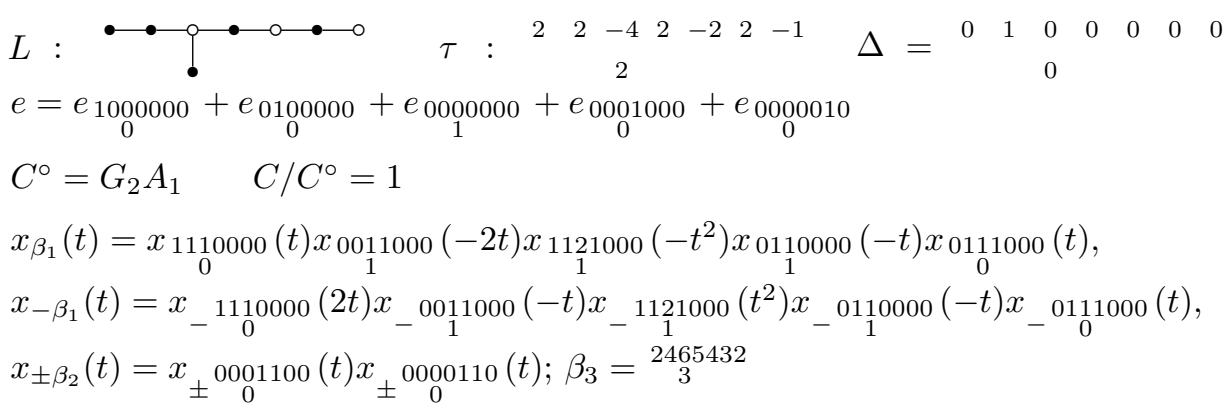

\begin{tabular}{|c|c|c|c|c|c|}
\hline$n$ & $m$ & $\lambda$ & $v$ & $\mathcal{Z}^{\natural}$ & $\mathcal{Z}$ \\
\hline \hline 4 & 1 & $\lambda_{2}+\lambda_{3}$ & $e_{2465431}$ & & \\
\hline 3 & 2 & $2 \lambda_{1}$ & $e_{2343210}$ & & \\
\hline 2 & 3 & $\lambda_{1}+\lambda_{3}$ & $e_{2454321}$ & & \\
\hline 1 & 2 & 0 & $e$ & $z_{2}$ & $z_{2}$ \\
& 4 & $\lambda_{1}$ & $e_{1222110}$ & & \\
\hline
\end{tabular}

$E_{8}$, orbit 10: $A_{2}{ }^{2}$

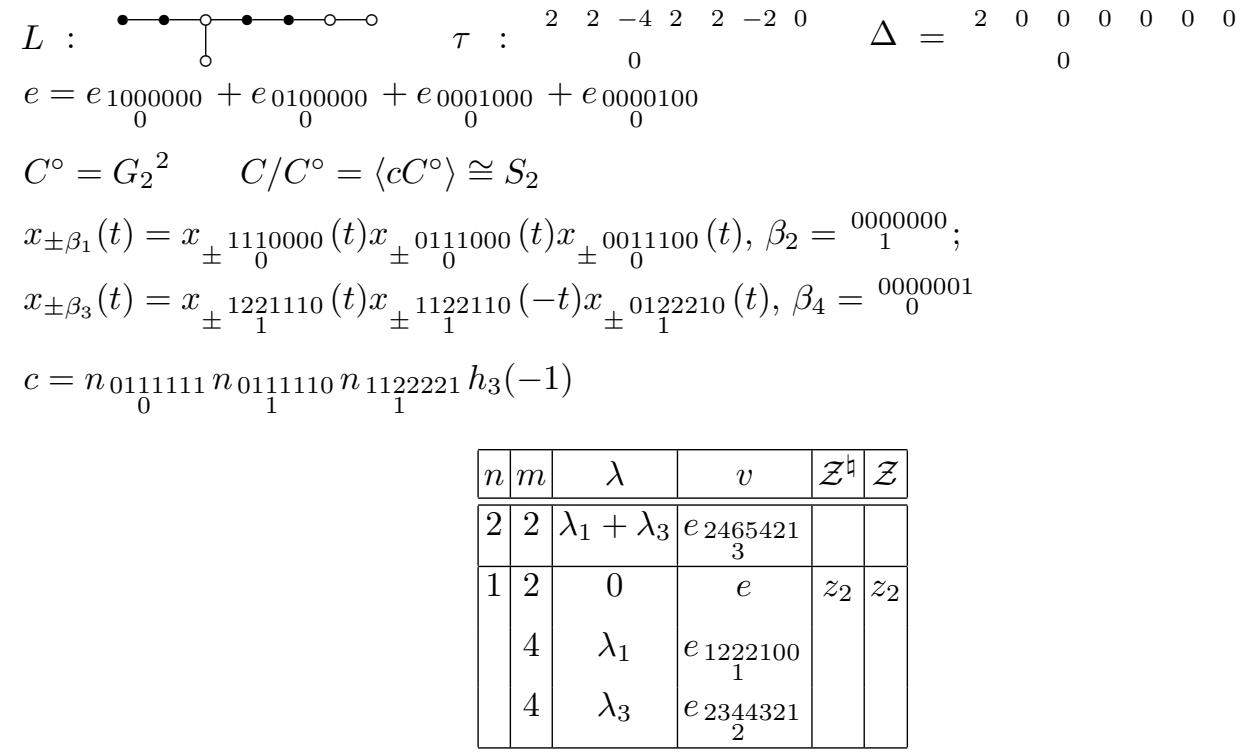


$E_{8}$, orbit 11: $A_{2}{ }^{2} A_{1}$

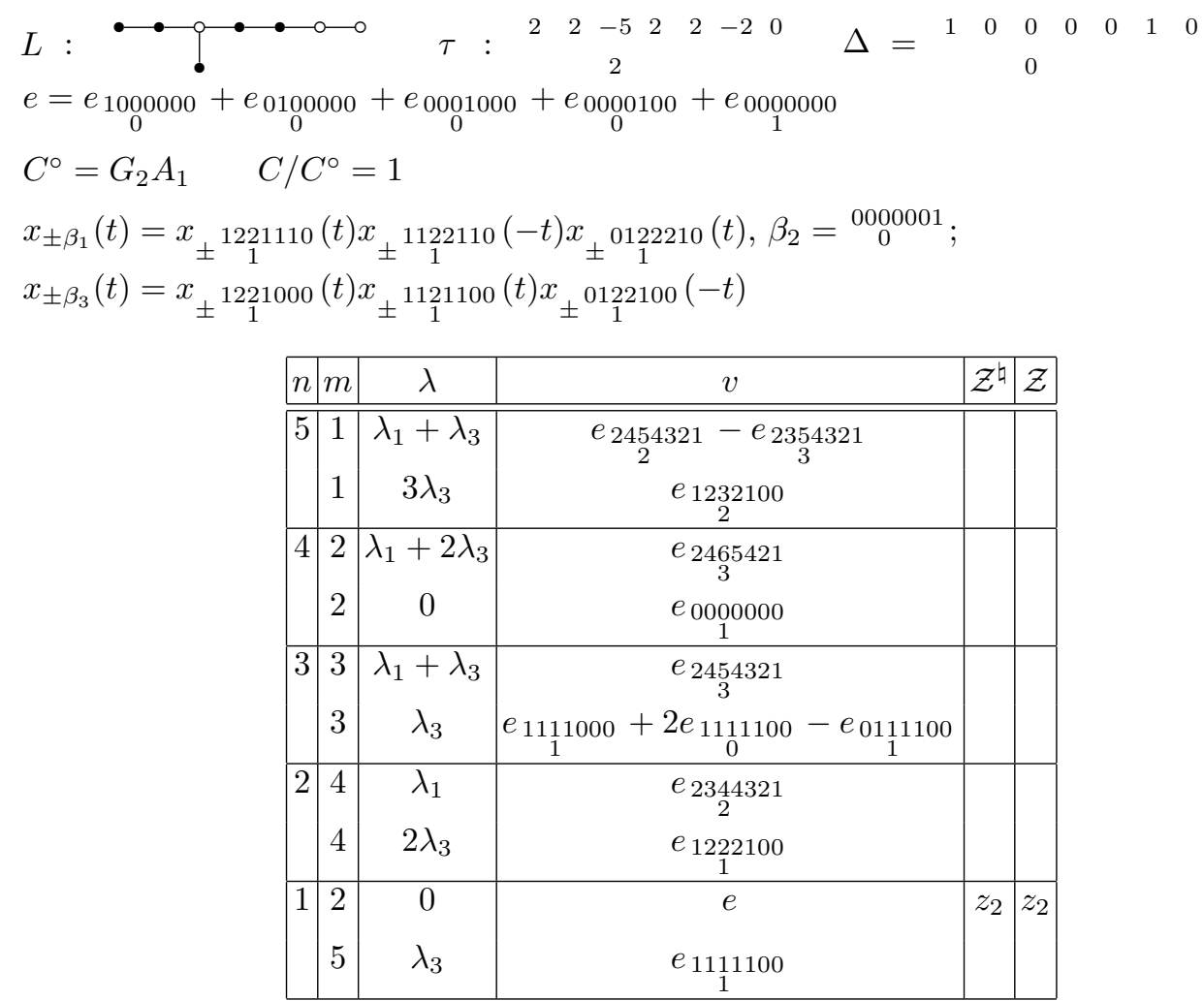


$E_{8}$, orbit $12: A_{3} A_{1}$

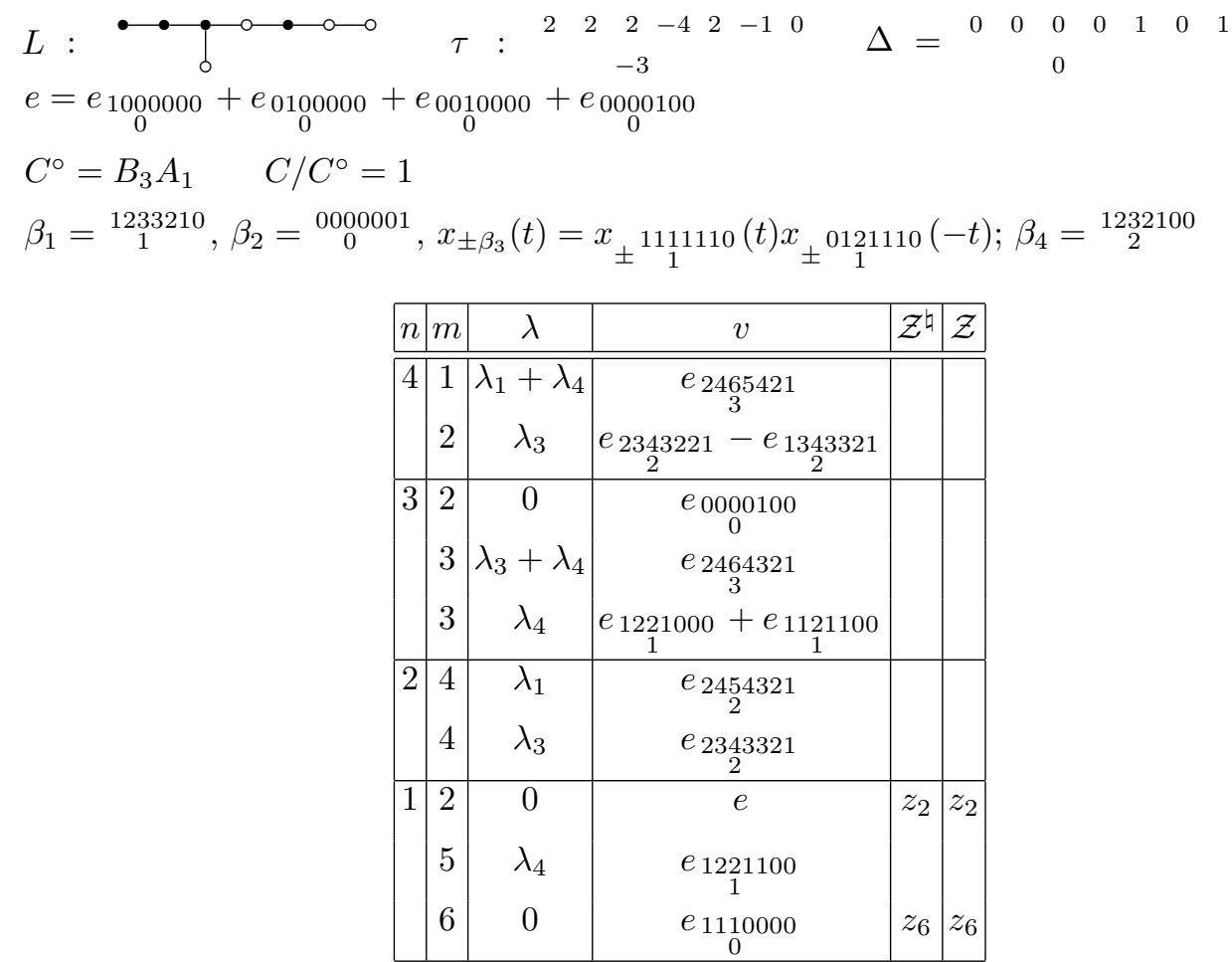


$E_{8}$, orbit 13: $D_{4}\left(a_{1}\right)$

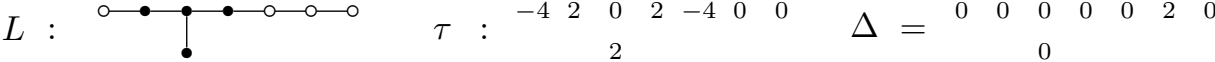

$$
\begin{aligned}
& e=\underset{0}{e 0100000}+e_{1}^{0010000}+e_{0}^{0011000}+e_{1}^{000000}+e_{0}^{0001000} \\
& C^{\circ}=D_{4} \quad C / C^{\circ}=\left\langle c_{1} C^{\circ}, c_{2} C^{\circ}\right\rangle \cong S_{3} \\
& \beta_{1}=\underset{0}{0000010}, \beta_{2}=\underset{0}{0000001}, \beta_{3}={ }_{1}^{0122210}, \beta_{4}={ }_{2}^{2343210} \\
& c_{1}=n_{1110000} n_{1111000} h_{2}(-1) \text {, } \\
& c_{2}=\left(n_{1221100} n_{1122100} h_{1}(-1) h_{2}(-1) h_{6}(-1)\right)^{g}, \\
& g=x_{0010000}\left(\frac{1}{3}\right) n_{0}^{0010000} h_{1}(4) h_{2}(-4) h_{3}(16) h_{4}(-48) h_{5}(16) h_{6}(-8) x_{0010000}\left(-\frac{1}{3}\right)
\end{aligned}
$$

\begin{tabular}{|c|c|c|c|c|c|}
\hline$n$ & $m$ & $\lambda$ & $v$ & $\mathcal{Z}^{\natural}$ & $\mathcal{Z}$ \\
\hline \hline 3 & 2 & $\lambda_{4}$ & $e_{2464321}+e_{2454321}$ & & \\
& 2 & $\lambda_{3}$ & $e_{1354321}-e_{13444321}$ & \\
& 2 & $\lambda_{1}$ & $e_{1233221}$ & & \\
& 2 & 0 & $2 e_{0000000}-e_{0010000}+e_{0110000}+e_{0011000}$ & & \\
& 2 & 0 & $e_{0000000}+e_{0001000}$ & \\
& 1 & & \\
\hline 2 & 4 & $\lambda_{4}$ & $e_{2465321}$ & \\
& 4 & $\lambda_{3}$ & $e_{1354321}$ & \\
& 4 & $\lambda_{1}$ & $e_{1343221}$ & \\
& 4 & 0 & $e_{0110000}+2 e_{0011000}-e_{0111000}$ & \\
& 1 & 2 & 0 & & \\
& 2 & $e$ & $z_{2}$ & $z_{2}$ \\
& 6 & 0 & $e_{0111000}$ & $z_{6}{ }^{1}$ & \\
& 6 & 0 & $e_{0121000}$ & $z_{6}{ }^{2}$ & \\
\hline
\end{tabular}


$E_{8}$, orbit $14: D_{4}$

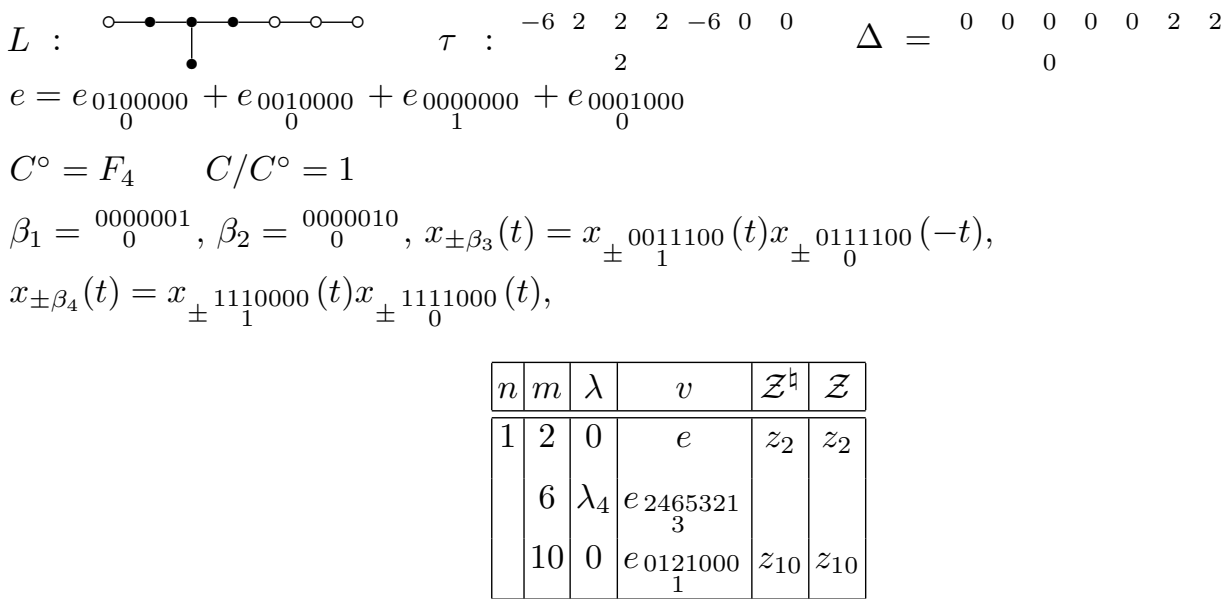

$E_{8}$, orbit 15: $A_{2}{ }^{2} A_{1}{ }^{2}$

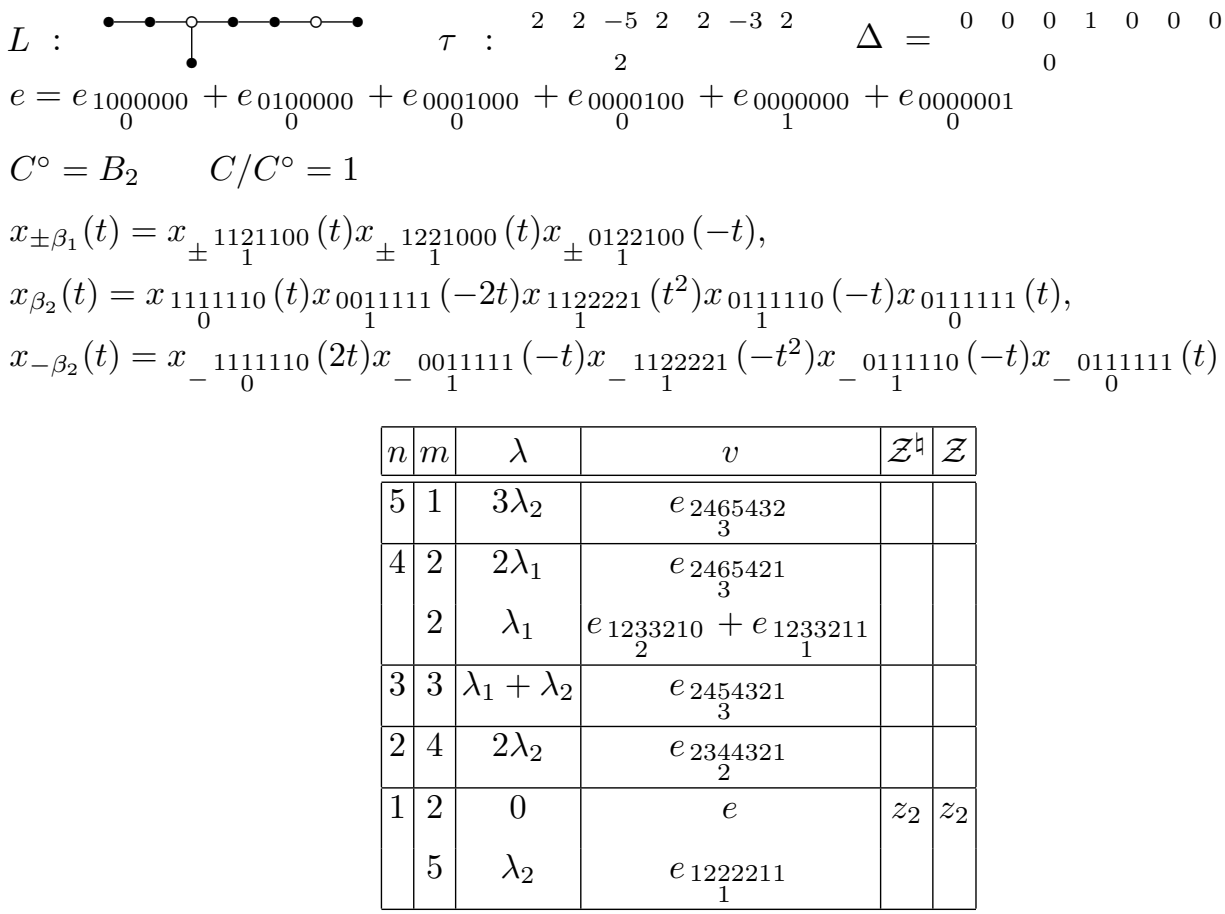


$E_{8}$, orbit 16: $A_{3} A_{1}{ }^{2}$

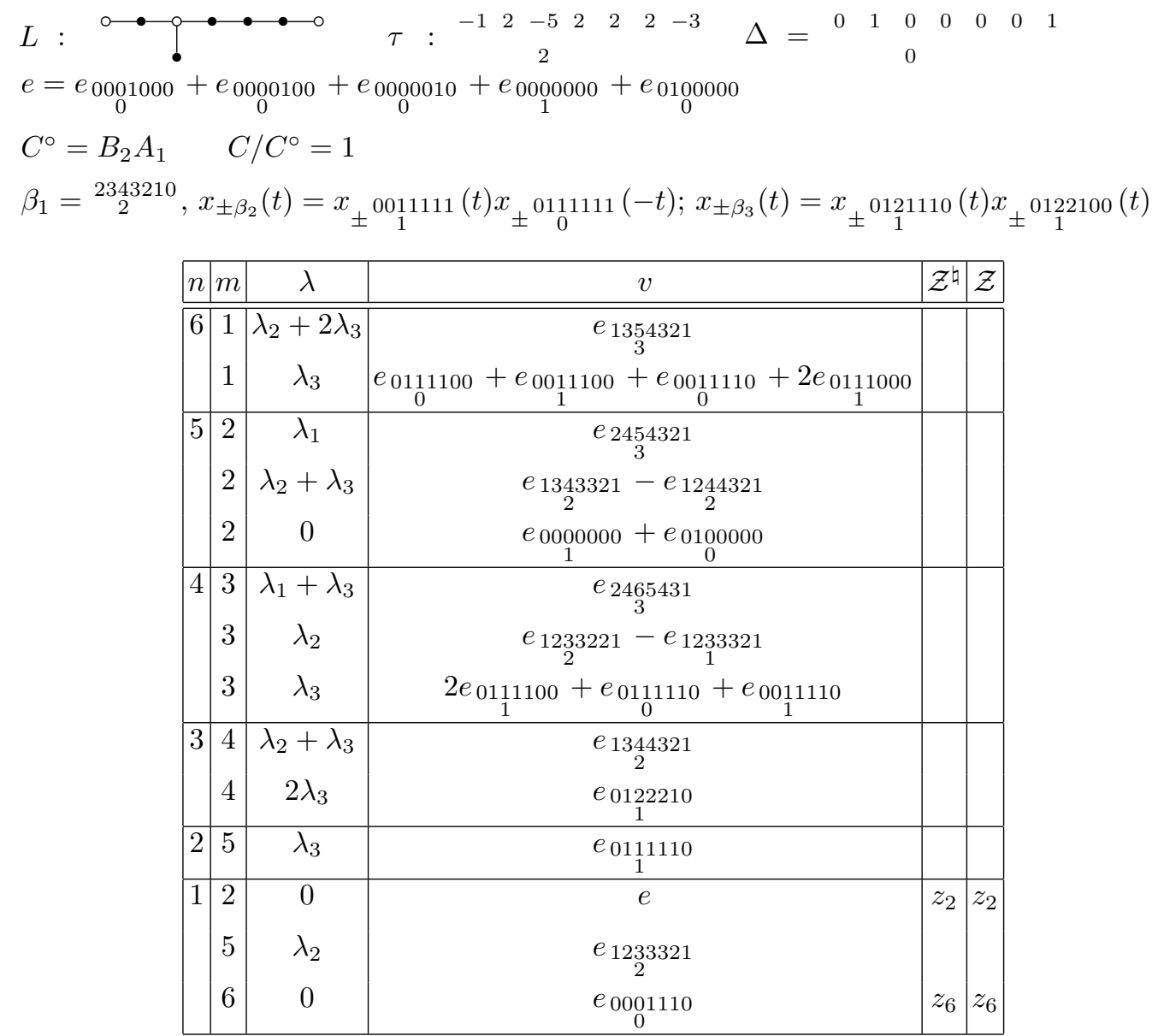

$E_{8}$, orbit 17: $D_{4}\left(a_{1}\right) A_{1}$

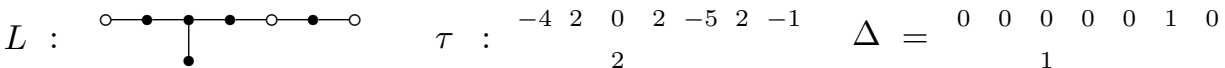

$$
\begin{aligned}
& e=e_{0}^{0100000}+e_{0010000}+e_{0}^{0011000}+e_{0000000}+e_{0}^{0001000}+e_{0000010} \\
& C^{\circ}=A_{1}{ }^{3} \quad C / C^{\circ}=\left\langle c_{1} C^{\circ}, c_{2} C^{\circ}\right\rangle \cong S_{3} \\
& \beta_{1}={ }_{1}^{0122210} ; \beta_{2}={ }_{2}^{2343210} ; \beta_{3}={ }_{3}^{2465432} \\
& c_{1}=n_{1110000} n_{1111000} h_{2}(-1) \text {, } \\
& c_{2}=\left(n_{1221111} n_{1122111} h_{3}(i) h_{5}(i)\right)^{g} \text {, } \\
& g=x_{0010000}\left(\frac{1}{3}\right) n_{0010000} h_{1}(4) h_{2}(-4) h_{3}(16) h_{4}(-48) h_{5}(16) h_{6}(-8) x_{0010000}\left(-\frac{1}{3}\right)
\end{aligned}
$$




\begin{tabular}{|c|c|c|c|c|c|}
\hline$n \mid$ & & $\lambda$ & $v$ & & $\mathcal{Z}$ \\
\hline \multirow[t]{4}{*}{$\overline{6}$} & 1 & $\overline{\lambda_{1}+\lambda_{2}+\lambda_{3}}$ & $e_{2465431}$ & & \\
\hline & 1 & $\lambda_{3}$ & $e_{1232221}-2 e_{1233211}+e_{1233221}$ & & \\
\hline & 1 & $\lambda_{2}$ & $e_{1221110}+e_{1232100}-2 e_{1122110}-e_{1222100}$ & & \\
\hline & 1 & $\lambda_{1}$ & $e_{0111100}+e_{0121100}+2 e_{0011110}-e_{0111110}$ & & \\
\hline 5 & 2 & $\lambda_{2}+\lambda_{3}$ & $e_{2454321}+e_{2464321}$ & & \\
\hline & 2 & $\lambda_{1}+\lambda_{3}$ & $e_{1344321}-e_{1354321}$ & & \\
\hline & 2 & $\lambda_{1}+\lambda_{2}$ & $e_{1233210}$ & & \\
\hline & 2 & 0 & $2 e_{0000000}-e_{0010000}+e_{0110000}+e_{0011000}$ & & \\
\hline & 2 & 0 & $e_{0000000}+e_{0001000}$ & & \\
\hline & 2 & 0 & $e_{0000010}$ & & \\
\hline 4 & 3 & $\lambda_{3}$ & $\begin{array}{c}0 \\
e_{1} 12020291\end{array}$ & & \\
\hline & & 83 & ${ }_{2}$ & & \\
\hline & 3 & $\lambda_{3}$ & $e_{1342}{ }_{2} 3211-e_{1243221}$ & & \\
\hline & 3 & $\lambda_{2}$ & $e_{1232100}+e_{1232110}$ & & \\
\hline & 3 & $\lambda_{2}$ & $e_{1222110}-e_{1232110}$ & & \\
\hline & 3 & $\lambda_{1}$ & $e_{0121110}+e_{0122100}$ & & \\
\hline & 3 & $\lambda_{1}$ & $e_{0121110}+e_{0111110}$ & & \\
\hline 3 & 4 & $\lambda_{2}+\lambda_{3}$ & & & \\
\hline & & & $\begin{array}{r}340321 \\
3\end{array}$ & & \\
\hline & 4 & $\lambda_{1}+\lambda_{3}$ & $\begin{array}{l}e_{1354321} \\
3\end{array}$ & & \\
\hline & 4 & $\lambda_{1}+\lambda_{2}$ & $e_{134}{ }_{2} 210$ & & \\
\hline & 4 & 0 & $e_{0110000}+2 e_{0011000}-e_{0}^{0111000}$ & & \\
\hline \multirow[t]{3}{*}{2} & 5 & $\lambda_{3}$ & $e_{1343221}$ & & \\
\hline & 5 & $\lambda_{2}$ & $e_{1232110}$ & & \\
\hline & 5 & $\lambda_{1}$ & $e_{0122110}$ & & \\
\hline \multirow[t]{3}{*}{1} & 2 & 0 & $e$ & $z_{2}$ & $z_{2}$ \\
\hline & 6 & 0 & $e_{0111000}$ & $z_{6}^{1}$ & \\
\hline & 6 & 0 & $e_{0121000}$ & $z_{6}^{2}$ & \\
\hline
\end{tabular}


$E_{8}$, orbit 18: $A_{3} A_{2}$

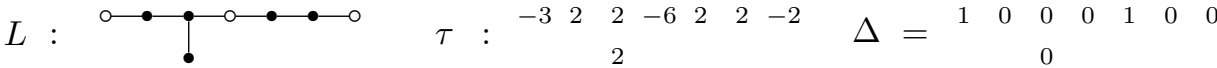

$$
\begin{aligned}
& e=\underset{0}{e 0100000}+\underset{0}{e 0010000}+e_{0}^{0000000}+\underset{0}{e 0000100}+\underset{0}{e 000010} \\
& C^{\circ}=B_{2} T_{1} \quad C / C^{\circ}=\left\langle c C^{\circ}\right\rangle \cong S_{2} \\
& \beta_{1}={ }_{2}^{2343210}, x_{ \pm \beta_{2}}(t)=x_{ \pm} 0011111(t) x_{ \pm}^{0111111}(-t) \\
& T_{1}=\left\{h_{2}\left(\mu^{3}\right) h_{3}\left(\mu^{3}\right) h_{4}\left(\mu^{6}\right) h_{5}\left(\mu^{6}\right) h_{6}\left(\mu^{4}\right) h_{7}\left(\mu^{2}\right): \mu \in k^{*}\right\} \\
& c=n_{1}^{001100} \underset{0}{0111100} n_{0122110} h_{2}(-1) h_{4}(-1) h_{5}(-1) h_{6}(-1) h_{7}(-1)
\end{aligned}
$$

\begin{tabular}{|c|c|c|c|c|c|}
\hline$n$ & $m$ & $\lambda$ & $v$ & & $\mathcal{Z}$ \\
\hline 6 & 1 & $\lambda_{2}$ & $e_{1232111}-e_{1232211}+e_{1222221}$ & & \\
\hline & 1 & $\lambda_{2}$ & $e_{1343211}-e_{1243221}+e_{1233321}$ & & \\
\hline 5 & 2 & $\lambda_{1}$ & $e_{2343321}$ & & \\
\hline & 2 & $\lambda_{1}$ & $e_{2465431}$ & & \\
\hline & 2 & 0 & $f_{0122100}$ & & \\
\hline & 2 & 0 & $e_{0122210}$ & & \\
\hline & 2 & 0 & $2 f_{0011100}+2 f_{0001110}+f_{0111000}+f_{0011000}$ & & \\
\hline & 2 & 0 & $2 e_{0121000}+2 e_{0111100}+e_{0111110}+e_{0011110}$ & & \\
\hline & 2 & 0 & $e_{0000100}+e_{0000010}$ & & \\
\hline 4 & 3 & $\lambda_{2}$ & $e_{1221111}$ & & \\
\hline & & & 1 & & \\
\hline & 3 & $\lambda_{2}$ & $e_{1354321}$ & & \\
\hline & 3 & $\lambda_{2}$ & $e_{1232211}-e_{1232221}$ & & \\
\hline & 3 & $\lambda_{2}$ & $e_{1343221}-e_{1243321}$ & & \\
\hline 3 & 4 & $\lambda_{1}$ & $e_{2464321}$ & & \\
\hline & 4 & 0 & $f_{0011000}+f_{0001100}$ & & \\
\hline & 4 & 0 & $e_{0121100}+e_{0111110}$ & & \\
\hline & 4 & 0 & $e_{0000110}$ & & \\
\hline 2 & 5 & $\lambda_{2}$ & $e_{1232221}$ & & \\
\hline & 5 & $\lambda_{2} \mid$ & $e_{1343321}$ & & \\
\hline & & & & & \\
\hline 1 & 2 & 0 & $e$ & $z_{2}$ & $z_{2}$ \\
\hline & 6 & 0 & $f_{0001000}$ & & \\
\hline & 6 & 0 & $e_{0121110}$ & & \\
\hline & 6 & 0 & $e_{0110000}$ & $z_{6}$ & $z_{6}$ \\
\hline
\end{tabular}


$E_{8}$, orbit 19: $A_{4}$

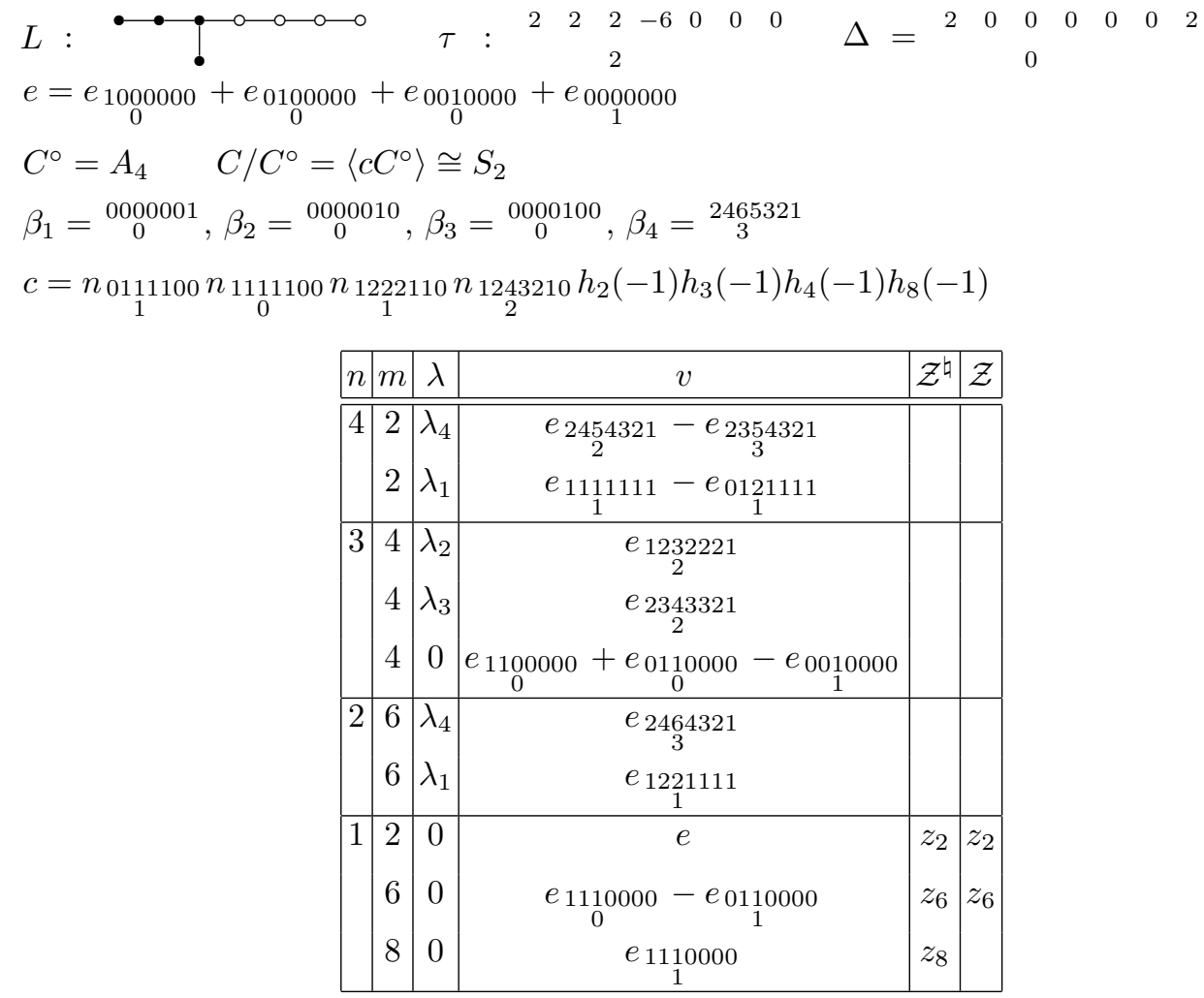

$E_{8}$, orbit 20: $A_{3} A_{2} A_{1}$

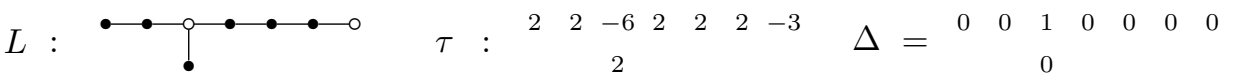

$e=\underset{0}{e 0001000}+\underset{0}{e 0000100}+e_{0}^{0000010}+\underset{0}{e} \underset{0}{1000000}+e_{0}^{0100000}+e_{0}^{000000}$

$C^{\circ}=A_{1}^{2} \quad C / C^{\circ}=1$

$\beta_{1}={ }_{3}^{2465432} ; x_{\beta_{2}}(t)=x_{0111000}(2 t) x_{001110}(t) x_{1110000}(3 t) x_{0}^{0111100}(t) x_{0122110}\left(t^{2}\right) \times$ $x_{1121110}\left(\frac{3}{2} t^{2}\right) x_{1221100}\left(-\frac{3}{2} t^{2}\right) x_{1232110}\left(-2 t^{3}\right) x_{1232210}\left(-t^{3}\right) x_{1343210}\left(\frac{9}{4} t^{4}\right) x_{0}^{1111000}(t) \times$ $x_{0011100}(t) x_{1}^{1122100}\left(-\frac{1}{2} t^{2}\right)$,

$x_{-\beta_{2}}(t)=x_{-}^{0111000}(2 t) x_{-}^{0011110}(6 t) x_{-} \underset{1}{1110000}(2 t) x_{-}^{0111100}(4 t) x_{-}^{0122110}\left(-6 t^{2}\right) \times$ $x_{-}^{1121110}\left(-6 t^{2}\right) x_{-}^{1221100}\left(4 t^{2}\right) x_{-}^{1232110}\left(-8 t^{3}\right) x_{-}^{1232210}\left(-16 t^{3}\right) x_{-}^{1343210}\left(-36 t^{4}\right) \times$ $x_{-} \underset{0}{1111000}(2 t) x_{-} 0011100(2 t) x_{-}^{1122100}\left(2 t^{2}\right)$ 


\begin{tabular}{|c|c|c|c|c|c|}
\hline$n$ & $m$ & $\lambda$ & $v$ & $\mathcal{Z}^{\natural}$ & $\mathcal{Z}$ \\
\hline 6 & 1 & $\lambda_{1}+4 \lambda_{2}$ & $e_{2454321}-e_{2354321}$ & & \\
\hline 5 & 2 & $8 \lambda_{2}$ & $e_{2343210}$ & & \\
\hline & 2 & $4 \lambda_{2}$ & $3 e_{1221110}+2 e_{1222100}+e_{0122210}-e_{1122110}$ & & \\
\hline 4 & 3 & $\lambda_{1}+6 \lambda_{2}$ & $\begin{array}{c}e_{2465431} \\
3\end{array}$ & & \\
\hline 3 & 4 & $6 \lambda_{2}$ & $e_{1233210}$ & & \\
\hline & 4 & $2 \lambda_{2}$ & $e_{1111100}-e_{1111110}+2 e_{0111110}$ & & \\
\hline 2 & 5 & $\lambda_{1}+2 \lambda_{2}$ & $e_{2344321}$ & & \\
\hline 1 & 2 & 0 & $e$ & $z_{2}$ & $z_{2}$ \\
\hline & 6 & $4 \lambda_{2}$ & $e_{1222210}$ & & \\
\hline
\end{tabular}

If $p=7$, the two summands with $m=2$ and high weights $8 \lambda_{2}$ and $4 \lambda_{2}$ are replaced by a single reducible tilting module $T_{A_{1}{ }^{2}}\left(8 \lambda_{2}\right)$ generated by the two given vectors together with $e_{1221110}+e_{1222100}$.

$$
\begin{aligned}
& E_{8} \text {, orbit 21: } D_{4} A_{1}
\end{aligned}
$$

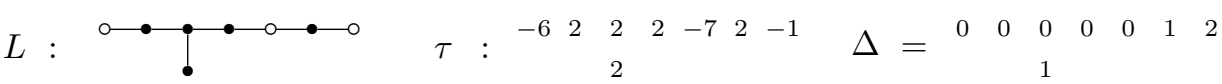

$$
\begin{aligned}
& e=\underset{0}{0100000}+e_{0}^{0010000}+e_{0000000}+e_{0}^{0001000}+e_{0000010}^{00} \\
& C^{\circ}=C_{3} \quad C / C^{\circ}=1 \\
& x_{ \pm \beta_{1}}(t)=x_{ \pm}^{0011111}(t) x_{ \pm}^{0111111}(-t), x_{ \pm \beta_{2}}(t)=x_{ \pm}{ }_{1}^{1110000}(t) x_{ \pm} 1111000(t), \beta_{3}={ }_{1}^{0122210}
\end{aligned}
$$

\begin{tabular}{|c|c|c|c|c|c|}
\hline$n$ & $m$ & $\lambda$ & $v$ & $\mathcal{Z}^{\natural}$ & $\mathcal{Z}$ \\
\hline \hline 3 & 1 & $\lambda_{3}$ & $e_{2465431}$ & & \\
& 5 & $\lambda_{1}$ & $e_{1343211}-e_{1243221}$ & & \\
\hline 2 & 2 & 0 & $e_{0000010}$ & & \\
& 6 & $\lambda_{2}$ & $e_{2465321}$ & & \\
\hline 1 & 2 & 0 & $e$ & $z_{2}$ & $z_{2}$ \\
& \multirow{2}{*}{7} & $\lambda_{1}$ & $e_{1343221}$ & & \\
& & & 2 & & \\
& 10 & 0 & $e_{0121000}$ & $z_{10}$ & $z_{10}$ \\
& & & & \\
\hline
\end{tabular}


$E_{8}$, orbit 22: $D_{4}\left(a_{1}\right) A_{2}$

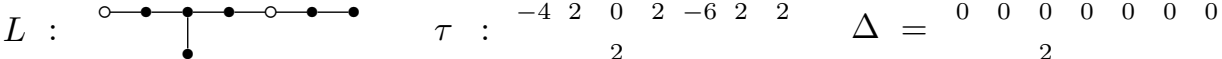

$$
\begin{aligned}
& e=\underset{0}{0} 0100000+e_{1}^{0010000}+\underset{0}{e 011000}+e_{0}^{0000000}+\underset{0}{e 0001000}+e_{0}^{0000010}+e_{000001}^{0} \\
& C^{\circ}=A_{2} \quad C / C^{\circ}=\left\langle c C^{\circ}\right\rangle \cong S_{2} \\
& x_{\beta_{1}}(t)=x_{0001111}(3 t) x_{0121100}(t) x_{0011111}(-t) x_{0111100}(t) x_{0122211}\left(-2 t^{2}\right) x_{0011110}(2 t) \times \\
& x_{0111110}(-t) \text {, } \\
& x_{-\beta_{1}}(t)=x_{-} \underset{0}{0001111}(t) x_{-} 0121100(3 t) x_{-} 0011111(-t) x_{-}^{0111100}(t) x_{-}^{0122211}\left(2 t^{2}\right) \times \\
& x_{-} 0011110(t) x_{-}{ }_{0}^{0111110}(-2 t) \text {, } \\
& x_{\beta_{2}}(t)=x_{1111111}(-3 t) x_{1232100}(-t) x_{1}^{121111}(-t) x_{1222100}(t) x_{2343211}\left(-2 t^{2}\right) x_{1122110}(2 t) \times \\
& x_{122110}(-t) \text {, }
\end{aligned}
$$

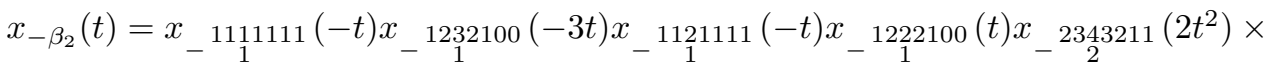

$$
\begin{aligned}
& x_{-} 1122110(t) x_{-}{ }_{1}^{1221110}(-2 t) \\
& c=n_{1111000} n_{1110000} h_{5}(-1)
\end{aligned}
$$

\begin{tabular}{|c|c|c|c|c|c|}
\hline$n$ & $m$ & $\lambda$ & $v$ & $\mathcal{Z}^{\natural}$ & $\mathcal{Z}$ \\
\hline \hline 3 & 2 & $2 \lambda_{1}+2 \lambda_{2}$ & $e_{2465432}$ & & \\
\hline 2 & 4 & $3 \lambda_{1}$ & $e_{1354321}$ & & \\
& & 3 & & \\
& 4 & $3 \lambda_{2}$ & $e_{2465321}$ & & \\
\hline 1 & 2 & 0 & $e$ & $z_{2}$ & $z_{2}$ \\
& & & & & \\
& 6 & $\lambda_{1}+\lambda_{2}$ & $e_{1343221}$ & & \\
\hline
\end{tabular}

$E_{8}$, orbit 23: $A_{4} A_{1}$

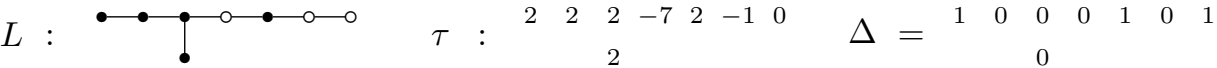

$$
\begin{aligned}
& e=\underset{0}{e_{1000000}}+e_{0}^{0100000}+e_{0010000}+e_{000000}+e_{0}^{0000100} \\
& C^{\circ}=A_{2} T_{1} \quad C / C^{\circ}=\left\langle c C^{\circ}\right\rangle \cong S_{2} \\
& \beta_{1}=\underset{0}{0000001}, \beta_{2}=\underset{3}{2465431} \\
& T_{1}=\left\{h_{1}\left(\mu^{4}\right) h_{2}\left(\mu^{6}\right) h_{3}\left(\mu^{8}\right) h_{4}\left(\mu^{12}\right) h_{5}\left(\mu^{10}\right) h_{6}\left(\mu^{5}\right): \mu \in k^{*}\right\} \\
& c=n_{1}^{011110} n_{1111110} n_{1222100} n_{1243210} h_{1}(-1) h_{3}(-1) h_{4}(-1) h_{6}(-1) h_{8}(-1)
\end{aligned}
$$




\begin{tabular}{|c|c|c|c|c|c|}
\hline$n$ & $m$ & $\lambda$ & $v$ & $\mathcal{Z}^{\natural}$ & $\mathcal{Z}$ \\
\hline \multirow[t]{4}{*}{8} & \begin{tabular}{|l|}
1 \\
\end{tabular} & $\lambda_{1}$ & $e_{0000111}$ & & \\
\hline & 1 & $\lambda_{2}$ & $e_{2465421}$ & & \\
\hline & 1 & 0 & $f_{0111000}+f_{0011100}-f_{0111100}-2 f_{0}^{1111000}$ & & \\
\hline & 1 & 0 & $e_{0111100}+e_{0121000}-e_{1111000}-2 e_{1}^{1111100}$ & & \\
\hline \multirow[t]{3}{*}{7} & 2 & $\lambda_{1}$ & $e_{1111111}-e_{0121111}$ & & \\
\hline & 2 & $\lambda_{2}$ & $e_{2454321}-e_{2354321}$ & & \\
\hline & 2 & 0 & $\begin{array}{c}e_{0000100} \\
0\end{array}$ & & \\
\hline \multirow[t]{4}{*}{6} & 3 & $\lambda_{1}$ & $e_{1232211}-e_{1232111}$ & & \\
\hline & 3 & $\lambda_{2}$ & $e_{2343221}-e_{1343321}$ & & \\
\hline & 3 & 0 & $f_{0111000}-f_{0011000}$ & & \\
\hline & 3 & 0 & $e_{1111100}-e_{0121100}$ & & \\
\hline \multirow[t]{5}{*}{5} & 4 & $\lambda_{2}$ & $e_{1232221}$ & & \\
\hline & 4 & $\lambda_{1}$ & $e_{234}^{234211}$ & & \\
\hline & 4 & 0 & $f_{0122100}$ & & \\
\hline & 4 & 0 & $e_{1232100}$ & & \\
\hline & 4 & 0 & $e_{1100000}+e_{0110000}-e_{0010000}$ & & \\
\hline \multirow[t]{4}{*}{4} & 5 & $\lambda_{1}$ & $e_{1232211}$ & & \\
\hline & 5 & $\lambda_{2}$ & $e_{2343321}$ & & \\
\hline & 5 & 0 & $f_{0011000}+f_{0001100}$ & & \\
\hline & 5 & 0 & $e_{1221000}+e_{1121100}$ & & \\
\hline \multirow[t]{3}{*}{3} & 6 & $\lambda_{1}$ & $e_{1221111}$ & & \\
\hline & 6 & $\lambda_{2}$ & $e_{2464321}$ & & \\
\hline & 6 & 0 & $e_{0}^{1110000}-e_{0110000}$ & & \\
\hline \multirow[t]{2}{*}{\begin{tabular}{|l|}
2 \\
\end{tabular}} & \begin{tabular}{|l|}
7 \\
\end{tabular} & 0 & $f_{0001000}$ & & \\
\hline & 7 & 0 & $e_{1221100}$ & & \\
\hline \multirow[t]{2}{*}{1} & 2 & 0 & $e$ & $z_{2}$ & $z_{2}$ \\
\hline & 8 & 0 & $e_{11}^{110000}$ & $z_{8}$ & \\
\hline
\end{tabular}


$E_{8}$, orbit $24: A_{3}{ }^{2}$

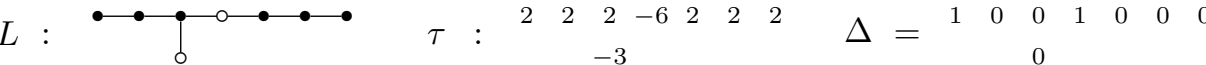

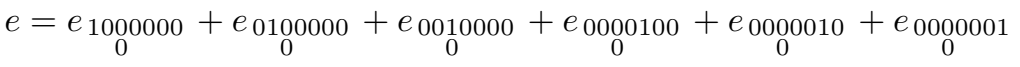

$$
\begin{aligned}
& C^{\circ}=B_{2} \quad C / C^{\circ}=1
\end{aligned}
$$

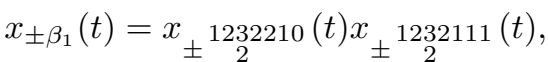

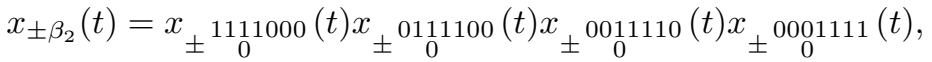

\begin{tabular}{|c|c|c|c|c|c|}
\hline$n$ & $m$ & $\lambda$ & $v$ & $\mathcal{Z}^{\natural}$ & $\mathcal{Z}$ \\
\hline 7 & 1 & $\lambda_{2}$ & $2 e_{0122221}-2 e_{1122211}+e_{1232110}-e_{1222210}+e_{1222111}$ & & \\
\hline 6 & 2 & $\lambda_{1}$ & $e_{2343211}-e_{1343221}+e_{1243321}$ & & \\
\hline 5 & 3 & $\lambda_{1}+\lambda_{2}$ & $e_{2465432}$ & & \\
\hline 4 & 4 & $2 \lambda_{2}$ & $e_{2454321}$ & & \\
\hline 3 & 5 & $\lambda_{2}$ & $e_{1232211}-e_{1222221}$ & & \\
\hline 2 & 6 & $\lambda_{1}$ & $e_{2343321}$ & & \\
\hline & 6 & 0 & $e_{0}^{1110000}+e_{0}^{0000111}$ & & \\
\hline 1 & 2 & 0 & $e$ & $z_{2}$ & $z_{2}$ \\
\hline & 7 & $\lambda_{2}$ & $e_{1232221}$ & & \\
\hline
\end{tabular}


$E_{8}$, orbit $25: D_{5}\left(a_{1}\right)$

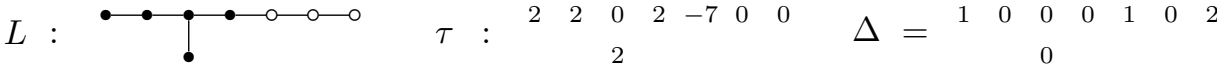

$$
\begin{aligned}
& e=\underset{0}{e} \underset{0}{1000000}+e_{0}^{0100000}+e_{1}^{0010000}+e_{0}^{0011000}+e_{0}^{0000000}+e_{0}^{0001000} \\
& C^{\circ}=A_{3} \quad C / C^{\circ}=\left\langle c C^{\circ}\right\rangle \cong S_{2} \\
& \beta_{1}={ }_{0}^{0000001}, \beta_{2}=\underset{0}{0000010}, \beta_{3}={ }_{3}^{2465421} \\
& c=n_{1233210} n_{1232210} h_{1}(-1) h_{2}(-1) h_{4}(-1)
\end{aligned}
$$

\begin{tabular}{|c|c|c|c|c|c|}
\hline$n$ & $m$ & $\lambda$ & $v$ & $\mathcal{Z}^{\natural}$ & $\mathcal{Z}$ \\
\hline 5 & 1 & $\lambda_{1}$ & $e_{111111}+e_{0122111}$ & & \\
\hline & 1 & $\lambda_{3}$ & $e_{2344321}-e_{1354321}$ & & \\
\hline & 4 & 0 & $e_{0}^{1110000}+e_{0}^{0111000}-e_{1}^{0110000}-2 e_{1}^{0011000}$ & & \\
\hline 4 & 2 & $\lambda_{2}$ & $e_{1233221}$ & & \\
\hline & 2 & 0 & $e_{0000000}+e_{0001000}^{0}$ & & \\
\hline & 5 & $\lambda_{1}$ & $e_{1222111}-e_{1232111}$ & & \\
\hline & 5 & $\lambda_{3}$ & $e_{2454321}+e_{2464321}$ & & \\
\hline \begin{tabular}{|l|}
3 \\
\end{tabular} & 6 & $\lambda_{2}$ & $e_{2343221}$ & & \\
\hline & 6 & 0 & $e_{0}^{1111000}-e_{0111000}+e_{0121000}$ & & \\
\hline & 6 & 0 & $e_{1110000}+e_{0111000}+e_{0121000}$ & & \\
\hline 2 & 7 & $\lambda_{1}$ & $e_{1232111}$ & & \\
\hline & 7 & $\lambda_{3}$ & $e_{2465321}$ & & \\
\hline 1 & 2 & 0 & e & $z_{2}$ & $z_{2}$ \\
\hline & 8 & 0 & $e_{1111000}$ & $z_{8}$ & \\
\hline & 10 & 0 & $e_{1221000}$ & $z_{10}$ & $z_{10}$ \\
\hline
\end{tabular}

$E_{8}$, orbit 26: $A_{4} A_{1}^{2}$

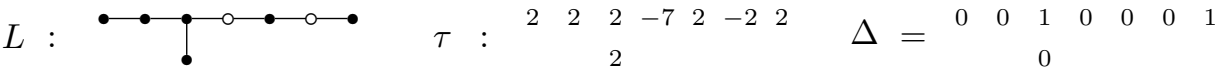

$$
\begin{aligned}
& e=\underset{0}{e} \underset{0}{1000000}+e_{0100000}^{e}+\underset{0}{e_{0010000}}+e_{0}^{0000000}+\underset{0}{e_{0}^{0000100}}+e_{000001} \\
& C^{\circ}=A_{1} T_{1} \quad C / C^{\circ}=\left\langle c C^{\circ}\right\rangle \cong S_{2} \\
& x_{ \pm \beta_{1}}(t)=x_{ \pm} 0000110(t) x_{ \pm}{ }_{0}^{0000011}(t) \\
& T_{1}=\left\{h_{1}\left(\mu^{8}\right) h_{2}\left(\mu^{12}\right) h_{3}\left(\mu^{16}\right) h_{4}\left(\mu^{24}\right) h_{5}\left(\mu^{20}\right) h_{6}\left(\mu^{15}\right) h_{7}\left(\mu^{10}\right) h_{8}\left(\mu^{5}\right): \mu \in k^{*}\right\} \\
& c=n_{122210} n_{1222111} n_{1354321} n_{2354321} h_{1}(-1) h_{3}(-1) h_{4}(-1) h_{6}(-1)
\end{aligned}
$$




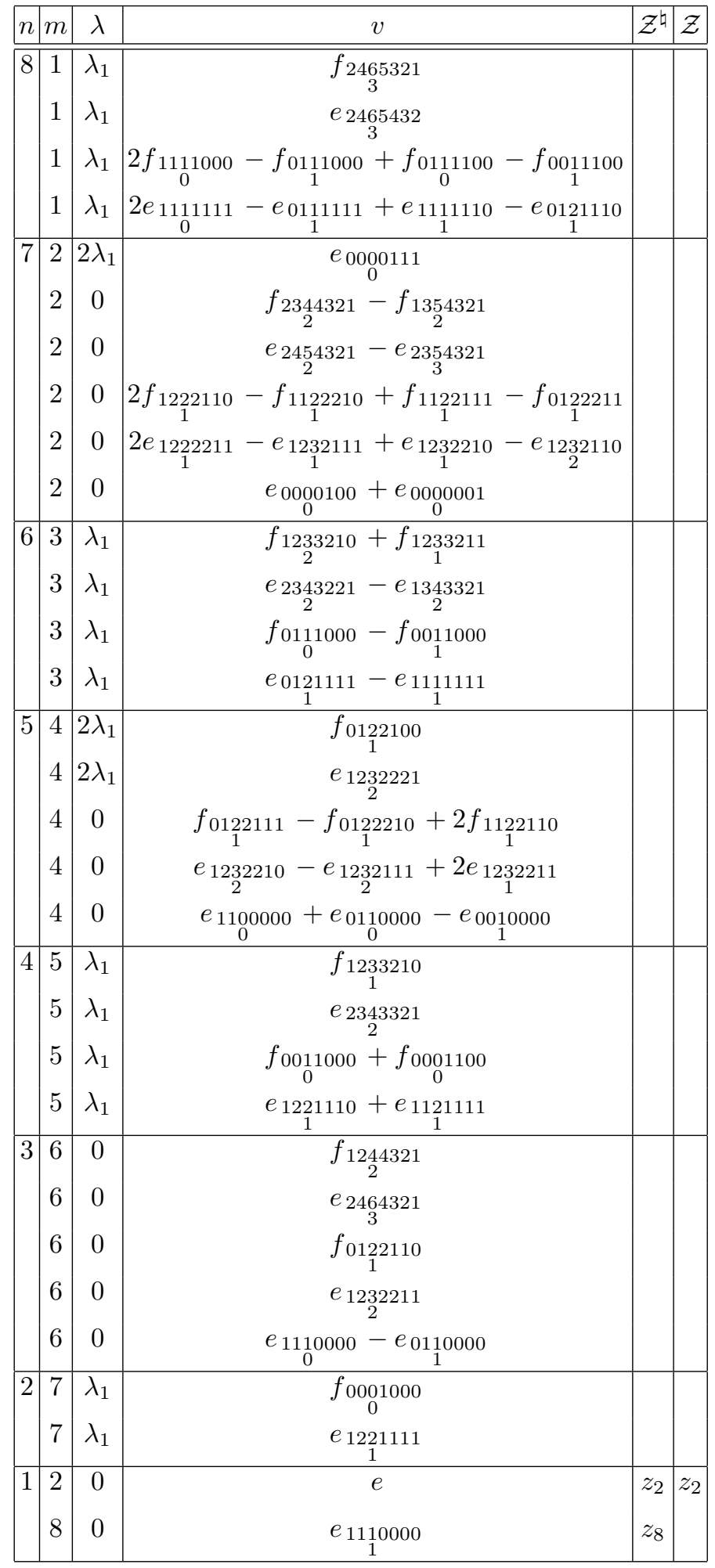


$E_{8}$, orbit 27: $A_{4} A_{2}$

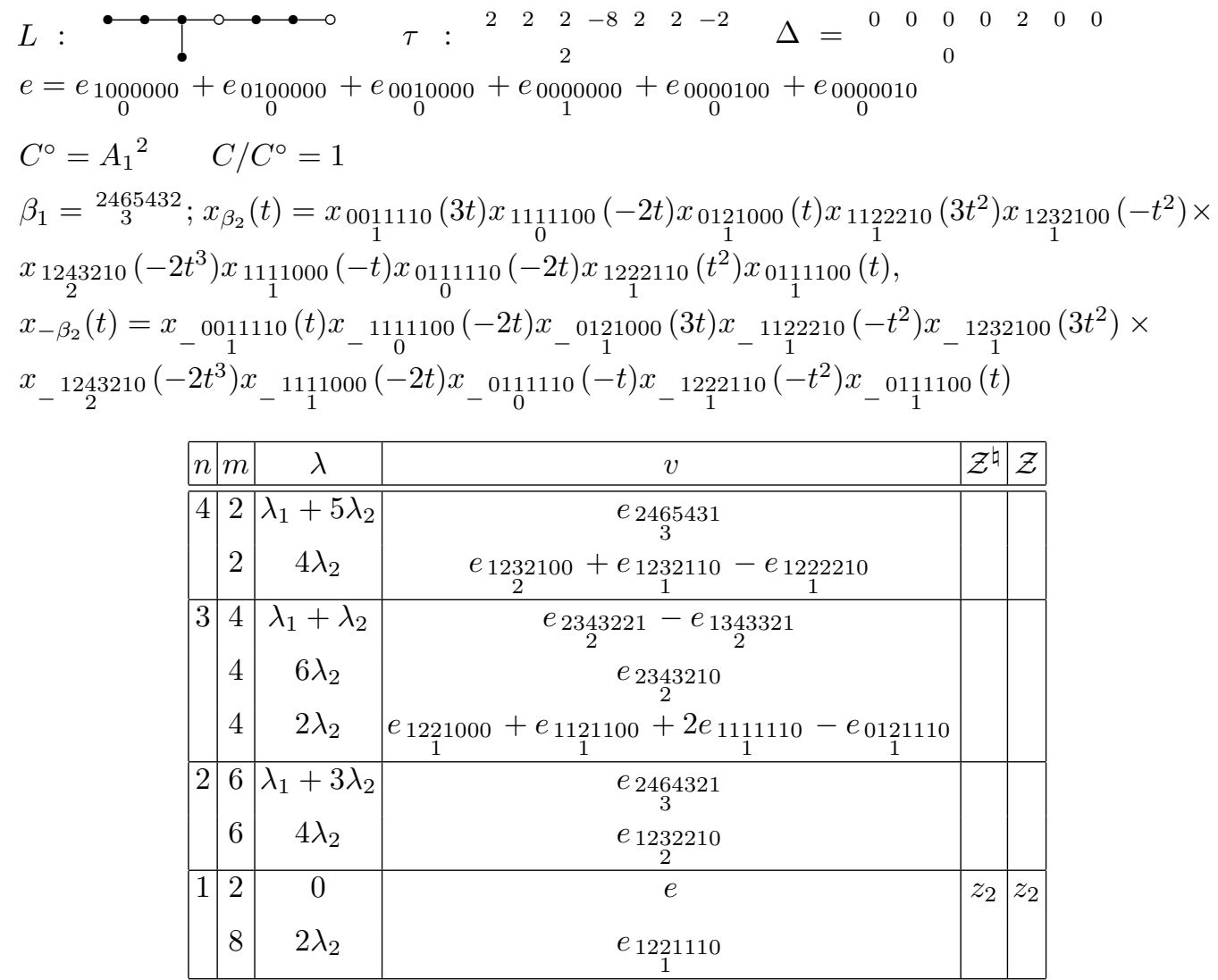

$E_{8}$, orbit 28: $A_{5}$

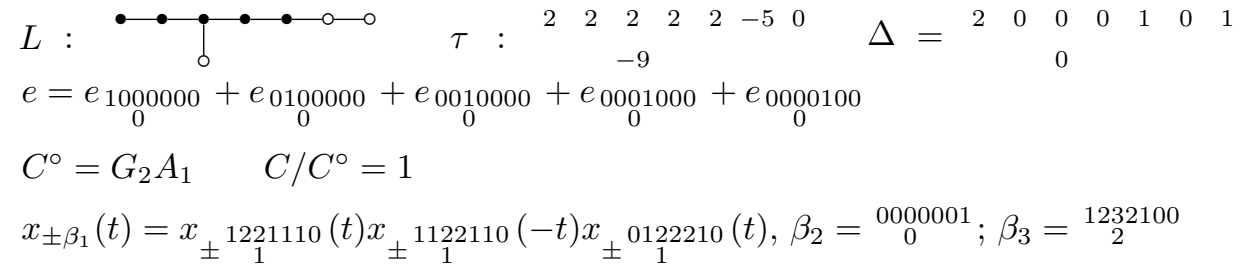




\begin{tabular}{|c|c|c|c|c|c|}
\hline$n$ & $m$ & $\lambda$ & $v$ & $\mathcal{Z}^{\natural}$ & $\mathcal{Z}$ \\
\hline 3 & \begin{tabular}{|l|}
3 \\
\end{tabular} & $\lambda_{3}$ & $e_{1221000}+e_{1121100}-e_{0122100}$ & & \\
\hline 2 & $\begin{array}{l}4 \\
5 \\
6\end{array}$ & $\begin{array}{c}\lambda_{1} \\
\lambda_{1}+\lambda_{3} \\
0\end{array}$ & $\begin{array}{c}e_{2344321}-e_{1354321} \\
2 \\
e_{2465421} \\
3 \\
e_{0} e_{0} 110000 \\
0 \\
0\end{array}$ & & \\
\hline 1 & $\begin{array}{l}2 \\
8\end{array}$ & $\begin{array}{c}0 \\
\lambda_{1} \\
\lambda_{3}\end{array}$ & $\begin{array}{c}e \\
e_{2454321} \\
e_{1232100}\end{array}$ & $z_{2}$ & $z_{2}$ \\
\hline & 10 & 0 & $e_{0} e_{0}$ & $z_{10}$ & $z_{10}$ \\
\hline
\end{tabular}

$E_{8}$, orbit 29: $D_{5}\left(a_{1}\right) A_{1}$

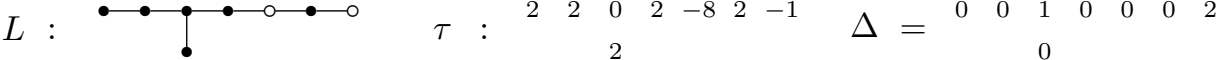

$$
\begin{aligned}
& e=\underset{0}{e_{1000000}}+e_{0}^{0100000}+e_{1}^{0010000}+e_{0}^{0011000}+e_{1}^{0000000}+e_{0}^{0001000}+e_{0}^{0000010} \\
& C^{\circ}=A_{1}^{2} \quad C / C^{\circ}=1 \\
& \beta_{1}={ }_{3}^{2465432} ; x_{\beta_{2}}(t)=x_{1111100}(t) x_{1}^{0121110}(t) x_{2}^{1232210}\left(\frac{1}{2} t^{2}\right) x_{1}^{0122100}(t) x_{0}^{1111110}(t) \times \\
& x_{1233210}\left(\frac{1}{2} t^{2}\right) \text {, } \\
& x_{-\beta_{2}}(t)=x_{-} 1111100(2 t) x_{-}{ }_{1}^{0121110}(2 t) x_{-}{ }_{2}^{1232210}\left(-2 t^{2}\right) x_{-} 0122100(2 t) x_{-}{ }_{1}^{11111110}(2 t) \times \\
& x_{-} 1233210\left(-2 t^{2}\right)
\end{aligned}
$$

\begin{tabular}{|c|c|c|c|c|c|}
\hline$n$ & $m$ & $\lambda$ & $v$ & $\mathcal{Z}^{\natural}$ & $\mathcal{Z}$ \\
\hline 5 & \begin{tabular}{l|}
1 \\
4
\end{tabular} & $\begin{array}{c}\lambda_{1}+4 \lambda_{2} \\
2 \lambda_{2}\end{array}$ & $\begin{array}{c}e_{2465431} \\
3 \\
e_{1221110}+e_{1232100}-e_{1222100}-2 e_{1122110} \\
\end{array}$ & & \\
\hline 4 & \begin{tabular}{l|}
2 \\
2 \\
5
\end{tabular} & $\begin{array}{c}4 \lambda_{2} \\
0 \\
\lambda_{1}+2 \lambda_{2}\end{array}$ & $\begin{array}{c}e_{1233210} \\
e_{0000000}+e_{0001000}+e_{0000010} \\
0 \\
e_{2454321}+e_{2464321}\end{array}$ & & \\
\hline 3 & $\begin{array}{l}3 \\
6 \\
6\end{array}$ & $\begin{array}{c}\lambda_{1} \\
4 \lambda_{2} \\
2 \lambda_{2}\end{array}$ & $\begin{array}{c}e_{1233221} \\
e_{2} e_{2} 3210 \\
e_{1232100}-e_{1} 1232110+2 e_{1222110} \\
\end{array}$ & & \\
\hline 2 & 7 & $\lambda_{1}+2 \lambda_{2}$ & $e_{2465321}$ & & \\
\hline 1 & $\begin{array}{c}2 \\
8 \\
10\end{array}$ & $\begin{array}{c}0 \\
2 \lambda_{2} \\
0\end{array}$ & $\begin{array}{c}e \\
e_{1232110} \\
e_{1221000}\end{array}$ & $z_{2}$ & $z_{2}$ \\
\hline
\end{tabular}


$E_{8}$, orbit 30: $A_{4} A_{2} A_{1}$

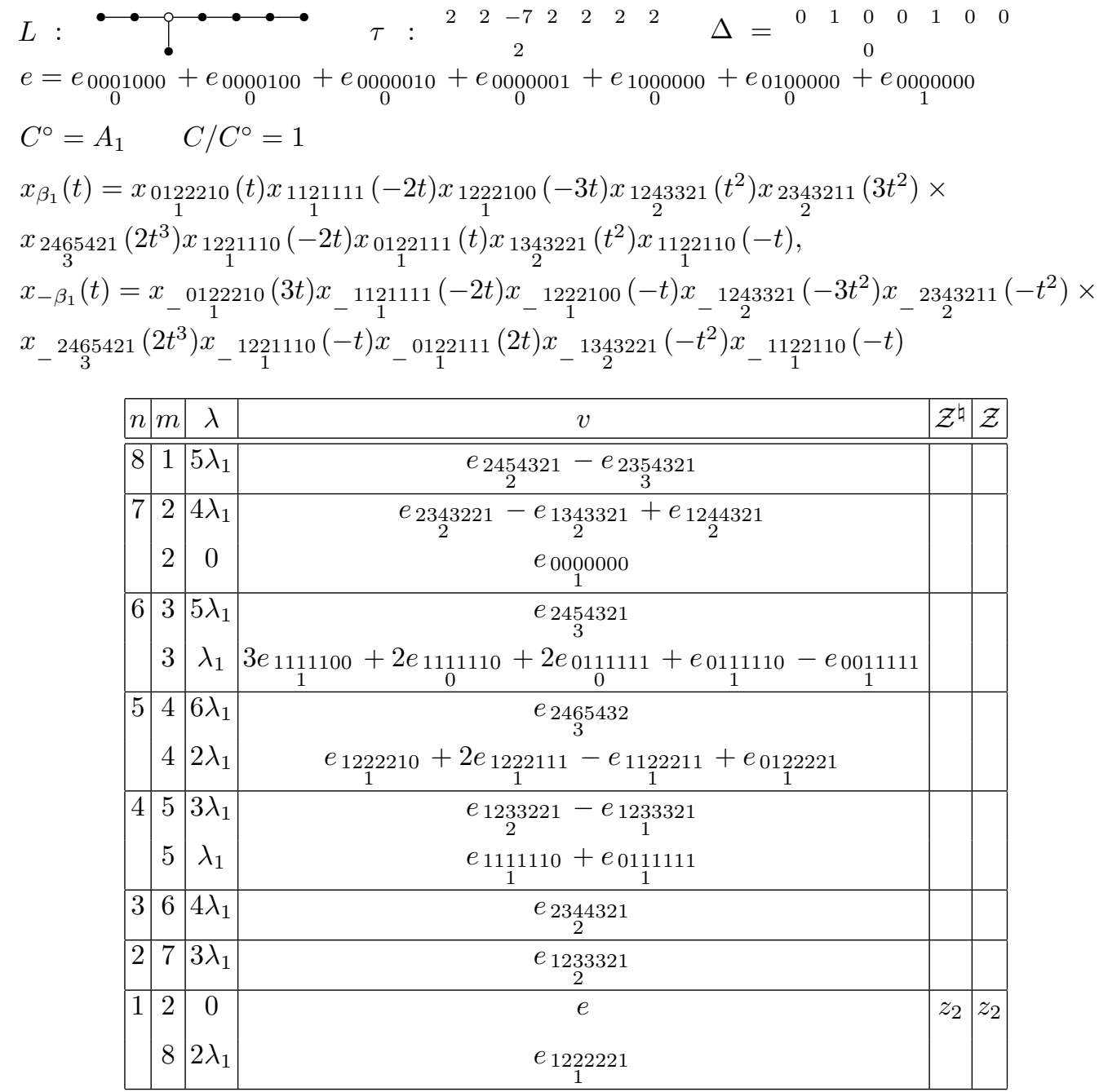


$E_{8}$, orbit 31: $D_{4} A_{2}$

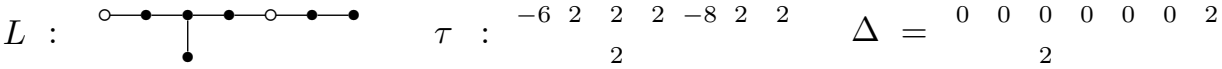

$$
\begin{aligned}
& e=\underset{0}{e} \underset{0}{0100000}+\underset{0}{e 010000}+\underset{0}{e 0000000}+e_{0}^{0001000}+e_{0}^{0000010}+\underset{0}{0000001} \\
& C^{\circ}=A_{2} \quad C / C^{\circ}=\left\langle c C^{\circ}\right\rangle \cong S_{2}
\end{aligned}
$$

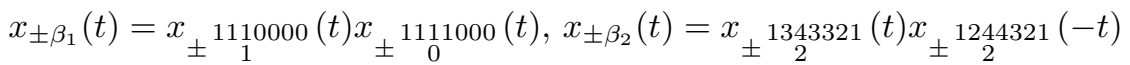

\begin{tabular}{|c|c|c|c|c|c|}
\hline$n$ & $m$ & $\lambda$ & $v$ & $\mathcal{Z}^{\natural}$ & $\mathcal{Z}$ \\
\hline 3 & 2 & $2 \lambda_{1}$ & $e_{2343221}$ & & \\
\hline & 2 & $2 \lambda_{2}$ & $e_{2465432}$ & & \\
\hline & 2 & 0 & $e_{0000010}+e_{0000001}$ & & \\
\hline & 4 & $\lambda_{1}$ & $e_{1232100}+e_{1232110}+e_{1222111}$ & & \\
\hline & 4 & $\lambda_{2}$ & $e_{1343210}+e_{1243211}-e_{1233221}$ & & \\
\hline 2 & 4 & 0 & $e_{0000011}$ & & \\
\hline & 6 & $\lambda_{1}+\lambda_{2}$ & $e_{2465321}$ & & \\
\hline & 6 & $\lambda_{1}$ & $e_{1232110}+e_{1232111}$ & & \\
\hline & 6 & $\lambda_{2}$ & $e_{1343211}-e_{1243221}$ & & \\
\hline 1 & 2 & 0 & $e$ & $z_{2}$ & $z_{2}$ \\
\hline & 8 & $\lambda_{1}$ & $e_{1232111}$ & & \\
\hline & 8 & $\lambda_{2}$ & $e_{1343221}$ & & \\
\hline & 10 & 0 & $e_{0121000}$ & $z_{10}$ & $z_{10}$ \\
\hline
\end{tabular}

$$
\begin{aligned}
& c=n_{1}^{001110} n_{0}^{011110} n_{0122211} h_{2}(-1) h_{4}(-1) h_{5}(-1) h_{6}(-1)
\end{aligned}
$$

$E_{8}$, orbit $32: E_{6}\left(a_{3}\right)$

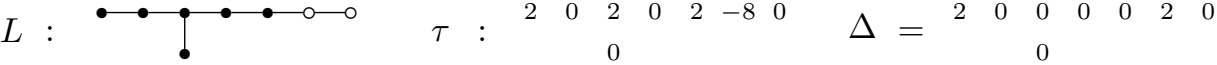

$$
\begin{aligned}
& e=e_{1}^{0110000}+e_{100000}+e_{0}^{0111000}+e_{0000100}+e_{1}^{0011000}+e_{0010000} \\
& C^{\circ}=G_{2} \quad C / C^{\circ}=\left\langle c C^{\circ}\right\rangle \cong S_{2} \\
& x_{ \pm \beta_{1}}(t)=x_{ \pm}{ }_{1}^{1221110}(t) x_{ \pm}{ }_{1}^{1122110}(t) x_{ \pm}{ }_{1}^{0122210}(-t), \beta_{2}=0000001 \\
& c=h_{4}(-1)
\end{aligned}
$$




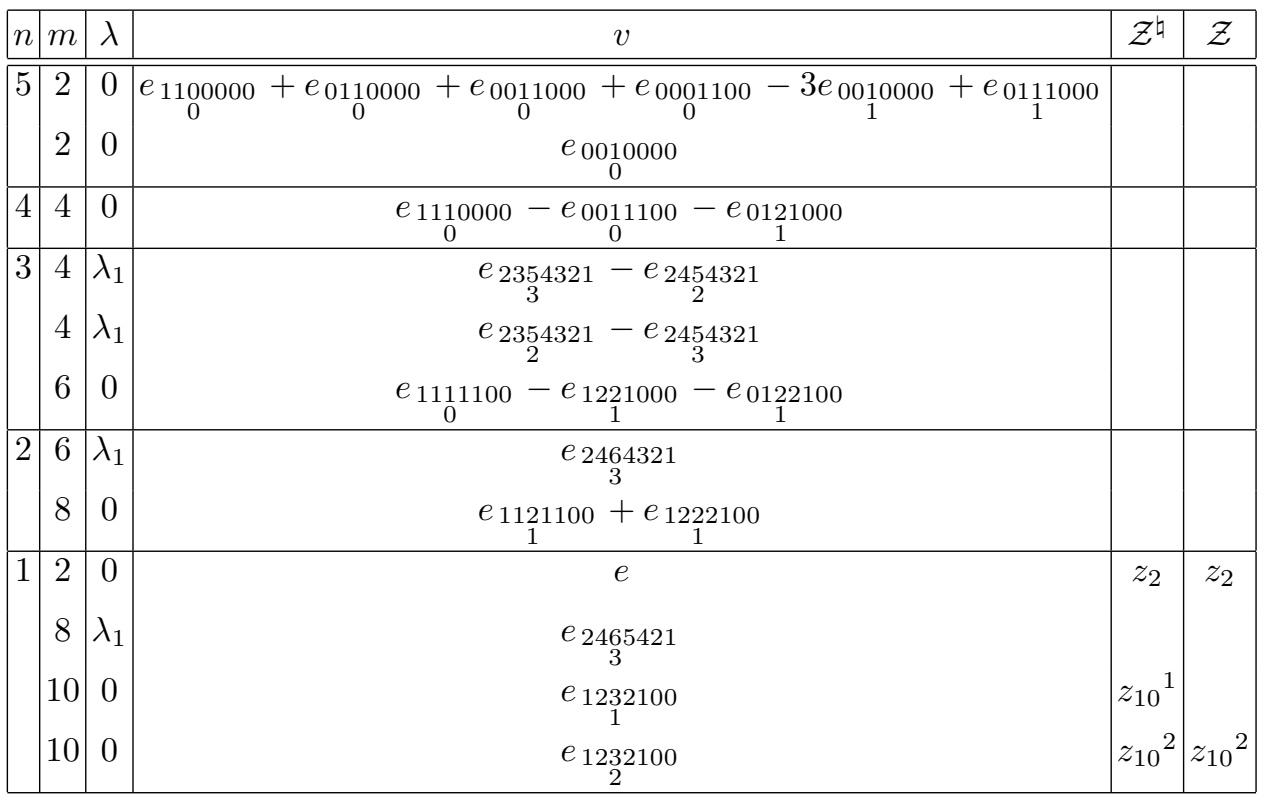

$E_{8}$, orbit $33: D_{5}$

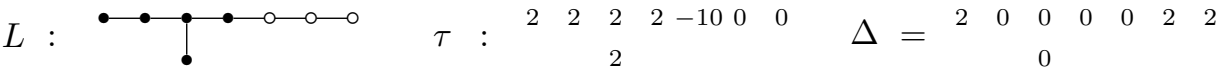

$$
\begin{aligned}
& e=e_{1000000}+e_{0100000}+e_{0010000}+e_{0000000}+e_{0001000} \\
& C^{\circ}=B_{3} \quad C / C^{\circ}=1 \\
& \beta_{1}=\underset{0}{0000010}, \beta_{2}=\underset{0}{0000001}, x_{ \pm \beta_{3}}(t)=x_{ \pm}^{1232210}(t) x_{ \pm}^{1233210}(-t)
\end{aligned}
$$

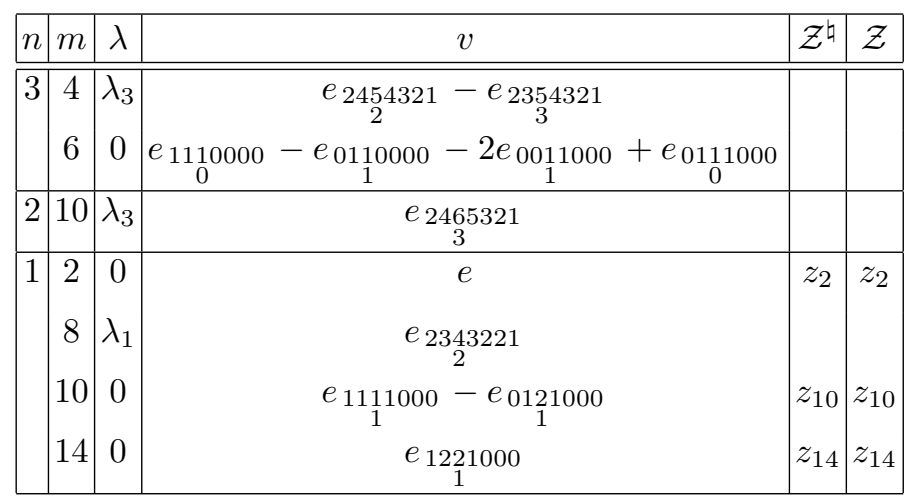


$E_{8}$, orbit $34: A_{4} A_{3}$

$$
\begin{aligned}
& L \text { : } \\
& \cdots \cdots \\
& e=\underset{0}{e} \underset{0}{1000000}+\underset{0}{e 0100000}+\underset{0}{e 0010000}+e_{0}^{0000000}+\underset{0}{e 0000100}+e_{0}^{0000010}+e_{0}^{0000001} \\
& C^{\circ}=A_{1} \quad C / C^{\circ}=1 \\
& x_{\beta_{1}}(t)=x_{1122211}(2 t) x_{1232110}(-t) x_{2354321}\left(-t^{2}\right) x_{1}^{0122221}(-2 t) x_{2}^{1232100}(-t) x_{1354321}\left(t^{2}\right) \times \\
& x_{1222210}(t) x_{1222111}(-t) \text {, }
\end{aligned}
$$

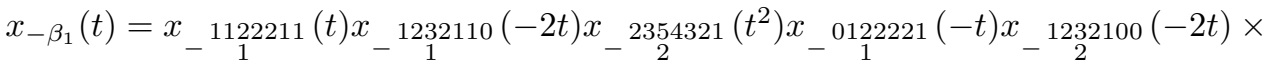

$$
\begin{aligned}
& x_{-} 1354321\left(-t^{2}\right) x_{-} 1222210(t) x_{-}{ }_{12} 22111(-t)
\end{aligned}
$$

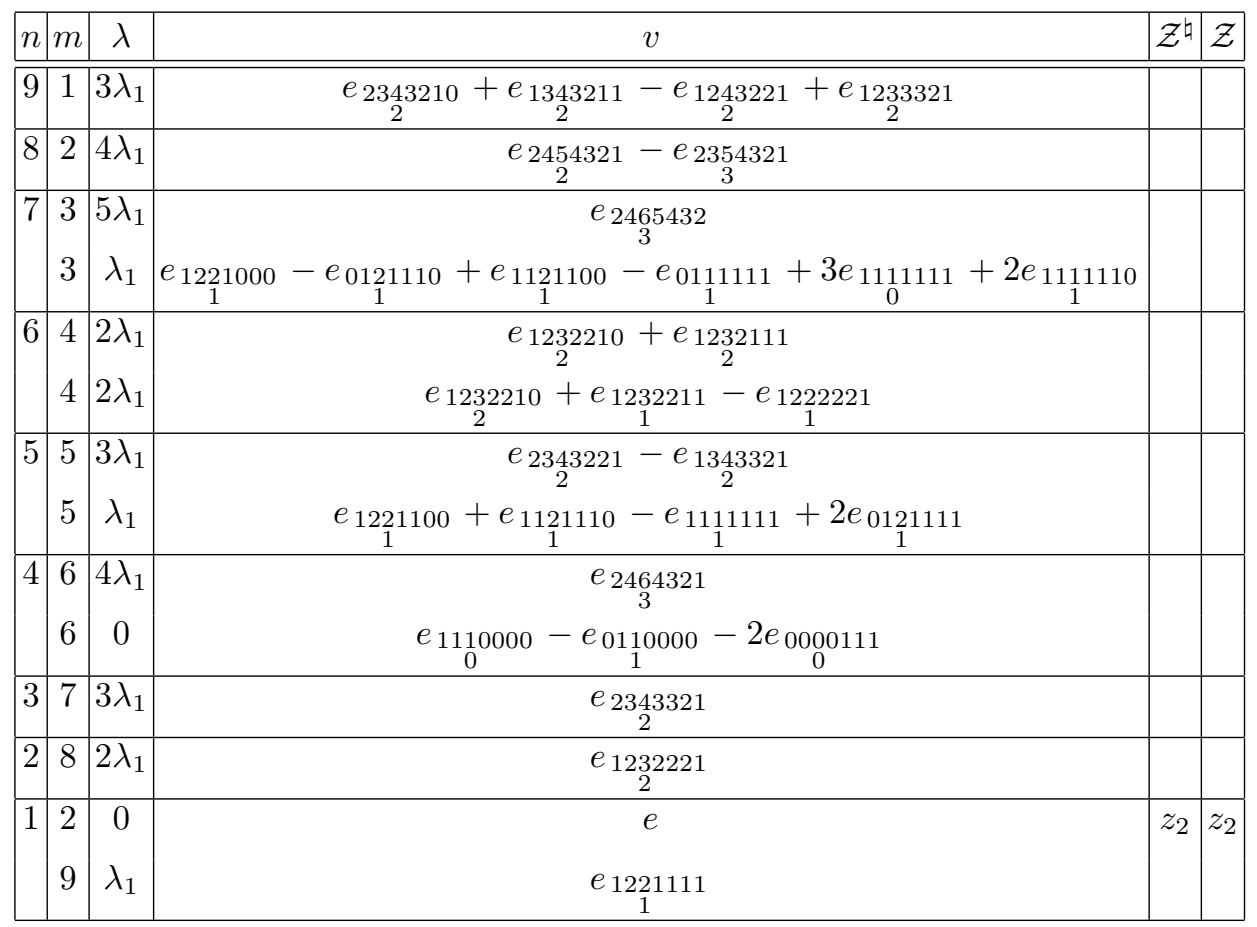


$E_{8}$, orbit $35: A_{5} A_{1}$

$$
\begin{aligned}
& L: \bullet \bullet \bullet \bullet \bullet \quad \tau: \begin{array}{lllllll}
2 & -9 & 2 & 2 & 2 & 2 & -5
\end{array} \quad \Delta=\begin{array}{lllllll}
1 & 0 & 1 & 0 & 0 & 0 & 1
\end{array} \\
& e=\underset{1}{e 0000000}+\underset{0}{e 0010000}+\underset{0}{e 0001000}+\underset{0}{00000100}+\underset{0}{e 000010}+e_{0}^{000000} \\
& C^{\circ}=A_{1}^{2} \quad C / C^{\circ}=1 \\
& \beta_{1}={ }_{3}^{2465432}, x_{ \pm \beta_{2}}(t)=x_{ \pm}{ }_{2}^{1232100}(t) x_{ \pm}{ }_{1}^{1232110}(t) x_{ \pm}{ }_{1}^{1222210}(-t)
\end{aligned}
$$

\begin{tabular}{|c|c|c|c|c|c|}
\hline$\left.n\right|_{1}$ & & $\lambda$ & $v$ & $\mathcal{Z}^{\natural}$ & $\mathcal{Z}$ \\
\hline \begin{tabular}{|l|}
5 \\
\end{tabular} & 1 & $3 \lambda_{2}$ & $e_{2343210}$ & & \\
\hline & 3 & $\lambda_{2}$ & $e_{1121100}-e_{0122100}-e_{0121110}+2 e_{1111110}$ & & \\
\hline \begin{tabular}{|l|}
4 \\
\end{tabular} & 2 & 0 & $e_{1000000}$ & & \\
\hline & 3 & $\lambda_{1}$ & $e_{1243211}-e_{1233221}+e_{1233321}$ & & \\
\hline & 4 & $\lambda_{1}+\lambda_{2}$ & $e_{2354321}-e_{1354321}$ & & \\
\hline & 4 & $2 \lambda_{2}$ & $e_{1233210}-e_{1232210}$ & & \\
\hline 3 & 5 & $\lambda_{1}+2 \lambda_{2}$ & $e_{2465431}$ & & \\
\hline & 5 & $\lambda_{2}$ & $e_{1122100}+e_{1121110}$ & & \\
\hline & 7 & $\lambda_{2}$ & $e_{1122110}-e_{0122210}$ & & \\
\hline 2 & 6 & $\lambda_{1}+\lambda_{2}$ & $e_{3}^{2354321}$ & & \\
\hline & 6 & 0 & $e_{0011000}^{0}+e_{0}^{0011100}+e_{0}^{0001110}$ & & \\
\hline & 8 & $2 \lambda_{2}$ & $e_{1243210}$ & & \\
\hline 1 & 2 & 0 & e & $z_{2}$ & $z_{2}$ \\
\hline & 9 & $\lambda_{1}$ & $e_{1244321}$ & & \\
\hline & 9 & $\lambda_{2}$ & $e_{1122210}$ & & \\
\hline & 10 & 0 & $e_{0011110}$ & $z_{10}$ & $z_{10}$ \\
\hline
\end{tabular}


$E_{8}$, orbit 36: $D_{5}\left(a_{1}\right) A_{2}$

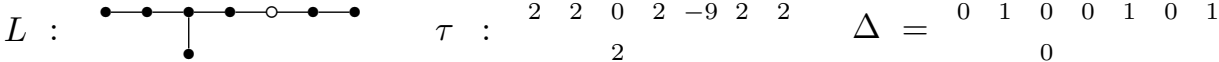

$$
\begin{aligned}
& e=\underset{0}{e} 1000000+e_{0}^{0100000}+e_{1}^{0010000}+e_{0}^{0011000}+e_{1}^{0000000}+e_{0}^{0001000}+e_{0}^{0000010}+e_{0}^{0000001} \\
& C^{\circ}=A_{1} \quad C / C^{\circ}=1 \\
& x_{\beta_{1}}(t)=x_{1232221}(t) x_{1233210}(-2 t) x_{2465431}\left(t^{2}\right) x_{1232211}(-t) x_{1233211}(-t) \text {, } \\
& x_{-\beta_{1}}(t)=x_{-} 1232221(2 t) x_{-} 1233210(-t) x_{-}{ }_{2}^{2465431}\left(-t^{2}\right) x_{-} 1232211(-t) x_{-}{ }_{1}^{1233211}(-t)
\end{aligned}
$$

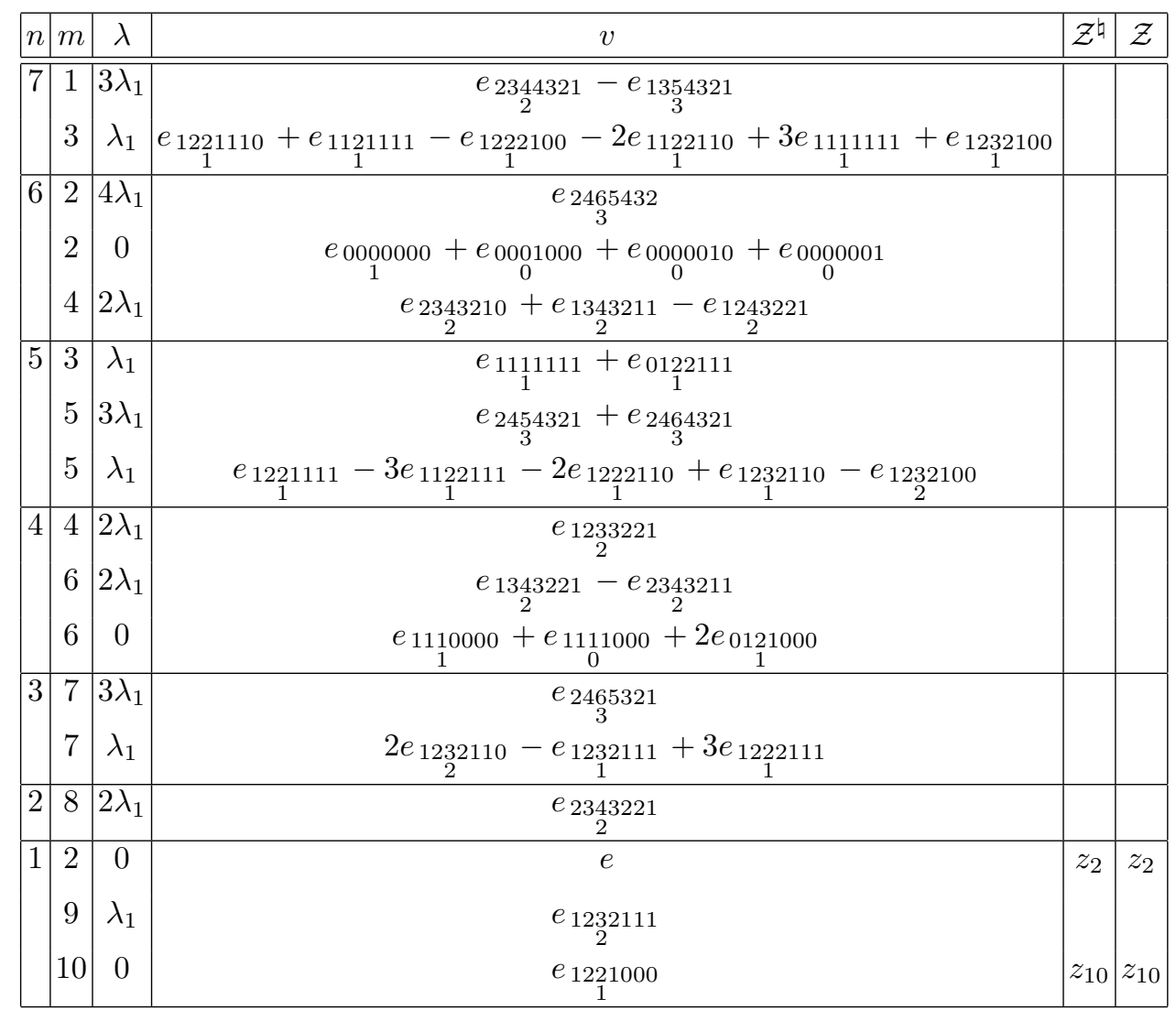


$E_{8}$, orbit $37: D_{6}\left(a_{2}\right)$

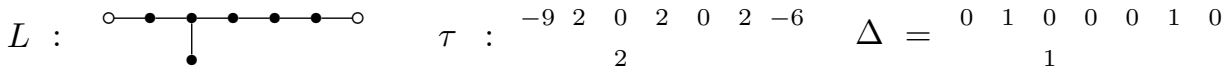

$$
\begin{aligned}
& e=\underset{0}{0000010}+\underset{0}{e 001100}+e_{0}^{0010000}-e_{0}^{0110000}+\underset{0}{e 011000}+e_{0}^{0000000}+e_{0100000}^{0} \\
& C^{\circ}=A_{1}{ }^{2} \quad C / C^{\circ}=\left\langle c C^{\circ}\right\rangle \cong S_{2} \\
& \beta_{1}={ }_{2}^{2343210}, \beta_{2}={ }_{3}^{2465432} \\
& c=n_{0011111} n_{0111111} h_{4}(-1) h_{5}(-1)
\end{aligned}
$$

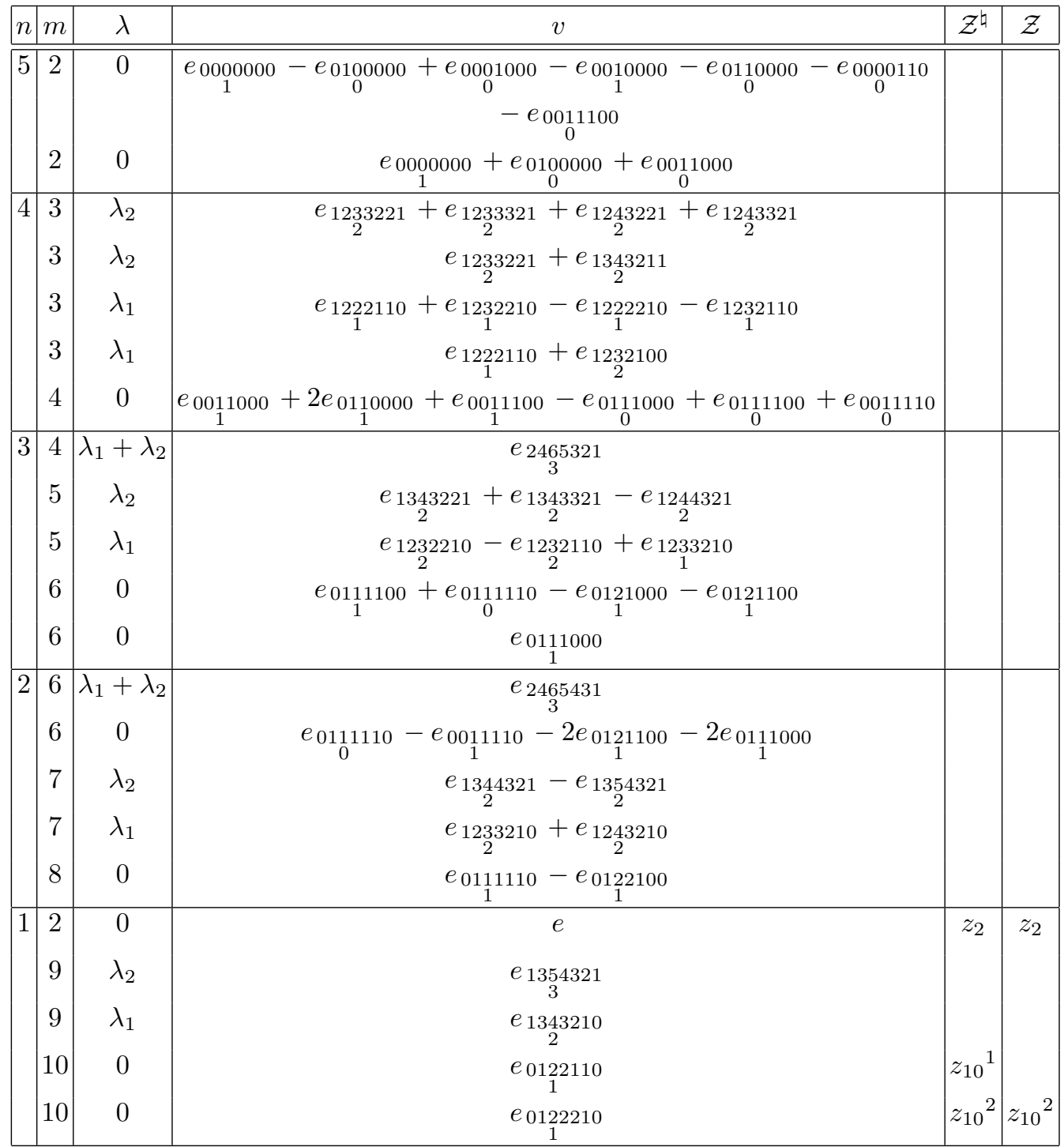


$E_{8}$, orbit $38: E_{6}\left(a_{3}\right) A_{1}$

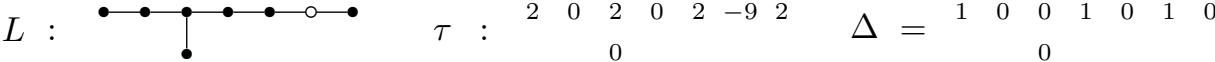

$$
\begin{aligned}
& e=\underset{1}{0.110000}+e_{0} 1000000+e_{0}^{0111000}+e_{0}^{0000100}+e_{1}^{0011000}+e_{0}^{0010000}+e_{000001}^{0} \\
& C^{\circ}=A_{1} \quad C / C^{\circ}=\left\langle c C^{\circ}\right\rangle \cong S_{2} \\
& x_{ \pm \beta_{1}}(t)=x_{ \pm \underset{2}{1244321}(t) x_{ \pm} 1343321}(t) x_{ \pm \underset{2}{2}}^{2343221}(-t) \\
& c=h_{4}(-1)
\end{aligned}
$$

\begin{tabular}{|c|c|c|c|c|c|}
\hline$n$ & $m$ & $\lambda$ & $v$ & $\mathcal{Z}^{\natural}$ & $\mathcal{Z}$ \\
\hline 5 & 1 & $3 \lambda_{1}$ & $e_{2465432}$ & & \\
\hline & 2 & 0 & $e_{0}^{1100000}+e_{0}^{0110000}+e_{0011000}^{00}+e_{0}^{0001100}-3 e_{0010000}+e_{0111000}^{0}$ & & \\
\hline & 2 & 0 & $e_{0010000}$ & & \\
\hline & 3 & $\lambda_{1}$ & $e_{1233210}+e_{1232210}+2 e_{1232111}-e_{1222211}$ & & \\
\hline & 3 & $\lambda_{1}$ & $e_{1233210}+e_{1232210}+2 e_{1232111}+e_{1122211}$ & & \\
\hline 4 & 2 & 0 & $e_{0}^{0000001}$ & & \\
\hline & 4 & $2 \lambda_{1}$ & $e_{2354321}-e_{2454321}$ & & \\
\hline & 4 & $2 \lambda_{1}$ & $e_{2354321}-e_{2454321}$ & & \\
\hline & 4 & 0 & $e_{1110000}-e_{0011100}-e_{0121000}$ & & \\
\hline & 5 & $\lambda_{1}$ & $e_{1232211}-e_{1243210}$ & & \\
\hline 3 & 5 & $\lambda_{1}$ & $e_{1232211}+e_{1233211}$ & & \\
\hline & 5 & $\lambda_{1}$ & $e_{1232211}+e_{1233211}$ & & \\
\hline & 6 & $2 \lambda_{1}$ & $e_{2464321}$ & & \\
\hline & 6 & 0 & $e_{1111100}-e_{1221000}-e_{0122100}$ & & \\
\hline & 7 & $\lambda_{1}$ & $e_{2343210}+e_{1343211}$ & & \\
\hline 2 & 7 & $\lambda_{1}$ & $e_{1243211}$ & & \\
\hline & 8 & $2 \lambda_{1}$ & $e_{2465421}$ & & \\
\hline & 8 & 0 & $e_{1121100}+e_{1222100}$ & & \\
\hline 1 & 2 & 0 & $e$ & $z_{2}$ & $z_{2}$ \\
\hline & 9 & $\lambda_{1}$ & $e_{2343211}$ & & \\
\hline & 10 & 0 & $e_{1232100}$ & $z_{10}{ }^{1}$ & \\
\hline & 10 & 0 & $e_{1232100}$ & $z_{10}^{2}$ & $z_{10}{ }^{2}$ \\
\hline
\end{tabular}


$E_{8}$, orbit 39: $E_{7}\left(a_{5}\right)$

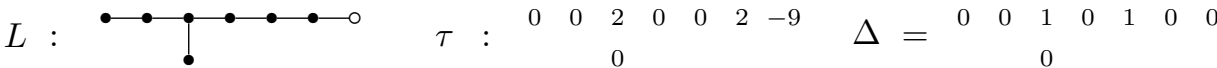

$$
\begin{aligned}
& e=\underset{0}{e} e_{111000}+e_{0}^{0011100}+e_{0}^{0000010}+e_{0}^{0111100}+e_{1110000}+e_{0}^{0010000}+e_{0111000} \\
& C^{\circ}=A_{1} \quad C / C^{\circ}=\left\langle c_{1} C^{\circ}, c_{2} C^{\circ}\right\rangle \cong S_{3} \\
& \beta_{1}={ }_{3}^{2465432} \\
& c_{1}=h_{2}(\omega) h_{3}(\omega) h_{5}(\omega) \text {, } \\
& c_{2}=n_{1} 0000000 \underset{0}{0100000} n_{0001000} h_{3}(-1) h_{4}(-1)
\end{aligned}
$$

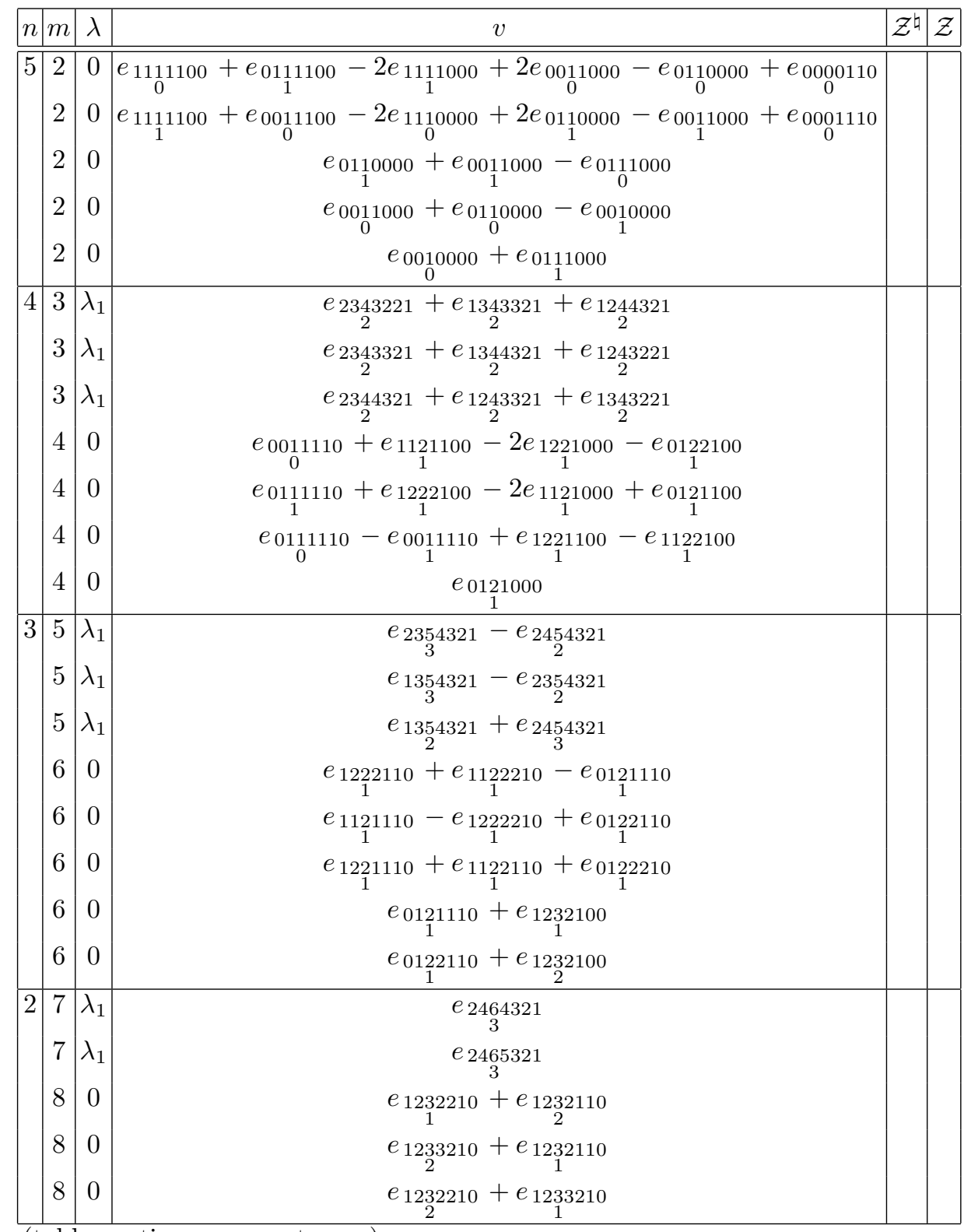

(table continues on next page) 


\begin{tabular}{|c|c|c|c|c|c|}
\hline$n$ & $m$ & $\lambda$ & $v$ & $\mathcal{Z}^{\natural}$ & $\mathcal{Z}$ \\
\hline 1 & 2 & 0 & $e$ & $z_{2}$ & $z_{2}$ \\
\hline & 9 & $\lambda_{1}$ & $e_{2465431}$ & & \\
\hline & 10 & 0 & $e_{1243210}$ & $z_{10}{ }^{1}$ & \\
\hline & 10 & 0 & $e_{1343210}$ & $z_{10}{ }^{2}$ & \\
\hline & 10 & 0 & $e_{2343210}$ & $z_{10}{ }^{3}$ & $z_{10}{ }^{3}$ \\
\hline
\end{tabular}

$E_{8}$, orbit 40: $D_{5} A_{1}$

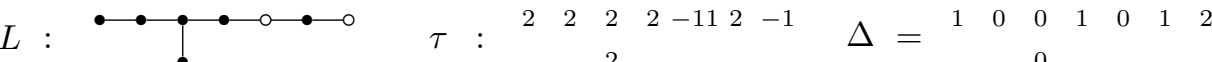

$$
\begin{aligned}
& e=e_{1000000}+e_{0100000}+e_{0010000}+e_{0000000}+e_{0001000}+e_{0000010} \\
& C^{\circ}=A_{1}^{2} \quad C / C^{\circ}=1 \\
& \beta_{1}={ }_{3}^{2465432}, x_{ \pm \beta_{2}}(t)=x_{ \pm \underset{2}{1232210}}(t) x_{ \pm \underset{1}{1233210}(-t)}
\end{aligned}
$$

\begin{tabular}{|c|c|c|c|c|c|}
\hline$n$ & $m$ & $\lambda$ & $v$ & $\mathcal{Z}^{\natural}$ & $\mathcal{Z}$ \\
\hline 6 & 1 & $\lambda_{1}+2 \lambda_{2}$ & $e_{2465431}$ & & \\
\hline & 3 & $\lambda_{2}$ & $e_{1221100}+e_{1121110}-e_{1122100}-2 e_{0122110}$ & & \\
\hline 5 & 2 & 0 & $\underset{0}{e 000010}$ & & \\
\hline & 4 & $\lambda_{1}+\lambda_{2}$ & $e_{2454321}-e_{2354321}$ & & \\
\hline & 6 & 0 & $e_{0} e_{1110000}-e_{0110000}-2 e_{0011000}+e_{0}^{0111000}$ & & \\
\hline 4 & 5 & $\lambda_{2}$ & $e_{1221110}-e_{1122110}$ & & \\
\hline & 7 & $\lambda_{1}$ & $e_{2343211}-e_{1342}$ & & \\
\hline & 9 & $\lambda_{2}$ & $e_{1232100}+e_{1232110}$ & & \\
\hline 3 & 8 & $2 \lambda_{2}$ & $e_{2}^{2343210}$ & & \\
\hline & 10 & $\lambda_{1}+\lambda_{2}$ & $e_{2465321}$ & & \\
\hline 2 & 11 & $\lambda_{2}$ & $e_{1232110}$ & & \\
\hline 1 & 2 & 0 & $e$ & $z_{2}$ & $z_{2}$ \\
\hline & 9 & $\lambda_{1}$ & $e_{2343221}$ & & \\
\hline & 10 & 0 & $e_{1111000}-e_{0121000}$ & $z_{10}$ & $z_{10}$ \\
\hline & 14 & 0 & $e_{1221000}$ & $z_{14}$ & $z_{14}$ \\
\hline
\end{tabular}


$E_{8}$, orbit 41: $E_{8}\left(a_{7}\right)$

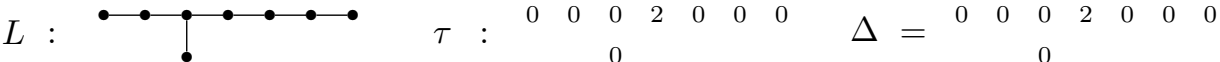

$$
\begin{aligned}
& e=e_{0}^{1111111}+e_{0121110}+e_{0}^{0001000}+e_{1121100}+e_{1221000}+e_{0011111}+e_{0111100}+e_{111110} \\
& C^{\circ}=1 \quad C / C^{\circ}=\left\langle c_{1} C^{\circ}, c_{2} C^{\circ}, c_{3} C^{\circ}\right\rangle \cong S_{5} \\
& c_{1}=h_{2}\left(\zeta^{2}\right) h_{3}\left(\zeta^{4}\right) h_{4}(\zeta) h_{6}\left(\zeta^{4}\right) h_{7}(\zeta) h_{8}\left(\zeta^{2}\right) \text {, } \\
& c_{2}=n_{0100000} n_{0010000} n_{0000000} n_{0000100} n_{0000010} n_{0000001} \\
& \times \stackrel{0}{h_{1}(-1) h_{3}(-1) h_{5}(-1) h_{6}(-1) h_{8}(-1),} \\
& c_{3}=\left(n_{1110000} n_{0110000} n_{0000111} n_{0000010} h\right)^{u} \text {, } \\
& h=h_{1}\left(\frac{2}{5}\right) h_{2}\left(-\frac{2}{5}\right) h_{3}\left(\frac{2(1-3 \phi)}{25}\right) h_{4}\left(\frac{2(1-3 \phi)}{25}\right) h_{6}\left(\frac{3+\phi}{5}\right) h_{7}\left(-\frac{3+\phi}{5}\right) h_{8}\left(-\frac{3+\phi}{5}\right) \text {, } \\
& u=x_{1110000}\left(-\frac{1}{2}+\frac{1}{2} \phi\right) x_{0110000}(-3-2 \phi) x_{10010000}(-3-2 \phi) x_{0000000}(1) x_{1110000}(1+\phi) \\
& \times x_{0110000}(5+3 \phi) x_{0}^{0010000}(4+2 \phi) x_{0}^{1100000}\left(1+\frac{1}{2} \phi\right) x_{0}^{0100000}(1+\phi) x_{0}^{1000000}(\phi) \\
& \times x_{0}^{0000111}(-\phi) x_{0}^{0000100}(\phi) x_{0}^{0000011}(-1+\phi) x_{0}^{0000010}(-\phi) x_{0000001}(-\phi)
\end{aligned}
$$

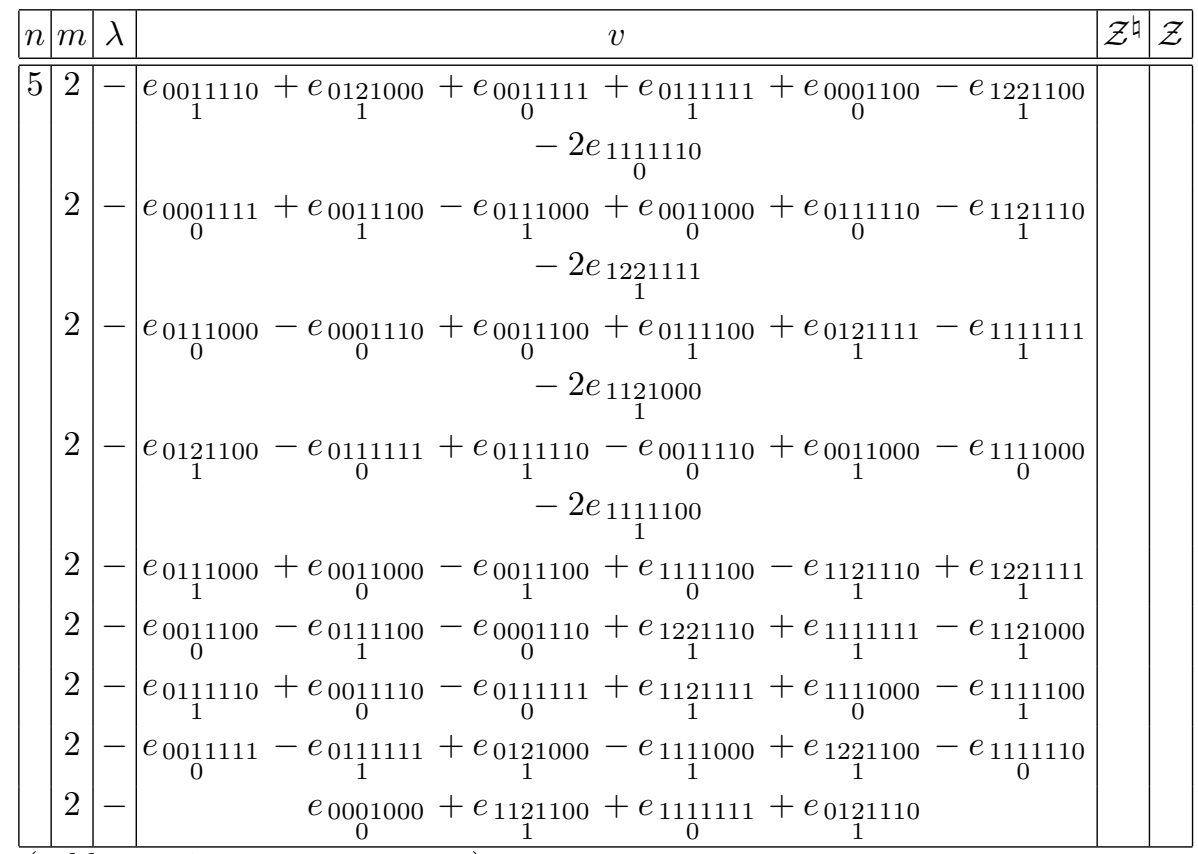

(table continues on next page) 


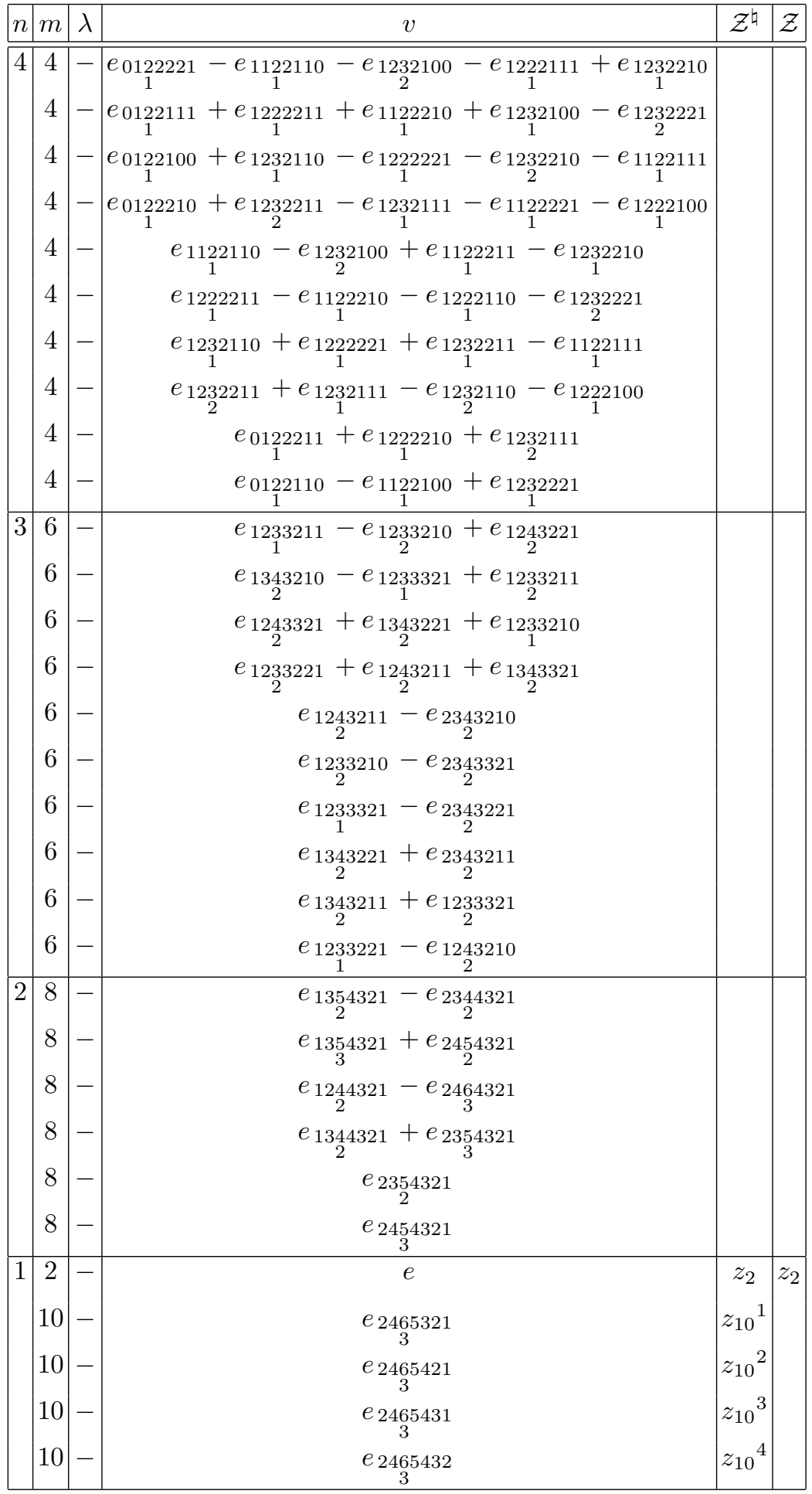


$E_{8}$, orbit $42: A_{6}$

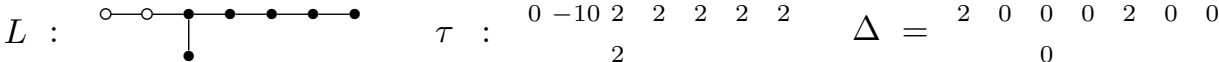

$$
\begin{aligned}
& e=\underset{1}{e 0000000}+\underset{0}{e 0010000}+\underset{0}{e 0001000}+\underset{0}{00000100}+\underset{0}{e 000010}+e_{0}^{000001} \\
& C^{\circ}=A_{1}^{2} \quad C / C^{\circ}=1 \\
& \beta_{1}={ }_{0}^{1000000} \text {; } \\
& x_{\beta_{2}}(t)=x_{2} 232111(2 t) x_{1233210}(-t) x_{2465321}\left(-t^{2}\right) x_{2}^{1232210}(t) x_{1222221}(t) x_{1232211}(-t) \text {, } \\
& x_{-\beta_{2}}(t)=x_{-} 1232111(t) x_{-} 1233210(-2 t) x_{-} 2465321\left(t^{2}\right) x_{-}{ }_{2}^{1232210}(t) x_{-}{ }_{1}^{1222221}(t) \times \\
& x_{-} 1232211(-t)
\end{aligned}
$$

\begin{tabular}{|c|c|c|c|c|c|}
\hline$n$ & $m$ & $\lambda$ & $v$ & $\mathcal{Z}^{\natural}$ & $\mathcal{Z}$ \\
\hline \hline 6 & 2 & $\lambda_{1}+\lambda_{2}$ & $e_{1122100}+e_{1121110}+e_{1111111}$ & & \\
\hline 5 & 4 & $2 \lambda_{2}$ & $e_{1243210}+e_{1233211}-e_{1232221}$ & & \\
\hline 4 & 6 & $\lambda_{1}+3 \lambda_{2}$ & $e_{2354321}$ & & \\
\hline 3 & 8 & $2 \lambda_{2}$ & $e_{1243221}-e_{1233321}$ & & \\
\hline 2 & 6 & $4 \lambda_{2}$ & $e_{2465432}$ & & \\
& 10 & $\lambda_{1}+\lambda_{2}$ & $e_{112221}$ & & \\
\hline 1 & 2 & 0 & $e$ & $z_{2}$ & $z_{2}$ \\
& 10 & 0 & $e_{0011110}+e_{0011111}$ & $z_{10}$ & $z_{10}$ \\
& 12 & $2 \lambda_{2}$ & $e_{1244321}$ & & \\
\hline
\end{tabular}


$E_{8}$, orbit 43: $D_{6}\left(a_{1}\right)$

$$
\begin{aligned}
& L: \longrightarrow ? \longrightarrow \longrightarrow \quad \tau: \begin{array}{ccccccccccccccc}
-11 & 2 & 0 & 2 & 2 & 2 & -8 & \Delta= \\
0 & 1 & 0 & 0 & 0 & 1 & 2
\end{array} \\
& e=\underset{0}{e 0000010}+\underset{0}{e 0000100}+e_{0}^{0001000}+e_{1}^{0010000}-e_{0}^{0110000}+e_{0}^{0000000}+e_{0}^{0100000} \\
& C^{\circ}=A_{1}{ }^{2} \quad C / C^{\circ}=\left\langle c C^{\circ}\right\rangle \cong S_{2} \\
& \beta_{1}={ }_{2}^{2343210}, \beta_{2}={ }_{3}^{2465432} \\
& c=n_{0011111} n_{0111111} h_{2}(i) h_{3}(i)
\end{aligned}
$$

\begin{tabular}{|c|c|c|c|c|c|}
\hline$n$ & $m$ & $\lambda$ & $v$ & $\mathcal{Z}^{\natural}$ & $\mathcal{Z}$ \\
\hline 5 & 2 & $\lambda_{1}+\lambda_{2}$ & $e_{2454321}$ & & \\
\hline & 2 & 0 & $e_{0000000}+e_{0}^{0100000}$ & & \\
\hline & 3 & $\lambda_{2}$ & $e_{1233221}-e_{1233321}+2 e_{1342}$ & & \\
\hline & 3 & $\lambda_{1}$ & $e_{1122210}-e_{1222110}-e_{1232110}-2 e_{1232100}$ & & \\
\hline 4 & 5 & $\lambda_{2}$ & $e_{1233321}+e_{13423221}$ & & \\
\hline & 5 & $\lambda_{1}$ & $e_{1222210}-e_{1232110}$ & & \\
\hline & 6 & 0 & $2 e_{0111000}+e_{0011100}+e_{0111100}+e_{0011110}$ & & \\
\hline & 6 & 0 & $2 e_{0121000}+e_{0011100}-e_{0111100}+e_{0001110}$ & & \\
\hline 3 & 8 & $\lambda_{1}+\lambda_{2}$ & $e_{2465431}$ & & \\
\hline & 8 & 0 & $2 e_{0111100}+e_{0}^{0111110}+e_{0011110}$ & & \\
\hline & 9 & $\lambda_{2}$ & $e_{1344321}-e_{1354321}$ & & \\
\hline & 9 & $\lambda_{1}$ & $e_{1233210}+e_{12423210}$ & & \\
\hline 2 & 11 & $\lambda_{2}$ & $e_{1354321}$ & & \\
\hline & 11 & $\lambda_{1}$ & $e_{1343210}$ & & \\
\hline 1 & 2 & 0 & $e$ & $z_{2}$ & $z_{2}$ \\
\hline & 10 & 0 & $e_{0121110}+e_{0122100}$ & $z_{10}{ }^{1}$ & $z_{10}^{1}$ \\
\hline & 10 & 0 & $e_{0111110}$ & $z_{10}^{2}$ & \\
\hline & 14 & 0 & $e_{012210}$ & $z_{14}$ & $z_{14}$ \\
\hline
\end{tabular}


$E_{8}$, orbit 44: $A_{6} A_{1}$

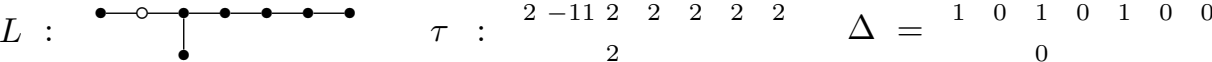

$$
\begin{aligned}
& e=\underset{1}{e 0000000}+\underset{0}{e 0010000}+\underset{0}{e 0001000}+\underset{0}{e 0000100}+\underset{0}{e 0000010}+e_{0}^{0000001}+e_{1000000} \\
& C^{\circ}=A_{1} \quad C / C^{\circ}=1 \\
& x_{\beta_{1}}(t)=x_{1232111}(2 t) x_{1233210}(-t) x_{2465321}\left(-t^{2}\right) x_{1232210}(t) x_{1222221}(t) x_{1232211}(-t) \text {, } \\
& x_{-\beta_{1}}(t)=x_{-\underset{2}{1232111}}(t) x_{-} 1233210(-2 t) x_{-}^{2465321}\left(t^{2}\right) x_{-}{ }_{2}^{1232210}(t) x_{-}{ }_{1}^{1222221}(t) \times \\
& x_{-} 1232211(-t)
\end{aligned}
$$

\begin{tabular}{|c|c|c|c|c|c|}
\hline$n$ & $m$ & $\lambda$ & $v$ & $\mathcal{Z}^{\natural}$ & $\mathcal{Z}$ \\
\hline 12 & \begin{tabular}{|l|}
1 \\
\end{tabular} & $\lambda_{1}$ & $e_{1121100}-e_{0111111}-e_{0122100}-e_{0121110}+2 e_{1111110}+3 e_{1111111}$ & & \\
\hline 11 & 2 & 0 & $\begin{array}{c}e_{100000} \\
0\end{array}$ & & \\
\hline 10 & 3 & $\lambda_{1}$ & $e_{1122100}+e_{1121110}+e_{1111111}$ & & \\
\hline \begin{tabular}{|l|}
9 \\
\end{tabular} & 4 & $2 \lambda_{1}$ & $e_{1243210}+e_{1233211}-e_{1232221}$ & & \\
\hline 8 & 5 & $3 \lambda_{1}$ & $e_{2354321}-e_{1354321}$ & & \\
\hline 7 & 6 & $4 \lambda_{1}$ & $e_{2465432}$ & & \\
\hline 6 & 7 & $3 \lambda_{1}$ & $e_{2354321}$ & & \\
\hline 5 & 8 & $2 \lambda_{1}$ & $e_{1243221}-e_{1233321}$ & & \\
\hline 4 & 9 & $\lambda_{1}$ & $e_{1122211}-e_{0122221}$ & & \\
\hline 3 & 10 & 0 & $e_{0011110}+e_{0011111}$ & & \\
\hline 2 & 11 & $\lambda_{1}$ & $e_{1122221}$ & & \\
\hline 1 & 2 & 0 & e & $z_{2}$ & $z_{2}$ \\
\hline & 12 & $2 \lambda_{1}$ & $e_{1244321}$ & & \\
\hline
\end{tabular}


$E_{8}$, orbit $45: E_{7}\left(a_{4}\right)$

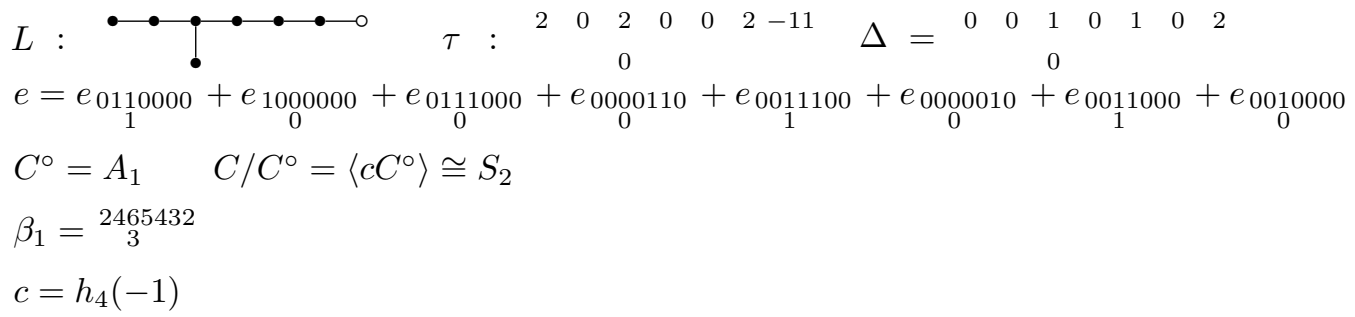

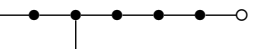

\begin{tabular}{|c|c|c|c|c|c|}
\hline$n$ & $m$ & $\lambda$ & $v$ & $\mathcal{Z}^{\natural}$ & $\mathcal{Z}$ \\
\hline 10 & \begin{tabular}{l|}
1 \\
2
\end{tabular} & \begin{tabular}{c|}
$\lambda_{1}$ \\
0
\end{tabular} & \begin{tabular}{|c}
$e_{1232221}+2 e_{1233221}-e_{1243211}$ \\
1 \\
$e_{1100000}+4 e_{0011000}+e_{0110000}-2 e_{0011100}+e_{0111000}+2 e_{0001110}$ \\
0 \\
0 \\
0 \\
0 \\
0
\end{tabular} & & \\
\hline 9 & $\begin{array}{l}2 \\
2 \\
3\end{array}$ & $\begin{array}{l}0 \\
0 \\
\lambda_{1}\end{array}$ & $\begin{array}{c}e_{0000010}+e_{0011000} \\
0 \\
e_{0010000} \\
0 \\
e_{1343321}+e_{1342}-e_{2343211}+2 e_{1244321}\end{array}$ & & \\
\hline 8 & $\begin{array}{l}3 \\
4 \\
4\end{array}$ & $\begin{array}{l}\lambda_{1} \\
0 \\
0\end{array}$ & $\begin{array}{c}e_{1243221} \\
e_{1110000}-e_{1111000}-e_{0121100}-e_{0111110} \\
e_{1} \\
e_{1111000}-e_{0121000}-2 e_{0011110}+e_{0111110}\end{array}$ & & \\
\hline 7 & $\begin{array}{l}5 \\
6 \\
6\end{array}$ & $\begin{array}{l}\lambda_{1} \\
0 \\
0\end{array}$ & 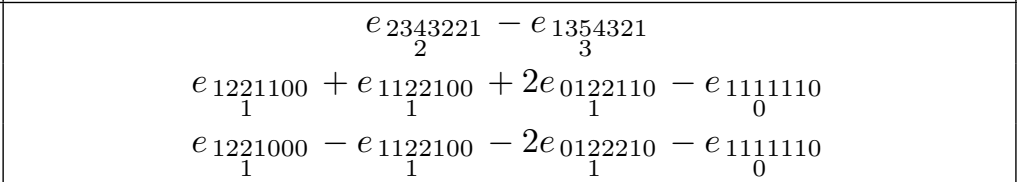 & & \\
\hline 6 & $\begin{array}{l}6 \\
7 \\
8\end{array}$ & $\begin{array}{l}0 \\
\lambda_{1} \\
0\end{array}$ & $\begin{array}{c}e_{1121000}+e_{0121110} \\
e_{2454321}-e_{2354321} \\
3 \\
2 e_{1121110}+e_{1222110}-e_{1222210}-e_{1232100}\end{array}$ & & \\
\hline 5 & $\begin{array}{l}8 \\
9\end{array}$ & $\begin{array}{c}0 \\
\lambda_{1}\end{array}$ & $\begin{array}{c}2 e_{1122110}+e_{1221110}-e_{1232100} \\
1 \\
e_{2465421}+e_{2465321} \\
3\end{array}$ & & \\
\hline 4 & $\begin{array}{c}9 \\
10\end{array}$ & \begin{tabular}{c|}
$\lambda_{1}$ \\
0
\end{tabular} & $\begin{array}{c}e_{2464321} \\
3 \\
e_{1232110}-e_{1232210} \\
\end{array}$ & & \\
\hline 3 & $\begin{array}{l}10 \\
10 \\
11\end{array}$ & $\begin{array}{c}0 \\
0 \\
\lambda_{1}\end{array}$ & $\begin{array}{c}3 e_{1232110}+2 e_{1233210} \\
e_{1232110} \\
2 \\
e_{2465431} \\
3\end{array}$ & & \\
\hline 2 & 12 & 0 & $e_{1243210}$ & & \\
\hline 1 & $\begin{array}{c}2 \\
10 \\
14\end{array}$ & $\begin{array}{l}0 \\
0 \\
0\end{array}$ & $\begin{array}{c}e \\
5 e_{1232110}+e_{1232210}+e_{1233210} \\
e_{2343210} \\
\end{array}$ & $\begin{array}{l}z_{2} \\
z_{10} \\
z_{14}\end{array}$ & $\begin{array}{l}z_{2} \\
z_{10} \\
z_{14}\end{array}$ \\
\hline
\end{tabular}


$E_{8}$, orbit 46: $E_{6}\left(a_{1}\right)$

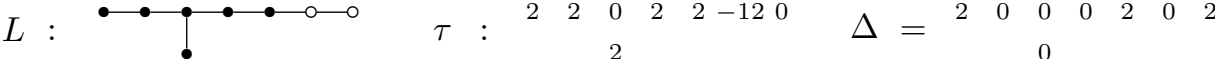

$$
\begin{aligned}
& e=\underset{0}{e} \underset{0}{1000000}+\underset{0}{e 0000100}+\underset{0}{e 0100000}+\underset{0}{e 0001000}+\underset{0}{e 011000}+e_{0}^{0110000}+e_{000000}^{0} \\
& C^{\circ}=A_{2} \quad C / C^{\circ}=\left\langle c C^{\circ}\right\rangle \cong S_{2} \\
& \beta_{1}=\underset{0}{0000001}, \beta_{2}={ }_{3}^{2465431} \\
& c=n_{0122210} n_{1122110} n_{1221110} h_{1}(-1) h_{2}(-1) h_{3}(-1) h_{5}(-1) h_{6}(-1) h_{8}(-1)
\end{aligned}
$$

\begin{tabular}{|c|c|c|c|c|c|}
\hline & & $\lambda$ & $v$ & $\mathcal{Z}^{\natural}$ & $\mathcal{Z}$ \\
\hline 4 & 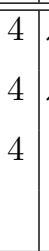 & $\begin{array}{l}\lambda_{1} \\
\lambda_{2} \\
0\end{array}$ & 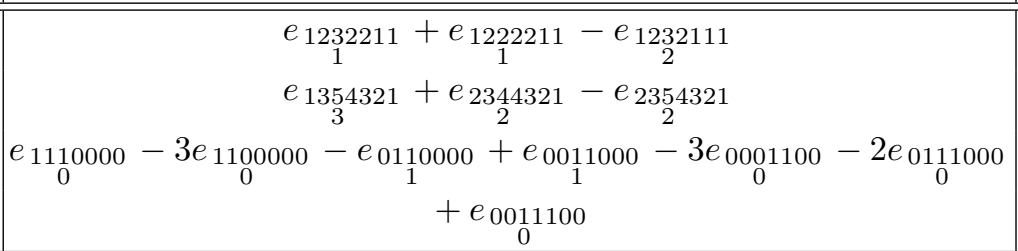 & & \\
\hline 3 & $\begin{array}{l}6 \\
8\end{array}$ & $\begin{array}{l}0 \\
\lambda_{1} \\
\lambda_{2} \\
0\end{array}$ & $\begin{array}{c}e_{1110000}+e_{1111000}-e_{0011100}+e_{0111100}+e_{0121000} \\
e_{1233211}-e_{1243211} \\
2 \\
e_{2454321}+e_{2464321} \\
3 \\
3 \\
e_{1111000}-e_{1121000}-e_{0111100}+2 e_{1111100}-e_{0121100} \\
1\end{array}$ & & \\
\hline 2 & $\begin{array}{l}10 \\
12 \\
12\end{array}$ & $\begin{array}{l}0 \\
\lambda_{1} \\
\lambda_{2}\end{array}$ & $\begin{array}{c}e_{1111100}+e_{1221000}+e_{0122100} \\
e_{2343211} \\
e_{2465421}\end{array}$ & & \\
\hline 1 & $\begin{array}{c}2 \\
10\end{array}$ & $\begin{array}{l}0 \\
0 \\
0 \\
0\end{array}$ & $\begin{array}{c}e \\
e_{1121100}+e_{1221000}-e_{0122100} \\
e_{1222100} \\
e_{1232100}\end{array}$ & \begin{tabular}{l|}
$z_{2}$ \\
$z_{10}$ \\
$z_{14}$ \\
$z_{16}$
\end{tabular} & $\begin{array}{l}z_{2} \\
z_{10} \\
z_{14}\end{array}$ \\
\hline
\end{tabular}


$E_{8}$, orbit $47: D_{5} A_{2}$

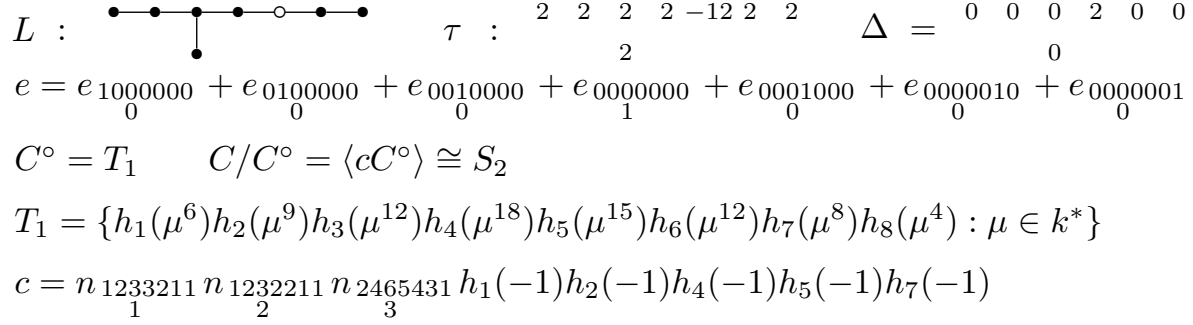

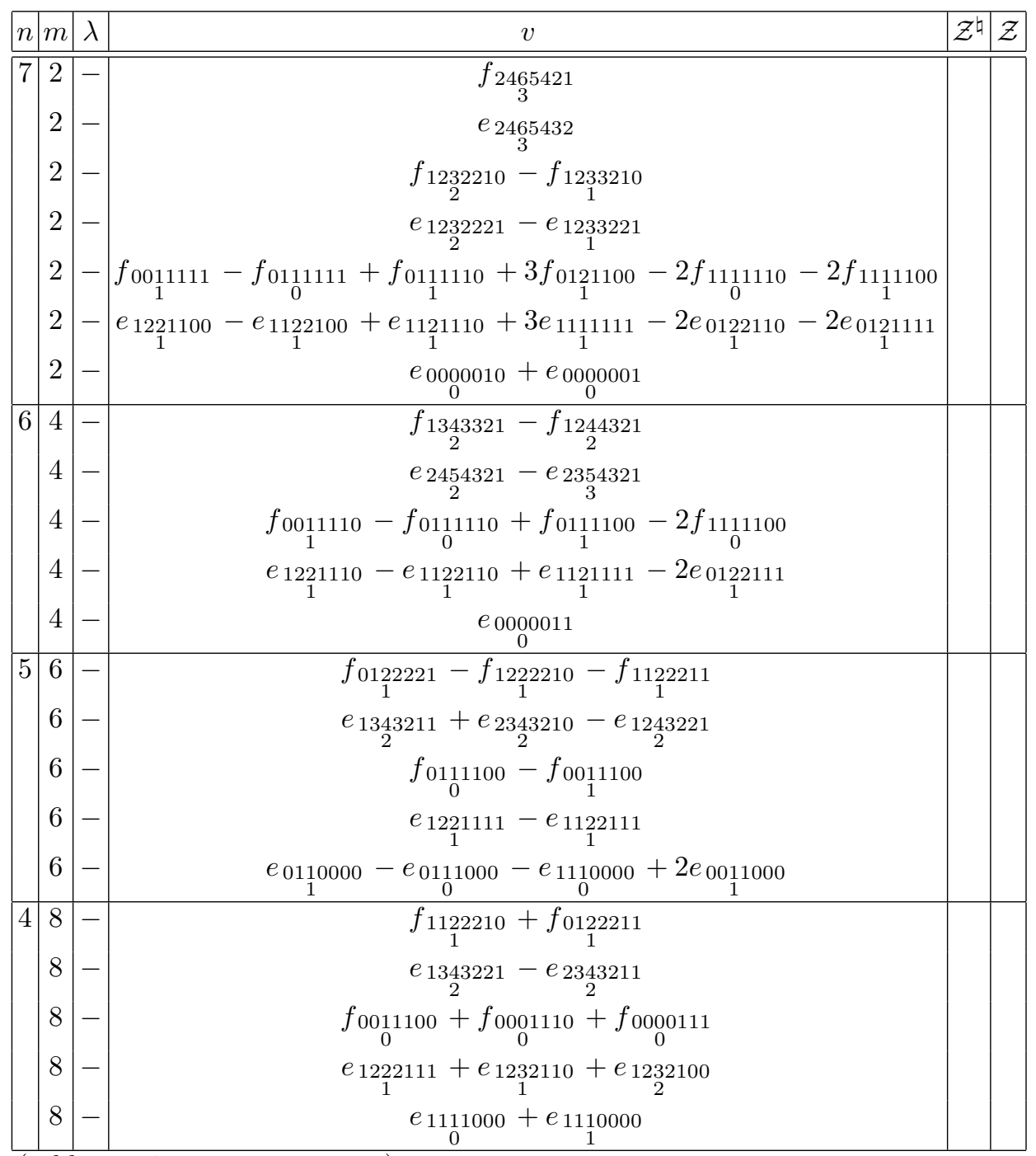

(table continues on next page) 


\begin{tabular}{|c|c|c|c|c|c|}
\hline$n$ & $m$ & $\lambda$ & $v$ & $\mathcal{Z}^{\natural}$ & $\mathcal{Z}$ \\
\hline 3 & 10 & -1 & f & & \\
\hline & 10 & - & $e_{2465321}$ & & \\
\hline & 10 & - & $f_{0122210}$ & & \\
\hline & 10 & - & $e_{2343221}$ & & \\
\hline & 10 & - & $f_{0001100}+f_{0000110}$ & & \\
\hline & 10 & - & $e_{1232110}+e_{1232111}$ & & \\
\hline & 10 & - & $e_{1111000}-e_{0121000}$ & & \\
\hline 2 & 12 & - & $f_{0000100}$ & & \\
\hline & 12 & - & $e_{1232111}$ & & \\
\hline 1 & 2 & - & $e$ & $z_{2}$ & $z_{2}$ \\
\hline & 14 & - & $e_{1221000}$ & $z_{14}$ & $z_{14}$ \\
\hline
\end{tabular}

$E_{8}$, orbit $48: D_{6}$

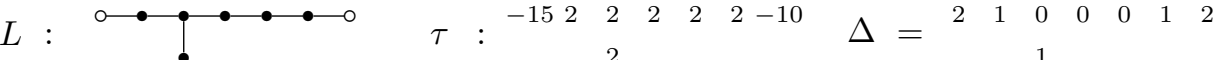

$$
\begin{aligned}
& e=e_{0}^{0000010}+e_{0000100}+e_{0001000}+e_{0010000}+e_{0000000}+e_{0100000} \\
& C^{\circ}=B_{2} \quad C / C^{\circ}=1 \\
& \beta_{1}={ }_{2}^{2343210}, x_{ \pm \beta_{2}}(t)=x_{ \pm} 0011111(t) x_{ \pm}^{0111111}(-t)
\end{aligned}
$$

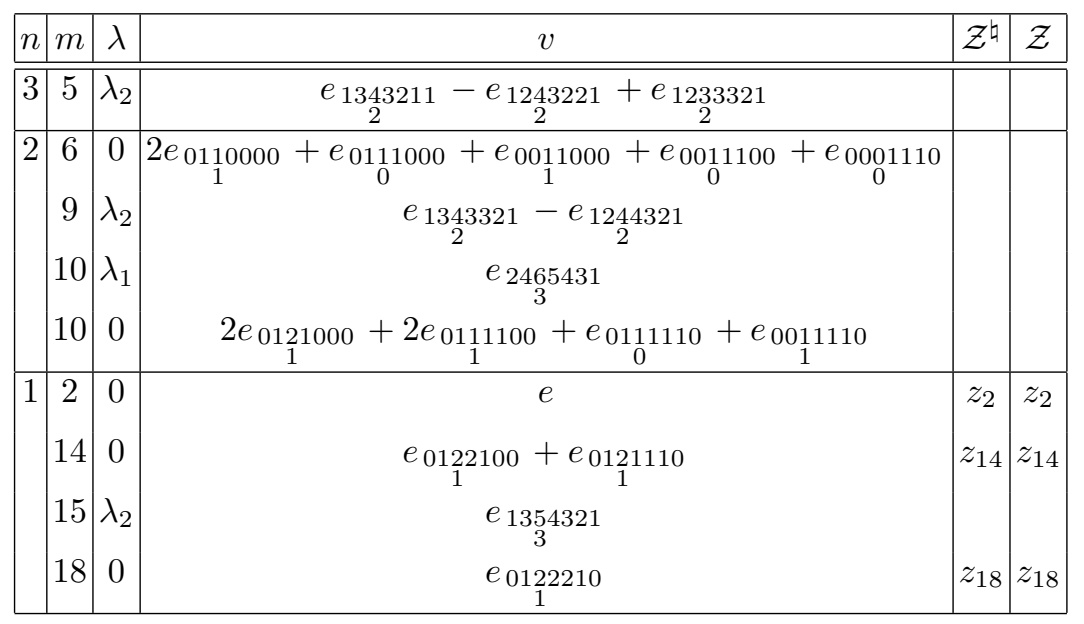


$E_{8}$, orbit 49: $E_{6}$

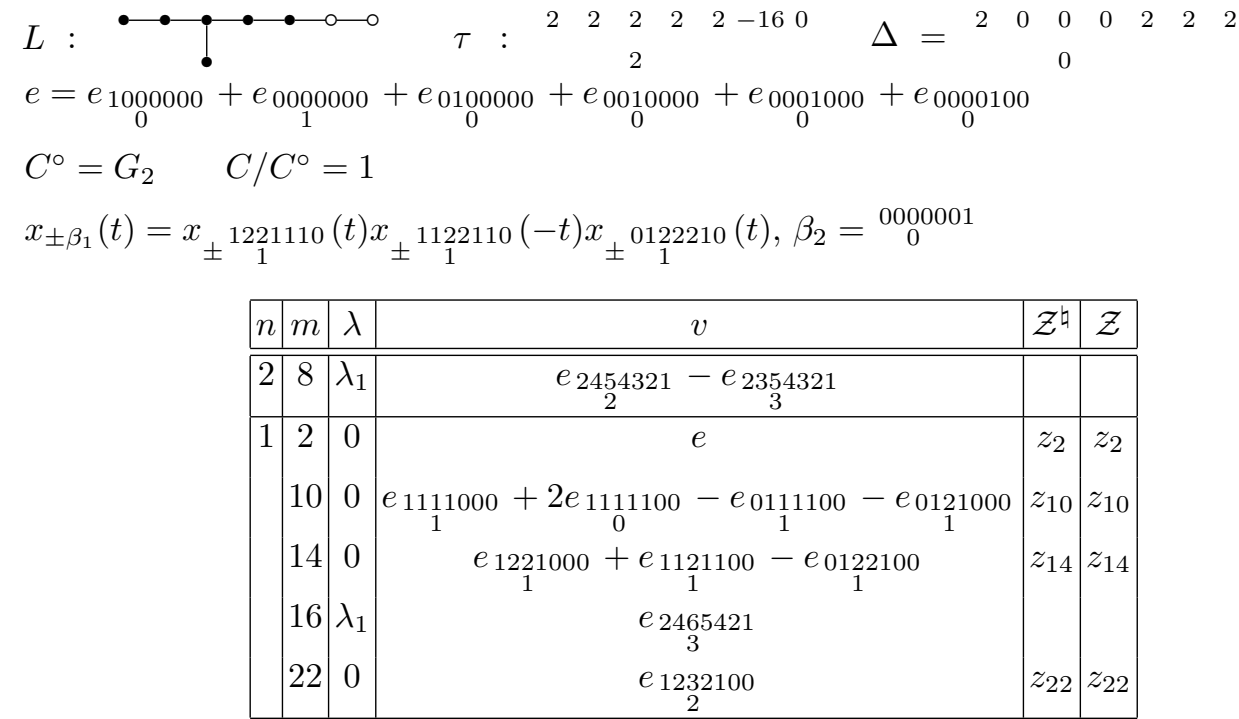

$E_{8}$, orbit 50: $D_{7}\left(a_{2}\right)$

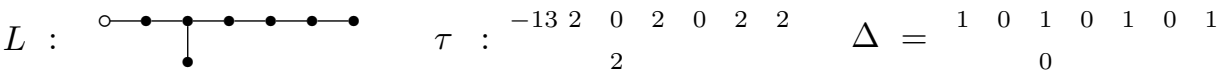

$e=e_{0}^{000001}+\underset{0}{e 000010}+e_{0001100}+e_{1}^{0010000}-e_{0}^{0110000}+e_{0}^{0011000}+e_{000000}+e_{0100000}$

$C^{\circ}=T_{1} \quad C / C^{\circ}=\left\langle c C^{\circ}\right\rangle \cong S_{2}$

$T_{1}=\left\{h_{1}\left(\mu^{4}\right) h_{2}\left(\mu^{5}\right) h_{3}\left(\mu^{7}\right) h_{4}\left(\mu^{10}\right) h_{5}\left(\mu^{8}\right) h_{6}\left(\mu^{6}\right) h_{7}\left(\mu^{4}\right) h_{8}\left(\mu^{2}\right): \mu \in k^{*}\right\}$

$c=n_{2354321} n_{2454321} h_{4}(-1) h_{5}(-1)$

\begin{tabular}{|c|c|c|c|c|c|}
\hline$n$ & $m$ & $\lambda$ & $v$ & $\mathcal{Z}^{\natural}$ & $\mathcal{Z}$ \\
\hline$\overline{14}$ & 1 & - & 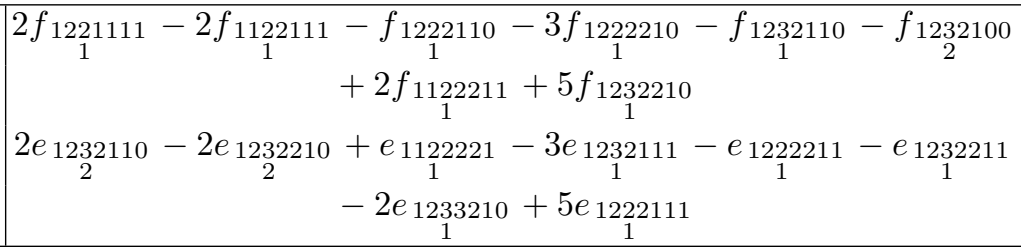 & & \\
\hline 13 & 2 & - & $e_{0000000}+e_{0100000}+e_{0011000}$ & & \\
\hline 12 & 3 & - & 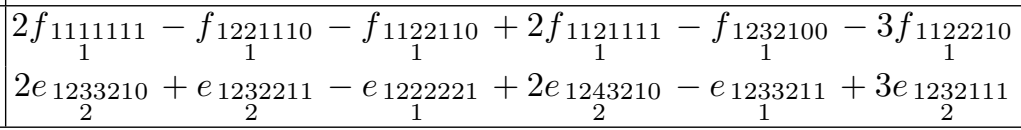 & & \\
\hline
\end{tabular}

(table continues on next page) 


\begin{tabular}{|c|c|c|c|c|c|}
\hline$n$ & $m$ & $\lambda$ & $v$ & $\mathcal{Z}^{\natural}$ & $\mathcal{Z}$ \\
\hline 111 & \begin{tabular}{l|}
4 \\
4 \\
4
\end{tabular} & $\begin{array}{l}- \\
- \\
-\end{array}$ & $\begin{array}{c}f_{2343321} \\
e_{2465321} \\
3321 \\
e_{0011000}+2 e_{0110000}+e_{001100}+e_{0000111}-e_{0}^{0111000}+e_{0}^{0111100} \\
+e_{0011110}\end{array}$ & & \\
\hline 10 & \begin{tabular}{|l|}
5 \\
5
\end{tabular} & - & $\begin{array}{l}f_{1111110}+2 f_{1111111}-f_{1221100}-f_{1121110} \\
e_{1233221}-e_{1233211}+2 e_{1343210}+e_{1243211} \\
e_{2}\end{array}$ & & \\
\hline 9 & $\begin{array}{l}6 \\
6 \\
6\end{array}$ & $\begin{array}{l}- \\
- \\
-\end{array}$ & $\begin{array}{c}2 e_{0111100}+e_{0111110}+e_{0011110}+e_{0011111}-2 e_{0121000} \\
0 \\
e_{0001111}-e_{0111110}+e_{001110}+2 e_{0121100} \\
0 \\
e_{0111000}\end{array}$ & & \\
\hline 8 & \begin{tabular}{|l|}
7 \\
7 \\
7 \\
7
\end{tabular} & $\begin{array}{l}- \\
- \\
- \\
-\end{array}$ & $\begin{array}{c}f_{1111100}-f_{1111000}+f_{1111110}-f_{1121000} \\
e_{1233221}+e_{1233321}+e_{1243221}+e_{1243321} \\
2 \\
f_{1111110}-f_{1121100} \\
e_{1233221}+e_{1343211} \\
2\end{array}$ & & \\
\hline 7 & $\begin{array}{l}8 \\
8 \\
8\end{array}$ & $\begin{array}{l}- \\
- \\
-\end{array}$ & $\begin{array}{c}f_{2343210} \\
e_{2465432} \\
3 \\
2 e_{0111110}+e_{1}^{0011111}+e_{0111111}-2 e_{0122100} \\
1\end{array}$ & & \\
\hline 6 & $\begin{array}{l}9 \\
9\end{array}$ & - & $\begin{array}{l}f_{1110000}-f_{1}^{1111000}+f_{1111100} \\
e_{0} \\
e_{1343221}+e_{2}^{1343321}-e_{2}^{1244321} \\
\end{array}$ & & \\
\hline 5 & $\begin{array}{l}10 \\
10\end{array}$ & - & $\begin{array}{c}e_{0111111}-e_{0122110} \\
1 \\
e_{0121111}-e_{0122210}^{0}\end{array}$ & & \\
\hline 4 & $\begin{array}{l}11 \\
11\end{array}$ & - & $\begin{array}{l}f_{1100000}+f_{1110000} \\
e_{1344321}-e_{1354321}\end{array}$ & & \\
\hline 3 & 12 & - & $e_{0122111}$ & & \\
\hline 2 & $\begin{array}{l}13 \\
13\end{array}$ & - & $\begin{array}{c}f_{1000000} \\
0 \\
e_{1354321} \\
3\end{array}$ & & \\
\hline 1 & $\begin{array}{c}2 \\
14\end{array}$ & - & $\begin{array}{c}e \\
e_{0122221} \\
\end{array}$ & \begin{tabular}{c|}
$z_{2}$ \\
$z_{14}$
\end{tabular} & $z_{2}$ \\
\hline
\end{tabular}


$E_{8}$, orbit 51: $A_{7}$

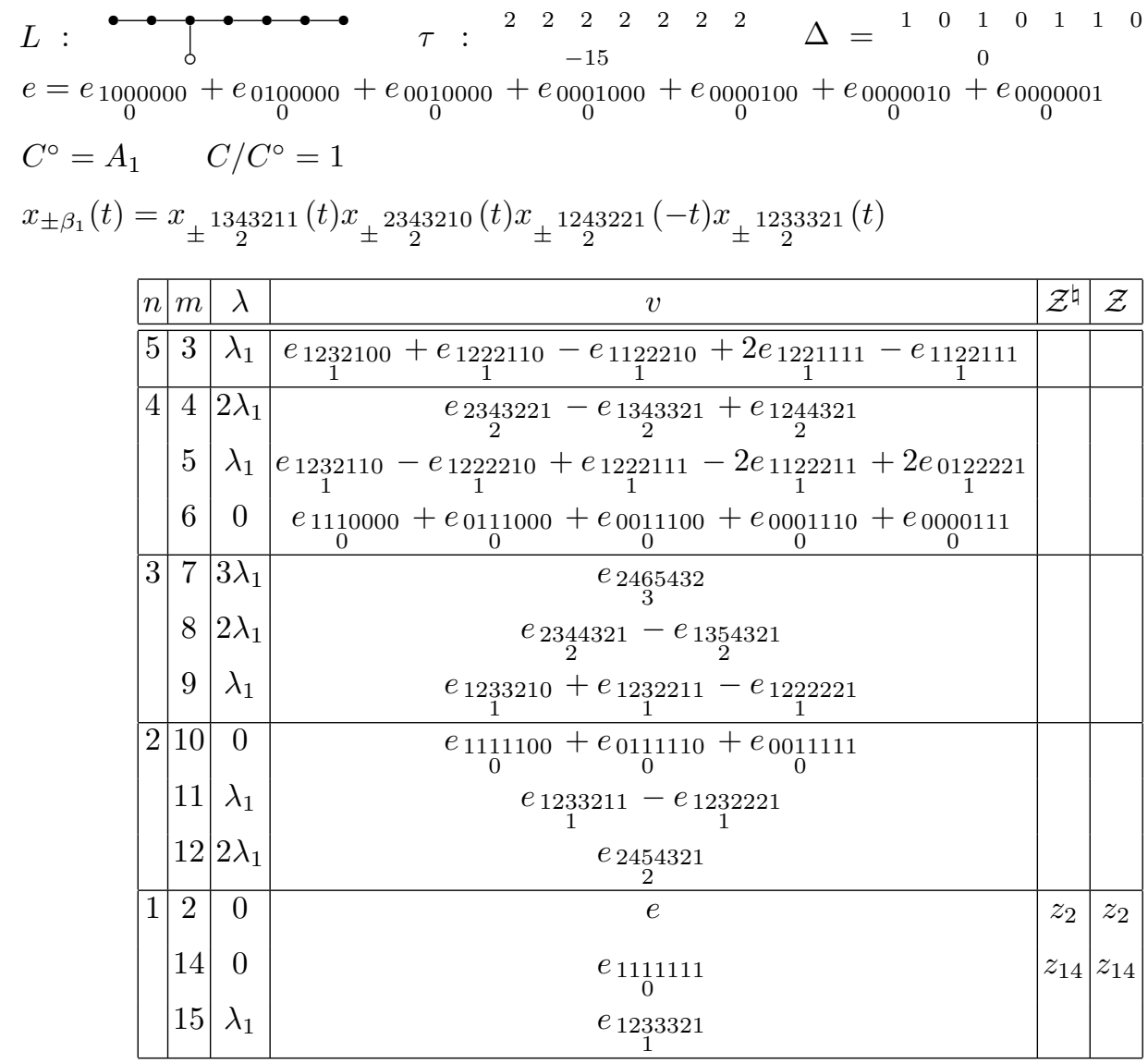

$E_{8}$, orbit 52: $E_{6}\left(a_{1}\right) A_{1}$

$$
\begin{aligned}
& L: \bullet \bullet \bullet \bullet-\bullet \quad \tau: \begin{array}{llllllll}
2 & 2 & 0 & 2 & 2 & -13 & 2
\end{array} \quad \Delta=\begin{array}{lllllll}
1 & 0 & 1 & 0 & 1 & 0 & 2
\end{array} \\
& e=e_{1000000}+e_{0000100}+e_{0100000}+e_{0001000}+e_{0011000}+e_{0110000}+e_{0000000}+e_{0000001} \\
& C^{\circ}=T_{1} \quad C / C^{\circ}=\left\langle c C^{\circ}\right\rangle \cong S_{2} \\
& T_{1}=\left\{h_{1}\left(\mu^{4}\right) h_{2}\left(\mu^{6}\right) h_{3}\left(\mu^{8}\right) h_{4}\left(\mu^{12}\right) h_{5}\left(\mu^{10}\right) h_{6}\left(\mu^{8}\right) h_{7}\left(\mu^{6}\right) h_{8}\left(\mu^{3}\right): \mu \in k^{*}\right\} \\
& c=n_{1244321} n_{1343321} n_{2343221} h_{1}(-1) h_{2}(-1) h_{3}(-1) h_{5}(-1) h_{6}(-1) h_{8}(-1)
\end{aligned}
$$




\begin{tabular}{|c|c|c|c|c|c|}
\hline$n$ & $m$ & $\lambda$ & $v$ & $\mathcal{Z}^{\natural}$ & $\mathcal{Z}$ \\
\hline 8 & $\begin{array}{l}1 \\
3 \\
3\end{array}$ & $\begin{array}{l}- \\
- \\
-\end{array}$ & $\begin{array}{c}f_{2465431} \\
e_{2465432} \\
3 \\
e_{1} \\
f_{1111110}-f_{0111111}+f_{1111111}-f_{0121111}+2 f_{0122110}-3 f_{1121110} \\
e_{1232110}-e_{1222210}+e_{1232111}-e_{1232210}+2 e_{1122211}-3 e_{1222111} \\
1 \\
\end{array}$ & & \\
\hline $7 \mid$ & $\begin{array}{l}2 \\
4 \\
4 \\
4\end{array}$ & $\begin{array}{l}- \\
- \\
- \\
-\end{array}$ & $\begin{array}{c}e_{0000001} \\
0 \\
f_{1233321}-f_{1233221}+f_{1243221} \\
1 \\
e_{1354321}+e_{2344321}-e_{2354321} \\
3 \\
2 \\
0 \\
e_{1110000}-3 e_{1100000}-e_{0110000}+e_{0011000}-3 e_{0001100}-2 e_{0111000} \\
1 \\
+e_{0011100} \\
0\end{array}$ & & \\
\hline 6 & \begin{tabular}{l|}
5 \\
5 \\
7 \\
7
\end{tabular} & $\begin{array}{l}- \\
- \\
-\end{array}$ & $\begin{array}{c}f_{0111110}-f_{1111110}+f_{0121110} \\
e_{1} \\
e_{1222211}-e_{1232111}+e_{1232211} \\
1 \\
2 \\
f_{0001111}+f_{0111110}-2 f_{0011110}-f_{0011111} \\
0 \\
e_{1233210}+2 e_{1233211}-e_{1232211}-e_{1243} \\
2\end{array}$ & & \\
\hline 5 & \begin{tabular}{l|}
6 \\
8 \\
8 \\
8
\end{tabular} & $\begin{array}{l}- \\
- \\
-\end{array}$ & $\begin{array}{c}e_{1110000}+e_{1111000}-e_{0011100}+e_{0111100}+e_{0121000} \\
0 \\
f_{1222221}+f_{1232221} \\
1 \\
e_{2454321}+e_{2464321} \\
3 \\
3 \\
e_{1111000}-e_{1121000}-e_{0111100}+2 e_{1111100}-e_{0121100} \\
1\end{array}$ & & \\
\hline 4 & $\begin{array}{l}9 \\
9 \\
11 \\
11\end{array}$ & $\begin{array}{l}- \\
- \\
- \\
-\end{array}$ & $\begin{array}{c}f_{0001110}-f_{0011110} \\
0 \\
e_{1233211}-e_{1243211} \\
2 \\
f_{0000110}+f_{0000011} \\
0 \\
e_{2343210}+e_{1343211} \\
2 \\
\end{array}$ & & \\
\hline 3 & \begin{tabular}{l|}
10 \\
10 \\
12 \\
12
\end{tabular} & $\begin{array}{l}- \\
- \\
- \\
-\end{array}$ & $\begin{array}{c}e_{1111100}+e_{1221000}+e_{0122100} \\
e_{1}+11100+e_{1221000}-e_{0122100} \\
1 \\
f_{0122221} \\
e_{1} \\
e_{2465421} \\
3\end{array}$ & & \\
\hline 2 & $\begin{array}{l}13 \\
13\end{array}$. & - & $\begin{array}{c}f_{0000010} \\
0 \\
e_{2343211} \\
\end{array}$ & & \\
\hline 1 & \begin{tabular}{l|}
2 \\
14 \\
16
\end{tabular} & - & $\begin{array}{c}e \\
e_{1222100} \\
e_{1232100}\end{array}$ & $\begin{array}{l}z_{2} \\
z_{14} \\
z_{16}\end{array}$ & \begin{tabular}{|c|}
$z_{2}$ \\
$z_{14}$
\end{tabular} \\
\hline
\end{tabular}


$E_{8}$, orbit 53: $E_{7}\left(a_{3}\right)$

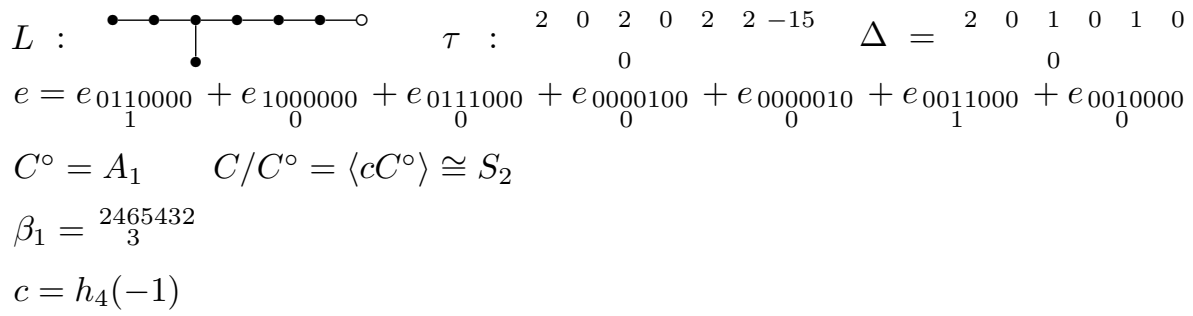

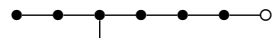

\begin{tabular}{|c|c|c|c|c|c|}
\hline$n$ & $m$ & $\lambda$ & $v$ & $\mathcal{Z}^{\natural}$ & $\mathcal{Z}$ \\
\hline 7 & \begin{tabular}{|l|}
1 \\
\end{tabular} & $\lambda_{1}$ & $e_{1232221}+e_{1243211}$ & & \\
\hline & 4 & 0 & $e_{0}^{0011100}+e_{1111000}+e_{0}^{0001110}-3 e_{0}^{1110000}+e_{0111100}+2 e_{0121000}$ & & \\
\hline 6 & \begin{tabular}{|l|}
2 \\
\end{tabular} & 0 & $\underset{0}{e 001000}$ & & \\
\hline & 5 & $\lambda_{1}$ & $e_{1244321}+e_{1343321}-e_{2343221}$ & & \\
\hline 5 & 6 & 0 & $e_{0011110}+e_{1121000}+e_{0121100}$ & & \\
\hline & 9 & $\lambda_{1}$ & $e_{2454321}-e_{2354321}$ & & \\
\hline 4 & 6 & 0 & $e_{1111100}+e_{0}^{0111110}-2 e_{0011110}-e_{1221000}-e_{0122100}$ & & \\
\hline & 8 & 0 & $e_{1111110}-e_{1121100}-2 e_{0121110}-e_{1222100}$ & & \\
\hline & 10 & 0 & $e_{1221110}+e_{1232100}$ & & \\
\hline & 10 & 0 & $e_{1221110}+e_{1122110}-e_{0122210}$ & & \\
\hline 3 & \begin{tabular}{|l|}
9 \\
\end{tabular} & $\lambda_{1}$ & $e_{2454321}-e_{2354321}$ & & \\
\hline & 11 & $\lambda_{1}$ & $e_{2464321}$ & & \\
\hline & 14 & 0 & $e_{1232210}+e_{1233210}$ & & \\
\hline 2 & 10 & 0 & $e_{1121110}-e_{1232100}$ & & \\
\hline & 15 & $\lambda_{1}$ & $e_{2465431}$ & & \\
\hline 1 & 2 & 0 & $e$ & $z_{2}$ & $z_{2}$ \\
\hline & 14 & 0 & $e_{1232} 210+e_{1233210}$ & $z_{14}$ & $z_{14}$ \\
\hline & 16 & 0 & $e_{1243210}$ & $z_{16}$ & \\
\hline & 18 & 0 & $e_{2343210}$ & $z_{18}$ & $z_{18}$ \\
\hline
\end{tabular}


$E_{8}$, orbit $54: E_{8}\left(b_{6}\right)$

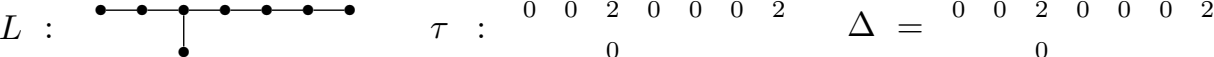

$$
\begin{aligned}
& e=e_{1}^{0011000}+e_{0}^{0010000}+e_{1111100}+e_{0111100}+e_{1}^{011110}-e_{1111110}+e_{0000001}+e_{0111000} \\
& +e_{1110000} \\
& C^{\circ}=1 \quad C / C^{\circ}=\left\langle c_{1} C^{\circ}, c_{2} C^{\circ}\right\rangle \cong S_{3} \\
& c_{1}=h_{1}(\omega) h_{2}(\omega) h_{5}\left(\omega^{2}\right) \text {, } \\
& c_{2}=n_{0}^{1000000} n_{0000000} n_{0001000} h_{2}(-1) h_{3}(-1) h_{4}(-1) h_{5}(-1) h_{8}(-1)
\end{aligned}
$$

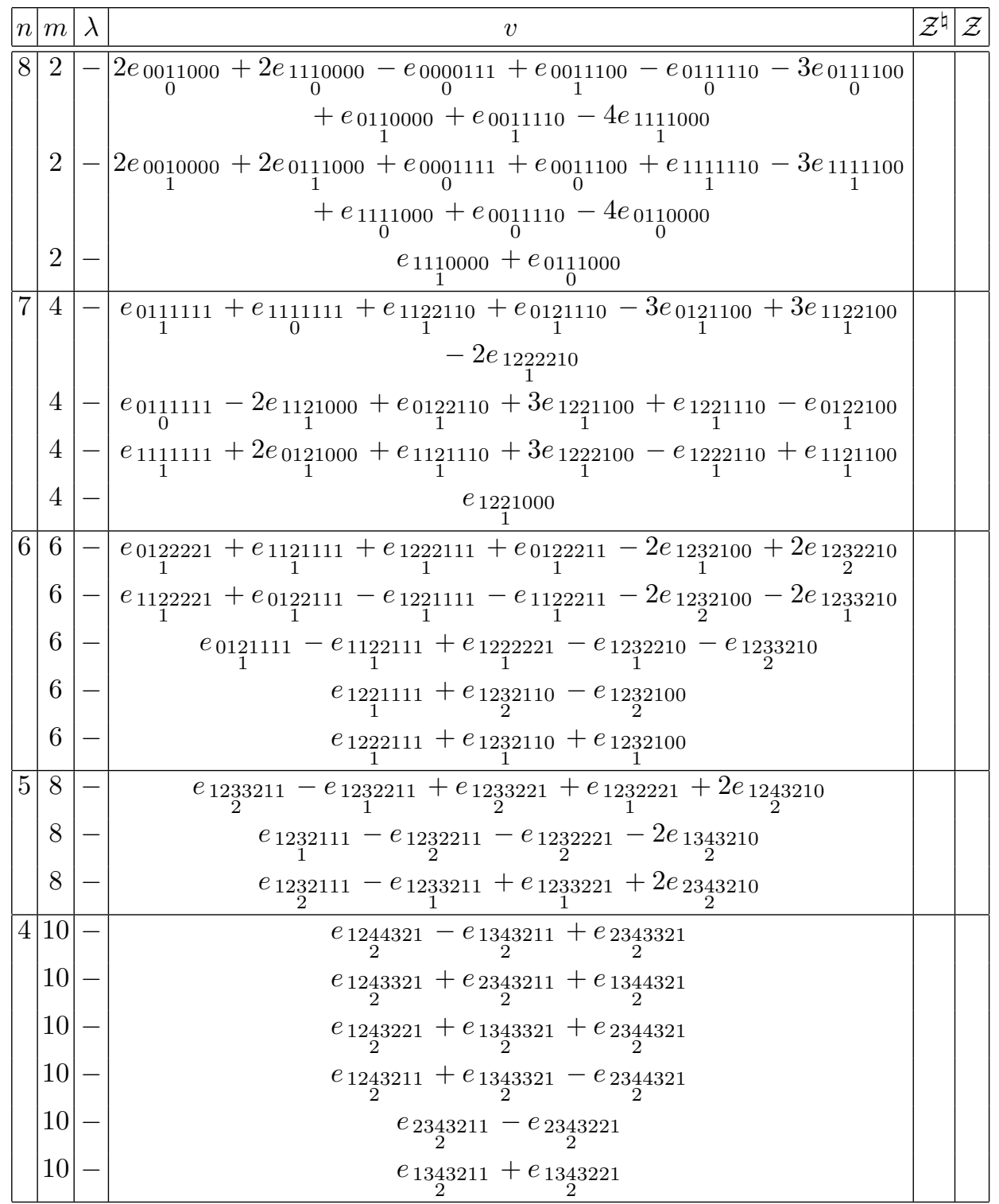

(table continues on next page) 


\begin{tabular}{|c|c|c|c|c|}
\hline \begin{tabular}{|l|l|}
$n$ & $m$ \\
\end{tabular} & $\lambda$ & $v$ & $\mathcal{Z}^{\natural}$ & $\mathcal{Z}$ \\
\hline \begin{tabular}{|l|l|}
3 & 12 \\
& 12 \\
\end{tabular} & $\begin{array}{l}- \\
-\end{array}$ & $\begin{array}{c}e_{2354321}-e_{2454321} \\
3 \\
e_{1354321}+e_{2454321} \\
e_{3}\end{array}$ & & \\
\hline \begin{tabular}{|l|l|}
2 & 14 \\
& 14 \\
\end{tabular} & - & $\begin{array}{c}e_{2464321} \\
3 \\
e_{2465321} \\
\end{array}$ & & \\
\hline \begin{tabular}{|l|c|}
1 & 2 \\
& 14 \\
& 16 \\
& \\
\end{tabular} & $\begin{array}{l}- \\
- \\
-\end{array}$ & $\begin{array}{c}e \\
e_{2465421} \\
3 \\
e_{2465432}\end{array}$ & $\begin{array}{c}z_{2} \\
z_{14} \\
z_{16}\end{array}$ & $\begin{array}{l}z_{2} \\
z_{14}\end{array}$ \\
\hline
\end{tabular}


$E_{8}$, orbit 55: $D_{7}\left(a_{1}\right)$

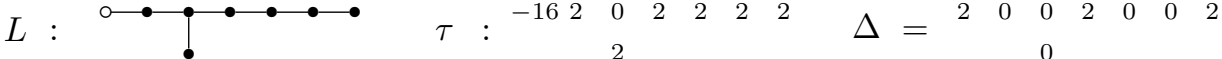

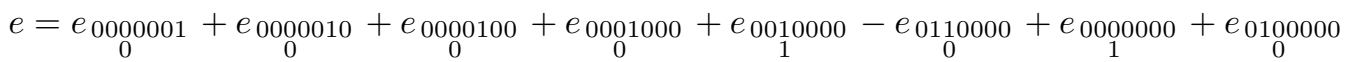

$$
\begin{aligned}
& C^{\circ}=T_{1} \quad C / C^{\circ}=\left\langle c C^{\circ}\right\rangle \cong S_{2} \\
& T_{1}=\left\{h_{1}\left(\mu^{4}\right) h_{2}\left(\mu^{5}\right) h_{3}\left(\mu^{7}\right) h_{4}\left(\mu^{10}\right) h_{5}\left(\mu^{8}\right) h_{6}\left(\mu^{6}\right) h_{7}\left(\mu^{4}\right) h_{8}\left(\mu^{2}\right): \mu \in k^{*}\right\} \\
& c=n_{2354321} n_{2454321} h_{2}(-1) h_{4}(-1) h_{6}(-1) h_{8}(-1)
\end{aligned}
$$

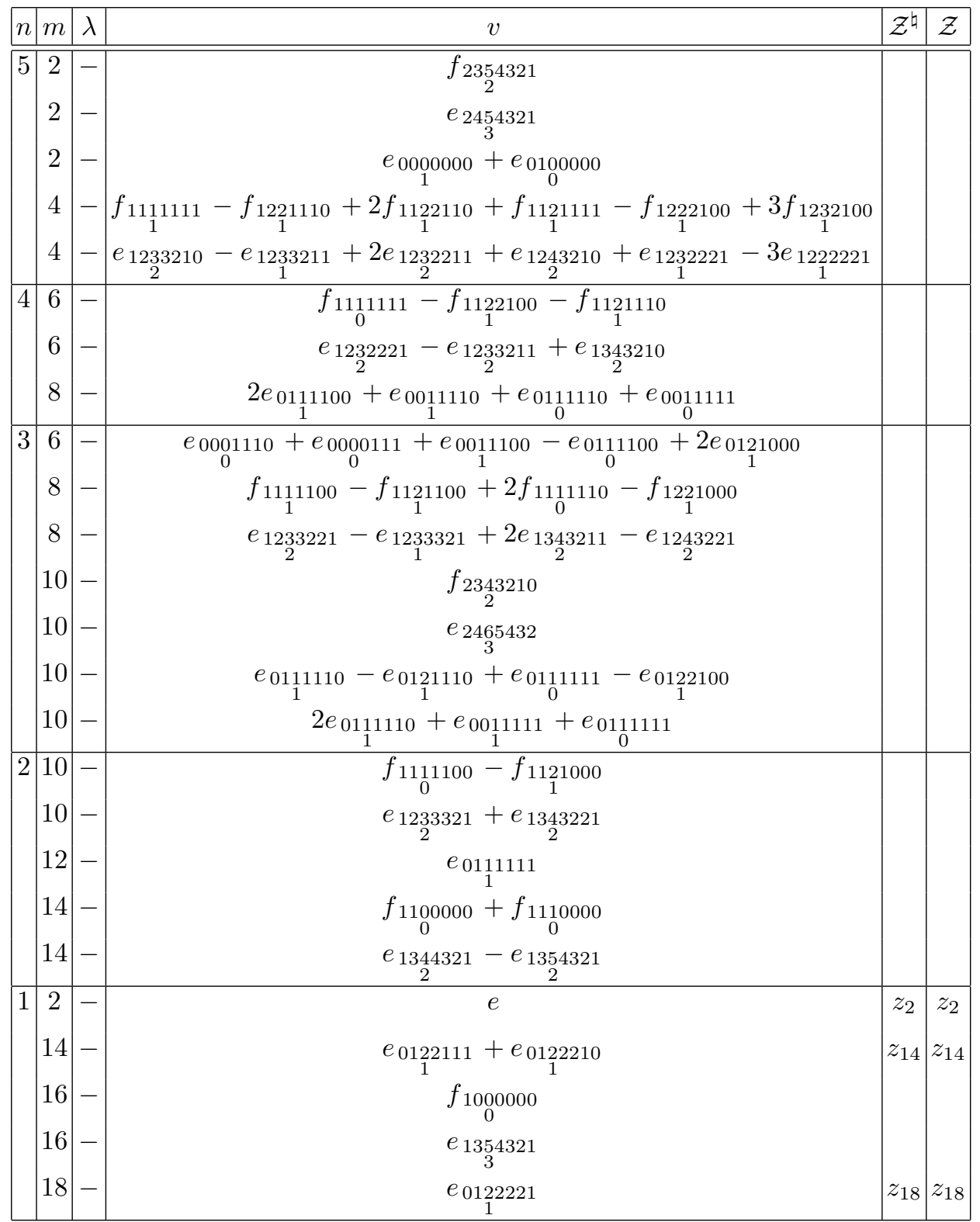


$E_{8}$, orbit 56: $E_{6} A_{1}$

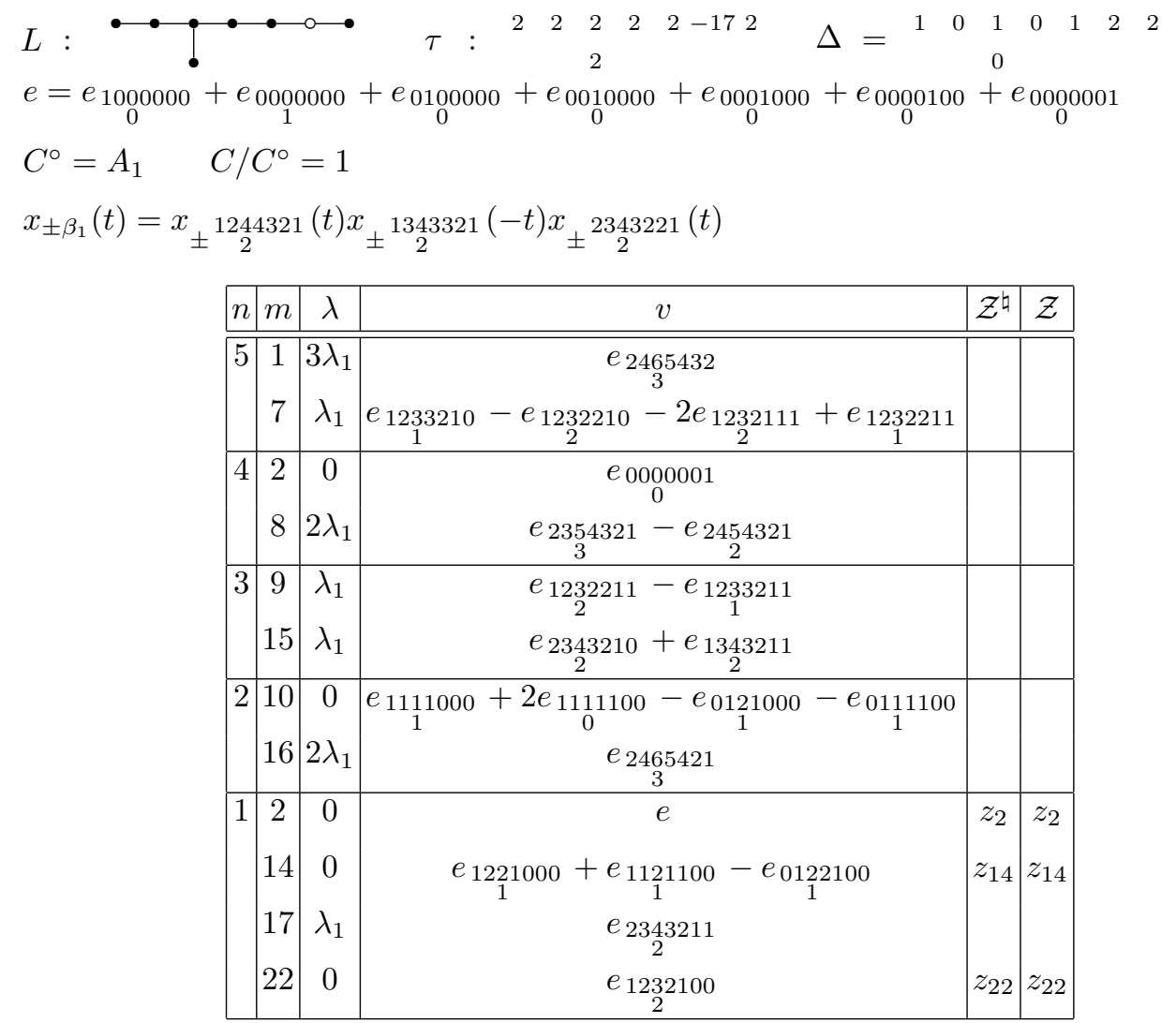


$E_{8}$, orbit $57: E_{7}\left(a_{2}\right)$

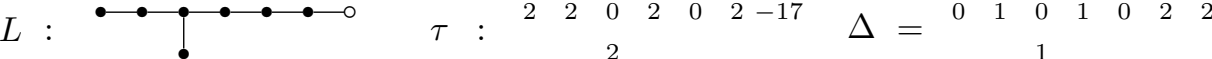

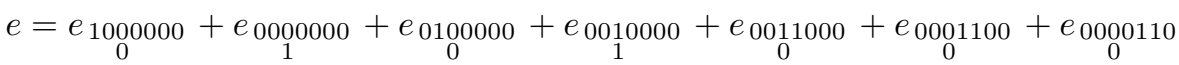

$$
\begin{aligned}
& C^{\circ}=A_{1} \quad C / C^{\circ}=1 \\
& \beta_{1}=\underset{3}{2465432}
\end{aligned}
$$

\begin{tabular}{|c|c|c|c|c|c|}
\hline$n$ & $m$ & $\lambda$ & $v$ & $\mathcal{Z}^{\natural}$ & $\mathcal{Z}$ \\
\hline 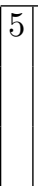 & 6 & \begin{tabular}{l|l}
0 \\
\end{tabular} & \begin{tabular}{|c}
$\begin{array}{c}e_{0000000}+e_{0001000}+e_{0000010} \\
1\end{array}$ \\
0 \\
$2 e_{1110000}+3 e_{1111100}-e_{1111000}+3 e_{0121100}+e_{0121000}-e_{0111100}$ \\
0 \\
$+e_{0111000}-e_{0111110}+2 e_{0011110}$ \\
1
\end{tabular} & & \\
\hline 4 & 3 & $\lambda_{1}$ & $\begin{array}{c}e_{1233221} \\
e_{2343321}-e_{2343221}-e_{1354321}+e_{1344321} \\
e_{1111100}-e_{1111000}-e_{1111110}+e_{0122100}-e_{0121110}\end{array}$ & & \\
\hline \begin{tabular}{|l|}
3 \\
\end{tabular} & 10 & $\lambda_{1}$ & $\begin{array}{c}e_{2344321}-e_{1354321} \\
2 \\
e_{1111110}+e_{0122110} \\
e_{1232100}+e_{1232210}\end{array}$ & & \\
\hline \begin{tabular}{|l|}
2 \\
\end{tabular} & $\begin{array}{l}10 \\
15 \\
16\end{array}$ & 0 & $\begin{array}{c}e_{1111110}+e_{0122110}+e_{1221000}+e_{1122100}+e_{1121110}+2 e_{0122210} \\
e_{2465421} \\
3 \\
e_{1232210}-e_{1232110}+e_{1233210}\end{array}$ & & \\
\hline 1 & \begin{tabular}{|c|}
2 \\
14 \\
\end{tabular} & 0 & $\begin{array}{c}e \\
e_{1232100}+e_{1232110}+e_{1222210}-e_{1222110} \\
e_{2465431}\end{array}$ & $\begin{array}{l}z_{2} \\
z_{14}\end{array}$ & $z_{2}$ \\
\hline & 18 & 0 & $e_{1233210}$ & $z_{18}$ & $z_{18}$ \\
\hline & 22 & 0 & $e_{2343210}$ & $z_{22}$ & $z_{22}$ \\
\hline
\end{tabular}

$$
\begin{aligned}
& E_{8} \text {, orbit 58: } E_{8}\left(a_{6}\right) \\
& L: \bullet \bullet \bullet \bullet \bullet \bullet \quad \tau: \begin{array}{lllllllllllllllll}
0 & 0 & 0 & 0 & 0 & 2 & 0 & \Delta= & 0 & 0 & 2 & 0 & 0 & 2 & 0
\end{array} \\
& e=e_{0011000}+e_{0} 1111100+e_{0000010}+e_{0111100}+e_{0010000}+e_{0001111}+e_{1110000}+e_{0111000} \\
& C^{\circ}=1 \quad C / C^{\circ}=\left\langle c_{1} C^{\circ}, c_{2} C^{\circ}\right\rangle \cong S_{3} \\
& c_{1}=h_{2}(\omega) h_{3}(\omega) h_{5}(\omega) h_{6}(\omega) h_{8}\left(\omega^{2}\right) \text {, } \\
& c_{2}=n_{1}^{0000000} n_{0}^{0100000} n_{0000001} n_{0001100} h_{2}(-1) h_{3}(-1) h_{4}(-1) h_{5}(-1) h_{7}(-1) h_{8}(-1)
\end{aligned}
$$




\begin{tabular}{|c|c|c|c|c|c|}
\hline$n$ & $m$ & $\lambda$ & $v$ & $\mathcal{Z}^{\natural}$ & $\mathcal{Z}$ \\
\hline 9 & 2 & - & 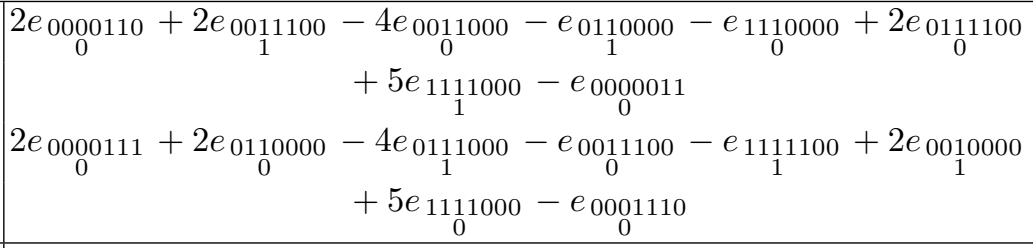 & & \\
\hline 8 & 4 & - & $\begin{array}{c}e_{0111110}-e_{0011111}-e_{1111110}+e_{1111111}-e_{1221000}-e_{1122100} \\
+e_{0121100} \\
1\end{array}$ & & \\
\hline 7 & 6 & - & 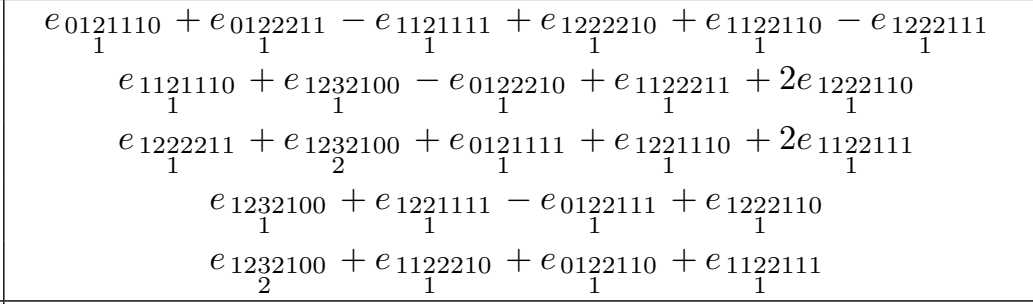 & & \\
\hline 6 & 8 & - & $\begin{array}{c}e_{1232210}+e_{1232211}+e_{1233210}+e_{1232111}-e_{0122221} \\
e_{2} \\
e_{1122221}+e_{1232210}+e_{1232110}-2 e_{1232111}-2 e_{1233211} \\
1 \\
e_{1222221}-e_{1232211}-e_{1233211}+2 e_{1233210}+2 e_{1232110} \\
1\end{array}$ & & \\
\hline 5 & $\begin{array}{l}10 \\
10\end{array}$ & $\begin{array}{l}- \\
-\end{array}$ & $\begin{array}{c}e_{1232221}-e_{1233321}-e_{1343211}-e_{1243210} \\
1 \\
e_{1233221}+e_{1343210}+e_{2343211} \\
1 \\
e_{1233221}-e_{1243211}-e_{2343210} \\
2\end{array}$ & & \\
\hline 4 & 12 & - & $\begin{array}{l}e_{1244321}+e_{1343221}+e_{2342} \\
2 \\
e_{1243321} \\
2 \\
e_{1343321}+e_{1344321}+e_{2} \\
2343243221 \\
2 \\
2\end{array}$ & & \\
\hline 3 & 14 & - & $\begin{array}{c}e_{1354321}-e_{2354321} \\
3 \\
e_{1354321}+e_{245} \\
3\end{array}$ & & \\
\hline 2 & 16 & - & $\begin{array}{c}e_{2465321} \\
3\end{array}$ & & \\
\hline 1 & $\begin{array}{c}2 \\
14\end{array}$ & $\begin{array}{l}- \\
-\end{array}$ & $\begin{array}{c}e \\
e_{2354321}-e_{2454321} \\
e_{2465431} \\
e_{2465432} \\
e_{3}\end{array}$ & \begin{tabular}{l|}
$z_{2}$ \\
$z_{14}$ \\
$z_{18}$ \\
$z_{18}$
\end{tabular} & $\begin{array}{l}z_{2} \\
z_{14}\end{array}$ \\
\hline
\end{tabular}


$E_{8}$, orbit 59: $D_{7}$

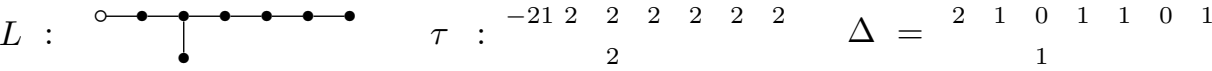

$$
\begin{aligned}
& e=\underset{0}{e 0000001}+\underset{0}{e 000010}+e_{0}^{0000100}+e_{0}^{0001000}+e_{0}^{0010000}+e_{0}^{0000000}+e_{0}^{0100000} \\
& C^{\circ}=A_{1} \quad C / C^{\circ}=1 \\
& x_{ \pm \beta_{1}}(t)=x_{ \pm}^{2354321}(t) x_{ \pm \underset{2}{2} 2454321}(-t)
\end{aligned}
$$

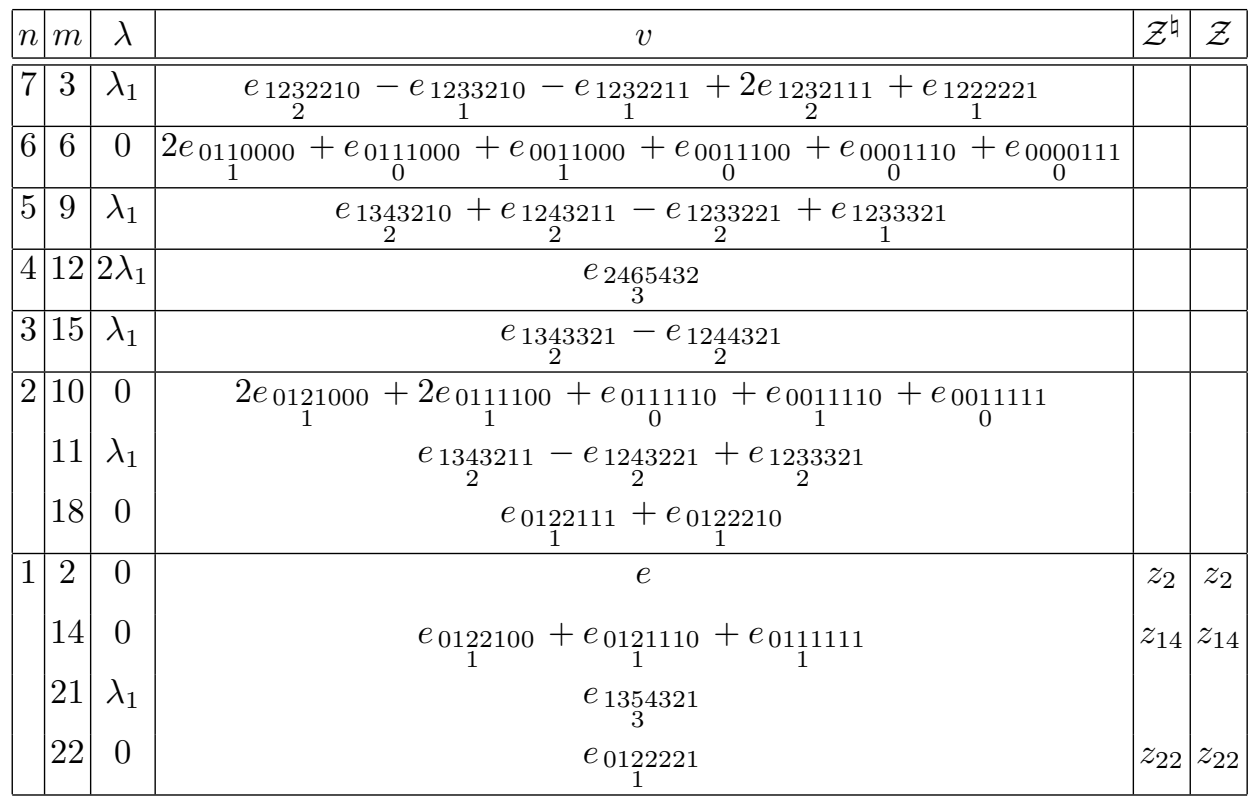


$E_{8}$, orbit $60: E_{8}\left(b_{5}\right)$

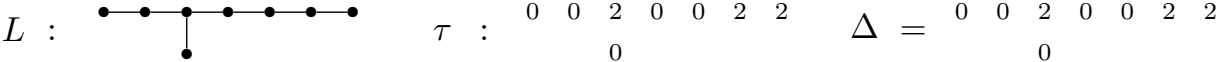

$$
\begin{aligned}
& e=e_{1}^{0011000}+e_{0}^{0000001}+e_{0}^{1111100}+e_{0}^{0000010}+e_{1}^{011100}+e_{0}^{0010000}+e_{0}^{0111000}+e_{1110000} \\
& C^{\circ}=1 \quad C / C^{\circ}=\left\langle c_{1} C^{\circ}, c_{2} C^{\circ}\right\rangle \cong S_{3} \\
& c_{1}=h_{1}(\omega) h_{2}(\omega) h_{5}\left(\omega^{2}\right) \text {, } \\
& c_{2}=n_{1000000} n_{0000000} n_{0001000} h_{1}(-1) h_{3}(-1) h_{4}(-1)
\end{aligned}
$$

\begin{tabular}{|c|c|c|c|c|c|}
\hline$n$ & $m$ & $\lambda$ & $v$ & $\mathcal{Z}^{\natural}$ & $\mathcal{Z}$ \\
\hline \multirow[t]{5}{*}{5} & 2 & - & $e_{0}^{1110000}-e_{1111000}-e_{1}^{0110000}$ & & \\
\hline & 2 & - & $e_{0111000}-e_{0}^{0110000}-e_{0}^{1111000}$ & & \\
\hline & 2 & - & $e_{1110000}+e_{0}^{0111000}$ & & \\
\hline & 6 & - & $e_{0011111}-2 e_{1111111}-e_{1121110}+e_{0122210}+3 e_{1232100}+2 e_{1222110}$ & & \\
\hline & 6 & - & $e_{0011111}-2 e_{0111111}-e_{0122110}-e_{1122210}-3 e_{1232100}-2 e_{1221110}$ & & \\
\hline \multirow[t]{4}{*}{4} & 4 & - & $e_{1221000}$ & & \\
\hline & 8 & - & $e_{1121111}+2 e_{1222111}+e_{1232110}+e_{1232210}$ & & \\
\hline & 8 & - & $e_{0122111}-2 e_{1221111}-e_{1232110}-e_{1233210}$ & & \\
\hline & 8 & - & $e_{1232210}+e_{1233210}-e_{0121111}+e_{1122111}$ & & \\
\hline \multirow[t]{5}{*}{3} & 10 & - & $e_{1222221}-e_{1232211}+e_{1} \underset{2}{233211}+\underset{2}{2 e_{1243210}}$ & & \\
\hline & 10 & - & $e_{1232111}-e_{1343210}$ & & \\
\hline & 10 & - & $e_{1232111}+e_{2343210}$ & & \\
\hline & 14 & - & $e_{1244321}+e_{1343221}+e_{2343321}$ & & \\
\hline & 14 & - & $e_{1243321}+e_{2343221}+e_{1344321}$ & & \\
\hline 2 & 16 & - & $e_{2354321}-e_{1354321}$ & & \\
\hline & 16 & - & $e_{2354321}-e_{2454321}$ & & \\
\hline & 16 & - & $e_{1354321}+e_{2454321}$ & & \\
\hline \multirow[t]{5}{*}{1} & 2 & - & $e$ & $z_{2}$ & $z_{2}$ \\
\hline & 14 & - & $e_{1243221}+e_{1343321}+e_{2344321}$ & $z_{14}$ & $z_{14}$ \\
\hline & 18 & - & $e_{2464321}$ & $z_{18}^{1}$ & \\
\hline & 18 & - & $e_{2465321}$ & $z_{18}{ }^{2}$ & \\
\hline & 22 & - & $e_{2465432}$ & $z_{22}$ & $z_{22}$ \\
\hline
\end{tabular}


$E_{8}$, orbit $61: E_{7}\left(a_{1}\right)$

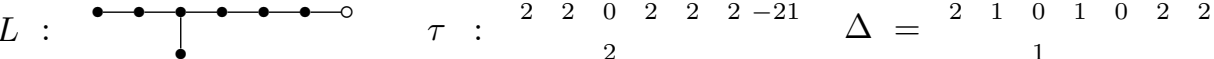

$$
\begin{aligned}
& e=\underset{0}{e} \frac{e_{0} 000000}{e 0100000}+\underset{0}{e 0110000}+e_{0}^{0010000}+\underset{0}{e 0001000}+e_{0}^{0000100}+e_{0}^{0000010} \\
& C^{\circ}=A_{1} \quad C / C^{\circ}=1 \\
& \beta_{1}={ }_{3}^{2465432}
\end{aligned}
$$

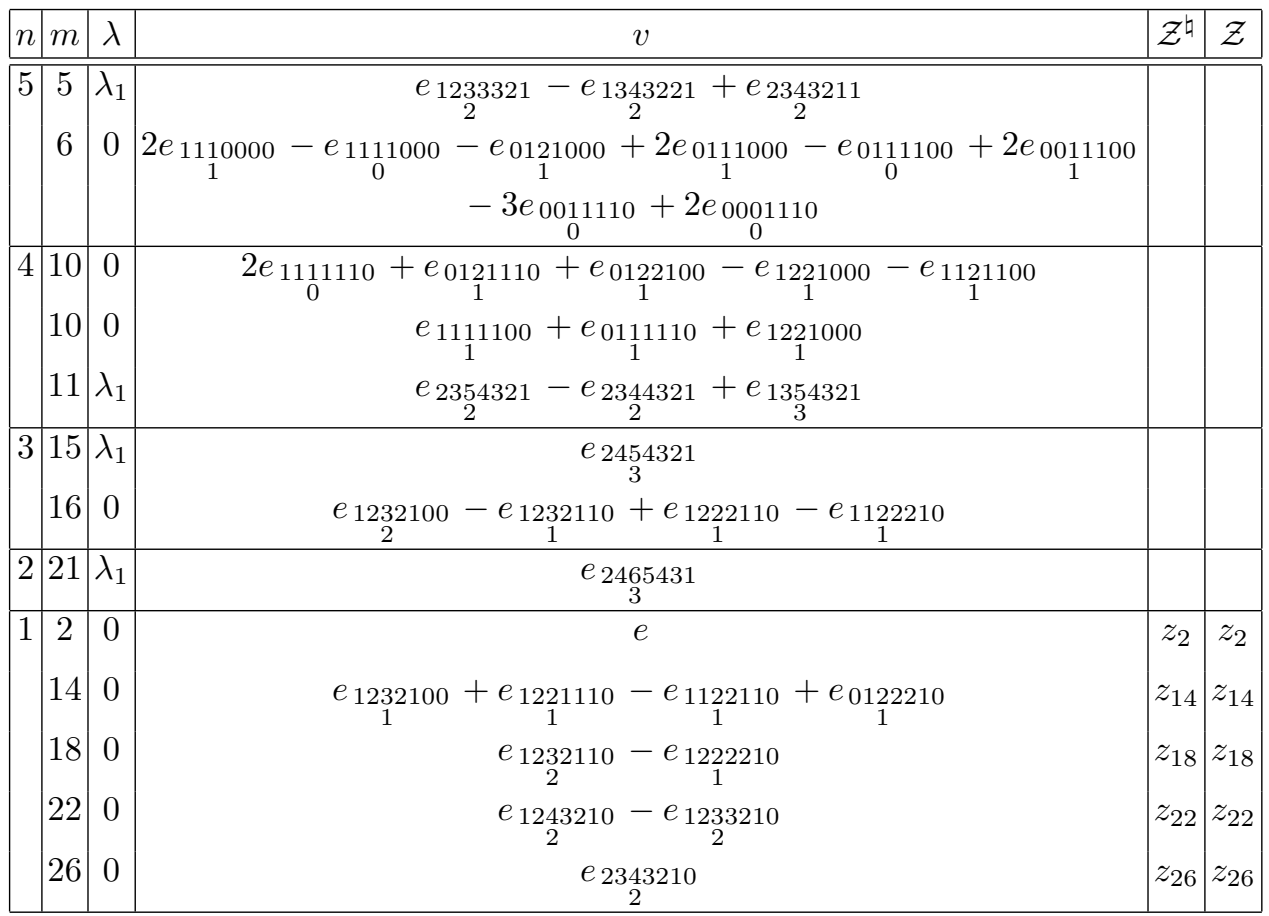


$E_{8}$, orbit $62: E_{8}\left(a_{5}\right)$

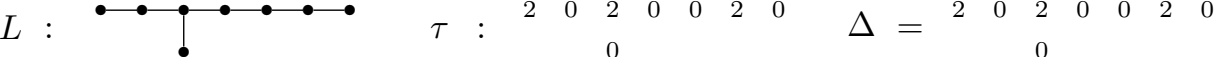

$$
\begin{aligned}
& e=e_{1}^{0011000}+e_{0}^{0000111}+e_{0}^{0111000}+e_{0} 000000+e_{0}^{0110000}+e_{0}^{0011100}+e_{0}^{0001110}+e_{0010000}^{00} \\
& +e_{0000010} \\
& C^{\circ}=1 \quad C / C^{\circ}=\left\langle c C^{\circ}\right\rangle \cong S_{2} \\
& c=h_{4}(-1) h_{7}(-1)
\end{aligned}
$$

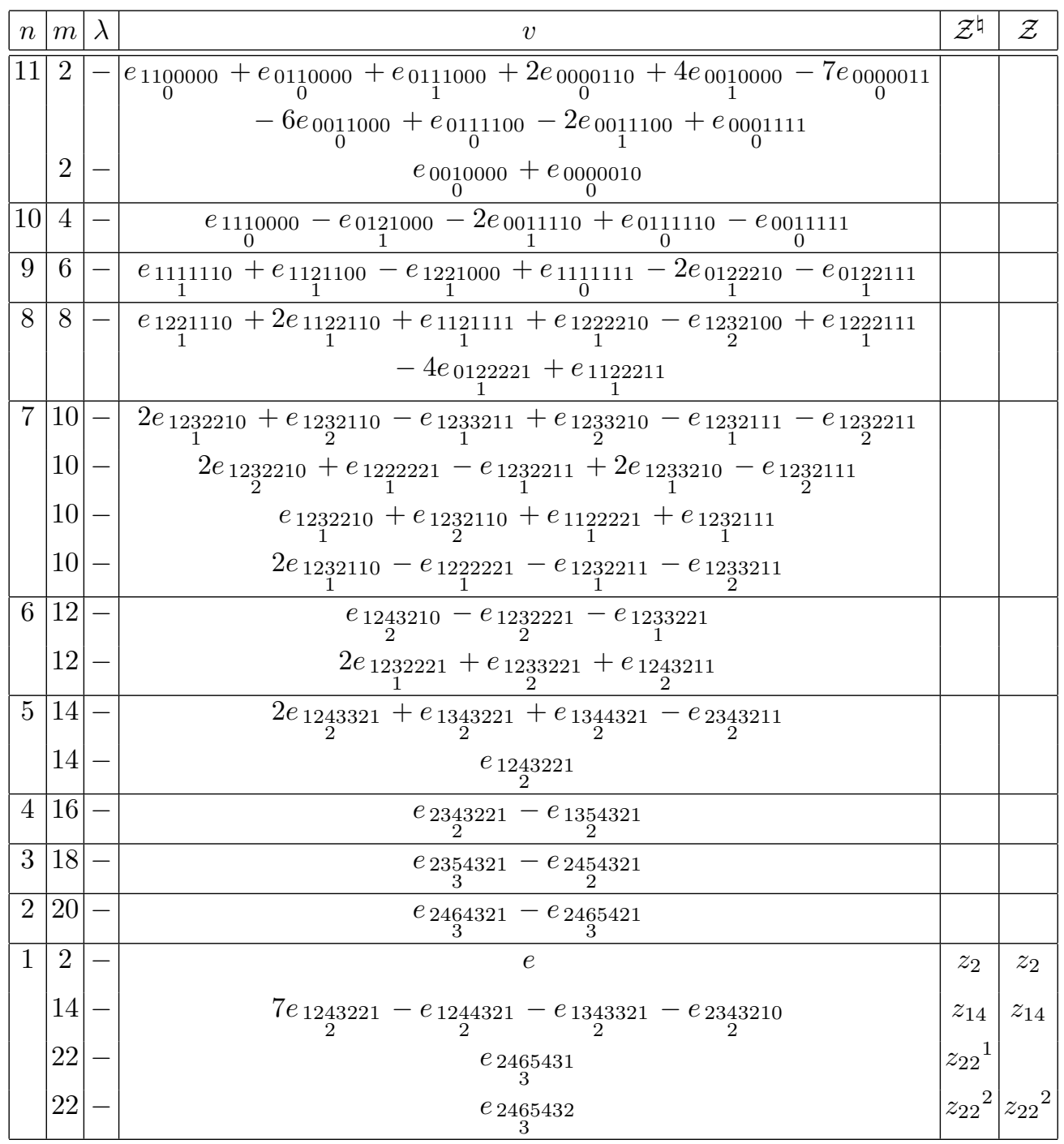


$E_{8}$, orbit $63: E_{8}\left(b_{4}\right)$

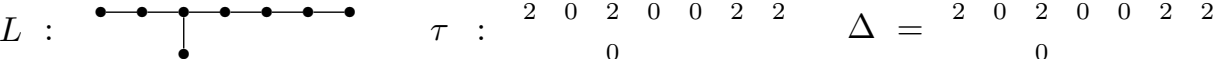

$$
\begin{aligned}
& e=\underset{0}{e 0000001}+e_{0}^{0000010}+e_{0}^{0000110}+e_{1}^{0011100}+e_{0}^{0111000}+e_{0}^{1000000}+e_{0110000}^{0}+e_{0}^{0010000} \\
& C^{\circ}=1 \quad C / C^{\circ}=\left\langle c C^{\circ}\right\rangle \cong S_{2} \\
& c=h_{4}(-1)
\end{aligned}
$$

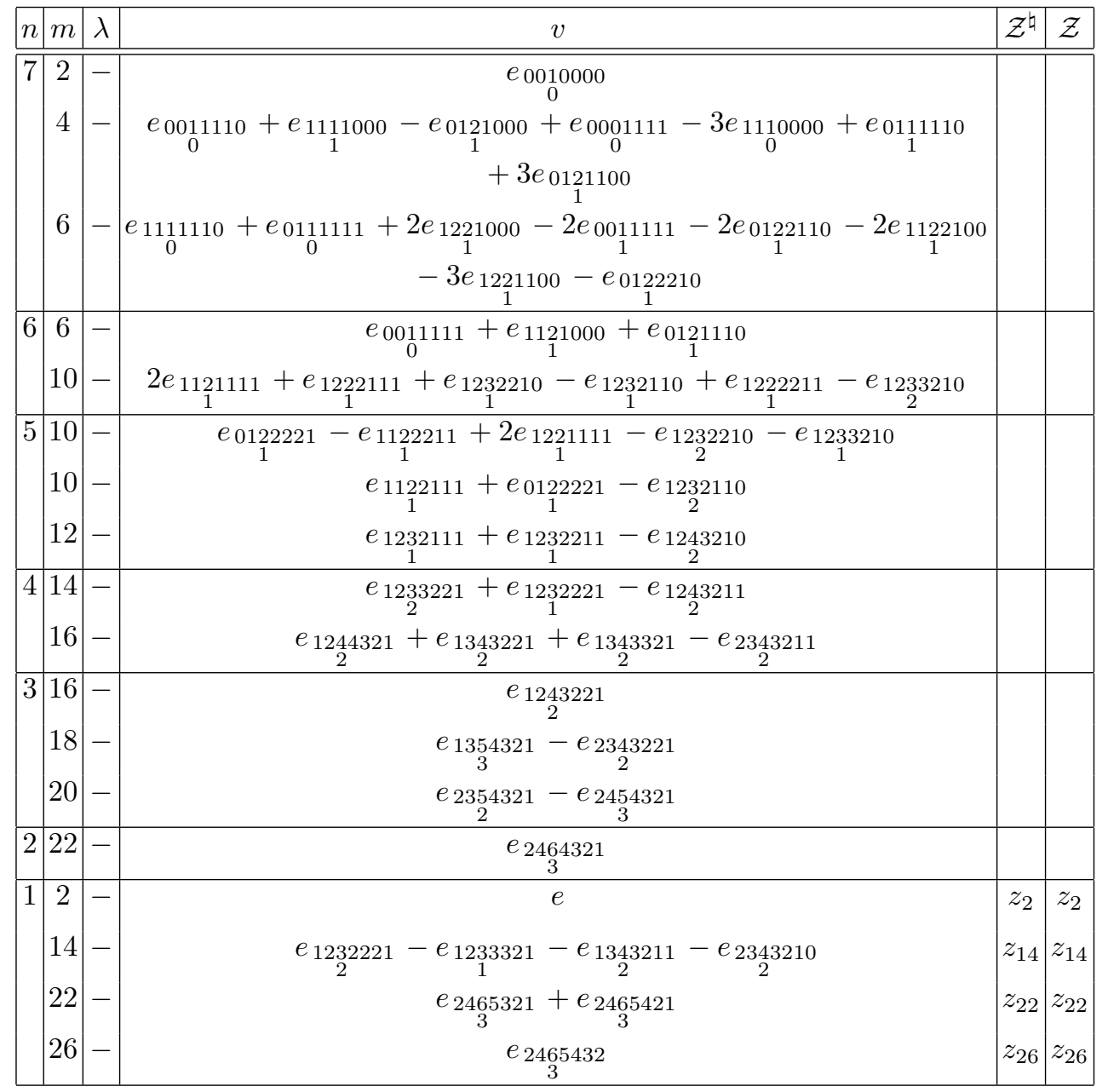


$E_{8}$, orbit $64: E_{7}$

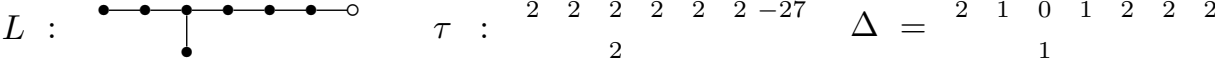

$$
\begin{aligned}
& e=\underset{0}{e} \underset{1}{1000000}+e_{0}^{0000000}+\underset{0}{e 100000}+\underset{0}{e 0010000}+\underset{0}{e 0001000}+e_{0}^{0000100}+e_{0}^{0000010} \\
& C^{\circ}=A_{1} \quad C / C^{\circ}=1 \\
& \beta_{1}={ }_{3}^{2465432}
\end{aligned}
$$

\begin{tabular}{|c|c|c|c|c|c|}
\hline$n$ & $m$ & $\lambda$ & $v$ & $\mathcal{Z}^{\natural}$ & $\mathcal{Z}$ \\
\hline 3 & 9 & $\bar{\lambda}$ & $e_{1244321}-e_{1343321}+e_{2343221}$ & & \\
\hline 2 & $\begin{array}{l}10 \\
17 \\
18\end{array}$ & $\begin{array}{c}0 \\
\lambda_{1} \\
0\end{array}$ & $\begin{array}{c}e_{1111000}+2 e_{1111100}-e_{0111100}-3 e_{0} 0011110 \\
1 \\
e_{2454321}-e_{2354321} \\
2 \\
e_{1221110}-e_{1122110}+e_{012211110}-e_{0121000} \\
1 \\
1\end{array}$ & & \\
\hline 1 & 2 & 0 & $e$ & $z_{2}$ & $z_{2}$ \\
\hline & 14 & 0 & $e_{1221000}+e_{1121100}+2 e_{1111110}-e_{0121110}-e_{0122100}$ & $z_{14}$ & $z_{14}$ \\
\hline & 22 & 0 & $e_{1232100}+e_{1232110}-e_{1222210}$ & $z_{22}$ & $z_{22}$ \\
\hline & 26 & 0 & $e_{1232210}-e_{1233210}$ & $z_{26}$ & $z_{26}$ \\
\hline & 27 & $\lambda_{1}$ & $e_{2465431}$ & & \\
\hline & 34 & 0 & $e_{2343210}$ & $z_{34}$ & $z_{34}$ \\
\hline
\end{tabular}


$E_{8}$, orbit $65: E_{8}\left(a_{4}\right)$

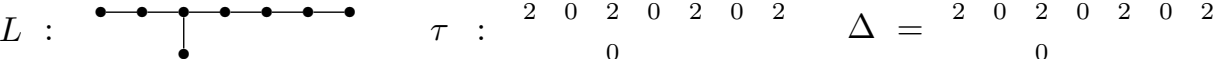

$$
\begin{aligned}
& e=e_{1}^{0011000}+e_{0}^{0000100}+\underset{0}{e 0111000}+e_{0} 0000000+e_{0}^{0110000}+e_{0}^{0001110}+e_{0}^{0010000}+e_{000001}^{0} \\
& C^{\circ}=1 \quad C / C^{\circ}=\left\langle c C^{\circ}\right\rangle \cong S_{2} \\
& c=h_{4}(-1) h_{8}(-1)
\end{aligned}
$$

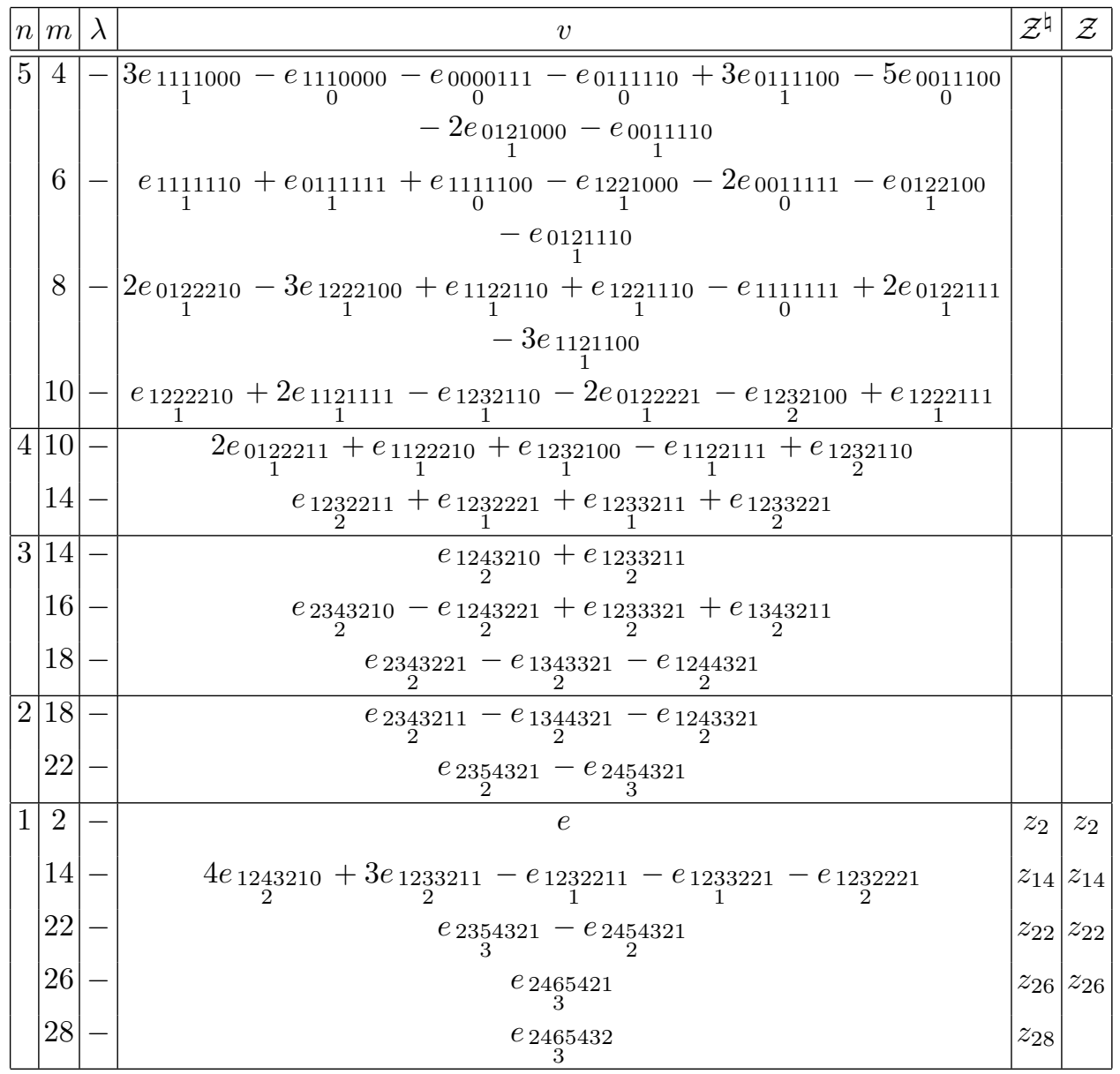


$E_{8}$, orbit $66: E_{8}\left(a_{3}\right)$

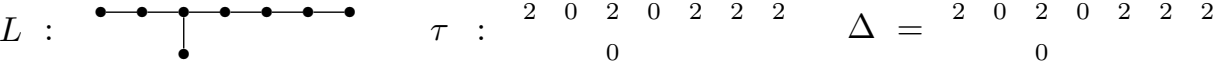

$$
\begin{aligned}
& e=\underset{0}{e 0000001}+e_{1}^{0011000}+e_{0}^{0000010}+e_{0}^{0000100}+e_{0}^{0111000}+e_{0}^{1000000}+e_{0110000}^{0}+e_{0}^{0010000} \\
& C^{\circ}=1 \quad C / C^{\circ}=\left\langle c C^{\circ}\right\rangle \cong S_{2} \\
& c=h_{4}(-1)
\end{aligned}
$$

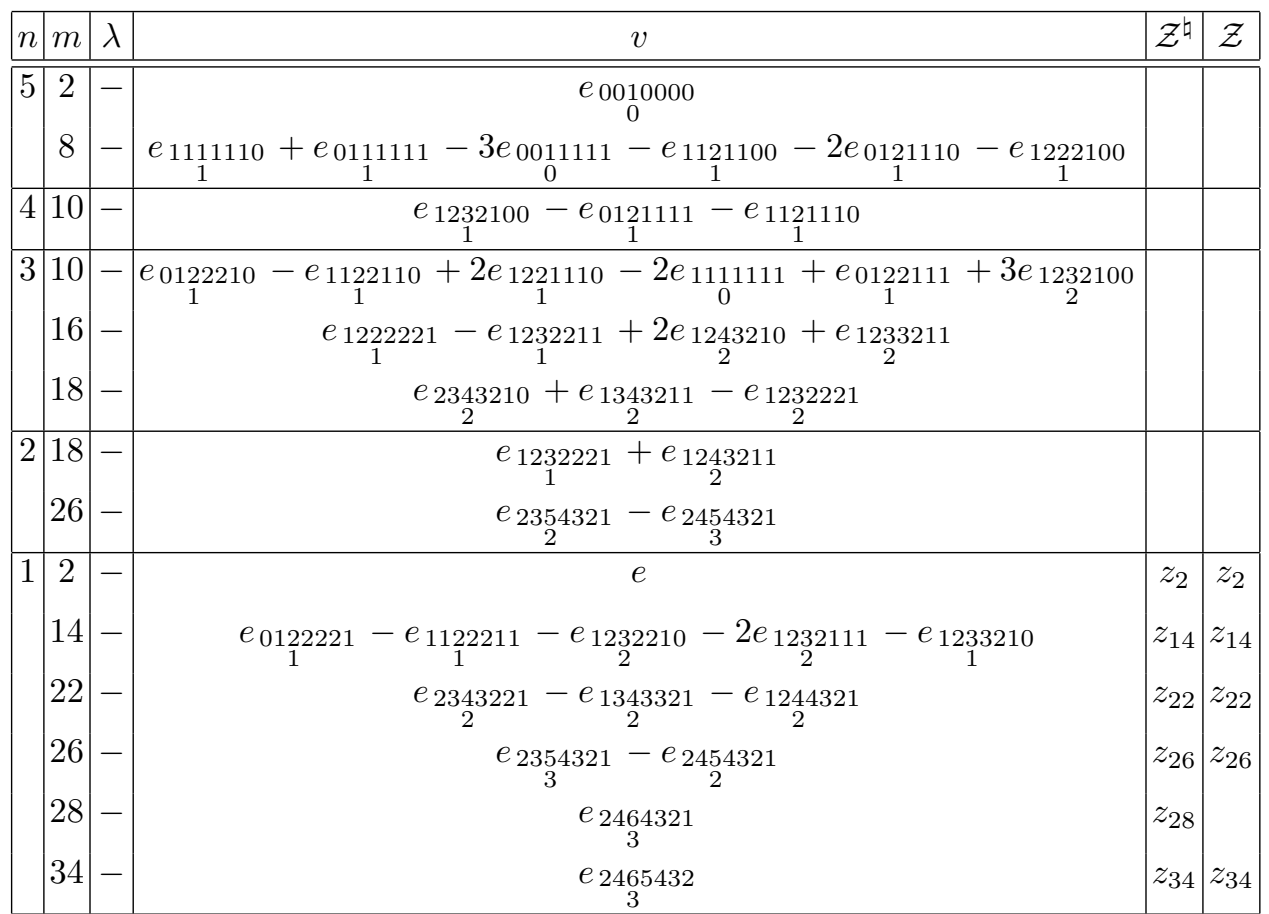


$E_{8}$, orbit $67: E_{8}\left(a_{2}\right)$

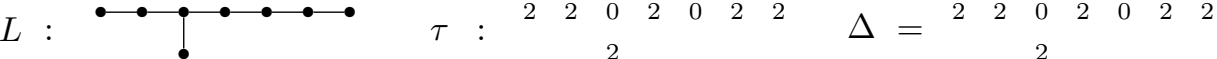

$$
\begin{aligned}
& e=\underset{0}{e} 1000000+e_{1}^{0000000}+e_{0}^{0100000}+e_{1}^{0010000}+e_{0}^{0011000}+e_{0}^{0001100}+e_{0}^{0000110}+e_{0}^{0000001} \\
& C^{\circ}=1 \quad C / C^{\circ}=1
\end{aligned}
$$

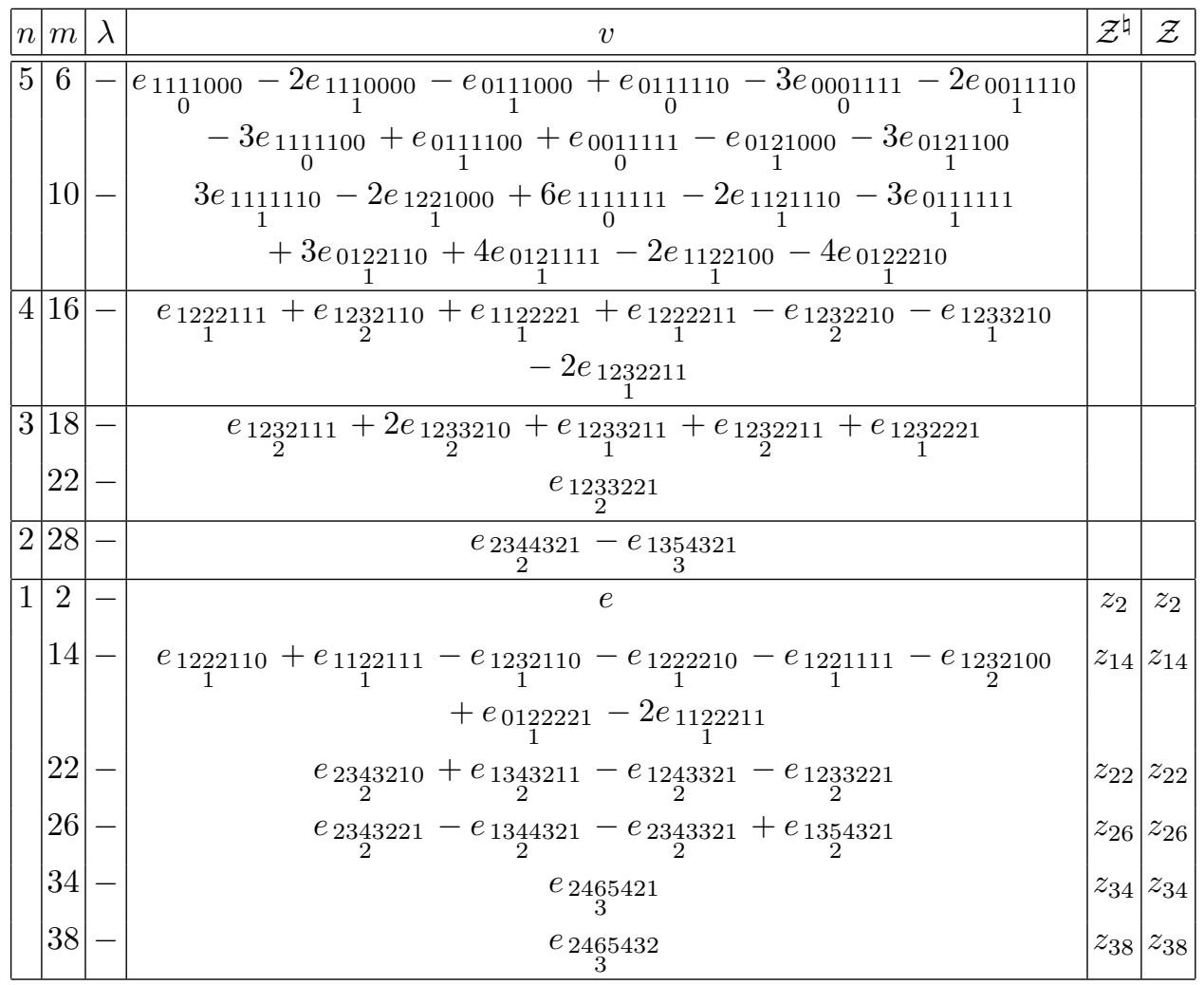

$E_{8}$, orbit $68: E_{8}\left(a_{1}\right)$

$$
\begin{aligned}
& L: \bullet \bullet \bullet \bullet \bullet \bullet \quad \tau: \begin{array}{lllllllllllllll}
2 & 2 & 0 & 2 & 2 & 2 & 2 & \Delta= \\
2 & 2 & 0 & 2 & 2 & 2 & 2
\end{array} \\
& e=e_{1000000}+e_{0000000}+e_{0010000}+e_{0110000}+e_{0001000}+e_{0000100}+e_{0000010}+e_{0000001} \\
& C^{\circ}=1 \quad C / C^{\circ}=1
\end{aligned}
$$




\begin{tabular}{|c|c|c|c|c|c|}
\hline$n$ & $m$ & $\lambda$ & $v$ & $\mathcal{Z}^{\natural}$ & $\mathcal{Z}$ \\
\hline 3 & 10 & - & 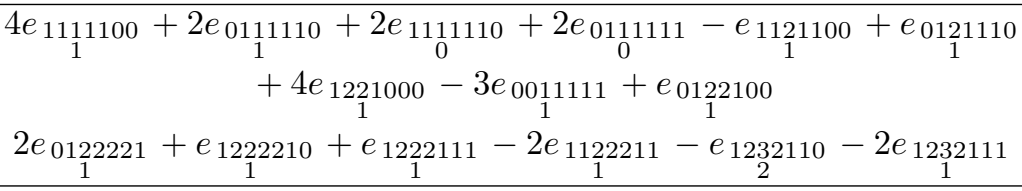 & & \\
\hline 2 & 28 & - & $e_{1343221}-e_{1233321}-e_{2343211}$ & & \\
\hline 1 & 14 & - & $\begin{array}{c}e \\
e \underset{1}{e} e_{0122210}-e_{1122110}-e_{1221110}-e_{1222100}+e_{1}^{0122111}-2 e_{1121111} \\
-e_{1111111}+e_{1232100}^{12321}\end{array}$ & $\begin{array}{l}z_{2} \\
z_{14}\end{array}$ & $z_{2}$ \\
\hline & 22 & - & $2 e_{1222221}-e_{1232221}+e_{1233211}-e_{1232211}-e_{1243210}$ & $z_{22}$ & $z_{22}$ \\
\hline & 26 & - & $e_{1233221}-e_{1233321}-e_{2}^{2343210}-e_{134}{ }_{2}{ }_{2}-e_{1243221}$ & $z_{26}$ & $z_{26}$ \\
\hline & 34 & - & $e_{1354321}-e_{2} 2354321$ & $z_{34}$ & $z_{34}$ \\
\hline & 38 & - & $e_{3}^{2454321}$ & $z_{38}$ & $z_{38}$ \\
\hline & 46 & - & $\begin{array}{l}e_{2465432} \\
3\end{array}$ & $z_{46}$ & $z_{46}$ \\
\hline
\end{tabular}

$E_{8}$, orbit 69: $E_{8}$
$L$ : $\tau:$ $\begin{array}{lllllll}2 & 2 & 2 & 2 & 2 & 2 & 2\end{array}$
$\Delta=$ $\begin{array}{lllllll}2 & 2 & 2 & 2 & 2 & 2 & 2\end{array}$
$e=e_{1000000}+e_{0000000}+e_{0100000}+e_{0010000}+e_{0001000}+e_{0000100}+$ $C^{\circ}=1 \quad C / C^{\circ}=1$

\begin{tabular}{|c|c|c|c|c|c|}
\hline$n$ & $m$ & $\lambda$ & $v$ & $\mathcal{Z}^{\natural}$ & $\mathcal{Z}$ \\
\hline 1 & 2 & - & $e$ & $z_{2}$ & $z_{2}$ \\
\hline & 14 & - & $\begin{array}{c}2 e_{1111110}-e_{0111111}+e_{1121100}-e_{0121110}+e_{1221000}+3 e_{1111111} \\
-e_{0122100}\end{array}$ & $z_{14}$ & $z_{14}$ \\
\hline & 22 & - & $e_{1222210}-e_{1232110}-2 e_{0122221}-e_{1232100}-e_{1222111}+2 e_{1122211}$ & $z_{22}$ & $z_{22}$ \\
\hline & 26 & - & $e_{1232210}+e_{1222221}-e_{1233210}-e_{1232211}+2 e_{1232111}$ & $z_{26}$ & $z_{26}$ \\
\hline & 34 & - & $e_{2343210}+e_{1343211}-e_{1243221}+e_{1233321}$ & $z_{34}$ & $z_{34}$ \\
\hline & 38 & - & $e_{2343221}-e_{1343321}+e_{1244321}$ & $z_{38}$ & $z_{3}$ \\
\hline & 46 & - & $e_{2354321}-e_{2454321}$ & $z_{46}$ & $z_{46}$ \\
\hline & 58 & - & $\begin{array}{l}e_{2465432} \\
3\end{array}$ & $z_{58}$ & $z_{5}$ \\
\hline
\end{tabular}




\section{References}

[1] P. Bala and R.W. Carter, "Classes of unipotent elements in simple algebraic groups, I", Math. Proc. Cambridge Philos. Soc. 79 (1976), 401-425.

[2] P. Bala and R.W. Carter, "Classes of unipotent elements in simple algebraic groups, II", Math. Proc. Cambridge Philos. Soc. 80 (1976), 1-18.

[3] A. Borel, Linear Algebraic Groups (second edition), Graduate Texts in Mathematics 126, Springer (1991).

[4] N. Bourbaki, Groupes et algèbres de Lie, IV, V, VI, Hermann, Paris (1968).

[5] R.W. Carter, Simple Groups of Lie Type, Wiley, London (1972).

[6] R.W. Carter, Finite Groups of Lie Type: Conjugacy Classes and Complex Characters, Wiley, Chichester (1985).

[7] B. Chang, "The conjugate classes of Chevalley groups of type $\left(G_{2}\right)$ ", J. Algebra 9 (1968), 190-211.

[8] C. Chevalley, "Sur certains groupes simples", Tohoku Math. J. 7 (1955), 14-66.

[9] C.W. Curtis, "Representations of Lie algebras of classical type with applications to linear groups", J. Math. Mech. 9 (1960), 307-326.

[10] E.B. Dynkin, "Semisimple subalgebras of semisimple Lie algebras", Selected Papers, Amer. Math. Soc., 175-308 (2000); corrections, 309-312.

[11] P.B. Gilkey and G.M. Seitz, "Some representations of exceptional Lie algebras", Geom. Dedicata 25 (1988), 407-416.

[12] J.E. Humphreys, Linear Algebraic Groups (corrected third printing), Graduate Texts in Mathematics 21, Springer (1987).

[13] J.E. Humphreys, Conjugacy Classes in Semisimple Algebraic Groups, Mathematical Surveys and Monographs 43, Amer. Math. Soc. (1995).

[14] J.C. Jantzen, Representations of Algebraic Groups (second edition), Mathematical Surveys and Monographs 107, Amer. Math. Soc. (2003).

[15] J.C. Jantzen, "Nilpotent orbits in representation theory", Part I of Lie Theory: Lie Algebras and Representations, Progress in Mathematics 228, Birkhäuser (2004).

[16] B. Kostant, "The principal three-dimensional subgroup and the Betti numbers of a complex simple Lie group", Amer. J. Math. 81 (1959), 973-1032.

[17] J.F. Kurtzke, "Centralizers of irregular elements in reductive algebraic groups", $P a-$ cific J. Math. 104 (1983), 133-154.

[18] R. Lawther, "Jordan block sizes of unipotent elements in exceptional algebraic groups", Comm. Algebra 23 (1995), 4125-4156; correction, Comm. Algebra 26 (1998), 2709.

[19] M.W. Liebeck and G.M. Seitz, Reductive Subgroups of Exceptional Algebraic Groups, Mem. Amer. Math. Soc. 580, 1996. 
[20] M.W. Liebeck and G.M. Seitz, The Maximal Subgroups of Positive Dimension in Exceptional Algebraic Groups, Mem. Amer. Math. Soc. 802, 2004.

[21] M.W. Liebeck and G.M. Seitz, Unipotent and nilpotent classes in algebraic groups and Lie algebras, preprint.

[22] F. Lübeck, "Small degree representations of finite Chevalley groups in defining characteristic", LMS J. Comput. Math. 4 (2001), 135-169.

[23] G.J. McNinch, "Sub-principal homomorphisms in positive characteristics", Math. Z. 244 (2003), 433-455.

[24] G.J. McNinch, "Optimal SL(2)-homomorphisms", Comment. Math. Helv. 80 (2005), $391-426$.

[25] G.J. McNinch and E. Sommers, "Component groups of unipotent centralizers in good characteristic", J. Algebra 260 (2003), 323-337.

[26] G.J. McNinch and D.M. Testerman, "Nilpotent centralizers and Springer's isomorphisms", preprint.

[27] K. Mizuno, "The conjugate classes of Chevalley groups of type $E_{6}$ ", J. Fac. Sci. Univ. Tokyo Sect. IA Math. 24 (1977), 525-563.

[28] K. Mizuno, "The conjugate classes of unipotent elements of the Chevalley groups $E_{7}$ and $E_{8} "$, Tokyo J. Math. 3 (1980), 391-461.

[29] K. Pommerening, "Über die unipotenten Klassen reduktiver Gruppen", J. Algebra 49 (1977), 525-536.

[30] K. Pommerening, "Über die unipotenten Klassen reduktiver Gruppen II", J. Algebra 65 (1980), 373-398.

[31] A. Premet, "Nilpotent orbits in good characteristic and the Kempf-Rousseau theory", J. Algebra 260 (2003), 338-366.

[32] A. Premet, "Special transverse slices and their enveloping algebras", Adv. Math. 170 (2002), 1-55.

[33] A. Premet, "Enveloping algebras of Slodowy slices and the Joseph ideal", J. Eur. Math. Soc. 9 (2007), 487-543.

[34] R. Proud, "Witt groups and unipotent elements in algebraic groups", Proc. London Math. Soc. 82 (2001), 647-675.

[35] R. Proud, "On centralizers of unipotent elements in algebraic groups", unpublished manuscript.

[36] R.W. Richardson, "Conjugacy classes in parabolic subgroups of semisimple algebraic groups", Bull. London Math. Soc. 6 (1974), 21-24.

[37] G.M. Seitz, "The maximal subgroups of classical algebraic groups", Mem. Amer. Math. Soc. 365, 1987.

[38] G.M. Seitz, "Unipotent elements, tilting modules, and saturation", Invent. Math. 141 (2000), 467-502. 
[39] G.M. Seitz, "Unipotent centralizers in algebraic groups", J. Algebra 279 (2004), $226-259$.

[40] K. Shinoda, "The conjugacy classes of Chevalley groups of type $\left(F_{4}\right)$ over finite fields of characteristic 2", J. Fac. Sci. Univ. Tokyo Sect. IA Math. 21 (1974), 133-159.

[41] T. Shoji, "The conjugacy classes of Chevalley groups of type $\left(F_{4}\right)$ over finite fields of characteristic $p \neq 2 "$, J. Fac. Sci. Univ. Tokyo Sect. IA Math. 21 (1974), 1-17.

[42] P. Slodowy, Simple singularities and simple algebraic groups, Lecture Notes in Mathematics 815, Springer (1980).

[43] E. Sommers, "A generalization of the Bala-Carter theorem for nilpotent orbits", Internat. Math. Res. Notices 11 (1998), 539-562.

[44] T.A. Springer, "A note on centralizers in semi-simple groups", Indag. Math. 28 (1966), $75-77$.

[45] T.A. Springer, "The unipotent variety of a semisimple group", Proc. Coll. in Algebraic Geometry (Tata Institute) (1969), 373-391.

[46] T.A. Springer, Linear Algebraic Groups (second edition), Birkhäuser, Boston (1998).

[47] R. Steinberg, Endomorphisms of Linear Algebraic Groups, Mem. Amer. Math. Soc. 80, 1968.

[48] U. Stuhler, "Unipotente und nilpotente Klassen in einfachen Gruppen und Lie algebren vom Typ $G_{2} "$, Indag. Math. 33 (1971), 365-378.

[49] D.M. Testerman, "A construction of certain maximal subgroups of the algebraic groups $E_{6}$ and $F_{4} "$, J. Algebra 122 (1989), 299-322.

[50] D.M. Testerman, " $A_{1}$-type overgroups of elements of order $p$ in semisimple algebraic groups and the associated finite groups", J. Algebra 177 (1995), 34-76.

[51] O. Yakimova, "Surprising properties of centralisers in classical Lie algebras", preprint. 